\title{
Estudio teórico de la reactividad y síntesis del fullereno $C_{60}$
}

Tesis para obtener el grado de Doctora en Ciencias (Química)

Martha Verónica Mojica Contreras

Francisco Méndez Ruiz

Profesor Titular $C$

Universidad Autónoma Metropolitana Iztapalapa
Julio Alfonso Alonso Martín Catedrático de Universidad Universidad de Valladolid 


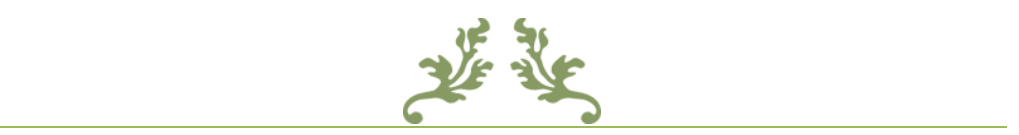

I DO NOT KNOW WHAT I MAY APPEAR TO THE WORLD; BUT TO MYSELF I SEEM TO HAVE BEEN ONLY LIKE A BOY

PLAYING ON THE SEASHORE, AND DIVERTING MYSELF IN NOW AND THEN FINDING A SMOOTHER PEBBLE OR A PRETTIER SHELL THAN ORDINARY, WHILST THE GREAT OCEAN OF TRUTH LAY ALL UNDISCOVERED BEFORE ME.

NEWTON (1642-1726) 


\section{Agradecimientos}

A mis asesores los Dr. Francisco Méndez Ruiz y Julio Alfonso Alonso Martín, por su apoyo y orientación constante.

A los Dr. Hugo Alejandro Jiménez Vázquez, Eduardo González Zamora, José Luis Gázquez Mateos y Nicola Batina, por tomarse el tiempo de leer y revisar mi trabajo a lo largo de su desarrollo y por sus invaluables comentarios y sugerencias que lo enriquecieron excepcionalmente.

A los Dr. María José López, Iván Cabria, Luis Miguel Molina y Víctor Manuel Rayon de la Universidad de Valladolid, por las extraordinarias charlas que mantuvimos durante mi estancia.

A mis padres por todo su apoyo y comprensión, no podría haberlo hecho sin ustedes.

A mi hermana Magda que me ayudo a escribir estos agradecimientos y a mi hermano Gus que me quitaba la computadora siempre que necesitaba trabajar. Mi vida sería demasiado aburrida sin ustedes.

A mi familia y amigos que me dan ánimos todo el tiempo, con ustedes he aprendido más de lo que podría imaginar.

A CONACYT por la beca nacional para estudios de posgrado (22893) asignada y por el apoyo derivado de la convocatoria de "Becas Mixtas 2012-2013".

A ti que estás leyendo esto.

Gracias 


\section{Índice}

Resumen 3

Introducción 4

Capítulo 1. Fullerenos: estructura y propiedades 6

1.1. Descubrimiento de los fullerenos 6

1.2. Estructura de los fullerenos 8

1.3. Propiedades químicas 9

1.4. Propiedades físicas 11

Capítulo 2. Síntesis de fullereno 13

2.1. Síntesis de fullerenos mediante vaporización de una fuente de carbono 13

2.2. Modelos que explican la síntesis de fullerenos 14

2.3. Síntesis química 18

2.4. Otros métodos 28

Capítulo 3. Nuevas estrategias de síntesis 32

3.1. Reacciones de Diels-Alder 33

3.2. Objetivos 38

$\begin{array}{ll}\text { 3.2.1. Generales } & 38\end{array}$

$\begin{array}{ll}\text { 3.2.2. Específicos } & 39\end{array}$

3.3. Metodología 39

Capítulo 4. Resultados y discusión 43

4.1. Primera parte: Análisis de la reacción de Diels-Alder entre los fragmentos 1 y 2 y etileno o butadieno. 43

4.1.1. Análisis de orbitales frontera 43

4.1.2. Parámetros cinéticos 45

4.1.3. Parámetros termodinámicos 48

4.1.4. Aromatización 49

4.1.5. Conclusiones de la primera parte 51

4.2. Segunda parte: Análisis de la reacción de Diels-Alder entre los 
fragmentos 1 y 2 substituidos y butadieno

4.2.1. Análisis de orbitales frontera

4.2.2. Parámetros cinéticos $\quad 56$

4.2.3. Parámetros termodinámicos 66

4.2.4. Aromatización 68

4.2.5. Conclusiones de la segunda parte $\quad 70$

4.3. Tercera parte: Análisis de la dimerización del fragmento 2

4.3.1. Conclusiones de la tercera parte 74

Capítulo 5: Reactividad química $\quad 75$

5.1. Criterios de reactividad basados en la teoría de funcionales de la $\begin{array}{ll}\text { densidad } & 75\end{array}$

5.2. Parámetros locales

5.3. Energía de interacción 92

Capítulo 6. Conclusiones y perspectivas 99

6.1.Conclusiones 99

6.2. Perspectivas 102

$\begin{array}{ll}\text { Referencias } & 103\end{array}$

Apéndice A. Cálculo de los parámetros termodinámicos 113

$\begin{array}{ll}\text { Anexo 1.Orbitales frontera } & 119\end{array}$

Anexo 2. Densidades orbitálicas, funciones de Fukui y descriptor dual 144

Anexo 3.Parámetros de reactividad global y funciones de Fukui condensadas 193 


\section{Resumen}

Con el propósito de analizar la factibilidad de la síntesis de fullerenos por dimerización de dos fragmentos $\mathrm{C}_{30} \mathrm{H}_{n}$, mediada por reacciones de Diels-Alder, se analizaron teóricamente las reacciones de Diels-Alder de los fragmentos triindenotrifenileno 1 y pentaciclopentacoranuleno 2 con etileno y butadieno. En este primer paso se observó que los fragmentos prefieren actuar como dienófilos y que la reacción del fragmento 2 con butadieno es la más favorecida.

Posteriormente se analizaron las reacciones de Diels-Alder entre el butadieno y los fragmentos 1 y 2 sustituidos con grupos electrodonadores y electroatractores. Se observó que las reacciones con los fragmentos sustituidos con grupos electroatractores son favorecidas cinética y termodinámicamente; sin embargo, los efectos estereoelectrónicos debidos a la presencia de los grupos sustituyentes conducen a que la reducción de la energía de activación de las reacciones propuestas no sea significativa.

Finalmente, analizamos dos de las probables rutas para la dimerización del fragmento 2 para dar $C_{60}$. Observamos que la ruta por pasos, en la que se forma de un primer cicloaducto por medio de una reacción de Diels-Alder seguida del cierre de la molécula mediante la formación de los 8 enlaces restantes, es más favorable cinéticamente que la ruta concertada en un solo paso, en la que se forman todos los enlaces necesarios a la vez.

Adicionalmente, analizamos los criterios de reactividad química basados en la teoría de funcionales de la densidad como son el potencial químico, la dureza, la blandura, la electrofilia y las funciones de Fukui de los fragmentos 1 y 2 mono- y disustituidos y sin sustituir. Calculamos también la energía de interacción para las reacciones entre los fragmentos y el butadieno. Los parámetros de reactividad obtenidos concuerdan con los parámetros cinéticos y termodinámicos calculados. 


\section{Introducción}

A partir del descubrimiento del $\mathrm{C}_{60}$ en 1985 por Kroto y colaboradores, ${ }^{1}$ los fullerenos se han convertido en una de las moléculas más estudiadas de la actualidad. Gracias a su capacidad de agregación ${ }^{2}$, reactividad ${ }^{3}$ y propiedades electrónicas, ${ }^{4}$ los fullerenos han sido empleados en una gran variedad de aplicaciones entre las que resaltan su empleo en nanomedicina, como transportadores de medicamentos ${ }^{5}$ o antioxidantes, ${ }^{6}$ en el campo de la nanotecnología y desarrollo de nuevos materiales empleándolos como nanosensores ${ }^{7}$ y componentes para celdas solares. ${ }^{8}$ Por ejemplo, la incorporación de fullerenos en polímeros permite la obtención de polímeros electroactivos, ${ }^{9}$ en los que las propiedades del fullereno se conservan y nuevas e interesantes propiedades surgen de la interacción entre ambas especies. ${ }^{10}$

En el diseño y desarrollo de estas aplicaciones así como de nuevos materiales y tecnologías basadas en fullerenos es indispensable el acceso a grandes cantidades de fullerenos isoméricamente puros; sin embargo los métodos tradicionales, además de dar lugar a una gran variedad de productos entre los que se encuentran los fullerenos con rendimientos muy bajos, son muy costosos, como lo demostraron Anctil y colaboradores, ${ }^{11}$ quienes analizaron el proceso de síntesis de fullerenos y cuantificaron las materias primas y la energía totales necesarias para su producción. En dicho análisis, observaron que el balance de materia y energía empleadas en la síntesis y la purificación de un kilogramo de $\mathrm{C}_{60}$ alcanzaba los $106.9 \mathrm{GJ} / \mathrm{kg}$, que es mayor a la energía eléctrica usada por una familia durante un año. ${ }^{12}$

Este gasto energético tan grande hace que los precios de los fullerenos sean elevados y aunque en la actualidad los precios son menores que en 1990 cuando estuvieron disponibles por primera vez en cantidades manejables, estos no descienden de los $\$ 7,660.00$ pesos por gramo de $C_{60}$ (más de 7 millones de pesos por $\mathrm{kg}$ de $\mathrm{C}_{60}$ sublimado, $99.9 \%$ pureza), $\$ 6,790.00$ pesos por $250 \mathrm{mg}$ de $\mathrm{C}_{70}$ (sublimado, pureza $\geq 99 \%$ ) y en el caso de fullerenos mayores como $C_{84}$ el precio es de $\$ 20,990.00$ pesos los $5 \mathrm{mg}$ (98\% pureza) mientras que el precio de $\mathrm{C}_{76}$ y $\mathrm{C}_{78}$ alcanza los $\$ 28,080.000$ pesos por cada $5 \mathrm{mg}\left(98 \%\right.$ pureza). ${ }^{13}$

La necesidad de nuevos métodos de síntesis que conduzcan a la formación de fullerenos isoméricamente puros, con rendimientos altos y fabricados empleando los principios fundamentales de la química orgánica nos llevó a proponer una nueva ruta de síntesis basada en la dimerización de dos hemifullerenos, mitades isométricas del $\mathrm{C}_{60}$. Los hemifullerenos 
triindenotrifenileno $\mathbf{1}$ y pentaciclopentaoranuleno $\mathbf{2}$ fueron escogidos como precursores. Analizando la forma en que estos fragmentos se aproximan para formar el $C_{60}$ observamos la formación de nuevos enlaces de 6 miembros (Figura 1), por lo que propusimos una dimerización mediada por reacciones de cicloadición de Diels-Alder.

Como primer paso analizamos la posibilidad de emplear la reacción de cicloadición de Diels-Alder sobre los fragmentos propuestos. Las reacciones entre los fragmentos propuestos y etileno o butadieno como complemento con el fin de aumentar el tamaño del fragmento, nos indicarán la reactividad de los fragmentos frente a las cicloadiciones propuestas. Posteriormente, el empleo de sustituyentes sobre los fragmentos $\mathbf{1}$ y $\mathbf{2}$ demostrarán los efectos que distintos tipos de sustituyentes (electroatractores y electrodonadores) tienen sobre la red aromática del fragmento y si estos facilitan o no las reacciones propuestas. Finalmente, el estudio de la dimerización de los fragmentos más favorecidos mediante reacciones de cicloadición de Diels-Alder se llevará a cabo, analizando todas las etapas posibles. Adicionalmente, los parámetros de reactividad derivados de la teoría del funcional de la densidad nos ayudarán a comprender el comportamiento de los fragmentos, tanto aislados como en las reacciones propuestas.

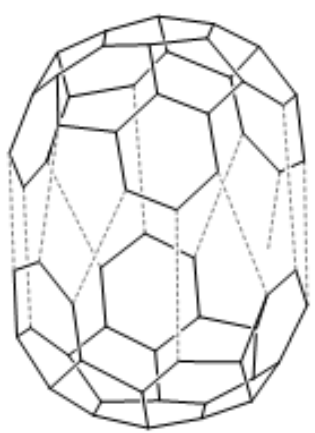

$1+1$

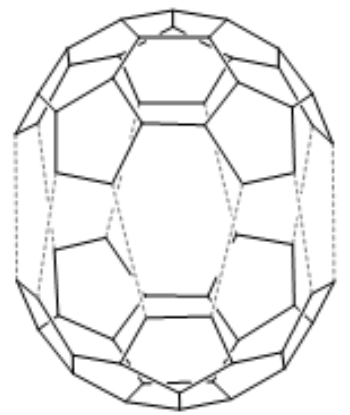

$2+2$
Figura 1. Dimerización de dos fragmentos de tipo $1(\mathbf{1 + 1})$ y dos fragmentos de tipo 2 (2+2). Se puede observar la formación de nuevos anillos de 6 miembros.

La presente tesis se organiza de la siguiente manera: en el primer capítulo resumimos las bases de nuestro objeto de estudio, es decir, los fullerenos; revisamos su descubrimiento, estructura y propiedades. En el segundo capítulo analizamos los métodos de síntesis tradicional y química más importantes, que nos condujeron al desarrollo de un nuevo método que expondremos en el capítulo 3. En el capítulo 4 exponemos resultados obtenidos del análisis cinético y termodinámico de las reacciones entre etileno, butadieno y los fragmentos 1 y $\mathbf{2}$ mono- y disustituidos y $\sin$ sustituir. En el capítulo 5 revisamos los parámetros de reactividad y en el 6 exponemos las conclusiones y perspectivas derivadas del presente trabajo. 


\section{Capítulo 1. Fullerenos: Estructura y propiedades}

\subsection{Descubrimiento de los fullerenos}

Con la intención de simular las reacciones químicas que tienen lugar en la atmósfera de una estrella de carbono ${ }^{14}$ y que conducen a la producción de cadenas largas de carbono, Kroto, Curl y Smalley vaporizaron grafito por irradiación laser. Como resultado observaron la formación de un agregado especialmente estable consistente de 60 átomos de carbono, identificado por espectrometría de masas (Figura 1.1). ${ }^{15}$

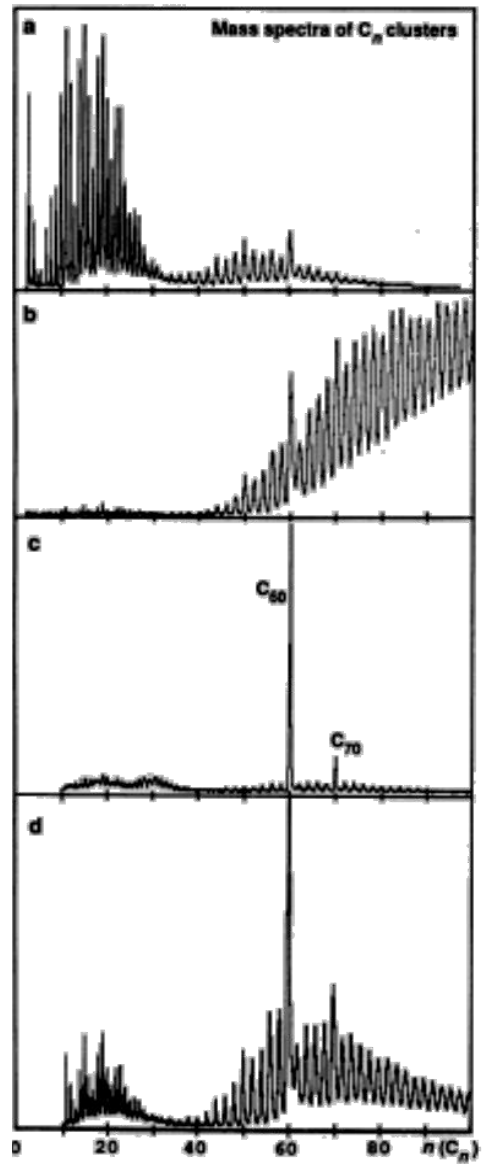

Propusieron que esta nueva especie súper-estable presentaba la estructura de un icosaedro truncado con los átomos de carbono en los vértices del poliedro, satisfaciendo todas sus valencias al formar dos enlaces sencillos y uno doble (Figura 1.2). Este agregado de estructura similar a un balón de futbol y aparentemente aromático, dominaba la distribución de agregados observados en los espectros; de hecho, más del $50 \%$ en abundancia se debía al $\mathrm{C}_{60}$.

Figura 1.1. Espectro de masas de agregados de carbono $C_{n}$. a) Agregación moderada: se observan cadenas poliínicas $(n<36)$ y fullerenos $(n>20)$. b) Agregación significante: se detectan solamente agregados con número de carbonos par. c) Agregación extensiva: $C_{60}$ y $C_{70}$ parecen predominar. d) Agregación similar a (c) pero con el láser aumentado 100 veces: fragmentación extensiva de agregados grandes en fullerenos $y$ cadenas precursoras. Tomado de la referencia 15 
Sugirieron el nombre de Bukminsterfullereno para esta estructura en honor al arquitecto Bukminster Fuller, diseñador de los domos geodésicos que inspiraron a Kroto y col. en la determinación de la estructura del $\mathrm{C}_{60}$, y consideraron que la familia de estructuras de "caja cerrada" o esféricas bien podrían llamarse fullerenos.

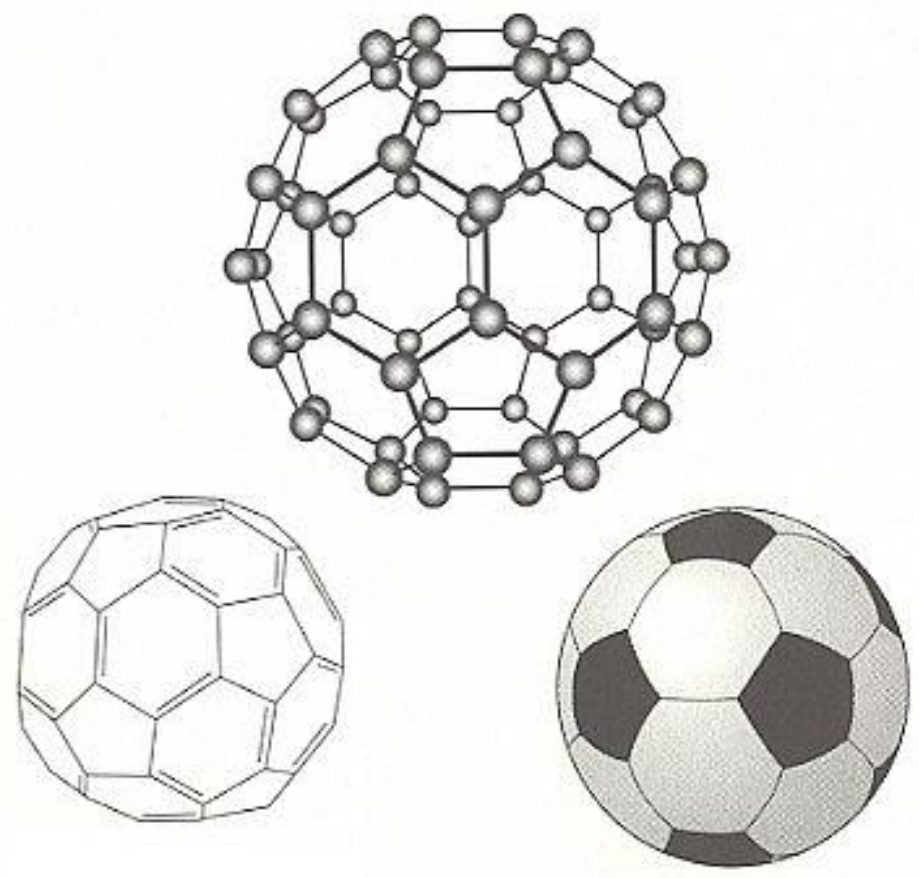

Figura 1.2. Estructura del $C_{60}$ y comparación con un balón de fútbol.

Dos eventos específicos dieron pie a la estructura de caja cerrada del $\mathrm{C}_{60}$, en el primero, el análisis de los espectros de masas condujo a la observación de que ciertos agregados con determinado número de átomos de carbono, llamados "números mágicos" eran los más estables. Estos números solo tenían sentido si las estructuras eran cajas cerradas. El segundo fue la encapsulación de otros átomos dentro del $\mathrm{C}_{60}$, primero La $\left(\mathrm{La}_{0} @ \mathrm{C}_{60}\right)^{16}$ y después $\mathrm{He}\left(\mathrm{He} @ \mathrm{C}_{60}\right)^{17}$, esto indicaba que la estructura de $\mathrm{C}_{60}$ era una caja cerrada que podía contener a otros átomos en su interior. La confirmación de dicha estructura se obtuvo con el análisis cristalográfico por rayos $\mathrm{X}^{18}$ las estructuras cristalinas observadas confirman el arreglo de caja cerrada o balón de futbol de los átomos en $\mathrm{C}_{60}$. 


\subsection{Estructura de los fullerenos}

Los fullerenos están constituidos por un número par de átomos de carbono con hibridación $s p^{2}$ que se ordenan formando una caja cerrada constituida por 12 anillos pentagonales y $m$ anillos hexagonales, donde $m=(n-20) / 2$ y $n$ es el número de átomos de carbono en la molécula. El $C_{60}$ (fullereno $\left.\left(C_{60} / h\right)[5,6]^{19}\right)$, es el fullereno más pequeño que obedece la regla del pentágono aislado ${ }^{20}$ que dice que los anillos pentagonales deben estar separados entre sí por hexágonos para reducir la inestabilidad inherente a los pentágonos fusionados. Los átomos de carbono en el $\mathrm{C}_{60}$ forman un icosaedro truncado, donde cada átomo se coloca en un vértice del poliedro. Este arreglo geométrico formado por 12 pentágonos y 20 hexágonos $(m=20, n=60)$ hace que todos los átomos de carbono en $\mathrm{C}_{60}$ sean equivalentes.

La determinación estructural mediante rayos $\mathrm{X}$ del $\mathrm{C}_{60}$ y algunos de sus derivados, revelan la existencia de dos tipos diferentes de enlaces, indicados en la Figura 1.3. Los enlaces 6-6 conectan átomos comunes a dos hexágonos adyacentes y tienen una longitud de $1.38 \AA$, mientras que los enlaces 5-6 conectan átomos comunes a un par pentágono-hexágono con una longitud de $1.45 \AA$. Los anillos pentagonales en la estructura dan lugar a la curvatura, que es necesaria para el cierre de la molécula. Dicha curvatura en la estructura de los fullerenos ocasiona que los tres enlaces entre cualquier átomo de carbono presente en la molécula y sus tres átomos vecinos se encuentren fuera del plano. ${ }^{21}$ Esta piramidalización modifica la hibridación de los átomos de carbono, de una hibridación $s p^{2}$ pura característica en el grafeno, a una hibridación intermedia entre $s p^{2}$ y $s p^{3}$. El resultado es una ganancia en el carácter $p$ que permite que los lóbulos $p$ se extiendan más sobre la superficie exterior que en el interior de la esfera; adicionalmente, los orbitales $\pi$ ganan cierto carácter $s .^{22}$ Estos cambios ejercen un efecto muy importante en un gran número de propiedades, contribuyendo por ejemplo, a la alta afinidad electrónica de la molécula. La piramidalización también es responsable del incremento en la reactividad química de los fullerenos frente a reacciones de adición, en comparación con la reactividad típica de una lámina plana de grafeno. ${ }^{23}$

Figura 1.3. Tipos de enlaces (6-6 y 5-6) presentes en $C_{60}$.

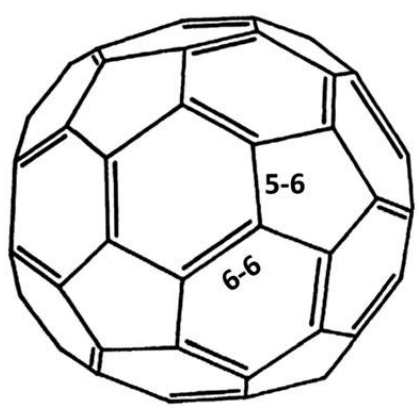




\subsection{Propiedades químicas}

En el $\mathrm{C}_{60}$ cada átomo de carbono aporta 3 orbitales híbridos y 3 electrones para formar 3 enlaces $\sigma$ localizados; el cuarto electrón aportado se encuentra en un orbital $\rho$, ortogonal a los orbitales $\sigma$ y forma un enlace $\pi$. Se calcula que existen 12500 estructuras de resonancia, ${ }^{24}$ de las cuales las principales se observan en la Figura 1.4.

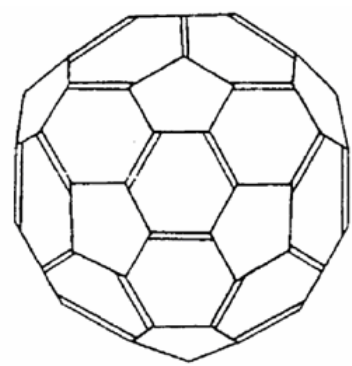

1a

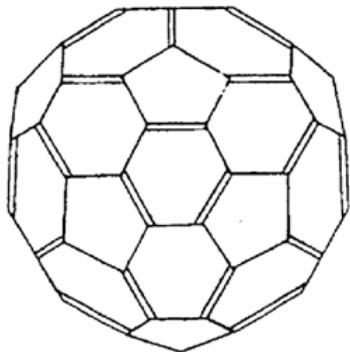

$1 b$

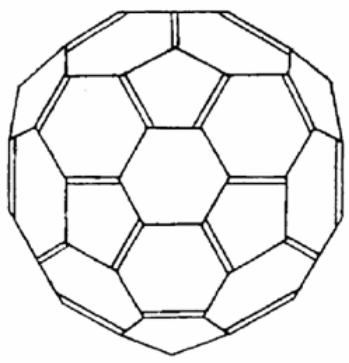

1c

Figura 1.4. Principales estructuras de resonancia del $C_{60}$. Tomado de la referencia 24

La estructura 1a es la que más contribuye y es la única estructura que no presenta dobles enlaces en los anillos de 5 miembros; los dobles enlaces se localizan en los anillos de seis miembros. ${ }^{25}$ Esto provoca que la deslocalización de los electrones sea pobre, lo que la transforma en una molécula muy reactiva, poco aromática, que se comporta como una poliolefina deficiente en electrones (con carácter fuertemente electroatractor), por lo que experimenta una gran variedad de reacciones químicas (Figura 1.5). ${ }^{26}$

Debido a su naturaleza deficiente en electrones, las principales reacciones que sufren los fullerenos son las de adición nucleofílica. Los fullerenos son excelentes dienófilos en reacciones de Diels-Alder, por lo que son ampliamente utilizadas en su funcionalización. Pueden reaccionar con especies oxigenadas y grupos arilo para formar epóxidos y sus análogos de carbono (fulleroides), también reaccionan con metales formando compuestos de coordinación $\eta 2$. Son especialmente reactivos frente a radicales, sufren polimerizaciones formando largas cadenas y en presencia de luz UV sufren coalescencia y pueden formar nanobalas o nanotubos. ${ }^{27}$ Solo los aniones de fullerenos o los fullerenos sustituidos son reactivos frente a electrófilos. 


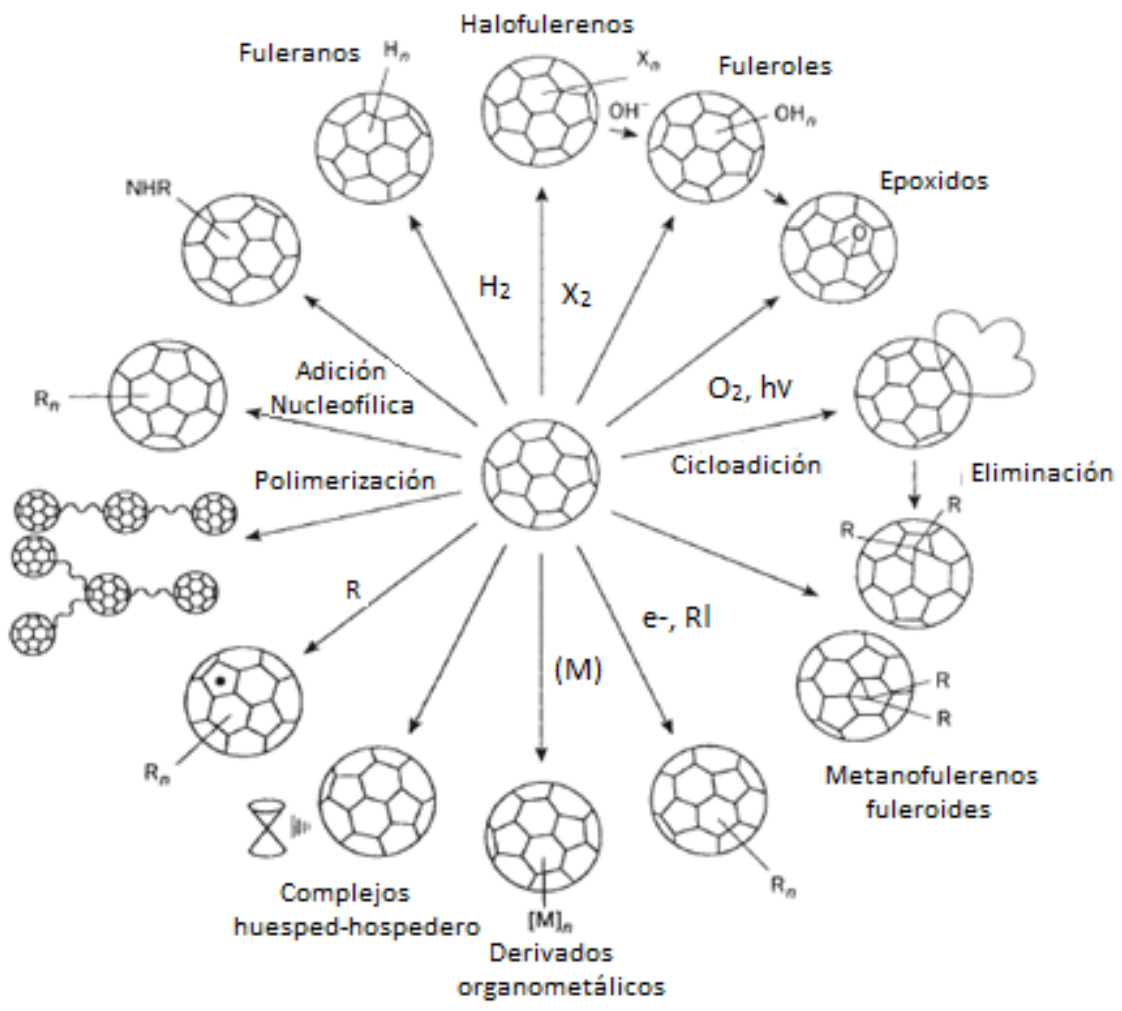

Figura 1.5. Diagrama de las principales reacciones de los fullerenos. Tomado de la referencia 26.

La reducción de fullerenos para formar hidruros se ha sugerido como un medio para almacenamiento de hidrógeno. En principio, debería ser posible el almacenamiento de 30 moles de $\mathrm{H}_{2}$ por mol de $\mathrm{C}_{60}$; sin embargo, solo se han obtenido especies con hasta 36 átomos de $\mathrm{H}$ unidos a la red de carbonos $\left(\mathrm{C}_{60} \mathrm{H}_{18}, \mathrm{C}_{60} \mathrm{H}_{28}, \mathrm{C}_{60} \mathrm{H}_{32}, \mathrm{C}_{60} \mathrm{H}_{36}\right)$ mediante la reacción de reducción de Birch ${ }^{28}$. El análisis de la reducción de $\mathrm{C}_{70}$ y su producto mayoritario $\mathrm{C}_{70} \mathrm{H}_{36}$ indica que la reacción se lleva a cabo sobre los anillos de 5 miembros (enlaces 6-5) por lo que el número máximo de átomos de $\mathrm{H}$ enlazados es igual en ambos fullerenos. De encontrarse una forma fácil de remover reversiblemente el hidrógeno de estos compuestos, se obtendría un método seguro de almacenamiento de hidrógeno como combustible.

La mayoría de las reacciones se llevan a cabo sobre los enlaces 6-6 que poseen mayor densidad electrónica; pueden formarse estructuras abiertas, anillos de 3 miembros que pueden conducir a la inserción de átomos de carbono o nitrógeno sobre los enlaces 6-5, anillos de 4 miembros, así como anillos de 5 y 6 miembros (Figura 1.6). ${ }^{29}$ Las estructuras abiertas se producen principalmente mediante adiciones $1,2{ }^{30}$ sin embargo, se han observado adiciones 1,4 en estructuras con impedimento estérico importante. La formación de anillos de 3 miembros usualmente involucra 
reacciones 1,3-dipolares seguidas del reordenamiento de los aductos obtenidos sobre los enlaces $6-5 .{ }^{31}$ Los derivados de ciclobuteno se obtienen mediante cicloadiciones $[2+2],{ }^{32}$ mientras que para la síntesis de los anillos de 5 miembros se emplean cicloadiciones [3+2] que pueden dar lugar a derivados cíclicos ${ }^{33}$ o heterocíclicos. ${ }^{34}$ Los anillos de 6 miembros se obtienen mediante cicloadiciones [4+2] sobre los enlaces 6-6. ${ }^{35}$ Adicionalmente, se pueden producir agujeros en la superficie de los fullerenos mediante adición selectiva de azidas, ${ }^{36}$ tratamiento térmico o fotooxidación. ${ }^{37}$
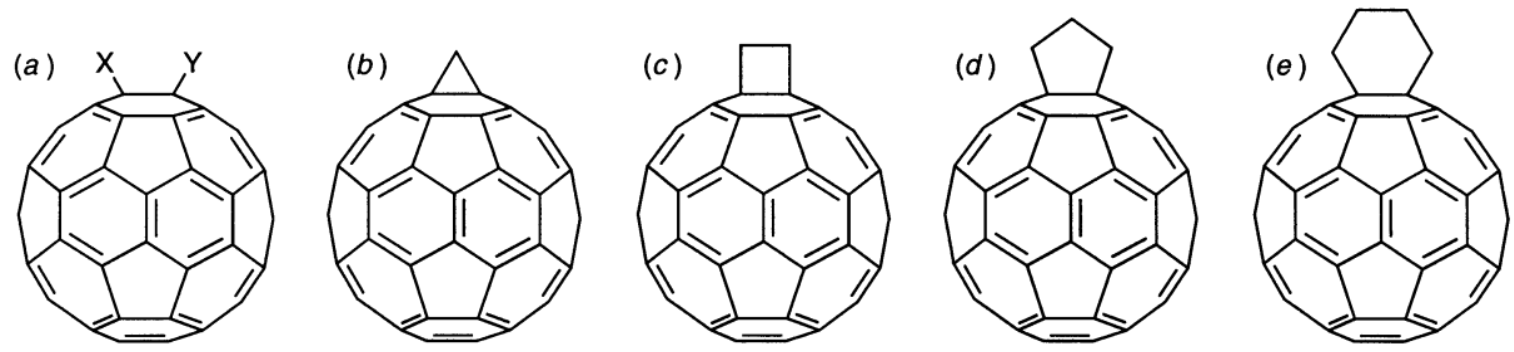

Figura 1.6. Productos de adición sobre un enlace 6-6. (a) Estructura abierta, (b) anillo de 3 miembros, (c) anillo de 4 miembros, (d) anillo de 5 miembros y (e) anillo de 6 miembros. Tomado de la referencia 29.

La funcionalización química de fullerenos permite su uso en diversos sistemas al modificar algunas de sus propiedades; por ejemplo la solubilidad en medios acuosos. De esta manera, derivados de fullerenos con cadenas laterales con grupos amino, solubles en agua, pueden emplearse como inhibidores potenciales de algunas proteasas del virus de inmunodeficiencia humana (VIH). ${ }^{38}$ Además, se ha sugerido que los fullerenos polihidroxilados poseen propiedades neuroprotectoras, al bloquear los receptores de glutamato y reducir los niveles de Ca intracelular. ${ }^{39}$

\subsection{Propiedades físicas}

Los fullerenos muestran un amplio rango de solubilidad en solventes orgánicos como tolueno o benceno; de hecho, son los únicos alótropos de carbono solubles en solventes comunes a temperatura ambiente. ${ }^{40}$ Basado en la solubilidad del $C_{60}$ en diversos solventes orgánicos se predijo su carácter lipofílico, así como su distribución en líquidos biológicos. ${ }^{41}$ En agua el $\mathrm{C}_{60}$ forma agregados fácilmente, el menor de los cuales contiene 13 moléculas de $\mathrm{C}_{60}{ }^{41}$ Debido a su empaquetamiento compacto, los fullerenos pueden ser usados como lubricantes. Se ha observado 
que films de $\mathrm{C}_{60}$ y $\mathrm{C}_{70}$ dopados con metales alcalinos conducen electricidad a temperatura ambiente $^{42}$ y son superconductores a $18 \mathrm{~K} .^{43}$

El análisis electroquímico del $\mathrm{C}_{60}$ mediante voltametría cíclica y polografía diferencial, revela la existencia de al menos 7 estados de oxidación $\left(C_{60}{ }^{n}: n=0,-1,-2,{ }^{44}-3,{ }^{45}-4,-5,{ }^{46}-6^{47}\right)$. Las 6 reducciones conocidas del $\mathrm{C}_{60}$ son reversibles y ocurren a intervalos igualmente espaciados de $200 \mathrm{mV}$. Se ha observado que las propiedades electroquímicas del $C_{60}$ se conservan en sus derivados, especialmente en los fuleroides y metanofullerenos. ${ }^{48}$

En cuanto a sus propiedades ópticas, diversos experimentos han detectado dos excitaciones colectivas en $\mathrm{C}_{60}$ : la primera, alrededor de $20 \mathrm{eV}$, corresponde al movimiento colectivo de los electrones en enlaces $\sigma$, y la segunda aproximadamente a $6 \mathrm{eV}$ indica el movimiento de los electrones en los enlaces $\pi$. En fullerenos mayores, cuya estructura se aleja de la esférica, las propiedades ópticas se vuelven más complejas. ${ }^{8 b}$ El sistema $p$ conjugado del $C_{60}$ permite su uso en aplicaciones de óptica no-lineal. ${ }^{37} \mathrm{En}$ el estado basal, el $\mathrm{C}_{60}$ presenta fuertes bandas de absorción en la región UV y absorción débil en la región visible (hasta 650nm). Adicionalmente, exhibe una absorción a 750nm caracterizada por una transición triplete-triplete con un coeficiente de absorción mayor que el del estado basal, responsable del comportamiento óptico limitante de las soluciones de $\mathrm{C}_{60}$ y $\mathrm{C}_{70}{ }^{49}$ Esta característica especial permite la aplicación de los fullerenos en matrices trasparentes como protectores contra la radiación de láseres de alta energía. La combinación de estas propiedades permite el uso del $C_{60}$ y sus derivados en el diseño de nuevos materiales electrónicos y dispositivos fotovoltaicos, en especial en el desarrollo de materiales para fotosíntesis artificial. ${ }^{50}$

El estudio detallado de las propiedades físicas, químicas y de reactividad del $C_{60}$ y fullerenos mayores (la química de fullerenos está actualmente confinada al $C_{60}$ ) requiere cantidades grandes de fullerenos. Además, el diseño y desarrollo de nuevos materiales, así como las nuevas tecnologías basadas en fullerenos requieren el empleo de fullerenos puros, así como de isómeros individuales en el caso de fullerenos mayores (se conocen 5 isómeros distintos de $\mathrm{C}_{78}$ que siguen la regla del pentágono aislado ${ }^{51}$ y el número de isómeros posibles aumenta al aumentar el tamaño de los fullerenos). 


\section{Capítulo 2. Síntesis de fullerenos}

Tradicionalmente los fullerenos son producidos mediante descarga de arco voltaico, procesos de combustión o por métodos de deposición de vapor, en los que se vaporiza grafito u otra fuente de carbono. Sin embargo, en la búsqueda de rutas sintéticas con mejores rendimientos y en las que se obtengan productos más puros, se han desarrollado múltiples métodos para sintetizar fullerenos y una gran cantidad de modelos que buscan explicar el o los mecanismos de formación de fullerenos, así como las aproximaciones a sistemas curvos extrapolables a las síntesis de $\mathrm{C}_{60} \mathrm{u}$ otros fullerenos mayores. Algunas de las contribuciones más importantes se detallan a continuación.

\subsection{Síntesis de fullerenos mediante vaporización de una fuente de carbono}

Cinco años después del descubrimiento de los fullerenos, Krätschmer y colaboradores ${ }^{52}$ desarrollaron el primer método pasa sintetizar $\mathrm{C}_{60}$. Este método consistía en la vaporización de grafito por calentamiento en atmósfera de helio. El trabajo de Krätschmer y colaboradores produjo un parteaguas en la ciencia de fullerenos. El acceso a cantidades mayores de $\mathrm{C}_{60}$ modificó la forma en que se estudiaban estos compuestos y permitió la aparición de la química de fullerenos. Sin embargo, este método no es muy eficiente, con rendimientos menores al $1 \%$ debido a las rigurosas condiciones de reacción (temperaturas mayores a $1300^{\circ} \mathrm{C}$ y presiones de 100 torr). ${ }^{53}$ Adicionalmente, este método solo permite obtener $C_{60}$ y $C_{70}$ en buenas cantidades, produciendo cantidades ínfimas de fullerenos mayores como el $\mathrm{C}_{84}$ junto con una gran variedad de moléculas de carbono que se producen durante la reacción, por lo que la purificación de las muestras requiere un gran esfuerzo (más de 20 ciclos de HPLC para $\mathrm{C}_{84}{ }^{54}$ ); además, debido al carácter incontrolable de la reacción no es posible obtener un solo tamaño de fullereno o un isómero específico en el caso de fullerenos mayores.

Otros métodos basados en la vaporización de una fuente de carbono como la pirólisis, plasma formado por radiofrecuencia o por descarga de arco voltaico, tienen las mismas desventajas que el método propuesto por Krätschmer. Por esto, han sido propuestos una gran variedad de métodos 
para sintetizar fullerenos, al igual que diversos modelos que intentan explicar el proceso de síntesis.

\subsection{Modelos que explican la formación de fullerenos}

La teoría general que explica el proceso de formación de fullerenos indica que, primero se vaporiza la fuente de carbono hasta las unidades más pequeñas posibles, es decir átomos de carbono y probablemente dímeros, que sufren una serie de reacciones en las que, dependiendo del intervalo de temperatura y la presión en el que se encuentren, se recombinan para dar lugar a los fullerenos; sin embargo, esta explicación no es satisfactoria, por lo que se han propuesto distintos modelos para explicar de forma más específica el proceso de formación de fullerenos.

El primer modelo que explica la formación de fullerenos a partir de vapor de carbono es el esquema de nucleación en espiral (icospiral particle nucleation scheme). ${ }^{55}$ En este modelo, mostrado en la Figura 2.1, la nucleación comienza a partir de una molécula tipo coranuleno $\left(\mathrm{C}_{20}\right)$, cuya estructura está formada por un pentágono rodeado de cinco hexágonos. Esta estructura altamente reactiva tiende a formar espirales "tipo nautilos" mediante la agregación de pequeños fragmentos de carbono por adsorción. ${ }^{56}$ El cierre ocasional de la red con la disposición correcta de pentágonos y hexágonos explica la formación de los fullerenos. Otras partículas crecen formando partículas de forma cuasi-espiral, como las encontradas por Iijima mediante microscopía de alta resolución (Figura 2.2), ${ }^{57}$ sin embargo, se ha encontrado evidencia en contra de esta hipótesis. ${ }^{58}$

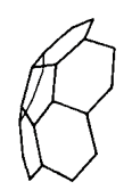

$a$

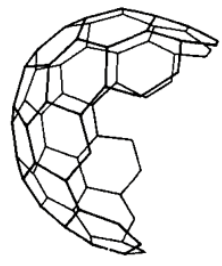

$b$

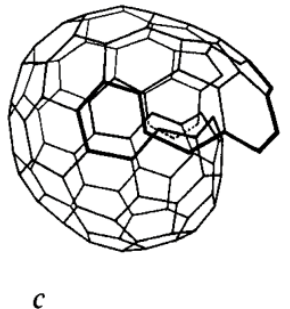

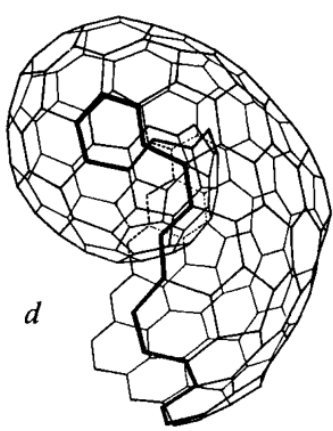

Figura 2.1. Esquema de nucleación en espiral. Tomado de la referencia 55b.

Adicionalmente, de acuerdo con el modelo, la formación de fullerenos en atmósfera de He tardaría $10^{-4} \mathrm{~s}$, mientras que los análisis experimentales concluyen que los fullerenos son formados en una escala de tiempo mucho menor, de $10^{-12}-10^{-9} \mathrm{~s}^{59}$ 
En 1991, Heath propuso un mecanismo en cuatro pasos para explicar la formación de fullerenos (Esquema 2.1). ${ }^{60}$ En el primer paso, los átomos de carbono, producto de la vaporización de grafito, forman cadenas de carbono de hasta diez átomos de longitud $\left(C_{10}\right)$. El segundo paso consiste en el crecimiento de las cadenas para formar anillos $\left(C_{10}-C_{20}\right)$. En el tercer paso, el crecimiento y producción de redes tridimensionales tiene lugar y finalmente, en el cuarto paso, la formación de fullerenos mediante el cierre de las estructuras previamente formadas da lugar al $\mathrm{C}_{60}, \mathrm{C}_{70} \mathrm{y}$ otros fullerenos mayores.

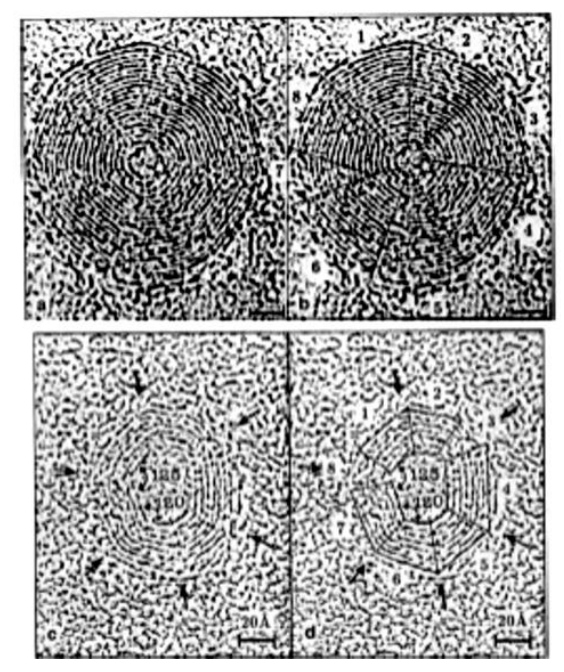

Figura 2.2. Partículas encontradas por lijima mediante microscopía de alta resolución. Las imágenes muestran evidencia de estructuras poliédricas concéntricas. Tomado de la referencia 57.

En 1992, Wakabayashi y colaboradores propusieron un mecanismo para el crecimiento de $\mathrm{C}_{60} \mathrm{Y}$ $\mathrm{C}_{70}{ }^{61}$ Ellos sugirieron que los fullerenos se forman siguiendo un modelo de "apilamiento de anillos" (ring-stacking model) en el que la estructura esférica cerrada típica de los fullerenos, se obtiene mediante el apilamiento de anillos formados por un número par de átomos de carbono como se muestra en la Esquema 2.2. Consideran el anillo $C_{10}$ como el precursor inicial que se deforma en una molécula formada por dos hexágonos con ocho enlaces reactivos. Sobre esta molécula se apilan anillos que eliminan los primeros enlaces reactivos y crean nuevos. En número de enlaces reactivos decrece al apilarse anillos de $C_{18}, C_{12}$ y $C_{2}$, formando finalmente el $C_{60}$. Un esquema similar fue propuesto para la formación de $\mathrm{C}_{70}$ y fullerenos mayores. 


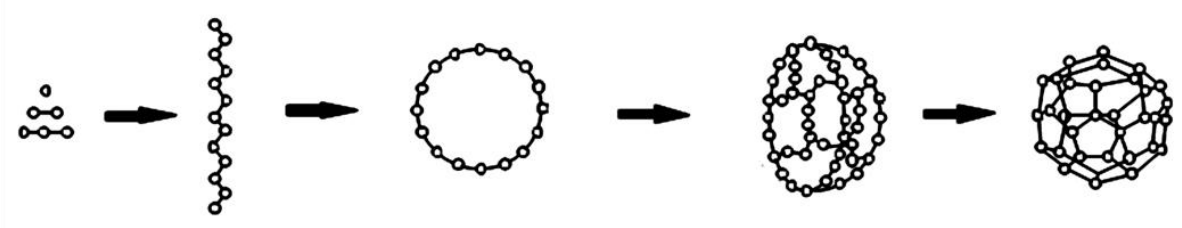

Esquema 2.1. Mecanismo en 4 etapas propuesto por Heath para la formación de fullerenos. Tomado de la referencia 60.

Otro esquema que explica la formación de fullerenos es el modelo de recocido de agregados de carbono (Esquema 2.3). ${ }^{62}$ Dichos agregados de carbono, pueden ser cadenas y anillos que se fusionan, ${ }^{63}$ biciclos o triciclos de 34 a 60 átomos de carbono, ${ }^{64}$ agregados mayores o incluso otros fullerenos. ${ }^{65}$ Dos mecanismos para el recocido de agregados de carbono fueron propuestos. En el primero, la formación de fullerenos ocurre mediante un proceso de transformaciones isoméricas secuenciales. El segundo involucra la cristalización de agregados líquidos. Lozovik y Popov ${ }^{63 b}$ propusieron la formación de agregados líquidos de carbono que cristalizan formando fullerenos, con la emisión de átomos y agregados muy pequeños. En sus experimentos, Hunter y colaboradores ${ }^{63 a}$ observaron que la vaporización láser de grafito genera agregados de iones $\mathrm{C}_{60}^{+}$. Una fracción de estos son fullerenos, mientras que el resto son una mezcla isomérica de policiclos planos. Durante el recocido, la mayoría de los iones $\mathrm{C}_{60}^{+}$planos se transforman en fullerenos; sin embargo, algunos se fragmentan.

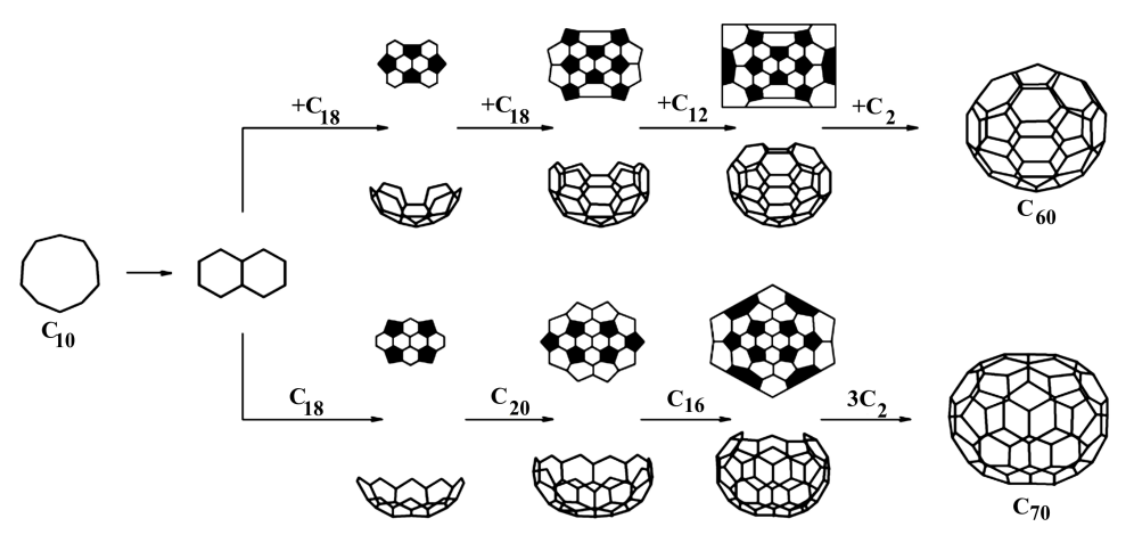

Esquema 2.2. Esquema del modelo de apilamiento de anillos para la obtención de $C_{60}$ y $C_{70}$. Tomado de la referencia $61 b$. 
Posteriormente, Askhabov propuso que los fullerenos surgen de una fase intermedia que solo existe al nivel nanoscópico. ${ }^{66}$ Askhabov propuso que agregados de esta fase intermedia, a los que llamó quatarons, se forman en condiciones de no-equilibrio. Los quatarons son considerados estructuras dinámicas con tamaños específicos, que cambian de forma constantemente. Físicamente, estos pueden definirse como agregados de precristalización de la fase intermedia. En este esquema, los quatarons líquidos surgen inicialmente en un medio supersaturado, para después, transformarse en agregados rígidos con distancias interatómicas fijas, como los fullerenos. Sin embargo, este controversial modelo no ha sido aceptado debido a la falta de evidencia que lo sostenga.

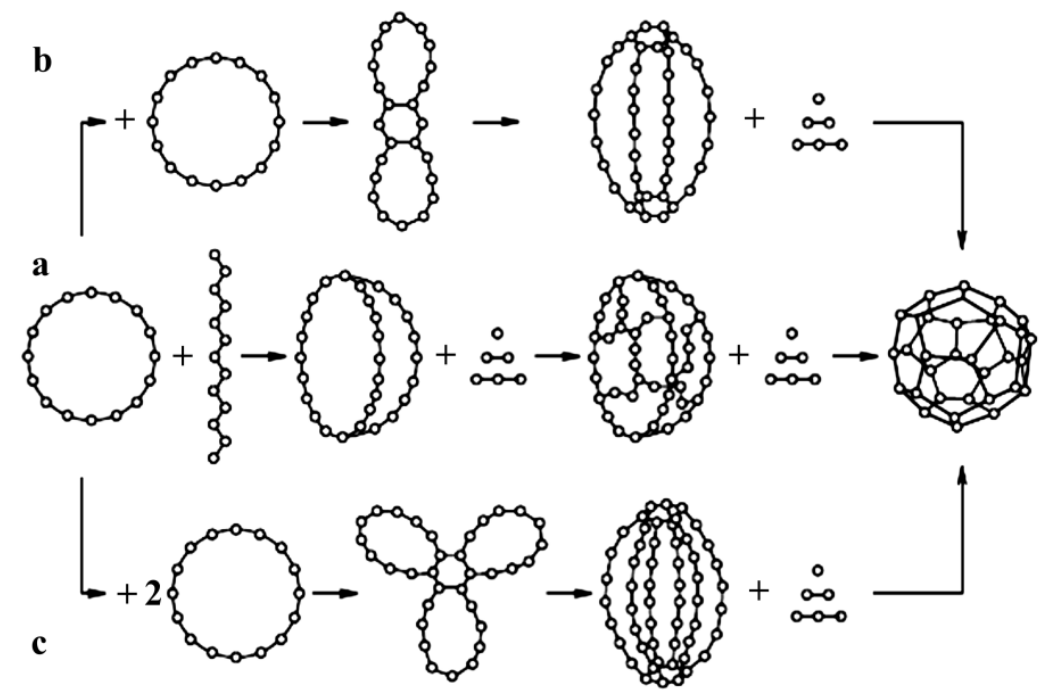

Esquema 2.3. Esquema del crecimiento de agregados de carbono de acuerdo con las siguientes etapas: cadenas-anillos-agregados policíclicos tridimensionales-fullerenos. Se muestran diferentes caminos que llevan a los agregados policíclicos tridimensionales. Tomado de la referencia $62 b$.

Con base en los resultados de simulaciones del autoensamblado molecular en medios de vaporización obtenidos mediante dinámica molecular, Irle y colaboradores ${ }^{67}$ propusieron un mecanismo llamado encogimiento de gigantes (shrinking hot giant (SHG)), en el que los fullerenos se forman por el encogimiento de fullerenos gigantes $\left(C_{n} n \geq 90\right)$. Este mecanismo se divide en dos etapas, la primera, o de crecimiento, consiste en la formación de fullerenos gigantes a partir del vapor de carbono siguiendo un esquema similar al propuesto por Heath. ${ }^{60}$ En la segunda etapa, o de reducción, los fullerenos gigantes se reducen hasta formar $C_{60}$ y $C_{70}$ mediante la pérdida irreversible de moléculas de $\mathrm{C}_{2}$, favorecida por la naturaleza altamente irregular y la gran cantidad de defectos de los fullerenos gigantes. Irle y colaboradores sugieren que este mecanismo combinado explica satisfactoriamente el proceso de formación de fullerenos en medios caóticos. 


\subsection{Síntesis química}

Muchos métodos han sido propuestos para sintetizar $\mathrm{C}_{60}$; desafortunadamente muy pocos han tenido éxito. Por ejemplo, una estrategia particularmente atractiva es el ensamblado de dos hidrocarburos hemisféricos idénticos. ${ }^{68}$ Sin embargo, la forma de unir dichas moléculas hemisféricas aún no existe.

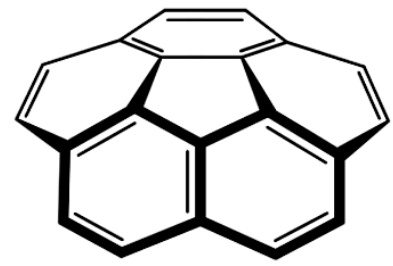

Figura 2.3. Estructura del coranuleno.

El mayor problema de la síntesis química de fullerenos es la introducción de la curvatura o piramidalización de los átomos de carbono en la molécula. Barth y Lawton fueron los primeros en sintetizar una molécula curva, el coranuleno (Figura 2.3). ${ }^{69}$ Sin embargo, dicha síntesis era complicada y los rendimientos muy bajos, así que fue olvidada hasta que en 1991 Scott y colaboradores ${ }^{70}$ propusieron una nueva síntesis del coranuleno que empleaba pirólisis (flash vacuum pirolisis, FVP) para cerrar los anillos e introducir la curvatura. Este método fue mejorado hasta la síntesis en tres pasos mostrada en la Esquema $2.4 .^{71}$ El primer paso es una reacción de Knovenagel-Diels-Alder entre acenaftoquinona, 2,4,6-triheptanona y norbornadieno para dar 7,10-diacetilfluoranteno, con un rendimiento del 7075\%. En el segundo paso, la conversión de los grupos acetilo en grupos clorovinilo, con un rendimiento del $80-85 \%$, se lleva a cabo. El último paso consiste en la formación in situ de alquinos terminales mediante eliminación térmica (FVP) de $\mathrm{HCl}$ para dar lugar al coranuleno, con un rendimiento de $35-40 \%$.

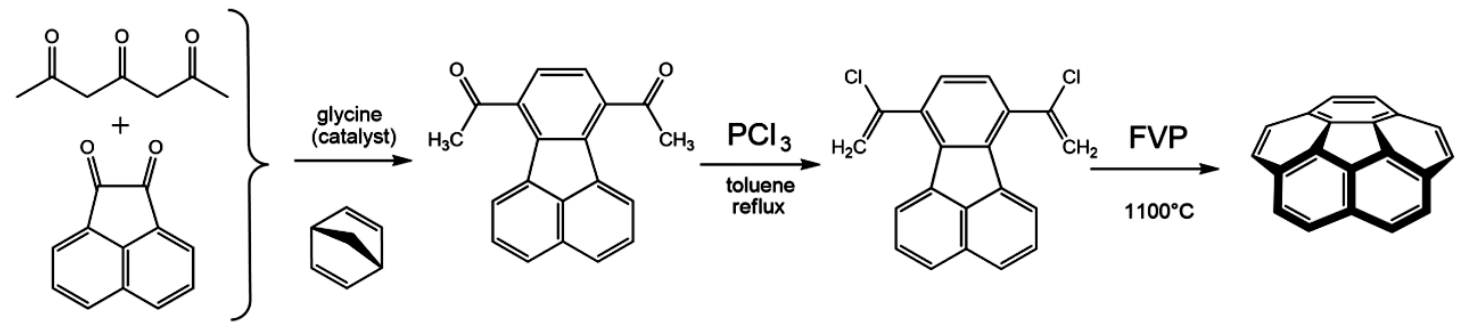

Esquema 2.4. Síntesis del coranuleno en 3 pasos. Tomado de la referencia $71 b$.

Scott y colaboradores estudiaron también otros fragmentos de fullerenos y propusieron que los fragmentos cuyo carácter electrónico era más parecido al del $\mathrm{C}_{60}$, favorecerían la formación de $\mathrm{C}_{60}{ }^{72}$ Analizaron la pirólisis del decacicleno a $1200-1300^{\circ} \mathrm{C}$ para obtener triacenaftotrifenileno 
$\left(\mathrm{C}_{36} \mathrm{H}_{12}\right)$ mediante la formación de tres enlaces intramoleculares, producto de la deshidrogenación del decacicleno (Esquema 2.5). En este intervalo de temperatura obtuvieron también los productos con uno y dos cierres, así como trazas de $\mathrm{C}_{60}$, y determinaron que la pirólisis a temperaturas más bajas (menores a $1100^{\circ} \mathrm{C}$ ) no provee la energía necesaria para la triple deshidrogenación del decacicleno. Además, observaron que el espectro de absorción ultravioleta del triacenaftotrifenileno es similar al del $C_{60}$ (Figura 2.4). Dicha similitud en el espectro podría ser una forma de medir que tanto se parece un fragmento al $\mathrm{C}_{60}$.

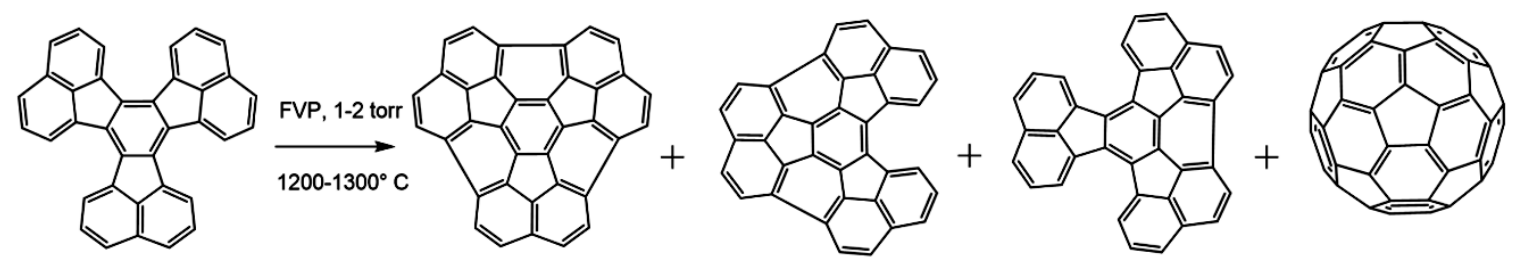

Esquema 2.5. Productos obtenidos por la pirólisis del decacicleno. Moléculas con tres $\left(C_{36} H_{12}\right)$, dos $\left(\mathrm{C}_{36} \mathrm{H}_{14}\right)$ y un $\left(\mathrm{C}_{36} \mathrm{H}_{16}\right)$ cierre y $\mathrm{C}_{60}$.
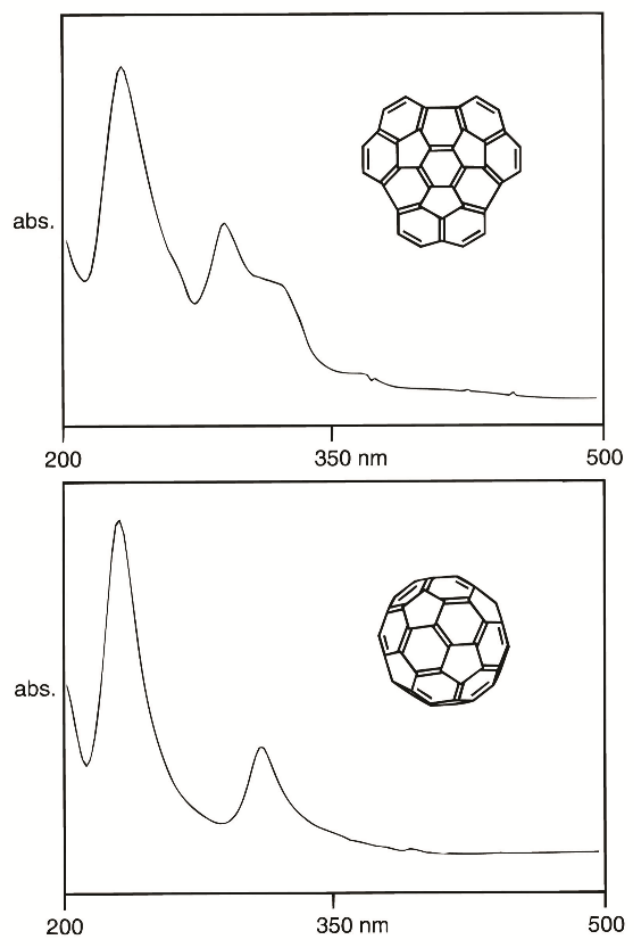

En 1997 Plater propuso un análisis retrosintético del $\mathrm{C}_{60}$ donde el fullereno se abre para dar lugar a un hidrocarburo policíclico aromático simétrico, relativamente libre de tensión. De esta manera, Plater observó una serie de fragmentos que podrían ser aislados y actuar como bloques para la construcción del $\mathrm{C}_{60}{ }^{73}$ Propuso tres fragmentos principales: decacicleno $\left(\mathrm{C}_{36} \mathrm{H}_{18}\right)$, tribenzodecacicleno $\left(\mathrm{C}_{48} \mathrm{H}_{24}\right)$ y trinaftodecacicleno $\left(\mathrm{C}_{60} \mathrm{H}_{30}\right)$ mostrados en la Figura 2.5, que reaccionarían por pirólisis para formar $\mathrm{C}_{60}$ y sugirió que los fragmentos halogenados reaccionarían con mayor facilidad. Estos precursores policíclicos sufrirían reacciones de acoplamiento intramoleculares (flechas y líneas punteadas en la Figura 2.5) para formar los fullerenos o fragmentos de fullerenos deseados.

Figura 2.4. Espectros de absorción UVVis del triacenaftotrifenileno y del $C_{60}$. Tomado de la referencia 72. 
Figura 2.5. Fragmentos propuestos por Plater a
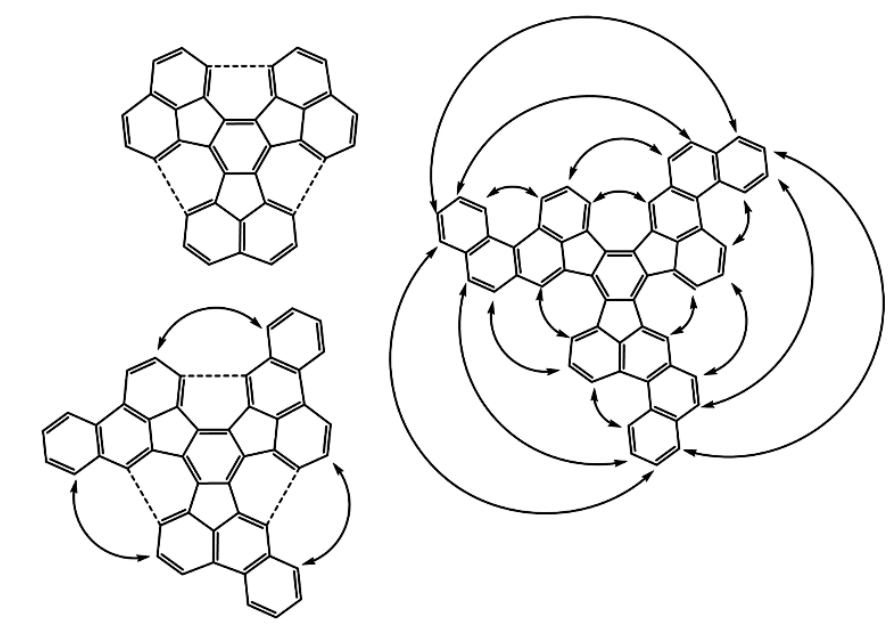

partir del análisis retrosintético del $C_{60}$. Tomado de la referencia $73 a$.

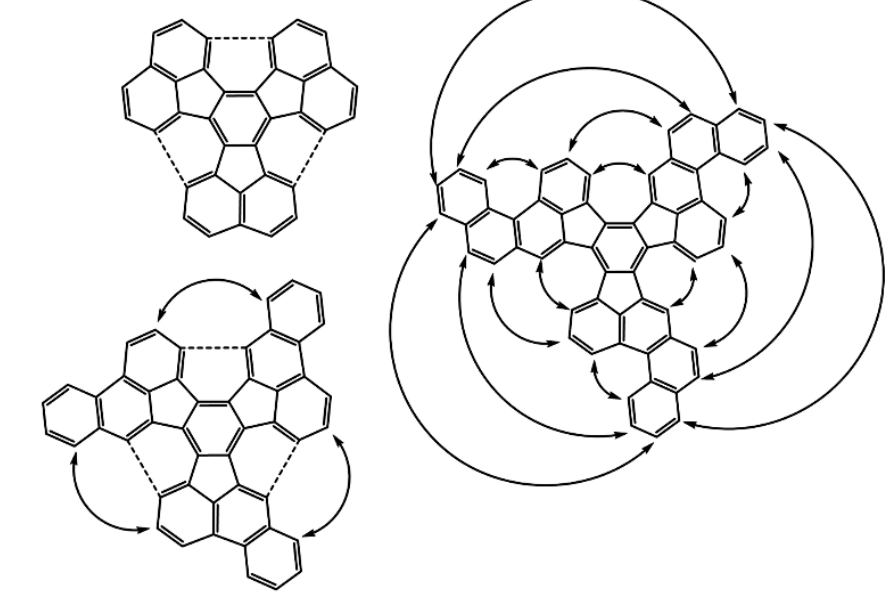

Posteriormente Ansems y Scott propusieron una ruta para la síntesis de triacenaftotrifenileno mediante la pirólisis de triclorodecacicleno, con un rendimiento del $30 \%{ }^{74}$ Este método fue empleado como prototipo para la síntesis de moléculas geodésicas de mayor tamaño a partir de hidrocarburos policíclicos aromáticos halogenados. Sugirieron que, una vez que la curvatura se introduce en un sistema aromático, los subsecuentes cierres por deshidrogenación ocurren, generalmente, sin la necesidad de promotores adicionales. Desde entonces, la pirólisis de diferentes precursores ha sido utilizada para sintetizar un gran número de hidrocarburos policíclicos aromáticos curvos, identificados como fragmentos de fullerenos (Figura 2.6). ${ }^{75}$ Sin embargo, las desventajas propias de la pirólisis, han conducido a la necesidad de desarrollar métodos no pirolíticos para sintetizar este tipo de estructuras, por ejemplo, los métodos de síntesis en solución.

Seiders y colaboradores propusieron la síntesis de coranuleno basado en el acoplamiento reductivo mediado por titanio (Esquema 2.6). ${ }^{76}$ En este método el coranuleno es formado mediante la ciclación interna de los precursores bromobencílicos por acoplamiento reductivo con $\mathrm{TiCl}_{3} / \mathrm{LiAlH}_{4}$ y la subsecuente deshidrogenación de la mezcla de cis-dimetiltetrahidrocoranulenos y trans-dimetiltetrahidrocoranulenos obtenida para dar lugar al dimetilcoranuleno, con un rendimiento del $18 \%$. Este método fue usado por Sygula y Rabideau para sintetizar el hemifullereno $\mathrm{C}_{30} \mathrm{H}_{12}$ (estructura 13 en la Figura 2.6). ${ }^{77}$ En este caso, el acoplamiento reductivo intramolecular del precursor dodecabromado conduce a la formación del hemifullereno $\mathrm{C}_{30} \mathrm{H}_{12}$, con un modesto rendimiento del $20 \%$ (Esquema 2.7). Las desventajas de este método radican en el requerimiento de técnicas de alta dilución, con tiempos de reacción de 2 a 4 días, además de la necesidad de una atmósfera limpia, libre de oxígeno y humedad, que hacen aún más difícil la aplicación de este método. 


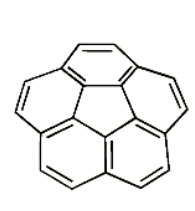

1

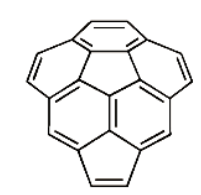

2

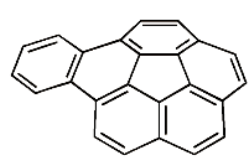

3

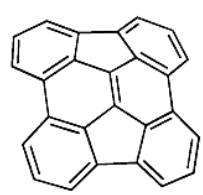

4

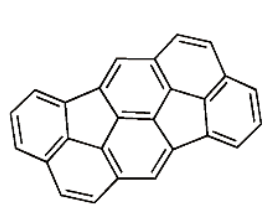

5

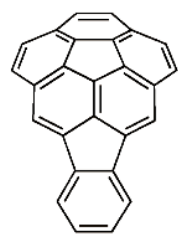

6

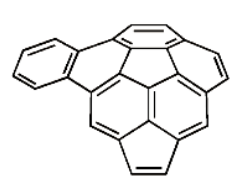

7

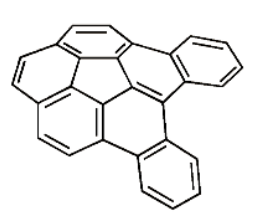

12

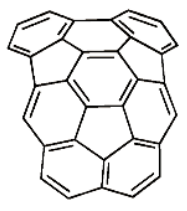

17

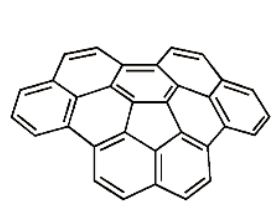

22

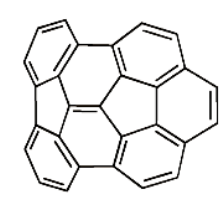

8

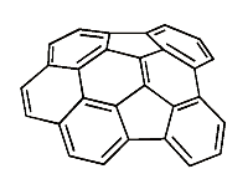

9

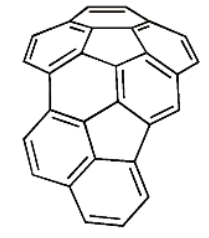

10

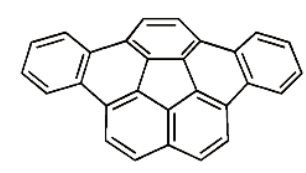

11

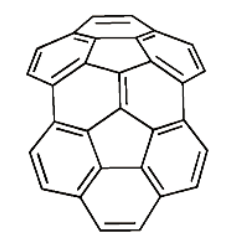

13

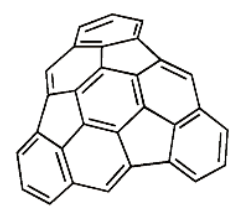

14

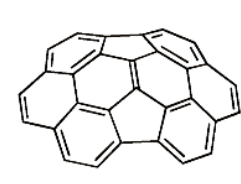

15

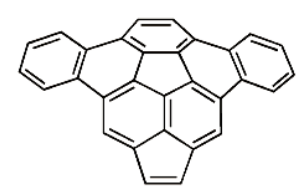

16

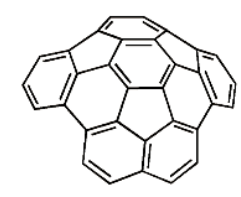

18

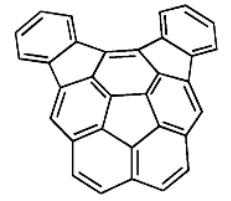

19

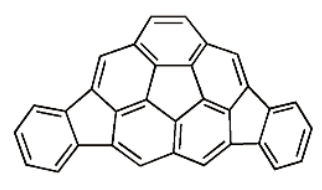

20

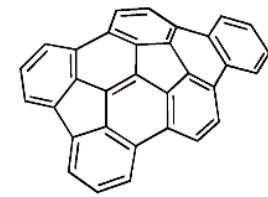

21

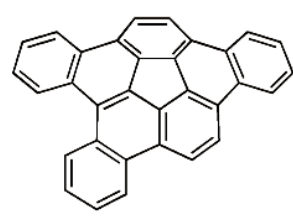

23

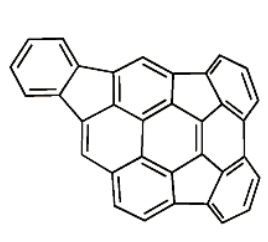

24

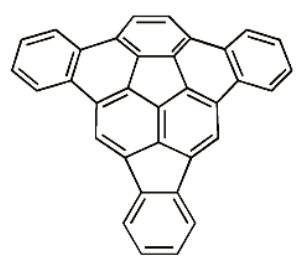

25

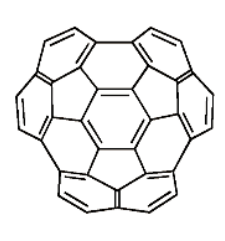

26

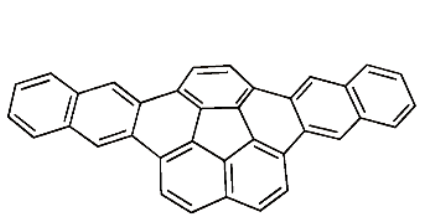

27

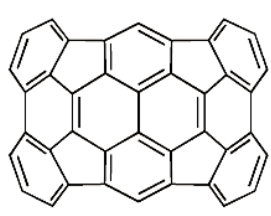

28

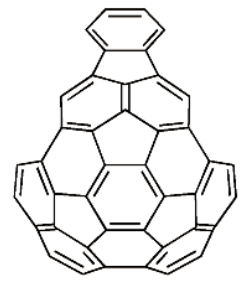

29

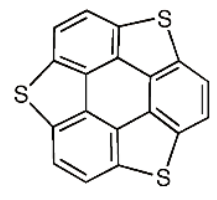

30

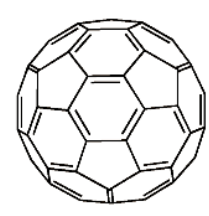

31

Figura 2.6. Hidrocarburos policíclicos aromáticos curvos sintetizados empleando pirólisis. Tomado de la referencia 75. 

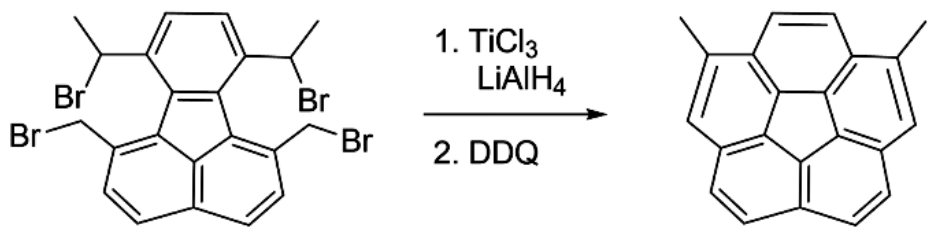

$(18 \%)$

Esquema 2.6. Síntesis de coranuleno propuesta por Seiders y colaboradores, basada en el acoplamiento reductivo de 1,6-bis(bromometil)-7,10-bis (1-bromometil) fluoranteno y su subsecuente reducción. Tomado de la referencia 76.

En 2002, Sygula y Rabideau descubrieron un nuevo método para sintetizar coranuleno. ${ }^{78}$ Este método, mostrado en la Esquema 2.8, se basa en el tratamiento de 1,6,7,10-tetrakis (dibromometil)fluoranteno con hidróxido de sodio y la subsecuente reducción para obtener coranuleno, con un rendimiento combinado de 55\%. En primer paso, el $\mathrm{NaOH}$ favorece la deshidrogenación de los grupos bromometilo, formando un carbanion que se transformaría en un bromocarbeno que se insertaría en el enlace $\mathrm{CBr}$ del grupo dibromometilo adyacente, terminando con la eliminación de $\mathrm{HBr}$. Los átomos de bromo en el tetrabromocoranuleno obtenido, pueden remplazarse por grupos metilo o trimetilsililacetileno, que permitirían la formación de nuevas estructuras. Posteriormente Sygula y colaboradores, propusieron otro método para sintetizar 1,2di(carboximetil)coranuleno (Esquema 2.9) que empleaba níquel como agente reductivo. ${ }^{79}$ Este método provee una forma sencilla de obtener coranulenos sustituidos al modificar o cambiar los grupos carboximetilo.

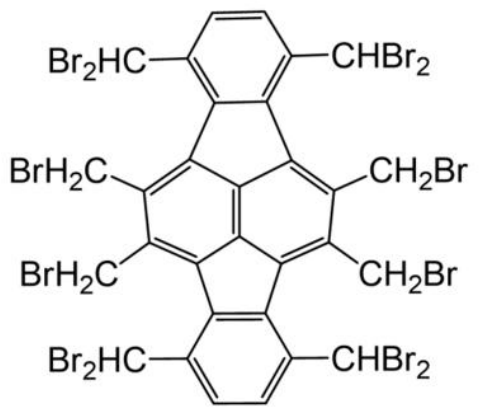
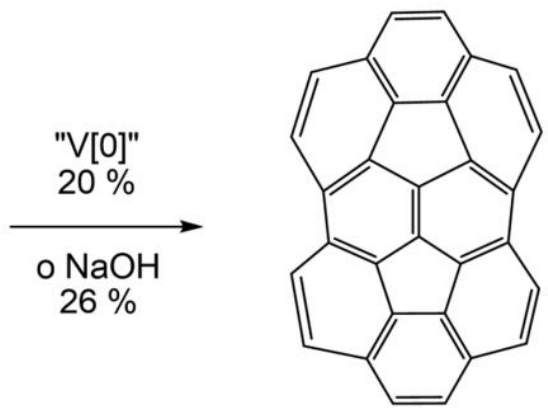

Esquema 2.7. Síntesis del hemifullereno $\mathrm{C}_{30} \mathrm{H}_{12}$ propuesta por Sygula y Rabideau, basada en el método de Seiders et al. Tomado de la referencia 77. 


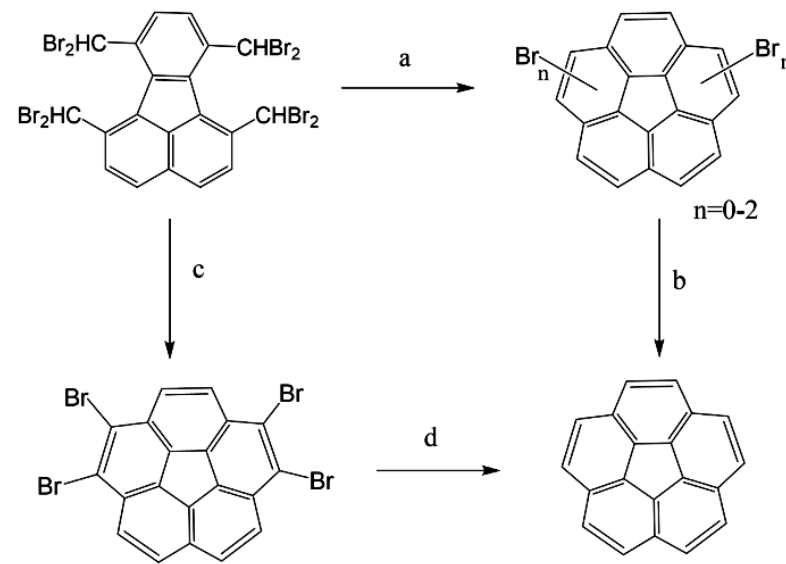

Esquema 2.8. Síntesis de coranuleno propuesta por Sygula y Rabideau.

Condiciones: (a) acetona/agua (3:1), $\mathrm{NaOH}$, reflujo por 1 hora. (b) $n-B u L i, T H F,-78^{\circ} \mathrm{C}, 30$ min, $\mathrm{HCl}$ diluido. El rendimiento de combinado de los dos pasos es de 50-55\%. (c) dioxano/agua (2.5:1), $\mathrm{NaOH}$, reflujo $15 \mathrm{~min}$, rendimiento del 83\%. (d) LiAlH4, DDQ, rendimiento del $30 \%$. Tomado de la referencia 78.

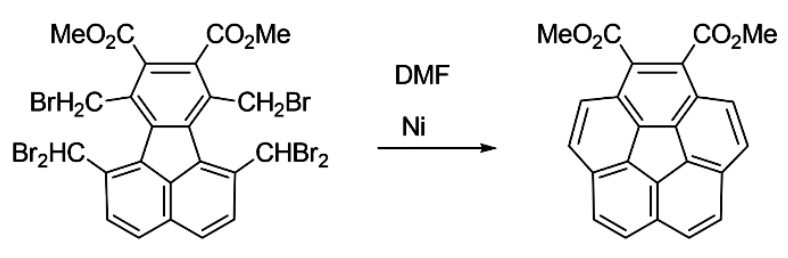

Esquema 2.9. Síntesis de 1,2di(carboximetil)coranuleno. Tomado de la referencia 79.

$(60 \%)$

Basados en la idea de la dimerización de dos fragmentos isométricos $C_{30}$ para obtener $C_{60}$, Geneste y colaboradores ${ }^{80}$ analizaron los fragmentos isométricos derivados de $\mathrm{C}_{60}$ usando el método de "la coupe du roi" (un método de segmentación empleado para dividir una manzana en dos mitades quirales) empleado por Anet y colaboradores en el estudio de la fragmentación y recombinación de diversas moléculas. ${ }^{81}$ Geneste y colaboradores encontraron 9 estructuras isométricas (Figura 2.7) entre las que se encuentran un fragmento heteroquiral (14), cuatro homoquirales (32-35) y cuatro aquirales (36-39). Calcularon la energía de estabilización de los fragmentos y determinaron que el fragmento más estable es el 14 seguido por la estructura 34, que además es el hemifullereno con mayor piramidalización. Sin embargo, no proponen una forma de unir los fragmentos para formar el $\mathrm{C}_{60}$. 


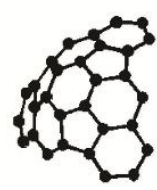

14

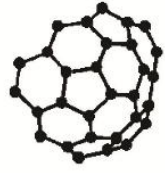

32

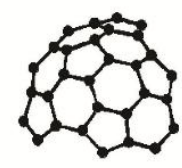

33

Figura 2.7. Estructura de los hemifullerenos isométricos encontrados por Geneste, et al. Tomado de la referencia 80.

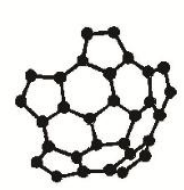

34

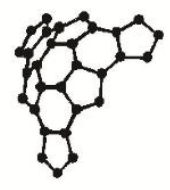

35

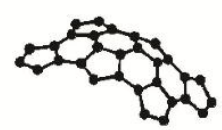

36

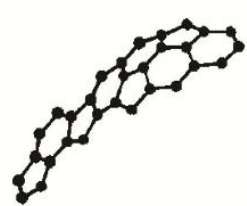

37

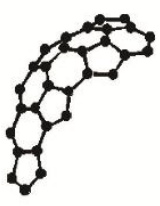

38

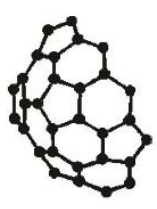

39

En 2002, Scott y colaboradores reportaron la primera síntesis racional de $\mathrm{C}_{60}{ }^{82}$ Ellos sintetizaron $\mathrm{C}_{60}$ a partir de la pirólisis de $\mathrm{C}_{60} \mathrm{H}_{27} \mathrm{Cl}_{3}$ (Esquema 2.10). La pirólisis de los derivados clorados a $1100^{\circ} \mathrm{C}$ induce la formación de los enlaces carbono-carbono mostrados por las flechas en la Esquema 2.10, mediados por la formación y reordenamiento de especies radicales (* en la Esquema 2.10) promovidos por la presencia de los átomos de cloro en la molécula. La unión de los brazos del precursor conduce específicamente a la formación del $\mathrm{C}_{60}$; ningún otro fullereno puede formarse en este proceso a partir del precursor utilizado. Lamentablemente, el rendimiento de la reacción es muy bajo, menor al $1 \%$, ya que la molécula reactiva se descompone debido a las condiciones extremas necesarias para la reacción, lo que llevo a Scott y colaboradores a sugerir que el uso de otros promotores de radicales podrían mejorar los rendimientos. En este sentido, Kabdulov y colaboradores, propusieron el uso de flúor, en lugar de cloro o bromo, como promotor de radicales sobre el mismo precursor $\mathrm{C}_{60} \mathrm{H}_{30} \cdot{ }^{83}$ La estructura de los precursores hexa- y nonafluorados se muestra en la Figura 2.8. En el precursor hexafluorado $(X=H)$ los átomos de flúor se eliminan para introducir la curvatura, que favorece la deshidrogenación térmica que da lugar al $\mathrm{C}_{60}$. En el caso de la molécula nonafluorada la pérdida de los primeros seis átomos de flúor acerca el resto de la molécula lo que permite la eliminación de los tres átomos de $\mathrm{F}$ restantes. El flúor debería mejorar los rendimientos de la reacción debido a que la introducción de este átomo no incrementa mucho el peso molecular del precursor, permitiendo la sublimación de la molécula. Además, el enlace CF es relativamente estable, lo que reduciría la descomposición del precursor en el medio de reacción, sin contar con que el tamaño del F permite su introducción en regiones impedidas estéricamente. 


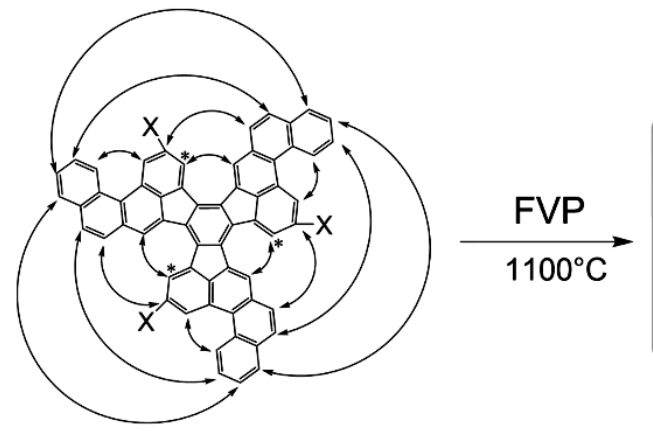

$\mathrm{X}=\mathrm{H}, \mathrm{Cl}$

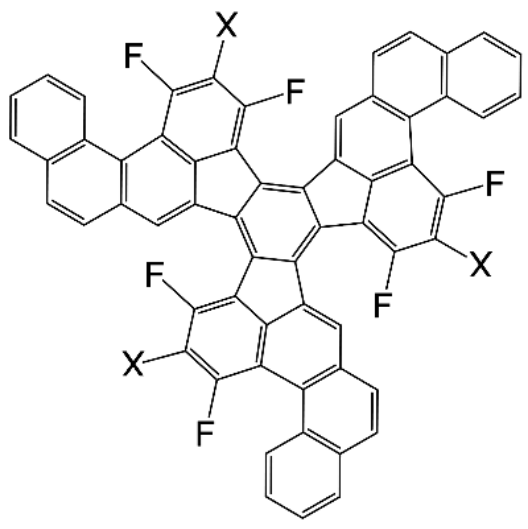

$\mathrm{X}=\mathrm{H}, \mathrm{F}$
Esquema 2.10. Paso final en la síntesis de $C_{60}$ propuesta por Scott, et al. las líneas curvas indican los sitios donde se formarán nuevos enlaces. Tomado de la referencia 82 .
Figura 2.8. Precursores hexafluorados $(X=H)$ y nonafluorados $(X=F)$ propuestos por Kabdulov et al. Tomado de la referencia 83.

En 2008, Otero y colaboradores propusieron el uso de superficies de Pt para catalizar la deshidrogenación del precursor plano $\mathrm{C}_{60} \mathrm{H}_{30}$, para mejorar los rendimientos de la reacción propuesta por Scott y colaboradores. ${ }^{84}$ El platino favorece la deshidrogenación de la molécula precursora, reduciendo la temperatura de reacción $\left(750 \mathrm{~K}=1023^{\circ} \mathrm{C}\right)$. Además, la interacción entre el precursor y la superficie de Pt fija a la molécula precursora a la superficie, eliminando las colisiones y los factores geométricos de la reacción. Esto permite obtener rendimientos cercanos al 100\%. La Figura 2.9 muestra el proceso por el cual una molécula de $\mathrm{C}_{57} \mathrm{H}_{33} \mathrm{~N}_{3}$ se transforma en el triazafullereno $\mathrm{C}_{57} \mathrm{~N}_{3}$ al calentar a $750 \mathrm{~K}\left(1023^{\circ} \mathrm{C}\right)$. Adicionalmente, Otero y colaboradores emplearon el método impulso de banda elástica $\left(\mathrm{NEB}^{85}\right.$ ) para calcular un mecanismo general para el cierre del $\mathrm{C}_{60} \mathrm{H}_{30}$ (Esquema 2.11). El proceso comienza con la formación de los enlaces centrales, lo que introduce la curvatura a la molécula, favoreciendo la formación de los enlaces restantes. 
a
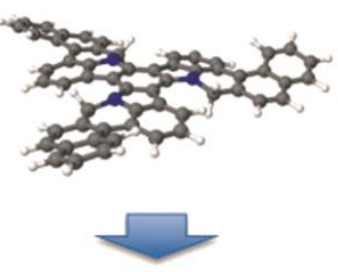

b
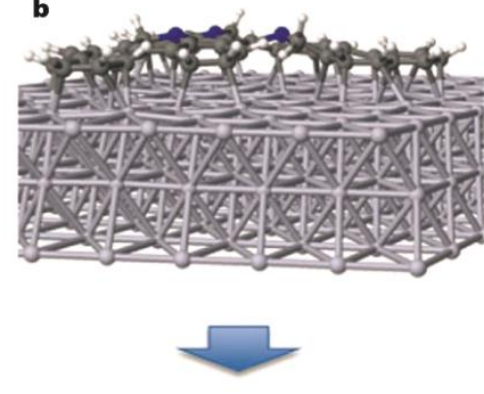

c

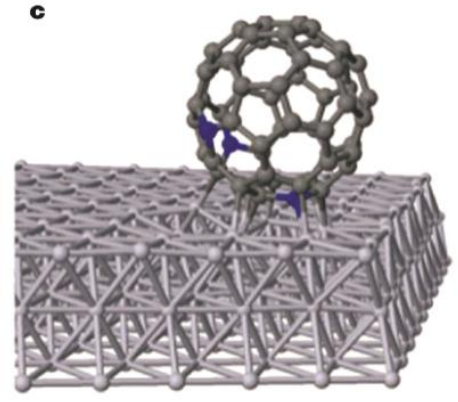

Figura 2.9. Formación del triazafullereno $C_{57} N_{3}$ a partir del precursor plano $\mathrm{C}_{57} \mathrm{H}_{33} \mathrm{~N}_{3}$, que primero es absorbido en una superficie de Pt (111) y después calentado a $750 \mathrm{~K}$. Las esferas azules representan los átomos de nitrógeno. Tomado de la referencia 84.

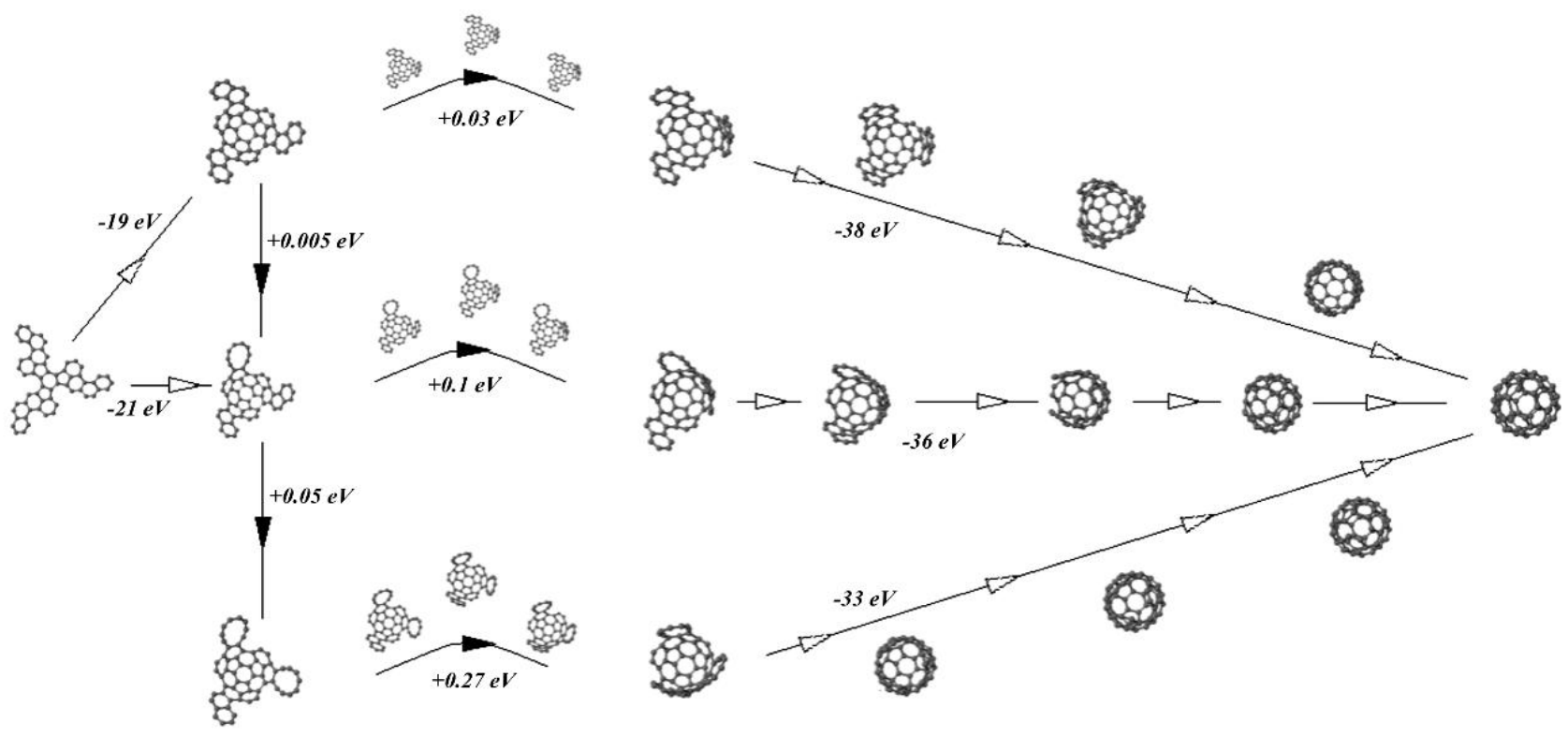

Esquema 2.11. Ruta propuesta por Otero, et al. donde se muestran los pasos clave para la formación del $C_{60}$ a partir del precursor completamente deshidrogenado. Tomado de la referencia 84. 
Amsharov y colaboradores analizaron la deshidrogenación de una serie de benzofenantrenos sustituidos para dar 2,13-benzofluoranteno. ${ }^{86}$ Esta reacción fue considerada una reacción prototipo en la formación de fullerenos. Amsharov et al. propusieron una serie de mecanismos basados en reacciones con radicales libres y probaron diferentes promotores de radicales, como se muestra en la Esquema 2.12. Usaron átomos de $\mathrm{Cl}(\mathbf{4 0 a})$ y $\mathrm{F}(\mathbf{4 0 d})$ como promotores de radicales, al igual que la ruptura homolítica del enlace $\mathrm{CH}$ de los grupos metilo (40b) y fenilo (40c). El reordenamiento de los radicales formados, que conduce a la formación del bezofluoranteno deseado (41), se muestra en dicha figura. También propusieron mecanismos no mediados por radicales. En el primer caso, la eliminación de HF de $\mathbf{4 0 d}$ conduce a la formación de las benzinas $\mathbf{4 9}$ y 50 que evolucionan a bezopireno (43) y benzofluoranteno (41) respectivamente. En el segundo caso la 1,5-eliminacion de HF de 40 e conduce directamente al benzofluoranteno (41). Datos experimentales de las reacciones mostradas en la Esquema 2.12 indican que la reacción de $40 \mathrm{e}$ a 41 es termodinámica y cinéticamente favorecida, contrario a las reacciones basadas en la producción y reordenamiento de radicales. La síntesis del 2,13-benzofluoranteno ofrece una guía para el análisis del último paso de la síntesis de fullerenos, permitiendo proponer y entender nuevos métodos de síntesis.

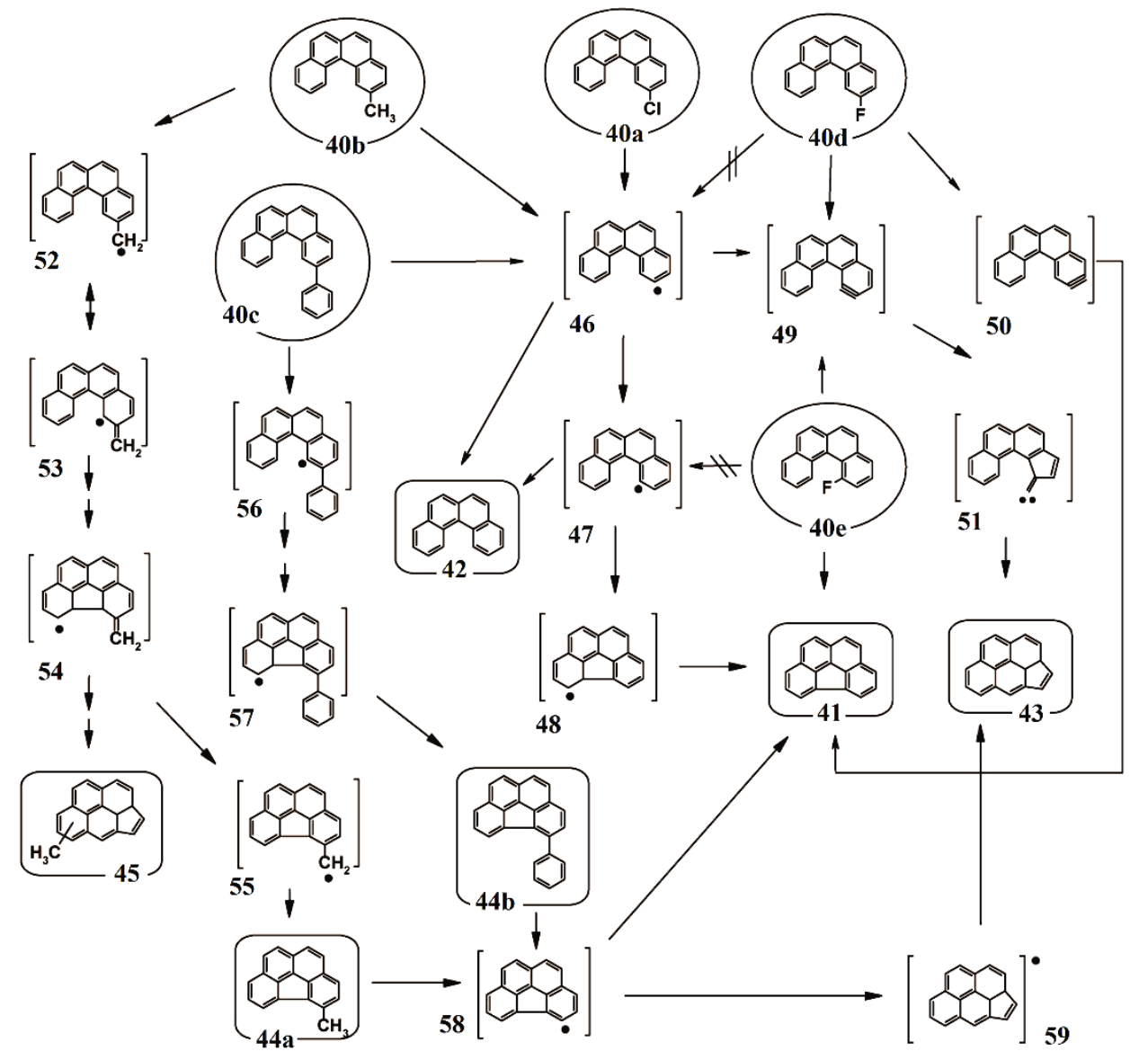

Esquema 2.12. Mecanismos propuestos para la condensación de benzofenantrenos sustituidos en benzofluoranteno mediante pirólisis. Tomado de la referencia 86. 


\subsection{Otros métodos}

Empleando las propiedades de simetría del $\mathrm{C}_{60}$, Yasuda ${ }^{87}$ propuso la formación de precursores tetraédricos formados por benceno y acetileno que se transformarían en $\mathrm{C}_{60}$ mediante múltiples reacciones intramoleculares. La síntesis de dichos precursores altamente reactivos se muestra en la Esquema 2.13 donde dos precursores tetraédricos son obtenidos mediante reacciones de acoplamiento entre bencenos halogenados y derivados de acetileno. Estos análogos presentan la misma simetría que el $\mathrm{C}_{60}$, así como todos los átomos de carbono necesarios para formarlo; adicionalmente, pueden ser sustituidos con heteroátomos, dando lugar a heterofullerenos como el $\mathrm{C}_{48} \mathrm{~N}_{12}$. La alta reactividad de los precursores que surgen de la interacción del benceno halogenado y los derivados de acetileno es la clave para el éxito de este método.
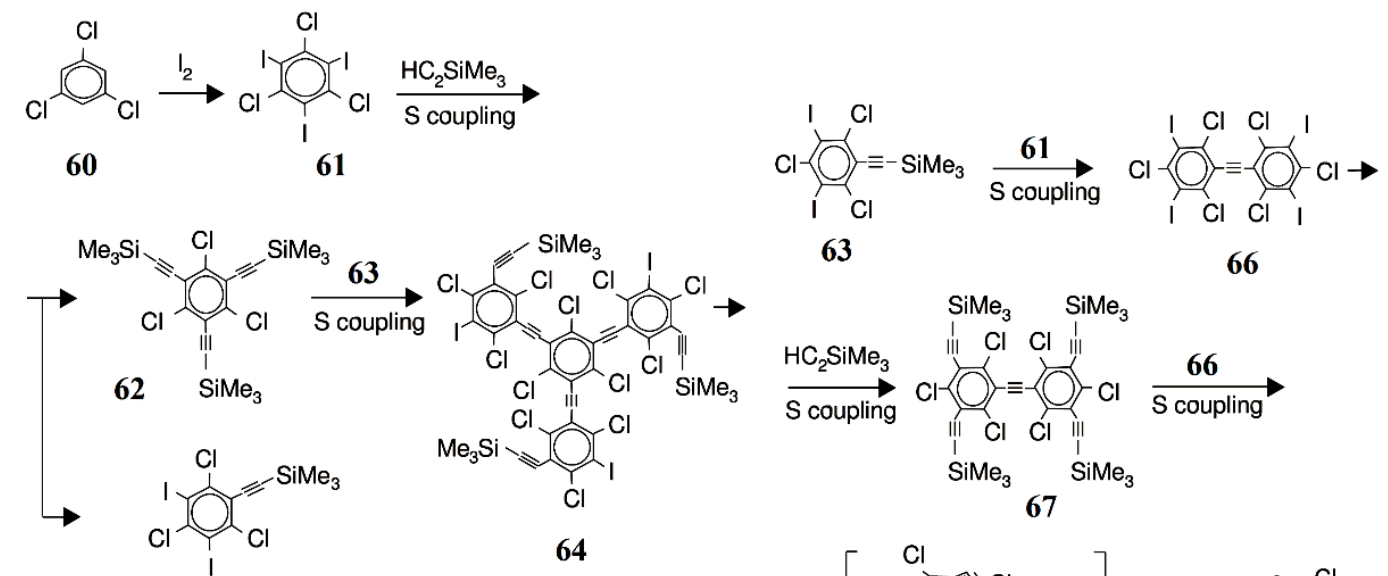

63

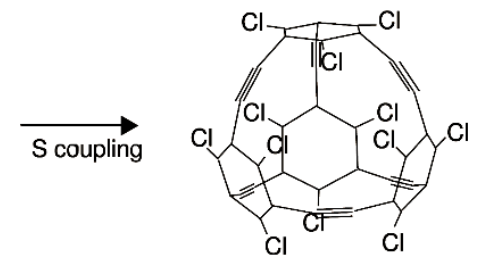

63

66

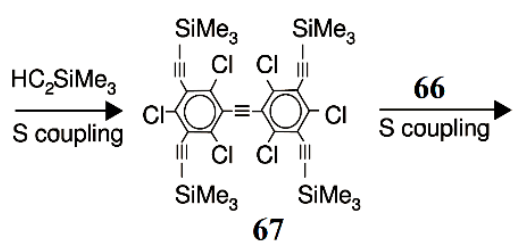

(a)

65

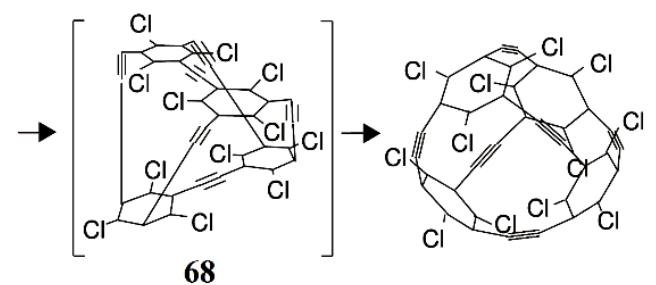

69

(b)

Esquema 2.13. Síntesis de dos análogos tetraédricos mediante reacciones de acoplamiento inter-e intra-moleculares. (a) síntesis del precursor 65 mediante reacciones de acoplamiento intramolecular de 1,3,5-tri(feniletinil)benceno (64). (b) síntesis del precursor 69 mediante reacciones de acoplamiento intermolecular entre derivados del 2-difeniletino (67). Tomado de la referencia 87. 
Rubin, et $a l^{88}$ y Tobe, et $a l^{89}{ }^{8}$ independientemente, propusieron el uso de macrociclos esféricos ricos en acetileno como precursores de fullerenos (Figura 2.10). Las ventajas de este método es que sólo involucra la formación de enlaces, reduciendo los costos energéticos debidos a la ruptura y reordenamiento de enlaces, procesos que se llevan a cabo en la síntesis de fullerenos a partir de vapor de carbono. ${ }^{90} \mathrm{~A}$ pesar de que algunos iones de fullerenos hayan sido identificados en espectros de masas por desorción laser de dichos precursores, los intentos de obtener $\mathrm{C}_{60}$ no han sido exitosos. Esto indica que la reorganización de los precursores es necesaria para la obtención de fullerenos. ${ }^{91}$

Figura 2.10. Macrociclos esféricos ricos en acetileno
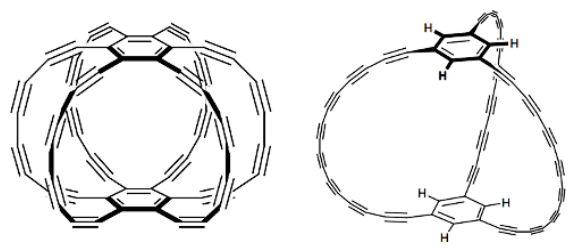
propuestos como precursores de fullerenos.

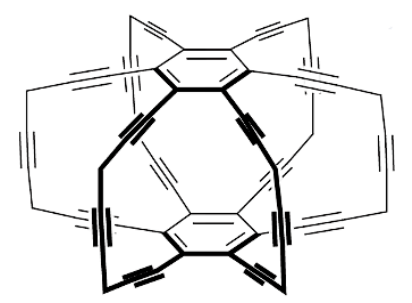

Viñes y Görling ${ }^{92}$ analizaron teóricamente, la posibilidad de sintetizar fullerenos a partir de precursores simples como metano o etileno, usando nanopartículas metálicas como moldes. En este caso la formación de fullerenos es inducida por la forma y la estructura electrónica de la partícula molde, que también actúa como catalizador. Para que la síntesis de fullerenos sea exitosa es necesario cumplir ciertas condiciones: (i) las moléculas precursoras deben ser completamente deshidrogenadas por las nanopartículas; (ii) tanto los átomos de carbono como los compuestos intermediarios deben permanecer unidos a las nanopartículas metálicas; y (iii) la formación de fullerenos debe ser energéticamente favorable, comparada con la de otras estructuras de carbono, especialmente fragmentos de grafeno. En el caso de la síntesis de fullerenos sobre partículas de Pt, las tres condiciones se cumplen. Los resultados del análisis de la formación de fullerenos sobre Pt se muestran en la Figura 2.11, se optimizaron diversas estructuras de carbono sobre partículas esféricas de $\mathrm{Pt}_{79}$, incluyendo átomos dispersos, hojuelas tipo grafeno y estructuras tipo fullereno. Cuando el número de átomos de carbono sobre la partícula de Pt es menor a 13 ( $n$ <13) los átomos de carbono prefieren dispersarse sobre las caras de la partícula, para números de átomos entre 13 y $24(13<n<24)$, se observan hojuelas tipo grafeno sobre las partículas, y cuando $n>30$ la estructura tipo fullereno es la preferida. 


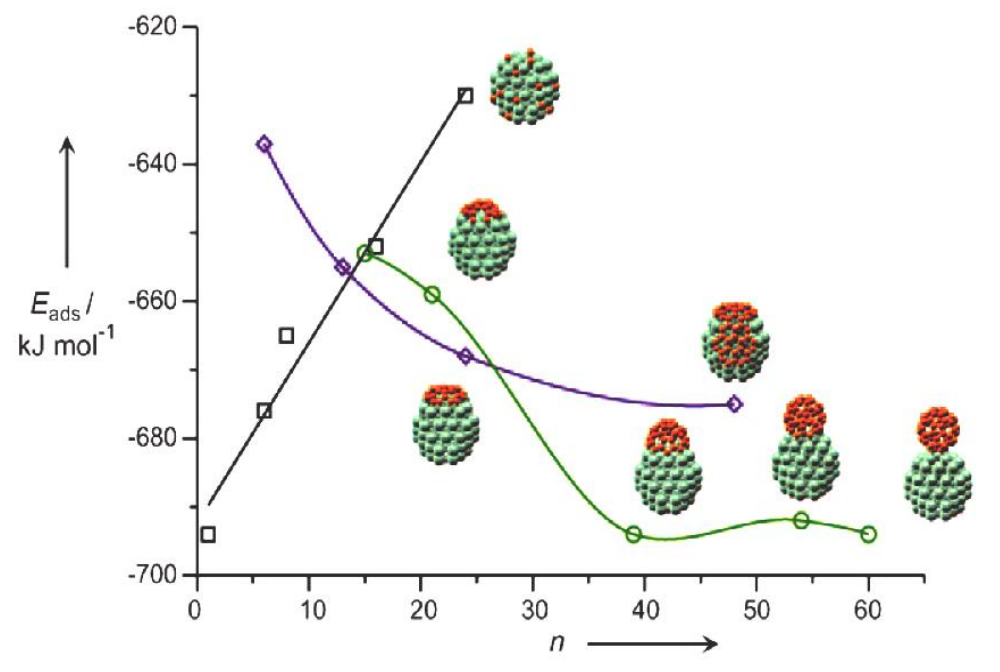

Figura 2.11. Estructuras de carbono sobre una partícula de $P t_{79}$. La gráfica muestra las energías de adsorción $E_{\text {ads }}$ por átomo de carbono en función del número $n$ de átomos de carbono de tres patrones distintos: átomos completamente dispersos (cuadrados), estructuras tipo grafeno (rombos) y estructuras tipo fullereno (círculos). Las esferas naranjas $y$ verdes representan átomos de carbono y platino respectivamente. Tomado de la referencia 92.

En base al hecho de que el $\mathrm{C}_{60}$, como la mayoría de los compuestos orgánicos, se convierte en $\mathrm{CO}_{2}$ bajo condiciones oxidantes, Chen y Lou ${ }^{93}$ propusieron sintetizar $\mathrm{C}_{60}$ mediante la reacción inversa, es decir la reducción de $\mathrm{CO}_{2}$ con litio metálico, obteniendo un rendimiento del $0.2 \%$ (Esquema 2.14). Estos autores demostraron que la conversión de $\mathrm{CO}_{2}$ a $\mathrm{C}_{60}$ depende altamente de la temperatura y presión, obteniendo diferentes productos como diamante, nanotubos de carbono, nanoesferas o carbono amorfo al modificar los intervalos de ambas variables. Además, sugieren que especies como carbenos, radicales metilo o radicales aniónicos de tipo $\mathrm{CO}_{2}{ }^{\bullet}$ actúan como intermediarios de la formación de fullerenos por este método. Aunque podría pensarse en el empleo de este método como una fuente verde de fullerenos al emplear el $\mathrm{CO}_{2}$ formado en diversos procesos industriales, los elevados costos lo convierten en una opción inviable.

Por otra parte, Chuvilin y colaboradores proponen la formación de fullerenos a partir de grafeno, empleando microscopía de transmisión electrónica (TEM). ${ }^{94}$ Durante el análisis de láminas de grafeno usando TEM, observaron que cuando la intensidad de los haces de electrones era de 80 $\mathrm{keV}$, los bordes de las láminas de grafeno se modificaban, dando lugar a la formación de $\mathrm{C}_{60}$. La energía del haz de electrones empleado se transfiere a los átomos de carbono causando la fractura de las láminas de grafeno y la producción de fullerenos. 


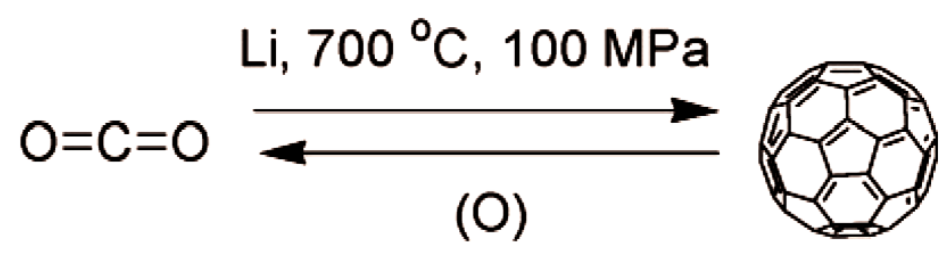

Esquema 2.14. Formación de $\mathrm{C}_{60}$ mediante la reducción de $\mathrm{CO}_{2}$ con litio metálico. Tomado de la referencia 93.

Los métodos tradicionales de síntesis de fullerenos no son muy eficientes, por lo que nuevas metodologías deben ser implementadas. Por estas razones, el estudio de nuevos métodos de síntesis es un factor clave en la evolución de la ciencia y tecnología de fullerenos.

Gracias al vanguardista trabajo de Scott y colaboradores, ha sido posible la síntesis del $\mathrm{C}_{60}$ siguiendo una serie de procesos químicos, sin embargo, aún no se ha descubierto un procedimiento práctico para la síntesis de fullerenos. En las palabras del propio Scott: "Claramente, aún queda un gran camino por recorrer antes de que la síntesis rutinaria de fullerenos, isoméricamente puros sea considerada un problema resuelto". ${ }^{95}$

En la actualidad la síntesis de fullerenos continúa siendo un proceso muy costoso que ha resistido todos los intentos de escalamiento, permitiendo solamente la síntesis de algunas decenas de gramos de $\mathrm{C}_{60}$ por hora. Los fullerenos mayores se producen en cantidades aún más pequeñas y son por lo tanto aún más costosos. 


\section{Capítulo 3: \\ Nuevas estrategias de síntesis}

Como mencionamos anteriormente, es necesario el desarrollo de nuevos métodos de síntesis que conduzcan a fullerenos isoméricamente puros y en grandes cantidades, para su aplicación en los nuevos materiales y las nuevas tecnologías basadas en fullerenos. Esta nueva estrategia no debería incluir la vaporización de carbono ni técnicas pirolíticas para la introducción de la curvatura, ya que en el primer caso, los bajos rendimientos y las dificultades para separar los productos obtenidos hacen la síntesis de fullerenos ineficiente y costosa, ${ }^{96}$ mientras que en el segundo caso el tratamiento pirolítico dificulta el escalamiento.

Una estrategia particularmente seductora es la polimerización de fragmentos de fullerenos, en especial la dimerización de dos hemifullerenos idénticos $\left(\mathrm{C}_{30} \mathrm{H}_{\mathrm{X}}\right)^{97-100}$ En este caso, en el que la curvatura ya se encuentra en los fragmentos, el principal reto consiste en encontrar el método para unir los fragmentos y dar lugar al fullereno. Obviamente, la elección de los fragmentos a utilizar es un paso clave para el éxito de esta metodología. Geneste y col., ${ }^{80}$ encontraron 9 fragmentos isométricos $C_{30}$ derivados de $C_{60}$ usando el método de "La Coupe du Roi" ${ }^{81}$ y determinaron que el triindenotrifenileno 1 y el pentaciclopentacoranuleno $\mathbf{2}$ eran los fragmentos más estables (Figura 3.1).

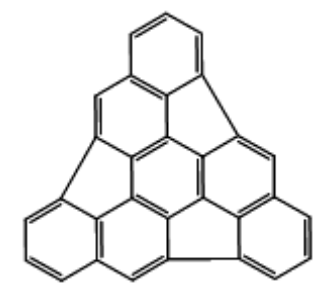

1

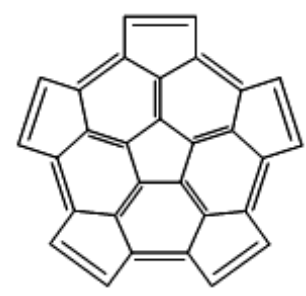

2

Figura 3.1. Triindenotrifenileno 1 y pentaciclopentacorenuleno 2. 
Observando cuidadosamente la estructura de los hemifullerenos y la forma en que se aproximan para unirse (Figura 3.2), podemos ver la formación de nuevos ciclos de 6 miembros, por lo que la cicloadición de Diels-Alder (DA) puede ser una forma efectiva de unir ambos fragmentos. Recientemente, Scott y colaboradores ${ }^{101}$ propusieron el uso de la reacción de DA para sintetizar nanotubos de carbono (CNT) de diámetro uniforme y una sola quiralidad, en solución. La adición de acetileno como dienófilo en los bordes de un fragmento de fullereno hemisférico o un cinturón aromático, conduce a la formación del CNT mediante la formación de nuevos anillos de 6 miembros. ${ }^{102}$ Calcularon la energía de activación $\left(E_{a}\right)$ para la adición de acetileno a diferentes hidrocarburos aromáticos y observaron que la $E_{a}$ descendía a medida que el tamaño de la molécula aromática aumentaba. Propusieron también la reacción de DA entre 7,14dimesitilbisanteno y acetileno para probar experimentalmente la metodología y encontraron que la reacción procede de forma relativamente fácil. Posteriormente propusieron el uso de un bencino como dienófilo para adicionar dos anillos de 6 miembros al esqueleto del nanotubo a la vez. ${ }^{103}$ Esta evidencia nos sugiere el uso de la reacción de DA en la síntesis de $\mathrm{C}_{60}$.

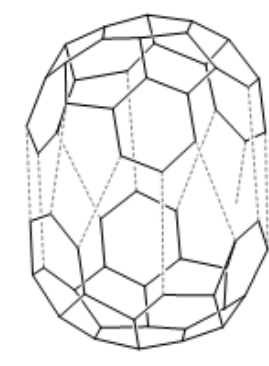

$1+1$

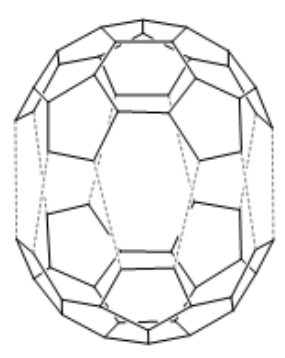

$2+2$

Figura 3.2. Dimerización de dos fragmentos de tipo $1(\mathbf{1 + 1})$ y dos fragmentos de tipo $\mathbf{2}(\mathbf{2 + 2})$.

\subsection{Reacciones de Diels-Alder}

La cicloadición de Diels-Alder es una de las reacciones orgánicas mejor conocidas y más ampliamente usadas para construir anillos de 6 miembros de manera regio- y estéreo-controlada, lo que la transforma en una reacción muy versátil que permite la obtención de una gran variedad de moléculas simples y complejas. ${ }^{104}$

La reacción de Diels-Alder se lleva a cabo entre un dieno conjugado y un dienófilo (Esquema 3.1). Los dienos conjugados reaccionan cuando los dobles enlaces tienen o pueden asumir una 
geometría cisoide. Los dienos cíclicos son generalmente más reactivos que los de cadena abierta. El efecto electrónico debido a la presencia de sustituyentes en el dieno tiene efectos directos sobre la velocidad de reacción. ${ }^{105}$ La presencia de grupos electrodonadores en el dieno acelera la reacción con dienófilos sustituidos con grupos electroatractores, ${ }^{106}$ mientras que dienos sustituidos con grupos electroatractores aumentan la velocidad de reacción cuando interactúan con dienófilos sustituidos con grupos electrodonadores. ${ }^{107}$ Las reacciones que son insensibles al efecto del sustituyente se denominan neutrales.

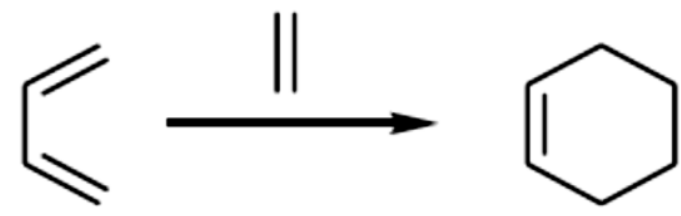

Esquema 3.1. Ejemplo de una reacción de cicloadición de Diels-Alder. Reacción entre el dienófilo y el dieno para dar lugar al cicloaducto. Tomado de la referencia 104.

La reacción se clasifica como una cicloadición $\left[{ }_{\pi} 4_{s} t_{\pi} 2_{s}\right]$, donde los números 4 y 2 identifican tanto el número de electrones $\pi$ involucrados en la reacción como el número de átomos que dan origen al nuevo anillo de 6 miembros. El subíndice $s$ indica que la reacción se lleva a cabo suprafacialmente en ambos componentes. La reacción de Diels-Alder puede ser intermolecular o intramolecular y puede ocurrir bajo una gran variedad de condiciones experimentales, además, cuando en el dieno y/o dienófilo hay uno o más heteroátomos la reacción se denomina heteroDiels-Alder. En las reacciones de Diels-Alder el proceso de formación de enlaces es concertado, pudiendo ser sincrónico cuando la formación de los nuevos enlaces ocurre simultáneamente ${ }^{108} \mathrm{o}$ asincrónico cuando uno de los nuevos enlaces se forma antes que el otro. ${ }^{109}$

La regioselectividad de estas reacciones se ha explicado en términos del efecto electrónico de los sustituyentes, quienes orientan el ataque de los reactivos generando cargas parciales, positivas y negativas, en el dieno y en el dienófilo. Generalmente, mientras mayor sea el efecto electrónico de los sustituyentes, mayor será la regioselectividad de la reacción. Por otro lado, en las reacciones de Diels-Alder se preserva la estereoquímica relativa de los sustituyentes presentes en el dieno y dienófilo. 
La estereoquímica relativa de los sustituyentes en los nuevos centros estereogénicos del aducto formado está determinada por dos posibles aproximaciones espaciales de los reactivos denominadas endo y exo. En la aproximación endo el dieno y dienófilo se colocan uno sobre el otro, contrario a la aproximación exo donde los reactivos se orientan hacia lados opuestos. Mientras que la orientación exo permite reducir la tensión estérica en el aducto formado, las reacciones endo son preferidas debido a la presencia de interacciones orbitálicas secundarias que estabilizan los estados de transición (Esquema 3.2).

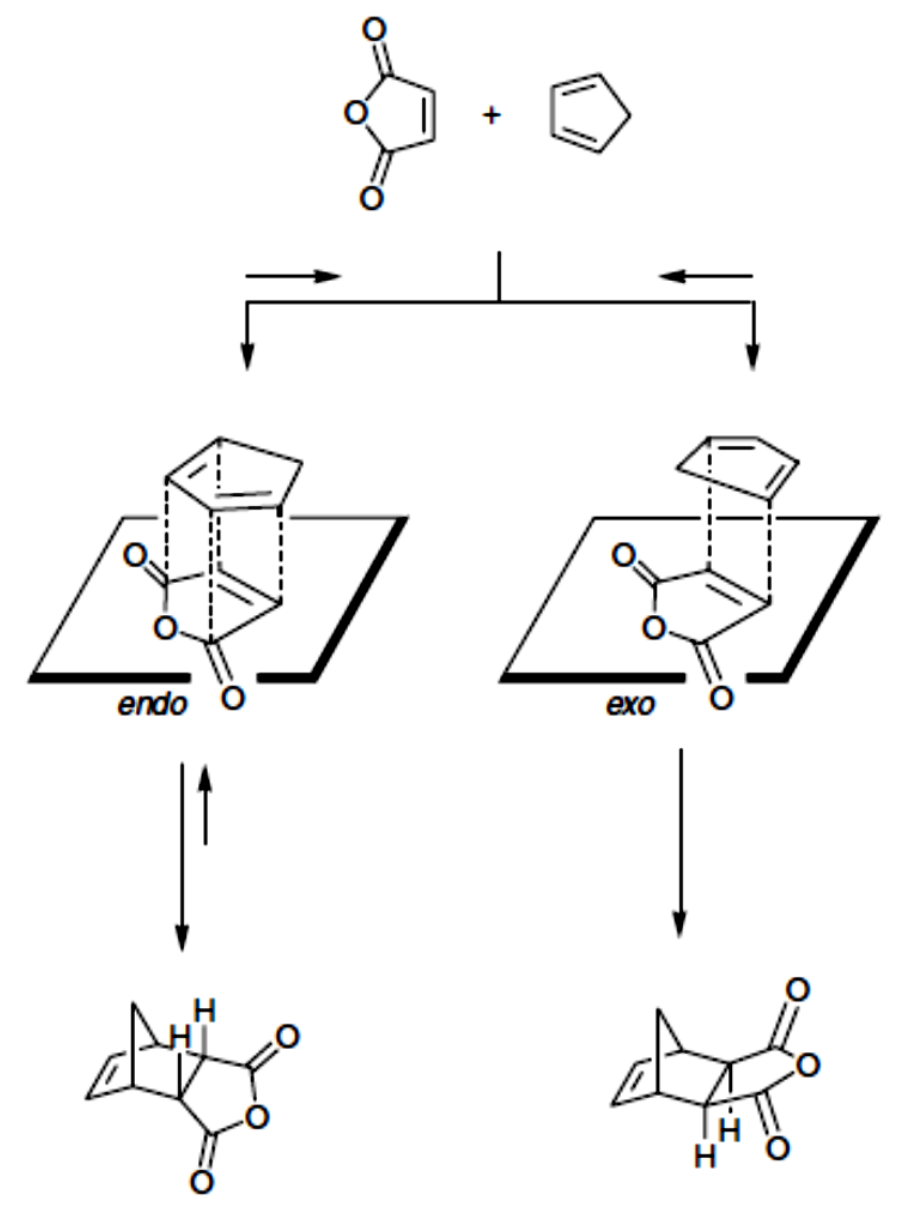

Esquema 3.2 Reacción de Diels-Alder entre el ciclopentadieno y el anhídrido maleico. Se observan los aductos endo y exo, así como la estabilización del estado de transición endo debida a interacciones orbitálicas secundarias. Tomado de la referencia 104.

La reacción de Diels-Alder es reversible y la dirección de cicloadición es favorecida debido a que dos enlaces $\pi$ son sustituidos por dos enlaces $\sigma$. La cicloreversión ocurre cuando el dieno o el dienófilo son especies particularmente estables o cuando uno de ellos puede removerse o ser 
consumido por una reacción subsecuente. La retro Diels-Alder usualmente requiere altas temperaturas para sortear la alta barrera de activación propia de la cicloreversión.

En términos de la teoría de orbitales frontera (FMO) la reactividad, regioselectividad y estereoselectividad de las reacciones de Diels-Alder son controladas por interacciones suprafacielas en fase entre el Orbital Ocupado de Mayor Energía (HOMO) de un componente y el Orbital Desocupado de Menor Energía (LUMO) del otro (Figura 3.3). Estos orbitales son los más cercanos en energía. Cuando la interacción se lleva a cabo entre el HOMO del dieno y el LUMO del dienófilo, la reacción se denomina reacción de DA de demanda electrónica normal (NED); en cambio si la reacción se produce entre el HOMO del dienófilo y el LUMO del dieno, la reacción se llama reacción de DA de demanda electrónica inversa (IED). ${ }^{110}$

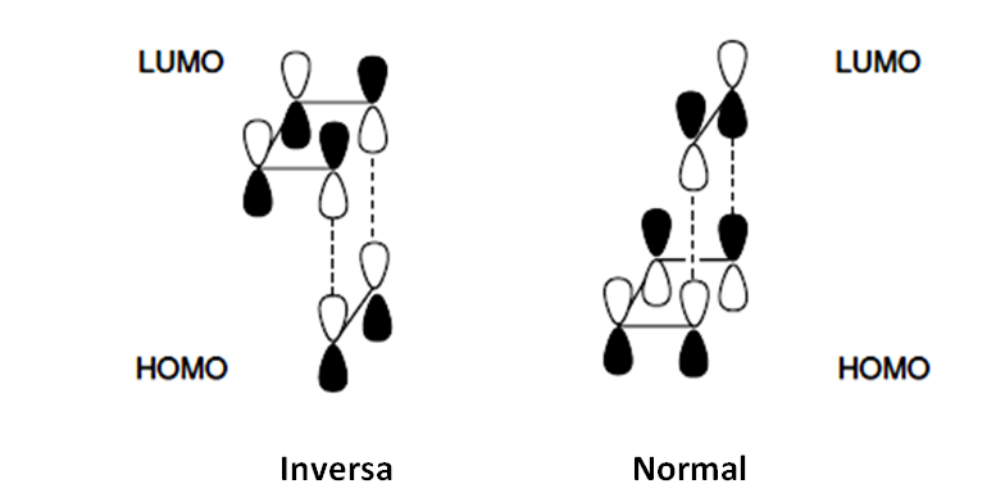

Figura 3.3. Interacciones orbitálicas dominantes en las reacciones de Diels-Alder. Tomado de la referencia 104.

La reactividad de una reacción de Diels-Alder depende de diferencia de energía entre los oribitales HOMO y LUMO (gap HOMO-LUMO) de los reactivos: mientras menor sea el gap HOMO-LUMO, menor será la energía del estado de transición. La presencia de sustituyentes electroatractores disminuye la energía de los orbitales HOMO y LUMO, mientras que los sustituyentes electrodonadores aumentan su energía. Las reacciones NED son favorecidas por la presencia de sustituyentes electrodonadores en el dieno y electroatractores en el dienófilo, mientras que sustituyentes elecrodonadores en el dienófilo y electroatractores en el dieno favorecen las reacciones IED. Las reacciones de demanda neutra son insensibles al efecto electrónico de los sustituyentes.

La regioselectividad está determinada por la superposición de los orbitales con los mayores coeficientes (lobulos más grandes en la Figura 3.4). Mientras mayor sea la diferencia entre los 
coeficientes de los orbitales de los átomos involucrados en la reacción, más regioselectiva será la cicloadición. Como se observa en la Figura 3.4 los dos nuevos enlaces $\sigma$ se forman en un solo paso, pero debido a la diferencia de tamaño de los orbitales uno se forma antes que el otro, por lo tanto las reacciones de Diels-Alder en las que participan reactivos no simétricos son concertadas pero asincrónicas. Mientras más asimétricos sean los reactivos, más asincrónicos serán los estados de transición observados.

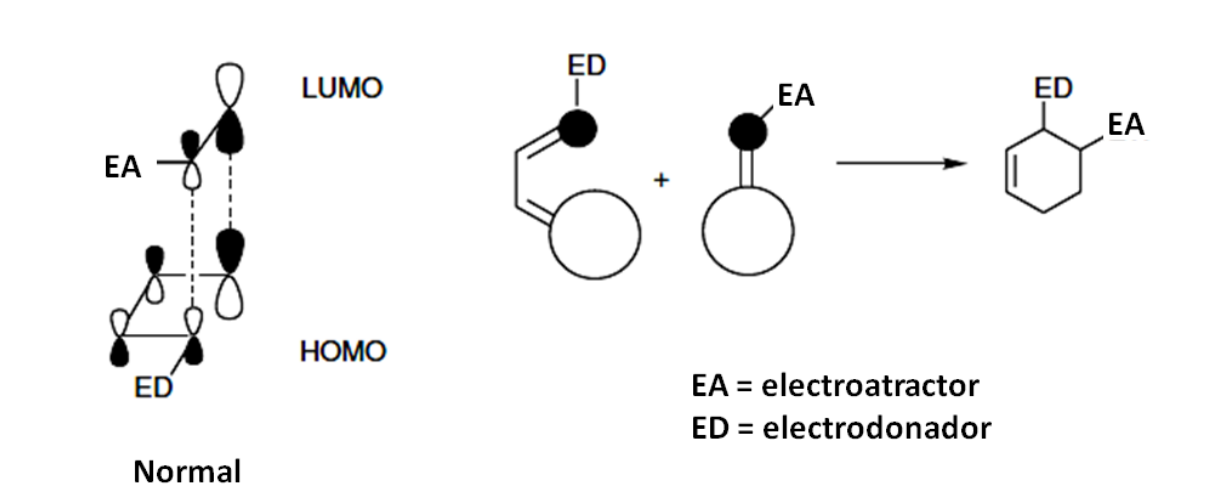

Figura 3.4. Interacciones orbitálicas en reacciones de Diels-Alder con reactivos no simétricos. Tomado de la referencia 104.

La FMO explica la preferencia de la aproximación endo al considerar interacciones no enlazantes adicionales. Las interacciones orbitálicas secundarias no dan lugar a la formación de nuevos enlaces, sin embargo, contribuyen a disminuir la energía del estado de transición endo con respecto al exo (Figura 3.5). Mientras más grandes sean los lóbulos de los orbitales interactuantes, mayor sea el traslape, más fuerte la interacción y más favorecida la formación del aducto endo.

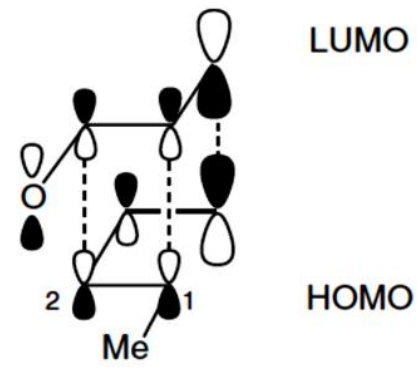

Figura 3.5. Interacciones orbitálicas secundarias en la reacción entre piperileno y acroleína. Tomado de la referencia 104. 
En las reacciones de cicloadición fotoquímicas un componente es excitado electrónicamente como consecuencia del movimiento de un electrón del HOMO al HOMO*. En este caso la interacción se lleva a cabo entre los orbitales HOMO*-LUMO* del componente en estado excitado y los orbitales HOMO-LUMO del otro componente en estado basal. Estas interacciones son enlazantes en cicloadiciones [2+2], pero antienlazantes en reacciones de Diels-Alder [4+2] (Figura 3.6). De acuerdo con las reglas de Woodward-Hoffmann las reacciones de Diels-Alder son térmicamente permitidas y fotoquímicamente no permitidas.
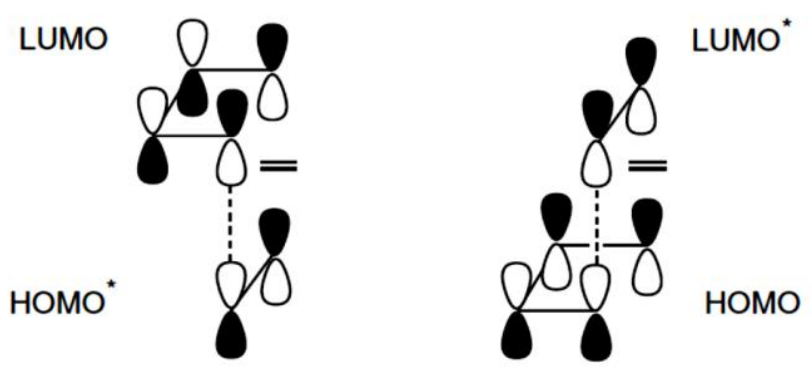

Figura 3.6. Reacciones de Diesl-Alder fotoquímicas no permitidas por simetría. Tomado de la referencia 104.

\subsection{Objetivos}

\subsubsection{Generales}

Analizar la síntesis de fulerenos por dimerización

Determinar la factibilidad del uso de la reacción de DA en la síntesis de fulerenos. 


\subsubsection{Especificos}

Determinar el uso de la reacción de Diels-Alder para aumentar el tamaño de los fragmentos 1 y 2.

Determinar el carácter de dieno y dienófilo de los hemifulerenos $\mathbf{1}$ y $\mathbf{2}$.

Analizar la reacción de Diels-Alder entre el etileno y los fragmentos 1 y 2 .

Analizar la reacción de Diels-Alder entre el butadieno y los fragmentos 1 y

2.

Determinar el efecto de la presencia de grupos electrodonadores en los fragmentos $\mathbf{1}$ y $\mathbf{2}$ sobre los parámetros cinéticos y termodinámicos de las reacciones propuestas.

Determinar el efecto de la presencia de grupos electroatractores en los fragmentos $\mathbf{1}$ y $\mathbf{2}$ sobre los parámetros cinéticos y termodinámicos de las reacciones propuestas.

Analizar la dimerización del fragmento $\mathbf{2}$ por medio de cicloadiciones de Diels-Alder.

Analizar los parámetros de reactividad derivados de la teoría de funcionales de la densidad de los fragmentos 1 y 2 sustituidos y $\sin$ sustituir.

\subsection{Metodología}

Para probar que la síntesis de $\mathrm{C}_{60}$ por dimerización de hemifullerenos mediada por reacciones de Diels-Alder es factible, debemos empezar por determinar la factibilidad de una cicloadición sobre los bordes de los hemifullerenos y si estos actúan como dienos o dienófilos (Figura 3.4). Analizando la estructura de los fragmentos 1 y 2 encontramos regiones con 2 y 4 electrones $\pi$ 
conjugados (regiones rojas y azules), que podrían reaccionar con dienos y dienófilos. El dieno por excelencia es el butadieno y el etileno es el dienófilo más sencillo, por lo que usaremos estas moléculas para probar la reacción de Diels-Alder sobre los fragmentos y determinar si los fragmentos prefieren actuar como dienos o como dienófilos.

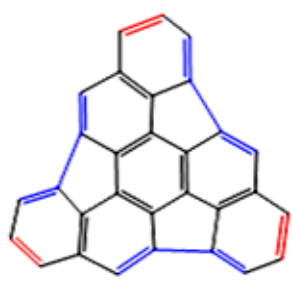

1

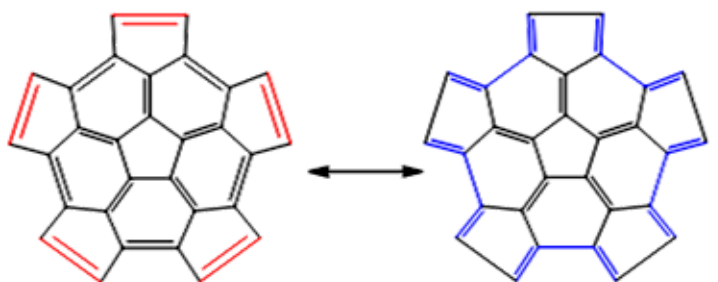

2b

Figura 3.4. Regiones de comportamiento diénico (azul) y dienofílico (rojo) de los fragmentos $\mathbf{1}$ y 2.

La reacción de Diels-Alder procede de manera más efectiva en sistemas activados, por lo que, una vez determinada la factibilidad de la reacción sobre los bordes de los fragmentos $\mathbf{1}$ y $\mathbf{2}$, activaremos los fragmentos al sustituirlos con grupos electroatractores y electrodonadores. El análisis de las reacciones con los fragmentos sustituidos nos permitirá encontrar el sustituyente adecuado que mejore los parámetros de reacción y haga más sencilla la cicloadición.

En reacciones de Diels-Alder los estados de transición endo son favorecidos debido a la presencia de interacciones orbitálicas secundarias, por lo que analizaremos solamente las aproximaciones endo de las reacciones propuestas.

Finalmente, se llevará a cabo el análisis de la dimerización de dos hemifullerenos para dar lugar al $\mathrm{C}_{60}$. Propondremos las reacciones involucradas en la dimerización en base a los resultados obtenidos en los análisis anteriores, escogiendo el o los fragmentos con mejores desempeños.

Los análisis propuestos se basan en la realización de cálculos de estructura electrónica de los fragmentos. En estos cálculos empleamos el método B3LYP y la función de base 6-31G (d, p), ampliamente utilizados en el estudio de fullerenos y fragmentos de fullerenos, como en el análisis de la reactividad de $C_{60}, C_{70}, C_{58}$ y coranuleno, al describir los sitios reactivos en estas moléculas ${ }^{111}$ o en la descripción de la reactividad local empleando el descriptor dual, ${ }^{112}$ así como en el análisis de la reacción entre el $\mathrm{C}_{60}$ y el radical $\mathrm{OH}^{113}$ y las interacciones entre metales alcalinos y moléculas curvas identificadas como fragmentos de $\mathrm{C}_{60} \cdot{ }^{114}$ Este nivel de cálculo describe satisfactoriamente las reacciones de DA sobre fullerenos y fragmentos de fullerenos, se ha utilizado en el estudio de la reacción entre ciclopentadieno y $\mathrm{C}_{60}{ }^{114}$ en el cálculo de energías orbitálicas y estados de 
transición de las reacciones entre fuleroides, metanofullerenos y $\mathrm{C}_{60}$ con ciclopentadieno y 2,3dimetil-1,3-butadieno ${ }^{116}$ o en el análisis de reacciones de cicloadición de butadieno y dipolos 1,3 a moléculas curvas, fullerenos y nanotubos, ${ }^{117}$ en el que los autores mencionan que el método B3LYP asociado a la función de base $6-31 G(d)$ proveen una descripción confiable de los mecanismos de reacción de las reacciones pericíclicas. Además, este método presenta buena concordancia con los parámetros geométricos obtenidos por difracción de rayos $\mathrm{X}$ de este tipo de estructuras. $^{118}$

Adicionalmente a la evidencia bibliográfica encontrada, realizamos una serie de cálculos con diferentes métodos para probar la validez del método escogido. Debido a la falta de información experimental de los fragmentos escogidos, analizamos los parámetros cinéticos obtenidos experimentalmente para reacción de Diels-Alder entre etileno y butadieno, ${ }^{119,120}$ con los obtenidos usando los funcionales B3LYP, PBE, PBE0, M06L y M06, así como el método MP2. Los valores obtenidos se muestran en la Tabla 1.3, podemos observar que el método que mejor describe al sistema, en términos de los valores experimentales, es B3LYP.

Tabla 3.1. Comparación de los valores experimentales de energía de activación $E_{a}$ energía libre de Gibbs estándar de activación $\Delta G^{\ddagger}$, entalpia estándar de activación $\Delta H^{\ddagger}$, y entropía estándar de activación $T \Delta S^{\ddagger}$ de la reacción de Diels-Alder entre etileno y butadieno, con los calculados con distintos métodos

\begin{tabular}{|c|c|c|c|c|}
\hline Método & $\begin{array}{c}\text { Ea } \\
\mathrm{kcal} / \mathrm{mol}\end{array}$ & $\begin{array}{c}\Delta^{\circ} \mathrm{G}^{\mp} \\
\mathrm{kcal} / \mathrm{mol}\end{array}$ & $\begin{array}{c}\Delta^{\circ} \mathrm{H}^{\mp} \\
\mathrm{kcal} / \mathrm{mol}\end{array}$ & $\begin{array}{c}\mathrm{T} \Delta^{\circ} \mathrm{S}^{\ddagger} \\
\mathrm{kcal} / \mathrm{mol}\end{array}$ \\
\hline B3LYP & 24.80 & 36.35 & 23.41 & -12.93 \\
\hline PBE & 15.72 & 27.09 & 14.47 & -12.62 \\
\hline M06L & 19.87 & 31.28 & 18.56 & -12.71 \\
\hline M06 & 20.89 & 32.35 & 19.53 & -12.82 \\
\hline PBEO & 19.34 & 30.85 & 18.00 & -12.85 \\
\hline MP2 & 19.39 & 30.98 & 18.00 & -12.98 \\
\hline Exp & $\begin{array}{c}27.5^{a} \\
24.2-26.7^{b} \\
25.9^{c}\end{array}$ & --- & $\begin{array}{l}24.2^{d} \\
24.9^{e}\end{array}$ & $\begin{array}{l}-13.32^{f} \\
-12.76^{g}\end{array}$ \\
\hline $\begin{array}{l}{ }^{a} E_{a} \text { Experim } \\
\text { experiment }\end{array}$ & $\begin{array}{l}{ }^{\mathrm{b}} \mathrm{E}_{\mathrm{a}} \text { Experim } \\
\text { a calculada ; }\end{array}$ & $\begin{array}{r}\text { la ciclorev } \\
\text { erimental ; } \\
\text { calculad }\end{array}$ & $\begin{array}{l}\text { ciclohexanc } \\
\text { ulada; }{ }^{\mathrm{f}} \mathrm{T} \Delta \mathrm{S}\end{array}$ & $\begin{array}{l}\text { or de reacción } \\
\text { nental, }{ }^{\mathrm{g}} \mathrm{T} \Delta \mathrm{S}\end{array}$ \\
\hline
\end{tabular}


Todas las geometrías fueron optimizadas y se calcularon los parámetros cinéticos y termodinámicos. Los estados de transición (TS) fueron localizados utilizando la opción QST2 y se comprobaron las frecuencias vibracionales de cada estructura (una sola frecuencia imaginaria en los TS y todas las frecuencias reales para las estructuras de mínima energía). Las frecuencias vibracionales se calculan determinando la segunda derivada de la energía con respecto a las coordenadas nucleares.

Empleando dichas frecuencias es posible calcular la función de partición canónica Z que permite obtener la energía del sistema en términos de la función de Helmholtz ( $F=-k T / n Z)$ a partir de la cual se calculan las propiedades termodinámicas (ver Apéndice A). ${ }^{121}$ Las propiedades cinéticas se obtienen tomando en cuenta la energía del estado de transición y de los productos y reactivos asociados al mismo.

Las coordenadas intrínsecas de reacción fueron determinadas a partir de los estados de transición correspondientes. Las geometrías se optimizaron en cada punto a lo largo de la coordenada de reacción y las constantes de fuerza se calcularon en cada caso. Las secciones hacia reactivos y productos fueron calculadas independientemente. ${ }^{122}$

En el caso del análisis de la dimerización del fragmento 2 se empleó el método de impulso de banda elástica (NEB) ${ }^{85}$ para generar las coordenadas de reacción propias de la dimerización. Las estructuras obtenidas de esta forma se optimizaron y se emplearon en la búsqueda de estados de transición adecuados.

Los cálculos de estructura electrónica y el cálculo de los parámetros cinéticos y termodinámicos se realizó empleando el programa Gaussian, ${ }^{123}$ mientras que los cálculos de coordenadas de reacción empleando el método NEB se realizaron con el programa DACAPO. ${ }^{124}$ 


\section{Capítulo 4. Análisis cinético y termodinámico}

\subsection{Primera parte: Análisis de la reacción de Diels-Alder entre los fragmentos 1 y 2 y etileno o butadieno.}

Basados en las estructuras de los fragmentos $\mathbf{1}$ y $\mathbf{2}$ y en las regiones en que los fragmentos se comportan como dieno o dienófilo, se propusieron dos reacciones base. En el primer caso la reacción de DA se produce entre 1,3-butadieno como el dieno y los fragmentos $\mathbf{1}$ y $\mathbf{2}$ como dienófilos (Figura 4.1., 1 y 2a). En el segundo caso la reacción de DA se lleva a cabo entre etileno como dienófilo y los fragmentos 1 y 2 actuando como dienos (Figura 4.1., 1 y 2b).

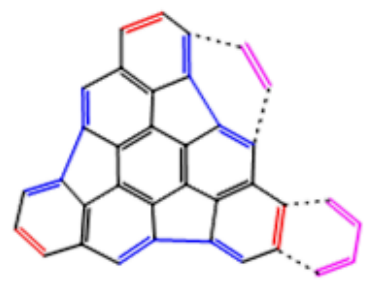

1

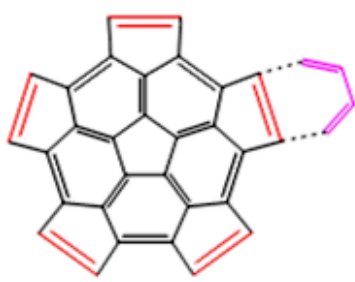

2a

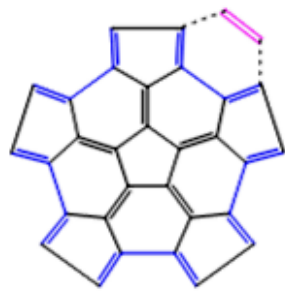

$2 \mathrm{~b}$

Figura 4.1. Reacción de DA de los fragmentos 1 y 2 con un dieno (1,3-butadieno) en las regiones con carácter de dienófilo (rojo) y con un dienófilo (etileno) en las regiones con carácter de dieno (azul).

\subsubsection{Análisis de Orbitales Frontera}

En la Teoría de Orbitales Frontera (FMO) la reacción de DA se describe como la interacción del Orbital Ocupado de Mayor Energía (HOMO) de una de las moléculas interactuantes, con el Orbital Desocupado de Menor Energía (LUMO) de la otra. Cuando la interacción se lleva a cabo entre el HOMO del dieno y el LUMO del dienófilo, la reacción se denomina reacción de DA de demanda electrónica normal (NED); en cambio si la reacción se produce entre el HOMO del dienófilo y el LUMO del dieno, la reacción se llama reacción de DA de demanda electrónica inversa (IED). ${ }^{110}$ La 
disminución en la diferencia de energía ente los dos orbitales involucrados (HOMO-LUMO gap) favorece la reacción.

Las energías de los orbitales HOMO y LUMO del etileno, butadieno y de los fragmentos $\mathbf{1}$ y $\mathbf{2}$ se presentan en la Tabla 4.1. La Tabla 4.2 muestra el gap HOMO-LUMO para las reacciones NED $\left(\Delta \mathrm{E}_{\mathrm{NED}}=\mathrm{E}_{\text {LUMOdienófilo }}-\mathrm{E}_{\text {HOMOdieno }}\right)$ y IED $\left(\Delta \mathrm{E}_{\mathrm{IED}}=\mathrm{E}_{\text {LUMOdieno }}-\mathrm{E}_{\text {HOMOdienófilo }}\right)$ así como la diferencia $\delta \Delta \mathrm{E}=\Delta \mathrm{E}_{\text {IED }}$ $\Delta \mathrm{E}_{\mathrm{NED}}$. Valores de $\delta \Delta \mathrm{E}<0$ indican que la reacción IED es favorecida mientras que valores de $\delta \Delta \mathrm{E}>0$ indican que la reacción NED es preferida.

Tabla 4.1. Energías de los orbitales HOMO y LUMO (en eV) del etileno, 1,3-butadieno y los fragmentos 1 y 2.

\begin{tabular}{ccc}
\hline Molécula & $\mathbf{E}_{\text {Hомо }}$ & $\mathbf{E}_{\text {LUMо }}$ \\
\hline Etileno & $-10.11(-10.07)^{\mathrm{a}}$ & $4.88(4.87)^{\mathrm{a}}$ \\
Butadieno & $-8.77(-8.82)^{\mathrm{a}}$ & $3.45(3.59)^{\mathrm{a}}$ \\
$\mathbf{1}$ & -7.28 & 1.38 \\
$\mathbf{2}$ & -7.18 & 0.67 \\
\hline \multicolumn{3}{c}{${ }^{\text {a }}$ tomado de la referencia 125 } \\
\hline
\end{tabular}

Podemos observar que los gap HOMO-LUMO de la reacción entre butadieno (b) y etileno (e) (reacción de referencia) son más grandes que los gaps para las reacciones que involucran a los fragmentos 1 y 2; por lo tanto, estas reacciones deberían proceder más fácilmente. Adicionalmente, las reacciones entre $\mathbf{b}$ o e con el fragmento $\mathbf{2}$ son preferidas sobre las reacciones correspondientes con el fragmento $\mathbf{1}$. Además, las reacciones entre los fragmentos $\mathbf{1}$ y $\mathbf{2}$ con $\mathbf{b}$ son preferidas sobre las reacciones entre los fragmentos $\mathbf{1}$ y $\mathbf{2}$ con $\mathbf{e}$.

Tabla 4.2. Gaps HOMO-LUMO (en eV) de las reacciones NED y IED de los fragmentos 1 y 2 con etileno y 1,3-butadieno y de la reacción entre etileno y 1,3-butadieno como referencia. $\Delta E_{N E D}$

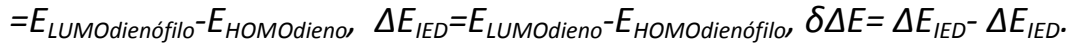

\begin{tabular}{cccc}
\hline Reacción & $\Delta \mathrm{E}_{\mathrm{NED}}$ & $\Delta \mathrm{E}_{\text {IED }}$ & $\boldsymbol{\delta} \mathrm{E}$ \\
\hline $\mathbf{e}+\mathbf{b}$ & $13.66(13.69)^{\mathrm{a}}$ & $13.56(13.66)^{\mathrm{a}}$ & -0.09 \\
$\mathbf{1}+\mathbf{e}$ & 12.17 & 11.49 & -0.67 \\
$\mathbf{2}+\mathbf{e}$ & 12.06 & 10.78 & -1.27 \\
$\mathbf{1}+\mathbf{b}$ & 10.15 & 10.73 & 0.58 \\
$\mathbf{2}+\mathbf{b}$ & 9.45 & 10.63 & 1.18 \\
\hline
\end{tabular}




\subsubsection{Parámetros cinéticos}

Los estados de transición de las reacciones propuestas se muestran en la Figura 4.2 y las distancias de transición $r_{1}$ y $r_{2}$, definidas como las distancias entre el butadieno y etileno o entre los fragmentos $\mathbf{1}$ y $\mathbf{2}$ y butadieno o etileno en el estado de transición, se muestran en la Tabla 4.3. Valores pequeños de $\Delta r=r 1-r 2$ sugieren reacciones sincrónicas, mientras que valores grandes de $\Delta r$ sugieren reacciones asincrónicas. Las reacciones de e sobre la superficie cóncava de los fragmentos $\mathbf{1}$ y $\mathbf{2}$ son las más asincrónicas, seguidas por las reacciones de b sobre las superficies cóncava y convexa del fragmento $\mathbf{1}$. La reacción de $\mathbf{b}$ sobre la superficie convexa del fragmento $\mathbf{2}$ es totalmente sincrónica $(\Delta r=0)$, al igual que la reacción entre e y $\mathbf{b}$, además las distancias de transición de ambas reacciones son iguales $(2.27 \AA$ A $)$ y concuerdan con las calculadas por Houk et al. ${ }^{126}$ para la reacción de e+b.

Tabla 4.3. Distancias de transición $r_{1}$ y $r_{2}$ (en Å) de los estados de transición mostrados en la figura 4.2. $\Delta r=r_{1}-r_{2}$.

\begin{tabular}{cccc}
\hline Reacción & $\boldsymbol{r}_{\mathbf{1}}$ & $\boldsymbol{r}_{\mathbf{2}}$ & $\boldsymbol{\Delta r}$ \\
\hline e + b & 2.27 & 2.27 & 0 \\
& $(2.21)^{\mathrm{a}}$ & $(2.21)^{\mathrm{a}}$ & \\
$\mathbf{1}+$ e cóncava & 2.09 & 2.07 & 0.02 \\
$\mathbf{2}+$ e cóncava & 2.07 & 2.06 & 0.01 \\
$\mathbf{1}+$ e convexa & 2.41 & 1.68 & 0.73 \\
$\mathbf{2}+$ e convexa & 2.31 & 1.63 & 0.67 \\
$\mathbf{1}+$ b cóncava & 2.49 & 1.92 & 0.58 \\
$\mathbf{2}+$ b cóncava & 2.28 & 2.25 & 0.03 \\
$\mathbf{1}+$ b convexa & 2.36 & 2.02 & 0.34 \\
$\mathbf{2}+$ b convexa & 2.27 & 2.27 & 0 \\
\hline \multicolumn{4}{c}{ a tomado de la referencia 126 } \\
\hline
\end{tabular}

En la Tabla 4.4 se muestran las energías de activación $E_{a}$, energías libres de Gibbs estándar de activación $\Delta G^{\ddagger}$, entalpías de activación $\Delta H^{\ddagger}$ y entropías de activación $\Delta S^{\ddagger}$ de los estados de transición de la Figura 4.2 y para la reacción entre e y b. Las interacciones con e son favorecidas sobre la superficie cóncava de los fragmentos, mientras que las interacciones con $\mathbf{b}$ son favorecidas sobre la superficie convexa de los fragmentos. Es bien sabido que la reacción de DielsAlder entre el $\mathbf{e}$ y $\mathbf{b}$ procede experimentalmente, por lo que es un buen punto de comparación 
para el resto de las reacciones propuestas. La $E_{a}$ calculada para la reacción $\mathbf{e}+\mathbf{b}$ es de $24.82 \mathrm{kcal}$ $\mathrm{mol}^{-1}$, por lo tanto las reacciones con menor o igual energía de activación deberían proceder fácilmente. La reacción de $\mathbf{b}$ sobre la superficie convexa del fragmento $\mathbf{2}$ presenta la $E_{a}$ más pequeña, $22.61 \mathrm{kcal} \mathrm{mol}^{-1}$, lo que sugiere que esta reacción es factible. La misma reacción pero sobre la superficie cóncava presenta una $E_{a}$ mayor debido a la repulsión estérica entre $\mathbf{b}$ y la nube electrónica en la superficie cóncava del fragmento. El resto de las reacciones presentan $E_{a}$ mayores por lo que requerirían mayores cantidades de energía para proceder, eso puede traducirse en temperaturas y presiones elevadas que harían las condiciones experimentales similares a las empleadas en los métodos tradicionales de síntesis de fullerenos. Considerando las reacciones entre $\mathbf{e}$ y $\mathbf{b}$ con los fragmento $\mathbf{1}$ y $\mathbf{2}$, la reactividad general predicha por los orbitales frontera es similar a lo predicho por la $E_{a}$, exceptuando el orden relativo de las reacciones entre e y los fragmentos $\mathbf{1}$ y $\mathbf{2}$. Las reacciones en las que el fragmento $\mathbf{2}$ actúa como un dieno presentan $E_{a}$ más altas comparadas con las reacciones en las que el fragmento 1 actúa como dieno.

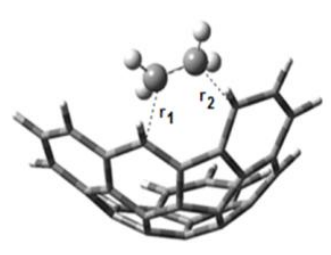

a

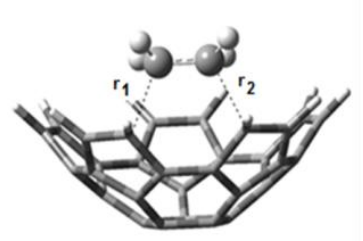

b

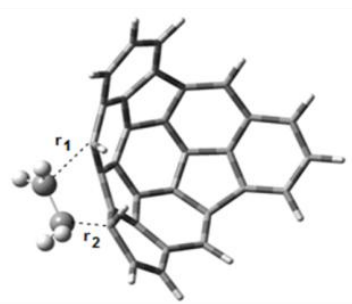

C

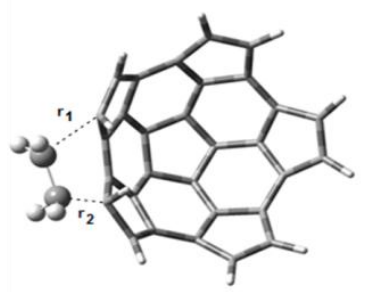

d

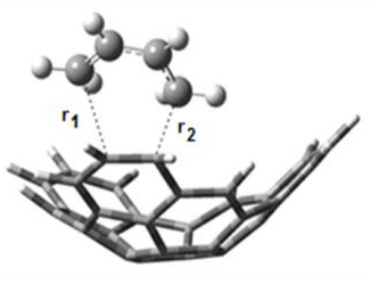

e

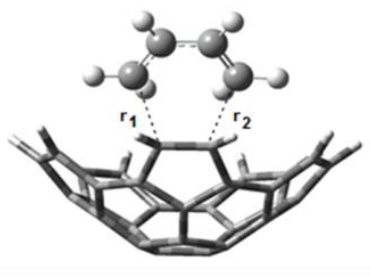

f

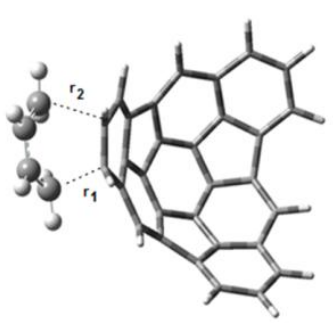

g

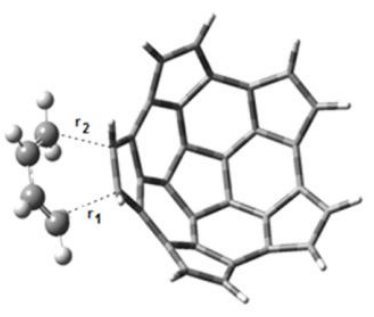

h

Figura 4.2. Estados de transición de las reacciones propuestas. a) e sobre la superficie cóncava del fragmento 1; b) e sobre la superficie cóncava del fragmento 2 ; c) e sobre la superficie convexa del fragmento 1; d) e sobre la superficie convexa del fragmento 2 ; e) b sobre la superficie cóncava del fragmento $1 ; f)$ b sobre la superficie cóncava del fragmento $2 ; g)$ b sobre la superficie convexa del fragmento $\mathbf{1} ; \mathrm{y}$ ) $\boldsymbol{b}$ sobre la superficie convexa del fragmento 2. 
Tabla 4.4. Energías de activación $E_{a}$ energía libre de Gibbs estándar de activación $\Delta G^{\ddagger}$, entalpía de activación $\Delta H^{\ddagger}$ y entropía de activación $T \Delta S^{\ddagger}$ para las reacciones de DA de e y $\boldsymbol{b}$ con los fragmentos $\mathbf{1}$ y $\mathbf{2}$, así como para la reacción de referencia $\boldsymbol{e}+\boldsymbol{b}$.

\begin{tabular}{|c|c|c|c|c|}
\hline Reacción & $\begin{array}{c}E_{a} \\
\left(\mathrm{kcal} \mathrm{mol}^{-1}\right)\end{array}$ & $\begin{array}{c}\Delta G^{\prime} \\
\left(\mathrm{kcal} \mathrm{mol}^{-1}\right)\end{array}$ & $\begin{array}{c}\Delta H^{t} \\
\left(\mathrm{kcal} \mathrm{mol}^{-1}\right)\end{array}$ & $\begin{array}{c}T \Delta S^{\prime} \\
\left(\mathrm{kcal} \mathrm{mol}^{-1}\right)\end{array}$ \\
\hline$e+b$ & $\begin{array}{c}24.8(27.5)^{\mathrm{a}} \\
(24.2-26.7)^{\mathrm{b}} \\
(25.9)^{\mathrm{c}}\end{array}$ & 36.35 & $\begin{array}{c}23.45(24.2)^{\mathrm{d}} \\
(24.9)^{\mathrm{e}}\end{array}$ & $\begin{array}{c}-12.91(-13.32)^{f} \\
(-12.76)^{\mathrm{g}}\end{array}$ \\
\hline $1+$ e cóncava & 40.71 & 52.88 & 39.62 & -13.25 \\
\hline 2 + e cóncava & 45.63 & 57.21 & 44.70 & -12.51 \\
\hline $1+$ e convexa & 67.89 & 80.22 & 66.75 & -13.47 \\
\hline $2+$ e convexa & 73.54 & 85.84 & 72.35 & -13.49 \\
\hline $1+$ b cóncava & 39.01 & 51.86 & 38.36 & -13.49 \\
\hline $2+$ b cóncava & 31.05 & 43.53 & 30.43 & -13.10 \\
\hline $1+b$ convexa & 33.11 & 46.03 & 32.47 & -13.56 \\
\hline $2+b$ convexa & 22.61 & 35.52 & 22.02 & -13.51 \\
\hline
\end{tabular}

Las coordenadas intrínsecas de reacción (IRC) de las reacciones propuestas se muestran en la Figura 4.3 donde el análisis de los mecanismos de reacción muestra que los estados de transición calculados (IRC=0) llevan a los cicloaductos DA en la dirección de "adelante" y a los reactivos en la dirección de "atrás"; además no se presentan evidencias de intermediarios o complejos activados a lo largo de las coordenadas de reacción. EI IRC mantiene las tendencias predichas por los orbitales frontera y las derivadas de la $E_{a}$. 


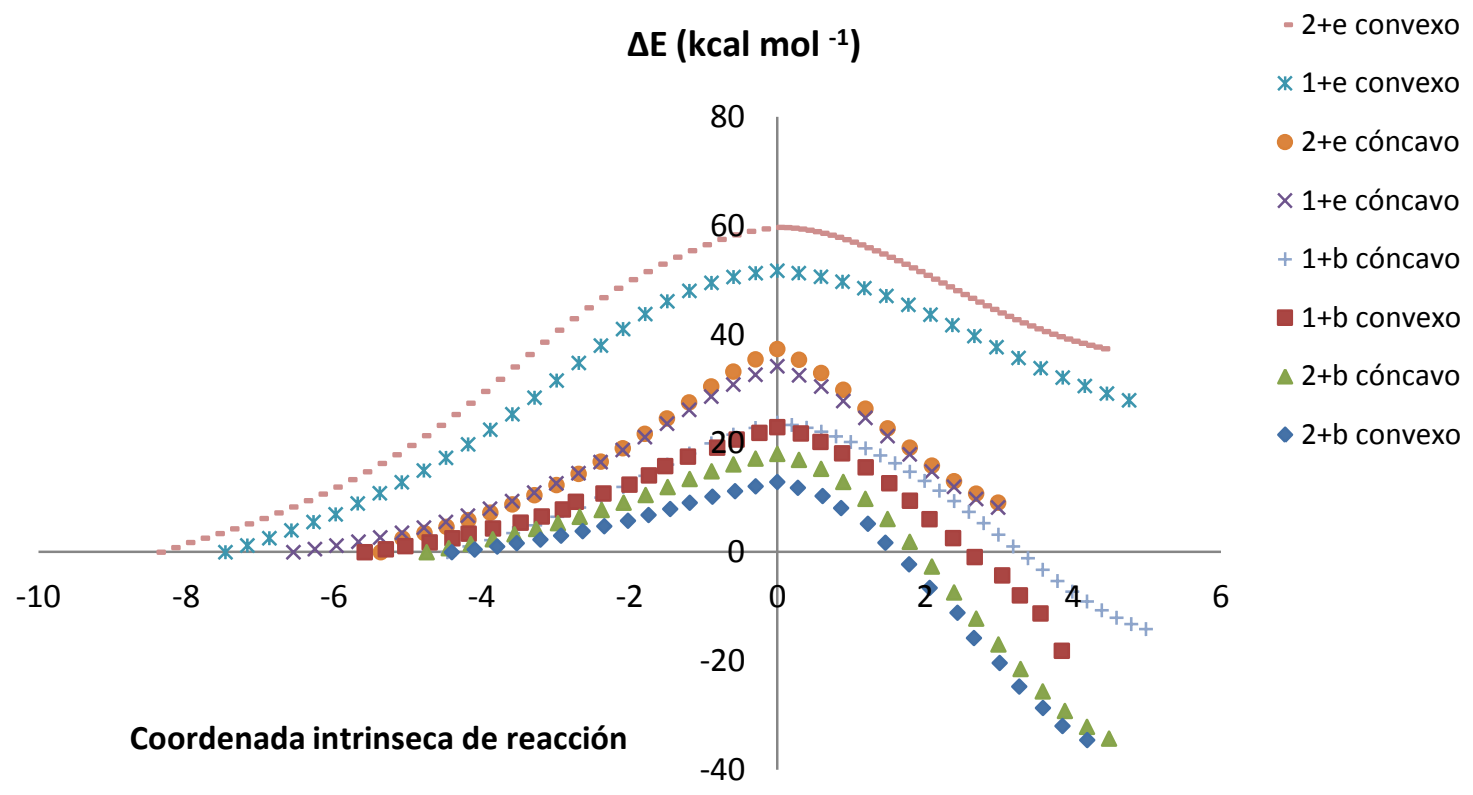

Figura 4.3. Gráfica de la energía ( $\mathrm{kcal} \mathrm{mol}^{-1}$ ) vs la coordenada intrínseca de reacción (en bohr uma $\left.{ }^{1 / 2}\right)$ para las reacciones propuestas.

\subsubsection{Parámetros termodinámicos}

Los parámetros termodinámicos de las reacciones estudiadas se muestran en la Tabla 4.5. Todas las reacciones son impedidas por entropía. Las reacciones de los fragmentos $\mathbf{1 y} \mathbf{2}$ con $\mathbf{b}$ son exotérmicas $\left(\Delta \mathrm{H}_{\mathrm{r}}\right.$ negativo), mientras que las reacciones con e son endotérmicas $\left(\Delta \mathrm{H}_{\mathrm{r}}\right.$ positivo). Solo la reacción de referencia, $\mathbf{e}+\mathbf{b}$ y la reacción entre el fragmento $\mathbf{2}$ y $\mathbf{b}$ son termodinámicamente favorecidas $\left(\Delta G_{r}<0\right)$. Este resultado refuerza la posibilidad de sintetizar fragmentos de fullerenos más grandes a partir del fragmento 2 y además sugiere la posibilidad de la dimerización del fragmento $\mathbf{2}$, es decir la reacción $\mathbf{2 + 2}$ como un método para sintetizar $\mathrm{C}_{60}$. 
Tabla 4.5. Energías libres de Gibbs estándar de reacción $\Delta \boldsymbol{G}_{r}$, entalpías de reacción $\Delta \boldsymbol{H}_{r}$ y entropías de reacción $T \Delta S_{r}$ de las reacciones propuestas.

\begin{tabular}{|c|c|c|c|}
\hline Reacción & $\Delta G_{r}\left(\mathrm{kcal} \mathrm{mol}^{-1}\right)$ & $\Delta H_{r}\left(\mathrm{kcal} \mathrm{mol}^{-1}\right)$ & $T \Delta S_{r}\left(\mathrm{kcal} \mathrm{mol}^{-1}\right)$ \\
\hline$e+b$ & -23.76 & $-37.82(-37.9)^{a}(-36.6)^{b}$ & $-14.06(-14.1)^{c}$ \\
\hline $1+$ e cóncava & 16.03 & 1.73 & -14.29 \\
\hline $2+$ e cóncava & 21.90 & 7.86 & -14.04 \\
\hline $1+$ e convexa & 49.53 & 35.36 & -14.17 \\
\hline $2+$ e convexa & 60.84 & 46.82 & -14.02 \\
\hline $1+$ b cóncava & 6.62 & -8.49 & -15.11 \\
\hline $2+$ b cóncava & -10.41 & -25.50 & -15.09 \\
\hline $1+b$ convexa & 10.34 & -4.28 & -14.62 \\
\hline $2+b$ convexa & -13.05 & -27.88 & -14.82 \\
\hline
\end{tabular}

\subsubsection{Aromatización}

Siguiendo el esquema propuesto por Scott en la síntesis de nanotubos, analizamos la reacción de deshidrogenación de los aductos formados mediante las reacciones de DA propuestas. La deshidrogenación conduce a la rearomatización de los aductos. Las reacciones de deshidrogenación se muestran en el Esquema 4.1 y las energías libre de Gibbs estándar de reacción $\Delta \mathrm{G}_{\mathrm{r}}$, entalpías de reacción $\Delta \mathrm{H}_{\mathrm{r}}$ y entropías de reacción $T \Delta \mathrm{S}_{\mathrm{r}}$ de dichas reacciones se muestran en la Tabla 4.6. Podemos observar que las reacciones con el fragmento 2 son endotérmicas ( $\Delta \mathrm{H}_{\mathrm{r}}$ positivo), excepto la reacción de e sobre la superficie convexa del fragmento, mientras que las reacciones con el fragmento 1 son exotérmicas ( $\Delta H_{r}$ negativo), excepto por la reacción de e sobre la superficie cóncava del fragmento, sin embargo, todas las reacciones son termodinámicamente favorables $\left(\Delta \mathrm{G}_{\mathrm{r}}\right.$ negativo), debido a la contribución favorable del término entrópico $\left(T \Delta \mathrm{S}_{\mathrm{r}} \approx 17 \mathrm{kcal} \mathrm{mol}^{-1}\right)$. Los valores de $\Delta \mathrm{G}_{\mathrm{r}}$ de las reacciones entre e y los fragmentos $\mathbf{1}$ y 2 son más negativos debido a la baja estabilidad de los aductos que forman. 
a
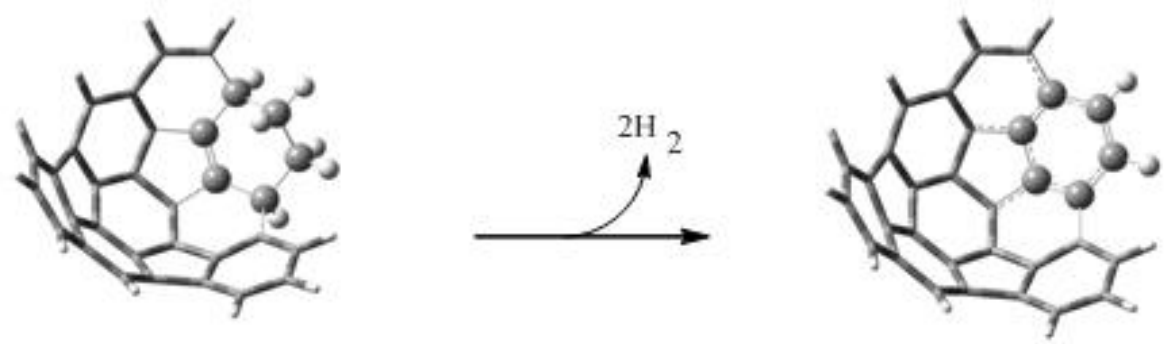

b
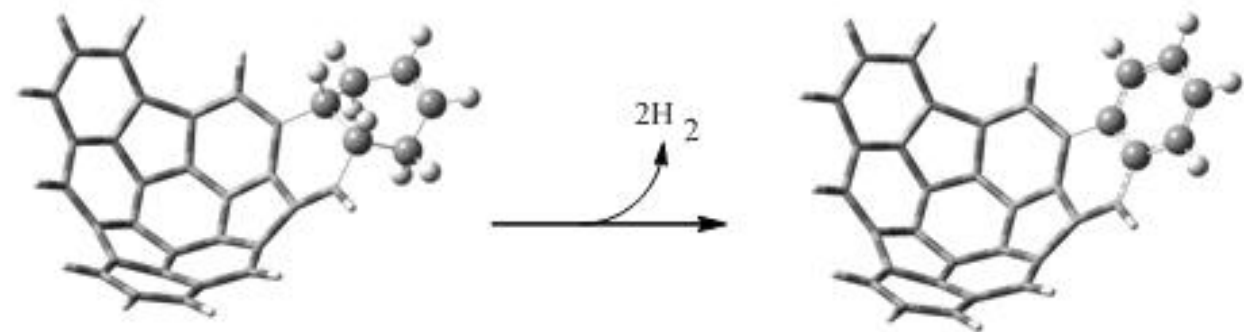

c
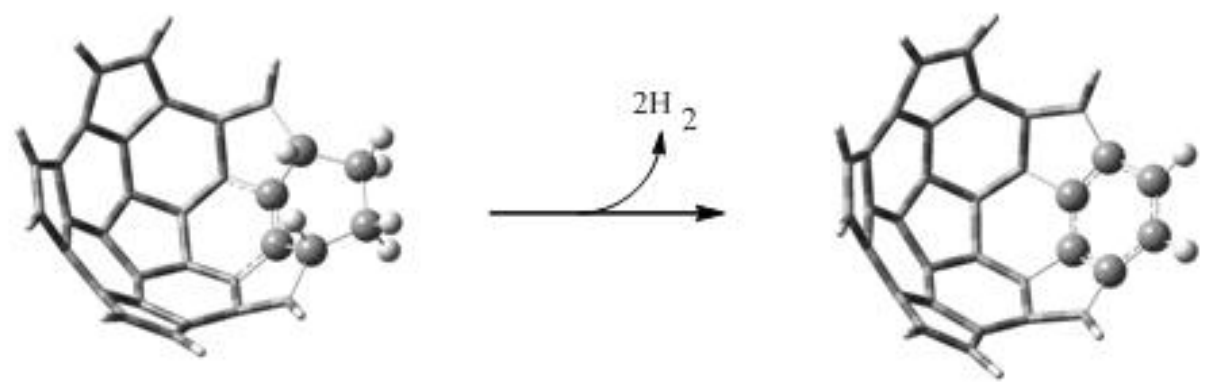

d
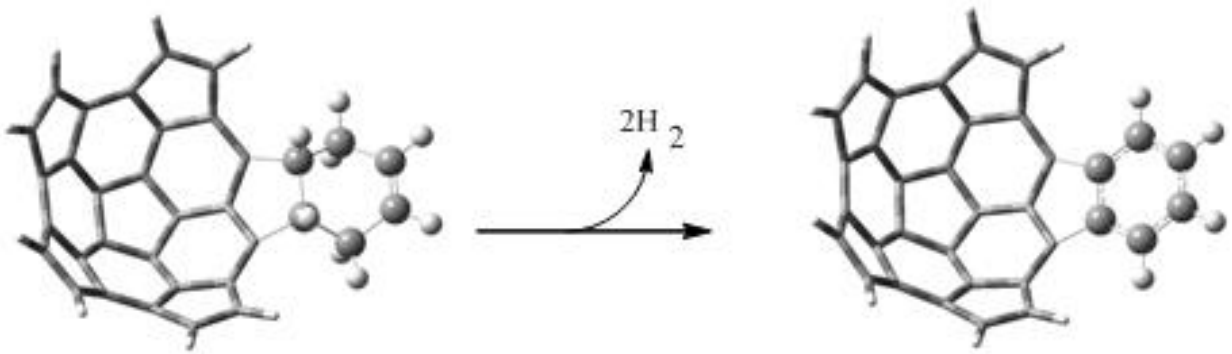

Esquema 4.1. Reacciones de deshidrogenación de los aductos formados. 
Tabla 4.6. Energías libres de Gibbs estándar de reacción $\Delta G_{r}$, entalpías de reacción $\Delta H_{r}$ y entropías de reacción $T \Delta S_{r}$ de las reacciones de deshidrogenación de los aductos formados.

\begin{tabular}{|c|c|c|c|}
\hline Sistema & $\Delta \mathrm{G}_{r}\left(\mathrm{kcal} \mathrm{mol}^{-1}\right)$ & $\Delta \mathrm{H}_{r}\left(\mathrm{kcal} \mathrm{mol}^{-1}\right)$ & $\mathrm{T} \Delta \mathrm{S}_{\mathrm{r}}\left(\mathrm{kcal} \mathrm{\textrm {mol } ^ { - 1 } )}\right.$ \\
\hline$e+b$ & 0.09 & $17.20(21.13)^{a}$ & 17.12 \\
\hline 1 + e cóncava & -11.34 & 5.93 & 17.27 \\
\hline 2 + e cóncava & -12.23 & 4.96 & 17.19 \\
\hline $1+$ e convexa & -44.85 & -27.70 & 17.15 \\
\hline $2+$ e convexa & -51.16 & -33.99 & 17.17 \\
\hline $1+$ b cóncava & -22.84 & -5.58 & 17.26 \\
\hline $2+$ b cóncava & -13.12 & 4.14 & 17.25 \\
\hline $1+b$ convexa & -26.56 & -9.80 & 16.77 \\
\hline $2+b$ convexa & -10.48 & 6.51 & 16.99 \\
\hline
\end{tabular}

\subsubsection{Conclusiones de la primera parte}

Los cálculos realizados muestran la posibilidad de utilizar la reacción de Diels-Alder como un método para incrementar el tamaño de fragmentos de fullerenos.

El análisis de los orbitales frontera indica que las reacciones en las que los fragmentos se comportan como dienófilos son preferidas y esta tendencia fue confirmada en el análisis de los parámetros cinéticos y termodinámicos. Los estados de transición indican que las interacciones del etileno son favorecidas sobre la superficie cóncava de los fragmentos, mientras que las interacciones con butadieno se prefieren sobre la superficie convexa de los fragmentos.

Aunque el fragmento $\mathbf{1}$ sea más estable que el fragmento $\mathbf{2}$ debido a su menor piramidalización, las reacciones de adición más favorables son las producidas por la interacción entre butadieno y el fragmento 2. En este caso particular, la barrera energética es menor que para las otras reacciones, además la energía libre de reacción es negativa mientras que para el resto de las reacciones es positiva. 


\subsection{Segunda parte: Análisis de la reacción de Diels-Alder entre butadieno y los fragmentos 1 y 2 sustituidos.}

Con la intención de encontrar una forma para facilitar la reacción de DA del butadieno sobre los bordes de los hemifullerenos, sustituimos los fragmentos $\mathbf{1}$ y $\mathbf{2}$ con grupos elecroatractores ( $F$, $\left.\mathrm{COOH}, \mathrm{CF}_{3}, \mathrm{CHO}, \mathrm{CN}, \mathrm{NO}_{2}\right)$ y electrodonadores $\left(\mathrm{NH}_{2}, \mathrm{OMe}, \mathrm{OH}, \mathrm{Me}\right.$, i-Pr) (Figura 4.4). Consideramos dos opciones para cada fragmento: a) por cuestiones de simetría, dos sustituyentes idénticos se colocaron en cada lado del doble enlace dienofílico $\left(R^{1}=R^{2}\right)$, y b) solamente se colocó un sustituyente en el fragmento para reducir la tensión estérica debida a la presencia de sustituyentes grandes $\left(R^{1} \neq R^{2}=H\right)$.

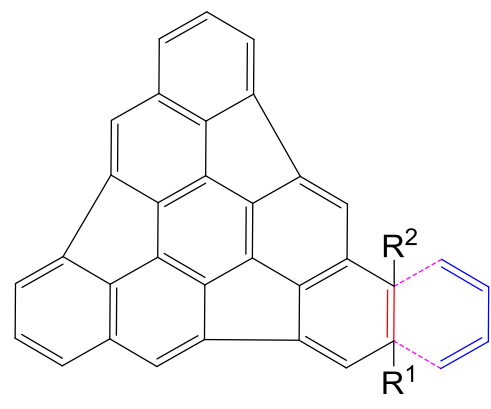

1-S

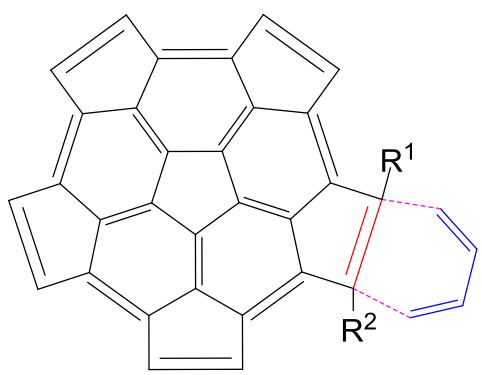

2-S

Figura 4.4. Trilndenotrifenileno 1-S y pentaciclopentacoranuleno 2-S sustituidos. a) $R^{1}=R^{2}=N H_{2}$, $\mathrm{OMe}, \mathrm{OH}, \mathrm{Me}, \mathrm{i}-\mathrm{Pr}, \mathrm{F}, \mathrm{COOH}, \mathrm{CF}_{3}, \mathrm{CHO}, \mathrm{CN}$ y NO $\mathrm{O}_{2}$ b) $\mathrm{R}^{1}=\mathrm{NH}_{2}, \mathrm{OMe}, \mathrm{OH}, \mathrm{Me}, \mathrm{i}-\mathrm{Pr}, \mathrm{F}, \mathrm{COOH}, \mathrm{CF}_{3}, \mathrm{CHO}$, $\mathrm{CN}$ y $\mathrm{NO}_{2} ; \mathrm{R}^{2}=\mathrm{H}$.

\subsubsection{Análisis de orbitales frontera}

Como mencionamos anteriormente, la reacción de DA puede ser interpretada en términos de la FMO como la interacción entre el HOMO de una de las moléculas y el LUMO de la otra. Cuando la interacción se da entre el HOMO del dieno y el LUMO del dienófilo, la reacción es llamada de demanda electrónica normal (NED), y cuando la reacción se lleva a cabo entre el HOMO del dienófilo y LUMO del dieno es llamada de demanda electrónica inversa (IED). ${ }^{110}$ La presencia de grupos electroatractores o electrodonadores en el dienófilo favorecerá la reacción NED o IED respectivamente. 
Los orbitales HOMO y LUMO de los fragmentos sustituidos se muestran en el Anexo 1. Los orbitales de los fragmentos sustituidos con $\mathrm{NH}_{2}$ y NO 2 se muestran en las Figuras 4.5 y 4.6 que corresponden a la sustitución de dos átomos de $H\left(R^{1}=R^{2}\right)$ y a la sustitución de un solo átomo de $H$ $\left(R^{1} \neq R^{2}\right)$ respectivamente. Consideramos primero la sustitución de dos hidrógenos, en la mayoría de los casos en los que los fragmentos se sustituyeron con grupos electrodonadores, el HOMO se encuentra localizado en los átomos involucrados en la reacción. Por otra parte, cuando los fragmentos son sustituidos con grupos electroatractores, son los orbitales LUMO los que se encuentran sobre los átomos involucrados en la reacción. Los fragmentos sustituidos con $\mathrm{F}$ y los fragmentos no sustituidos actúan como casos límite separando ambos grupos de sustituyentes. En estos casos se observa una considerable deslocalización de los orbitales HOMO y LUMO. En el caso de la sustitución de un solo hidrógeno, las formas de los orbitales HOMO y LUMO son similares a las observadas en los fragmentos disustituidos, sin embargo, los orbitales se encuentran menos localizados alrededor de los átomos involucrados en la reacción. Estas características concuerdan con la teoría que indica que los fragmentos sustituidos con grupos electrodonadores prefieren reacciones de tipo IEDDA, mientras que los fragmentos sustituidos con grupos electroatractores prefieren reacciones de NEDDA.

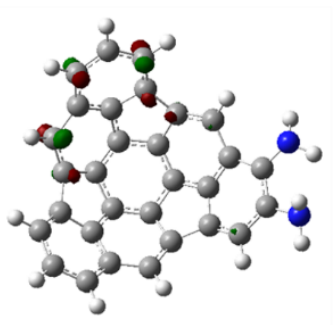

LUMO 1- $\mathrm{NH}_{2}$ (0.08 ua)

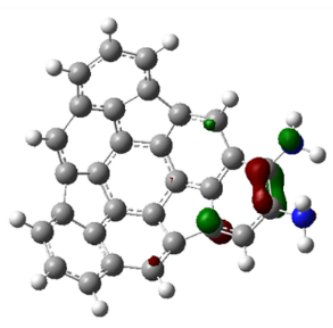

HOMO

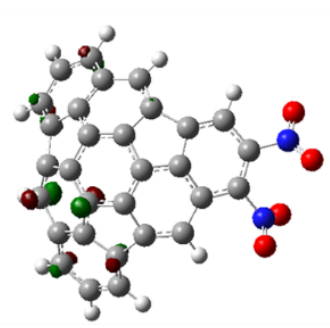

HOMO

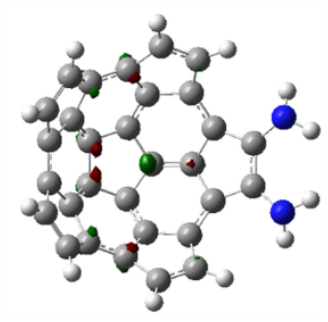

LUMO

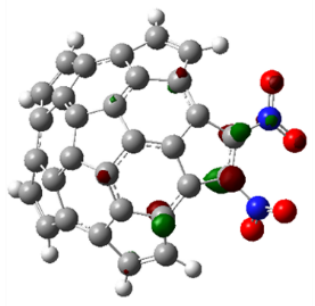

LUMO

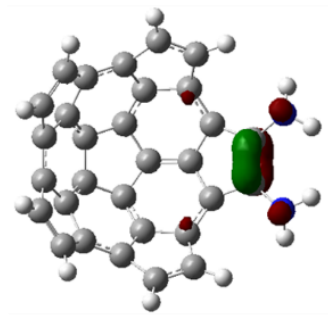

HOMO 2- $\mathrm{NH}_{2} \quad$ (0.08 ua)

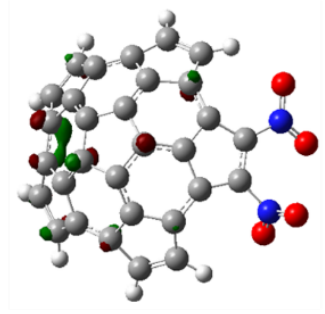

HOMO 1- $\mathrm{NO}_{2}$ (0.08 ua) 2- $\mathrm{NO}_{2}$ (0.08 ua)

Figura 4.5. Orbitales HOMO y LUMO de los fragmentos 1 y 2 sustituidos con dos grupos $\mathrm{NH}_{2}$ y $\mathrm{NO}_{2}$. 


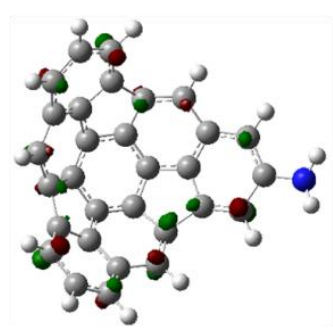

LUMO 1- $\mathrm{NH}_{2} \quad$ (0.07 ua)

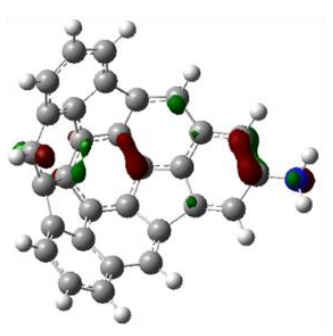

HOMO

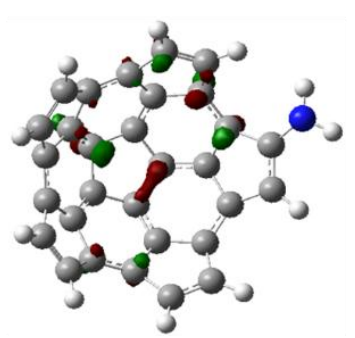

LUMO

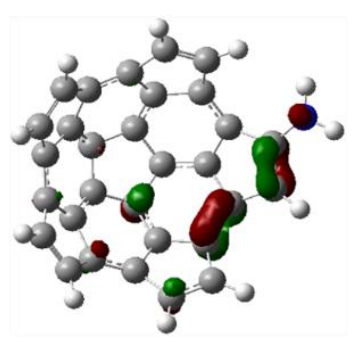

HOMO 2- $\mathrm{NH}_{2} \quad$ (0.07 ua)

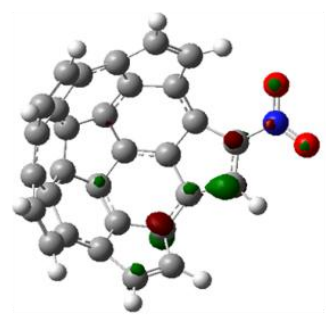

LUMO

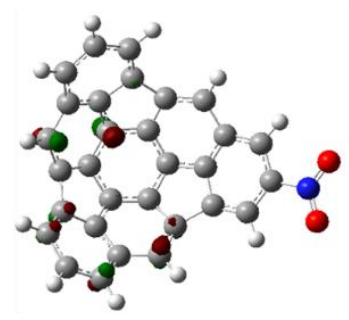

HOMO 1- $\mathrm{NO}_{2} \quad$ (0.08 ua)

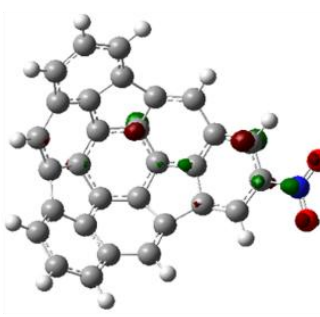

LUMO

Figura 4.6. Orbitales HOMO y LUMO de los fragmentos 1 y 2 sustituidos con un grupo $\mathrm{NH}_{2}$ y $\mathrm{NO}_{2}$.

Las energías de los orbitales HOMO y LUMO de los fragmentos sustituidos, al igual que los gaps HOMO-LUMO para las reacciones NEDDA calculadas como $\Delta \mathrm{E}_{\text {NEDDA }}=\mathrm{E}_{\text {LUMOdienófilo }}-\mathrm{E}_{\text {HOMOdieno }} \mathrm{Y}$ para las reacciones IEDDA calculadas como $\triangle \mathrm{E}_{\mathrm{IEDDA}}=\mathrm{E}_{\mathrm{LUMOdieno}}-\mathrm{E}_{\text {HOMOdienófilo }}$ se muestran en las Tablas 4.7 y 4.8. El $\delta \Delta E=\Delta E_{\text {IEDDA }}-\Delta E_{N E D D A}$ indica si la reacción NEDDA $(\delta \Delta E>0)$ o la reacción IEDDA $(\delta \Delta E<0)$ es favorecida. Como referencia, incluimos los valores para las reacciones con los fragmentos sin sustituir $\left(R^{1}=R^{2}=H\right)$.

Tres puntos importantes pueden concluirse de las Tablas 4.7 y 4.8. Primero, en las reacciones con los fragmentos sustituidos con grupos electrodonadores el gap HOMO-LUMO de la reacción IED $\left(\Delta \mathrm{E}_{\mathrm{IED}}\right)$ es menor que el $\Delta \mathrm{E}_{\mathrm{IED}}$ de las reacciones con los fragmentos sin sustituir; por otro lado, el gap HOMO-LUMO de la reacción NED $\left(\Delta \mathrm{E}_{\mathrm{NED}}\right)$ para las reacciones con los fragmentos sustituidos con grupos electroatractores es menor que $\Delta \mathrm{E}_{\mathrm{NED}}$ para las reacciones con los fragmentos no sustituidos. Cabe resaltar que, aun cuando la reacción NED es preferida en todos los casos (excepto en la reacción 1- $\mathrm{NH}_{2}$ ), la diferencia con las reacciones IED $(\delta \Delta \mathrm{E})$ es menor para los fragmentos sustituidos con grupos electrodonadores; además, el gap HOMO-LUMO de las reacciones IED es menor para éstos fragmentos mientras que el gap HOMO-LUMO para reacciones NED es menor para las reacciones con fragmentos sustituidos con grupos 
electroatractores, especialmente la reacción con los fragmentos sustituidos con grupos $\mathrm{NO}_{2}$, que presentan el gap HOMO-LUMO más pequeño. Finalmente la tendencia observada no se modifica en el caso de los fragmentos monosustituidos sin embargo, los valores de los gaps HOMO-LUMO son mayores que los obtenidos para los fragmentos disustituidos y más cercanos a los obtenidos para los fragmentos sin sustituir, lo que resalta el efecto electrónico debido a la presencia de dos sustituyentes.

Tabla 4.7. Energía de los orbitales HOMO y LUMO (en eV) del fragmento 1 mono- y disustituido y

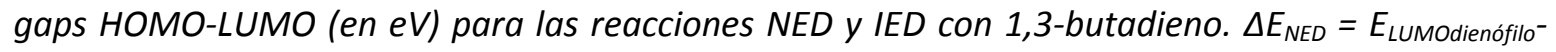
$E_{\text {HOMOdieno }}$ and $\Delta E_{I E D}=E_{\text {LUMOdieno }}-E_{\text {HOMOdienófilo, }} \delta \Delta E=\Delta E_{I E D}-\Delta E_{N E D}$. Los valores para las reacciones del fragmento sin sustituir $\left(R^{1}=R^{2}=H\right)$ se incluyen para comparación.

\begin{tabular}{|c|c|c|c|c|c|c|c|c|c|c|}
\hline \multirow[t]{2}{*}{ Reacción } & \multicolumn{2}{|c|}{$\mathbf{E}_{\text {номо }}$} & \multicolumn{2}{|c|}{$\mathrm{E}_{\mathrm{LUMO}}$} & \multicolumn{2}{|c|}{$\Delta \mathrm{E}_{\mathrm{NED}}$} & \multicolumn{2}{|c|}{$\Delta \mathrm{E}_{\text {IED }}$} & \multicolumn{2}{|c|}{$\delta \Delta \mathrm{E}$} \\
\hline & $R^{1}=R^{2}$ & $R^{1} \neq R^{2}$ & $R^{1}=R^{2}$ & $R^{1} \neq R^{2}$ & $R^{1}=R^{2}$ & $R^{1} \neq R^{2}$ & $R^{1}=R^{2}$ & $R^{1} \neq R^{2}$ & $R^{1}=R^{2}$ & $R^{1} \neq R^{2}$ \\
\hline 1- $\mathrm{NH}_{2}$ & -6.70 & -6.95 & 1.48 & 1.38 & 10.26 & 10.16 & 10.15 & 10.40 & -0.11 & 0.25 \\
\hline 1-OMe & -7.01 & -7.10 & 1.39 & 1.31 & 10.16 & 10.09 & 10.46 & 10.55 & 0.29 & 0.46 \\
\hline $1-\mathrm{OH}$ & -7.02 & -7.17 & 1.38 & 1.27 & 10.16 & 10.04 & 10.47 & 10.62 & 0.31 & 0.58 \\
\hline 1-Me & -7.13 & -7.23 & 1.42 & 1.38 & 10.20 & 10.16 & 10.58 & 10.67 & 0.39 & 0.52 \\
\hline $1-i-\operatorname{Pr}$ & -7.15 & -7.21 & 1.47 & 1.38 & 10.18 & 10.15 & 10.60 & 10.66 & 0.42 & 0.50 \\
\hline 1- $\mathrm{H}$ & \multicolumn{2}{|c|}{-7.28} & \multicolumn{2}{|c|}{1.38} & \multicolumn{2}{|c|}{10.15} & \multicolumn{2}{|c|}{10.73} & \multicolumn{2}{|c|}{0.58} \\
\hline $1-F$ & -7.50 & -7.38 & 1.08 & 1.16 & 9.42 & 9.87 & 10.96 & 10.83 & 1.53 & 0.96 \\
\hline $1-\mathrm{COOH}$ & -7.53 & -7.43 & 0.71 & 1.09 & 9.48 & 9.86 & 10.98 & 10.88 & 1.50 & 1.02 \\
\hline $1-\mathrm{CF}_{3}$ & -7.67 & -7.51 & 0.65 & 1.10 & 9.42 & 9.87 & 11.12 & 10.96 & 1.70 & 1.08 \\
\hline 1-CHO & -7.57 & -7.48 & 0.32 & 1.02 & 9.10 & 9.80 & 11.02 & 10.92 & 1.92 & 1.13 \\
\hline $1-\mathrm{CN}$ & -7.85 & -7.61 & 0.17 & 0.90 & 8.94 & 9.67 & 11.29 & 11.05 & 2.35 & 1.38 \\
\hline $1-\mathrm{NO}_{2}$ & -7.94 & -7.67 & -0.03 & 0.67 & 8.75 & 9.44 & 11.39 & 11.11 & 2.64 & 1.67 \\
\hline
\end{tabular}


Tabla 4.8. Energía de los orbitales HOMO y LUMO (en eV) del fragmento 2 mono- y disustituido y gaps HOMO-LUMO (en eV) para las reacciones NED y IED con 1,3-butadieno. $\triangle E_{N E D}=E_{L_{U M} \text { dienófilo }}$ $E_{\text {HOMOdieno }}$ and $\Delta E_{I E D}=E_{L U M O d i e n o}-E_{\text {HOMOdienófilo, }} \delta \Delta E=\Delta E_{I E D}-\Delta E_{N E D}$. LOS valores para las reacciones del fragmento sin sustituir $\left(R^{1}=R^{2}=H\right)$ se incluyen para comparación.

\begin{tabular}{|c|c|c|c|c|c|c|c|c|c|c|}
\hline \multirow[t]{2}{*}{ Reacción } & \multicolumn{2}{|c|}{$\mathrm{E}_{\text {номо }}$} & \multicolumn{2}{|c|}{$\mathrm{E}_{\text {LUMO }}$} & \multicolumn{2}{|c|}{$\Delta \mathrm{E}_{\mathrm{NED}}$} & \multicolumn{2}{|c|}{$\Delta \mathrm{E}_{\text {IED }}$} & \multicolumn{2}{|c|}{$\delta \Delta \mathrm{E}$} \\
\hline & $R^{1}=R^{2}$ & $R^{1} \neq R^{2}$ & $R^{1}=R^{2}$ & $R^{1} \neq R^{2}$ & $R^{1}=R^{2}$ & $R^{1} \neq R^{2}$ & $R^{1}=R^{2}$ & $R^{1} \neq R^{2}$ & $R^{1}=R^{2}$ & $R^{1} \neq R^{2}$ \\
\hline 2- $\mathrm{NH}_{2}$ & $\begin{array}{l}-6.51 \\
\end{array}$ & $\begin{array}{l}-6.78 \\
\end{array}$ & 0.84 & 0.72 & 9.62 & 9.49 & 9.96 & 10.23 & 0.35 & 0.73 \\
\hline 2-OMe & -7.03 & -7.06 & 0.66 & 0.62 & 9.43 & 9.39 & 10.48 & 10.51 & 1.05 & 1.12 \\
\hline $2-\mathrm{OH}$ & -6.97 & -7.05 & 0.65 & 0.53 & 9.43 & 9.31 & 10.42 & 10.50 & 0.99 & 1.20 \\
\hline 2-Me & -7.08 & -7.10 & 0.72 & 0.68 & 9.49 & 9.45 & 10.53 & 10.55 & 1.04 & 1.10 \\
\hline 2-i-Pr & -7.06 & -7.09 & 0.70 & 0.68 & 9.48 & 9.46 & 10.51 & 10.53 & 1.03 & 1.08 \\
\hline $2-\mathrm{H}$ & \multicolumn{2}{|c|}{-7.18} & \multicolumn{2}{|c|}{0.67} & \multicolumn{2}{|c|}{9.45} & \multicolumn{2}{|c|}{10.63} & \multicolumn{2}{|c|}{1.18} \\
\hline $2-F$ & -7.40 & -7.26 & 0.37 & 0.48 & 9.15 & 9.25 & 10.85 & 10.71 & 1.70 & 1.46 \\
\hline $2-\mathrm{COOH}$ & -7.40 & -7.31 & -0.01 & 0.26 & 8.77 & 9.03 & 10.85 & 10.75 & 2.08 & 1.72 \\
\hline $2-\mathrm{CF}_{3}$ & -7.62 & -7.42 & -0.05 & 0.33 & 8.72 & 9.11 & 11.07 & 10.87 & 2.35 & 1.76 \\
\hline 2-CHO & -7.48 & -7.35 & -0.37 & 0.19 & 8.40 & 8.96 & 10.93 & 10.80 & 2.53 & 1.83 \\
\hline $2-\mathrm{CN}$ & -7.79 & -7.52 & -0.52 & 0.09 & 8.24 & 8.86 & 11.24 & 10.96 & 3.00 & 2.10 \\
\hline $2-\mathrm{NO}_{2}$ & -7.91 & -7.60 & -0.73 & -0.18 & 8.04 & 8.59 & 11.36 & 11.04 & 3.31 & 2.45 \\
\hline
\end{tabular}

\subsubsection{Parámetros cinéticos}

Las estructuras de transición de las reacciones propuestas se muestran en las Figuras 4.7 y 4.8 , y las distancias de transición $\mathrm{r} 1$ y $\mathrm{r} 2$ en las Tablas 4.9 y 4.10. En general, la presencia de sustituyentes hacen la reacción más asincrónica; sin embargo, las reacciones con el fragmento 1 sustituidos con dos grupos $i-\mathrm{Pr}, \mathrm{CF}_{3}$ y $\mathrm{CN}$, y el fragmento 2 sustituido con dos $\mathrm{Me}, \mathrm{F} \mathrm{CF}_{3}$ y $\mathrm{CN}$, mantienen la sincronía de los las reacciones con los fragmentos no sustituidos. Los fragmentos monosustituidos siguen la misma tendencia que los fragmentos disustituidos y la sincronía de las reacciones con los fragmentos no sustituidos es mantenida para los mismos grupos. Estas similitudes pueden entenderse como un efecto del tamaño y forma de los sustituyentes. El átomo de $\mathrm{F}$ puede considerarse como un " $\mathrm{H}$ grande" y el grupo $\mathrm{CN}$ como un enlace "C-H grande". Por otra parte, la geometría tetraédrica de los grupos $\mathrm{CF}_{3}$ y $\mathrm{Me}$ hacen que estos sustituyentes ocupen un espacio circular homogéneo, como si se tratara de un solo átomo grande. El grupo i-Pr también presenta geometría tetraédrica pero el efecto, que podría considerarse similar al del Me, solo es observado en el fragmento 1 en el que la ubicación espacial de los dobles enlaces dienofílicos permite un mejor ajuste de los grupos sustituyentes. 

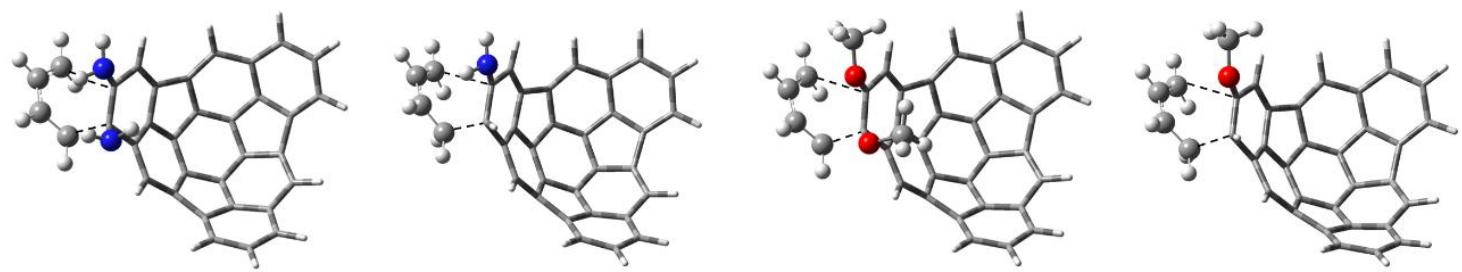

a
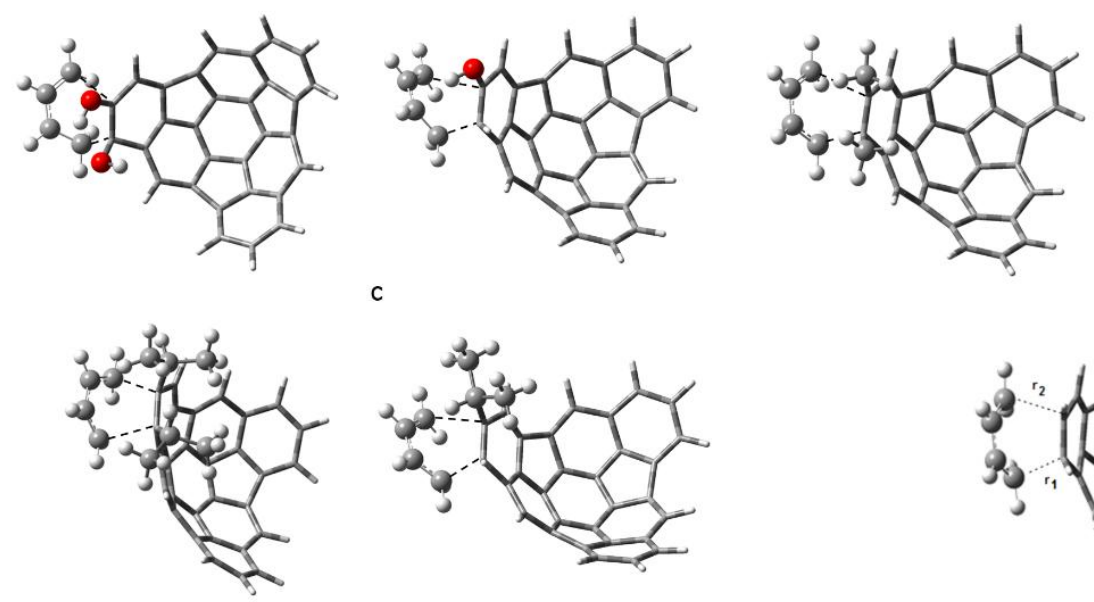

b
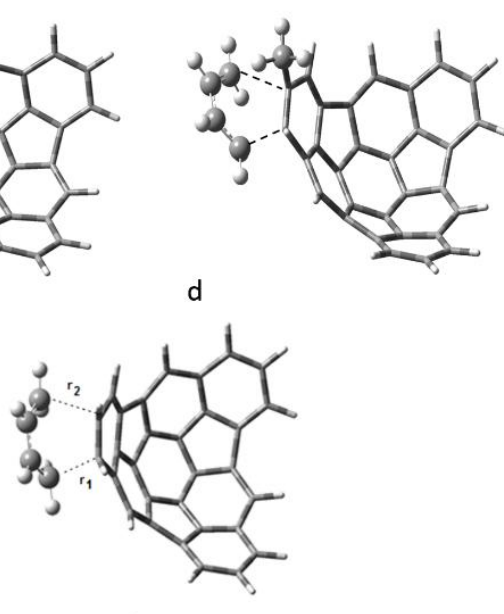

e
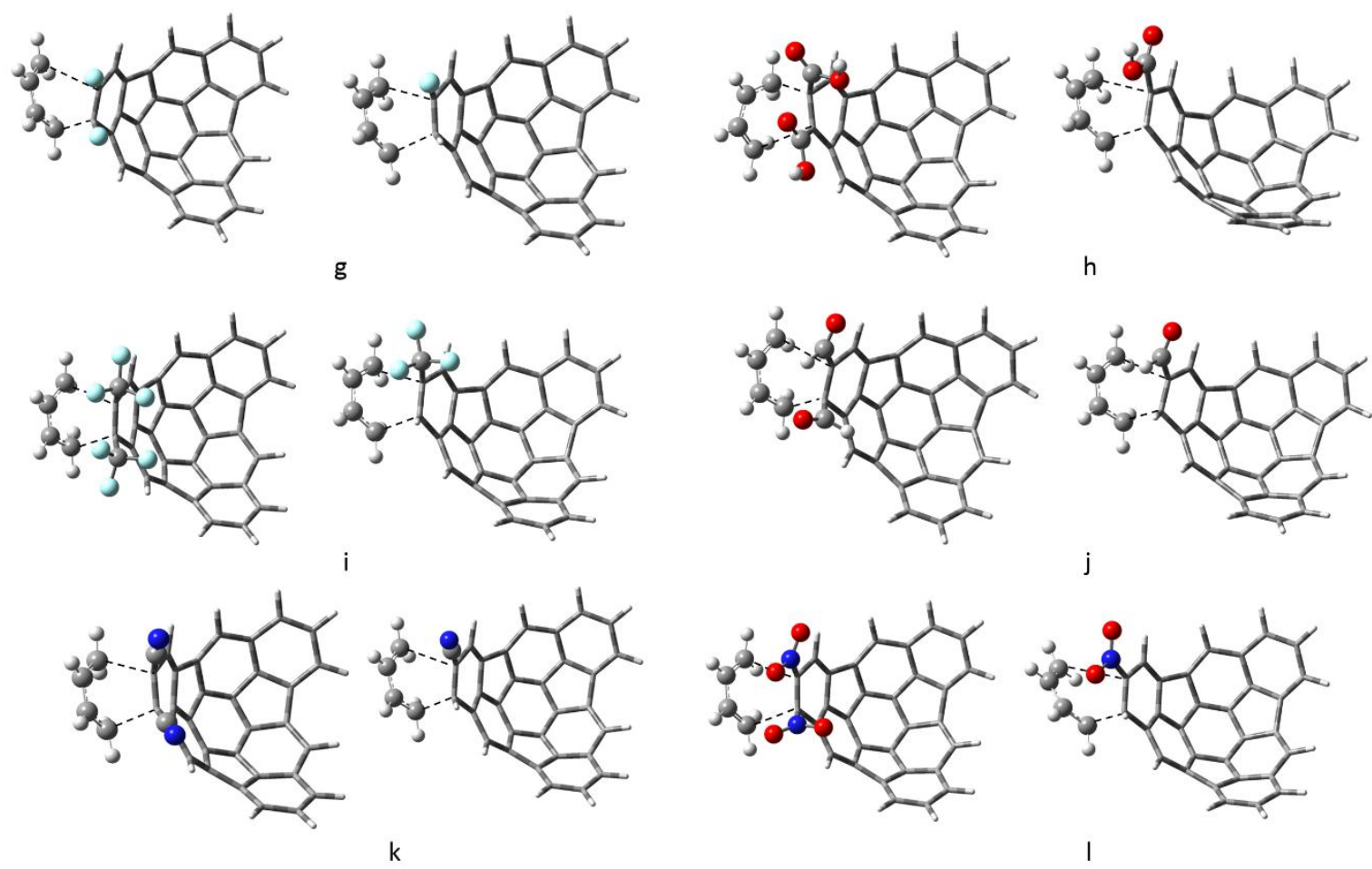

Figura 4.7. Estructuras de transición para la reacción entre el butadieno y el fragmento 1 di- y monosustituido. a) $\mathrm{NH}_{2}$, b) $\mathrm{OMe}$, c) $\mathrm{OH}$, d) $\mathrm{Me}$, e) $\left.\left.\mathrm{i}-\mathrm{Pr}, \mathrm{f}\right) \mathrm{H}, \mathrm{g}\right) \mathrm{F}$, h) $\mathrm{COOH}$, i) $\mathrm{CF}_{3}$, j) $\left.\left.\mathrm{CHO}, \mathrm{k}\right) \mathrm{CN}, \mathrm{I}\right)$ $\mathrm{NO}_{2}$ 


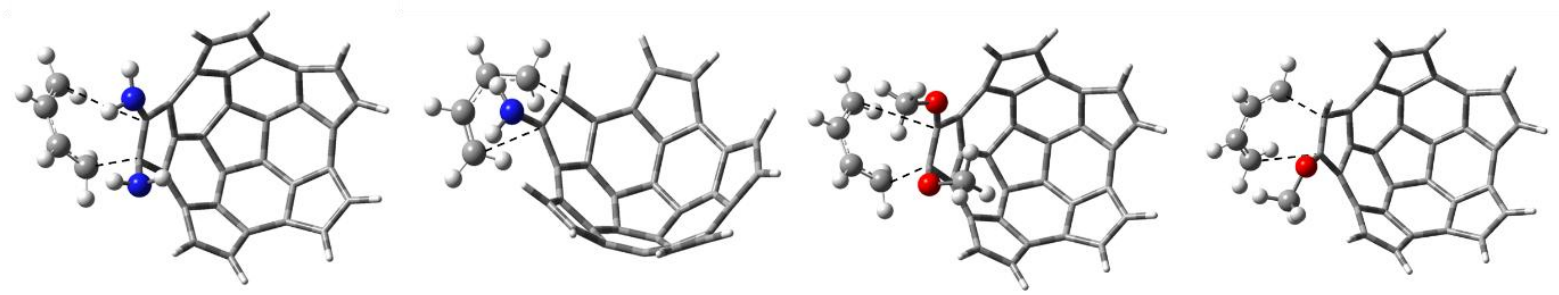

a

b
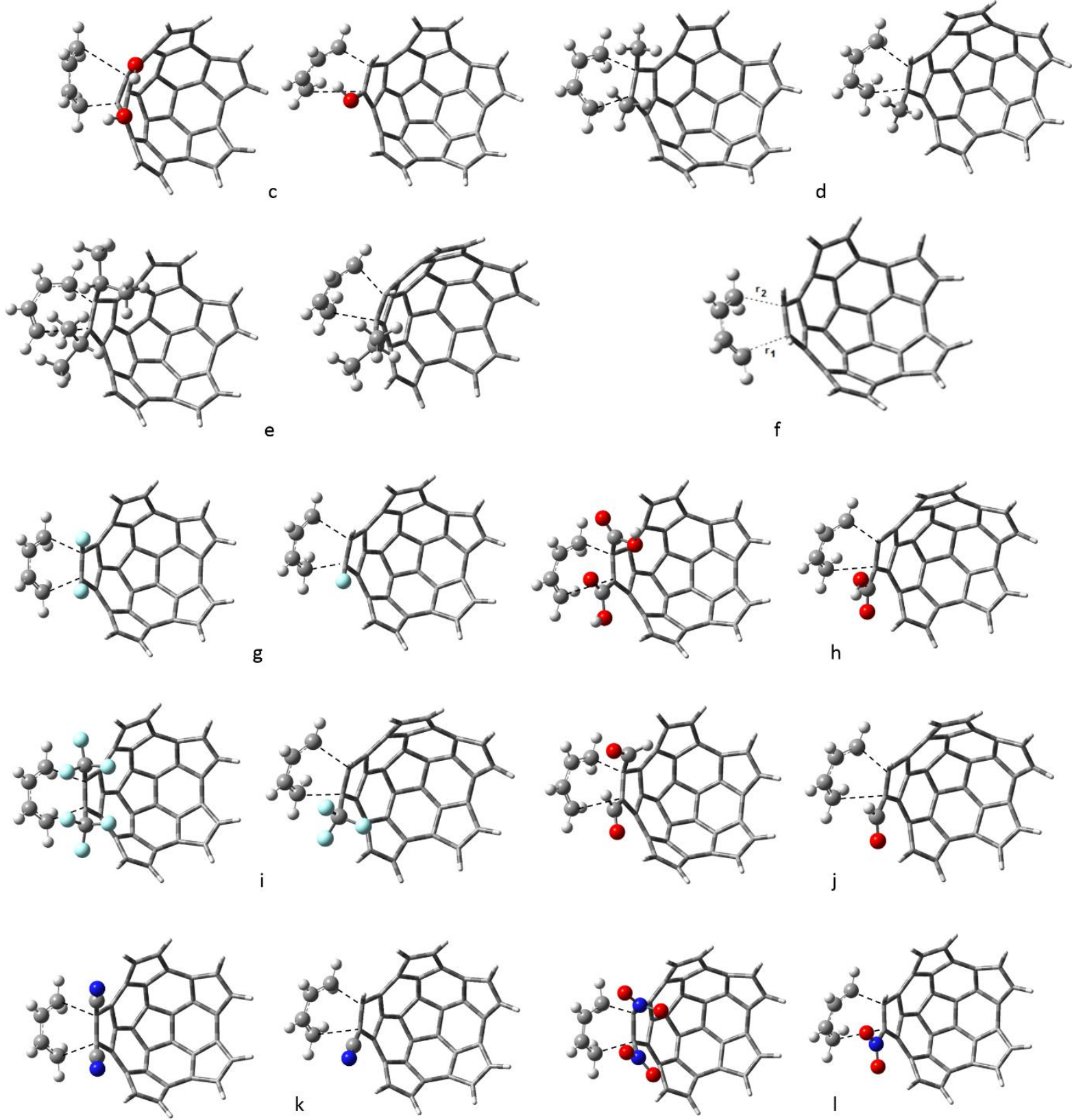

Figura 4.8. Estructuras de transición para la reacción entre el butadieno y el fragmento 2 di- y monosustituido. a) $\mathrm{NH}_{2}$, b) $\left.\mathrm{OMe}, \mathrm{c}\right) \mathrm{OH}$, d) $\mathrm{Me}$, e) $\left.\left.\mathrm{i}-\mathrm{Pr}, \mathrm{f}\right) \mathrm{H}, \mathrm{g}\right) \mathrm{F}$, h) $\mathrm{COOH}$, i) $\mathrm{CF}_{3}$, j) $\mathrm{CHO}, \mathrm{k}$ ) $\mathrm{CN}$, l) $\mathrm{NO}_{2}$ 
Tabla 4.9. Distancias $r 1$ y r2 (en Å) para los estados de transición mostrados en la Figura 4.2.6. $\Delta r=r 1-r 2$.

\begin{tabular}{|c|c|c|c|c|c|c|}
\hline \multirow{2}{*}{ TS } & \multicolumn{2}{|c|}{$r_{1}$} & \multicolumn{2}{|c|}{$r_{2}$} & \multicolumn{2}{|c|}{$\Delta r$} \\
\hline & $R^{1}=R^{2}$ & $R^{1} \neq R^{2}$ & $\mathrm{R}^{1}=\mathrm{R}^{2}$ & $R^{1} \neq R^{2}$ & $R^{1}=R^{2}$ & $\mathbf{R}^{1} \neq \mathbf{R}^{2}$ \\
\hline $1-\mathrm{NH}_{2}$ & 2.61 & 2.59 & 1.67 & 1.87 & 0.74 & 0.72 \\
\hline 1-OMe & 2.63 & 2.63 & 1.67 & 1.70 & 0.96 & 0.93 \\
\hline $1-\mathrm{OH}$ & 2.76 & 2.72 & 1.68 & 1.71 & 1.08 & 1.01 \\
\hline 1-Me & 2.55 & 2.58 & 1.86 & 1.86 & 0.69 & 0.72 \\
\hline 1-i-Pr & 2.36 & 2.66 & 1.99 & 1.80 & 0.37 & 0.86 \\
\hline 1- $\mathrm{H}$ & \multicolumn{2}{|c|}{2.36} & \multicolumn{2}{|c|}{2.02} & \multicolumn{2}{|c|}{0.34} \\
\hline $1-\mathrm{F}$ & 2.84 & 2.36 & 1.72 & 2.01 & 1.12 & 0.35 \\
\hline $1-\mathrm{COOH}$ & 2.74 & 2.61 & 1.76 & 1.84 & 0.98 & 0.77 \\
\hline $1-\mathrm{CF}_{3}$ & 2.36 & 2.36 & 2.04 & 2.03 & 0.32 & 0.33 \\
\hline 1-CHO & 2.67 & 2.61 & 1.83 & 1.85 & 0.84 & 0.76 \\
\hline $1-\mathrm{CN}$ & 2.23 & 2.64 & 2.08 & 1.84 & 0.15 & 0.80 \\
\hline $1-\mathrm{NO}_{2}$ & 2.55 & 3.20 & 2.42 & 1.73 & 0.13 & 1.47 \\
\hline
\end{tabular}

Tabla 4.10. Distancias $r 1$ y r2 (en Å) para los estados de transición mostrados en la Figura 4.2.7. $\Delta r=r 1-r 2$.

\begin{tabular}{|c|c|c|c|c|c|c|}
\hline \multirow{2}{*}{ TS } & \multicolumn{2}{|c|}{$r_{1}$} & \multicolumn{2}{|c|}{$r_{2}$} & \multicolumn{2}{|c|}{$\Delta r$} \\
\hline & $R^{1}=R^{2}$ & $R^{1} \neq R^{2}$ & $R^{1}=R^{2}$ & $\mathbf{R}^{1} \neq \mathbf{R}^{2}$ & $R^{1}=R^{2}$ & $\mathbf{R}^{1} \neq \mathbf{R}^{2}$ \\
\hline 2- $\mathrm{NH}_{2}$ & 3.48 & 3.28 & 1.88 & 1.91 & 1.60 & 1.37 \\
\hline 2-OMe & 3.27 & 2.98 & 1.88 & 1.93 & 1.39 & 1.05 \\
\hline $2-\mathrm{OH}$ & 2.94 & 3.34 & 1.91 & 1.91 & 1.03 & 1.43 \\
\hline 2-Me & 2.26 & 2.38 & 2.26 & 2.17 & 0 & 0.21 \\
\hline 2-i-Pr & 2.93 & 2.44 & 1.79 & 2.11 & 1.14 & 0.33 \\
\hline 2- $\mathrm{H}$ & \multicolumn{2}{|c|}{2.27} & \multicolumn{2}{|c|}{2.27} & \multicolumn{2}{|c|}{0} \\
\hline $2-F$ & 2.30 & 2.33 & 2.27 & 2.22 & 0.03 & 0.11 \\
\hline $2-\mathrm{COOH}$ & 3.06 & 2.68 & 1.97 & 1.99 & 1.09 & 0.69 \\
\hline $2-\mathrm{CF}_{3}$ & 2.27 & 2.34 & 2.27 & 2.21 & 0.01 & 0.13 \\
\hline 2- $\mathrm{CHO}$ & 2.69 & 2.69 & 1.97 & 1.98 & 0.72 & 0.71 \\
\hline $2-C N$ & 2.24 & 2.64 & 2.24 & 2.02 & 0 & 0.62 \\
\hline $2-\mathrm{NO}_{2}$ & 2.19 & 3.04 & 1.78 & 1.96 & 0.41 & 1.08 \\
\hline
\end{tabular}


Las Tablas 4.11 y 4.12 muestran las energías de activación $E_{a}$, energías libres de Gibbs estándar de activación $\Delta \mathrm{G}^{\ddagger}$, entalpías de activación $\Delta \mathrm{H}^{\ddagger}$ y entropías de activación $\Delta \mathrm{S}^{\ddagger}$ de las reacciones propuestas. La energía de activación de las reacciones con los fragmentos $\mathbf{1}$ y $\mathbf{2}$ mono- y disustituidos sigue la misma tendencia. Todos los valores para las reacciones disustituidas son mayores que los obtenidos para las reacciones con los fragmentos sin sustituir excepto para los fragmentos sustituidos con $\mathrm{NO}_{2}$. En el caso de los fragmentos monosustituidos los valores son cercanos a los obtenidos para los fragmentos no sustituidos. El fragmento 1 sustituido con un grupo $\mathrm{NH}_{2}, \mathrm{OMe}, \mathrm{OH}, \mathrm{COOH}, \mathrm{CHO}$ y $\mathrm{NO}_{2}$ presentan Ea menores que el fragmento 1 sin sustituir, mientras que en el caso del fragmento 2 sustituido con un grupo $\mathrm{COOH}, \mathrm{CHO}$ and $\mathrm{NO}_{2}$ presentan un barrera energética menor que la obtenida para el fragmento $\mathbf{2}$ sin sustituir. Las reacciones con los fragmentos 1 y $\mathbf{2}$ mono- y di-sustituidos con grupos $\mathrm{NO}_{2}$, presentan las barreras de activación más bajas observadas.

Tabla 4.11. Energías de activación $E_{a}$ energías libres de Gibbs estándar de activación $\Delta G^{*}$, entalpias estándar de activación $\Delta H^{\ddagger}$, y entropías estándar de activación $T \Delta S^{\ddagger}$ de las reacciones de Diels-Alder entre el butadieno y el fragmento 1 sustituido. Los valores de la reacción con el fragmento sin sustituir (1-H) se incluyen como referencia.

\begin{tabular}{|c|c|c|c|c|c|c|c|c|}
\hline \multirow[t]{2}{*}{ Reacción } & \multicolumn{2}{|c|}{$\begin{array}{c}E_{a} \\
\left(\mathrm{kcal} \mathrm{mol}^{-1}\right)\end{array}$} & \multicolumn{2}{|c|}{$\begin{array}{c}\Delta G^{\ddagger} \\
\left(\mathrm{kcal} \mathrm{mol}^{-1}\right)\end{array}$} & \multicolumn{2}{|c|}{$\begin{array}{c}\Delta H^{\ddagger} \\
\left(\mathrm{kcal} \mathrm{mol}^{-1}\right)\end{array}$} & \multicolumn{2}{|c|}{$\begin{array}{c}T \Delta S^{\ddagger} \\
\left(\mathrm{kcal} \mathrm{mol}^{-1}\right)\end{array}$} \\
\hline & $R^{1}=R^{2}$ & $R^{1} \neq R^{2}$ & $\mathbf{R}^{1}=\mathbf{R}^{2}$ & $R^{1} \neq R^{2}$ & $R^{1}=R^{2}$ & $R^{1} \neq R^{2}$ & $\mathrm{R}^{1}=\mathrm{R}^{2}$ & $\mathbf{R}^{1} \neq \mathbf{R}^{2}$ \\
\hline $1-\mathrm{NH}_{2}$ & 38.94 & 31.29 & 53.03 & 45.14 & 37.73 & 30.15 & -15.29 & -14.99 \\
\hline 1-OMe & 34.82 & 32.51 & 49.55 & 46.44 & 33.59 & 31.55 & -15.97 & -14.90 \\
\hline $1-\mathrm{OH}$ & 35.06 & 31.24 & 49.16 & 45.05 & 33.85 & 30.13 & -15.31 & -14.92 \\
\hline 1-Me & 40.37 & 35.51 & 54.30 & 49.08 & 39.31 & 34.68 & -14.99 & -14.39 \\
\hline 1-i-Pr & 54.40 & 36.88 & 68.53 & 50.79 & 53.41 & 36.09 & -15.12 & -14.70 \\
\hline 1- $\mathrm{H}$ & \multicolumn{2}{|c|}{33.11} & \multicolumn{2}{|c|}{46.03} & \multicolumn{2}{|c|}{32.47} & \multicolumn{2}{|c|}{-13.56} \\
\hline $1-\mathrm{F}$ & 33.50 & 34.23 & 47.08 & 47.03 & 32.73 & 33.67 & -14.35 & -13.36 \\
\hline 1-COOH & 35.98 & 32.61 & 50.39 & 46.25 & 35.07 & 31.90 & -15.32 & -14.35 \\
\hline $1-\mathrm{CF}_{3}$ & 37.65 & 34.10 & 51.87 & 47.79 & 36.80 & 33.40 & -15.06 & -14.39 \\
\hline $1-\mathrm{CHO}$ & 35.96 & 32.58 & 49.66 & 45.98 & 35.16 & 31.88 & -14.51 & -14.10 \\
\hline $1-\mathrm{CN}$ & 38.52 & 33.64 & 51.80 & 46.77 & 37.87 & 33.03 & -13.93 & -13.74 \\
\hline $1-\mathrm{NO}_{2}$ & 32.79 & 28.71 & 47.19 & 42.52 & 31.88 & 27.90 & -15.31 & -14.62 \\
\hline
\end{tabular}


Tabla 4.12. Energías de activación $E_{a}$ energías libres de Gibbs estándar de activación $\Delta G^{\ddagger}$, entalpias estándar de activación $\Delta H^{\ddagger}$, y entropías estándar de activación $T \Delta S^{\ddagger}$ de las reacciones de Diels-Alder entre el butadieno y el fragmento 2 sustituido. Los valores de la reacción con el fragmento sin sustituir (1-H) se incluyen como referencia.

\begin{tabular}{|c|c|c|c|c|c|c|c|c|}
\hline \multirow[t]{2}{*}{ Reacción } & \multicolumn{2}{|c|}{$\begin{array}{c}E_{a} \\
\left(\mathrm{kcal} \mathrm{mol}^{-1}\right)\end{array}$} & \multicolumn{2}{|c|}{$\begin{array}{c}\Delta G^{\ddagger} \\
\left(\mathrm{kcal} \mathrm{mol}^{-1}\right)\end{array}$} & \multicolumn{2}{|c|}{$\begin{array}{c}\Delta \boldsymbol{H}^{\ddagger} \\
\left(\mathrm{kcal} \mathrm{mol}^{-1}\right)\end{array}$} & \multicolumn{2}{|c|}{$\begin{array}{c}T \Delta S^{\ddagger} \\
\left(\mathrm{kcal} \mathrm{mol}^{-1}\right)\end{array}$} \\
\hline & $R^{1}=R^{2}$ & $R^{1} \neq R^{2}$ & $R^{1}=R^{2}$ & $R^{1} \neq R^{2}$ & $R^{1}=R^{2}$ & $R^{1} \neq R^{2}$ & $R^{1}=R^{2}$ & $R^{1} \neq R^{2}$ \\
\hline 2- $\mathrm{NH}_{2}$ & 28.06 & 24.03 & 41.38 & 36.61 & 27.28 & 23.54 & -14.11 & -13.07 \\
\hline 2-OMe & 29.21 & 26.82 & 42.63 & 40.32 & 28.40 & 25.87 & -14.22 & -14.45 \\
\hline $2-\mathrm{OH}$ & 27.99 & 24.03 & 40.70 & 36.46 & 27.37 & 23.47 & -13.34 & -12.99 \\
\hline 2-Me & 30.73 & 26.60 & 44.42 & 39.92 & 29.86 & 25.91 & -14.55 & -14.01 \\
\hline 2-i-Pr & 46.86 & 31.34 & 61.52 & 45.17 & 45.67 & 30.52 & -15.85 & -14.66 \\
\hline 2- H & \multicolumn{2}{|c|}{22.61} & \multicolumn{2}{|c|}{35.52} & \multicolumn{2}{|c|}{22.02} & \multicolumn{2}{|c|}{-13.51} \\
\hline $2-F$ & 25.60 & 24.67 & 38.39 & 37.65 & 25.12 & 24.14 & -13.27 & -13.51 \\
\hline $2-\mathrm{COOH}$ & 23.60 & 22.88 & 37.52 & 36.34 & 22.98 & 22.25 & -14.54 & -14.09 \\
\hline $2-\mathrm{CF}_{3}$ & 25.23 & 23.22 & 39.30 & 37.32 & 24.42 & 22.50 & -14.88 & -14.82 \\
\hline 2-CHO & 27.54 & 22.98 & 40.88 & 36.14 & 26.88 & 22.37 & -14.01 & -13.78 \\
\hline $2-\mathrm{CN}$ & 27.18 & 23.55 & 40.58 & 36.58 & 26.56 & 23.03 & -14.03 & -13.56 \\
\hline 2- $\mathrm{NO}_{2}$ & 19.80 & 19.03 & 35.78 & 32.18 & 18.22 & 18.51 & -17.56 & -13.67 \\
\hline
\end{tabular}

Las coordenadas intrínsecas de reacción IRC de las reacciones propuestas se muestran en las Figuras 4.9 a 4.12. El análisis de las coordenadas de reacción muestran que las estructuras de los estados de transición obtenidos (IRC=0) conducen a los productos (hacia la derecha) y reactivos (hacia la izquierda) deseados, sin evidencias de la existencia de complejos activados o intermediarios. 

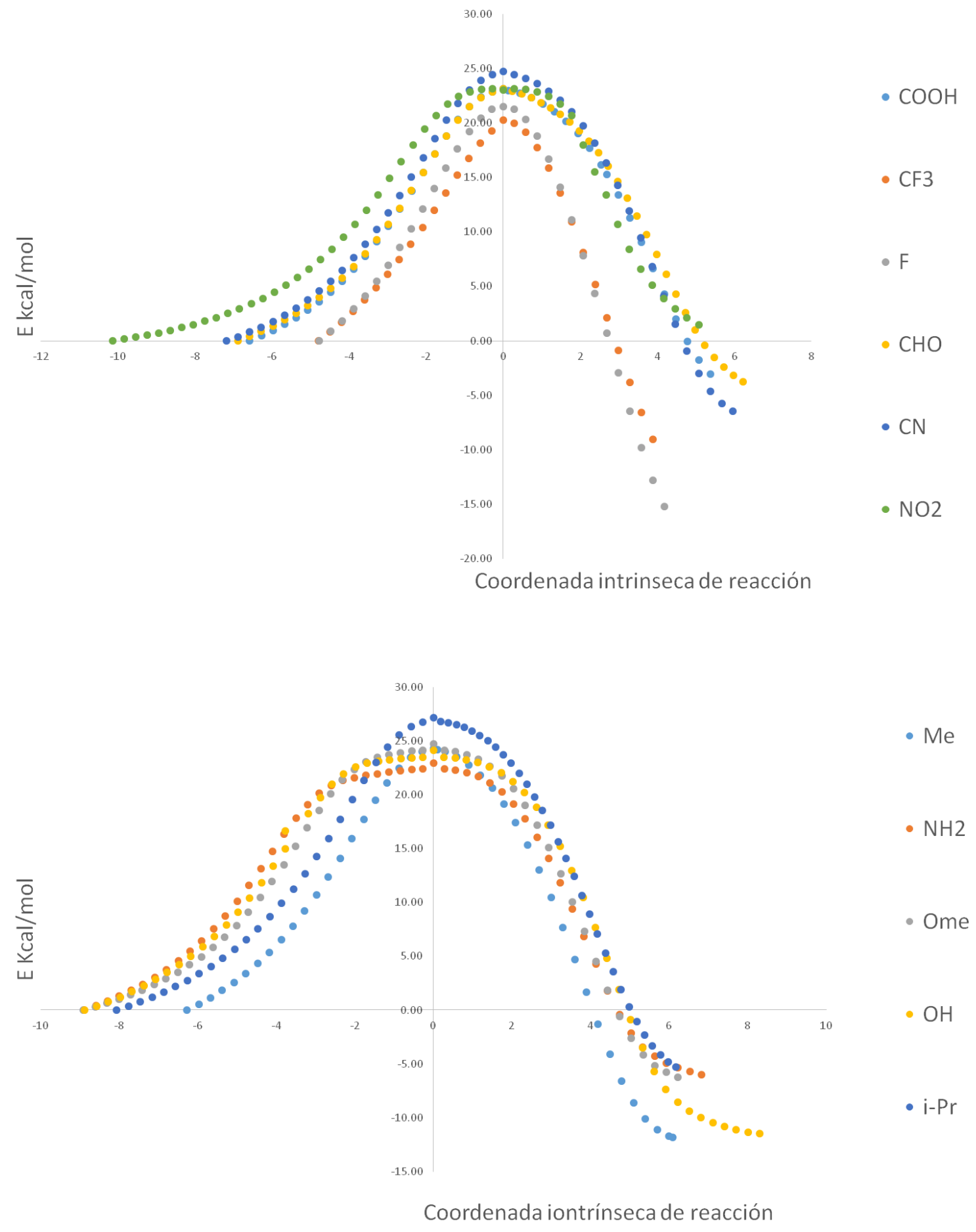

Figura 4.9. Coordenadas intrínsecas de reacción para las reacciones con el fragmento 1 monosustituido. 

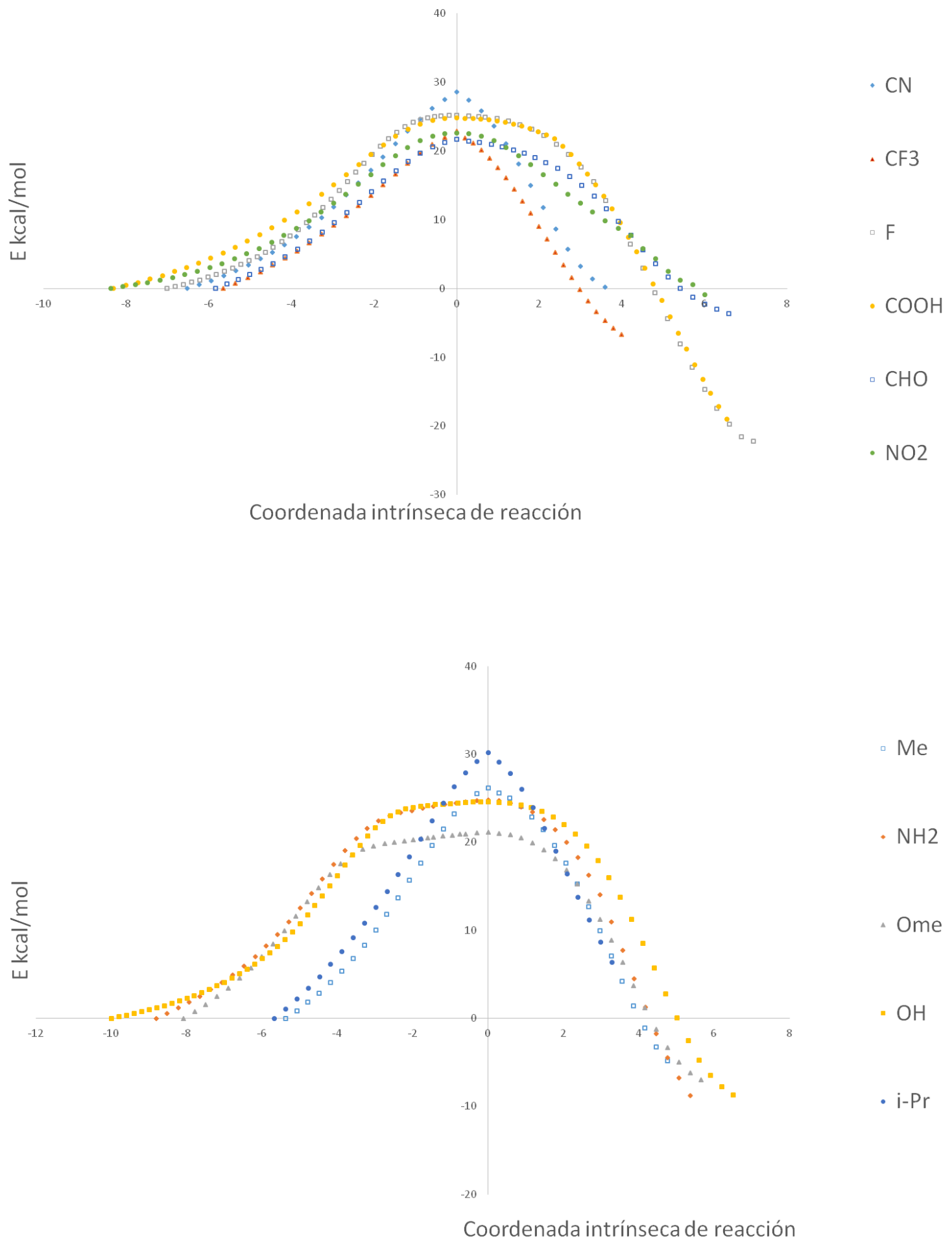

Figura 4.10. Coordenadas intrínsecas de reacción para las reacciones con el fragmento 1 disustituido. 


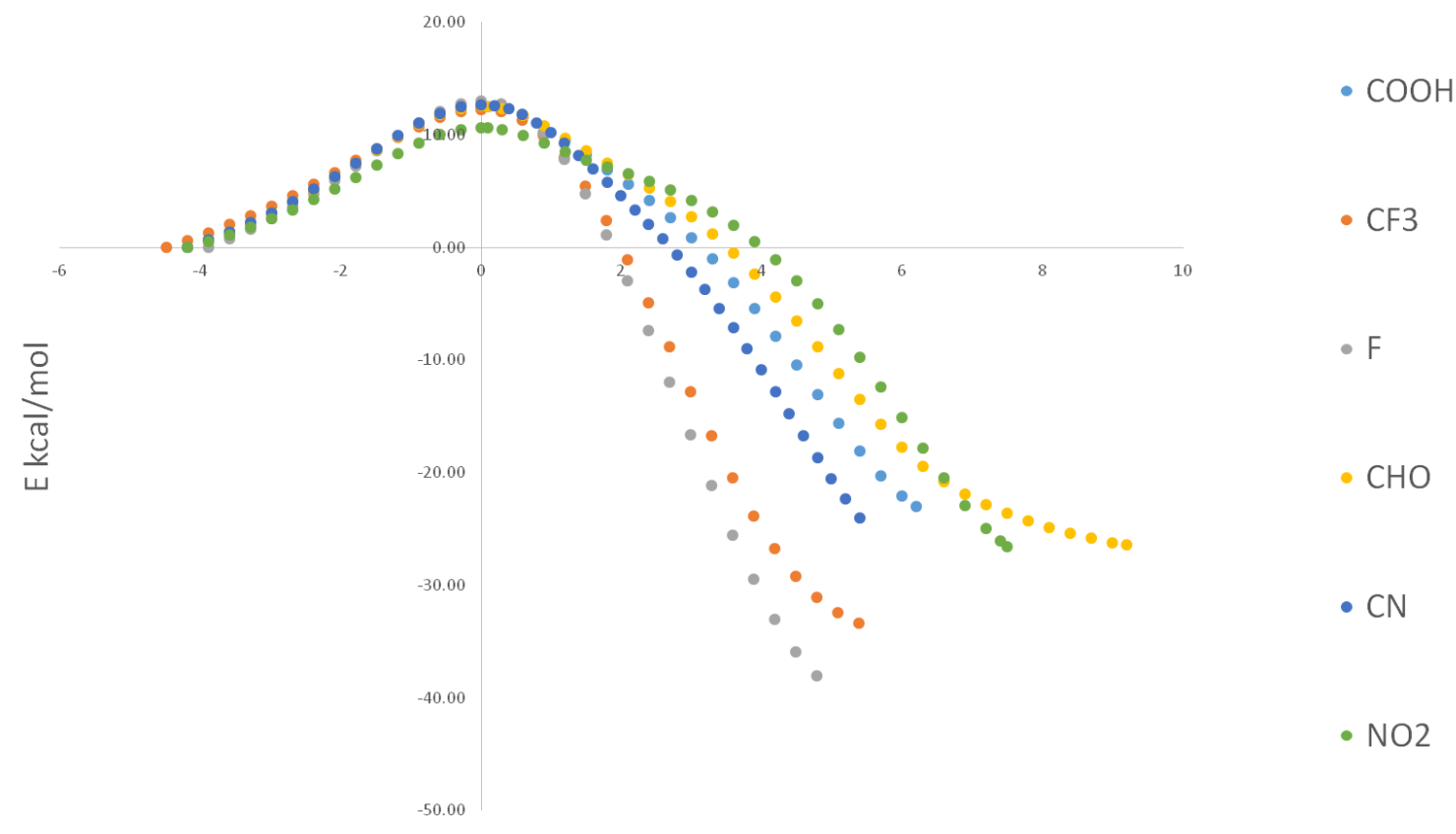

Coordenada intrínseca de reacción

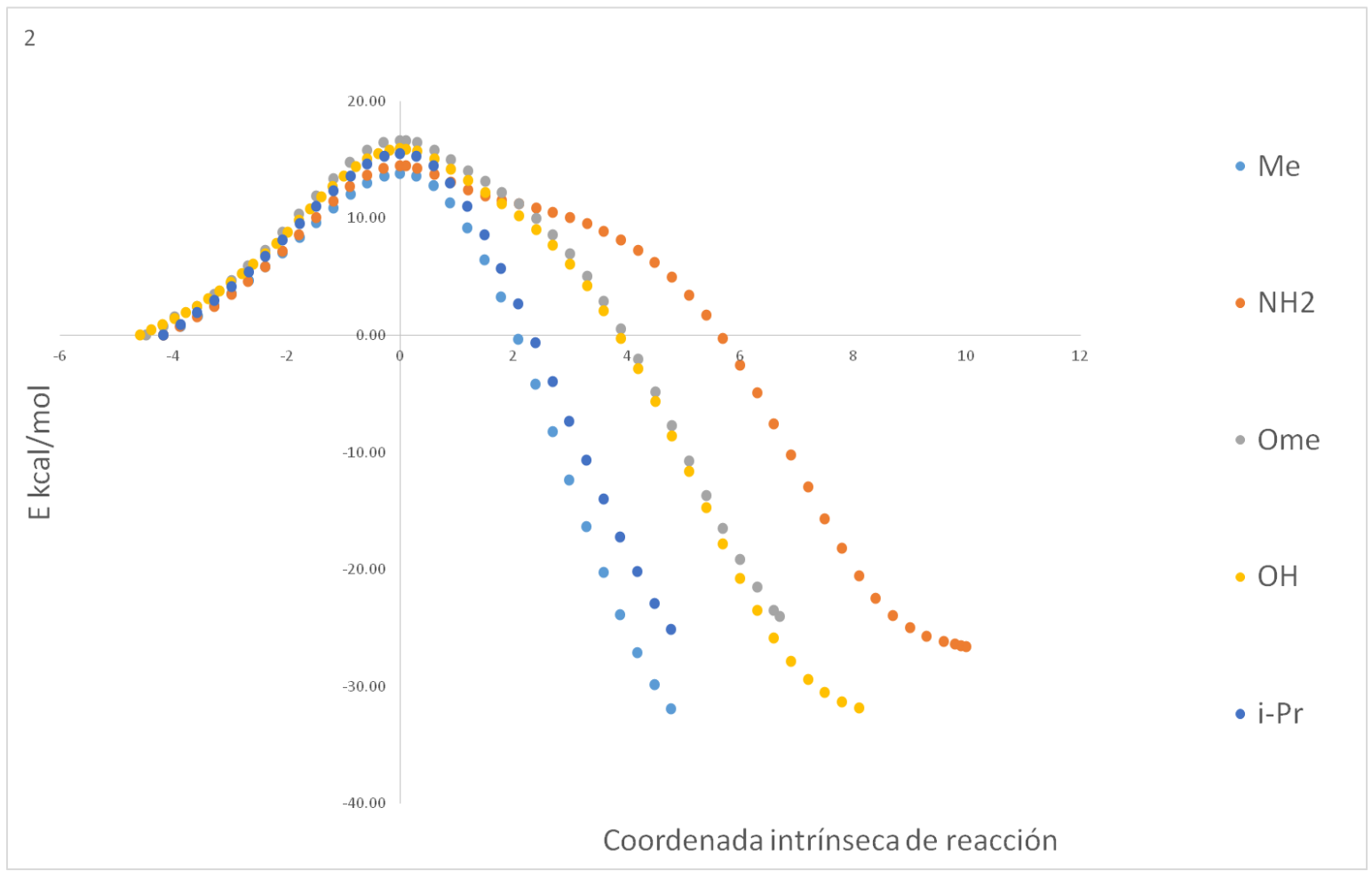

Figura 4.11. Coordenadas intrínsecas de reacción para las reacciones con el fragmento 2 monosustituido. 


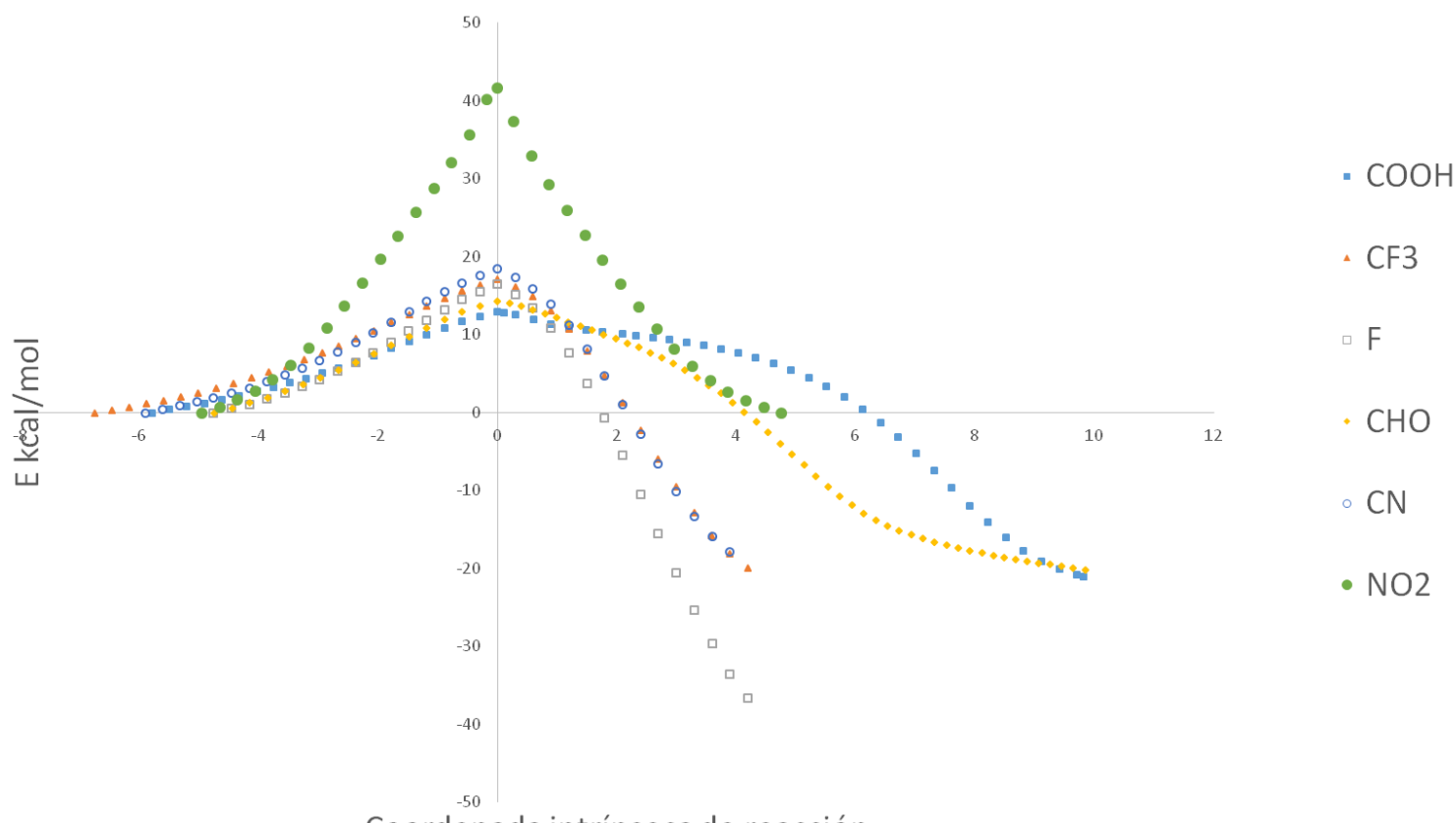

Coordenada intrínseca de reacción

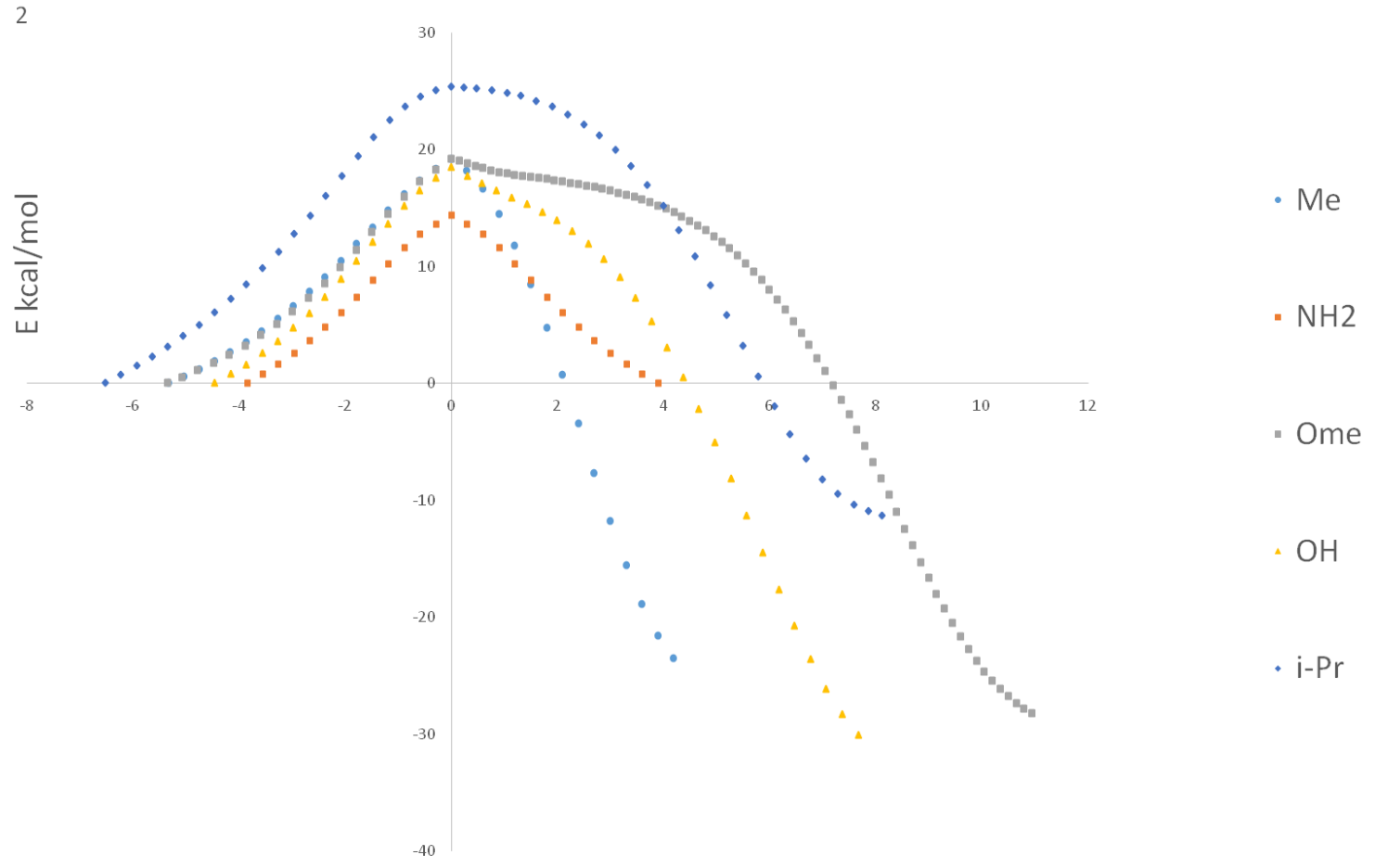

Coordenada intrínseca de reacción

Figura 4.12. Coordenadas intrínsecas de reacción para las reacciones con el fragmento 2 disustituido. 


\subsubsection{Parámetros termodinámicos}

El análisis termodinámico de las reacciones globales nos permite determinar la estabilidad de los productos obtenidos y por lo tanto, la factibilidad de la reacción. Las reacciones propuestas se muestran en la Esquema 4.2 y las energías de Gibbs estándar $\Delta G r$, entalpía estándar $\Delta H r$, y entropía estándar $T \Delta S r$ de reacción se muestran en las Tablas 4.13 y 4.14 .

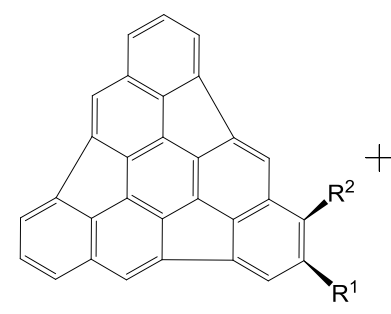

1-S

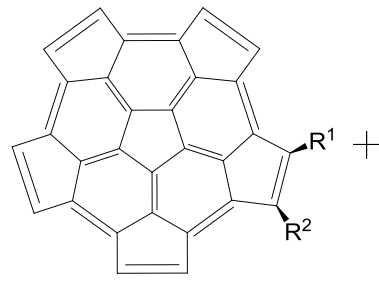

2-S

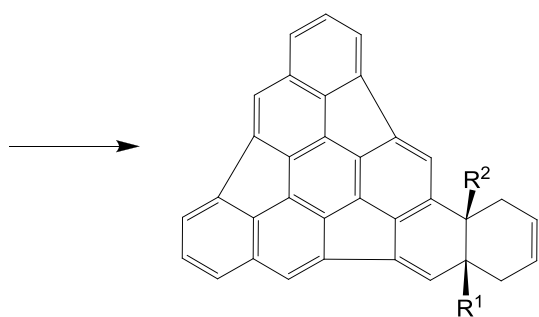

3

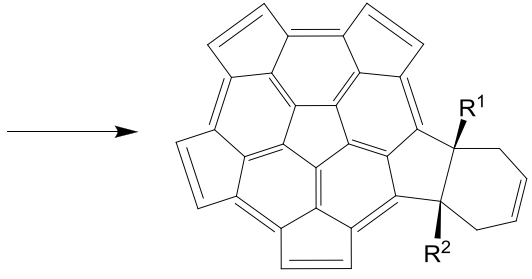

4

Esquema 4.2. Reacciones globales propuestas. a) $R^{1}=R^{2}=\mathrm{NH}_{2}, \mathrm{OMe}, \mathrm{OH}, \mathrm{Me}, \mathrm{i}-\mathrm{Pr}, \mathrm{F}, \mathrm{COOH}, \mathrm{CF}_{3}$, $\mathrm{CHO}, \mathrm{CN}$ y $\mathrm{NO}_{2}$, b) $\mathrm{R}^{1}=\mathrm{NH}_{2}, \mathrm{OMe}, \mathrm{OH}, \mathrm{Me}, i-\mathrm{Pr}, \mathrm{F}, \mathrm{COOH}, \mathrm{CF}_{3}, \mathrm{CHO}, \mathrm{CN}$ y NO $\mathrm{N}_{2} ; \mathrm{R}^{2}=\mathrm{H}$.

Todas las reacciones son impedidas por entropía. En el caso de fragmento 1, solo las reacciones con el fragmento sustituido con los grupos $\mathrm{OH}, \mathrm{Me}, \mathrm{F}, \mathrm{CF}_{3}$ y NO $\mathrm{NO}_{2}$ son exotérmicas $(\Delta \mathrm{Hr}<0)$ y todas las reacciones no son favorecidas termodinámicamente $\left(\Delta G^{\circ}>0\right)$ con valores más grandes que los obtenidos para los fragmentos no sustituidos, excepto para las reacciones con los fragmentos sustituidos $\mathrm{F}$ y $\mathrm{NO}_{2}$; en estos casos los fragmentos disustituidos son menos favorecidas termodinámicamente que los fragmentos monosustituidos, para el resto de los sustituyentes la tendencia se invierte. En el caso del fragmento 1 la tendencia de los parámetros termodinámicos no se modifica por el número de sustituyentes, sin embargo, los valores numéricos de las reacciones con los fragmentos mono-sustituidos son menores que los observados para los fragmentos disustituidos. 
Tabla 4.13. Energías de Gibbs estándar $\Delta G r$, entalpías estándar $\Delta H r$, y entropías estándar $T \Delta S r$ de las reacciones entre el butadieno y el fragmento 1 sustituido.

\begin{tabular}{|c|c|c|c|c|c|c|}
\hline \multirow[t]{2}{*}{ Reacción } & \multicolumn{2}{|c|}{$\Delta G_{r}\left(\mathrm{kcal} \mathrm{mol}^{-1}\right)$} & \multicolumn{2}{|c|}{$\Delta H_{r}\left(\mathrm{kcal} \mathrm{mol}^{-1}\right)$} & \multicolumn{2}{|c|}{$T \Delta S_{r}\left(\mathrm{kcal} \mathrm{mol}^{-1}\right)$} \\
\hline & $\mathrm{R}^{1}=\mathrm{R}^{2}$ & $\mathbf{R}^{1} \neq \mathbf{R}^{2}$ & $\mathbf{R}^{1}=\mathbf{R}^{2}$ & $\mathbf{R}^{1} \neq \mathbf{R}^{2}$ & $\mathbf{R}^{1}=\mathbf{R}^{2}$ & $\mathbf{R}^{1} \neq \mathbf{R}^{2}$ \\
\hline 1- $\mathrm{NH}_{2}$ & 25.06 & 17.78 & 9.56 & 2.83 & -15.50 & -15.88 \\
\hline 1-OMe & 22.26 & 15.45 & 6.18 & 0.58 & -16.08 & -14.87 \\
\hline $1-\mathrm{OH}$ & 15.05 & 12.03 & -0.62 & -2.74 & -15.67 & -14.78 \\
\hline 1-Me & 16.03 & 12.19 & -0.27 & -3.69 & -16.30 & -15.88 \\
\hline 1-i-Pr & 26.27 & 16.40 & 9.25 & 0.49 & -17.01 & -15.91 \\
\hline 1- $\mathrm{H}$ & \multicolumn{2}{|c|}{10.34} & \multicolumn{2}{|c|}{-4.28} & \multicolumn{2}{|c|}{-14.62} \\
\hline $1-F$ & 5.97 & 7.88 & -9.00 & -6.74 & -14.97 & -14.62 \\
\hline 1-COOH & 15.33 & 15.24 & -0.33 & 0.51 & -15.65 & -14.74 \\
\hline $1-\mathrm{CF}_{3}$ & 11.32 & 10.73 & -5.16 & -5.13 & -16.48 & -15.86 \\
\hline 1- $\mathrm{CHO}$ & 17.05 & 16.27 & 2.18 & 1.56 & -14.87 & -14.71 \\
\hline $1-\mathrm{CN}$ & 23.95 & 16.52 & 9.14 & 1.91 & -14.82 & -14.61 \\
\hline $1-\mathrm{NO}_{2}$ & 3.83 & 10.55 & -12.19 & -4.59 & -16.02 & -15.14 \\
\hline
\end{tabular}

En el caso del fragmento $\mathbf{2}$ se observa una clara diferencia entre las reacciones con los fragmentos mono- y disustituidos. Aun cuando todas las reacciones son exotérmicas $(\Delta \mathrm{Hr}<0)$, solo las reacciones con los fragmentos disustituidos con los grupos $\mathrm{OH}, \mathrm{F}, \mathrm{CF}_{3}$ y $\mathrm{NO}_{2}$ son favorecidas termodinámicamente $\left(\Delta G_{r}<0\right)$, mientras que todas las reacciones con los fragmentos monosustituidos son favorecidas $\left(\Delta G_{r}<0\right)$ excepto las reacciones del fragmento sustituido con $i$ $\operatorname{Pr}$ que no son termodinámicamente favorables $\left(\Delta G_{r}>0\right)$. Solamente las reacciones con los fragmentos (mono- y di-) sustituidos con $\mathrm{F}$ son más favorecidas termodinámicamente que las reacciones con los fragmentos sin sustituir, aun cuando se esperaba que las reacciones con los fragmentos (mono-y di-) sustituidos con $\mathrm{NO}_{2}$ fueran más favorables. 
Tabla 4.14. Energías de Gibbs estándar $\Delta G r$, entalpías estándar $\Delta H r$, y entropías estándar $T \Delta S r$ de las reacciones entre el butadieno y el fragmento 2 sustituido.

\begin{tabular}{|c|c|c|c|c|c|c|}
\hline \multirow[t]{2}{*}{ Reacción } & \multicolumn{2}{|c|}{$\Delta G_{r}\left(k^{2 a l ~ m o l}{ }^{-1}\right)$} & \multicolumn{2}{|c|}{$\Delta H_{r}\left(\mathrm{kcal} \mathrm{mol}^{-1}\right)$} & \multicolumn{2}{|c|}{$T \Delta S_{r}\left(\mathrm{kcal} \mathrm{mol}^{-1}\right)$} \\
\hline & $R^{1}=R^{2}$ & $R^{1} \neq R^{2}$ & $R^{1}=R^{2}$ & $R^{1} \neq R^{2}$ & $R^{1}=R^{2}$ & $\mathbf{R}^{1} \neq \mathbf{R}^{2}$ \\
\hline 2- $\mathrm{NH}_{2}$ & 2.43 & -3.93 & -13.87 & -19.10 & -16.30 & -15.17 \\
\hline 2-OMe & 1.44 & -4.64 & -15.29 & -19.79 & -16.72 & -15.15 \\
\hline $2-\mathrm{OH}$ & -9.10 & -9.33 & -24.94 & -24.51 & -15.84 & -15.18 \\
\hline 2-Me & 2.43 & -6.54 & -13.38 & -21.75 & -15.80 & -15.21 \\
\hline 2-i-Pr & 15.88 & 2.04 & -1.07 & -14.18 & -16.96 & -16.22 \\
\hline 2- $\mathrm{H}$ & \multicolumn{2}{|c|}{-13.05} & \multicolumn{2}{|c|}{-27.88} & \multicolumn{2}{|c|}{-14.82} \\
\hline $2-\mathrm{F}$ & -18.56 & -15.38 & -33.99 & -30.30 & -15.43 & -14.93 \\
\hline 2- $\mathrm{COOH}$ & 3.66 & -5.01 & -12.41 & -20.06 & -16.07 & -15.05 \\
\hline $2-\mathrm{CF}_{3}$ & -1.23 & -8.47 & -17.54 & -24.26 & -16.30 & -15.78 \\
\hline 2-CHO & 4.30 & -4.82 & -10.47 & -19.43 & -14.77 & -14.61 \\
\hline $2-\mathrm{CN}$ & 3.45 & -5.43 & -11.90 & -20.46 & -15.36 & -15.02 \\
\hline $2-\mathrm{NO}_{2}$ & -7.70 & -10.50 & -23.81 & -25.46 & -16.10 & -14.96 \\
\hline
\end{tabular}

\subsubsection{Aromatización}

Analizamos el proceso de aromatización en el que la pérdida de los sustituyentes de los aductos formados conduce a la formación de un nuevo anillo aromático en la red del fragmento. Las reacciones propuestas para la aromatización de los fragmentos $\mathbf{3}$ and $\mathbf{4}$ se muestran en la Esquema 4.3 y las energías de Gibbs estándar $\Delta G r$, entalpías estándar $\Delta H r$ y entropías estándar $T \Delta S r$ de aromatización se muestran en las Tablas 4.15 y 4.16.

Todas las reacciones de aromatización son termodinámicamente favorables $\left(\Delta G^{\circ}<0\right)$, exotérmicas $(\Delta \mathrm{H} r<0)$ y favorecidas por entropía $(T \Delta S r>0)$, lo que indica que los aductos formados tienden a aromatizarse para ganar estabilidad. Estos valores propician el uso de sustituyentes en la reacción debido a que su eliminación del producto final es favorable. 

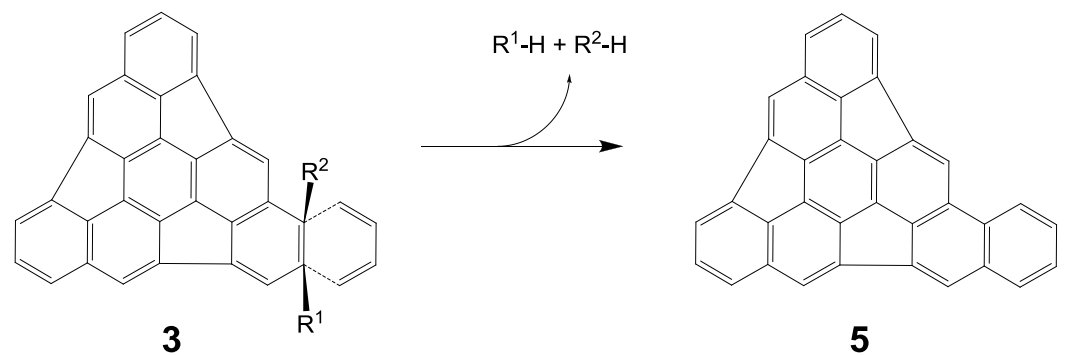

5

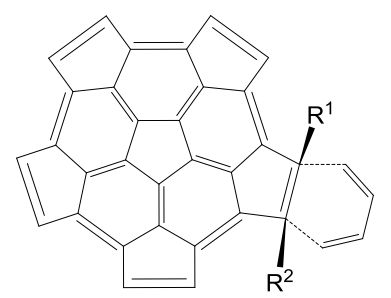

4
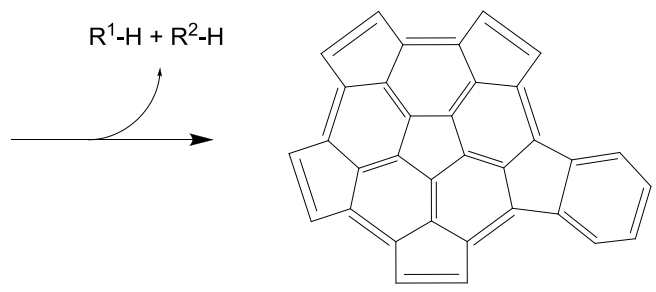

6

Esquema 4.3. Reacciones globales de aromatización. a) $R 1=R^{2}=\mathrm{NH}_{2}, \mathrm{OMe}, \mathrm{OH}, \mathrm{Me}, \mathrm{i}-\mathrm{Pr}, \mathrm{F}, \mathrm{COOH}$, $\left.\mathrm{CF}_{3}, \mathrm{CHO}, \mathrm{CN}_{\text {y NO}}, b\right) \mathrm{R}^{1}=\mathrm{NH}_{2} \mathrm{OMe}, \mathrm{OH}, \mathrm{Me}, \mathrm{i}-\mathrm{Pr}, \mathrm{F}, \mathrm{COOH}, \mathrm{CF}_{3}, \mathrm{CHO}, \mathrm{CN}$ y NO $\mathrm{O}_{2} ; \mathrm{R}^{2}=\mathrm{H}$.

Tabla 4.15. Energías de Gibbs estándar $\Delta G r$, entalpías estándar $\Delta H r$ y entropías estándar $T \Delta S r$ de las reacciones de aromatización de 3.

\begin{tabular}{|c|c|c|c|c|c|c|}
\hline \multirow[t]{2}{*}{ Reacción } & \multicolumn{2}{|c|}{$\Delta G_{r}\left(\mathrm{kcal} \mathrm{mol}^{-1}\right)$} & \multicolumn{2}{|c|}{$\Delta H_{r}\left(\mathrm{kcal} \mathrm{mol}^{-1}\right)$} & \multicolumn{2}{|c|}{$T \Delta S_{r}\left(\mathrm{kcal} \mathrm{mol}^{-1}\right)$} \\
\hline & $R^{1}=R^{2}$ & $R^{1} \neq R^{2}$ & $R^{1}=R^{2}$ & $\mathbf{R}^{1} \neq \mathbf{R}^{2}$ & $R^{1}=R^{2}$ & $R^{1} \neq R^{2}$ \\
\hline 1- $\mathrm{NH}_{2}$ & -65.89 & -46.18 & -41.55 & -25.75 & 24.33 & 20.43 \\
\hline 1-OMe & -68.02 & -44.73 & -42.34 & -23.76 & 25.68 & 20.97 \\
\hline $1-\mathrm{OH}$ & -54.80 & -40.80 & -31.14 & -20.85 & 23.66 & 19.95 \\
\hline 1-Me & -64.19 & -42.89 & -39.18 & -21.85 & 25.01 & 21.04 \\
\hline 1-i-Pr & -83.86 & -47.47 & -55.81 & -25.55 & 28.05 & 21.92 \\
\hline 1- $\mathrm{H}$ & \multicolumn{2}{|c|}{-26.56} & \multicolumn{2}{|c|}{-9.80} & \multicolumn{2}{|c|}{16.77} \\
\hline $1-\mathrm{F}$ & -43.77 & -32.55 & -22.97 & -13.84 & 20.79 & 18.71 \\
\hline 1-COOH & -38.94 & -29.87 & -12.84 & -8.86 & 26.10 & 21.02 \\
\hline $1-\mathrm{CF}_{3}$ & -49.38 & -32.76 & -22.94 & -11.30 & 26.43 & 21.47 \\
\hline $1-\mathrm{CHO}$ & -39.14 & -31.57 & -15.01 & -10.88 & 24.14 & 20.69 \\
\hline $1-\mathrm{CN}$ & -45.23 & -33.42 & -23.57 & -14.24 & 21.66 & 19.17 \\
\hline $1-\mathrm{NO}_{2}$ & -45.84 & -31.90 & -20.39 & -10.91 & 25.45 & 20.99 \\
\hline
\end{tabular}


Tabla 4.16. Energías de Gibbs estándar $\Delta G r$, entalpías estándar $\Delta H r$, y entropías estándar $T \Delta S r$ de las reacciones de aromatización de 4.

\begin{tabular}{|c|c|c|c|c|c|c|}
\hline \multirow[t]{2}{*}{ Reacción } & \multicolumn{2}{|c|}{$\Delta G_{r}\left(\mathrm{kcal} \mathrm{mol}^{-1}\right)$} & \multicolumn{2}{|c|}{$\Delta H_{r}\left(\mathrm{kcal} \mathrm{mol}^{-1}\right)$} & \multicolumn{2}{|c|}{$T \Delta S_{r}\left(\mathrm{kcal} \mathrm{mol}^{-1}\right)$} \\
\hline & $\mathrm{R}^{1}=\mathrm{R}^{2}$ & $R^{1} \neq R^{2}$ & $\mathbf{R}^{1}=\mathrm{R}^{2}$ & $R^{1} \neq R^{2}$ & $R^{1}=R^{2}$ & $\mathbf{R}^{1} \neq \mathbf{R}^{2}$ \\
\hline 2- $\mathrm{NH}_{2}$ & -48.33 & -28.37 & -24.23 & -7.66 & 24.11 & 20.71 \\
\hline 2-OMe & -54.12 & -31.89 & -28.05 & -10.85 & 26.07 & 21.04 \\
\hline $2-\mathrm{OH}$ & -38.72 & -25.92 & -14.94 & -5.58 & 23.78 & 20.35 \\
\hline 2-Me & -53.70 & -30.18 & -29.28 & -9.46 & 24.42 & 20.72 \\
\hline 2-i-Pr & -74.94 & 39.90 & -46.76 & -17.25 & 28.18 & 22.65 \\
\hline 2- H & \multicolumn{2}{|c|}{-10.48} & \multicolumn{2}{|c|}{6.51} & \multicolumn{2}{|c|}{16.99} \\
\hline $2-\mathrm{F}$ & -28.48 & -17.54 & -7.38 & 1.42 & 21.10 & 18.96 \\
\hline 2-COOH & -30.95 & -16.12 & -4.73 & 5.32 & 26.22 & 21.44 \\
\hline $2-\mathrm{CF}_{3}$ & -39.97 & -20.27 & -13.68 & 1.06 & 26.29 & 21.33 \\
\hline 2-CHO & -28.53 & -16.68 & -3.95 & 4.04 & 24.57 & 20.72 \\
\hline $2-\mathrm{CN}$ & -28.48 & -17.17 & -6.31 & 2.40 & 22.17 & 19.57 \\
\hline $2-\mathrm{NO}_{2}$ & -40.66 & -19.16 & -15.27 & 1.79 & 25.40 & 20.96 \\
\hline
\end{tabular}

\subsubsection{Conclusiones de la segunda parte}

Mientras que la presencia de dos sustituyentes incrementa el efecto electrónico reduciendo los gaps HOMO-LUMO y por lo tanto favoreciendo las reacciones, la tensión estérica producida por la aglomeración de átomos en los fragmentos disustituidos aumenta la $E_{a}$, haciendo estas reacciones menos favorables que las reacciones con los fragmentos sin sustituir. Las reacciones con los fragmentos monosustituidos parecen mejorar, la $E_{a}$ es similar o incluso menor (cuando los sustituyentes son los grupos $\mathrm{COOH}, \mathrm{CHO}$ and $\mathrm{NO}_{2}$ ) que en las reacciones con los fragmentos no sustituidos.

Esta evidencia nos hace pensar que, aun cuando el efecto electrónico de los grupos electrodonadores y electroatractores es transcendental en una reacción de DA típica, en este caso donde el sistema unido al dienófilo es grande, la tensión estérica de los sustituyentes juega un rol más importante, que en resumen hace que las reacciones con los fragmentos sustituidos ganen tan poco en energía, que las barreras de activación son comparables con aquellas de los fragmentos sin sustituir. 


\subsection{Tercera parte: Análisis de la dimerización del fragmento 2.}

Como mencionamos anteriormente, el análisis de los orbitales frontera de los fragmentos $\mathbf{1}$ y $\mathbf{2}$ muestra que la dimerización de dos unidades de $C_{30}$, en este caso triindenotrifenilieno 1 o pentaciclopentacoranuleno $\mathbf{2}$, mediada por reacciones de Diels-Alder es factible en términos de la teoría de orbitales frontera. ${ }^{110}$ Como se observa en la Tabla 4.17 los gaps HOMO-LUMO de la reacción de dimerización $(\mathbf{1 + 1} \circ \mathbf{2 + 2})$ son menores que el gap HOMO-LUMO de la reacción entre butadieno y etileno, por lo que la dimerización de cualquiera de estos fragmentos ( $\mathbf{1}$ ○ 2 ) debería ser factible y -de hecho- más fácil. Nos concentraremos en la dimerización del pentaciclopentacorenuleno $\mathbf{2}$, es la que presenta el gap HOMO-LUMO más bajo.

Tabla 4.17. Gaps HOMO-LUMO (en eV) de las reacciones dimerización de los fragmentos $\mathbf{1}$ y $\mathbf{2}$ y de la reacción entre etileno y 1,3-butadieno como referencia.

\begin{tabular}{cc}
\hline Reacción & Gap HOMO-LUMO \\
\hline etileno + butadieno & $13.66(13.69)^{\mathrm{a}}$ \\
$\mathbf{1}+\mathbf{1}$ & 8.66 \\
$\mathbf{2}+\mathbf{2}$ & 7.85 \\
\hline${ }^{\text {a }}$ tomado de la referencia & 114. \\
orbitales HOMO y valores de los \\
fragmentos $\mathbf{1}$ y $\mathbf{2}$ se muestran en la Tabla 4.1.1 \\
\hline
\end{tabular}

La dimerización de dos fragmentos por medio de la reacción de cicloadición de Diels-Alder requiere la formación de 5 cicloaductos (Figura 4.13). Estos pueden formarse todos al mismo tiempo de manera concertada o por pasos, formando primero un cicloaducto que acercaría los dos fragmentos y subsecuentemente, el resto de los enlaces necesarios para dar lugar al fullereno.

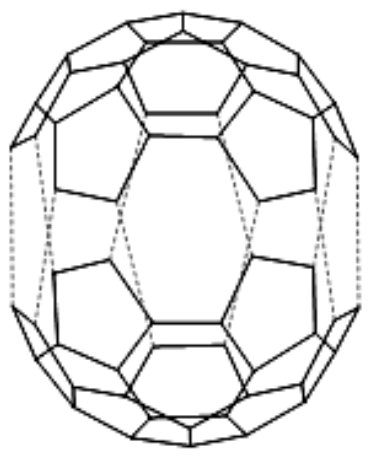

Figura 4.13. Dimerización de dos unidades de pentaciclopentacoranuleno $2 \mathrm{C}_{30} \mathrm{H}_{10}$ para dar lugar al $\mathrm{C}_{60}$. 
Utilizando el método NEB ${ }^{80}$ (Nudge Elastic Band) generamos una serie de coordenadas de reacción que describieran la unión de dos fragmentos $C_{30}$ idénticos para formar un fullereno $C_{60}$. Como primer caso consideramos la formación de los 5 cicloaductos necesarios de manera concertada (Figura 4.14). Encontramos que en este mecanismo es necesario superar una barrera de 115 $\mathrm{kcal} / \mathrm{mol}$ (correspondiente a la energía de activación, $E_{a}$ ) para dar lugar a una molécula de $\mathrm{C}_{60} \mathrm{H}_{20}$, que posteriormente perdería 20 átomos de hidrógeno conduciendo finalmente al fullereno $\mathrm{C}_{60}$. La reacción global es exotérmica y termodinámicamente favorecida $\left(\Delta H^{\circ}=-103.31 \mathrm{kcal} / \mathrm{mol}\right.$ y $\Delta G^{\circ}=$ $-78.95 \mathrm{kcal} / \mathrm{mol}$ ), sin embargo la deshidrogenación no lo es, lo que indicaría que el fullereno hidrogenado es más estable que el fullereno prístino y requiere condiciones oxidativas para deshidrogenarse.

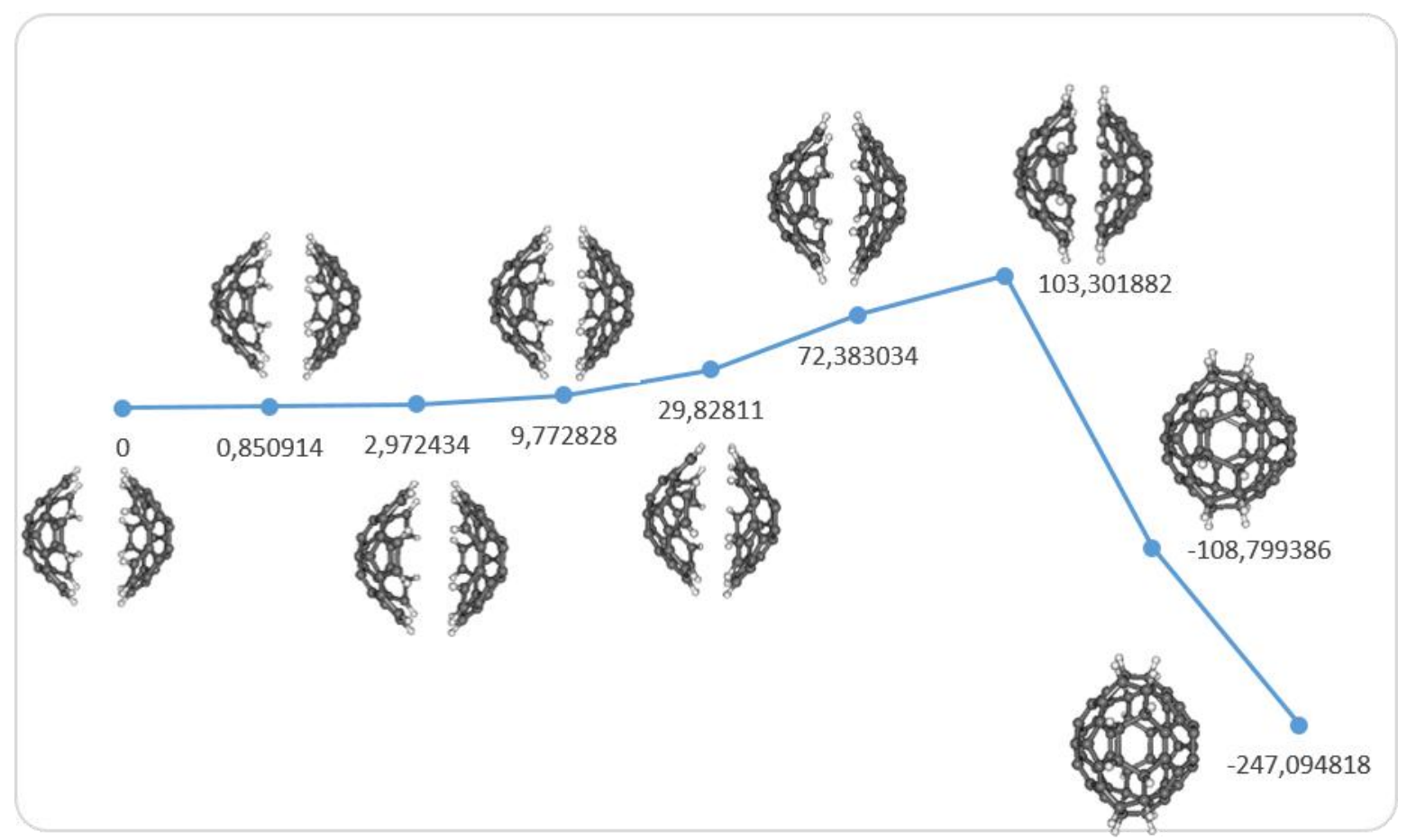

Figura 4.14. Coordenada de reacción de la formación de 5 cicloaductos de manera concertada.

Analizamos también el mecanismo de dimerización "no concertado" en el que los 5 cicloaductos no se forman al mismo tiempo, si no primero uno que une a los fragmentos y después el resto siguiendo el orden de cerrado. En este caso podemos dividir el proceso completo en dos pasos, el primero involucra la formación del primer cicloaducto entre los dos fragmentos (Figura 4.15). En este primer paso la reacción no presenta barrera aparente y la deshidrogenación del aducto formado es espontánea $\left(\Delta \mathrm{G}^{\circ}=-20 \mathrm{kcal} / \mathrm{mol}\right)$. 


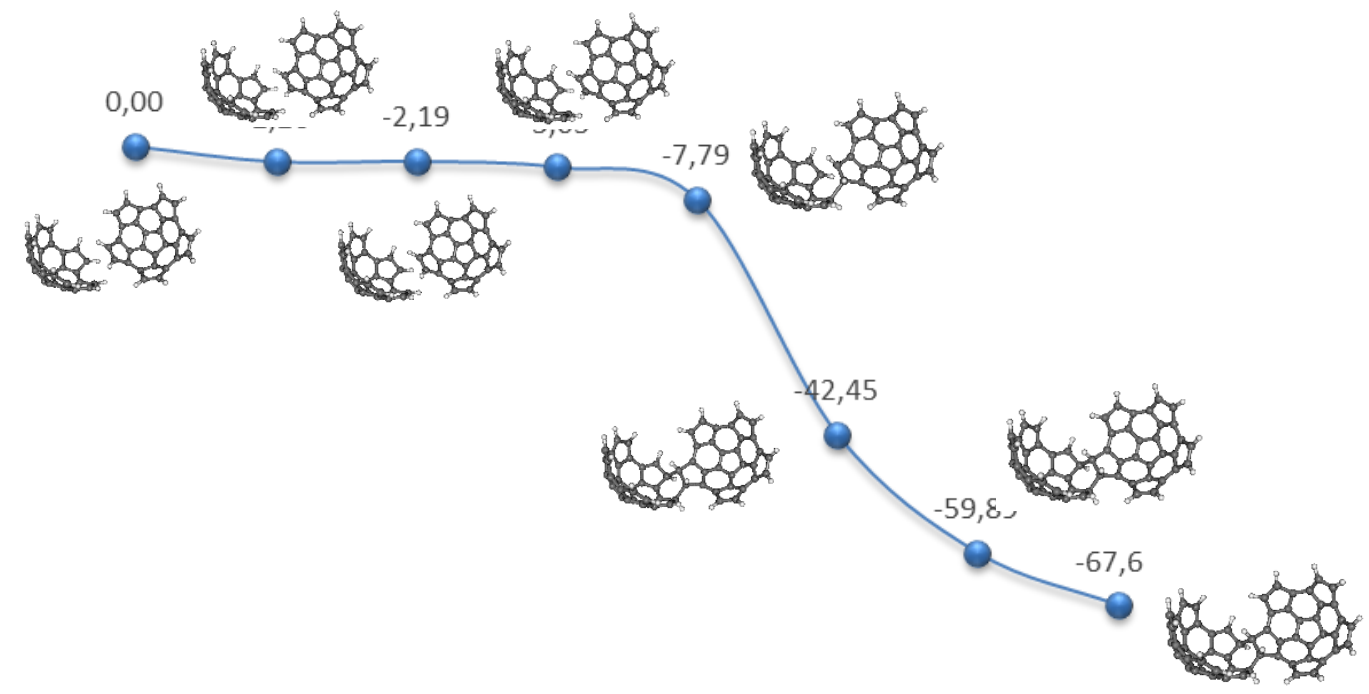

Figura 4.15. Coordenada de reacción para la formación de 1 cicloaducto, primer paso de la dimerización no concertada.

En el segundo paso se parte del primer aducto aromatizado y se requiere la formación de los 8 enlaces restantes para dar lugar al fullereno (Figura 4.16). En este caso la energía de activación es de $E_{a}=37.17 \mathrm{kcal} / \mathrm{mol}$ y la deshidrogenación final que da lugar al $C_{60}$ prístino es favorecida termodinámicamente $\left(\Delta G^{\circ}=-61.48 \mathrm{kcal} / \mathrm{mol}\right)$.

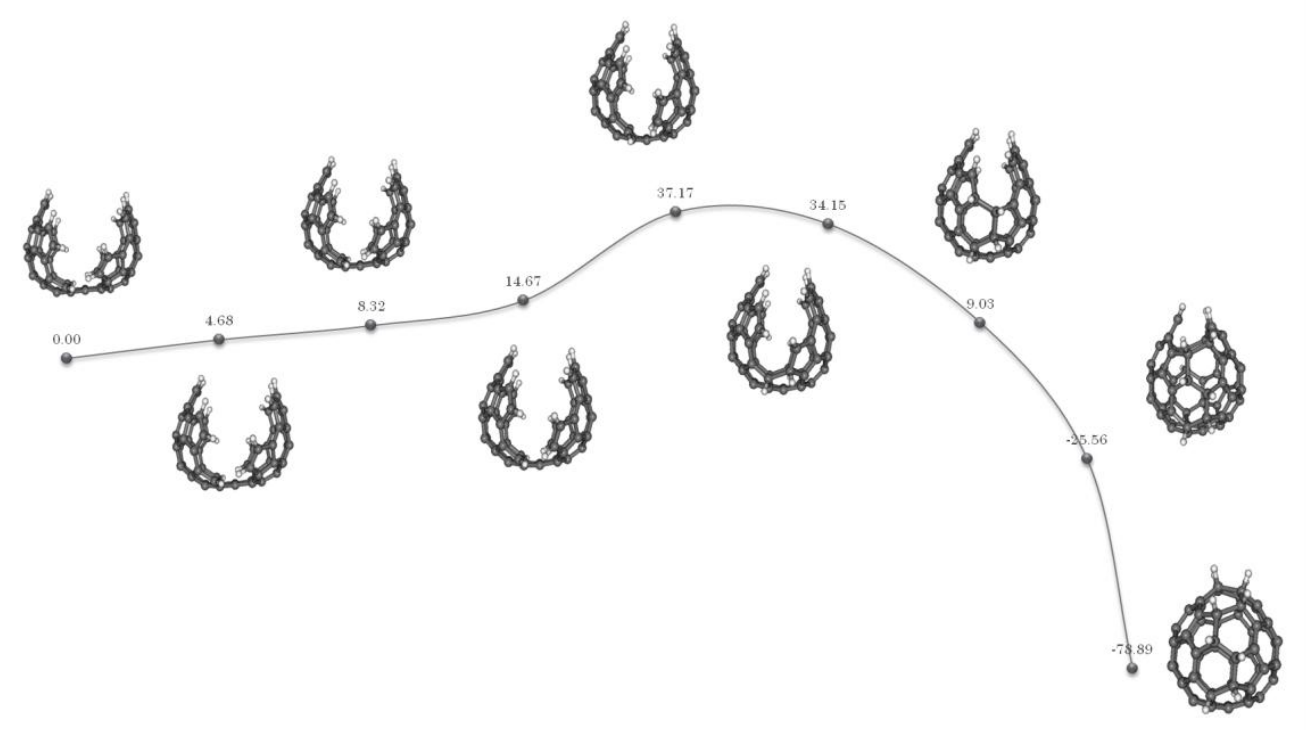

Figura 4.16. Coordenada de reacción de la dimerización a partir del primer aducto formado. 


\subsubsection{Conclusiones de la tercera parte}

La dimerización del fragmento $\mathbf{2}$ mediada por reacciones de Diels-Alder es factible. El mecanismo propuesto por pasos es termodinámica y cinéticamente favorecido sobre el mecanismo concertado en un solo paso. En este caso observamos que la formación del primer cicloaducto y su deshidrogenación son espontáneas, mientras que la formación del resto de los enlaces para dar lugar al $\mathrm{C}_{60}$ presenta una barrera de activación que podría ser superada calentando la mezcla de reacción. 


\section{Capítulo 5 Reactividad química}

Los principios de reactividad química nos permiten agrupar y racionalizar la gran cantidad de información sobre las diferentes reacciones químicas. Los principios de reactividad basados en la estructura electrónica determinan evolución de una reacción por el cambio en las propiedades electrónicas, como la electronegatividad, la dureza, o la naturaleza de los orbitales frontera, de las moléculas involucradas en la reacción. En este capítulo nos centraremos en el análisis de la reactividad en términos de las propiedades electrónicas, derivadas de la teoría de funcionales de la densidad, de los fragmentos $\mathbf{1}$ y $\mathbf{2}$ prístinos y mono- y disustituidos, así como del etileno y butadieno.

\subsection{Criterios de reactividad basados en la teoría de funcionales de la densidad.}

Los criterios de reactividad basados en la teoría de funcionales de la densidad nos permiten analizar la reactividad de una molécula en términos de sus propiedades iniciales. Sus principales exponentes son el potencial químico $\mu$ y la dureza $\eta$. En el marco de la teoría de funcionales de la densidad (DFT), el potencial químico $\mu$ se define como la derivada parcial de la energía con respecto al número de electrones a potencial externo constante ${ }^{128}$

$$
\mu=\left(\frac{\partial E}{\partial N}\right)_{v(r)}
$$

que por diferencias finitas puede calcularse como

$$
\mu=-\frac{I+A}{2}
$$


donde I y A son el potencial de ionización y la afinidad electrónica del sistema respectivamente. De esta forma el potencial químico puede identificarse como el negativo de la electronegatividad de Mülliken $\chi_{M}$

$$
\chi_{M}=\frac{I+A}{2}=-\mu
$$

Mientras que el potencial químico mide la tendencia de una molécula a ceder electrones, la dureza es una medida de la resistencia a cambiar de configuración electrónica. La dureza se define como la derivada del potencial químico con respecto al número de electrones a potencial externo constante o como la segunda derivada de la energía con respecto al número de electrones a potencial externo constante

$$
\eta=\left(\frac{\partial \mu}{\partial N}\right)_{v(r)}=\left(\frac{\partial^{2} E}{\partial N^{2}}\right)_{v(r)}
$$

En el esquema de diferencias finitas la dureza se obtiene como

$$
\eta=I-A
$$

Los conceptos de dureza $\eta$ y su inverso, la blandura $S(S=1 / \eta)$, fueron propuestos por Pearson en base a las estabilidades de los productos de reacción de diversas reacciones ácido-base. ${ }^{129}$ Pearson clasificó una serie de ácidos y bases de Lewis como "duros" o "blandos", de acuerdo a una variedad de criterios entre los que se incluían energías de enlace, constantes de equilibrio y velocidades de reacción, entre otros. En términos generales un ácido blando es una especie grande, con una carga positiva pequeña y muy polarizable, mientras que un ácido duro es una especie pequeña, con una gran carga positiva y poco polarizable. De la misma manera una base blanda es una especie poco electronegativa, muy polarizable y fácilmente oxidable, mientras que una base dura es una especie muy electronegativa, poco polarizable y difícilmente oxidable.

En base a sus observaciones, Pearson enunció el principio de ácidos y bases duros y blandos HSAB (Hard and Soft Acids and Bases) y el principio de máxima dureza MHP. ${ }^{130}$ El principio HSAB dice que los ácidos duros prefieren interactuar con las bases duras, mientras que los ácidos blandos prefieren reaccionar con bases blandas. Este principio indica que existe una estabilización extra en combinaciones duro-duro o blando-blando que, por ejemplo, reduciría la entalpía de reacción $(\Delta H<0)$ en reacciones de sustitución. 
Por otra parte, el principio de máxima dureza afirma que los sistemas moleculares tienden en el equilibrio a un estado de máxima dureza. Este principio conduce a que, en una reacción química, los productos sean los sistemas con mayor dureza mientras que los estados de transición deben ser las especies menos duras a lo largo del camino de reacción.

La electrofilia ${ }^{131}$ o potencia electrofílica $\omega$ también es un índice de reactividad global. Esta nos indica la capacidad de la molécula de aceptar electrones, evaluando la disminución de la energía de la especie en cuestión frente a un flujo máximo de electrones, que puede ser mayor o menor a uno. La electrofilia $\omega$ se define como el cuadrado del potencial químico $\mu$ entre el doble de la dureza $\eta$.

$$
\omega=\frac{\mu^{2}}{2 \eta}
$$

Y por diferencias finitas como

$$
\omega=\frac{(I+A)^{2}}{8(I-A)}
$$

Con la intención de diferenciar la respuesta del sistema al aceptar o donar electrones, y considerando que la dirección del flujo de electrones es definida, fundamentalmente, por el potencial químico, Gázquez y colaboradores ${ }^{132}$ consideraron las derivadas de la energía por la derecha y por la izquierda con respecto al número de electrones

$$
\begin{aligned}
& \mu^{+}=\left(\frac{\partial E}{\partial N}\right)_{v}^{+}=-\frac{1}{4}(I+3 A) \\
& \mu^{-}=\left(\frac{\partial E}{\partial N}\right)_{v}^{-}=-\frac{1}{4}(3 I+A)
\end{aligned}
$$

Definiendo así la potencia electroatractora $\omega^{+}$y la potencia electrodonadora $\omega^{-}$como

$$
\begin{aligned}
& \omega^{+}=\frac{\left(\mu^{+}\right)^{2}}{2 \eta}=\frac{(I+3 A)^{2}}{16(I-A)} \\
& \omega^{-}=\frac{\left(\mu^{-}\right)^{2}}{2 \eta}=\frac{(3 I+A)^{2}}{16(I-A)}
\end{aligned}
$$


De esta manera es posible cuantificar, en términos del potencial químico y la dureza, la cantidad de carga donada o aceptada por el sistema, considerando que valores grandes de $\omega^{+}$indican una mayor capacidad de aceptar electrones, mientras que valores pequeños de $\omega^{-}$indican una mayor capacidad de donar electrones.

En las Tablas 5.1 y 5.2 se muestran el potencial químico $\mu$, dureza $\eta$, blandura $S$, electrofilia $\omega$, potencia electroaceptora $\omega^{+}$y potencia electrodonadora $\omega^{-}$de los fragmentos 1 y 2 mono- $\left(R^{1} \neq R^{2}\right)$ y di- $\left(R^{1}=R^{2}\right)$ sustituidos. Podemos observar que el potencial químico es más negativo para los fragmentos sustituidos con grupos electroatractores que para los fragmentos sustituidos con grupos electrodonadores. En una reacción química el flujo de electrones se lleva a cabo de la especie con mayor potencial químico a la de menor potencial químico. En el caso de la reacción Diels-Alder podemos considerar dos opciones; para una reacción de demanda normal (NEDDA) el flujo de electrones parte del dieno hacia el dienófilo, mientras que en el caso de una reacción de demanda inversa (IEDDA) los electrones se mueven del dienófilo al dieno.

Considerando que el potencial químico del butadieno es mayor que el de los fragmentos, excepto en el caso de los fragmentos $\mathbf{1}$ y $\mathbf{2}$ disustituidos con grupos $\mathrm{NH}_{2}$, el flujo de electrones se llevaría a cabo del butadieno hacia los fragmentos, favoreciendo las reacciones de demanda normal, o de los fragmentos 1 y 2 disustituidos con grupos $\mathrm{NH}_{2}$ hacia el butadieno, favoreciendo una reacción de demanda inversa. Estas tendencias concuerdan con lo observado en el análisis de los orbitales frontera realizado en capítulos anteriores, donde observamos que las reacciones NED están favorecidas sobre las reacciones IED.

Observamos también que los fragmentos sin sustituir $(\mathrm{R}=\mathrm{H})$ son las especies más duras, seguidas por los fragmentos mono- y disustituidos con $F$, lo que indica que, de acuerdo con el principio $\mathrm{HSAB}$, las reacciones con estos fragmentos son preferidas sobre las reacciones con el resto de los fragmentos. En general, los fragmentos monosustituidos son más duros que los fragmentos disustituidos.

La diferencia de dureza entre los fragmentos no sustituidos y los mono- y disustituidos es muy pequeña, lo que se aprecia mejor al observar la blandura. En el caso del fragmento 1 la blandura del fragmento sin sustituir es igual que la de los fragmentos monosustituidos, a excepción del fragmento sustituido con $\mathrm{NH} 2$, y que la de los fragmentos disustituidos con los grupos $\mathrm{Me}, i-\mathrm{Pr}, \mathrm{F}$ y CF3, y solo una centésima de eV menos que el resto de los fragmentos. Por otra parte, la blandura del fragmento 2 sin sustituir es igual a la de los fragmentos mono-sustituidos con los grupos Me, $i$ $\operatorname{Pr}$ y $\mathrm{F}$ y los di-sustituidos con Me y $\mathrm{F}$, lo que indicaría que las reacciones con esos fragmentos serían las más favorecidas. 
Tabla 5.1. Potenciales químicos $\mu$, durezas $\eta$, blanduras $S$, electrofilias $\omega$, potencias electroaceptoras $\omega^{+}$y potencias electrodonadoras $\omega^{-}$del fragmento 1 mono- y disustituido. Los valores del fragmento sin sustituir (1-H), así como del butadieno y etileno se incluyen como referencia.

\begin{tabular}{|c|c|c|c|c|c|c|c|c|c|c|c|c|}
\hline \multirow[t]{2}{*}{ Molécula } & \multicolumn{2}{|c|}{$\mu \mathrm{eV}$} & \multicolumn{2}{|c|}{$\eta \mathrm{Ev}$} & \multicolumn{2}{|c|}{ S eV } & \multicolumn{2}{|c|}{$\omega \mathrm{eV}$} & \multicolumn{2}{|c|}{$\omega^{+} \mathrm{eV}$} & \multicolumn{2}{|c|}{$\omega^{-} \mathrm{eV}$} \\
\hline & $R^{1}=R^{2}$ & $R^{1} \neq R^{2}$ & $\mathbf{R}^{1}=\mathbf{R}^{2}$ & $R^{1} \neq R^{2}$ & $R^{1}=R^{2}$ & $R^{1} \neq R^{2}$ & $\mathrm{R}^{1}=\mathrm{R}^{2}$ & $R^{1} \neq R^{2}$ & $R^{1}=R^{2}$ & $R^{1} \neq R^{2}$ & $R^{1}=R^{2}$ & $\mathbf{R}^{1} \neq \mathbf{R}^{2}$ \\
\hline $1-\mathrm{NH}_{2}$ & -3.35 & -3.55 & 5.81 & 6.01 & 0.17 & 0.17 & 0.97 & 1.05 & 0.62 & 0.70 & 3.97 & 4.25 \\
\hline 1-OMe & -3.57 & -3.67 & 6.00 & 6.09 & 0.17 & 0.16 & 1.06 & 1.10 & 0.72 & 0.75 & 4.29 & 4.42 \\
\hline $1-\mathrm{OH}$ & -3.56 & -3.73 & 6.05 & 6.16 & 0.17 & 0.16 & 1.05 & 1.13 & 0.69 & 0.78 & 4.25 & 4.51 \\
\hline 1-Me & -3.68 & -3.76 & 6.22 & 6.32 & 0.16 & 0.16 & 1.09 & 1.12 & 0.73 & 0.75 & 4.41 & 4.51 \\
\hline $1-i-\operatorname{Pr}$ & -3.69 & -3.75 & 6.15 & 6.26 & 0.16 & 0.16 & 1.11 & 1.12 & 0.76 & 0.76 & 4.45 & 4.51 \\
\hline 1- $\mathrm{H}$ & \multicolumn{2}{|c|}{-3.80} & \multicolumn{2}{|c|}{6.39} & \multicolumn{2}{|c|}{0.16} & \multicolumn{2}{|c|}{1.13} & \multicolumn{2}{|c|}{0.76} & \multicolumn{2}{|c|}{4.56} \\
\hline $1-F$ & -3.96 & -3.91 & 6.31 & 6.30 & 0.16 & 0.16 & 1.24 & 1.21 & 0.90 & 0.86 & 4.86 & 4.77 \\
\hline 1-COOH & -4.16 & -3.97 & 6.03 & 6.29 & 0.17 & 0.16 & 1.44 & 1.25 & 1.17 & 0.91 & 5.34 & 4.88 \\
\hline $1-\mathrm{CF}_{3}$ & -4.24 & -4.02 & 6.13 & 6.32 & 0.16 & 0.16 & 1.47 & 1.28 & 1.20 & 0.94 & 5.44 & 4.96 \\
\hline $1-\mathrm{CHO}$ & -4.41 & -4.06 & 5.95 & 6.24 & 0.17 & 0.16 & 1.64 & 1.32 & 1.44 & 1.00 & 5.86 & 5.06 \\
\hline $1-\mathrm{CN}$ & -4.56 & -4.15 & 5.87 & 6.25 & 0.17 & 0.16 & 1.77 & 1.38 & 1.63 & 1.07 & 6.19 & 5.23 \\
\hline $1-\mathrm{NO}_{2}$ & -4.67 & -4.28 & 5.74 & 6.09 & 0.17 & 0.16 & 1.90 & 1.50 & 1.83 & 1.24 & 6.50 & 5.52 \\
\hline Butadieno & \multicolumn{2}{|c|}{-3.54} & \multicolumn{2}{|c|}{10.43} & \multicolumn{2}{|c|}{0.10} & \multicolumn{2}{|c|}{0.60} & \multicolumn{2}{|c|}{0.083} & \multicolumn{2}{|c|}{3.62} \\
\hline Etileno & \multicolumn{2}{|c|}{-3.54} & \multicolumn{2}{|c|}{13.68} & \multicolumn{2}{|c|}{0.07} & \multicolumn{2}{|c|}{0.46} & \multicolumn{2}{|c|}{0.001} & \multicolumn{2}{|c|}{3.54} \\
\hline
\end{tabular}

También observamos que el valor de la dureza del etileno es más del doble que la dureza de los fragmentos sin sustituir o sustituidos; por lo tanto las reacciones con etileno no se ven favorecidas, lo que concuerda con los parámetros cinéticos y termodinámicos discutidos en capítulos anteriores.

En el caso de la electrofilia $\omega$, podemos observar que los valores son mayores para los fragmentos sustituidos con grupos electroatractores que para los fragmentos sustituidos con grupos electrodonadores, lo que concuerda con el efecto de los sustituyentes sobre la capacidad de los fragmentos $\mathbf{1}$ y $\mathbf{2}$ de aceptar electrones. Observamos también que el fragmento $\mathbf{2}$ presenta un valor mayor de $\omega$ que el fragmento 1, lo que indicaría que el fragmento $\mathbf{2}$ puede aceptar más electrones que el fragmento 1. 
Tabla 5.2. Potenciales químicos $\mu$, durezas $\eta$, blanduras $S$, electrofilias $\omega$, potencias electroaceptoras $\omega^{+}$y potencias electrodonadoras $\omega^{-}$del fragmento 2 mono- y disustituido. Los valores del fragmento sin sustituir $(2-H)$, así como del butadieno y etileno se incluyen como referencia.

\begin{tabular}{|c|c|c|c|c|c|c|c|c|c|c|c|c|}
\hline \multirow[t]{2}{*}{ Molécula } & \multicolumn{2}{|c|}{$\mu \mathrm{eV}$} & \multicolumn{2}{|c|}{$n \mathrm{eV}$} & \multicolumn{2}{|c|}{$\mathrm{SeV}$} & \multicolumn{2}{|c|}{$\omega \mathrm{eV}$} & \multicolumn{2}{|c|}{$\omega+\mathrm{eV}$} & \multicolumn{2}{|c|}{$\omega-\mathrm{eV}$} \\
\hline & $R^{1}=R^{2}$ & $R^{1} \neq R^{2}$ & $R^{1}=R^{2}$ & $R^{1} \neq R^{2}$ & $\mathbf{R}^{1}=\mathbf{R}^{2}$ & $R^{1} \neq R^{2}$ & $R^{1}=R^{2}$ & $R^{1} \neq R^{2}$ & $R^{1}=R^{2}$ & $R^{1} \neq R^{2}$ & $R^{1}=R^{2}$ & $R^{1} \neq R^{2}$ \\
\hline 2- $\mathrm{NH}_{2}$ & -3.43 & -3.72 & 5.24 & 5.44 & 0.19 & 0.18 & 1.12 & 1.27 & 0.86 & 1.02 & 4.29 & 4.73 \\
\hline 2-OMe & -3.83 & -3.94 & 5.55 & 5.63 & 0.18 & 0.18 & 1.32 & 1.38 & 1.08 & 1.14 & 4.90 & 5.08 \\
\hline 2-OH & -3.79 & -3.95 & 5.51 & 5.57 & 0.18 & 0.18 & 1.30 & 1.40 & 1.05 & 1.18 & 4.84 & 5.13 \\
\hline 2-Me & -3.92 & -3.97 & 5.75 & 5.77 & 0.17 & 0.17 & 1.34 & 1.36 & 1.07 & 1.10 & 4.99 & 5.07 \\
\hline 2-i-Pr & -3.91 & -3.96 & 5.62 & 5.72 & 0.18 & 0.17 & 1.36 & 1.37 & 1.12 & 1.12 & 5.03 & 5.07 \\
\hline 2- H & \multicolumn{2}{|c|}{-4.02} & \multicolumn{2}{|c|}{5.86} & \multicolumn{2}{|c|}{0.17} & \multicolumn{2}{|c|}{1.38} & \multicolumn{2}{|c|}{1.11} & \multicolumn{2}{|c|}{5.13} \\
\hline $2-\mathrm{F}$ & -4.17 & -4.11 & 5.78 & 5.76 & 0.17 & 0.17 & 1.51 & 1.47 & 1.29 & 1.24 & 5.46 & 5.35 \\
\hline $2-\mathrm{COOH}$ & -4.40 & -4.27 & 5.41 & 5.58 & 0.18 & 0.18 & 1.79 & 1.63 & 1.72 & 1.48 & 6.13 & 5.74 \\
\hline $2-\mathrm{CF}_{3}$ & -4.52 & -4.23 & 5.59 & 5.83 & 0.18 & 0.17 & 1.82 & 1.54 & 1.74 & 1.32 & 6.25 & 5.55 \\
\hline $2-\mathrm{CHO}$ & -4.68 & -4.36 & 5.18 & 5.53 & 0.19 & 0.18 & 2.11 & 1.71 & 2.21 & 1.60 & 6.89 & 5.95 \\
\hline $2-\mathrm{CN}$ & -4.84 & -4.44 & 5.34 & 5.61 & 0.19 & 0.18 & 2.19 & 1.76 & 2.30 & 1.65 & 7.14 & 6.09 \\
\hline $2-\mathrm{NO}_{2}$ & -4.93 & -4.60 & 5.25 & 5.45 & 0.19 & 0.18 & 2.31 & 1.94 & 2.49 & 1.92 & 7.42 & 6.52 \\
\hline Butadieno & \multicolumn{2}{|c|}{-3.54} & \multicolumn{2}{|c|}{10.43} & \multicolumn{2}{|c|}{0.10} & \multicolumn{2}{|c|}{0.60} & \multicolumn{2}{|c|}{0.08} & \multicolumn{2}{|c|}{3.62} \\
\hline Etileno & \multicolumn{2}{|c|}{-3.54} & \multicolumn{2}{|c|}{13.68} & \multicolumn{2}{|c|}{0.07} & \multicolumn{2}{|c|}{0.46} & \multicolumn{2}{|c|}{0.001} & \multicolumn{2}{|c|}{3.54} \\
\hline
\end{tabular}

Con la intención de observar la magnitud del efecto electroatractor o electrodonador de los sustituyentes propuestos, calculamos las potencias electroaceptora $\omega^{+}$y electrodonadora $\omega^{-}$de los fragmentos mono- y disustituidos. Como era de esperarse los valores de $\omega^{+}$son mayores para los fragmentos sustituidos con grupos electroatractores que para los fragmentos sustituidos con grupos electrodonadores. De igual forma los valores de $\omega^{-}$son menores para los fragmentos sustituidos con grupos electrodonadores que para los fragmentos sustituidos con grupos electroatractores.

Observamos también el efecto en las potencias electroaceptora y electrodonadora debido a la presencia de uno o dos sustituyentes. En el caso de los sustituyentes electroatractores los valores de $\omega^{+}$son mayores para los fragmentos disustituidos que para los monosustituidos. Análogamente los valores de $\omega^{-}$son menores para los fragmentos disustituidos con grupos electrodonadores que 
para los fragmentos monosustituidos con los mismos grupos. Estas diferencias en la magnitud de $\omega^{+}$y $\omega^{-}$indican que el efecto electrónico (electroatractor o electrodonador) debido a la presencia de dos grupos sustituyentes en el fragmento favorece la reacción con el butadieno, lo que debería reflejarse en la reducción de la energía de interacción entre el butadieno y los fragmentos.

\subsection{Parámetros locales}

Los parámetros de reactividad local, en particular la función de Fukui, permiten determinar la naturaleza de los sitios reactivos en una molécula e inferir su comportamiento frente a otras especies.

La función de Fukui $f(r)^{133}$ se define como la derivada de la densidad $\rho(r)$ respecto al número de electrones $N$ a potencial externo $v(r)$ fijo, o como la derivada funcional del potencial químico $\mu$ respecto al potencial externo cuando $N$ es constante.

$$
f(r)=\left(\frac{\partial \rho(r)}{\partial N}\right)_{v(r)}=\left(\frac{\delta \mu}{\delta v(r)}\right)_{N}
$$

Usando el esquema de diferencias finitas para evaluar la derivada de $\rho(r)$ con respecto de $N$ obtenemos

$$
\begin{gathered}
f^{+}(r)=\rho_{N+1}(r)-\rho_{N}(r) \\
f^{-}(r)=\rho_{N}(r)-\rho_{N-1}(r) \\
f^{0}(r)=\frac{\rho_{N+1}(r)-\rho_{N-1}(r)}{2}
\end{gathered}
$$

donde $\rho_{N+1}(r), \rho_{N}(r)$ y $\rho_{N-1}(r)$ son las densidades electrónicas de los sistemas con $N+1, N$ y $N-1$ electrones y $f^{+}(r), f(r)$ y $f^{0}(r)$ son las funciones de Fukui para un ataque nucleofílico, electrofílico y por radicales libres respectivamente. 
La función de Fukui involucra la densidad electrónica de un átomo o molécula a través de la región de valencia, y por lo tanto está íntimamente relacionada con los orbitales frontera. Si ignoramos los efectos de relajación asociados a la adición o sustracción de electrones, entonces

$$
\begin{aligned}
& f^{+}(r) \approx \rho_{\text {LUMO }}(r) \\
& f^{-}(r) \approx \rho_{\text {HOMO }}(r)
\end{aligned}
$$

donde $\rho_{\text {LUMO }}(r)$ y $\rho_{\text {HOMO }}(r)$ son las densidades del orbital molecular desocupado de menor energía (LUMO) y del orbital molecular ocupado de mayor energía (HOMO) respectivamente.

Las funciones de Fukui condensadas se definen como

$$
\begin{gathered}
f_{i}^{+}(r)=q_{i}(N)-q_{i}(N+1) \\
f_{i}^{-}(r)=q_{i}(N-1)-q_{i}(N) \\
f_{i}^{0}(r)=\frac{q_{i}(N-1)-q_{i}(N+1)}{2}
\end{gathered}
$$

donde $q_{i}(N), q_{i}(N+1)$ y $q_{i}(N-1)$ es la carga del átomo $i$ en la molécula con $N, N+1$ y $N-1$ electrones respectivamente.

Adicionalmente, si definimos la blandura local $s(r)$ como

$$
s(r)=\left(\frac{\partial \rho(r)}{\partial \mu}\right)_{v(r)}
$$

y considerando la identidad

$$
\left(\frac{\partial \rho(r)}{\partial \mu}\right)_{v(r)}=\left(\frac{\delta \rho(r)}{\delta N}\right)_{v(r)}=\left(\frac{\delta N}{\delta \mu}\right)_{v(r)}
$$


encontramos que la blandura se relaciona con la función de Fukui como

$$
s(r)=f(r) S
$$

Esto nos indica que las funciones de Fukui $f(r)$ y la blandura local $s(r)$ contienen la misma información acerca de los sitios reactivos en una molécula; por lo tanto, las funciones de Fukui indican el esquema de reactividad en base a interacciones blando-blando y las funciones de Fukui condensadas pueden explicarse en términos de blandura.

En la misma dirección, Morell y cols. proponen un índice, al que llamaron descriptor dual, ${ }^{134}$ para describir la nucleofilia y electrofilia de una molécula en términos de la variación de la dureza $\eta$ con respecto al potencial externo $v(r)$. Este descriptor dual se define como la diferencia entre las funciones de Fukui nucleofílica $f^{+}(r)$ y electrofílica $f(r)$

$$
\Delta f(r)=f^{+}(r)-f^{-}(r)
$$

Si $\Delta f(r)>0$ el sitio es susceptible a ataques nucleofílicos, mientras que si $\Delta f(r)<0$ el sitio es reactivo frente a electrófilos. De esta manera el descriptor dual puede usarse para detectar simultáneamente las regiones nucleofílicas y electrofílicas de una molécula.

En el Anexo 2 se muestran las densidades de los orbitales HOMO $\left(\rho_{N}^{H O M O}(r)\right)$ y LUMO $\left(\rho_{N}^{L U M O}(r)\right)$, las funciones de Fukui para ataques por electrófilos $(f(r))$ y por nucleófilos $\left(f^{+}(r)\right)$ y el descriptor dual $(\Delta f(r))$ de los fragmentos 1 y $\mathbf{2}$ mono- y disustituidos y sin sustituir, así como del etileno y butadieno. De acuerdo con la definición de la reacción de Diels-Alder en términos de orbitales frontera la interacción se lleva a cabo entre el HOMO de una de las moléculas involucradas y el LUMO de la otra. En el caso de la reacción con los fragmentos sustituidos con grupos electroatractores se esperaría que la interacción favorecida fuera entre el LUMO de los fragmentos y el homo del butadieno (NEDDA), mientras que en el caso de los fragmentos sustituidos con grupos electrodonadores la interacción entre el HOMO del fragmento y el LUMO del butadieno (IEDDA) es la esperada, por lo que analizaremos las funciones de Fukui para un ataque nucleofílico $\left(f^{+}(r)\right)$ de los fragmentos sustituidos con grupos electroatractores y las funciones de Fukui para ataques por electrófilos $(f(r))$ en el caso de los fragmentos sustituidos con grupos electrodonadores. 
Podemos observar que en el caso del etileno y butadieno los sitios activos se encuentran bien localizados sobre los átomos de carbono, aunque, el etileno prefiere actuar como donador de electrones y el butadieno como aceptor de electrones, en ambos casos los átomos de carbono presentan carácter ambifílico (reactivos tanto frente a electrófilos como a nucleófilos), lo que concuerda con lo descrito en capítulos anteriores por los orbitales frontera, donde se resalta que esta reacción (entre etileno y butadieno) es de Demanda Electrónica Neutra.

De acuerdo con lo observado en capítulos anteriores, las reacciones entre los fragmentos $\mathbf{1}$ y $\mathbf{2}$ y el butadieno son de demanda normal, por lo que el orbital LUMO de los fragmentos es el involucrado en la reacción y la $f^{+}(r)$ es la que describiría la reactividad esperada. En el caso del fragmento 1 observamos que los sitios reactivos frente a electrófilos $(f(r))$ se encuentran deslocalizados en el cuerpo del fragmento, mientras que los sitios reactivos frente a nucleófilos $\left(f^{+}(r)\right)$ se localizan sobre los bordes del fragmento. Si aumentamos el valor del corte al máximo posible, en el que aún observamos contribuciones (Figura 5.1) encontramos que la función de Fukui para ataques nucloefílicos $\left(f^{+}(r)\right)$ es simétrica y se encuentra específicamente sobre los anillos de 6 miembros en el borde del fragmento, donde esperaríamos que se llevara a cabo la reacción con butadieno; mientras que la función de Fukui para ataques por electrófilos $(f(r))$ no es simétrica, si no que se encuentra orientada hacia un extremo del fragmento (en este caso hacia arriba) y fuera de los sitios donde esperaríamos la reacción. Esto confirma el carácter NEDDA de la reacción con butadieno.

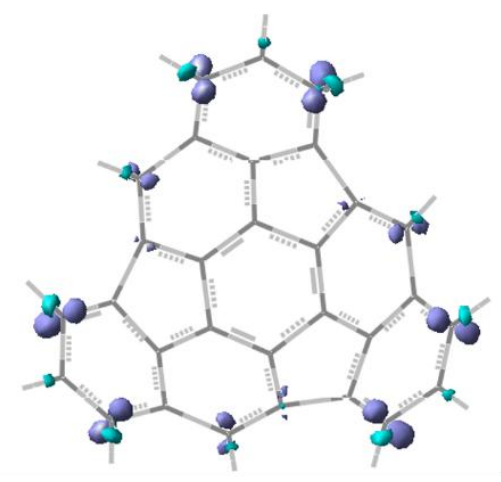

$f^{+}(r) \quad 0.005$ ua

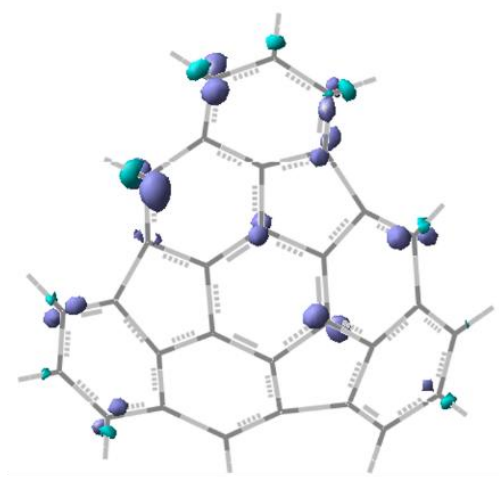

$f \cdot(r) \quad 0.005$ ua

Figura 5.1. Funciones de Fukui para ataques por nucleófilos $f^{+}(r)$ y electrófilos $f^{-}(r)$ del fragmento 1 


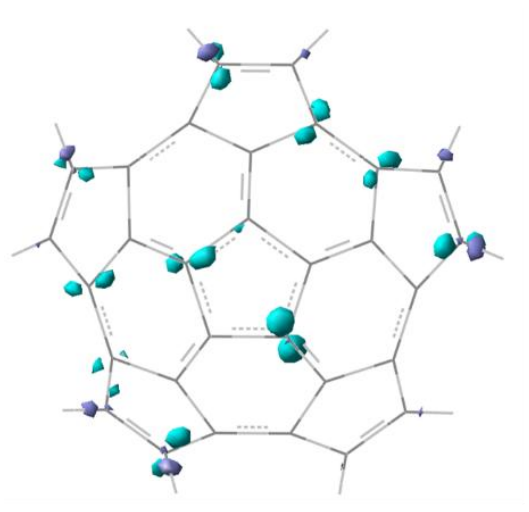

$f^{+}(r) \quad 0.005$ ua

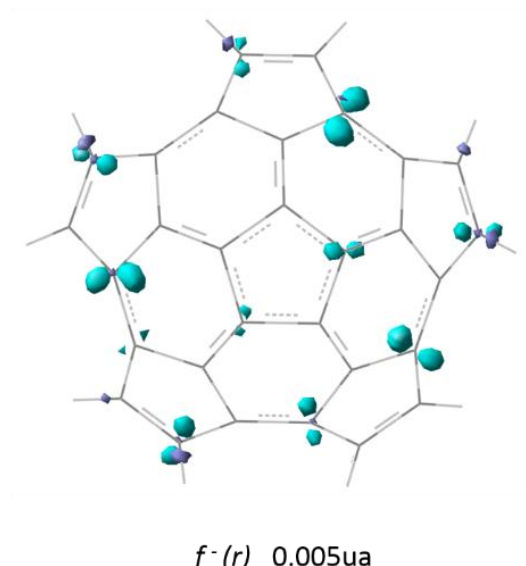

$f^{-}(r)$ 0.005ua

Figura 5.2. Funciones de Fukui para ataques por nucleófilos $f^{+}(r)$ y electrófilos $f^{-}(r)$ del fragmento 2

Por otra parte, aunque en el fragmento 2 se observan los sitios reactivos frente a electrófilos $(f(r))$ deslocalizados sobre el fragmento y los sitios reactivos frente a nucleófilos $\left(f^{+}(r)\right)$ en los bordes del fragmento, el efecto de una curvatura mayor en el fragmento, debida a la presencia del anillo de 5 miembros central, ocasiona que los sitios aceptores se deslocalicen, afectando también al anillo central. Si aumentamos nuevamente el nivel del corte (Figura 5.2) observamos que podemos encontrar contribuciones de las funciones de Fukui para ataques por nucleófilos $\left(f^{+}(r)\right)$ en los átomos asociados a los dobles enlaces del borde el fragmento donde se llevaría a cabo la reacción con butadieno,y contribuciones de la función de Fukui electrofílica sobre los anillos de 5 miembros del fragmento. En ambos casos se mantienen los sitios reactivos presentes en el anillo de 5 miembros central. Este tipo de reactividad, centrada en los anillos de 5 miembros, correlaciona con la observada en los fullerenos completos, específicamente en $\mathrm{C}_{60}$.

En el caso de los fragmentos mono- y disustituidos observamos que, contrario a lo esperado, el efecto electrónico (electroatractor o electrodonador) debido a la presencia de los grupos sustituyentes, no localiza la carga en los átomos involucrados en la reacción, sino que conduce a una deslocalización de los electrones sobre todo el cuerpo del fragmento, ocasionando el aumento de los sitios reactivos frente a nucleófilos y electrófilos, a tal grado que en algunos casos, prácticamente todos los átomos del fragmento son igualmente reactivos, como podemos ver en el caso de los fragmentos 1 y $\mathbf{2}$ mono-sustituidos con grupos $\mathrm{NO}_{2}$ o el fragmento $\mathbf{2}$ disustituido con grupos $\mathrm{CF}_{3}$. 
Para analizar con mayor detalle el efecto de la presencia de uno o dos grupos electrodonadores o electroatractores sobre los fragmentos, analizaremos las funciones de Fukui nucleofílica y electrofílica de los fragmentos 1 y $\mathbf{2}$ mono- y disustituidos con $\mathrm{NH}_{2}$ y $\mathrm{NO}_{2}$. En el primer caso, fragmento 1 sustituido con $\mathrm{NH}_{2}$ (Figura 5.3), se observa que la reactividad descrita por las funciones de Fukui para ataques por nucleófilos $\left(f^{+}(r)\right)$ se desplaza hacia el extremo opuesto al sustituyente, lo que es más claro en el caso del fragmento di-sustituido, mientras que las contribuciones de la función de Fukui para un ataque por electrófilos se mantienen en las cercanías del sustituyente, observándose un desplazamiento hacia el lado opuesto del fragmento o sobre el borde cuando hay uno o dos sustituyentes presentes respectivamente. El comportamiento aceptor observado al lado opuesto del fragmento y no sobre los átomos vecinos al sustituyente, donde habíamos propuesto se llevaría a cabo la reacción con butadieno, puede explicar las barreras de activación obtenidas anteriormente.

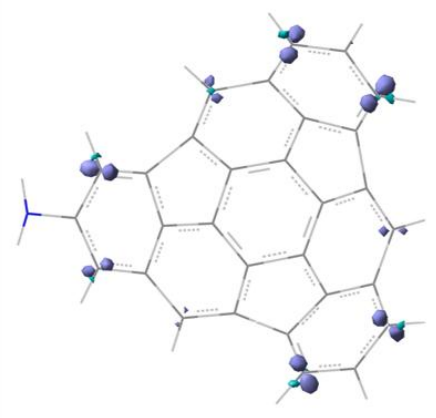

$f^{+}(r)$ 0.006ua

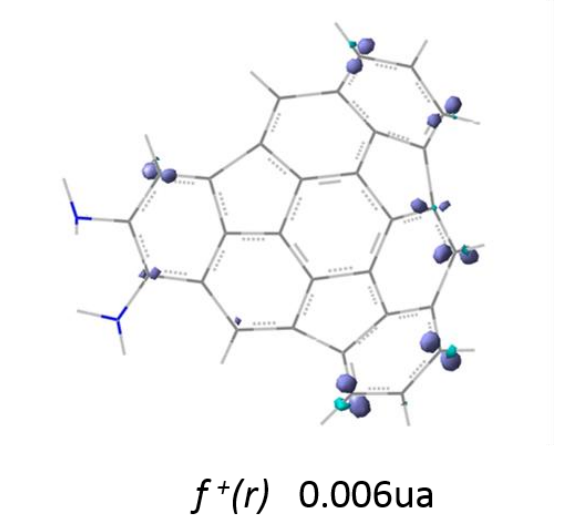

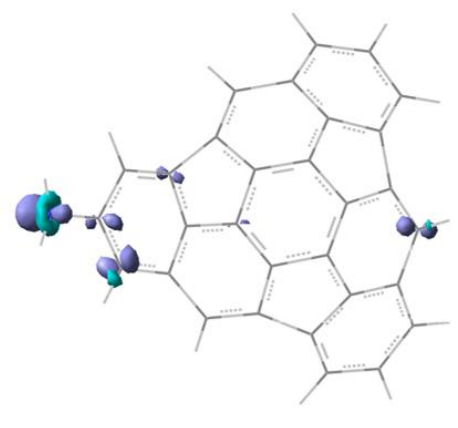

$f^{-}(r)$ 0.006ua

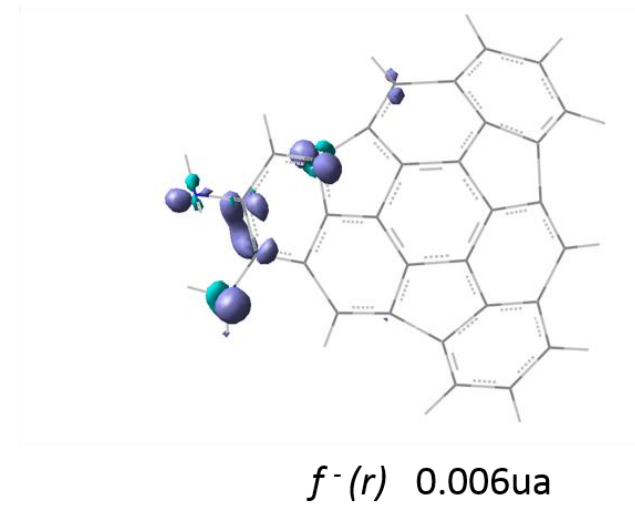

Figura 5.3. Funciones de Fukui para ataques por nucleófilos $f^{+}(r)$ y electrófilos $f^{-}(r)$ del fragmento 1 mono- y disustituido con $\mathrm{NH}_{2}$. 
En la Figura 5.4 se muestran las funciones de Fukui para ataques por nucleófilos y por electrófilos del fragmento 2 mono- y disustituido con $\mathrm{NH}_{2}$. En este caso observamos que en el fragmento monosustituido la reactividad frente a nucleófilos se encuentra desplazada hacia abajo de la posición del sustituyente en el fragmento, mientras que en el fragmento disustituido la reactividad de encuentra en el lado opuesto al sitio donde se encuentra el sustituyente. Por otro lado las contribuciones de las funciones de Fukui para ataques por electrófilos se encuentran en los átomos vecinos al sustituyente y desplazadas hacia arriba, en el caso del fragmento monosustituido, y simétricamente hacia arriba y abajo en el fragmento disustituido. Nuevamente las regiones con carácter aceptor de electrones se encuentran fuera de los átomos sobre los que propusimos las reacciones con butadieno.

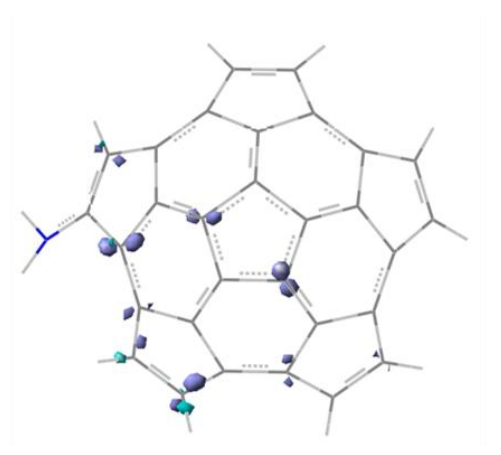

$f^{+}(r) \quad 0.006 u a$

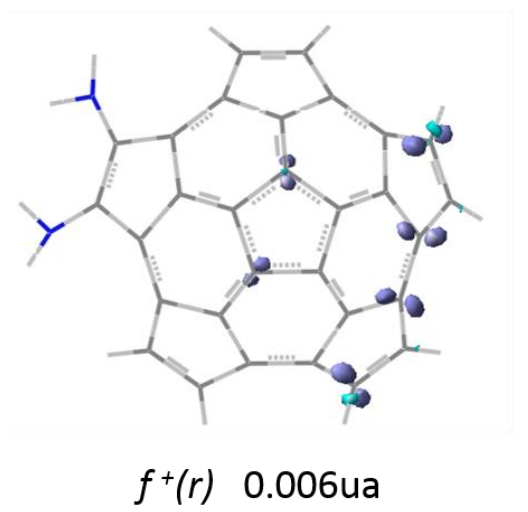

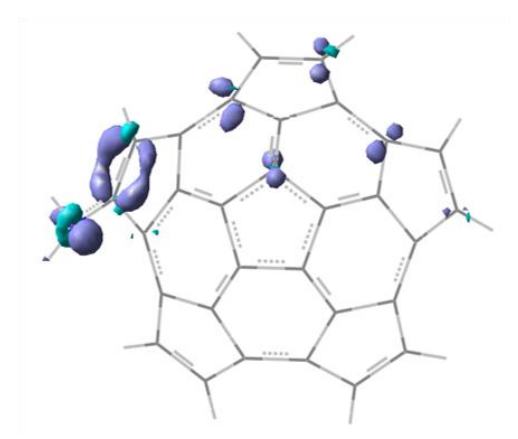

$f^{-}(r)$ 0.006ua

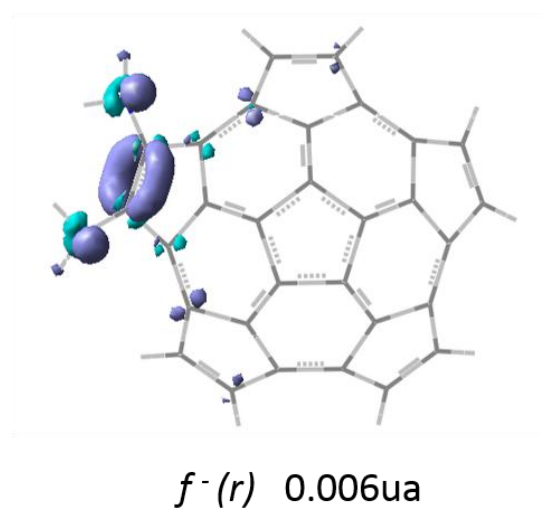

Figura 5.4. Funciones de Fukui para ataques por nucleófilos $f^{+}(r)$ y electrófilos $f^{-}(r)$ del fragmento 2 mono- y disustituido con $\mathrm{NH}_{2}$.

En el caso de los fragmentos sustituidos con $\mathrm{NO}_{2}$ observamos que para el fragmento 1 (Figura 5.5) las funciones de Fukui para un ataque por nucleófilos se encuentran sobre el grupo sustituyente, el átomo de carbono vecino y dispersas sobre el fragmento, en el anillo de 6 miembros central y 
en el sitio opuesto al sustituyente en el fragmento monosustituido y en el borde superior del fragmento disustituido, mientras que las funciones de Fukui para ataques electrofílicos se encuentran desplazadas hacia el extremo opuesto al sustituyente en ambos casos (mono- y disustituidos).

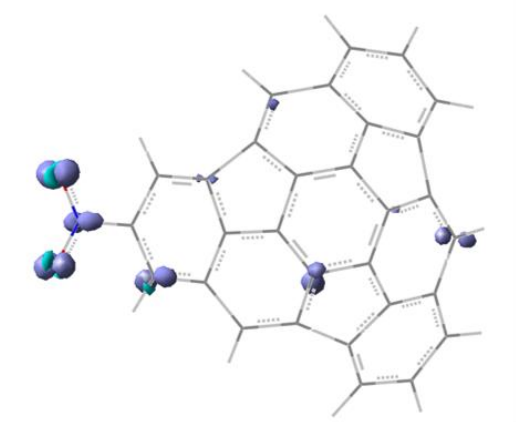

$f^{+}(r) \quad 0.006$ ua

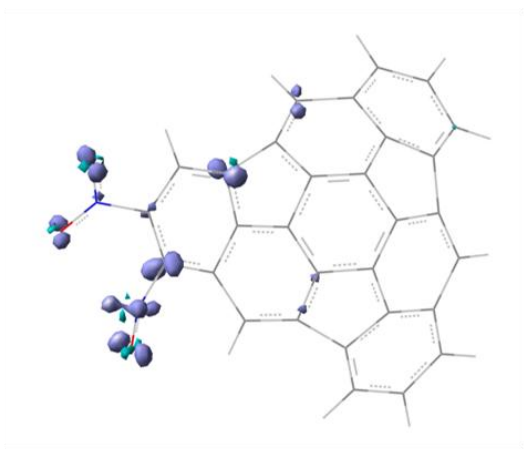

$f^{+}(r)$ 0.006ua

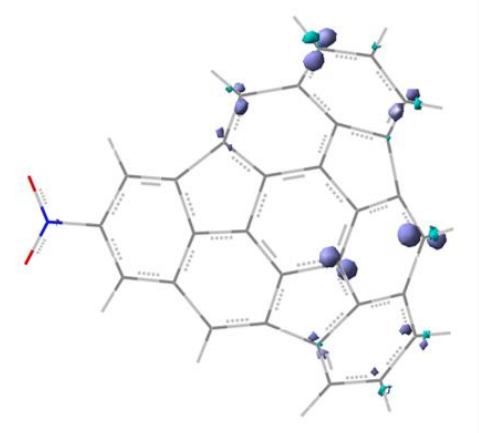

$f^{-}(r)$ 0.006ua

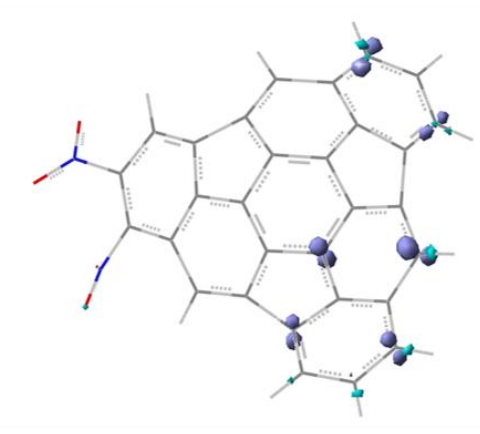

$f^{-}(r)$ 0.006ua

Figura 5.5. Funciones de Fukui para ataques por nucleófilos $f^{+}(r)$ y electrófilos $f^{-}(r)$ del fragmento 1 mono- y disustituido con $\mathrm{NO}_{2}$.

Similarmente, observamos que en el fragmento $\mathbf{2}$ (Figura 5.6) las funciones de Fukui para ataques nucleofílicos se encuentran, además de en los grupos sustituyentes, en los átomos vecinos a los sustituyentes y dispersas sobre los bordes del fragmento a ambos lados de la posición del sustituyente, mientras que las contribuciones de las funciones de Fukui para ataques por electrófilos se encuentran en el extremo opuesto a la posición de los sustituyentes en el fragmento, con pequeñas contribuciones en el resto del fragmento cuando éste está monosustituido. 
En este caso, aunque el efecto electroatractor de los grupos sustituyentes conduce a que las regiones aceptoras se localicen en los átomos sobre los que se llevarían a cabo las reacciones propuestas, el carácter aceptor del grupo sustituyente presente en el fragmento interviene en la reacción, propiciando que el butadieno reaccione con uno de los oxígenos del grupo $\mathrm{NO}_{2}$ en lugar de reaccionar con los carbonos del fragmento a los que está unido el sustituyente. Este efecto se observó en el análisis de los estados de transición obtenidos anteriormente, donde se observaban interacciones con los oxígenos de los sustituyentes y en la elevación de la energía de activación cuando se forzaba al butadieno a reaccionar con ambos átomos de carbono.

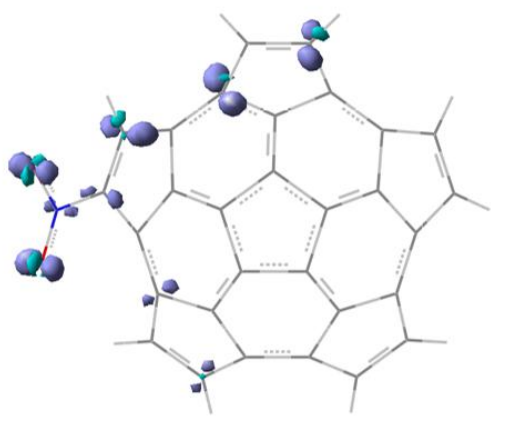

$f^{+}(r)$ 0.006ua

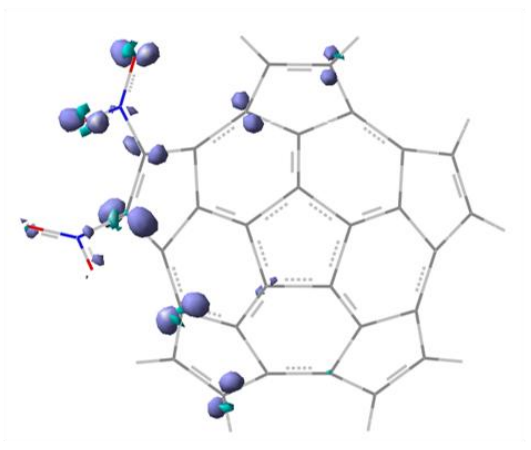

$f^{+}(r)$ 0.006ua

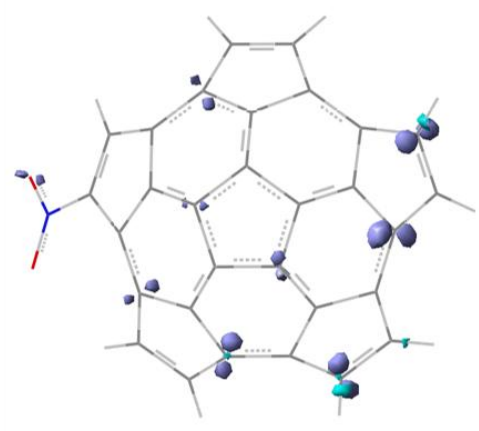

$f^{-}(r)$ 0.006ua

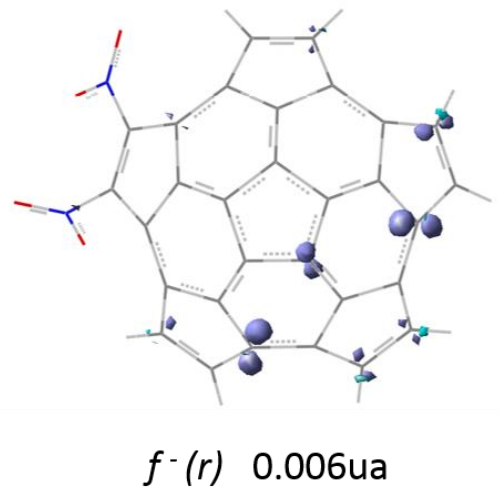

Figura 5.6. Funciones de Fukui para ataques por nucleófilos $f^{+}(r)$ y electrófilos $f^{-}(r)$ del fragmento 1 mono- $y$ di-sustituido con $\mathrm{NO}_{2}$.

Observamos también que la deslocalización de la densidad es mayor para los fragmentos sustituidos con grupos electroatractores, lo que indica la gran capacidad de los fragmentos de reorganizar la carga, tanto al aceptar como al perder electrones. Lo anterior podría explicar el hecho de que la presencia de los grupos sustituyentes no reduce sustancialmente la energía de activación obtenida en capítulos anteriores. Adicionalmente, si consideramos que al haber 
múltiples sitios activos sobre los que es posible llevar a cabo la reacción, la probabilidad de obtener el producto deseado se reduce, por lo que el rendimiento de la reacción, si pudiera llevarse a cabo, sería muy bajo.

Con la intención de analizar a más detalle los sitios activos de los fragmentos propuestos, obtuvimos las funciones de Fukui condensadas $\left(f_{i}(r)\right)$. Las gráficas de las funciones de Fukui condensadas de los fragmentos $\mathbf{1}$ y $\mathbf{2}$ mono- y disustituidos y sin sustituir, así como de etileno y butadieno, se muestran en el Anexo 3.

Las $f_{i}(r)$ de butadieno, etileno y los fragmentos 1 y 2 prístinos se obtuvieron empleando los esquemas de cargas de Mülliken y Hirshfeld. Observamos que las $f_{i}(r)$ calculadas con el esquema de Mülliken subestiman la reactividad de los átomos de carbono y sobreestiman los valores de reacivodad de los hidrógenos, haciendo que estos últimos sean los átomos más reactivos en los fragmentos. Estos resultados nos llevan a usar el esquema de Hirshfeld en el análisis de las funciones de Fukui condensadas.

En el caso del etileno y butadieno observamos que los átomos más blandos son los involucrados en la reacción (C1 y C4 en el caso del etileno y C1 y C7 en el caso del butadieno). Las magnitudes de las funciones de Fukui condensadas para ataques por electrófilos y por nucleófilos son similares, siendo ligeramente mayor el carácter donador de los átomos de carbono analizados.

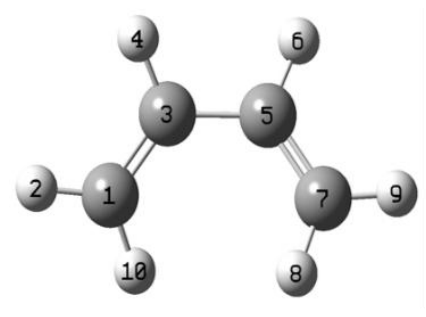

Butadieno

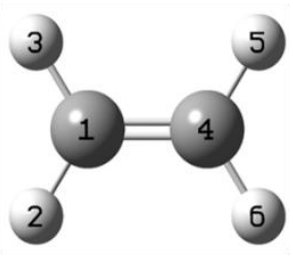

Etileno

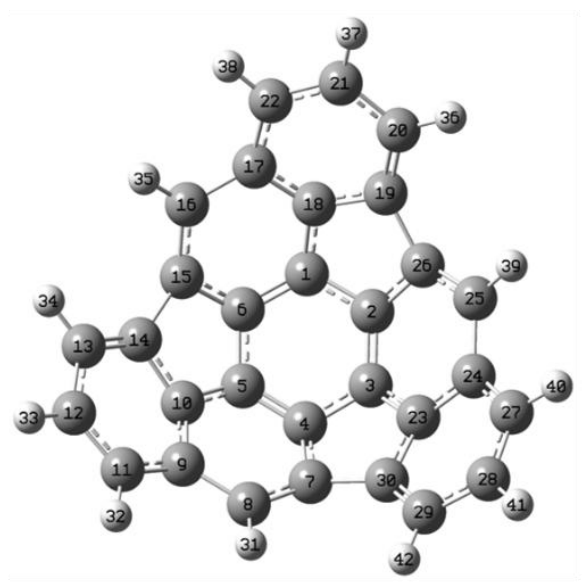

Fragmento 1

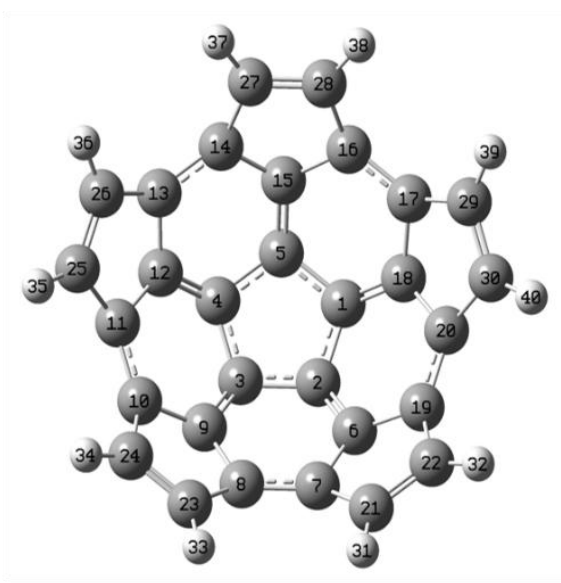

Fragmento 2

Figura 5.7. Estructuras numeradas de etileno, butadieno y los fragmentos $\mathbf{1}$ y 2. 
En el caso del fragmento 1 observamos que los sitios preferidos para ataques por nucleófilos son los carbonos C11, C13, C20, C22, C27 y C29 (ver Figura 5.7 para numeración), localizados en el borde del fragmento y sobre los cuales esperamos ocurran las reacciones propuestas. En cuanto a carácter donador, el átomo más reactivo es $\mathrm{C25}$, que forma parte de una de las regiones propuestas con comportamiento de dieno. Análogamente, los sitios más reactivos frente a taques nucleofílicos en el fragmento 2 son los átomos C4, C24 y C27, el primero pertenece al anillo de 5 miembros central y los segundos forman parte de las regiones dienofílicas propuestas, mientras que los sitios más reactivos frente a electrófilos son los átomos $\mathrm{C} 7$ en el cuerpo del fragmento y C27 y C30 en los bordes; estos últimos forman parte de las regiones con carácter de dieno propuestas. Debido a la simetría del fragmento 2 esperábamos encontrar valores más simétricos de las funciones de Fukui, como los observados en el fragmento 1, sin embargo, los valores obtenidos concuerdan con la baja energía de activación obtenida en el análisis de la dimerización analizado en capítulos anteriores.

Si analizamos el fragmento 1 monosustituido observamos que el efecto del grupo sustituyente sobre el fragmento se puede ver en 3 puntos específicos (Figura 5.8). El primero (en azul) está en el átomo C28, sobre el que se encuentra el sustituyente, y los átomos vecinos C27 y C29. En este punto se observa el efecto directo del grupo sustituyente, es decir, cuando el sustituyente es electroatractor los carbonos 27 y 28 generalmente presentan carácter aceptor, mientras que cuando el sustituyente es electrodonador estos carbonos tienen carácter donador principalmente. El carácter donador o aceptor del átomo C29 depende de la fuerza electroaceptora y electrodonadora del sustituyente. El segundo punto (en verde), está sobre el átomo C16 que se encuentra en el lado opuesto del fragmento. Este átomo presenta siempre carácter donador. El tercer punto (en rojo) son los átomos C11 y C20; estos átomos generalmente presentan carácter contrario al del sustituyente, es decir si el grupo sustituyente es electrodonador los átomos C11 y C20 presentan carácter aceptor y si el sustituyente es electroatractor los átomos tienen carácter donador. Análogamente, para el fragmento 2 se observan dos puntos principales de influencia. El primero lo conforman los átomos $\mathrm{C} 29$, al que está unido el grupo sustituyente, y su vecino $\mathrm{C} 30$, en ellos se refleja directamente el efecto del sustituyente. El segundo punto está sobre el átomo C10, que se encuentra en el extremo del fragmento opuesto al sustituyente y que presenta, generalmente, carácter donador.

En el caso del fragmento 1 disustituido se observa que las 3 áreas de influencia se mantienen, solamente cambia el carácter del átomo C16 que ahora depende del sustituyente, siendo donador cuando el sustituyente es electroatractor y aceptor cuando el sustituyente es electrodonador. Lo mismo ocurre para el fragmento 2 disustituido; en este caso el área 2 (en verde) abarca los átomos $\mathrm{C} 10$ y $\mathrm{C} 11$ que se comportan como donadores, excepto cuando los grupos sustituyentes son $\mathrm{NH}_{2}$, $\mathrm{OMe} \mathrm{y} \mathrm{OH}$. 

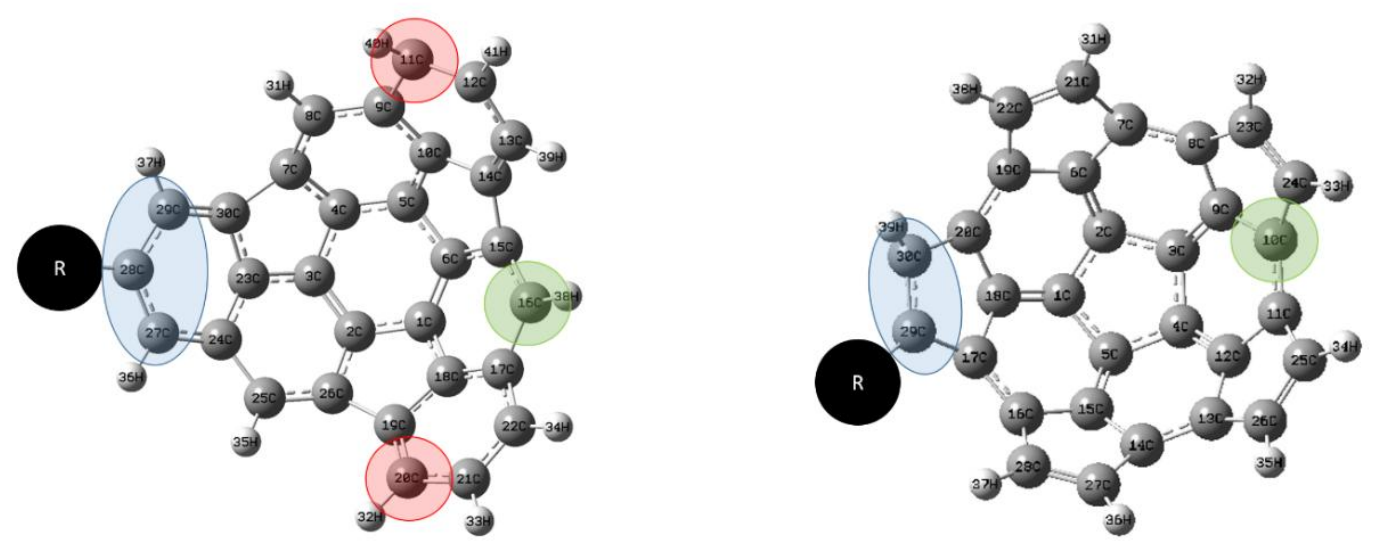

Figura 5.8. Áreas de influencia del grupo sustituyente $R$ en los fragmentos $\mathbf{1}$ y $\mathbf{2}$ monosustituidos. Área 1 en azul, área 2 en verde y área 3 en rojo.

\section{Energía de interacción}

Considerando la interacción entre dos moléculas A y B con NA y NB electrones respectivamente, la energía de interacción puede escribirse como:

$$
\Delta E_{\text {int }}=E\left[\rho_{A B}\right]-E\left[\rho_{A}\right]-E\left[\rho_{B}\right]
$$

Donde $\rho_{A B}, \rho_{A}$ y $\rho_{B}$ son las densidades de los sistemas $A B, A$ y $B$ respectivamente.

En términos del principio HSAB local propuesto por Méndez y Gázquez, ${ }^{135}$ la energía de interacción se lleva a cabo en dos pasos. En el primer paso la interacción se da a través de la igualación del potencial químico de los sistemas A y B a potencial externo constante, ${ }^{136}$ mientras que en el segundo se producen cambios en la densidad electrónica total a partir de cambios en el potencial externo a potencial químico constante. ${ }^{137}$ El primer paso es una manifestación del principio de igualación de electronegatividades, ${ }^{138}$ mientras que el segundo pone de manifiesto el principio de máxima dureza. ${ }^{130}$

De esta forma se define la energía de interacción como: 


$$
\Delta E_{\text {int }}=\Delta E_{v}+\Delta E_{\mu}
$$

donde:

$$
\begin{gathered}
\Delta E_{v} \approx-\frac{1}{2}\left(\frac{\left(\mu_{A}-\mu_{B}\right)^{2}}{\eta_{A}+\eta_{B}}\right) \\
\Delta E_{\mu} \approx-\frac{1}{2} N^{2}\left(\eta_{A B}-\eta_{A B}^{*}\right)
\end{gathered}
$$

donde $\eta_{A B}$ es la dureza del sistema $A B$ en el equilibrio y $\eta_{A B}^{*}$ es la dureza del sistema cuando los componentes $A$ y $B$ se encuentran separados al infinito.

En términos de blandura las ecuaciones anteriores pueden escribirse como:

$$
\begin{gathered}
\Delta E_{v} \approx-\frac{1}{2}\left(\frac{\left(\mu_{A}-\mu_{B}\right)^{2}}{S_{A}+S_{B}}\right)\left(S_{A} S_{B}\right) \\
\Delta E_{\mu} \approx-\frac{1}{2}\left(\frac{\lambda}{S_{A}+S_{B}}\right)
\end{gathered}
$$

Donde $\lambda$ es una constante de proporcionalidad que relaciona la blandura total del sistema en equilibrio $S_{A B}$ y del sistema $S_{A B}^{*}$ con la blandura de los sistemas A y B aislados $\left(S_{A B}=k\left(S_{A}+S_{B}\right)\right.$ y $\left.S_{A B}^{*}=k^{\prime}\left(S_{A}+S_{B}\right)\right)$ y el número de electrones del sistema $\mathrm{N}_{\mathrm{AB}}$

$$
\lambda=N^{2} K
$$

donde:

$$
K=\frac{1}{k-k^{\prime}}
$$

$\lambda$ representa el comportamiento dinámico de la nube de electrones durante la reacción y se ha relacionado físicamente con el número efectivo de electrones de valencia involucrados en la reacción. Se ha observado que en el caso de interacciones fuertes, como son los enlaces covalentes formados en reacciones de cicloadición, $\lambda$ toma el valor de $1(\lambda=1){ }^{139}$

Desde un punto de vista local, si la interacción se lleva a cabo entre el i-ésimo átomo de A y el sistema B, se puede expresar la energía de interacción como: 


$$
\Delta E_{\text {int }} \approx-\frac{1}{2}\left(\frac{\left(\mu_{A}-\mu_{B}\right)^{2}}{S_{A} f_{A i}+S_{B}}\right)\left(S_{A} f_{A i} \cdot S_{B}\right)-\frac{1}{2}\left(\frac{\lambda}{S_{A} f_{A i}+S_{B}}\right)
$$

Donde $f_{A i}$ es la función de Fukui del sitio i en A.

Los dos términos de la ecuación que describe la energía de interacción (ecuación 5.32) pueden interpretarse como las contribuciones a la energía debidas a la transferencia de carga $(\Delta E v)$ y a la reorganización electrónica $(\Delta \mathrm{E} \mu)$. Para reacciones neutrales, como es el caso de las reacciones de cicloadición de Diels-Alder, la magnitud de la contribución debida al primer término es muy pequeña, cercana a cero, mientras que la contribución debida al segundo término alcanza hasta el 99\% de la energía de interacción total.

Obtuvimos las energías de interacción para las reacciones de dimerización de los fragmentos $\mathbf{1}$ y $\mathbf{2}$ y las reacciones de los mismos con etileno y butadieno desde un punto de vista global y local. Los resultados de muestran en la Tabla 5.3.

Podemos observar que la contribución del término de transferencia de carga es cero para las reacciones de dimerización $(\mathbf{1 + 1}$ y $\mathbf{2 + 2})$ debido a que no hay cambio en el potencial químico de las especies interactuantes al ser estas iguales. La energía de interacción depende solamente del cambio en la dureza al pasar de los fragmentos individuales al fullereno $C_{60}$. En términos globales, esta estabilización de la energía debida al cambio de dureza es del orden de $30 \mathrm{kcal} / \mathrm{mol}$, siendo mayor para la reacción con el fragmento 1 (36.8 kcal/mol) que para las reacciones con el fragmento $2(33.8 \mathrm{kcal} / \mathrm{mol})$.

En el caso de las reacciones con etileno y butadieno, observamos que la contribución del primer término, aunque aún es muy pequeño, es mayor para las reacciones con etileno que con butadieno, esto indica que, globalmente, la transferencia de carga es mayor del etileno a los fragmentos $\mathbf{1}$ y $\mathbf{2}$, que de butadieno a los mismos. Adicionalmente, la diferencia entre los aductos formados durante las reacciones propuestas y los sistemas separados es mayor que la diferencia entre los fragmentos y el $\mathrm{C}_{60}$, alrededor de $10 \mathrm{kcal} / \mathrm{mol}$ para las reacciones con butadieno y 15 $\mathrm{kcal} / \mathrm{mol}$ para las reacciones con etileno. Esto se observa también en la dureza global presentada anteriormente, donde observamos que la diferencia de dureza entre el etileno y los fragmentos es mayor que la diferencia de dureza entre el butadieno y los fragmentos.

En el caso de la descripción local, empleamos los esquemas de cargas de Mülliken y Hirshfeld para calcular las funciones de Fukui condensadas de los átomos de los fragmentos. Como 
determinamos anteriormente, las reacciones propuestas son de demanda normal, es decir se llevan a cabo entre el HOMO del butadieno y el LUMO del fragmento, por lo que utilizamos las funciones de Fukui para ataques nucleofílicos $\left(f_{i}^{+}(r)\right)$ para calcular la blandura local de los átomos involucrados en la reacción. Observamos que la energía de interacción calculada con el esquema de Mülliken es más negativa, alrededor de $2 \mathrm{kcal} / \mathrm{mol}$ en el caso de las reacciones de dimerización y entre 5 y $10 \mathrm{kcal} / \mathrm{mol}$ para las reacciones con butadieno y etileno.

Tabla 5.3. Energías de interacción de los fragmentos 1 y $\mathbf{2}$ con ellos mismos y con etileno y butadieno desde un punto de vista global y local, empleando los esquemas de Mülliken y Hirshfeld para el cálculo de las funciones de Fukui necesarias.

\begin{tabular}{|c|c|c|c|c|c|}
\hline Reacción & & $\begin{array}{c}\Delta \mathrm{Ev} \\
(\mathrm{kcal} / \mathrm{mol})\end{array}$ & $\begin{array}{c}\Delta \mathrm{E} \mu \\
\text { (kcal/mol) }\end{array}$ & $\begin{array}{c}\Delta \mathrm{E} \text { int } \\
\text { (kcal/mol) }\end{array}$ & $\lambda$ \\
\hline & Global & 0 & -36.857 & -36.857 & 1 \\
\hline \multirow[t]{3}{*}{$1+1$} & Mülliken & 0 & -73.988 & -73.988 & 1 \\
\hline & Hirshfeld & 0 & -71.886 & -71.886 & 1 \\
\hline & Global & -0.048 & -45.699 & -45.747 & 1 \\
\hline \multirow[t]{3}{*}{$1+b$} & Mülliken & 0.000 & -120.979 & -120.978 & 1 \\
\hline & Hirshfeld & -0.003 & -115.459 & -115.462 & 1 \\
\hline & Global & -0.040 & -50.243 & -50.283 & 1 \\
\hline \multirow[t]{3}{*}{$1+e$} & Mülliken & 0.000 & -159.055 & -159.055 & 1 \\
\hline & Hirshfeld & -0.003 & -149.650 & -149.653 & 1 \\
\hline & Global & 0 & -33.802 & -33.802 & 1 \\
\hline \multirow[t]{3}{*}{$2+2$} & Mülliken & 0 & -67.083 & -67.083 & 1 \\
\hline & Hirshfeld & 0 & -66.178 & -66.178 & 1 \\
\hline & Global & -0.166 & -43.275 & -43.441 & 1 \\
\hline \multirow[t]{3}{*}{$2+b$} & Mülliken & -0.004 & -118.607 & -118.611 & 1 \\
\hline & Hirshfeld & -0.010 & -115.806 & -115.816 & 1 \\
\hline & Global & -0.139 & -47.328 & -47.467 & 1 \\
\hline \multirow[t]{2}{*}{$2+e$} & Mülliken & -0.004 & -154.981 & -154.985 & 1 \\
\hline & Hirshfeld & -0.010 & -150.233 & -150.243 & 1 \\
\hline
\end{tabular}


Tabla 5.4. Energías de interacción del fragmento 1 mono- y disustituido con butadieno.

\begin{tabular}{|c|c|c|c|c|c|}
\hline & & $\begin{array}{c}\Delta \mathrm{Ev} \\
\text { (kcal/mol) }\end{array}$ & $\begin{array}{c}\Delta \mathrm{E} \mu \\
\text { (kcal/mol) }\end{array}$ & $\begin{array}{c}\Delta \mathrm{E} \text { int } \\
\text { (kcal/mol) }\end{array}$ & $\lambda$ \\
\hline \multirow{2}{*}{ 1- $\mathrm{NH}_{2}$} & $\mathbf{R}^{1}=\mathrm{R}^{2}$ & -0.001 & -116.316 & -116.317 & 1 \\
\hline & $\mathbf{R}^{1} \neq \mathbf{R}^{2}$ & 0.000 & -116.319 & -116.319 & 1 \\
\hline \multirow[t]{2}{*}{ 1-OMe } & $R^{1}=R^{2}$ & 0.000 & -116.692 & -116.692 & 1 \\
\hline & $R^{1} \neq R^{2}$ & -0.001 & -116.203 & -116.203 & 1 \\
\hline \multirow{2}{*}{$1-\mathrm{OH}$} & $\mathbf{R}^{1}=\mathbf{R}^{2}$ & 0.000 & -116.276 & -116.276 & 1 \\
\hline & $R^{1} \neq R^{2}$ & -0.001 & -116.140 & -116.141 & 1 \\
\hline \multirow{2}{*}{ 1-Me } & $\mathbf{R}^{1}=\mathbf{R}^{2}$ & -0.001 & -116.828 & -116.829 & 1 \\
\hline & $\mathbf{R}^{1} \neq \mathbf{R}^{2}$ & -0.002 & -116.445 & -116.447 & 1 \\
\hline \multirow[t]{2}{*}{$1-i-\operatorname{Pr}$} & $R^{1}=R^{2}$ & -0.001 & -116.922 & -116.923 & 1 \\
\hline & $\mathbf{R}^{1} \neq \mathbf{R}^{2}$ & -0.002 & -116.443 & -116.445 & 1 \\
\hline 1- $\mathrm{H}$ & $\mathrm{R}^{1}=\mathrm{R}^{2}$ & -0.003 & -115.459 & -115.462 & 1 \\
\hline \multirow{2}{*}{$1-\mathrm{F}$} & $R^{1}=R^{2}$ & -0.007 & -115.899 & -115.906 & 1 \\
\hline & $\mathbf{R}^{1} \neq \mathbf{R}^{2}$ & -0.006 & -115.779 & -115.784 & 1 \\
\hline \multirow{2}{*}{$1-\mathrm{COOH}$} & $\mathbf{R}^{1}=\mathbf{R}^{\mathbf{2}}$ & -0.025 & -113.409 & -113.434 & 1 \\
\hline & $\mathbf{R}^{1} \neq \mathbf{R}^{2}$ & -0.012 & -113.335 & -113.346 & 1 \\
\hline \multirow[t]{2}{*}{$1-\mathrm{CF}_{3}$} & $\mathbf{R}^{1}=\mathbf{R}^{2}$ & -0.028 & -114.140 & -114.168 & 1 \\
\hline & $R^{1} \neq R^{2}$ & -0.009 & -116.050 & -116.059 & 1 \\
\hline \multirow{2}{*}{ 1- $\mathrm{CHO}$} & $\mathbf{R}^{1}=\mathbf{R}^{\mathbf{2}}$ & -0.055 & -112.522 & -112.577 & 1 \\
\hline & $R^{1} \neq R^{2}$ & -0.016 & -113.909 & -113.925 & 1 \\
\hline \multirow{2}{*}{$1-\mathrm{CN}$} & $\mathbf{R}^{1}=\mathbf{R}^{2}$ & -0.074 & -112.605 & -112.679 & 1 \\
\hline & $\mathbf{R}^{1} \neq \mathbf{R}^{2}$ & -0.019 & -114.806 & -114.825 & 1 \\
\hline \multirow{2}{*}{$1-\mathrm{NO}_{2}$} & $R^{1}=R^{2}$ & -0.079 & -113.657 & -113.736 & 1 \\
\hline & $R^{1} \neq R^{2}$ & -0.029 & -114.427 & -114.457 & 1 \\
\hline
\end{tabular}

En las Tablas 5.4 y 5.5 se muestran las energías de interacción para las reacciones con los fragmentos 1 y $\mathbf{2}$ y butadieno, considerando las interacciones locales. El esquema de cargas de Hirshfeld se empleó para calcular las funciones de Fukui para ataques por nucleófilos, necesarias para la descripción de estos sistemas. Podemos observar que, aunque el término de transferencia de carga sique siendo muy pequeño (menor al $1 \%$ de la energía de interacción total), esta contribución es mayor para las reacciones con los fragmentos sustituidos con grupos 
electroatractores, especialmente los fragmentos disustituidos. Esto implicaría que en los fragmentos sustituidos con grupos electroatractores la transferencia de carga es mayor que para los fragmentos sustituidos con grupos electrodonadores

Tabla 5.5. Energías de interacción del fragmento 2 mono- y disustituido con butadieno.

\begin{tabular}{|c|c|c|c|c|c|}
\hline & & $\begin{array}{c}\Delta \mathrm{Ev} \\
(\mathrm{kcal} / \mathrm{mol})\end{array}$ & $\begin{array}{c}\Delta \mathrm{E} \mu \\
\text { (kcal/mol) }\end{array}$ & $\begin{array}{c}\Delta \mathrm{E} \text { int } \\
\text { (kcal/mol) }\end{array}$ & $\lambda$ \\
\hline \multirow{2}{*}{ 2- $\mathrm{NH}_{2}$} & $R^{1}=R^{2}$ & 0.000 & -115.540 & -115.541 & 1 \\
\hline & $R^{1} \neq R^{2}$ & -0.001 & -116.934 & -116.935 & 1 \\
\hline \multirow{2}{*}{ 2-OMe } & $R^{1}=R^{2}$ & -0.004 & -114.886 & -114.891 & 1 \\
\hline & $R^{1} \neq R^{2}$ & -0.005 & -116.873 & -116.878 & 1 \\
\hline \multirow{2}{*}{$2-\mathrm{OH}$} & $R^{1}=R^{2}$ & -0.003 & -114.409 & -114.412 & 1 \\
\hline & $R^{1} \neq R^{2}$ & -0.005 & -116.905 & -116.910 & 1 \\
\hline \multirow{2}{*}{ 2-Me } & $R^{1}=R^{2}$ & -0.005 & -116.587 & -116.592 & 1 \\
\hline & $R^{1} \neq R^{2}$ & -0.006 & -116.883 & -116.889 & 1 \\
\hline \multirow[t]{2}{*}{ 2-i-Pr } & $R^{1}=R^{2}$ & -0.006 & -115.657 & -115.663 & 1 \\
\hline & $R^{1} \neq R^{2}$ & -0.005 & -116.903 & -116.908 & 1 \\
\hline 2- H & $R^{1}=R^{2}$ & -0.010 & -115.806 & -115.816 & 1 \\
\hline \multirow{2}{*}{$2-\mathrm{F}$} & $R^{1}=R^{2}$ & -0.025 & -113.490 & -113.515 & 1 \\
\hline & $R^{1} \neq R^{2}$ & -0.012 & -116.095 & -116.107 & 1 \\
\hline \multirow{2}{*}{ 2- $\mathrm{COOH}$} & $R^{1}=R^{2}$ & -0.227 & -112.125 & -112.351 & 1 \\
\hline & $R^{1} \neq R^{2}$ & -0.048 & -110.523 & -110.571 & 1 \\
\hline \multirow{2}{*}{$2-\mathrm{CF}_{3}$} & $R^{1}=R^{2}$ & -0.094 & -109.569 & -109.663 & 1 \\
\hline & $R^{1} \neq R^{2}$ & -0.073 & -117.514 & -117.587 & 1 \\
\hline \multirow{2}{*}{$2-\mathrm{CHO}$} & $R^{1}=R^{2}$ & -0.131 & -109.325 & -109.456 & 1 \\
\hline & $R^{1} \neq R^{2}$ & -0.057 & -111.080 & -111.137 & 1 \\
\hline \multirow{2}{*}{$2-C N$} & $R^{1}=R^{2}$ & -0.171 & -109.256 & -109.427 & 1 \\
\hline & $R^{1} \neq R^{2}$ & -0.076 & -110.189 & -110.265 & 1 \\
\hline \multirow{2}{*}{$2-\mathrm{NO}_{2}$} & $\mathbf{R}^{1}=\mathbf{R}^{2}$ & -0.186 & -109.834 & -110.020 & 1 \\
\hline & $\mathbf{R}^{1} \neq \mathbf{R}^{2}$ & -0.088 & -111.835 & -111.923 & 1 \\
\hline
\end{tabular}

Observamos también que en el caso de los fragmentos sustituidos con grupos electrodonadores la estabilización de la energía debida a la reorganización de carga es mayor que en el caso de los fragmentos sustituidos con grupos electroatractores, esto nos indicaría que el efecto de 
redistribución de carga es más importante en los fragmentos en los que el carácter donador se encuentra exacerbado.

Contrario a lo observado para los fragmentos prístinos, observamos que en el caso de los fragmentos sustituidos el efecto de redistribución de carga es mayor para las reacciones con los fragmentos 1 sustituidos que para las que involucran los fragmentos $\mathbf{2}$ sustituidos, lo que indicaría que el efecto electrónico debido a la presencia de los sustituyentes es mayor en el fragmento 1 que en el fragmento 2 .

Las energías de interacción observadas para la reacción entre el butadieno y los fragmentos $\mathbf{1}$ y $\mathbf{2}$ mono- y disustituidos son del orden de la energía necesaria para la formación de un enlace C-C. 


\section{Capítulo 6. Conclusiones y perspectivas.}

\subsection{Conclusiones}

1. La reacción de Diels-Alder puede ser empleada para incrementar el tamaño de fragmentos policíclicos aromáticos curvos, identificados como fragmentos de fullerenos.

2. El triindenotrifenileno (fragmento 1) y el pentaciclopentacoranuleno (fragmento2) constituyen buenos puntos de partida para la síntesis de fullerenos por dimerización empleando reacciones de Diels-Alder.

3. En reacciones de Diels-Alder, los fragmentos $\mathbf{1}$ y $\mathbf{2}$ prefieren actuar como dienófilos que como dienos. El gap HOMO-LUMO de las reacciones entre los fragmentos $\mathbf{1}$ y $\mathbf{2}$ y el butadieno es menor que el gap HOMO-LUMO de las reacciones entre los fragmentos $\mathbf{1}$ y $\mathbf{2}$ y el etileno, al igual que las energías de activación, que son menores cuando los fragmentos $\mathbf{1}$ y $\mathbf{2}$ reaccionan con butadieno. Esto nos indica que los fragmentos $\mathbf{1}$ y $\mathbf{2}$ reaccionarían más fácilmente con butadieno que con etileno.

4. Las reacciones con etileno son favorecidas sobre la superficie cóncava de los fragmentos, mientras que las reacciones con butadieno son favorecidas sobre la superficie convexa de los fragmentos.

5. Las reacciones más favorables son las producidas entre el fragmento $\mathbf{2}$ y el butadieno sobre su superficie convexa.

6. En general el gap HOMO-LUMO de la reacción de Diels-Alder de demanda normal NEDDA entre los fragmentos $\mathbf{1}$ y $\mathbf{2}$ sustituidos es menor que el gap HOMO-LUMO de la reacción de Diels-Alder de demanda inversa IEDDA. 
7. Los gaps HOMO-LUMO de las reacciones NEDDA con los fragmentos $\mathbf{1}$ y $\mathbf{2}$ sustituidos con grupos electroatractores son menores que los gaps HOMO-LUMO de las reacciones con los fragmentos sin sustituir.

8. Las energías de activación de las reacciones con los fragmentos $\mathbf{1}$ y $\mathbf{2}$ disustituidos son mayores que las de los fragmentos sin sustituir, mientras que las energías de activación del fragmento 1 monosustituido con los grupos $\mathrm{NH}_{2}, \mathrm{OMe}, \mathrm{OH}, \mathrm{COOH}, \mathrm{CHO}$ y NO , y del fragmento 2 monosustituido con los grupos $\mathrm{COOH}, \mathrm{CHO}$ and $\mathrm{NO}_{2}$ son menores que las de los fragmentos $\mathbf{1}$ y $\mathbf{2}$ sin sustituir respectivamente.

9. La pobre reducción de la energía de activación debida a la presencia de los grupos electroatractores y electrodonadores en los fragmentos $\mathbf{1}$ y $\mathbf{2}$, contrario a lo predicho por los orbitales frontera, indica la influencia de efectos estéreoelectrónicos en las reacciones propuestas.

10. El análisis de la reacción de dimerización del fragmento $\mathbf{2}$ indica que el uso de la reacción de cicloadición de Diels-Alder en la síntesis de fullerenos por dimerización es factible.

11. La alta energía de activación de la dimerización del fragmento $\mathbf{2}$ en un solo paso concertado, indica que la reacción no concertada es favorecida.

12. El primer paso del mecanismo de dimerización no concertado es la formación de un cicloaducto mediante una reacción de Diels-Alder entre los dos fragmentos, seguido de la aromatización de dicho aducto. La formación de este primer cicloaducto sigue lo observado en el análisis de la reacción entre el fragmento $\mathbf{2}$ sin sustituir y el butadieno o etileno.

13. Los fragmentos $\mathbf{1}$ y $\mathbf{2}$ sin sustituir son más duros que los fragmentos mono- y disustituidos, por lo que reaccionarían más fácilmente con butadieno. Eso explicaría el esquema de energías de activación observado.

14. La electrofilia es mayor para los fragmentos sustituidos con grupos electroatractores y menor para los fragmentos sustituidos con grupos electrodonadores, lo que describe correctamente el efecto el sustituyente sobre el fragmento.

15. Las potencias electrodonadora y electroaceptora diferencian a los fragmentos monosustituidos de los di-sustituidos por la magnitud del efecto del sustituyente, siendo 
menores para los fragmentos disustituidos con grupos electrodonadores y mayores para los fragmentos sustituidos con grupos electroaceptores respectivamente.

16. La contribución de las funciones de Fukui para ataques por nucleófilos se encuentra sobre los átomos involucrados en la reacción en el caso de los fragmentos prístinos, mientras que se encuentra dispersa sobre el esqueleto del fragmento en el caso de los fragmentos mono- y disustituido. De esto podemos inferir que las energías de activación no descienden considerablemente para los estados de transición calculados debido a la presencia de sitios más reactivos en los fragmentos.

17. La deslocalización de la carga en los fragmentos sustituidos debida a efectos de resonancia conduce a la aparición de tres sitios de influencia en el fragmento $\mathbf{1}$ y a dos sitios de influencia en el fragmento $\mathbf{2}$, donde se ve reflejado el efecto de los grupos sustituyentes.

18. La energía de interacción para las reacciones entre los fragmentos y el butadieno nos indica que el efecto principal en estas interacciones es el de reordenamiento de carga y no el de transferencia de carga. La magnitud de la energía de interacción obtenida es equivalente a la energía necesaria para la formación de un enlace C-C. 


\subsection{Perspectivas}

1. Analizar detalladamente el efecto estéreoelectrónico de las reacciones con los fragmentos 1 y 2 mono- y disustituidos.

2. Analizar la simetría de los orbitales involucrados en las reacciones propuestas.

3. Analizar las reacciones exo entre el butadieno y los fragmentos $\mathbf{1}$ y $\mathbf{2}$ mono- y disustituidos

4. Analizar detalladamente la formación de los 8 enlaces restantes en la síntesis de $\mathrm{C}_{60}$ por dimerización.

5. Analizar la dimerización del fragmento 1.

6. Realizar cálculos de dinámica molecular de las reacciones de dimerización.

7. Analizar las estructuras de resonancia de los fragmentos $\mathbf{1}$ y $\mathbf{2}$ prístinos y mono- y disustituidos y localizar las estructuras resonantes con mayor contribución al híbrido de resonancia mediante el cálculo de las energías de cada una de las estructuras propuestas empleando el método de Huckel o la teoría de enlace de valencia.

8. Analizar la aromaticidad de los fragmentos $\mathbf{1}$ y $\mathbf{2}$ mono- y disustituidos y sin sustituir.

9. Analizar profundamente el efecto de redistribución de carga en los fragmentos $\mathbf{1}$ y $\mathbf{2}$ mono- y disustituidos y sin sustituir

10. Sintetizar $\mathrm{C}_{60}$ mediante la dimerización del fragmento $\mathbf{2}$. 


\section{Referencias}

1. H. W. Kroto, J. R. Heath, S. C. O'Brien, R. F. Curl, R. E. Smalley, Nature, 1985, 318, 162.

2. R. Taylor, J. P. Hare, A. K. Abdul-Sada, H. W. Kroto, J. Chem. Soc. Chem. Comm., 1990, 20, 1423.

3. D. Sh. Sabirov, R. G. Bulakov, S. L. Khufsan, ARKIVOC, 2011, (VIII), 200.

4. a) I. Yanov, J. Leszczynski, Theoretical and Computational Chemistry, Vol.15: Computational Materials Science (Ed: J. Leszczynski), Elsevier, Amsterdam, 2004, p.85.; b) J. A. Alonso, Structure and Properties of Atomic Nanoclusters, Imperial College Press, London, 2005, p. 333-354.

5. a)M. Gallo, A. Favila, D. Glossman-Mitnik, Chemical Physics Letters,2007, 447, 105; b) K. A. Gonzalez, L. J. Wilson, W. Wu, G. H. Nancollas, Bioorganic and Medicinal Chemistry,2002,10, 1991.

6. a) C. Fumelli, A. Marconi, S. Salvioli, E. Straface, W. Malorni, A. M. Offidani, R. Pellicciari, G. Schettini, A. Giannetti, D. Monti, C. Franceschi, C. Pincelli, The Journal of Investigative Dermatology, 2000, 115, 835; b) M. Lens, Recent Patents on Biotechnology, 2011, 5, 67.

7. A. Modi, N. Koratkar, E. Lass, B. Wei, P. M. Ajayan, Nature, 2003, 424, 171.

8. a) S-R. Chae, M. Therezien, J. F. Budarz, L. Wessel, S. Lin, Y. Xiao, M. R. Wiesner, Journal of Nanoparticle Research, 2011, 13, 5121; b) D. Ray, C. Belin, F. Hui, B. Fabre, P. Hapiot, D. M. Bassani, Chemical Communications, 2011, 47, 2547.

9. Y. Kojima, T. Matsuoka, H. Yakajashi, T. Kirachi, Macromolecules, 1995, 28, 8868.

10. T. Benincori, E. Brenna, F. Sannicolo`, L. Trimarco, G. Zotti, P. Sozzani, Angew. Chem., Int. Ed. Engl., 1996, 35, 648.

11. A. Anctil, C. W. Babbitt, R. P. Rafaelle, B. Landi, J. Environ. Sci. Technol., 2011, 45, 2353. 
12. U. S. Energy Information Administration.

http://www.eia.gov/tools/faqs/faq.cfm?id=97\&t=3

13. Precios tomados del catalogo de Sigma Aldrich.

http://www.sigmaaldrich.com/catalog/search?interface=All\&term=Fullerenes\&N=0\&focus $=$ product\&lang=es\&region=MX

14. Las estrellas de carbono son estrellas rojas gigantes, frías, con temperaturas de alrededor de $3000^{\circ} \mathrm{C}$ en su superficie. Sus espectros exhiben bandas correspondientes a C2 anormalmente intensas, así como otras bandas que involucran al elemento carbono, como las correspondientes a CN y CH. R. D. McClure, J. Roy. Astron. Soc. Can., 1985, 79, 277.

15. H. W. Kroto, Angew. Chem. Int. Ed. Engl., 1992, 31, 111.

16. J. R. Heath, R. S. O’Brien, Q. Zhang, Y. Liu, R. F. Curl, H. W. Kroto, F. K. Tittel, R. E. Smalley, J. Am. Chem. Soc., 1985, 107, 7779.

17. M. Saunders, H. A. Jiménez-Vázquez, R. J. Cross, R. S. Poreda, Science, 1993, 259, 1428.

18. a) J. M. Hawkins, A. Meyer, T. A. Lewis, S. Loren, F. J. Hollander, Science, 1991, 252, 312; b) P. J. Fragan, J. C. Calabrese, B. Malone, Science, 1991, 252, 1160.

19. W. H. Powell, F. Cozzi, G. P. Moss, C. Thilgen, R. J.-R. Hwu, and A. Yerin, Pure Appl. Chem., 2002, 74, 629.

20. a) T. G. Schmalz, W. A. Seitz, D. J. Klein, G. E. Hite, Chem. Phys. Lett., 1986, 130, 203; b) D. Vukicevic, M. Randic, Chem. Phys. Let., 2005, 401, 446; c) M. Randic, H. W. Kroto, D. Vukicevic, J. Chem. Inf. Model., 2007, 487, 897.

21. R. C. Haddon, J. Am. Chem. Soc., 1997, 119, 1797.

22. R. C. Haddon, Science, 1993, 261, 1545.

23. T. C. Dinadayalane, J. Leszczynski, Struct. Chem., 2010, 21, 1155.

24. D. J. Klein, T. G. Schmalz, G. E. Hite, W. A. Seitz, J. Am. Chem. Soc., 1986, 108, 1301.

25. N. Matsuzawa, D. A. Dixon, T. Fukunaga, J Phys. Chem., 1992, 96, 7594. 
26. R. Taylor, D. M. R. Walton, Nature, 1993,363, 685.

27. M. Koshino, Y. Niimi, E. Nakamura, H. Kataura, T. Okazaki, K. Suenaga, S. lijima, Nature Chemistry, 2010, 2, 117.

28. R. E. Haufler, J. Conceicao, L. P. F. Chibante, Y. Chai, N. E. Byrne, S. Flanagan, M. M. Haley, S. C. O’Brien, C. Pan, Z. Xiao, W. E. Billups, M. A. Cuifolini, R. H. Hauge, L. J. Margrave, L. J. Wilson, R. F. Curl, R. E Smalley, J. Phys. Chem., 1990, 94, 8634.

29. M. Prato, J. Matter. Chem., 1997, 7, 1097.

30. M. Keshavarz-K, B. Knigth, G. Sardanov, F. Wudl, J. Am. Chem. Soc., 1995, 117, 11371.

31. a) F. Wudl, Acc. Chem. Ress., 1992, 25, 157; b) F. Diedrich, L. Isaacs, D. Philp, Chem. Soc. Rev., 1994, 23, 243.

32. a) S. H. Hoke II, J. Molstad, D. Dilettato, M. J. Jay, D. Carlson, B. Kahr, R. G. Cooks, J. Org. Chem., 1992, 57, 5069; b) M. Tsuda, T. Ishida, T. Nogami, S. Kurono, M. Ohasi, Tetrahedron. Lett., 1993, 34, 6911.

33. a) M. Prato, T. Suzuki, H. Foroudian, Q. Li, K. Khemani, F. Wudl, J. Leonetti, R. D. Little, T. White, B. Rickborn, S. Yamago, E. Nakamura, J. Am. Chem. Soc., 1993, 115, 1594; b) L. -L. Shiu, T. -I. Lin, S. -M. Peng, G. -R. Her, D. D. Ju, S. -K. Lin, J. -H. Hwang, C. M. L. Mou T.-Y., J. Chem. Soc., Chem. Commun., 1994, 5, 647.

34. a) X. Zheng, C. S. Foote, J. Am. Chem. Soc., 1995, 117, 4271; b) M. S. Meier, M. Poplawska, J. Org. Chem., 1993, 58, 4524; c) S. Muthu, P. Maruthamuthu, R. Ragunatha, P. R. Vasudeva Rao, C. K. Mathews, Tetrahedron Lett., 1994, 35, 1763; d) N. Jagerovic, J. Elgero, J. L. Aubagnac, J. Chem. Soc., Perkin Trans. 1, 1996, 6, 499.

35. a) Y. Rubin, S. Khan, D. I. Freedberg, C. Yeretzian, J. Am. Chem. Soc., 1993, 115, 344; b) P. Belik, A. Gügel, J. Spickermann, K. Müllen, Angew. Chem., Int. Ed. Engl., 1993, 32, 78.

36. T. Grösser, M. Prato, V. Lucchini, A. Hirsch, F. Wudl, Angew, Chem., Int. Ed. Engl., 1995, 34, 1343.

37. C. Taliani, G. Ruani, R. Zamboni, R. Danieli, F. Orlandi, F. Zerbetto, J. Chem. Soc., Chem. Commun., 1993, 3, 220. 
38. G. L. Marconi, T. Da Ros, S. Costellano, G. Stefancich, I. Bonin, S. Miertus, M. Prato, Org. Lett., 2000, 2, 3955.

39. H. Jin, W. Q. Chen, X. W. Tang, L. Y. Yang, J. V. Schloss, J. Y. Wu, J. Neurosci. Res., 2000, 62, 600.

40. A. Mostofizadeh, Y. Li, B. Song, Y. Huang, Journal of Nanomaterials, 2011, 685081.

41. M. H. Abraham, C. E. Green, W. E. Acreeu Jr., J. Chem. Soc., Perkin Trans. 2, 2000, 2, 281.

42. R. C. Haddon, A. F. Hebard, M. J. Rosseinsky, D. W. Murphy, S. J. Duclos, K. B. Lyons, B. Miller, J. M. Rosamila, R. M. Fleming, A. R. Kortan, S. H. Glarum, A. V. Makhija, A. J. Muller, R. H. Eick, S. M. Zahurak, R. Tycko, G. Dabbagh, F. A. Thiel, Nature, 1991, 350, 320.

43. a) C. C. Chen, S. P. Kelty, C. M. Lieber, Science, 1991, 253, 886; b) A. F. Hebard, M. J. Rosseinsky, R. C. Haddon, D. W. Murphy, S. H. Glarum, T. T. M. Palstra, A. P. Ramirez, A. R. Kortan, Nature, 1991, 350, 600; c) M. J. Rosseinsky, A. P. Ramirez, S. H. Glarum, D. W. Murphy, R. C. Haddon, A. F. Hebard, T. T. M. Palstra, A. R. Kortan, S. M. Zahurak, A. V. Makhija, Physical Review letters, 1991, 66, 2830.

44. R. E. Haufler, J. Conciecao, Y. Chai, L. P. F. Chibante, N. E. Byrne, S. Flanagan, M. M. Haley, S. C. O’Brien, C. Pan, Z. Xiao, W. E. Billups, M. A. Cuifolini, R. H. Hauge, J. M. Margrave, L. J. Wilson, R. F. Curl, R. E. Smalley, J. Phys. Chem., 1990, 94, 8634.

45. a) P. -M. Allemand, A. Kock, F. Wudl, Y. Rubin, F. Diedrich, M. M. Alvarez, S. J. Anz, R. L. Whetten, J. Am. Chem. Soc., 1991, 113, 1051; b) D. M. Cox, S. Behal, M. Disko, S. M. Gorum, M. Greaney, C. S. Hus, E. B. Kollin, J. Millar, J. Robbins, W. Robbins, R. D. Sherwood, P. Tindall, J. Am. Chem. Soc., 1991, 113, 2940.

46. a) D. Dubois, K. M. Kadish, S. Flanagan, R. E. Haufler, L. P. F. Chibante, L. J. Wilson, J. Am. Chem. Soc., 1991, 113, 2940; b) C. Jehoulet, A. Bard, F. Wudl, J. Am. Chem. Soc., 1991, 113, 5456; c) D. Dubois, K. M. Kadish, S. Flanagan, L. J. Wilson, J. Am. Chem. Soc., 1991, 113, 7773.

47. Q. Xie, E. Pérez-Cordero, L. Echegoyen, J. Am. Chem. Soc., 1992, 114, 3978.

48. a) M. Eiermann, F. Wudl, M. Prato, M. Maggini, J. Am. Chem. Soc., 1994, 116, 8364; b) T. Suzuki, Q. Li, K. C. Khermani, F. Wudl, Ö. Almarsson, J. Am. Chem. Soc., 1992, 114, 7300. 
49. L. W. Tutt, A. Kost, Nature, 1992, 356, 225.

50. a) Y. Wang, Nature, 1992, 356, 585; b) N. S. Sariciftci, L. Smilowitz, A. J. Heeger, F. Wudl, Science, 1992, 258, 1474; c) S. Morita, A. A. Zakidov, K. Yoshino, Solid State Commun., $1992,82,249$.

51. K. Y. Amsharov, M. Jansen, J. Org. Chem., 2008, 73, 2931.

52. W. Krätschmer, L. D. Lamb, K. Fostiropoulos, D. R. Huffman, Nature, 1990, 347, 354.

53. W. Krätschmer, K. Fostiropoulos, D. R. Huffman, Chem. Phys. Lett., 1990, 170, 167.

54. T. J. S. Dennis, T. Kai, T. Tomiyama, T. H. Shinohara, T. Yoshida, Y. Kobayashi, H. Ishiwatari, Y. Miyake, K. Kikuchi, Y. Achiba, J. Phys. Chem. A, 1999, 103, 8747.

55. a) Q. L. Zhang, S. C. O'Brien, J. R. Heath, Y. Liu, R. F. Curl, H. W. Kroto, R. E. Smalley, J. Phys. Chem., 1986, 90, 525; b) R. F. Curl, R. E. Smalley, Science, 1988, 242, 1017.

56. a) H. W. Kroto, Science, 1988, 242, 1139; b) H. W. Kroto, K. McKay, Nature, 1988, 331, 328.

57. S. lijima, J. Cryst. Growth, 1980, 50, 675.

58. L. B. Ebert, Science, 1989, 247, 1468.

59. G. Brinkmalm, D. Barofsky, P. Demirev, D. Feny, P. Hakansson, R. E. Johnson, C. T. Reimann, B. U. R. Sundqvist, Chem. Phys. Lett., 1992, 191, 345.

60. J. R. Heath, Fullerenes: Synthesis, Properties and Chemistry of Large Carbon Clusters (Eds: G. S. I. Hammond, V. J. Kuck), American Chemical Society, Washington, DC, 1991, p. 1.

61. a) T. Wakabayashi, Y. Achiba, Chem. Phys. Lett., 1992, 190, 465; b) T. Wakabayashi, H. Shiromaru, K. Kikuchi, Y. Achiba, Chem. Phys. Lett., 1993, 201, 470.

62. a) J. Hunter, J. Fye, M. F. Jarrold, Science, 1993, 260, 784; b) Y. E. Lozovik, A. M. Popov, Phys. Usp., 1997, 40, 717.

63. S. W. McElvany, M. M. Ross, N. S. Goroff, F. Diederich, Science, 1993, 259, 1594. 
64. a) G. von Helden, N. G. Gotts, M. T. Bowers, Nature, 1993, 363, 60; b) J. Hunter, J. Fye, M. F. Jarrold, J. Phys. Chem., 1993, 97, 3460.

65. C. Yeretzian, K. Hansen, F. Diederich, R. L. Whetten, Nature, 1992, 359, 44.

66. a) A. M. Askhabov, Phys. Solid State, 2005, 47, 1186; b) A. M. Askhabov, Zap. Vseross. Mineral. O-va., 2004, 133, 108.

67. S. Irle, G. Zheng, Z. Wang, K. Morokuma, J. Phys. Chem. B, 2006, 110, 14531.

68. L. T. Scott, Angew. Chem. Int. Ed., 2004, 43, 4994.

69. W. E. Barth, R. G. Lawton, J. Am. Chem. Soc., 1966, 88, 380.

70. L. T. Scott, M. M. Hasheim, D. T. Meyer, H. B. Warren, J. Am. Chem. Soc., 1991, 113, 7082.

71. L. T. Scott, P. Ch. Cheng, M. M. Hasheim, M. S. Bratcher, D. T. Meyer, H. B. Warren, J. Am. Chem. Soc., 1997, 119, 10963.

72. L. T. Scott, M. S. Bratcher, S. Hagen, J. Am. Chem. Soc., 1996, 118, 8743.

73. a) M. J. Plater, J. Chem. Soc. Perkin Trans. 1, 1997, 19, 2897; b) M. J. Plater, J. Chem. Soc. Perkin Trans. 1, 1997, 19, 2903.

74. R. B. M. Ansems, L. T. Scott, J. Am. Chem. Soc., 2000, 122, 2719.

75. V. M. Tsefrikas, L. T. Scott, Chem. Rev., 2006, 106, 4868.

76. T. J. Seiders, K. K. Baldridge, J. S. Siegel, J. Am. Chem. Soc., 1996, 118, 2754.

77. A. Sygula, P. W. Rabideau, J. Am. Chem. Soc., 1998, 120, 12666.

78. A. Sygula, P. Rabideau, J. Am. Chem. Soc., 2000, 122, 6323.

79. A. Sygula, S. D. Karlen, R. Sygula, P. W. Rabideau, Org. Lett., 2002, 4, 3135.

80. F. Geneste, A. Moradpour, G. Dive, D. Peeters, J. Malthête, J. F. Sadoc, J. Org. Chem., 2002, 67,605 . 
81. F. A. L. Anet, S. S. Miura, J. Siegel, K. Mislow, J. Am. Chem. Soc., 1983, 105, 1419.

82. L. T. Scott, M. M. Boorum, B. J. McMahon, S. Hagen, J. Mack, J. Blank, H. Wegner, A. de Meijere, Science, 2002, 295, 1500.

83. M. A. Kabdulov, K. Y. Amsharov, M. Jansen, Tetrahedron, 2010, 66, 8587.

84. G. Otero, G. Biddau, C. Sánchez-Sánchez, R. Caillard, M. F. López, C. Rogero, F. J. Palomares, N. Cabello, M. A. Basanta, J. Ortega, J. Méndez, A. M. Echavarren, R. Pérez, B. Gómez-Lor, J. A. Martín-Gago, Nature, 2008, 454, 865.

85. G. Henkelman, B. P. Ubesuaga, H. J. Jonsson, J. Chem. Phys., 2000, 113, 9901.

86. K. Y. Amsharov, M. A. Kabdulov, M. Jansen, Eur. J. Org. Chem., 2009, 36, 6328.

87. A. Yasuda, Carbon, 2005, 43, 889.

88. Y. Rubin, T. C. Parker, S. J. Pastor, S. Jalisatgi, C. Boulle, C. C. Wilkins, Angew. Chem. Int. Ed., 1998, 37, 1226.

89. Y. Tobe, N. Nakagawa, K. Naemura, T. Wakabayashi, T. Shida, Y. Achiba, J. Am. Chem. Soc., 1998, 120, 4544.

90. Y. Rubin, Chem. Eur. J., 1997, 3, 1009.

91. U. H. F. Bunz, Y. Rubin, Y. Tobe, Chem. Soc. Rev., 1999, 28, 107.

92. F. Viñes, A. Görling, Angew. Chem. Int. Ed., 2011, 50, 4611.

93. C. Chen, Z. Lou, J. Supercrit. Fluids, 2009, 50, 42.

94. A. Chuvilin, U. Kaiser, E. Bichoutskaia, N. A. Besley, A. N. Khlobystov, Nat. Chem., 2010, 2, 450.

95. L. T. Scott, M. A. Petrukhina, Preface in Fragments of Fullerenes and Carbon Nanotubes: Designed Synthesis, Unusual Reactions and Coordination Chemistry, (Eds: L. T. Scott, M. A. Petrukhina), John Wiley \& Sons, Inc, Hoboken, New Jersey, 2012, p. vii-x. 
96. W. Krätschmer, L. D. Lamb, K. Fostiropoulos, D. R Huffman, Nature, 1990, 347, 354.

97. L. T. Scott, Angew. Chem. Int. Ed., 2004, 43, 4994.

98. V. M Tsefrikas, L. T. Scott, Chem. Rev., 2006, 106, 4868.

99. A. H. Abdourazak, Z. Marcinow, A. Sygula, R. Sygula, P. W. Rabideau, J. Am. Chem. Soc., $1995,117,6410$.

100. S. Hagen, M. S. Bratcher, M. S. Erickson, G. Zimmermann, L. T. Scott, Angew. Chem., Int. Ed. 1997, 36, 406.

101. L. T. Scott, Polycyclic Aromat. Compd., 2010, 30, 247.

102. E. H. Fort, P. M. Donovan, L. T. Scott, J. Am. Chem. Soc. 2009, 131, 16006.

103. E. H. Fort, L. T. Scott, Tetrahedron Lett. 2011, 52, 2051.

104. F. Fringuelli, A. Taticchi, The Diels-Alder Reaction: Selected Practical Methods. John Wiley \& Sons Ltd: West Sussex, England, 2002; pp. 1-25.

105. J. Saver, Angew. Chem., Int. Ed. 1967, 6, 16.

106. F. Fringuelli, L. Minuti, F. Pizzo, A. Taticchi, Acta Chem. Scand., 1993, 47, 255.

107. G. J. Bodwell, Z. Pi, Tetrahedron Lett., 1997, 38, 309.

108. M. J. S. Dewar, S. Olivella, J. J. P. Stewart, J. Am. Chem. Soc. 1986, 108, 5771.

109. E. C. Angell, F. Fringuelli, F. Pizzo, B. Porter, A. Taticchi, E. Wenkert, J. Org. Chem., 1986, $51,2642$.

110. I. Flemig, Molecular Orbitals and Organic Chemical Reactions; John Wiley \& Sons Ltd: London, UK, 2009; pp. 224-242.

111. K. H. Lee, C. Lee, J. Kang, S. S. Park, J. Lee, S. K. Lee, D. K. Bohme, J. Phys. Chem. A 2006, $110,11730$.

112. J. I. Martínez, J. L. Moncada, J. M. Larenas, J. Mol. Model., 2010, 16, 1825. 
113. H. Tachikawa, T. Iyama, S. Abe, Physics Procedia, 2011, 14, 139.

114. J. A. Carranza-García, J. Rodríguez-Otero, E. M. Cabaleiro-Lago, J. Phys. Chem. B, 2011, 115, 2774.

115. S. Osuna, J. Morera, M. Cases, K. Morokuma, M. Solà, J. Phys. Chem. A, 2009, 113, 9721.

116. N. Ikuma, Y. Susami, T. Oshima, Org. Biomol. Chem. 2010, 8, 1394.

117. S. Osuna, K. N. Houk, Chem. Eur. J., 2009, 15, 13219.

118. M. A. Petrukhina, K. W. Andreini, J. Mack, L. T. Scott, J. Org. Chem. 2005,70, 5713.

119. K. N. Houk, R. J. Loncharich, J. F. Blake, W. L. Jorgensen, J. Am.Chem. Soc. 1989, 111, 9172.

120. V. Guner, K. S. Khuong, A. G. Leach, P. S. Lee, M. D. Bartberger, K. N. Houk, J. Phys. Chem. A 2003, 107, 11445

121. L. García-Colín Scherer, Introducción a la física estadística, El Colegio Nacional, México, 2011, pp 247-273.

122. Gaussian 03 user's reference

123. Gaussian 03, Revision B.04, M. J. Frisch, G. W. Trucks, H. B. Schlegel, G. E. Scuseria, M. A. Robb, J. R. Cheeseman, J. A. Montgomery, Jr., T. Vreven, K. N. Kudin, J. C. Burant, J. M. Millam, S. S. Iyengar, J. Tomasi, V. Barone, B. Mennucci, M. Cossi, G. Scalmani, N. Rega, G. A. Petersson, H. Nakatsuji, M. Hada, M. Ehara, K. Toyota, R. Fukuda, J. Hasegawa, M. Ishida, T. Nakajima, Y. Honda, O. Kitao, H. Nakai, M. Klene, X. Li, J. E. Knox, H. P. Hratchian, J. B. Cross, C. Adamo, J. Jaramillo, R. Gomperts, R. E. Stratmann, O. Yazyev, A. J. Austin, R. Cammi, C. Pomelli, J. W. Ochterski, P. Y. Ayala, K. Morokuma, G. A. Voth, P. Salvador, J. J. Dannenberg, V. G. Zakrzewski, S. Dapprich, A. D. Daniels, M. C. Strain, O. Farkas, D. K. Malick, A. D. Rabuck, K. Raghavachari, J. B. Foresman, J. V. Ortiz, Q. Cui, A. G. Baboul, S. Clifford, J. Cioslowski, B. B. Stefanov, G. Liu, A. Liashenko, P. Piskorz, I. Komaromi, R. L. Martin, D. J. Fox, T. Keith, M. A. Al-Laham, C. Y. Peng, A. Nanayakkara, M. Challacombe, P. M. W. Gill, B. Johnson, W. Chen, M. W. Wong, C. Gonzalez, and J. A. Pople, Gaussian, Inc., Pittsburgh PA, 2003. 
124. https://wiki.fysik.dtu.dk/dacapo/Dacapo

125. Y. S. Park, B.-S. Lee, I. Lee, New J. Chem. 1999, 27, 707.

126. K. N. Houk, Y.-T. Lin, F.K. Brown, J. Am. Chem. Soc. 1986, 108, 554.

127. G. B. Kistiakowsky, J. R. Ruhoff, H. A. Smith, W. E. Vaughan, J. Am. Chem. Soc. 1936, 58, 146.

128. R. Parr, W. Yang, Density-Functional Theory of Atoms and Molecules, Oxford University Press, 1989, New York, pg.70.

129. R. G. Pearson, Chemical Hardness, Wiley-VCH Verlag GmbH, 1997, London.

130. R. G. Pearson, J. Chem. Educ., 1987, 64, 561.

131. R. G. Parr, L. v. Szentpály, S. Liu, J. Am. Chem. Soc., 1999, 121, 1922.

132. J. L. Gázquez, A. Cedillo, A. Vela, J. Phys. Chem. A, 2007, 111, 1966.

133. R. Parr, W. Yang, J. Am. Chem. Soc. 1984, 106, 4049.

134. C. Morell, A. Grand, A. Toro-Labbé, J. Phys. Chem. A, 2005, 109, 205.

135. a) F. Méndez, J. L. Gázquez, J. Am. Chem. Soc., 1994, 116, 9298; b) J. L. Gázquez, F. Méndez, Proc. Indian Acad. Sci., 1994, 106, 183.

136. J. L. Gázquez, F. Méndez, J. Phys. Chem., 1994, 98, 4591.

137. J. L. Gázquez, A. Martínez, F. Méndez, J. Phys. Chem., 1993, 97, 4059.

138. R. T. Sanderson, Science 1951, 114, 670.

139. F. Méndez, J. Tamariz, P. Geerlings, J. Phys. Chem. A, 1998, 102, 6292. 


\section{Apéndice A. Cálculo de los parámetros termodinámicos.}

En termodinámica nos enseñan que las propiedades térmicas de un sistema macroscópico compuesto por un gran número de partículas están caracterizadas por un número relativamente pequeño de descriptores como la energía, temperatura, entropía, volumen, presión, etc. Sin embargo, la termodinámica fue desarrollada sin ninguna hipótesis de las propiedades microscópicas de la materia; de hecho, la descripción de las propiedades macroscópicas a partir de las propiedades microscópicas individuales de cada partícula que forma el sistema en estudio es prácticamente imposible. Esto conduce a dejar de lado la aproximación individual y confiar en una aproximación estadística.

Esta aproximación estadística, conocida como mecánica estadística, está basada en la hipótesis de que la materia, sin importar el estado en el que se encuentre, está compuesta por entidades microscópicas con características específicas. El estado de cada una de estas entidades cambia con el tiempo, sin embargo las propiedades colectivas permanecen estáticas.

Consideremos un sistema con volumen $V$ y número de partículas $N$, en contacto con un reservorio térmico que define la temperatura $T$. Suponiendo que: a) El reservorio y el sistema constituyen un sistema cerrado, y b) el reservorio es mucho más grande que el sistema. En este caso la energía $E$ del sistema no es fijada por el reservorio, sino que puede fluctuar sobre todas las posibles energías del sistema, es decir, obtenemos un conjunto de $\mathrm{N}$ sistemas en equilibrio térmico entre sí llamado conjunto canónico.

Si suponemos que tenemos una colección arbitrariamente grande de sistemas macroscópicos idénticos, es decir en equilibrio térmico ( $T$ fija), con el mismo volumen $V$ y composición $N$, sus energías E estarán dadas por las posibles soluciones de la ecuación

$$
\mathrm{H}_{N} \Psi_{i(N)}=E_{i} \Psi_{i(N)}
$$

Dado que el número de sistemas $\mathrm{N}$ es arbitrario y el espectro de energías es el mismo, entonces 


$$
\sum_{i} N_{i} E_{i}=\varepsilon=c t e
$$

donde $\mathrm{N}_{i}$ es el número de sistemas con energía $E_{i}$.

Si llamamos $\Omega_{i}$ a la degeneración de cada nivel del sistema de $N$ partículas podemos definir $Z$, la función de partición canónica, como

$$
Z=\sum_{i} \Omega_{i} e^{E_{i} / k T}
$$

y a la probabilidad de encontrar un sistema tomado al azar de este conjunto como

$$
P_{i}=\frac{\Omega_{i} e^{E_{i} / k T}}{Z}
$$

Por lo tanto la energía promedio $<E>$ de un sistema de dicho conjunto estará dada por

$$
\begin{gathered}
\langle E\rangle=\sum E_{i} P_{i} \\
\langle E\rangle=k T^{2}\left[\frac{d L n Z}{d T}\right]_{N, V}
\end{gathered}
$$

De esta manera $\Omega i$ equivale al número total de estados de un sistema compatible con una energía dada $E i, V$ y $N$. Debido a que $\Omega$ es un número muy grande podemos escribir

$$
Z=\int_{0}^{\infty} \Omega(E, V, N) e^{-\beta T} d E
$$

donde $\Omega e^{-B E}$ tiene un valor máximo para cierto valor de $E=\tilde{E}$ ( $\tilde{E}$ =energía máxima del sistema).

Si suponemos que en el equilibrio la entropía $S$ es una función de $\Omega$ cuando $E=\tilde{E}$

$$
S=k \operatorname{Ln} \Omega(\tilde{\mathrm{E}}, V, N)
$$

entonces 


$$
\left(\frac{\partial S}{\partial E}\right)_{E=\tilde{\mathrm{E}}}=\left(\frac{\partial S}{\partial U}\right)_{N, V}=k \beta=\frac{1}{T}
$$

donde $\tilde{E}$ es igual a la energía interna del sistema $U$, y por lo yanto al valor promedio de la energía $<E>$.

Evaluando $Z$ en $E=\tilde{E}$ obtenemos

$$
\operatorname{Ln} Z=\operatorname{Ln} \Omega(\tilde{\mathrm{E}}, V, N)-\beta \tilde{\mathrm{E}}
$$

o bien

$$
k L n Z=S(U, V, N)-\frac{U}{T}
$$

y por lo tanto

$$
F=-k T \operatorname{Ln} Z
$$

Donde $F$ es la energía libre de Helmholtz definida como $F=U-T S$.

Conocida $F$ las propiedades calorimétricas y la ecuación de estado pueden obtenerse directa y fácilmente mediante la ecuación

$$
d F=-p d V-S d T
$$

y eliminando el volumen usando la función de Gibbs

$$
G=F+p V=U-T S+p V
$$

obtenemos

$$
d G=V d p-S d T
$$

En el límite clásico $Z=3^{N} / N$ ! y por lo tanto

$$
F=-k T \operatorname{Ln} \frac{3^{N}}{N !}
$$

donde zes la función de partición molecular que obtendremos a continuación. 
Si consideramos que la energía de una molécula se puede descomponer en la energía translacional mas la energía interna que proviene de sus grados de libertad internos

$$
\varepsilon=\varepsilon^{(\text {trans })}+\varepsilon^{(i n t)}
$$

entonces

$$
z=z^{(\text {trans })} \cdot z^{(\text {int })}
$$

La función de partición translacional se define como

$$
\mathrm{z}^{(\text {trans })}=V\left[\frac{2 \pi \mu k T}{h^{2}}\right]^{3 / 2}
$$

Donde $\mu$ es la masa del centro de masa de la molécula.

Para el cálculo de la $z^{(i n t)}$ es necesario considerar todos los grados de libertad internos, es decir los rotacionales, vibracionales, electrónicos y nucleares. Considerando valida la hipótesis de la separabilidad de los grados de libertad internos tenemos que

$$
\varepsilon^{(i n t)}=\varepsilon^{(r o t)}+\varepsilon^{(v i b)}+\varepsilon^{(e l)}+\varepsilon^{(n u c l)}
$$

y por lo tanto

$$
3^{(i n t)}=3^{(r o t)} \cdot 3^{(v i b)} \cdot 3^{(e l)} \cdot 3^{(n u c l)}
$$

La contribución electrónica esta descrita en términos del momento angular y el espín electrónico como

$$
z^{e l}=\omega^{e l}, \quad \omega^{e l}=2 j+1=2 l+1
$$

donde $j$ es el número cuántico rotacional y l es el número cuántico electrónico.

La contribución nuclear está dada en términos del espín nuclear $I$

$$
3^{n u c l}=g^{\text {nucl }}, \quad g^{\text {nucl }}=2 I+1
$$


En el caso de moléculas diatómicas las funciones de partición rotacionales y vibracionales se definen como

$$
\mathrm{z}^{(r o t)}=\frac{T}{\sigma \Theta_{r}}
$$

donde $\Theta_{r}=\frac{\hbar^{2}}{2 I k} \quad$ y $\quad I=\mu r_{0}^{2}$

y $\sigma$ es el número de veces que la molécula retorna a su configuración inicial durante una rotación y

$$
3^{(v i b)}=\frac{e^{-\frac{1}{2} \Theta_{v} / T}}{1-e^{-\Theta_{v} / T}}
$$

donde $\Theta_{v}=\frac{h v}{k} \quad \mathrm{y} \quad v=\frac{1}{2 \pi} \sqrt{\frac{k}{\mu}}$

En el caso de moléculas poliatómicas las funciones de partición rotacional y vibracional se definen como

$$
\begin{gathered}
z^{(r o t)}=\frac{T}{\sigma \Theta_{r}} \\
z^{(v i b)}=\prod_{i=1}^{3 n-6} \frac{1}{1-e^{-{ }^{\Theta_{v} / T}}}
\end{gathered}
$$

para moléculas lineales, y como

$$
\begin{gathered}
3^{(r o t)}=\frac{(\pi A B C)^{1 / 2}}{\sigma}\left[\frac{2 k T}{\hbar^{2}}\right]^{3 / 2} \\
z^{(v i b)}=\prod_{i=1}^{3 n-5} \frac{1}{1-e^{-{ }^{\theta_{v} / T}}}
\end{gathered}
$$


para moléculas no lineales, donde $A, B$ y $C$ son los parámetros de inercia con respecto a los 3 ejes principales.

Así la función de partición total se obtiene como

$$
z_{(m o l)}=V\left[\frac{2 \pi \mu k T}{h^{2}}\right]^{3 / 2} \frac{T}{\sigma \Theta_{r}} \prod_{i=1}^{3 n-6} \frac{1}{1-e^{-\Theta_{v} / T}} \omega_{0} \prod_{j}^{r} g_{j}
$$

para moléculas lineales y

$$
\mathrm{z}_{(\mathrm{mol})}=V\left[\frac{2 \pi \mu k T}{h^{2}}\right]^{3 / 2} \frac{(\pi A B C)^{1 / 2}}{\sigma}\left[\frac{2 k T}{\hbar^{2}}\right]^{3 / 2} \prod_{i=1}^{3 n-5} \frac{1}{1-e^{-{ }^{\theta_{v} / T}}} \omega_{0} \prod_{j}^{r} g_{j}
$$

para moléculas no lineales. 


\section{Anexo 1.}

\section{Orbitales frontera}

Orbitales HOMO

Orbitales LUMO

Código de colores: Los lóbulos de signo positivo se muestran en rojo, mientras que los lóbulos de signo negativo se muestran en verde. 


\section{Etileno}
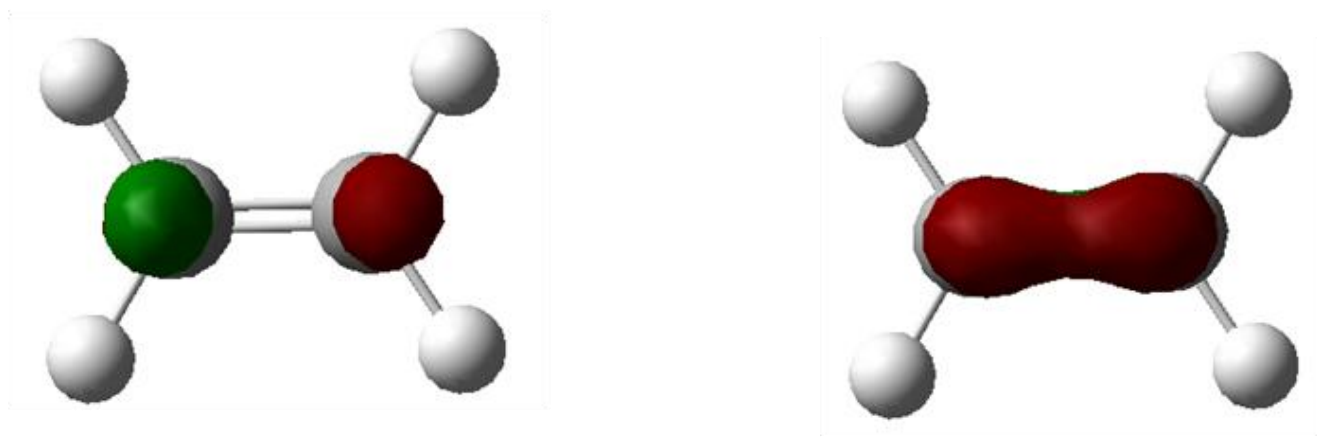

LUMO

HOMO

e (0.15ua)

\section{Butadieno}

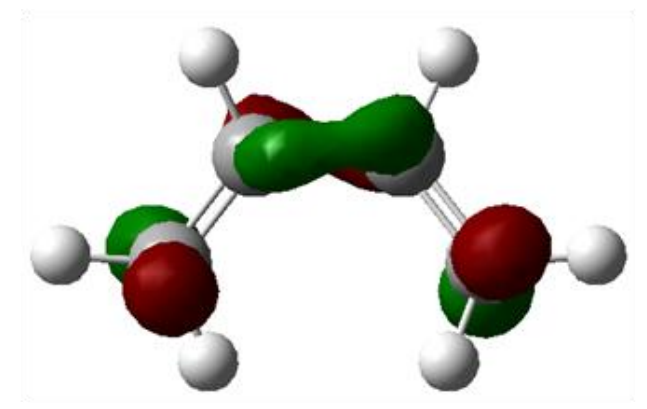

LUMO

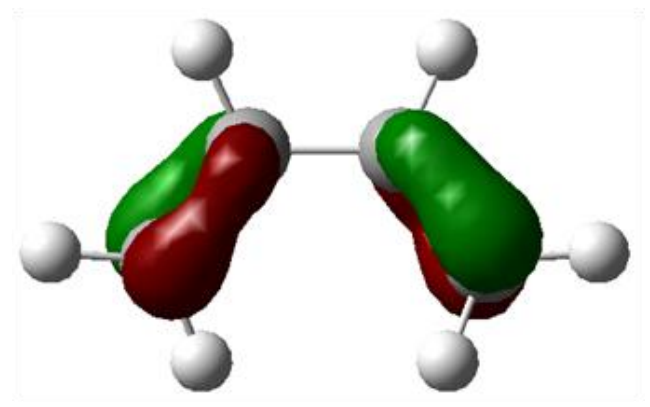

HOMO
b (0.1ua) 


\section{Fragmento 1}

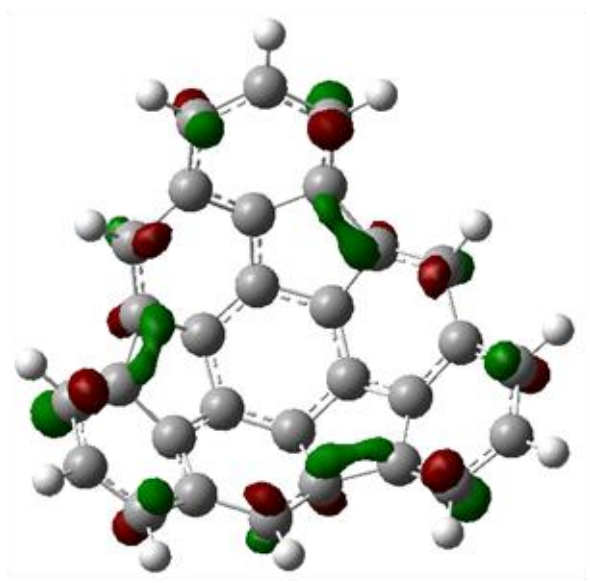

LUMO

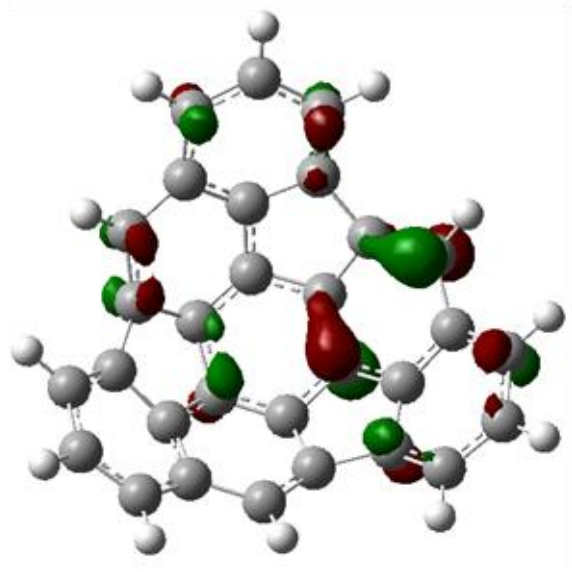

HOMO

a (0.06ua)

\section{Fragmento 2}

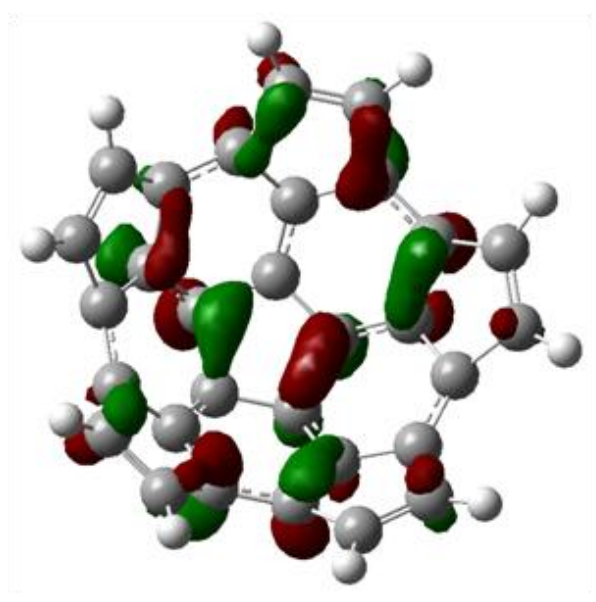

LUMO

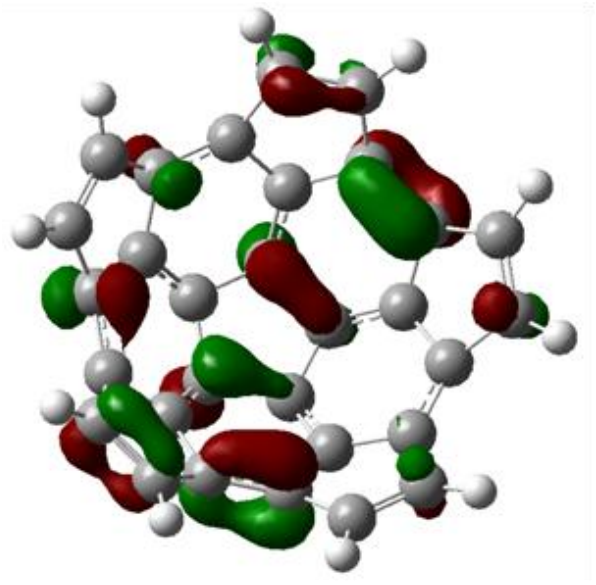

HOMO

$d$ (0.05ua) 
Fragmento 1 monosustituido con $\mathrm{CF}_{3}$
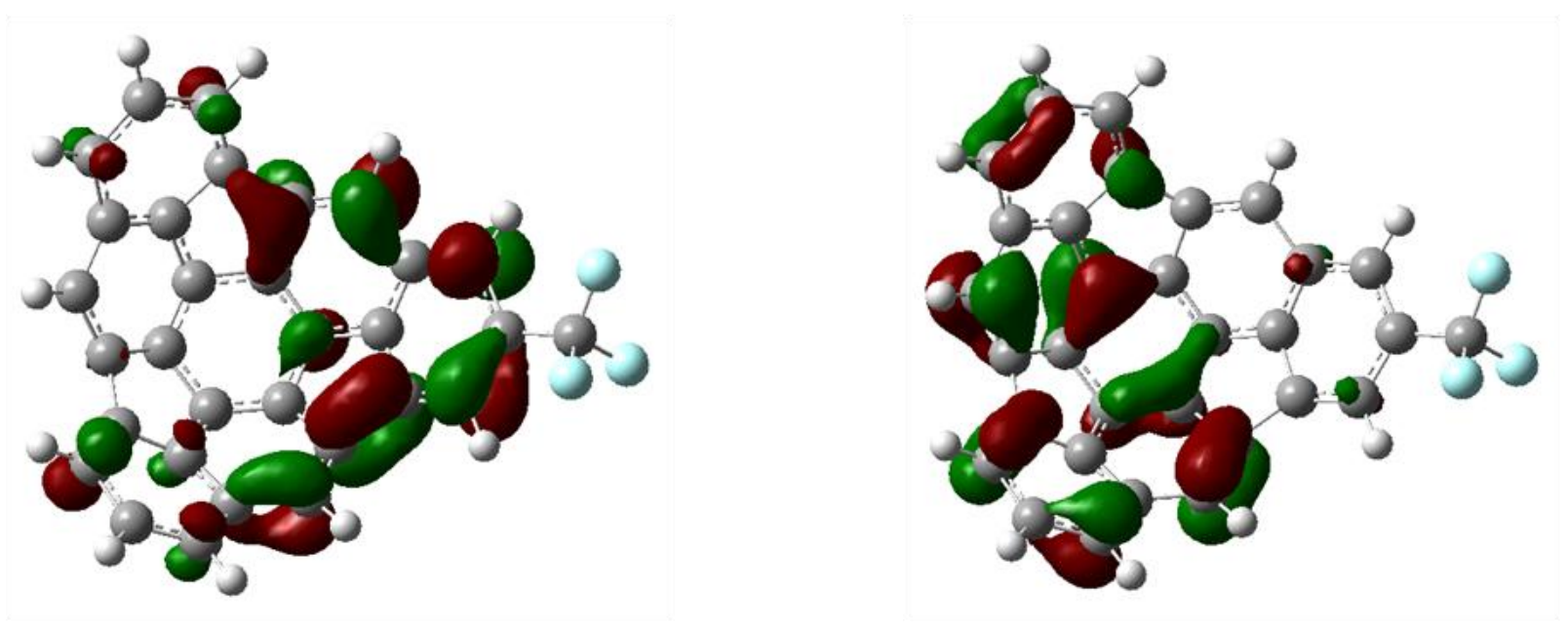

LUMO

HOMO

d (0.04ua)

Fragmento 1 disustituido con $\mathrm{CF}_{3}$

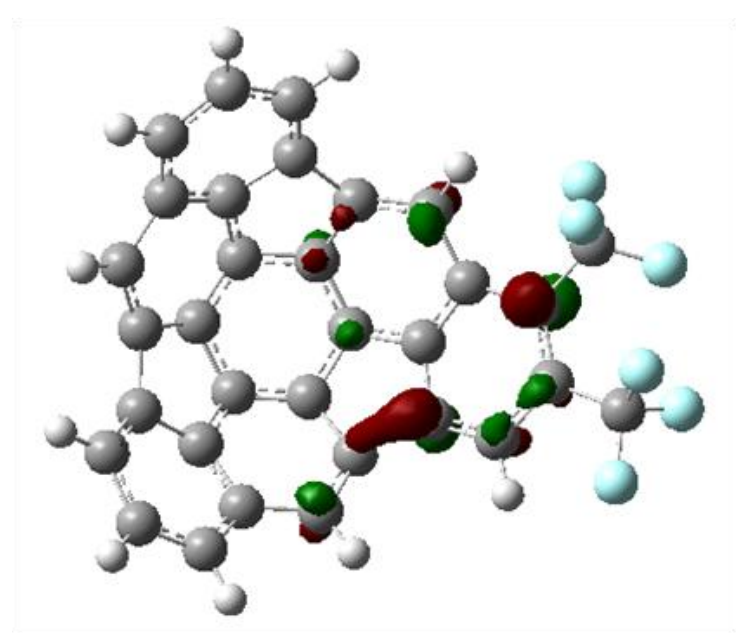

LUMO

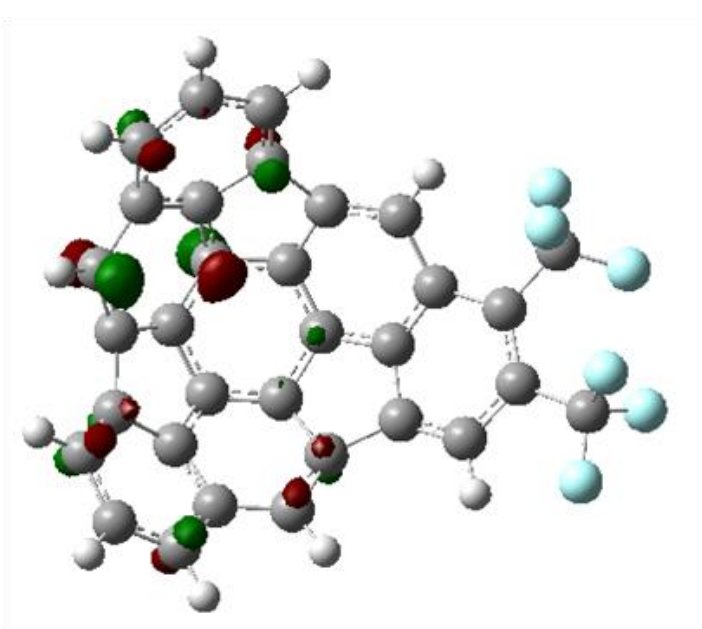

HOMO

d (0.07ua) 
Fragmento 1 monosustituido con $\mathrm{CHO}$
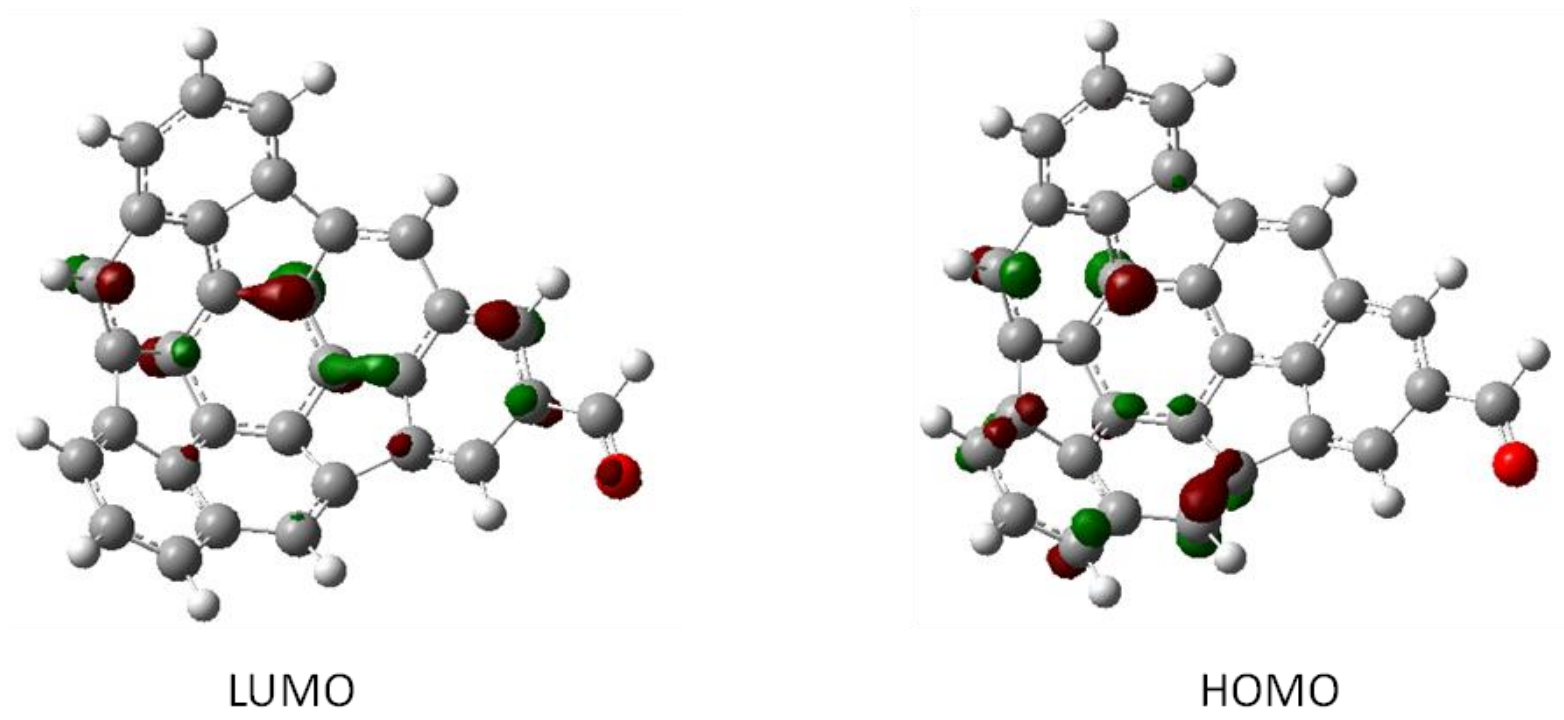

d (0.07ua)

HOMO

Fragmento 1 disustituido con $\mathrm{CHO}$

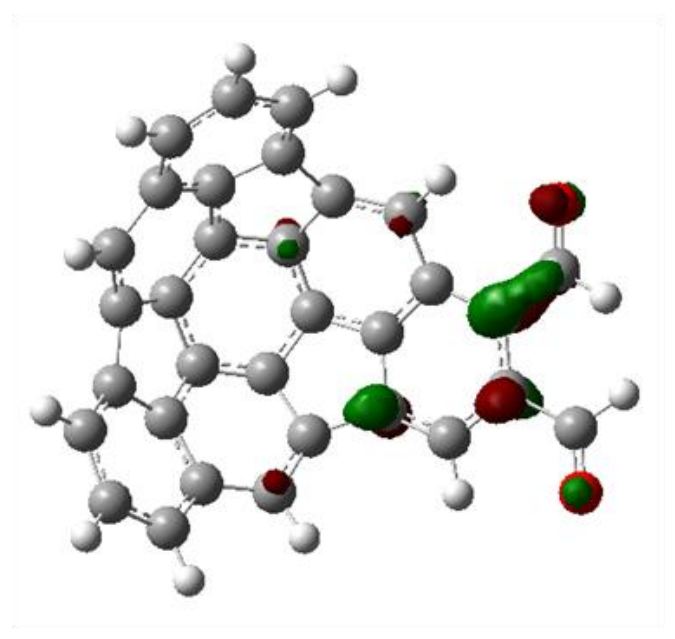

LUMO

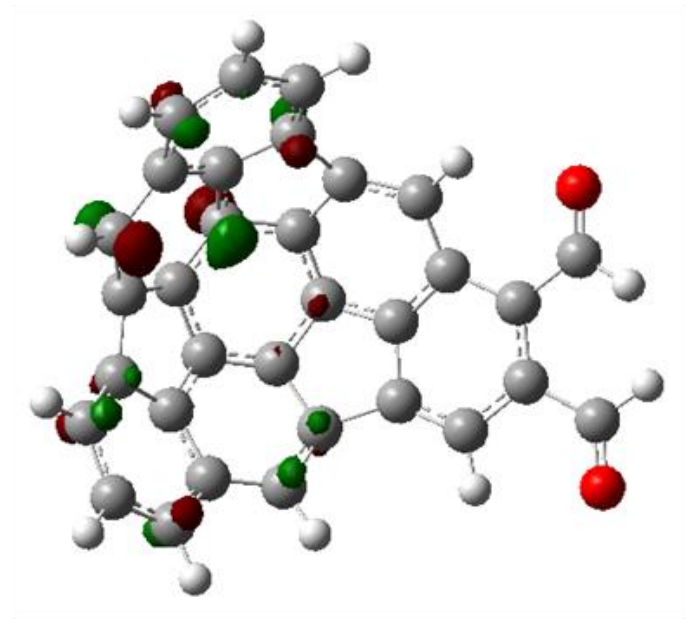

HOMO

d (0.07ua) 


\section{Fragmento 1 monosustituido con $\mathrm{CN}$}

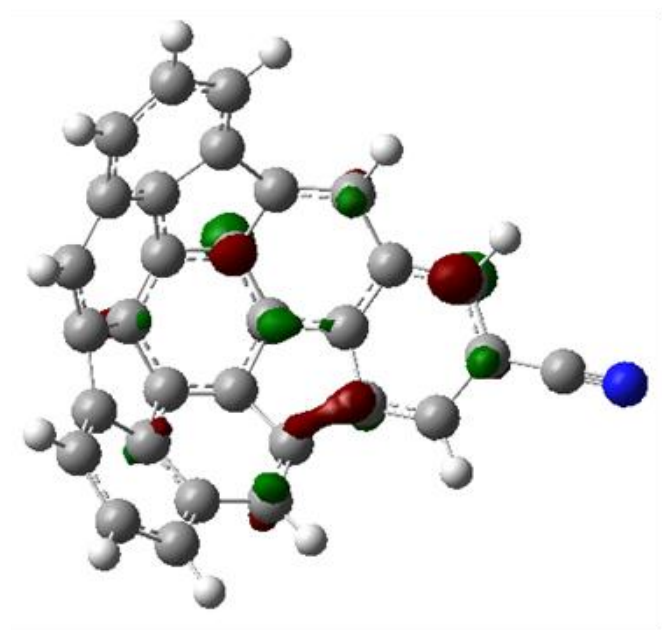

LUMO

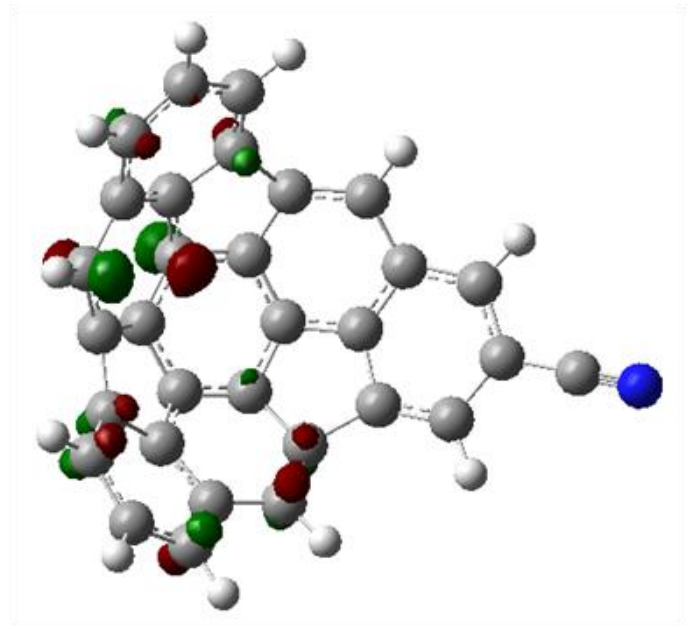

HOMO

d (0.08ua)

Fragmento 1 disustituido con $\mathrm{CN}$

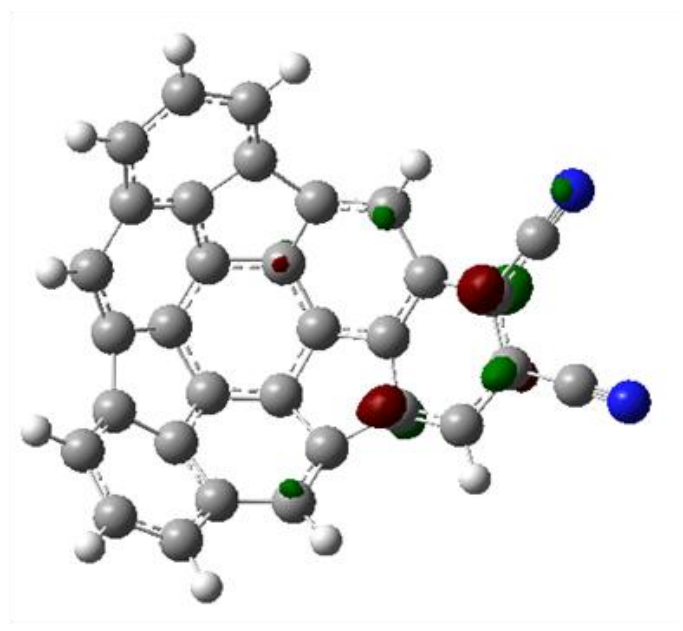

LUMO

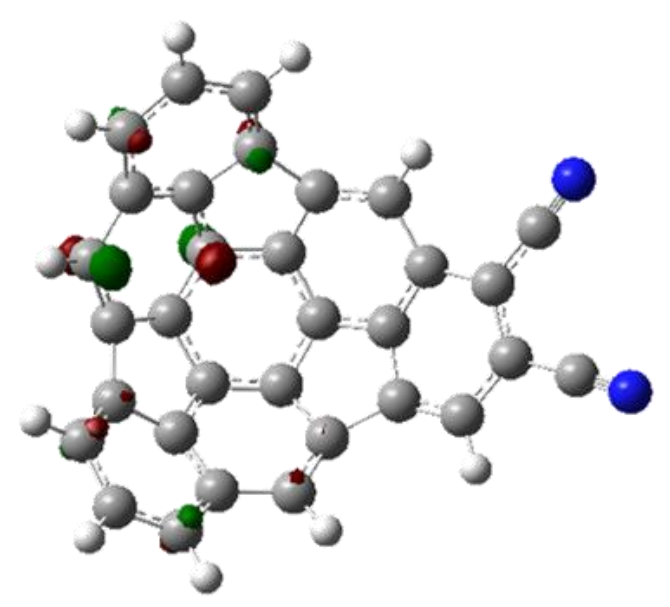

HOMO

d (0.08ua) 
Fragmento 1 monosustituido con $\mathrm{COOH}$

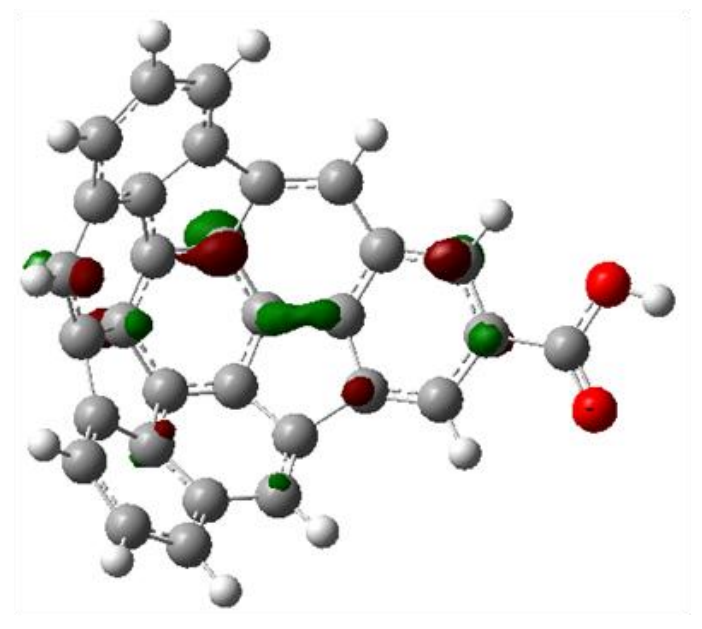

LUMO

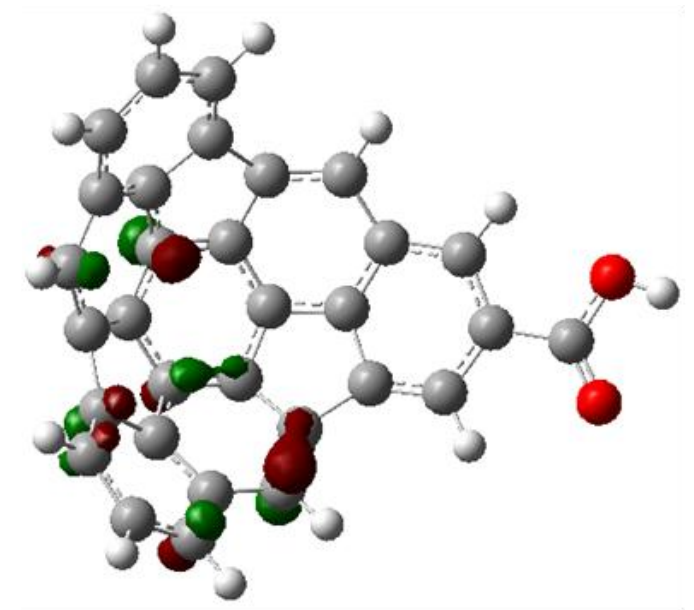

HOMO

d (0.07ua)

Fragmento 1 disustituido con $\mathrm{COOH}$

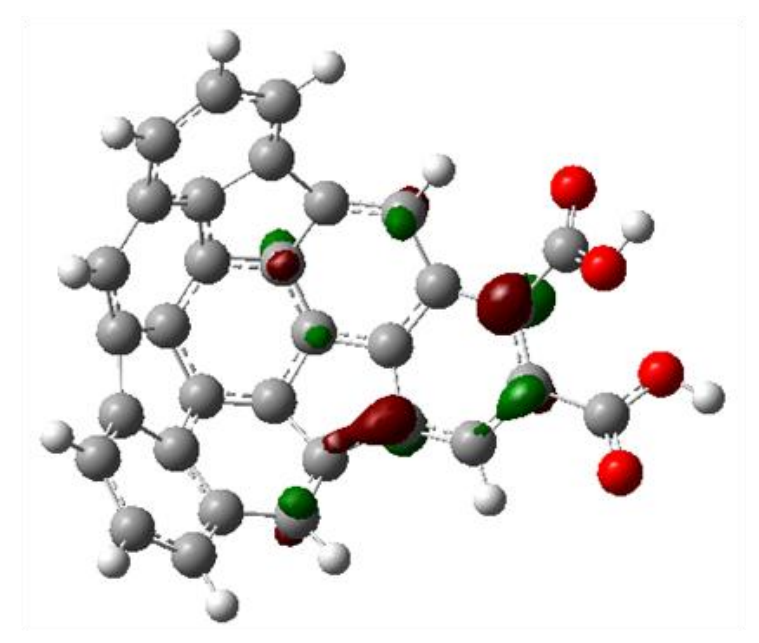

LUMO

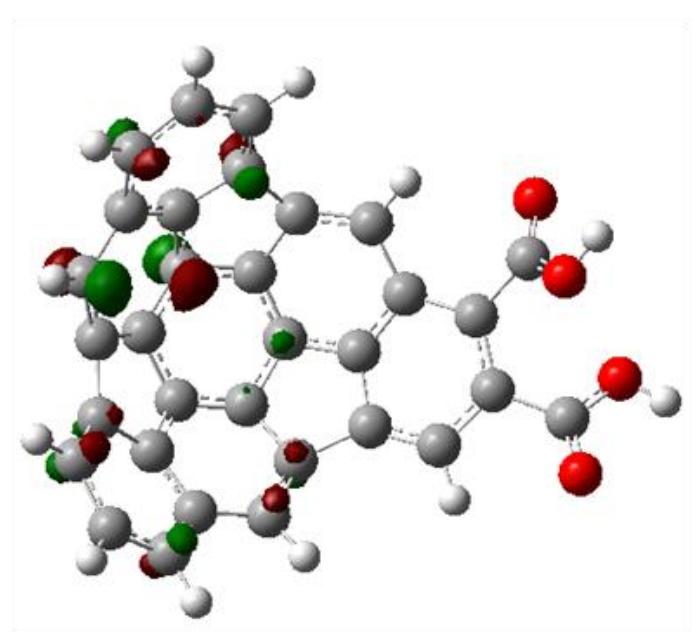

HOMO

d (0.07ua) 
Fragmento 1 monosustituido con $F$

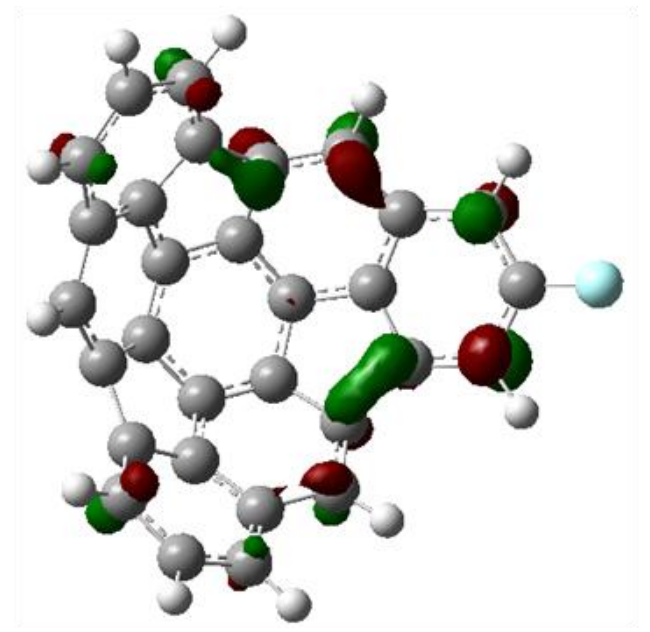

LUMO

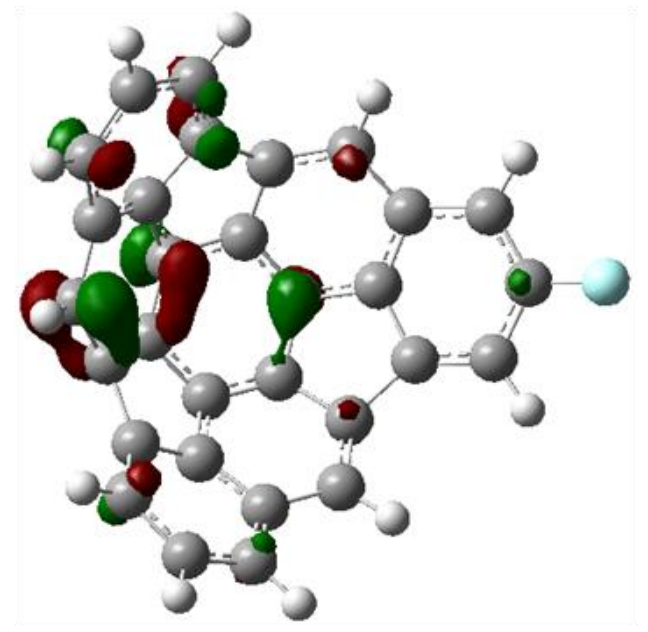

HOMO

d (0.06ua)

Fragmento 1 disustituido con $\mathrm{F}$

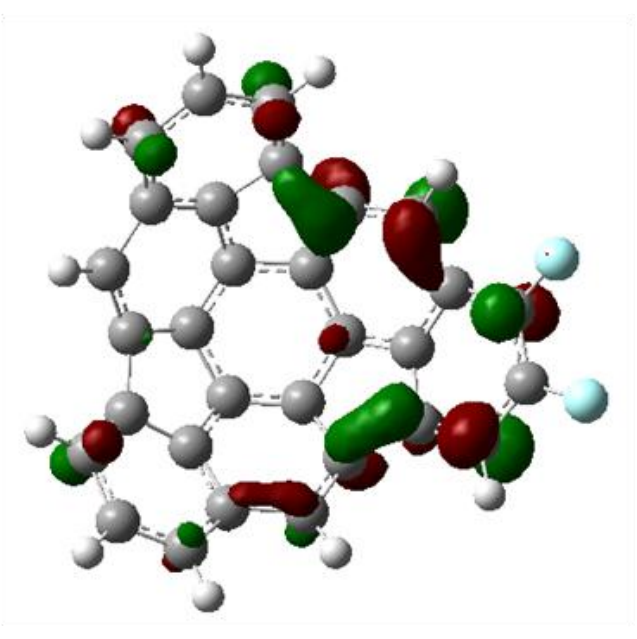

LUMO

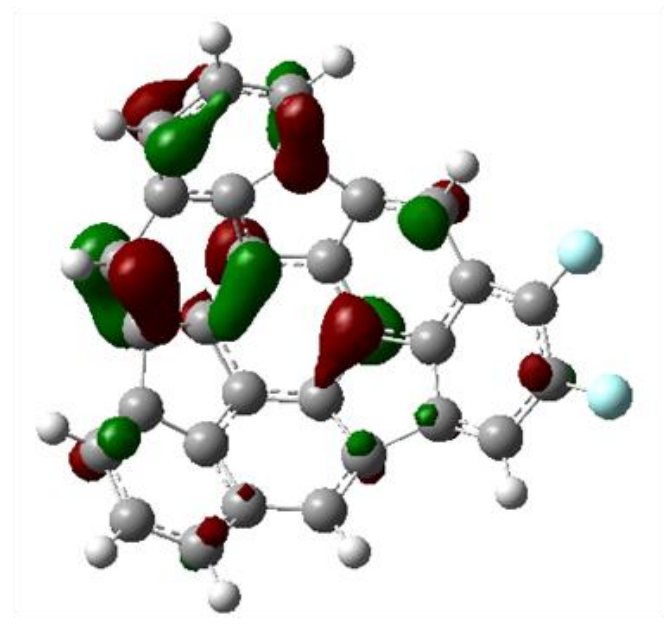

HOMO

d (0.05ua) 
Fragmento 1 monosustituido con $i-\mathrm{Pr}$

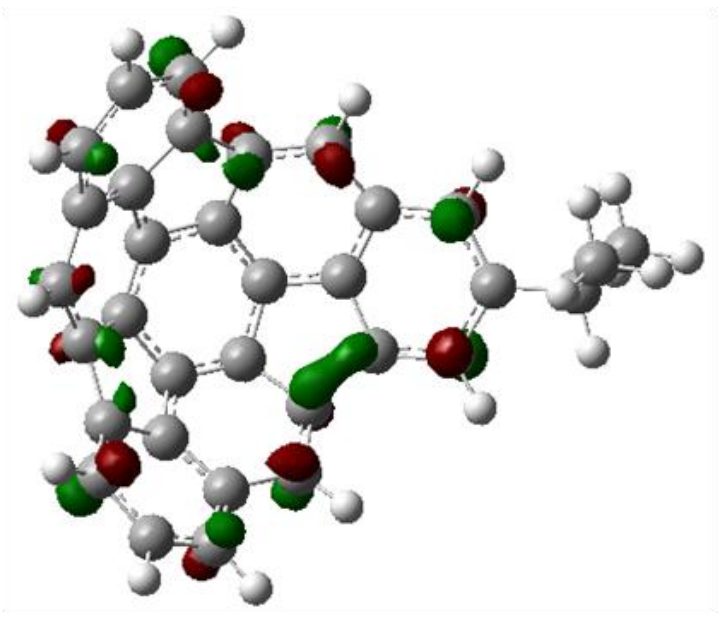

LUMO

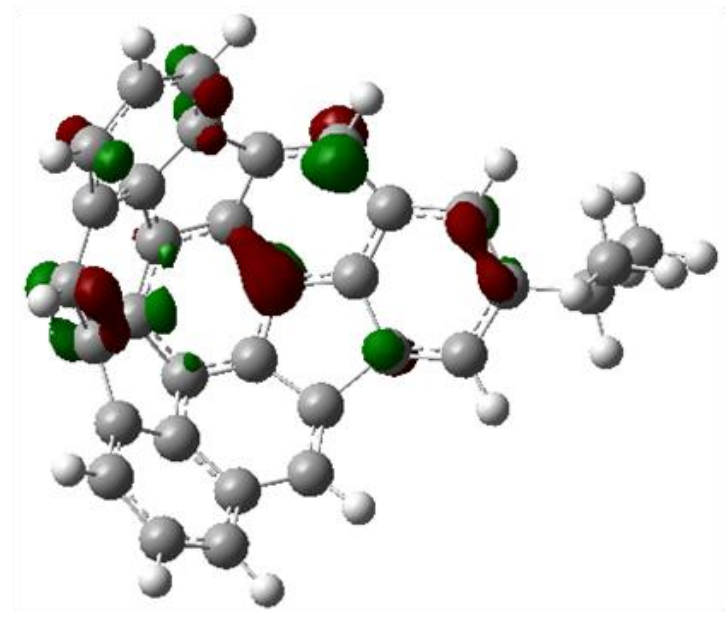

HOMO

d (0.06ua)

Fragmento 1 disustituido con $i-\operatorname{Pr}$

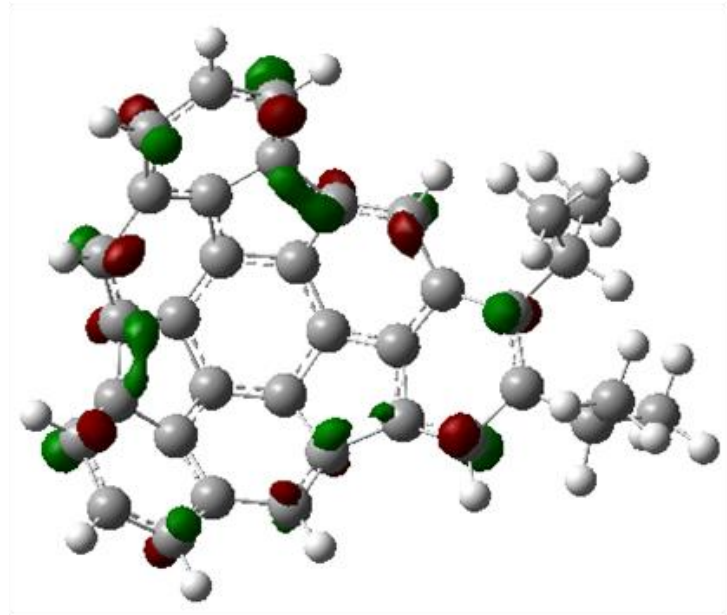

LUMO

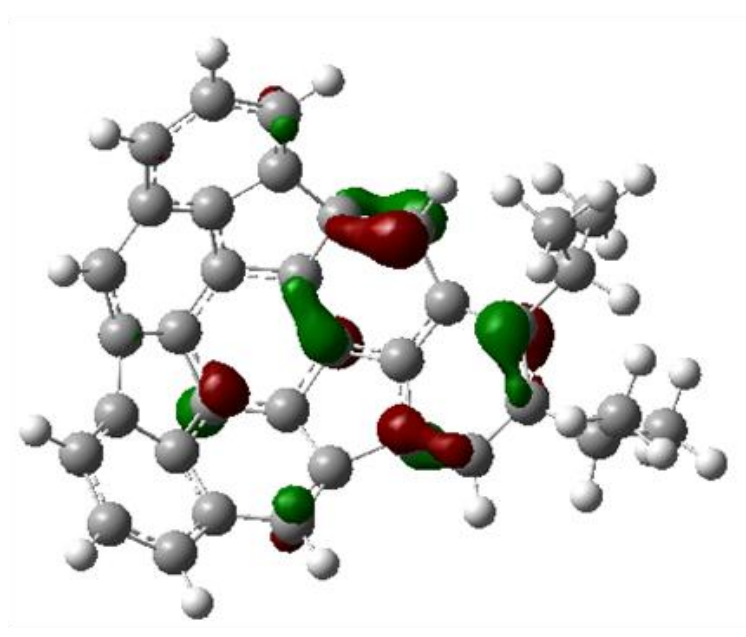

HOMO

d (0.06ua) 
Fragmento 1 monosustituido con Me

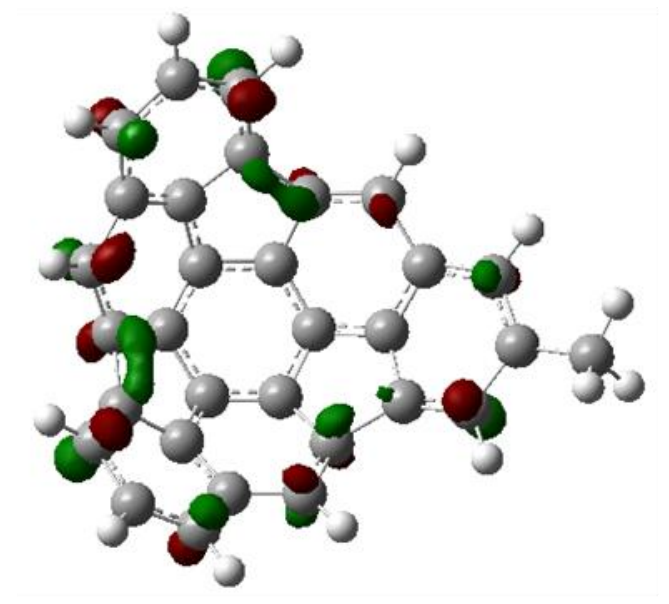

LUMO

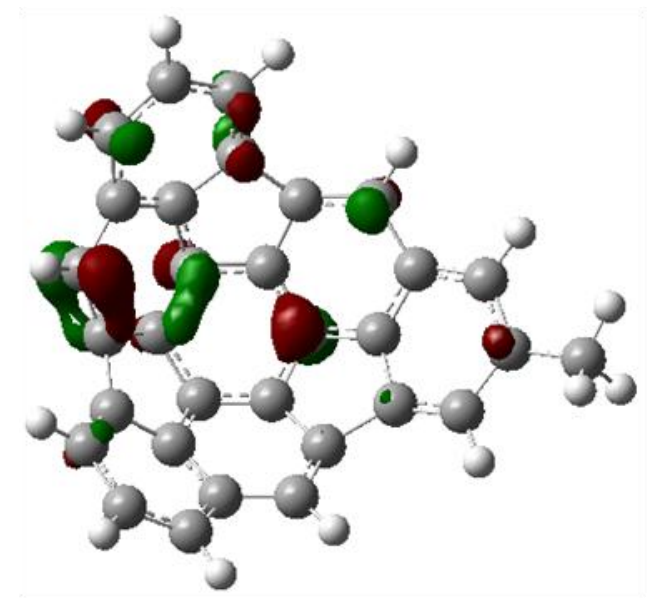

HOMO

d (0.06ua)

Fragmento 1 disustituido con Me

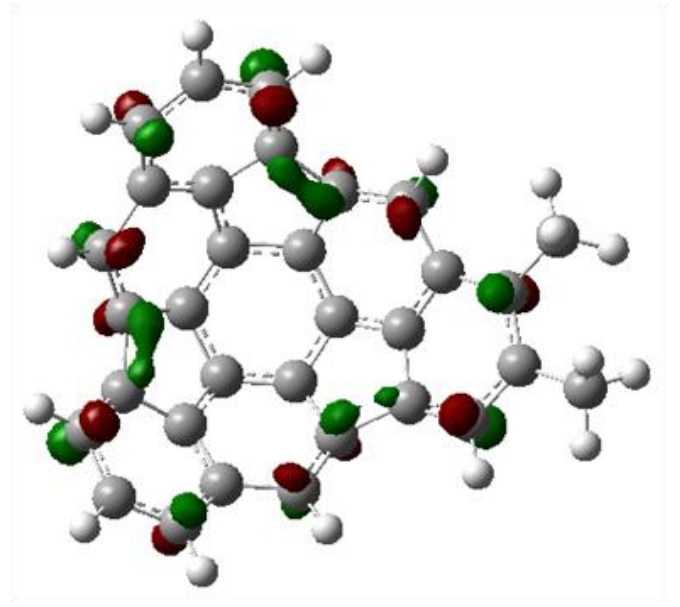

LUMO

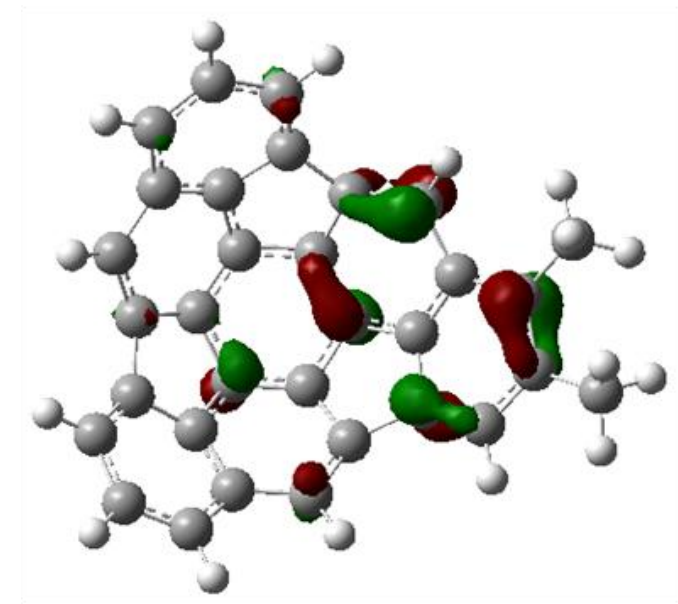

HOMO

d (0.06ua) 
Fragmento 1 monosustituido con $\mathbf{N H}_{2}$

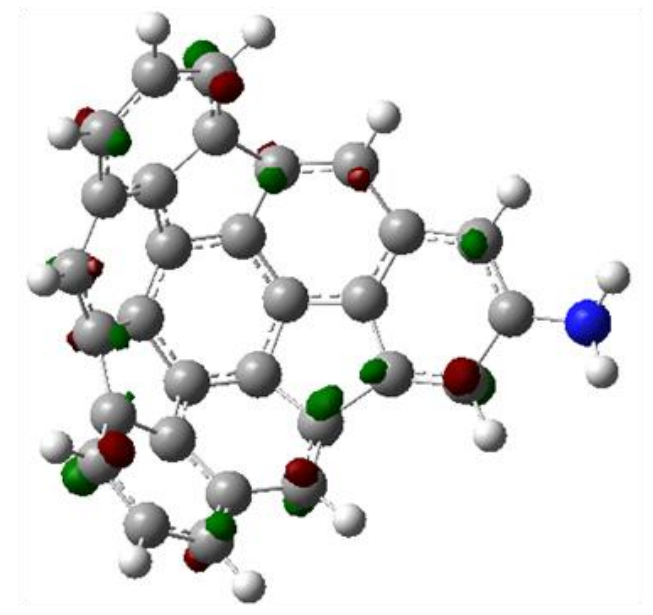

LUMO

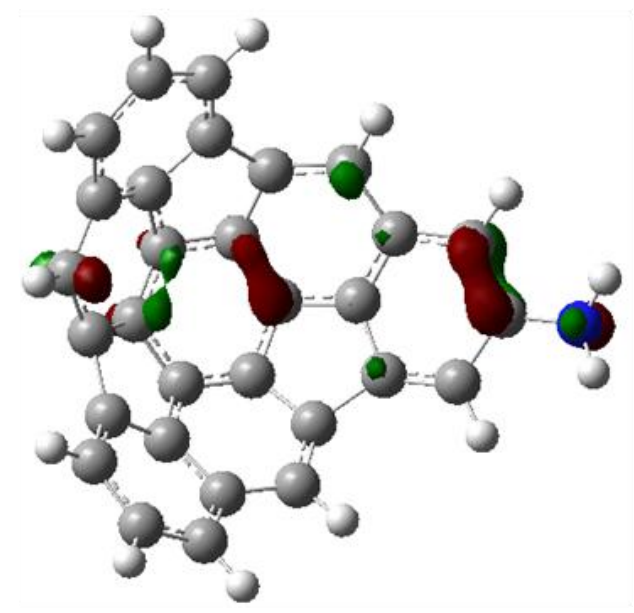

HOMO

$$
\text { d (0.07ua) }
$$

Fragmento 1 disustituido con $\mathrm{NH}_{2}$

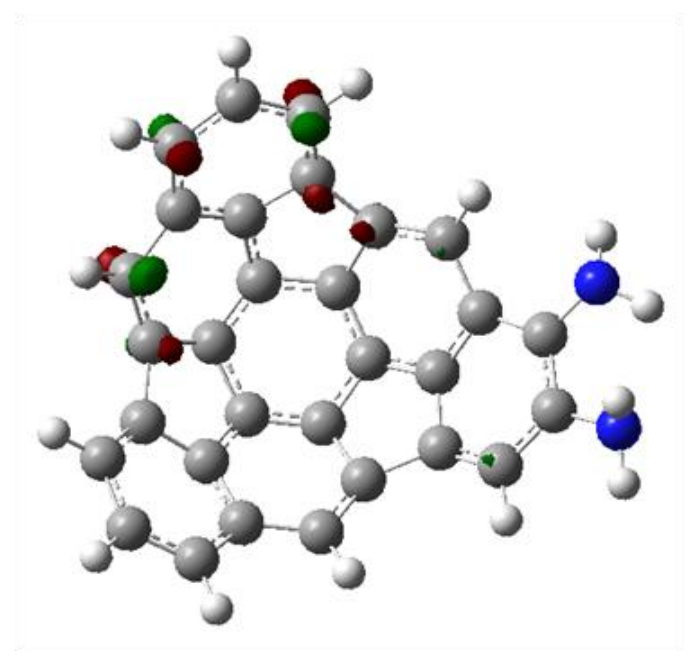

LUMO

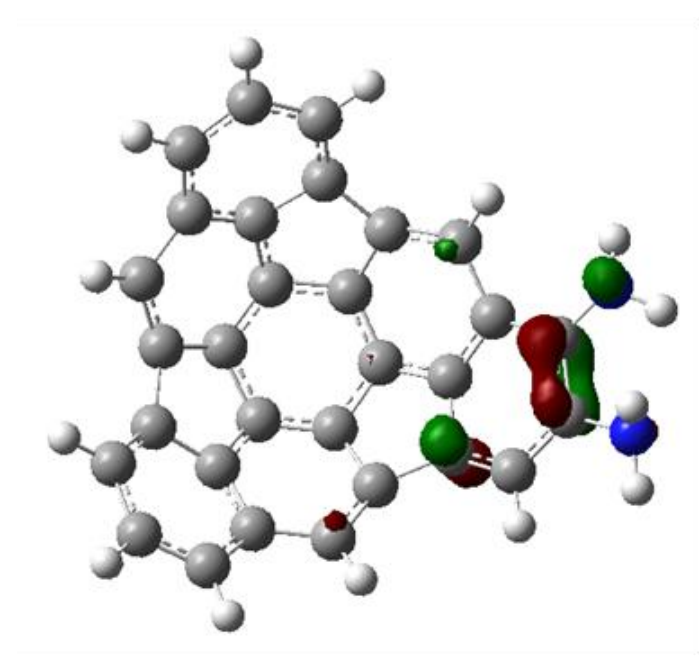

HOMO

a (0.08ua) 
Fragmento 1 monosustituido con $\mathrm{NO}_{2}$

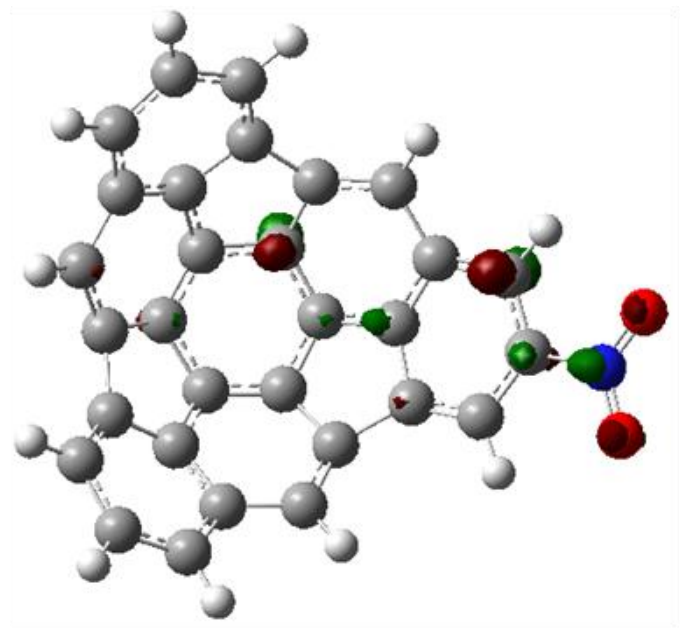

LUMO

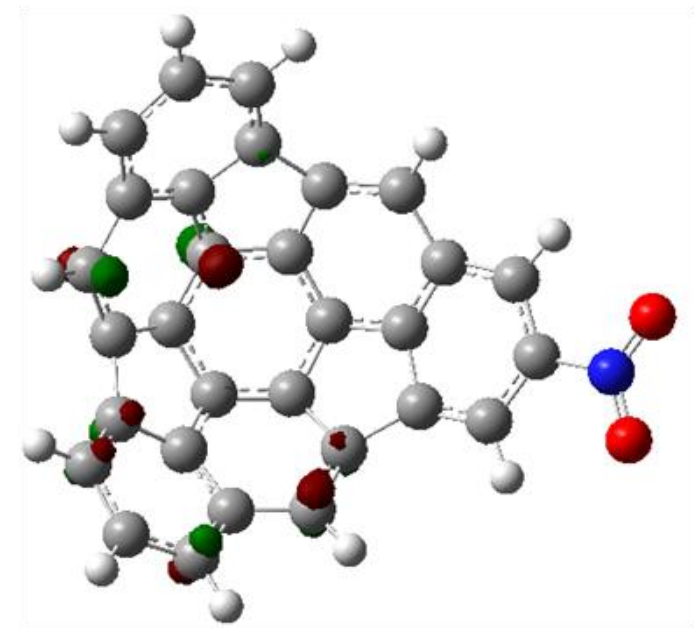

HOMO

d (0.08ua)

Fragmento 1 disustituido con $\mathrm{NO}_{2}$

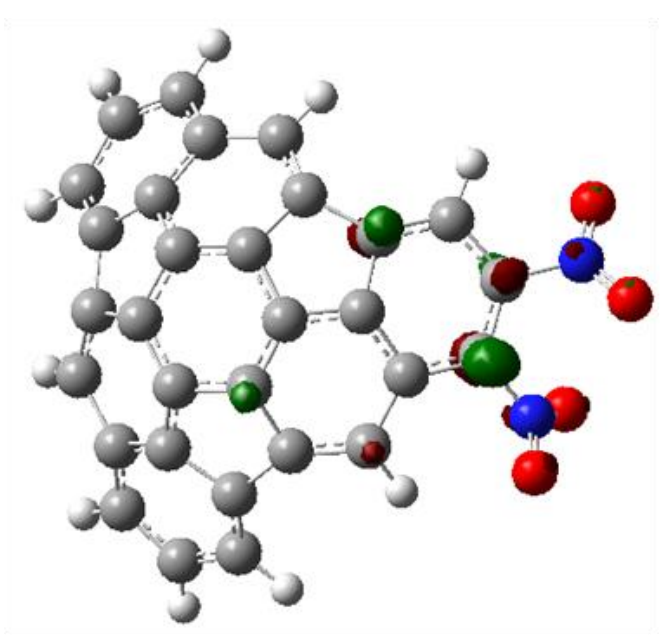

LUMO

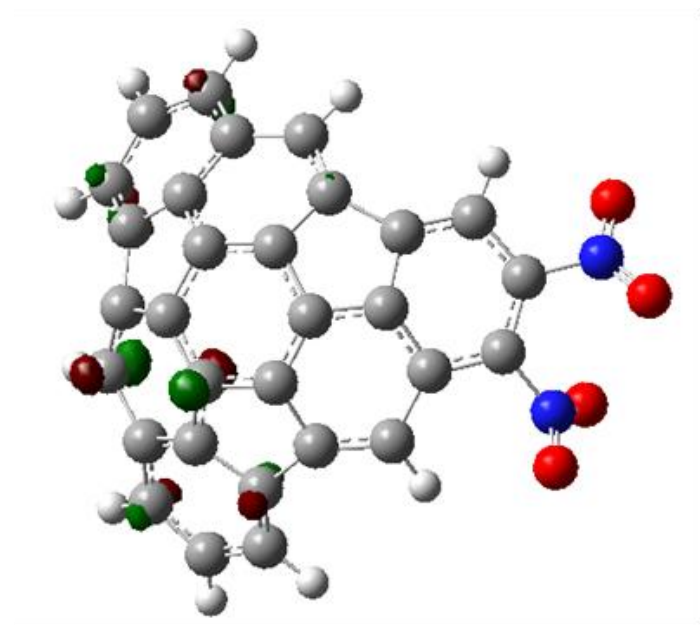

HOMO

d (0.08ua) 
Fragmento 1 monosustituido con $\mathrm{OH}$

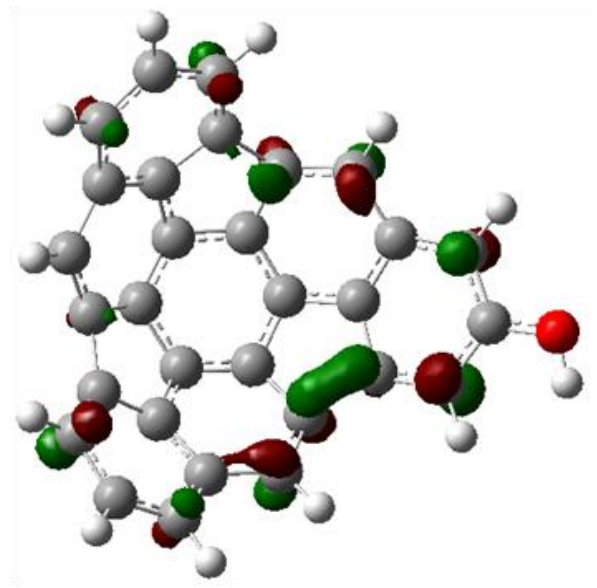

LUMO

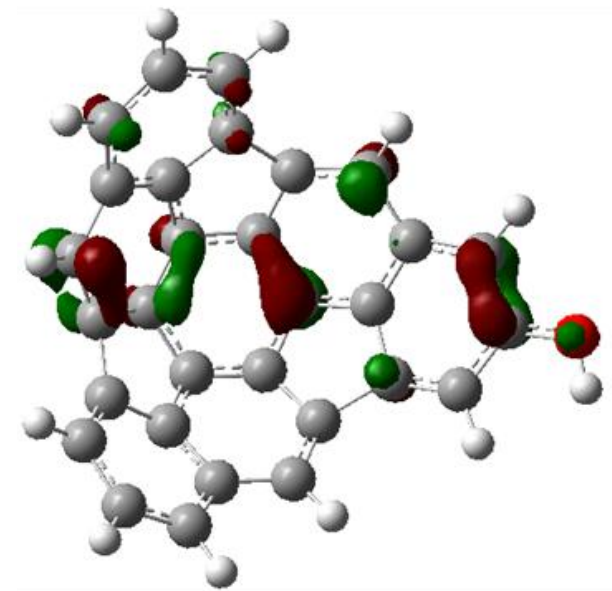

HOMO

d (0.06ua)

Fragmento 1 disustituido con $\mathrm{OH}$

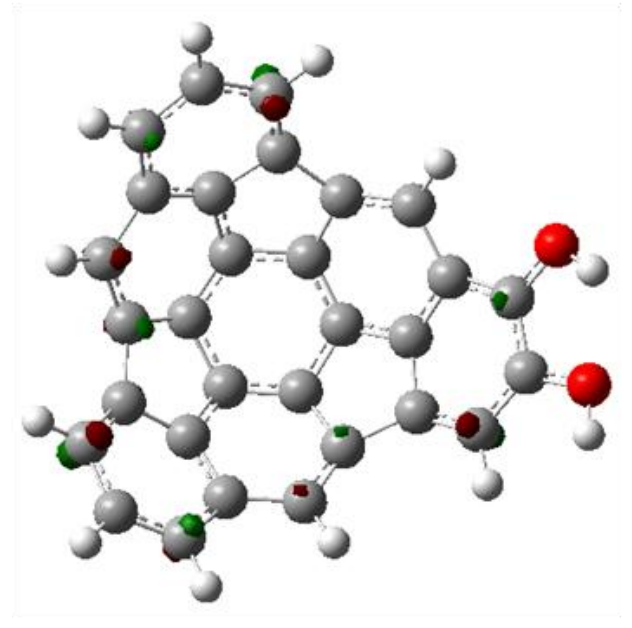

LUMO

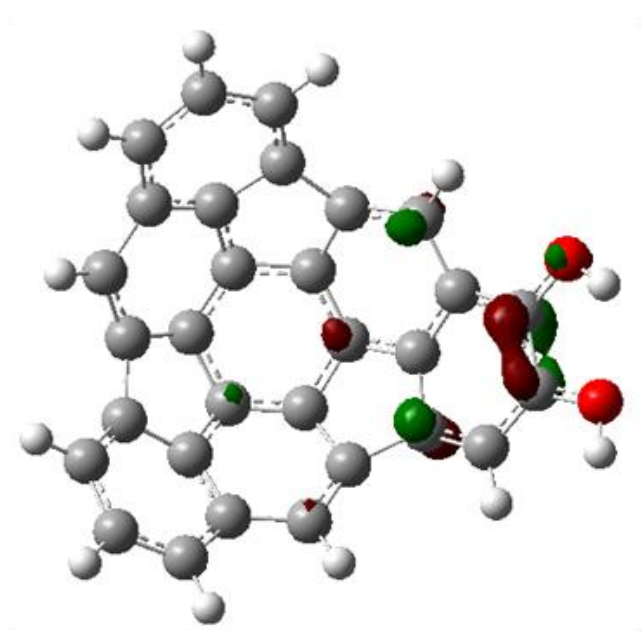

HOMO

d (0.08ua) 
Fragmento 1 monosustituido con OMe

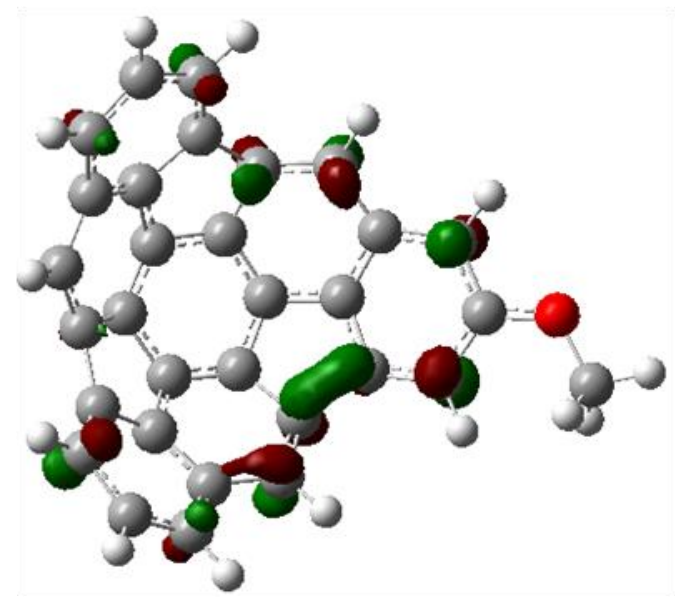

LUMO

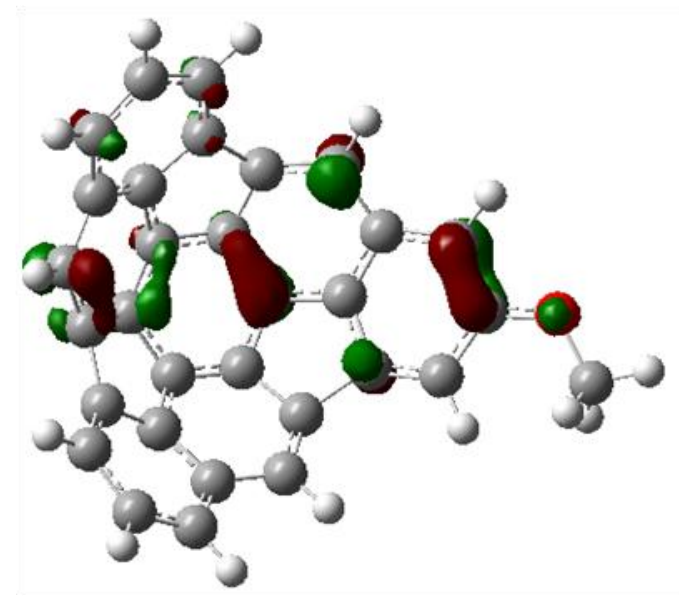

HOMO

d (0.06ua)

Fragmento 1 disustituido con OMe

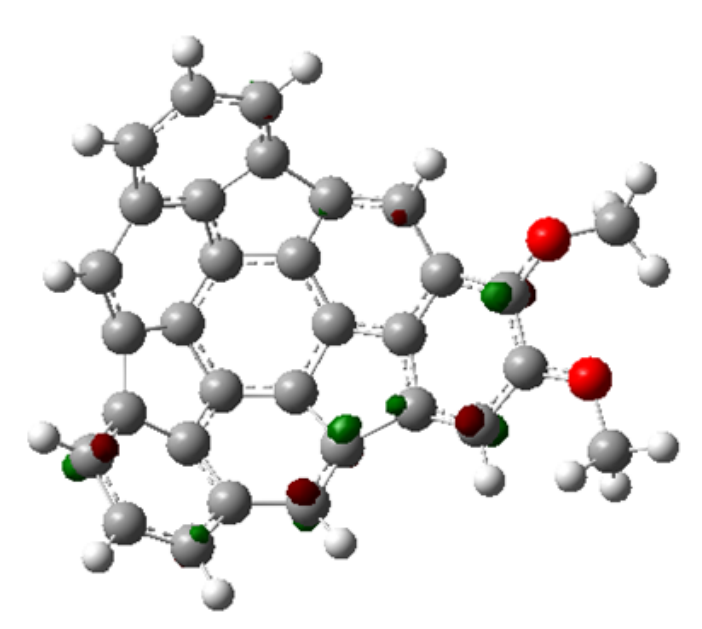

LUMO

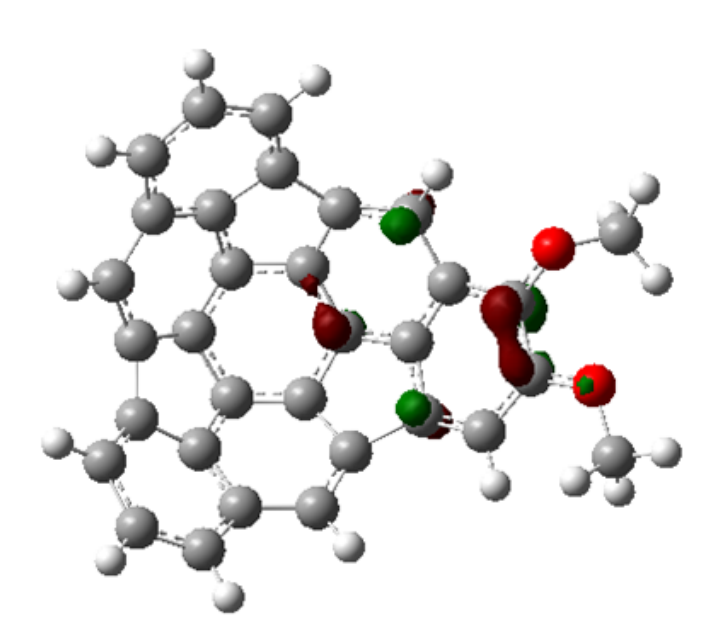

HOMO

d (0.08ua) 
Fragmento 2 monosustituido con $\mathrm{CF}_{3}$

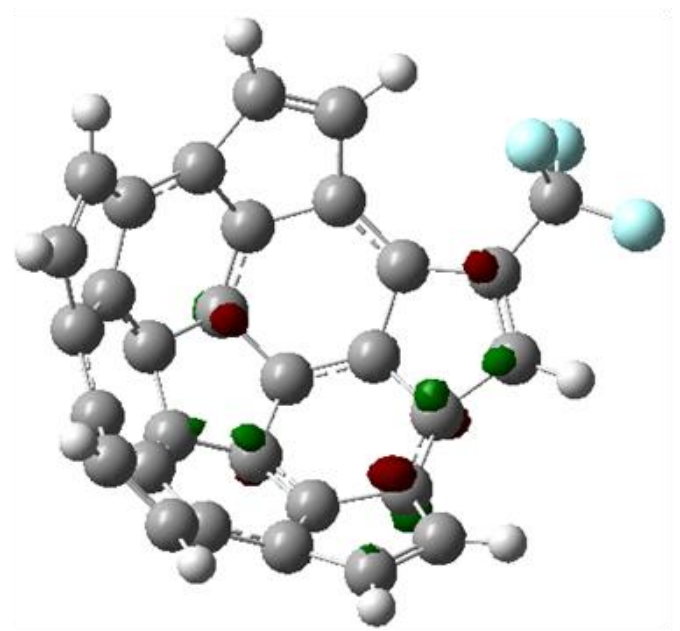

LUMO

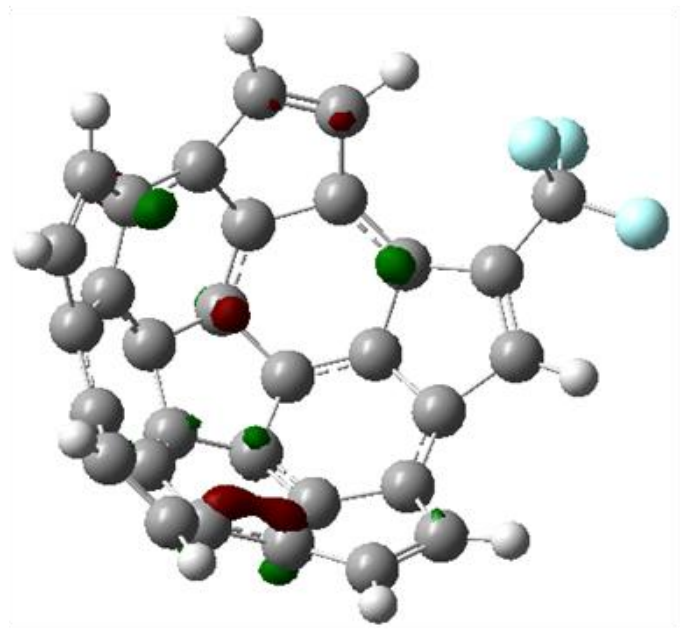

HOMO

d (0.08ua)

Fragmento 2 disustituido con $\mathrm{CF}_{3}$

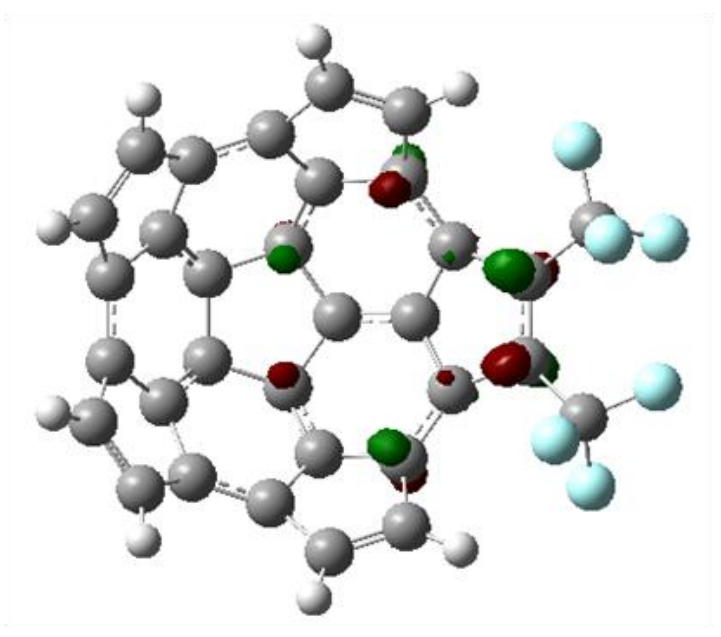

LUMO

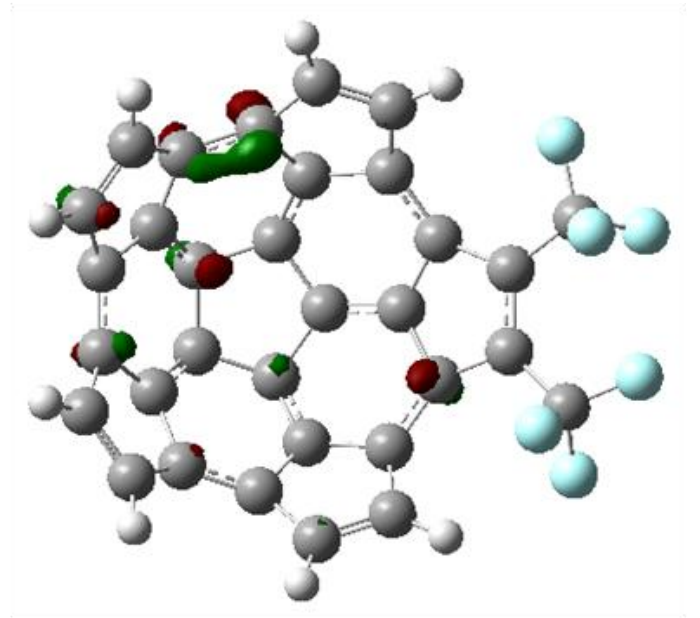

HOMO

d (0.08ua) 
Fragmento 2 monosustituido con $\mathrm{CHO}$

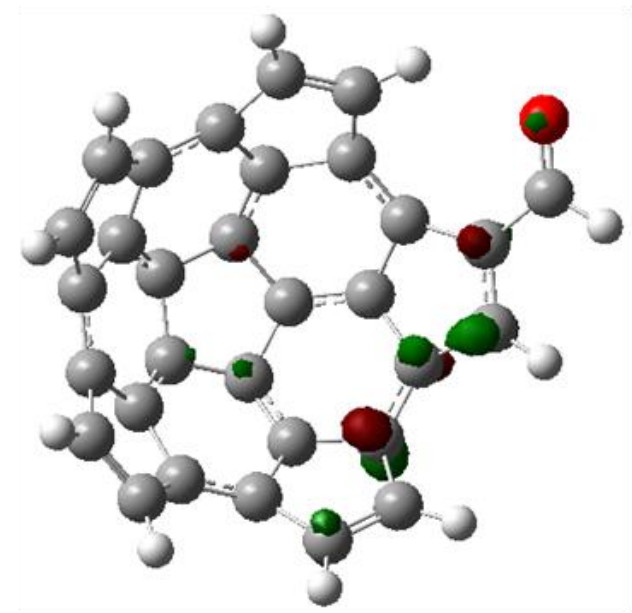

LUMO

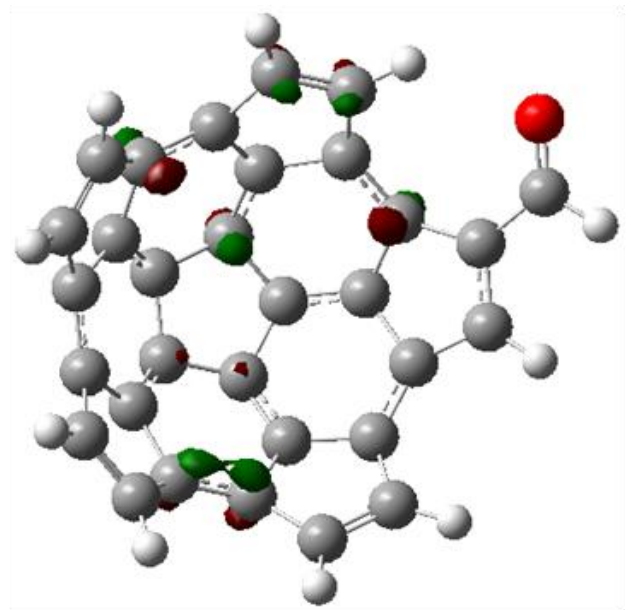

HOMO

$d$ (0.08ua)

Fragmento 2 disustituido con $\mathrm{CHO}$

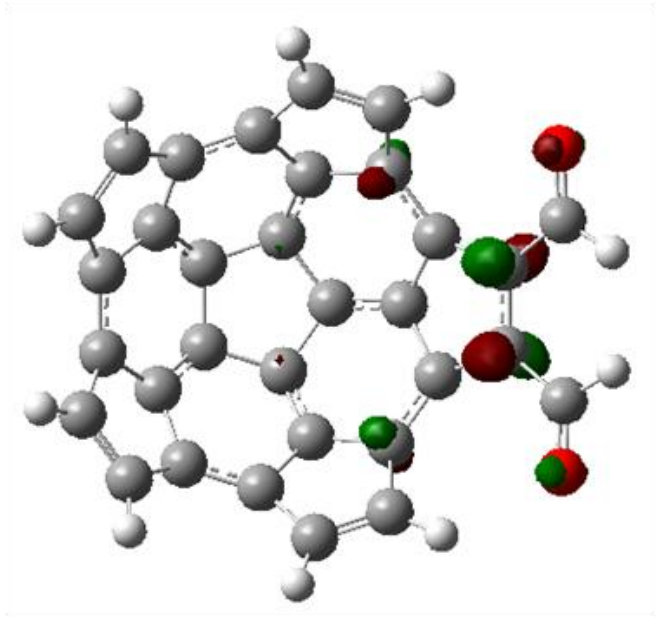

LUMO

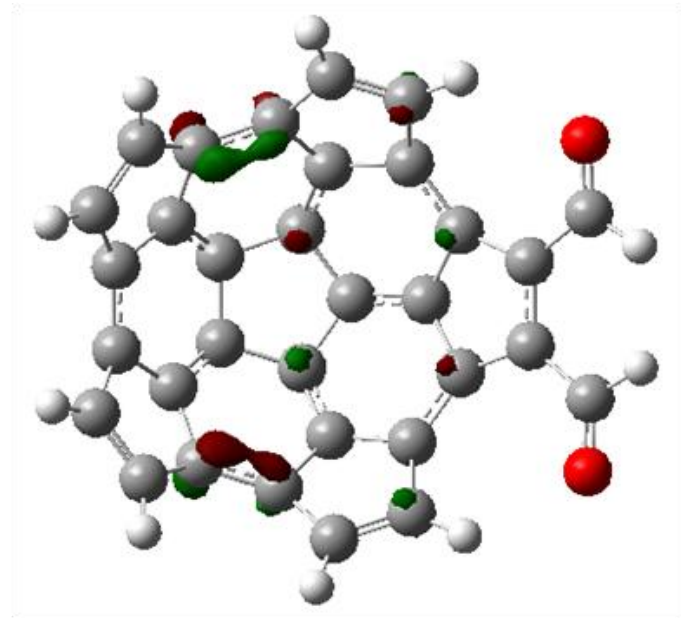

HOMO

d (0.08ua) 
Fragmento 2 monosustituido con $\mathrm{CN}$

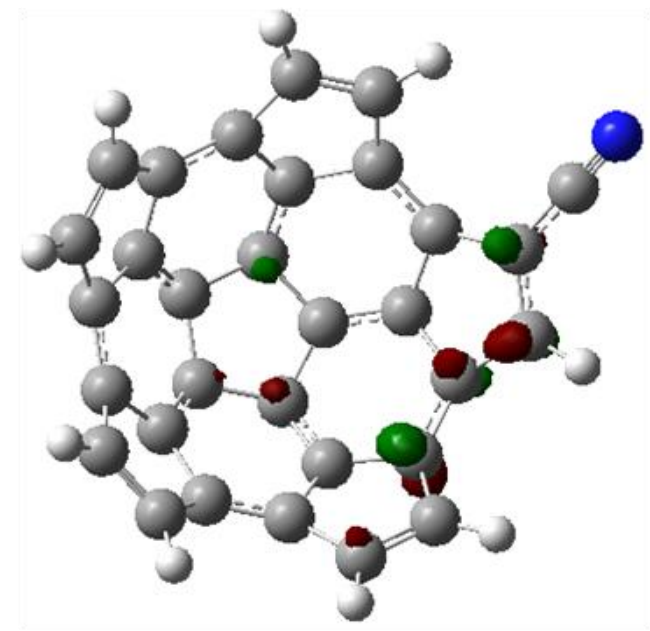

LUMO

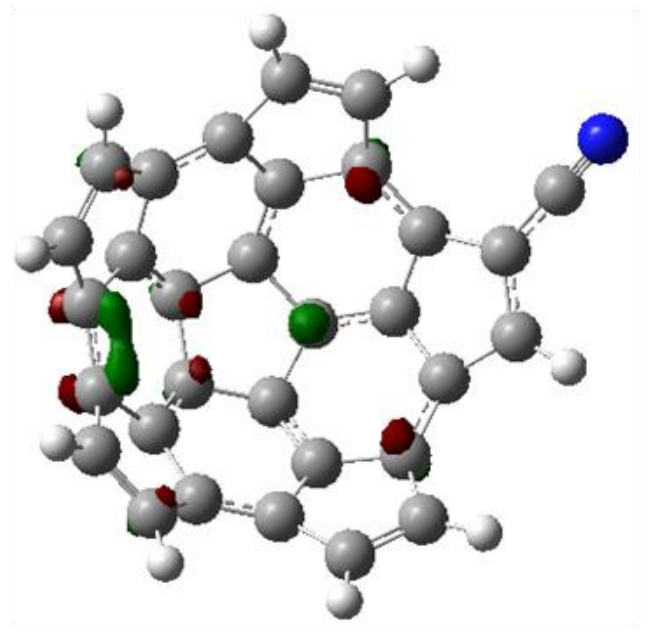

HOMO

d (0.08ua)

Fragmento 2 disustituido con CN

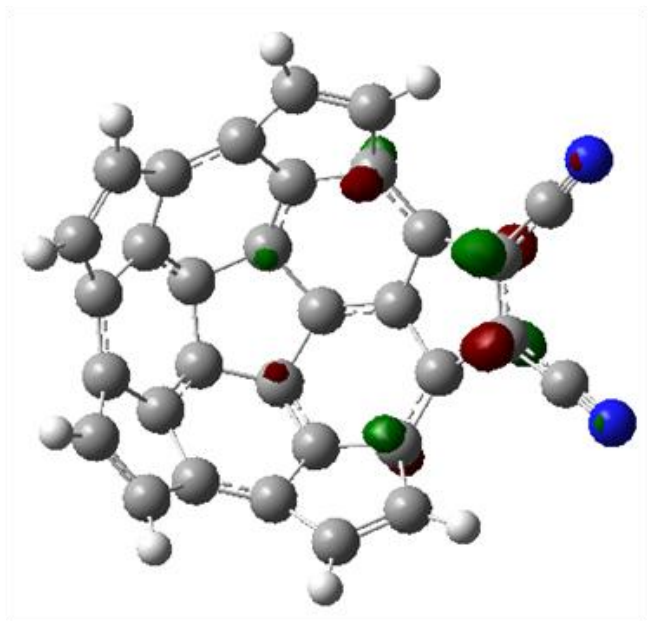

LUMO

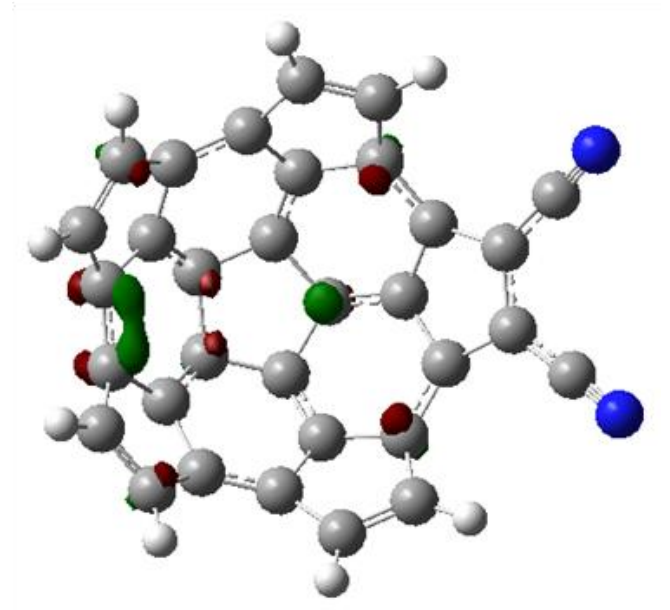

HOMO

d (0.08ua) 
Fragmento 2 monosustituido con $\mathrm{COOH}$

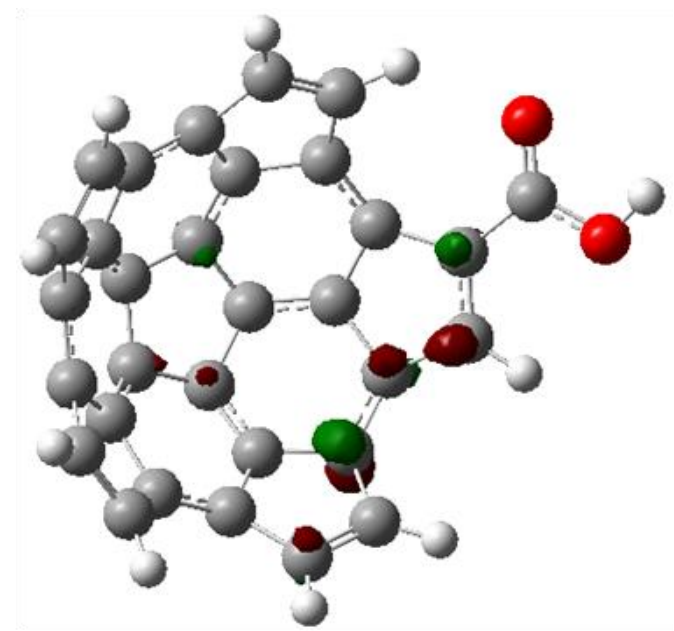

LUMO

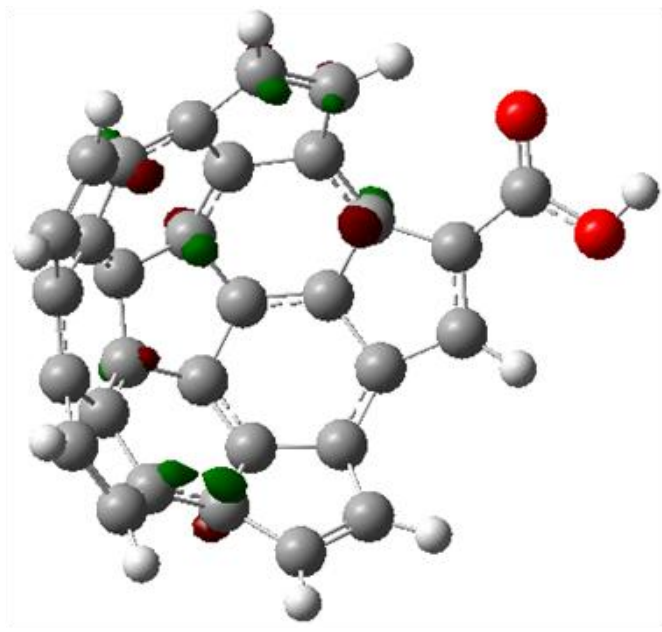

HOMO

d (0.08ua)

Fragmento 2 disustituido con $\mathrm{COOH}$

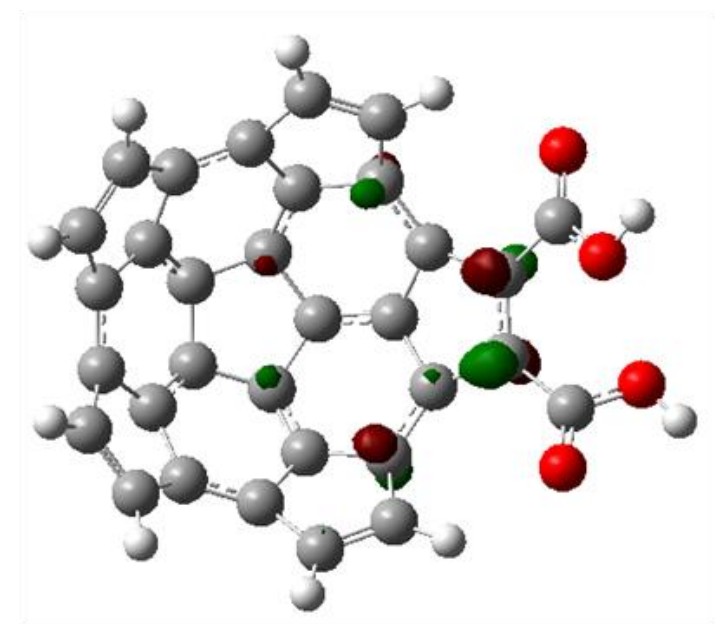

LUMO

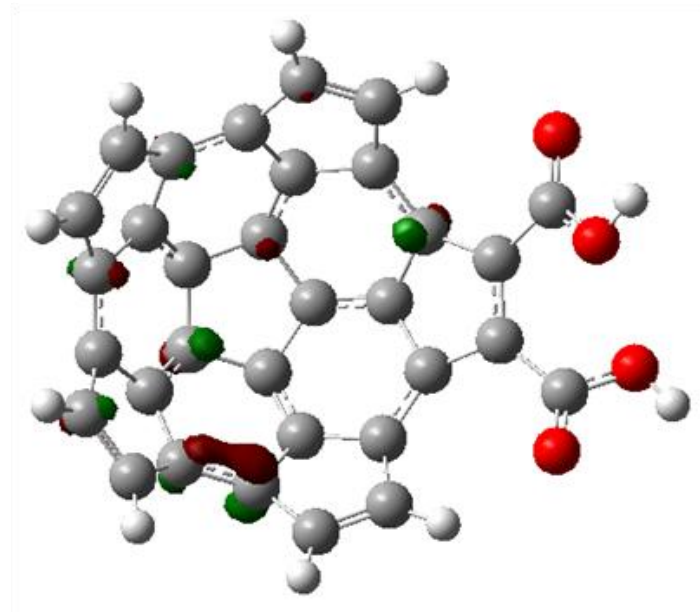

HOMO

d (0.08ua) 
Fragmento 2 monosustituido con $\mathrm{F}$

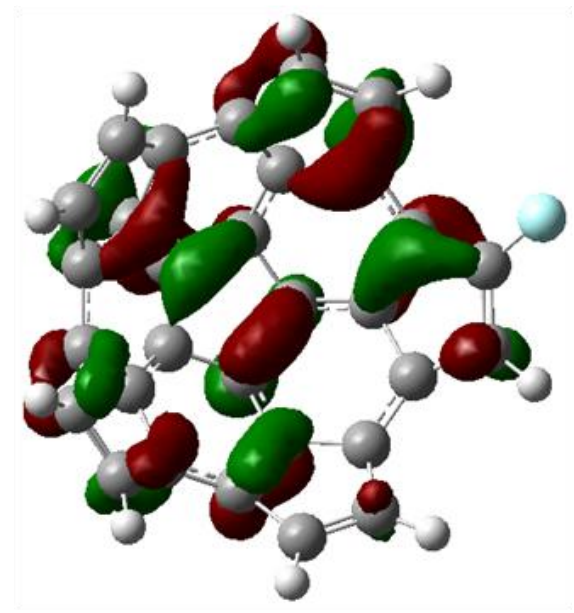

LUMO

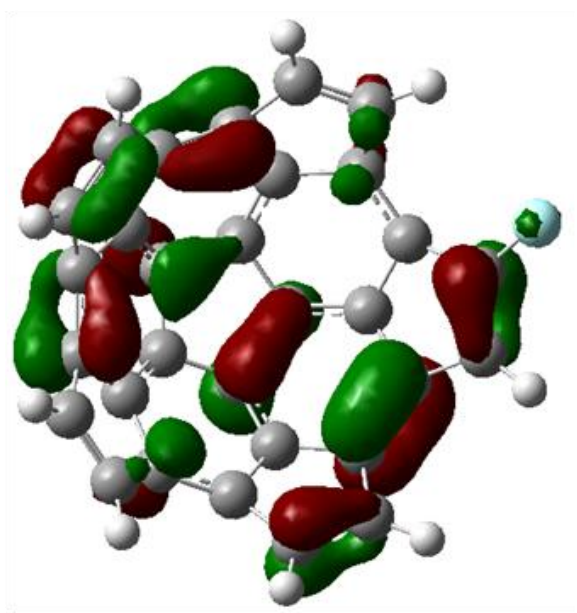

HOMO

d (0.04ua)

Fragmento 2 disustituido con $F$

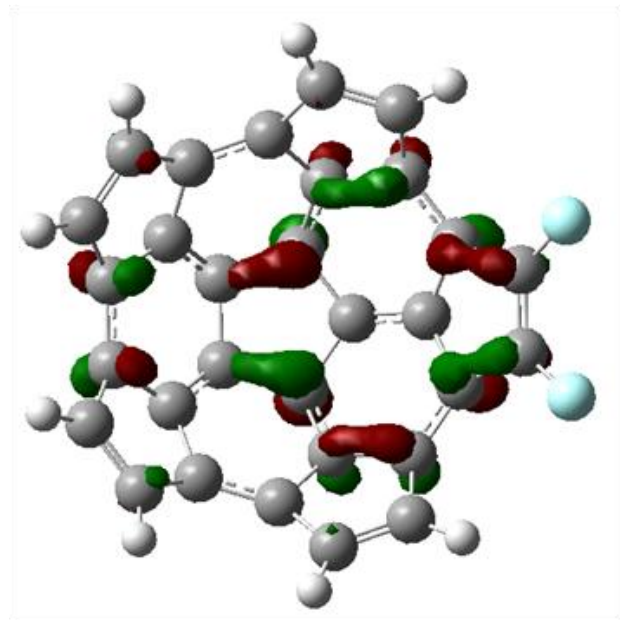

LUMO

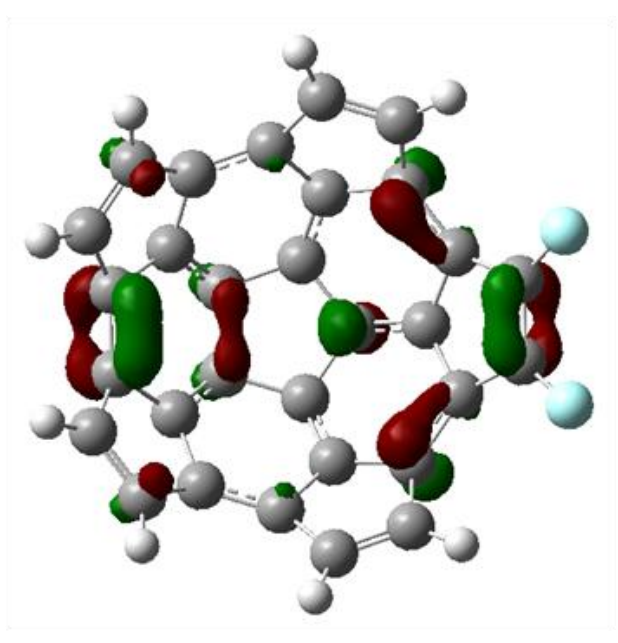

HOMO

d (0.06ua) 
Fragmento 2 monosustituido con $i-P r$
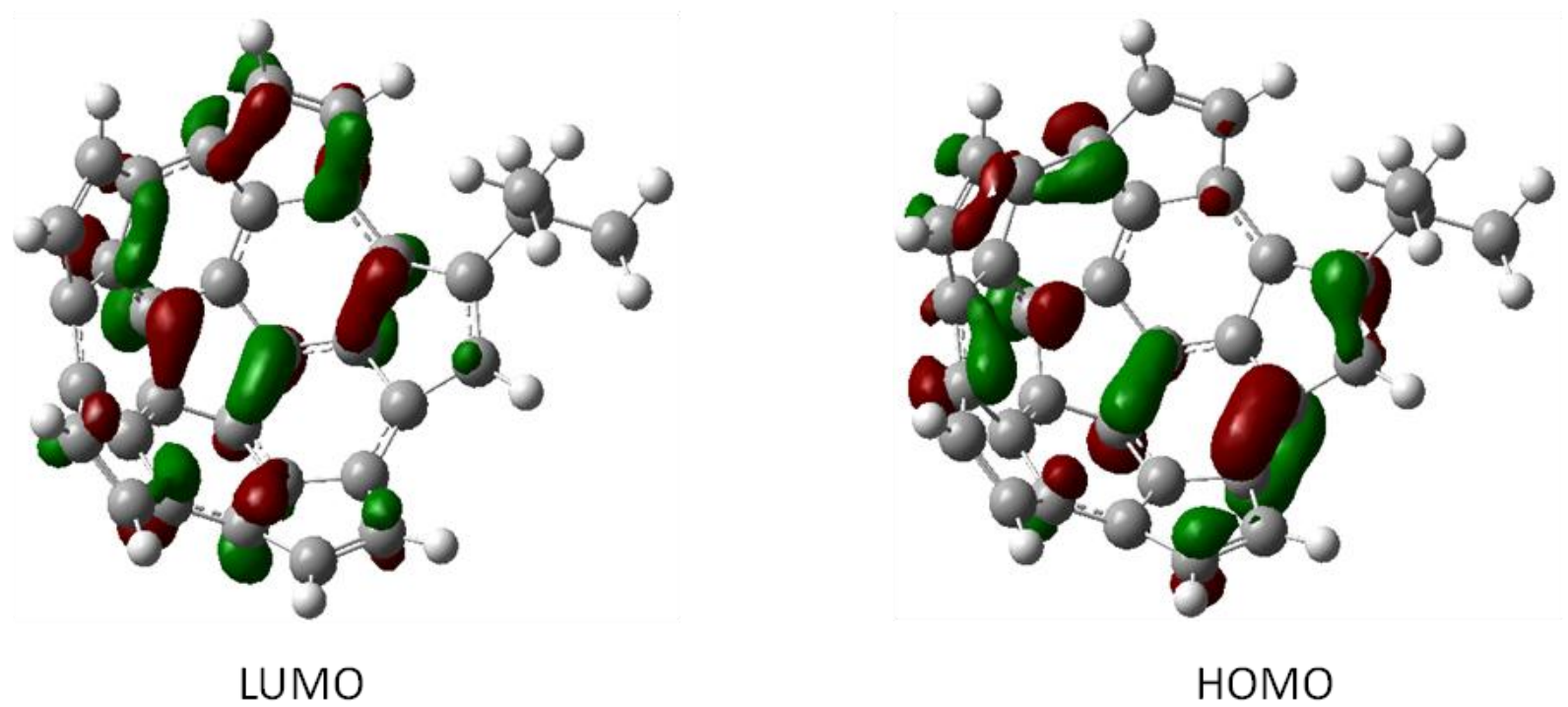

d (0.05ua)

Fragmento 2 disustituido con $i-P r$

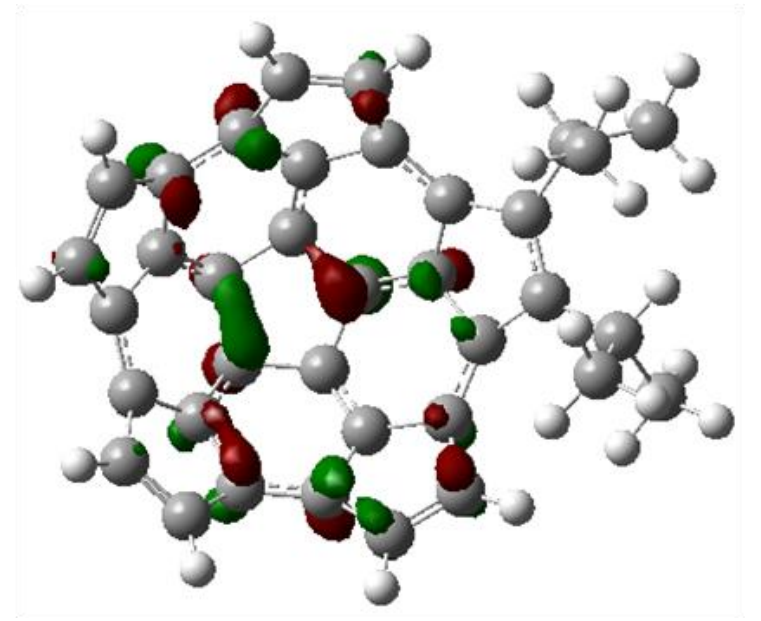

LUMO

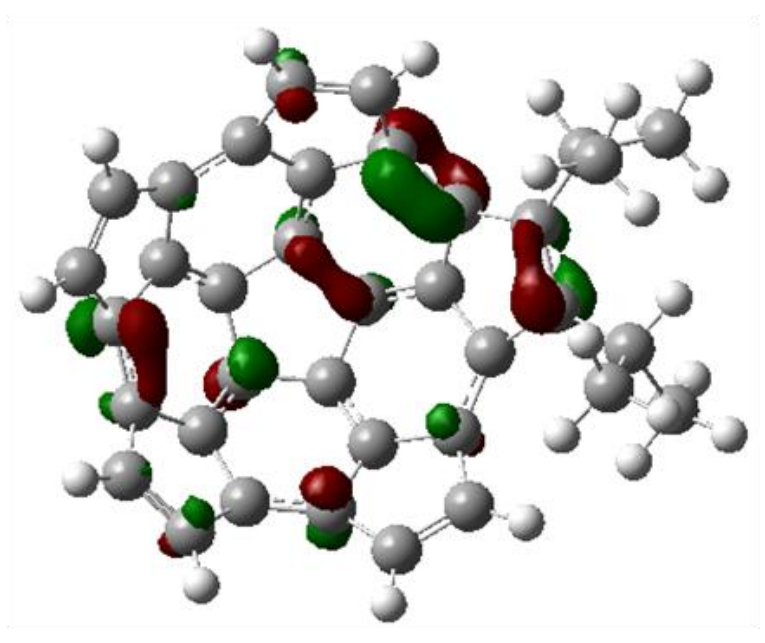

HOMO

d (0.06ua) 
Fragmento 2 monosustituido con Me

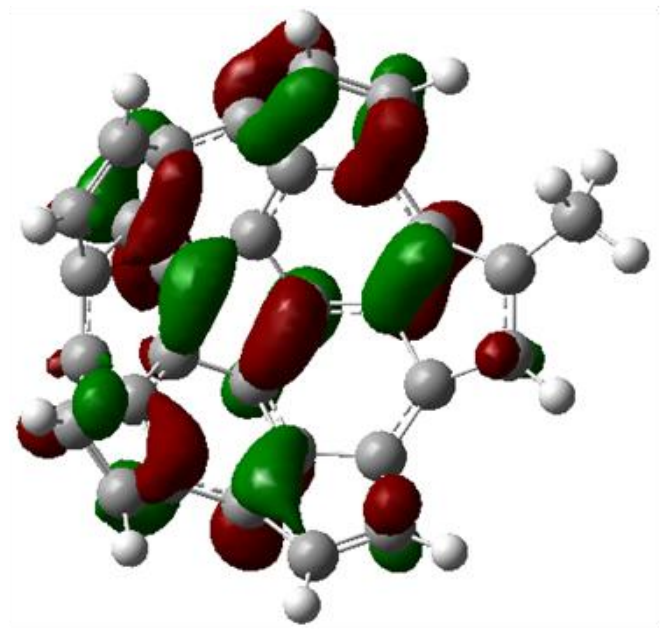

LUMO

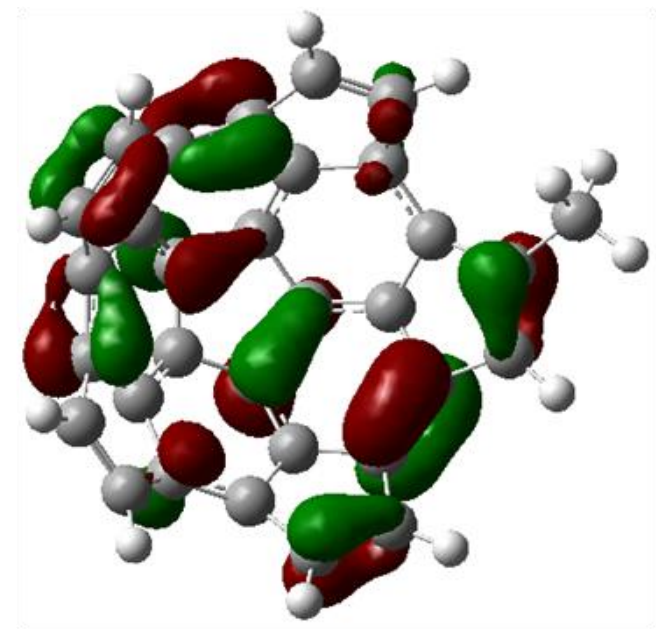

HOMO

d (0.04ua)

Fragmento 2 disustituido con Me

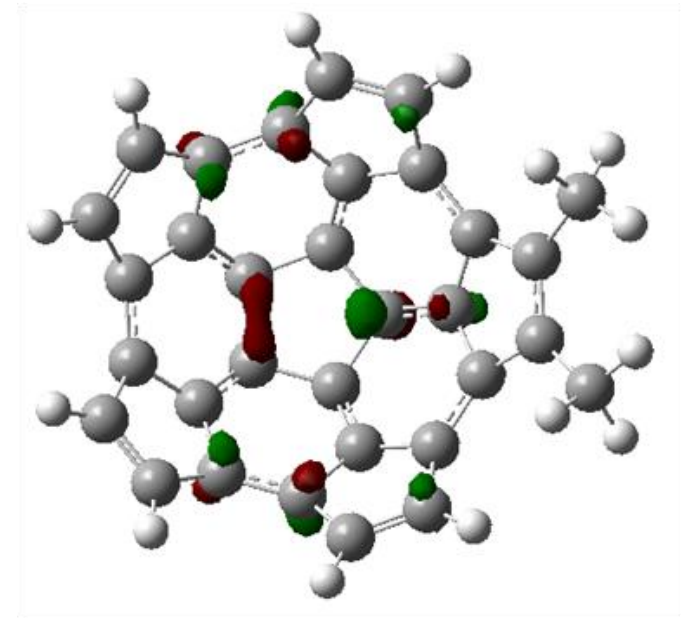

LUMO

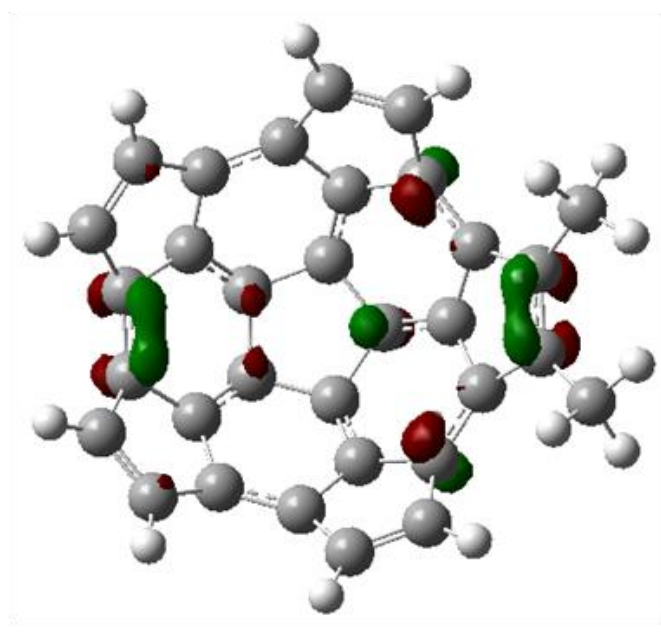

HOMO

$d$ (0.07ua) 
Fragmento 2 monosustituido con $\mathrm{NH}_{2}$

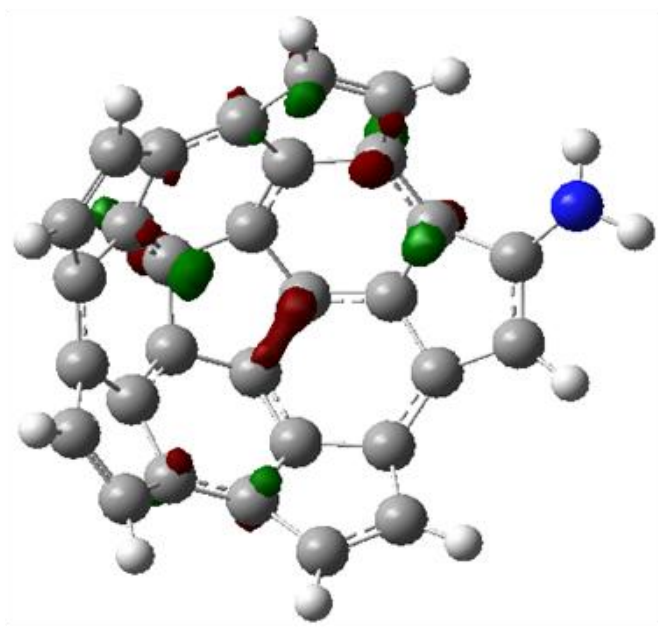

LUMO

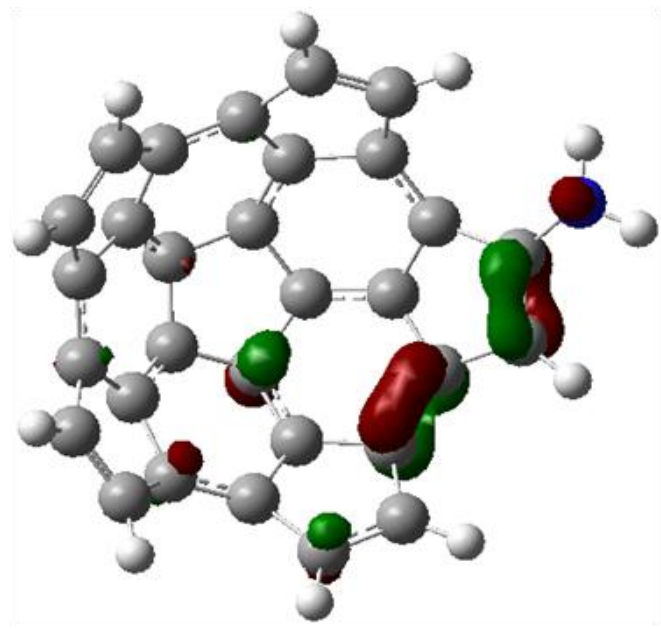

HOMO

d (0.07ua)

Fragmento 2 disustituido con $\mathrm{NH}_{2}$

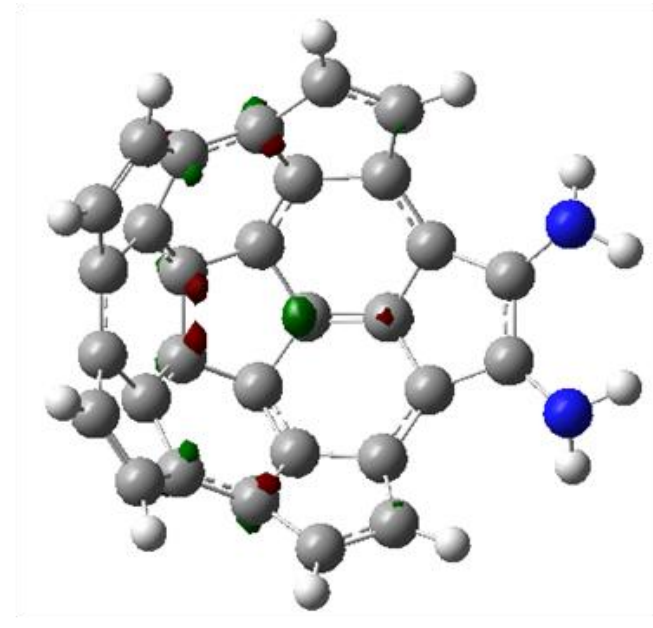

LUMO

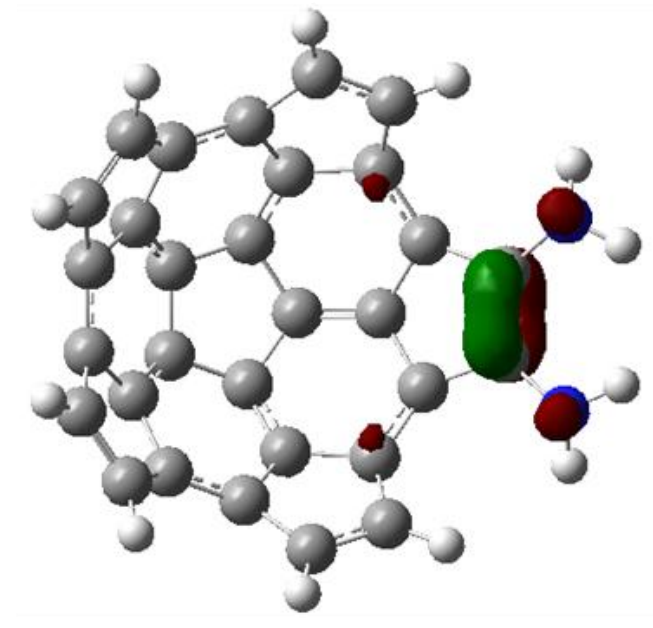

HOMO

d (0.08ua) 
Fragmento 2 monosustituido con $\mathrm{NO}_{2}$
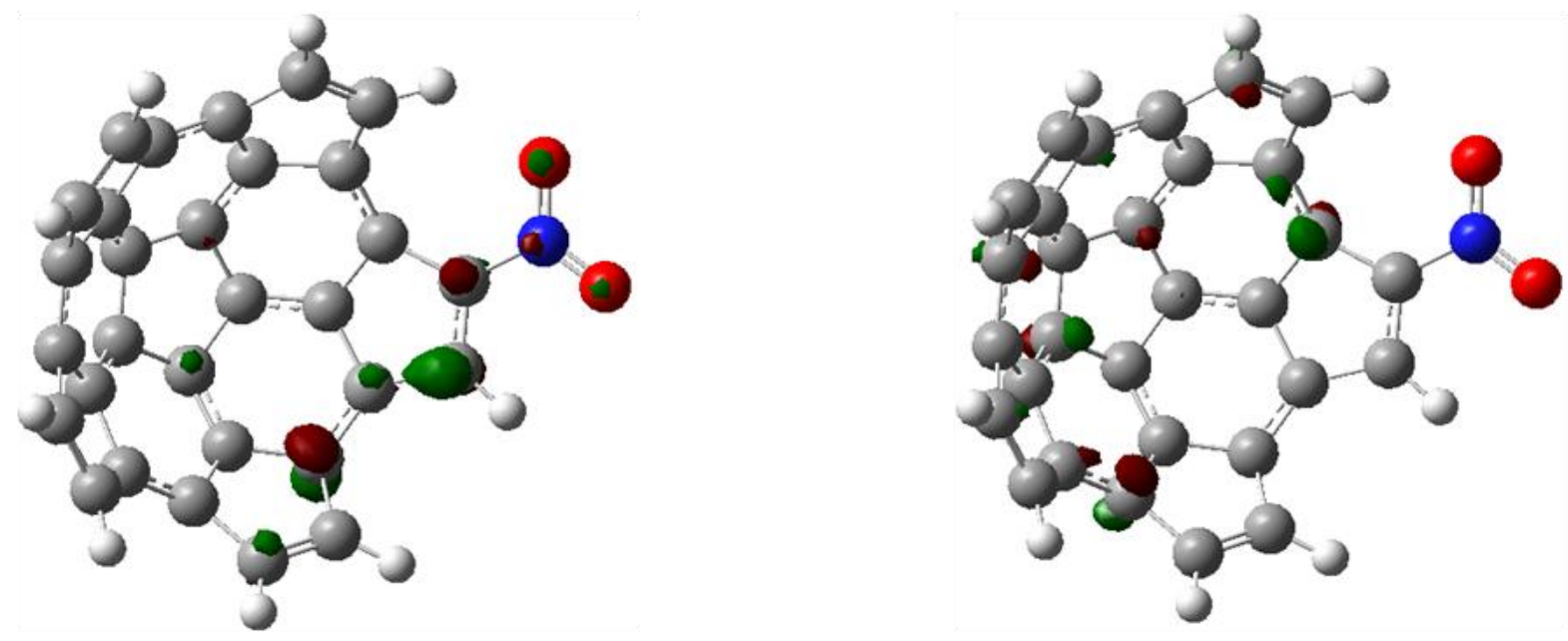

LUMO

HOMO

d (0.08ua)

Fragmento 2 disustituido con $\mathrm{NO}_{2}$

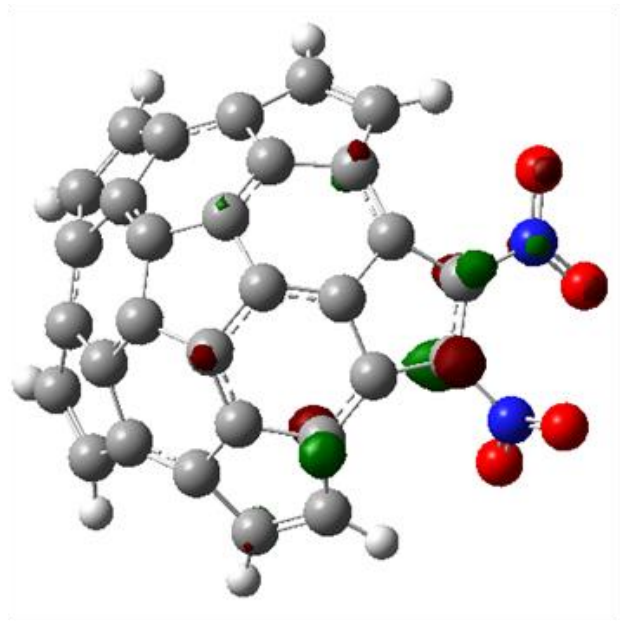

LUMO

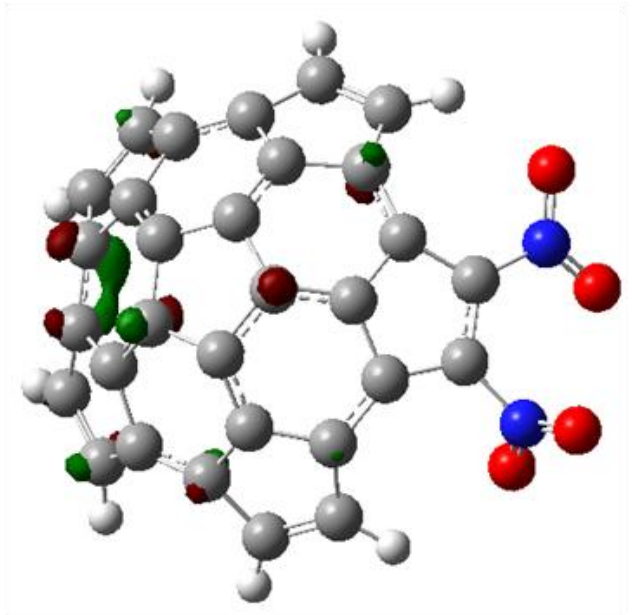

HOMO

d (0.08ua) 
Fragmento 2 monosustituido con $\mathrm{OH}$

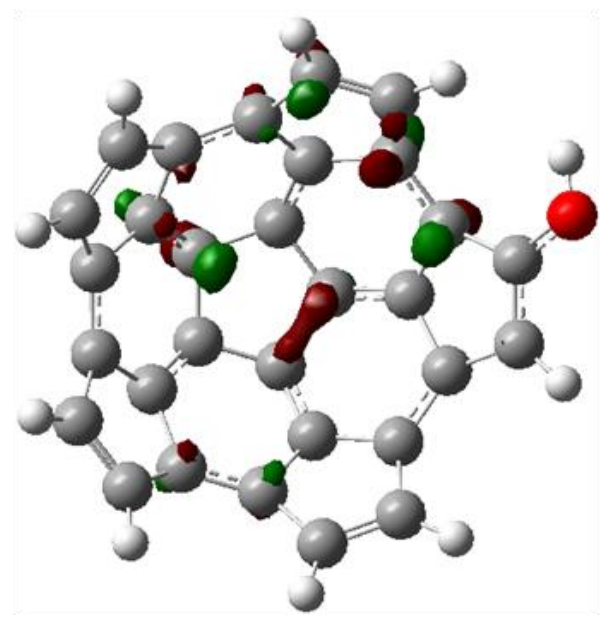

LUMO

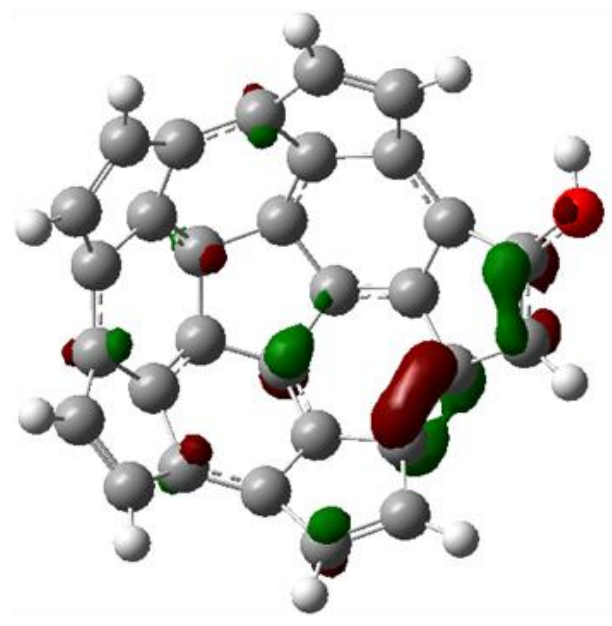

HOMO

d (0.07ua)

Fragmento 2 disustituido con $\mathrm{OH}$

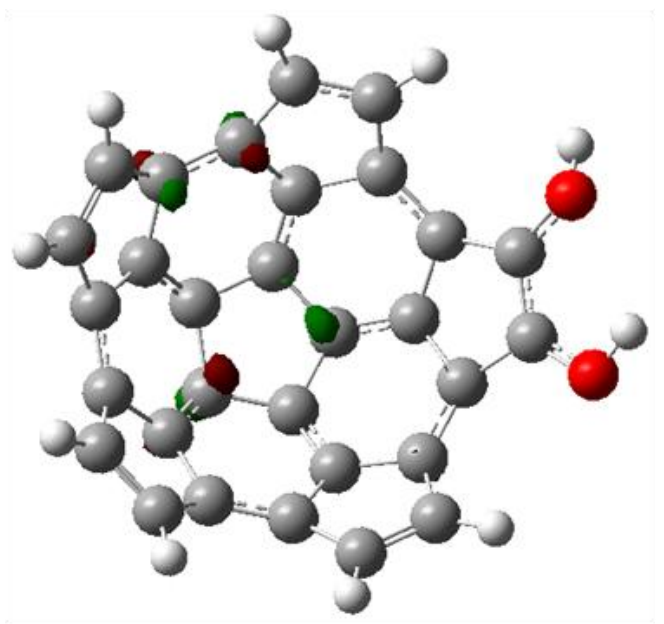

LUMO

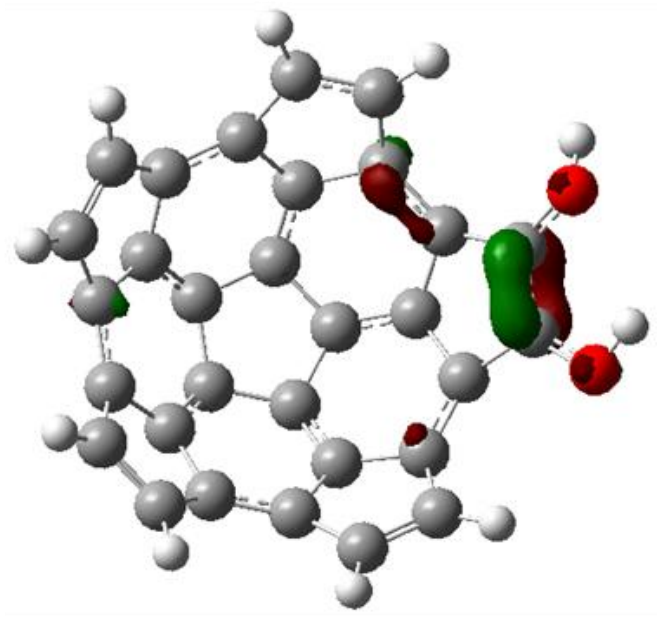

HOMO

d (0.08ua) 
Fragmento 2 monosustituido con OMe

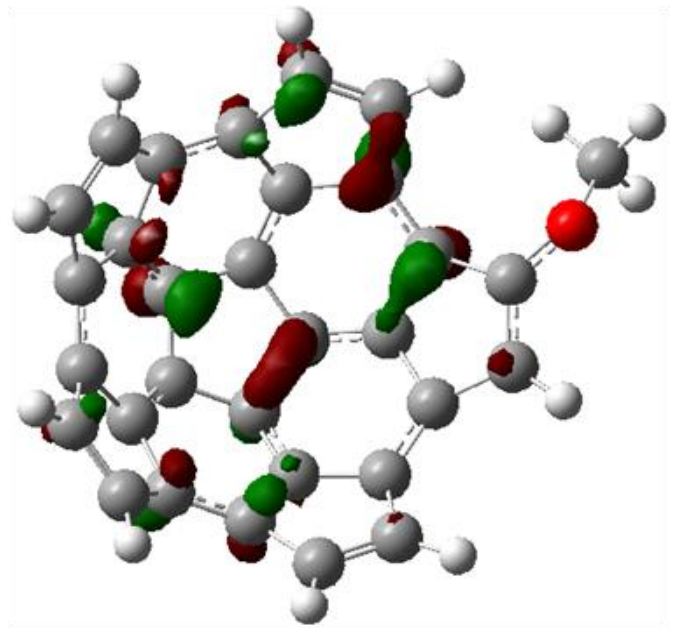

LUMO

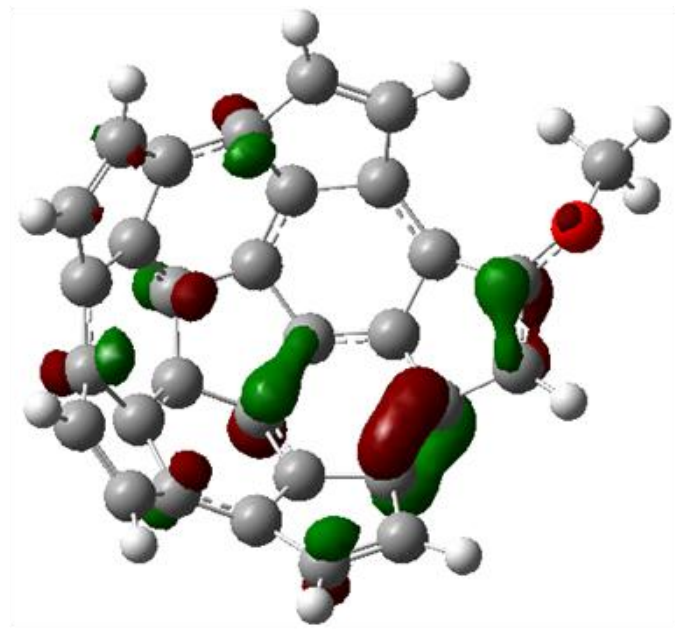

HOMO

$d$ (0.06ua)

Fragmento 2 disustituido con OMe

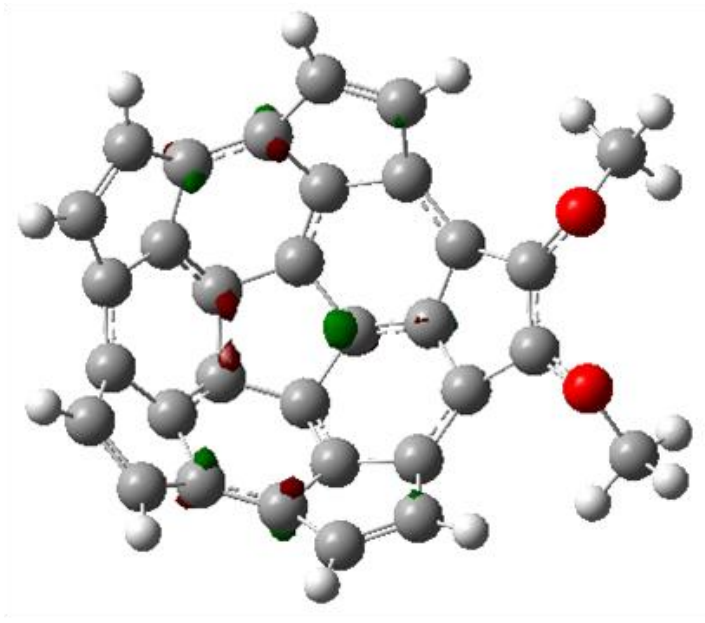

LUMO

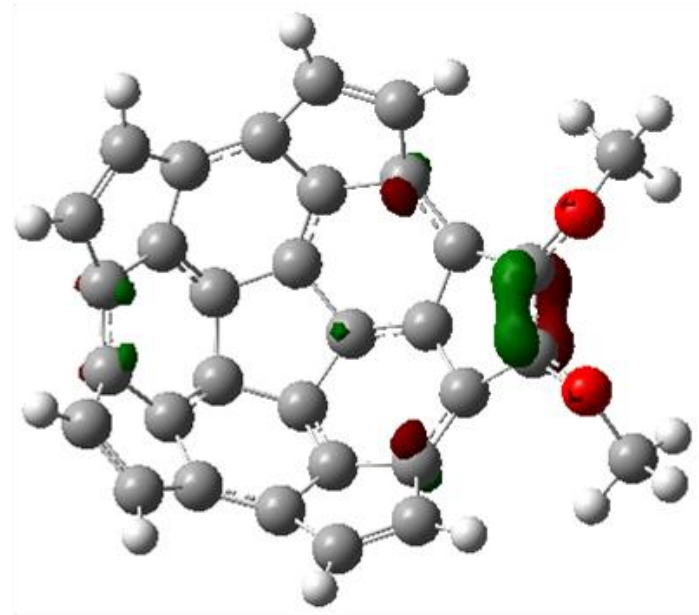

HOMO

d (0.08ua) 


\section{Densidades orbitálicas}

Funciones de Fukui

Densidades de los orbitales HOMO $\rho_{N}^{\text {HOMO }}(r)$

Densidades de los orbitales LUMO $\rho_{N}^{L U M O}(r)$

Funciones de Fukui para ataques electrofílicos $f^{-}(r)$

Funciones de Fukui para ataques nucleofílicos $f^{+}(r)$

Descriptor dual $\Delta f(r)$

Código de colores: Las densidades orbitálicas se obtuvieron elevando al cuadrado los orbitales HOMO y LUMO presentados en el anexo anterior, al ser siempre positivos se muestran en un solo color, rojo. Las contribuciones positivas y negativas de las funciones de Fukui se muestran en morado y azul respectivamente. En el caso del descriptor dual, las áreas amarillas son las más propensas a ataques por nucleófilos, es decir presentan carácter aceptor; mientras que las áreas verdes son más propensas a ataques por electrófilos, es decir presentan carácter donador. 


\section{Butadieno}
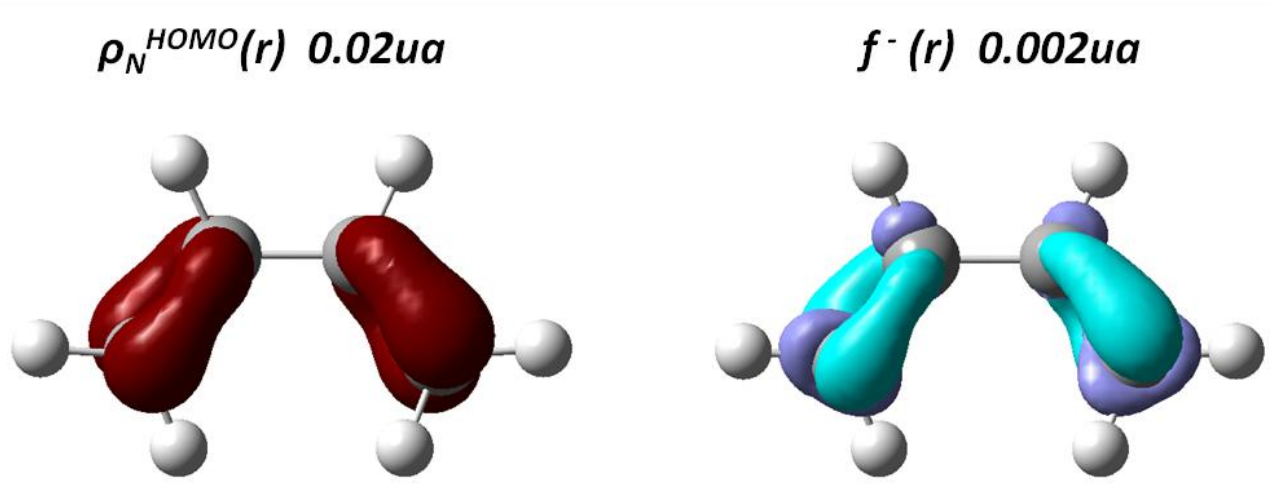

$\rho_{N}{ }^{L U M O}(r) 0.02 u a$

$f^{+}(r) 0.002 u a$
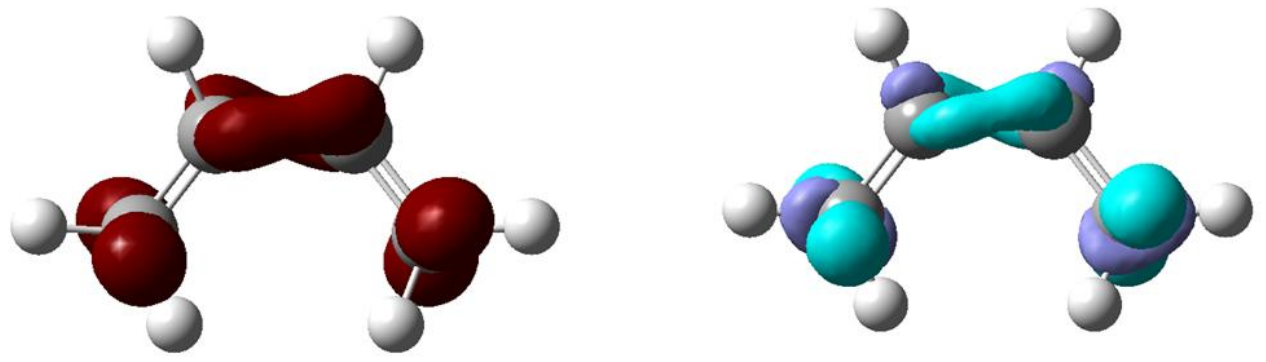

$\Delta f(r) 0.01 u a$

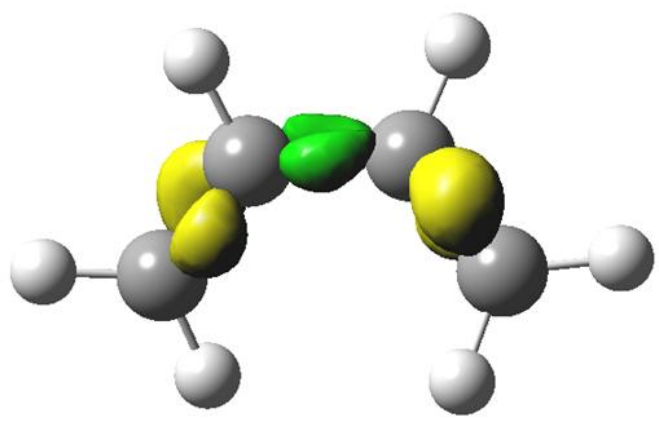




\section{Etileno}

$$
\rho_{N}{ }^{\mathrm{HOMO} O}(r) 0.02 u a
$$

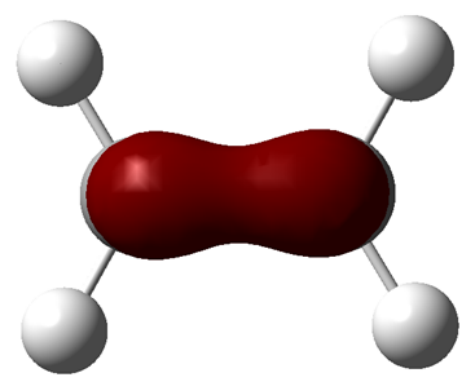

$\rho_{N}{ }^{\mathrm{LUMO}}(r) 0.02 \mathrm{ua}$

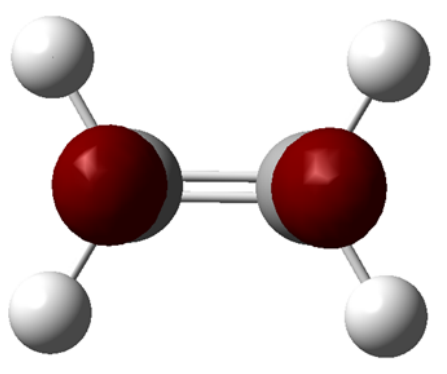

$$
f^{-} \text {(r) 0.002ua }
$$

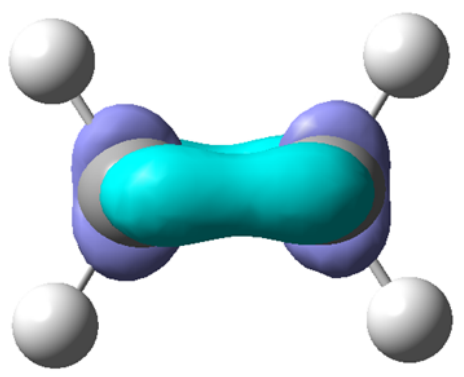

$f^{+}(r) 0.002 u a$

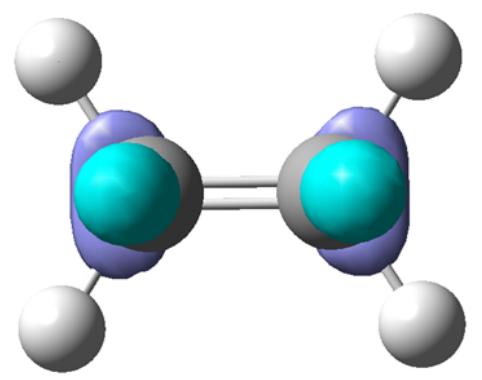

\section{$\Delta f(r) 0.01 u a$}

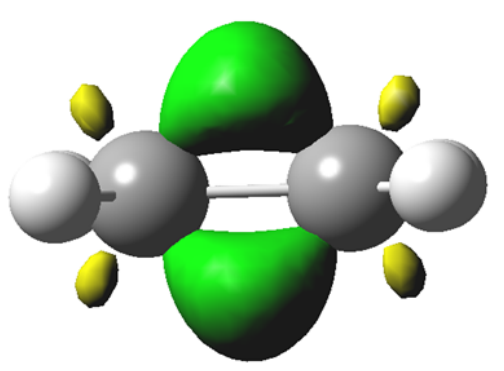




\section{Fragmento 1}
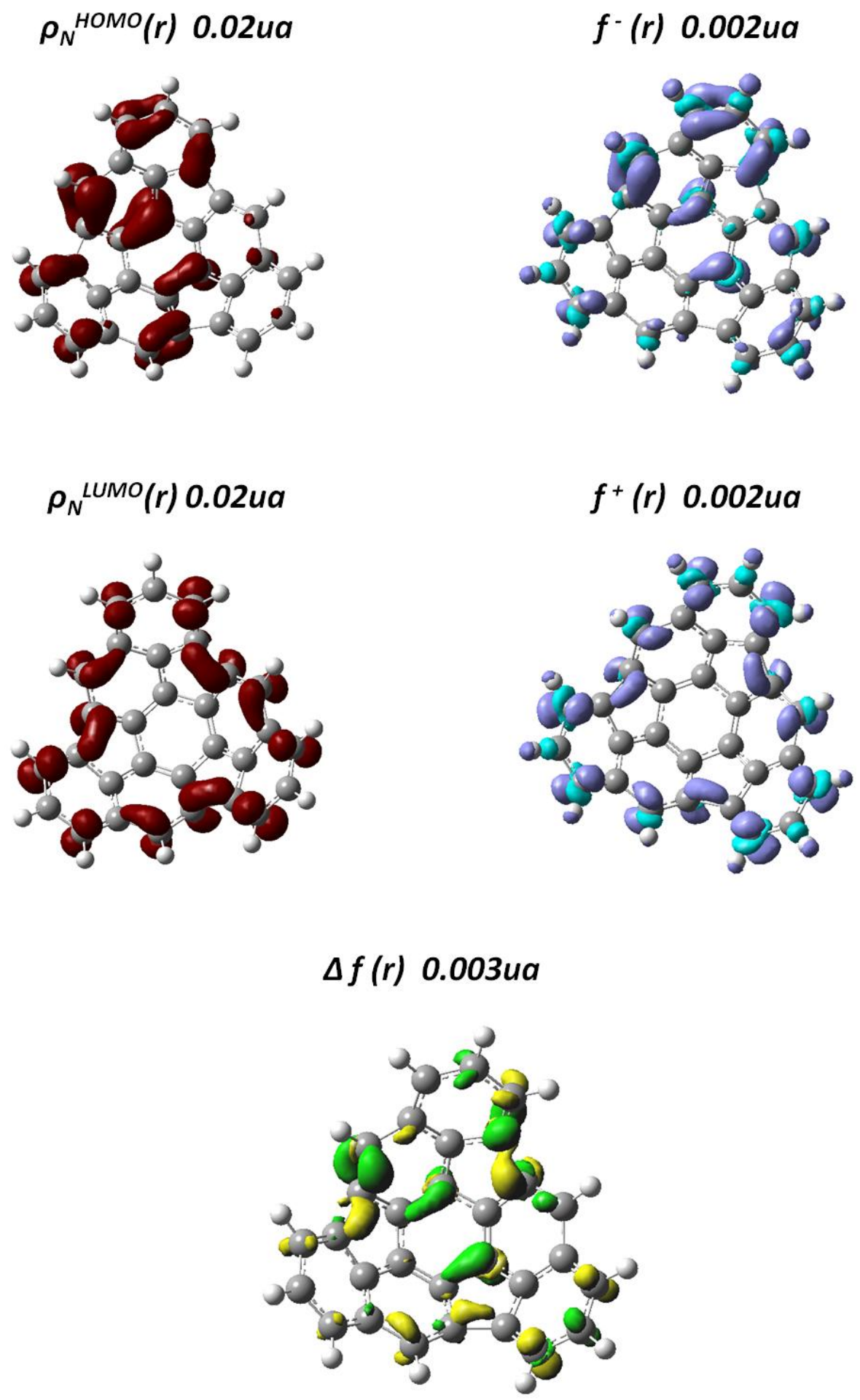


\section{Fragmento 2}
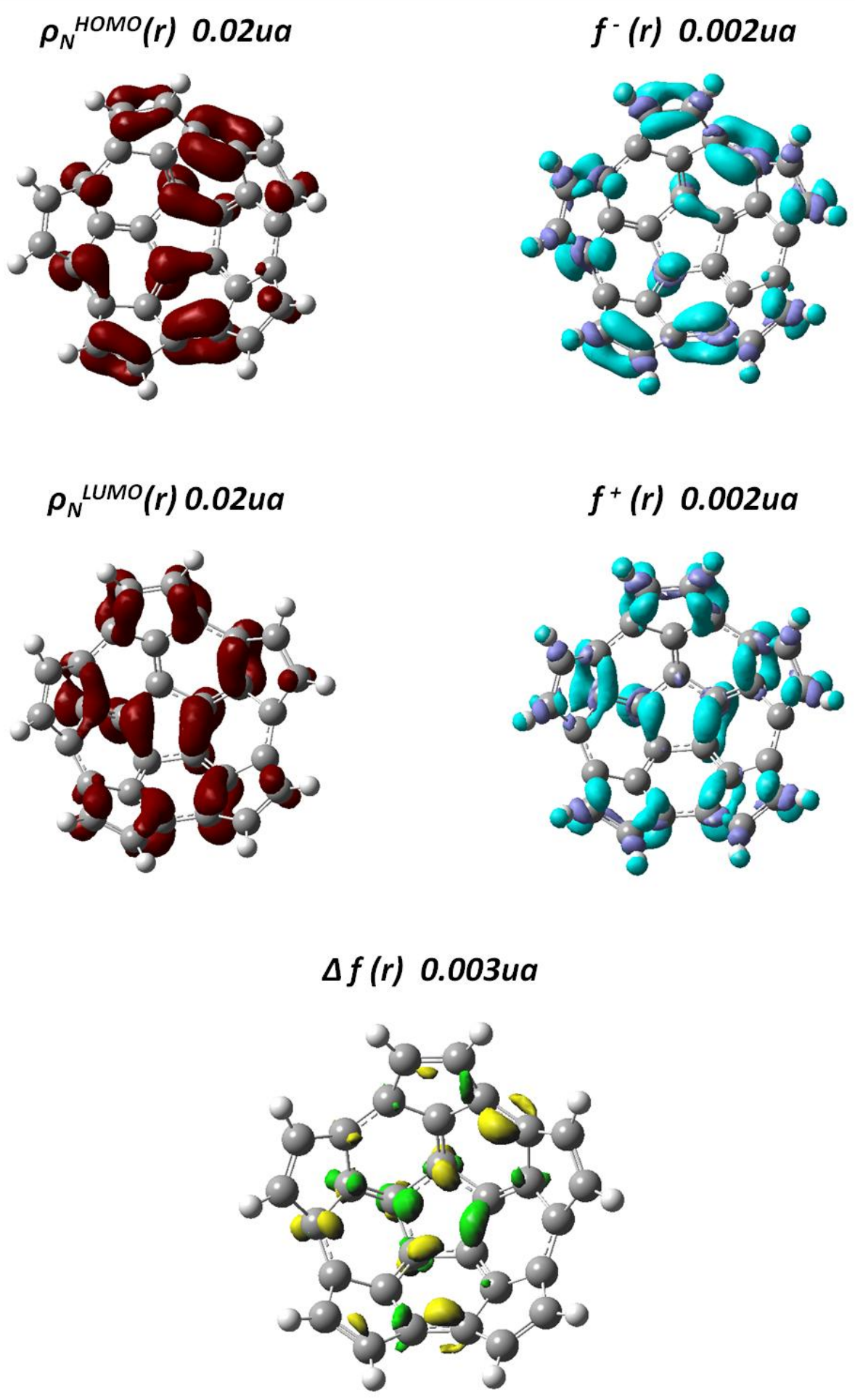
Fragmento 1 mono-sustituido con $\mathrm{CF}_{3}$
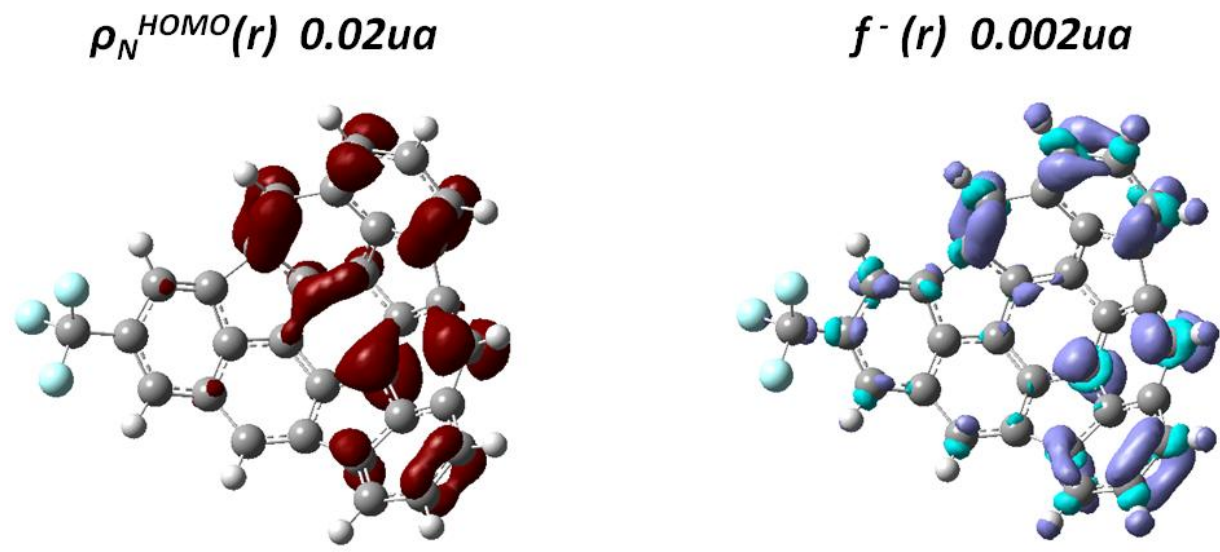

$$
\rho_{N}{ }^{L U M O}(r) 0.02 u a
$$

$$
f^{+}(r) 0.002 u a
$$
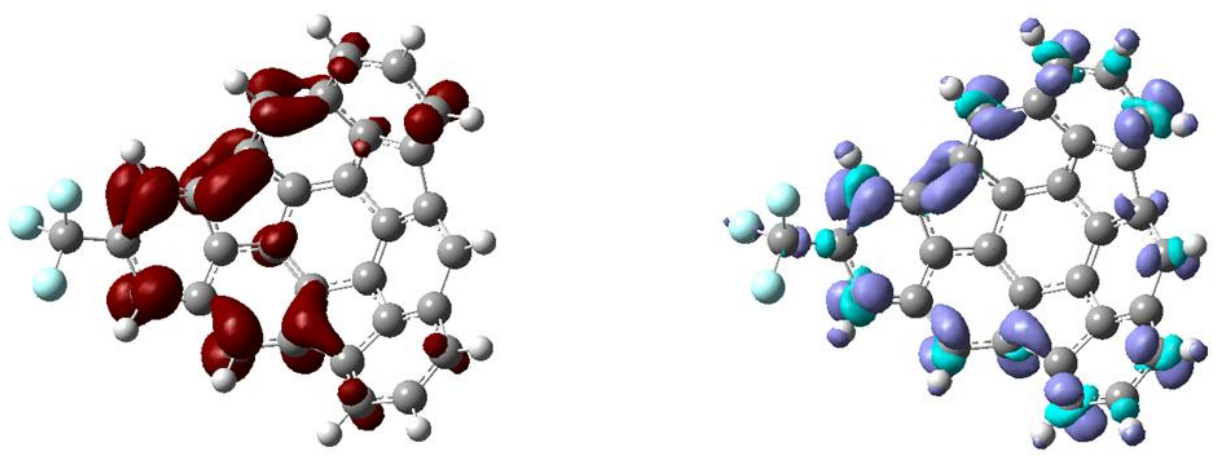

$\Delta f(r) 0.003 u a$

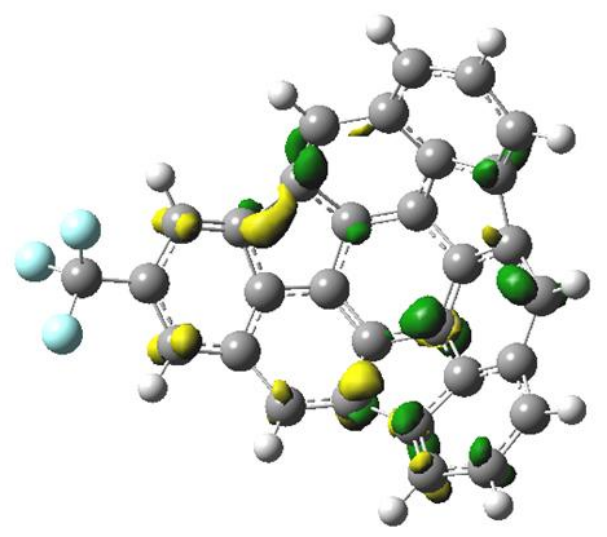


Fragmento 1 mono-sustituido con $\mathrm{CHO}$
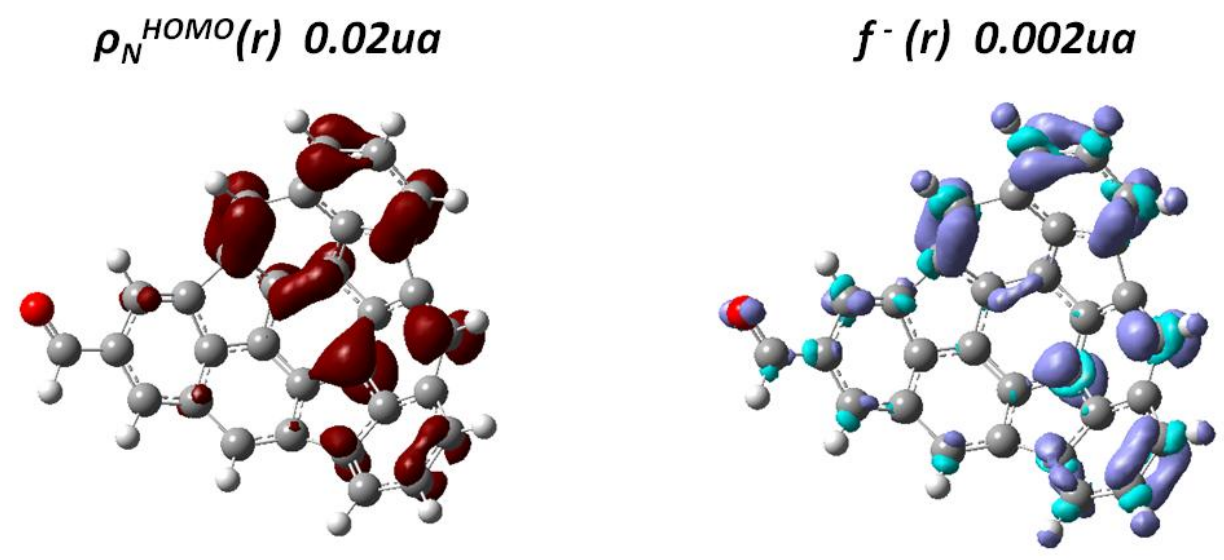

$\rho_{N}{ }^{\mathrm{LUMO}}(r) 0.02 \mathrm{ua}$

$$
f^{+}(r) 0.002 u a
$$
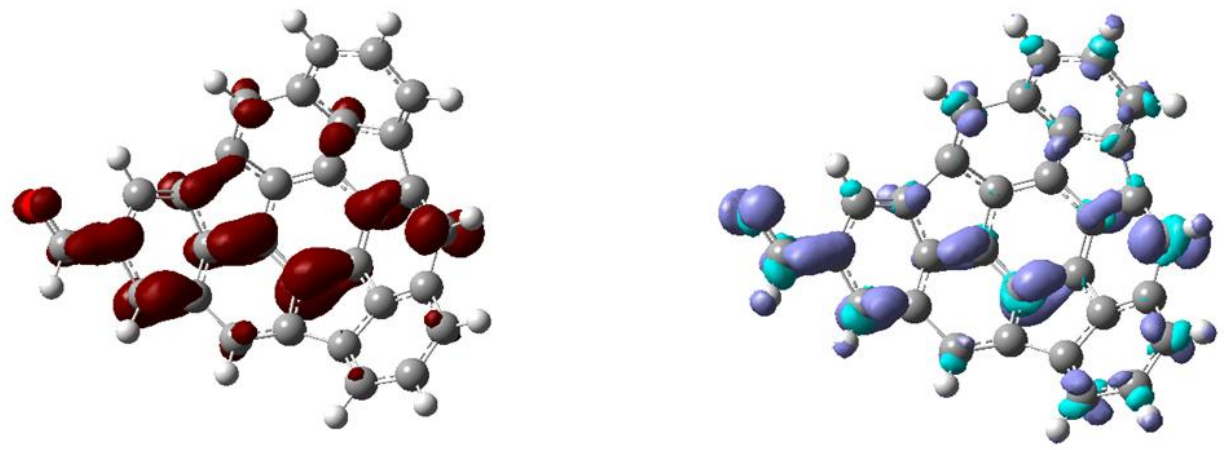

$\Delta f(r) 0.003 u a$

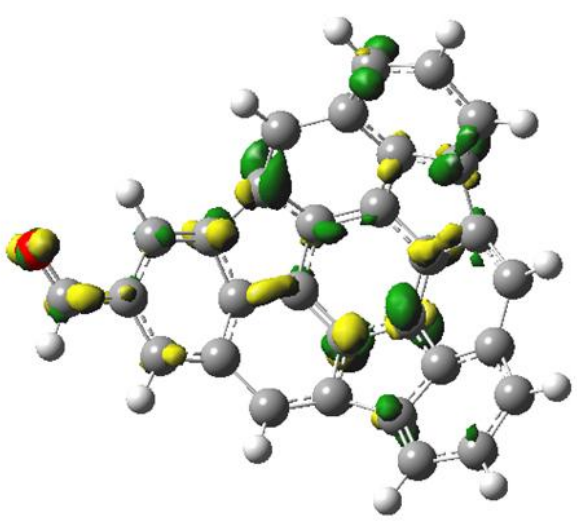




\section{Fragmento 1 mono-sustituido con $\mathrm{CN}$}
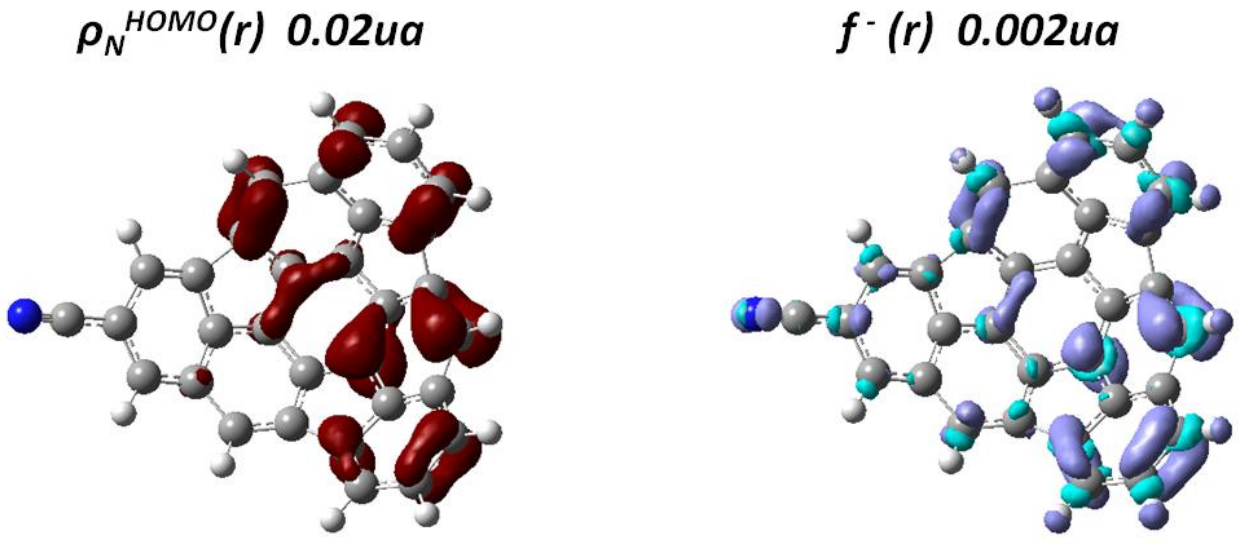

$\rho_{N}{ }^{L U M O}(r) 0.02 u a$

$f^{+}(r) 0.002 u a$
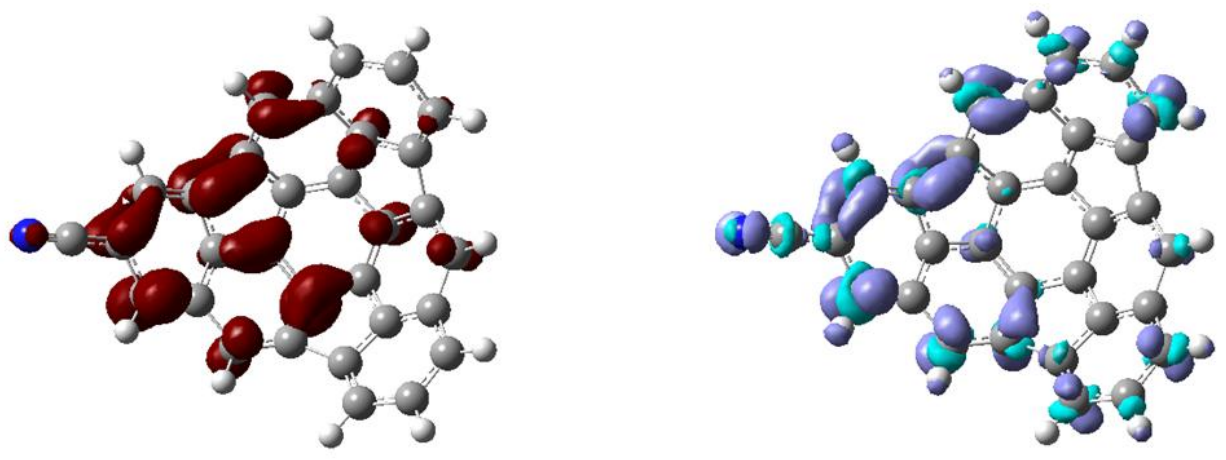

$\Delta f(r) 0.003 u a$

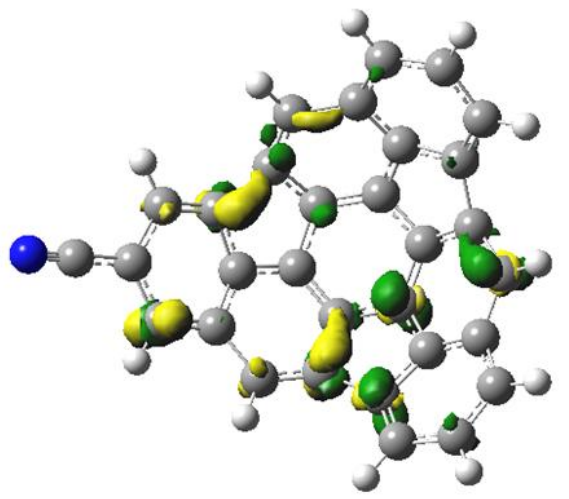




\section{Fragmento 1 mono-sustituido con $\mathrm{COOH}$}
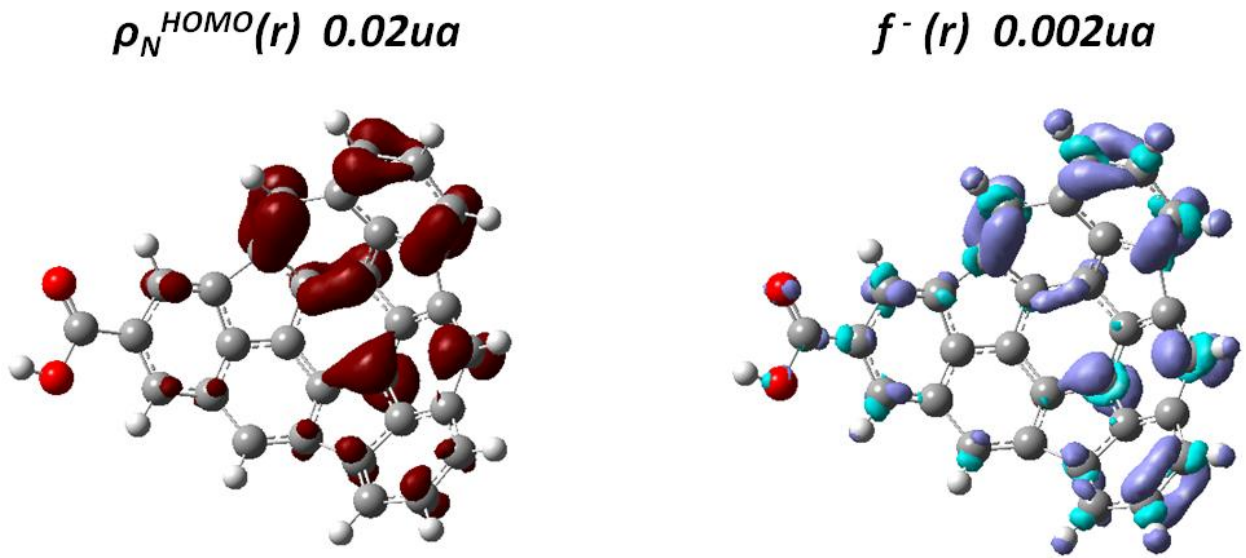

$\rho_{N}{ }^{L U M O}(r) 0.02 u a$

$$
f^{+}(r) 0.002 u a
$$
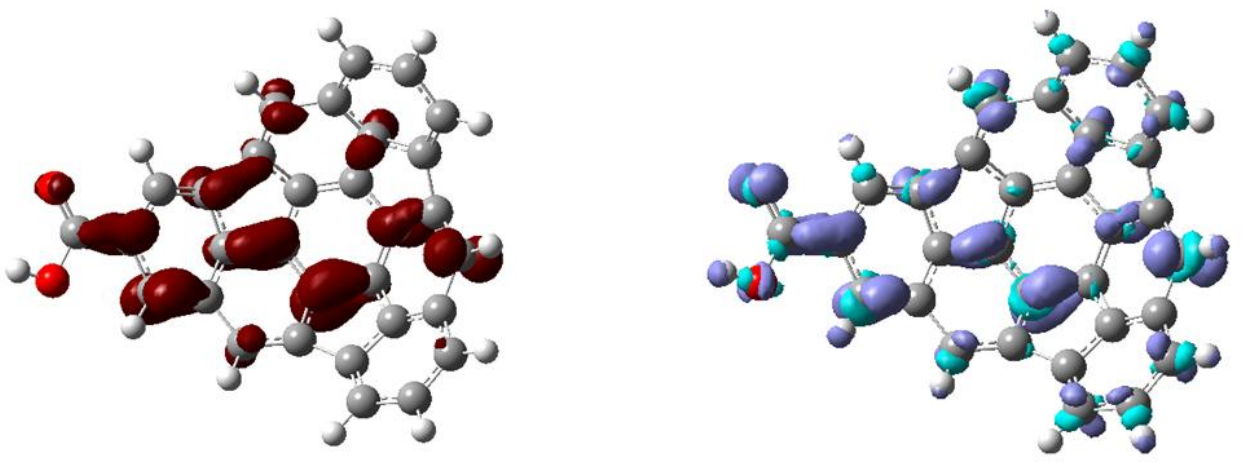

$\Delta f(r) 0.003 u a$

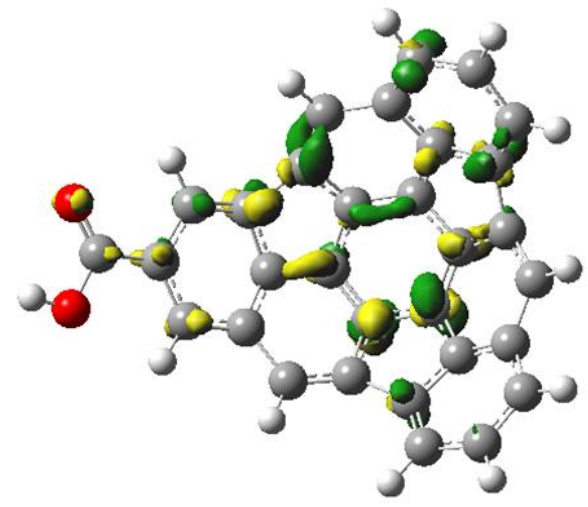


Fragmento 1 mono-sustituido con $\mathrm{F}$

$$
\rho_{N}{ }^{\mathrm{HOMO}}(r) 0.02 u a
$$

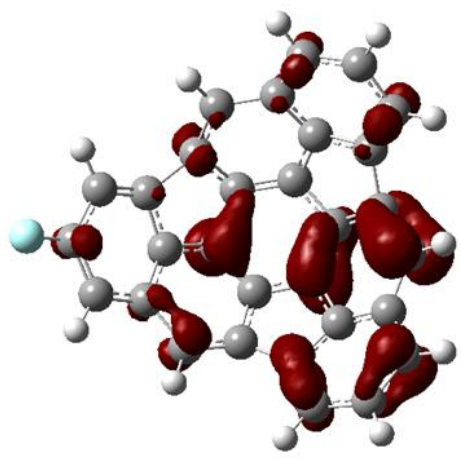

$\rho_{N}{ }^{\mathrm{LUMO}}(r) 0.02 \mathrm{ua}$

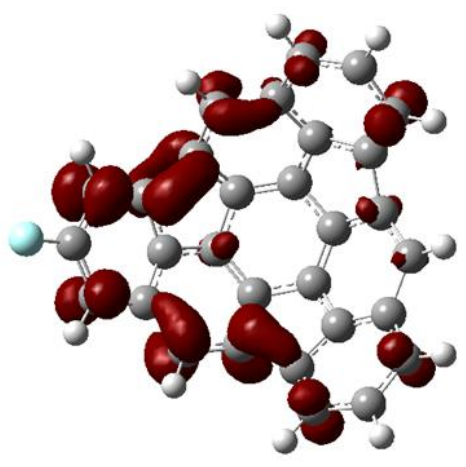

$f^{-}(r) 0.002 u a$

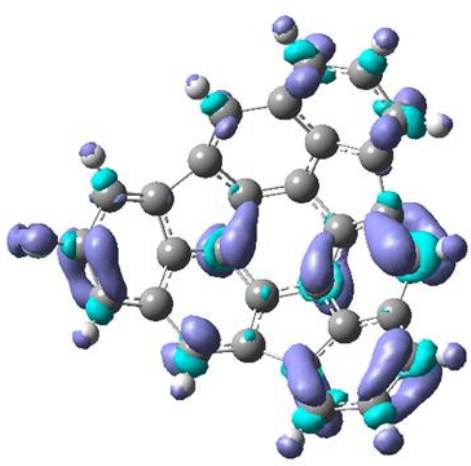

$f^{+}(r)$ 0.002ua

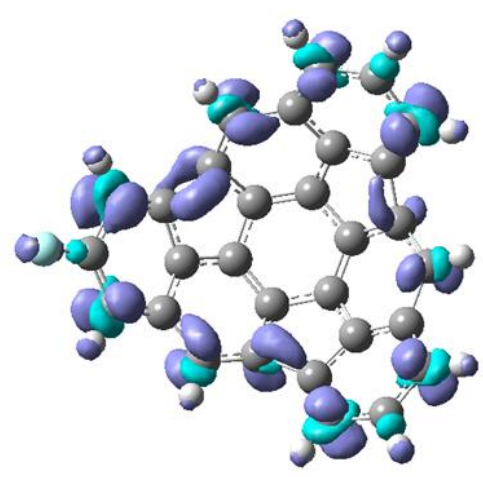

$\Delta f(r) 0.003 u a$

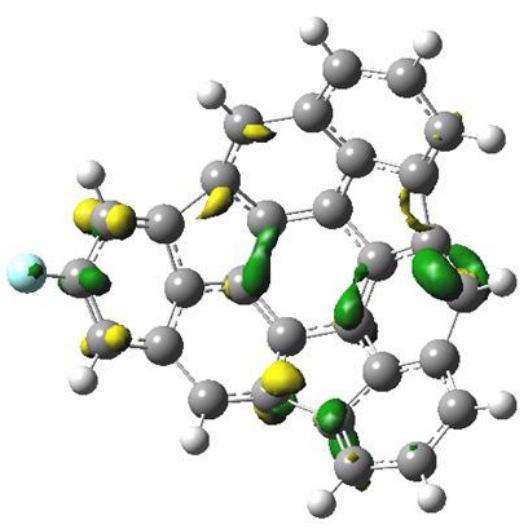


Fragmento 1 mono-sustituido con $i-\operatorname{Pr}$

$$
\rho_{N}{ }^{\text {HOMO }}(r) 0.02 u a
$$

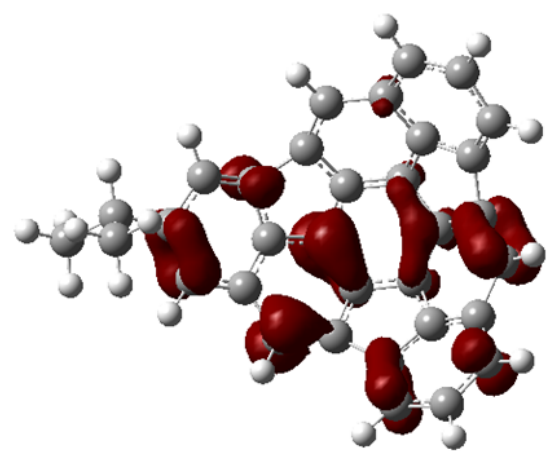

$\rho_{N}{ }^{\mathrm{LUMO}}(r) 0.02 \mathrm{ua}$

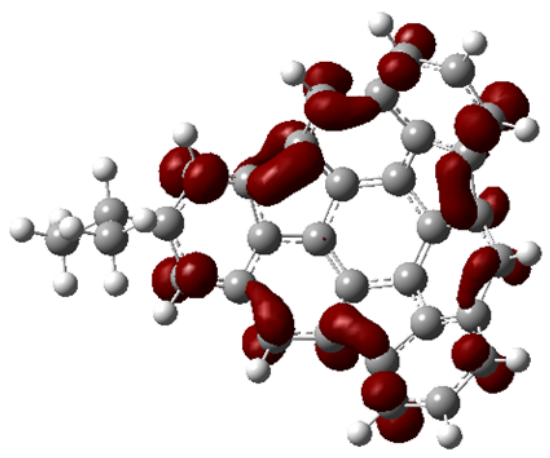

$$
f^{-} \text {(r) 0.002ua }
$$

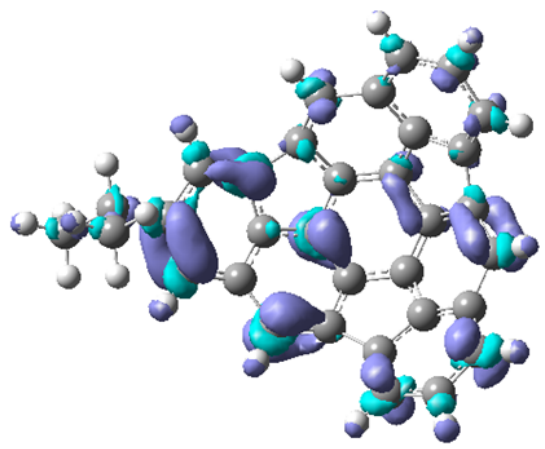

$f^{+}(r) 0.002 u a$

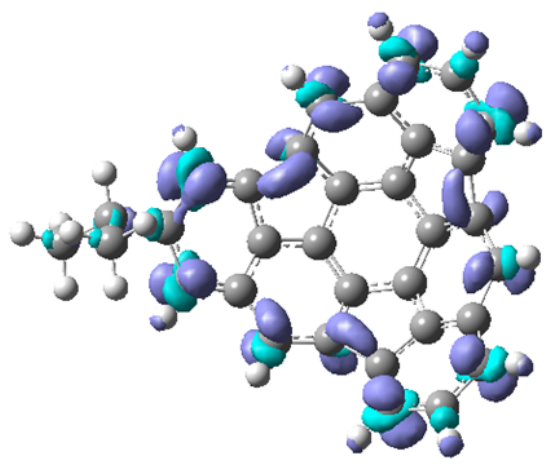

$\Delta f(r) 0.003 u a$

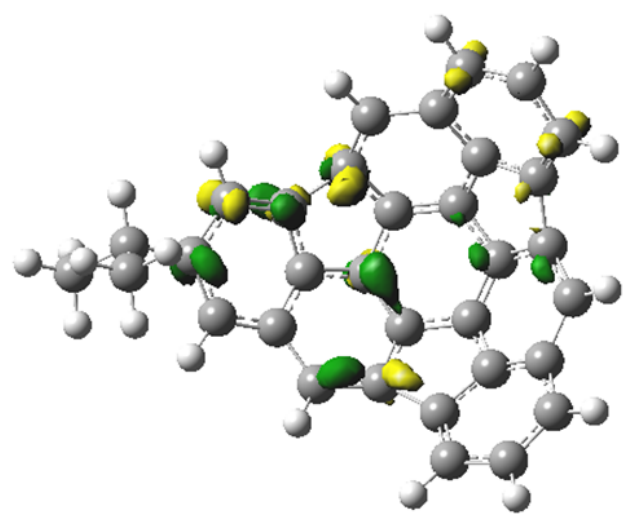




\section{Fragmento 1 mono-sustituido con Me}
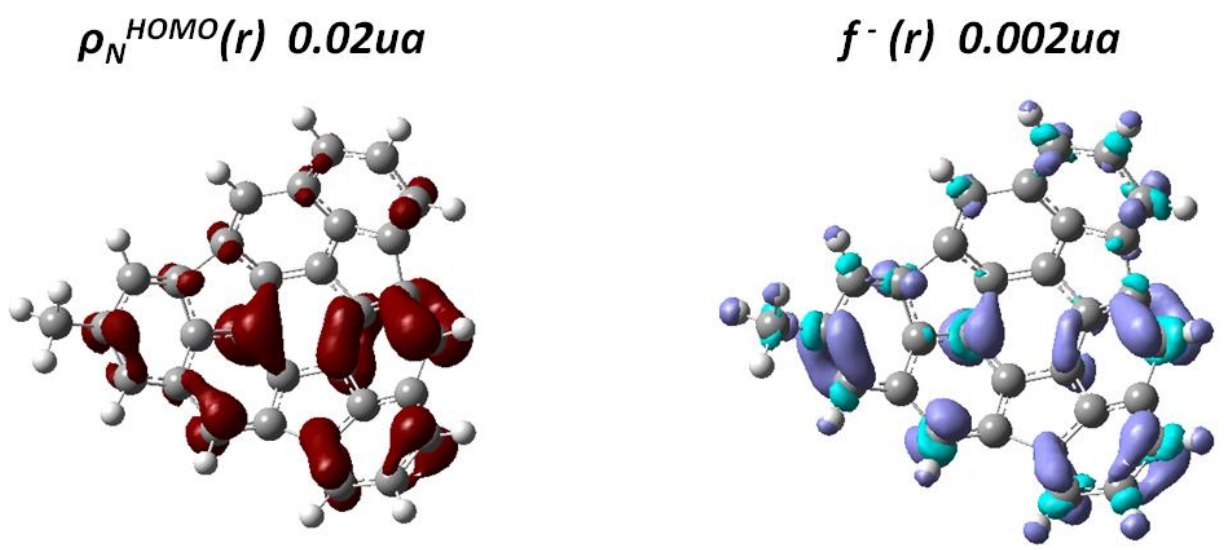

$$
\rho_{N}^{\text {LUMO }}(r) 0.02 u a
$$

$$
f^{+}(r) 0.002 u a
$$
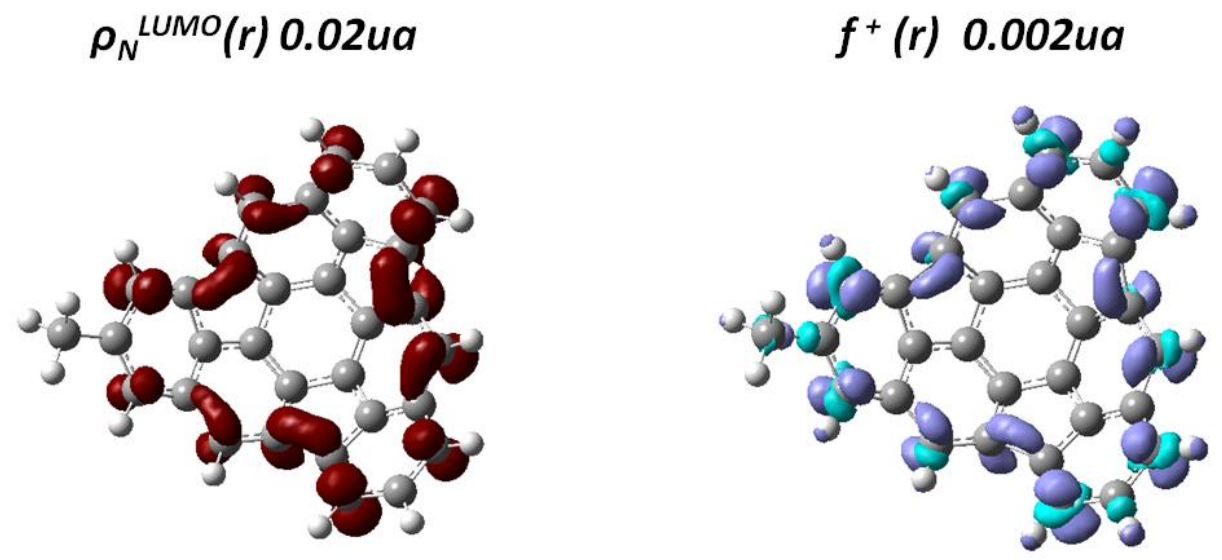

$\Delta f(r) 0.003 u a$

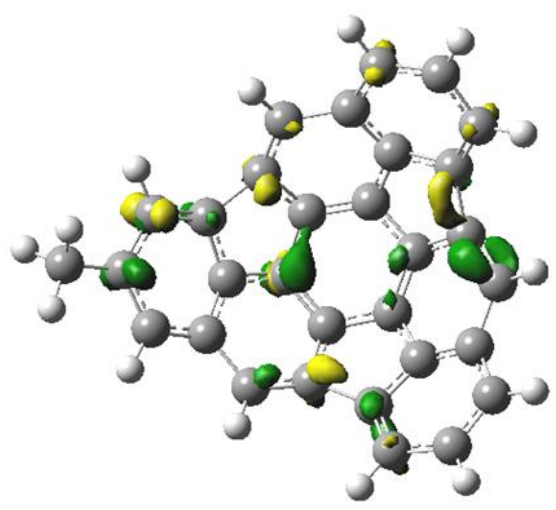


Fragmento 1 mono-sustituido con $\mathrm{NH}_{2}$
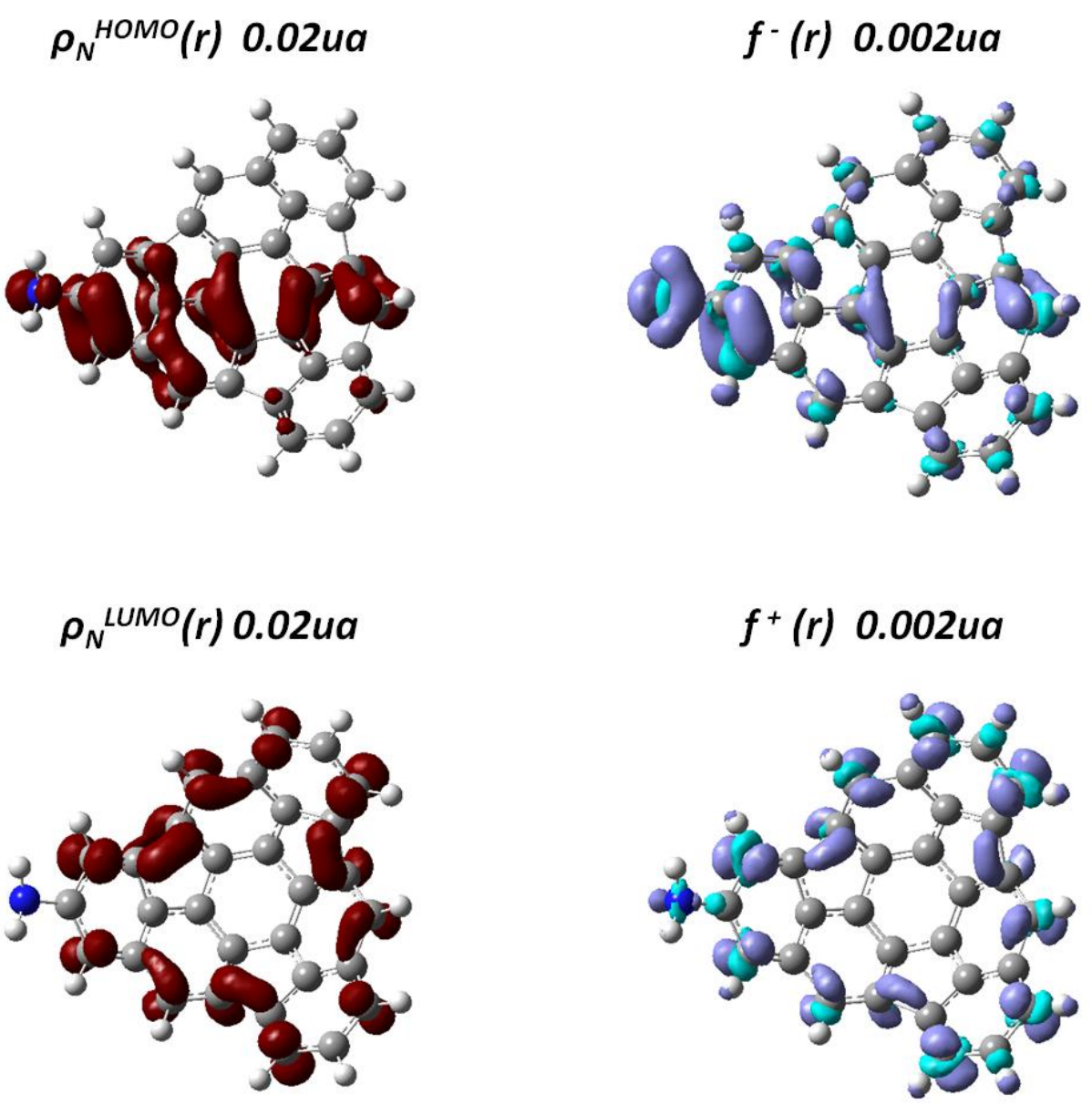

$\Delta f(r) 0.003 u a$

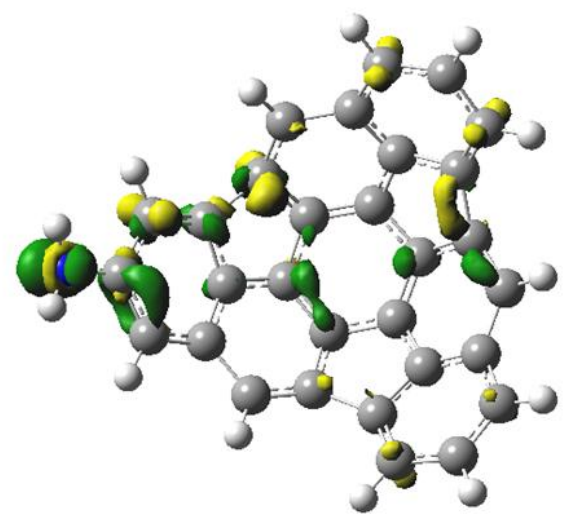


Fragmento 1 mono-sustituido con $\mathrm{NO}_{2}$
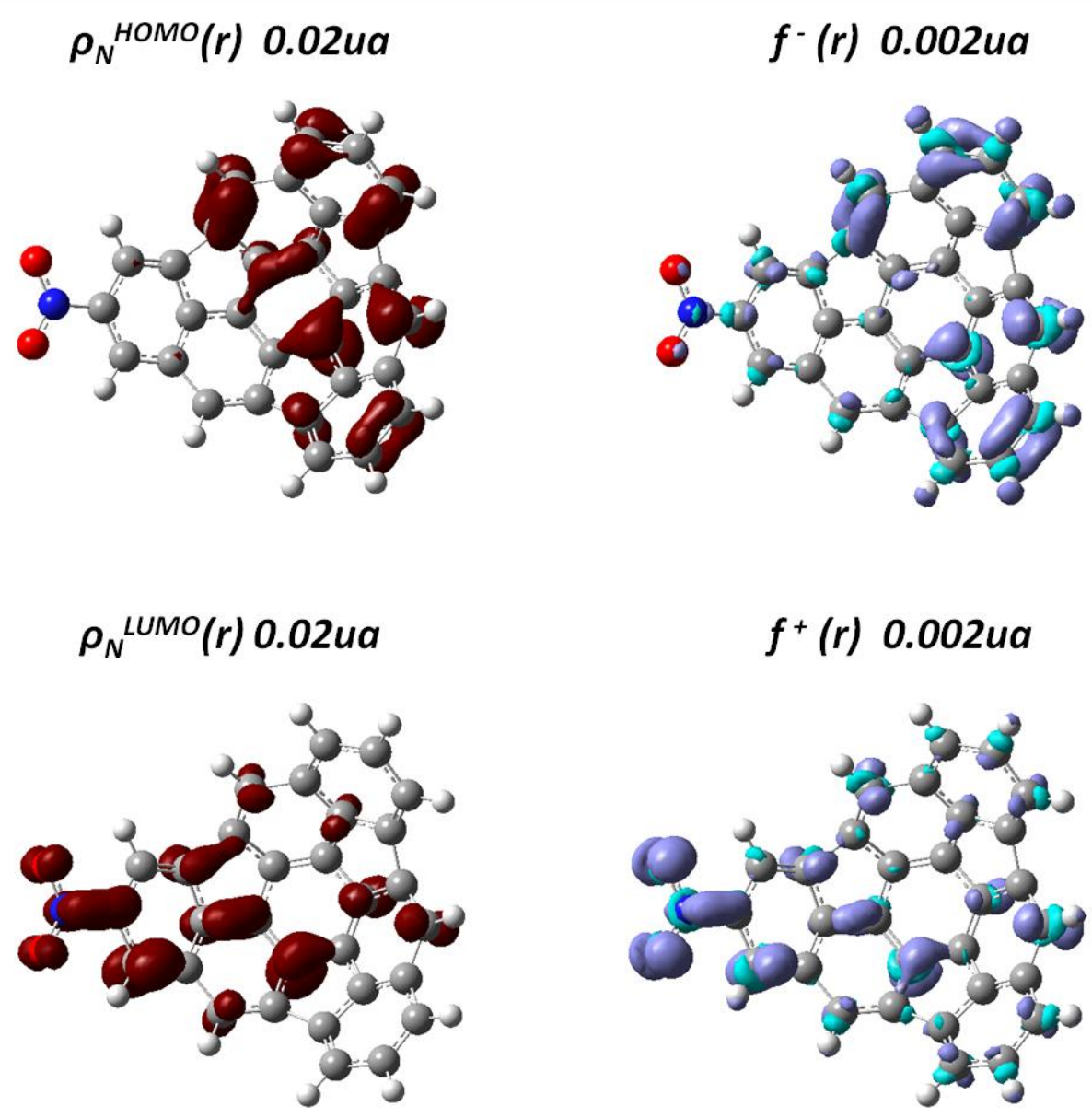

$\Delta f(r) 0.003 u a$

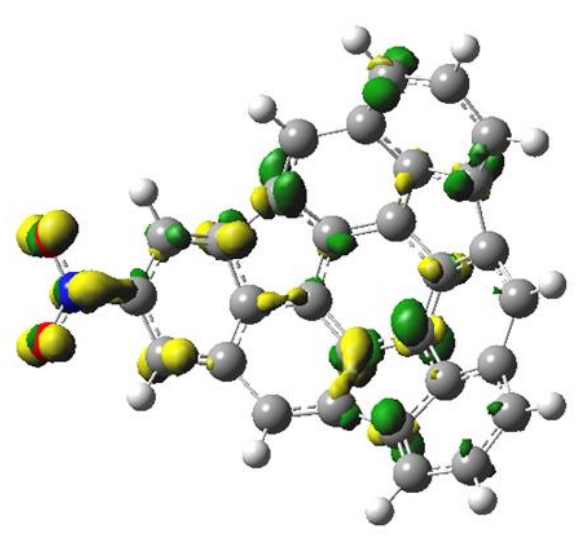


Fragmento 1 mono-sustituido con $\mathrm{OH}$
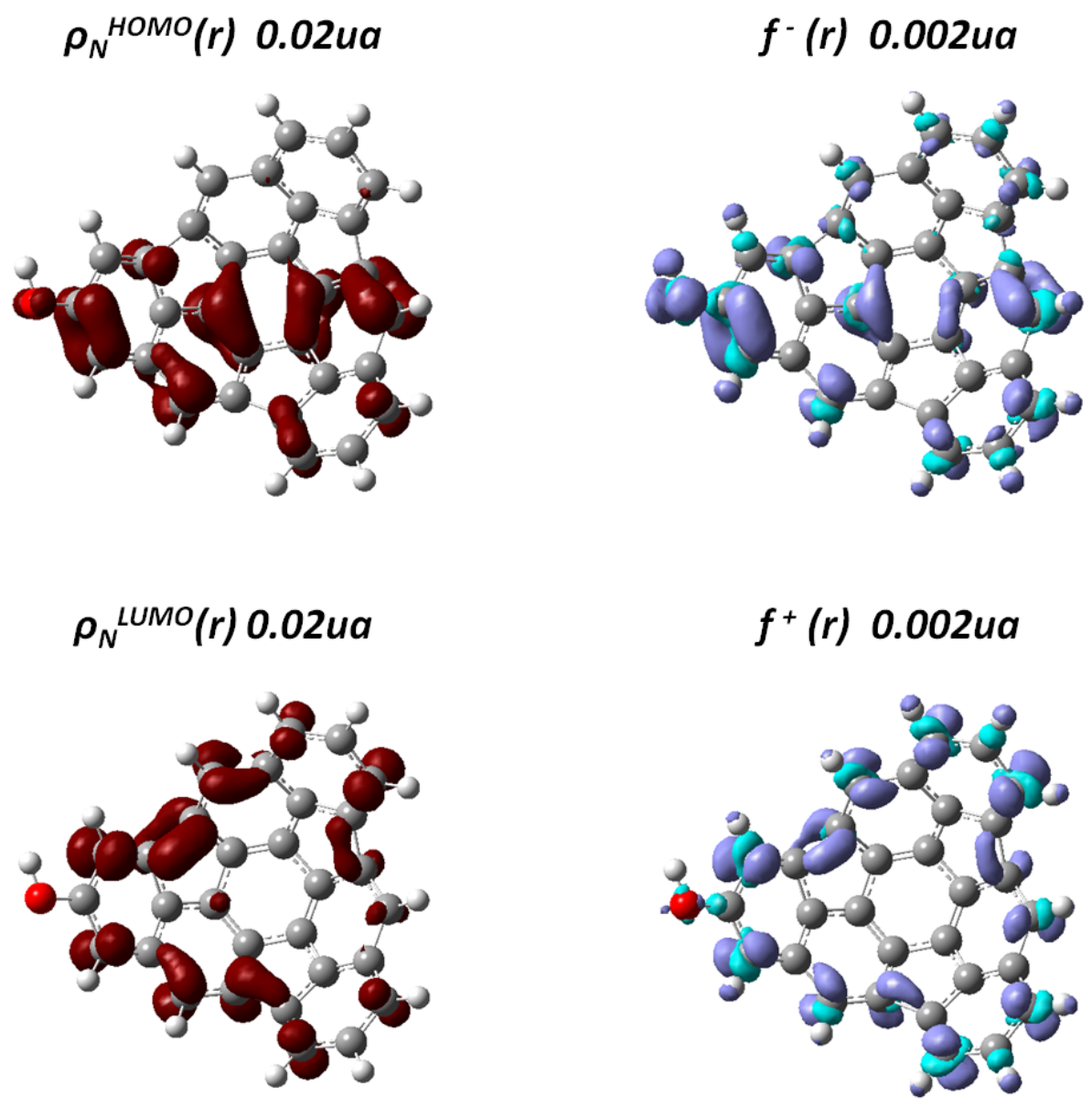

$\Delta f(r) 0.003 u a$

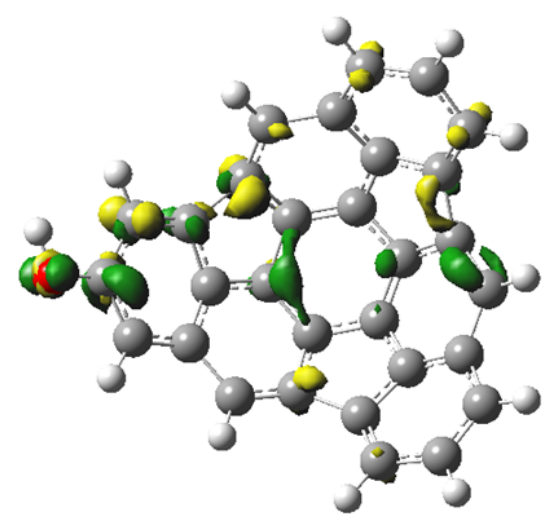


Fragmento 1 mono-sustituido con OMe
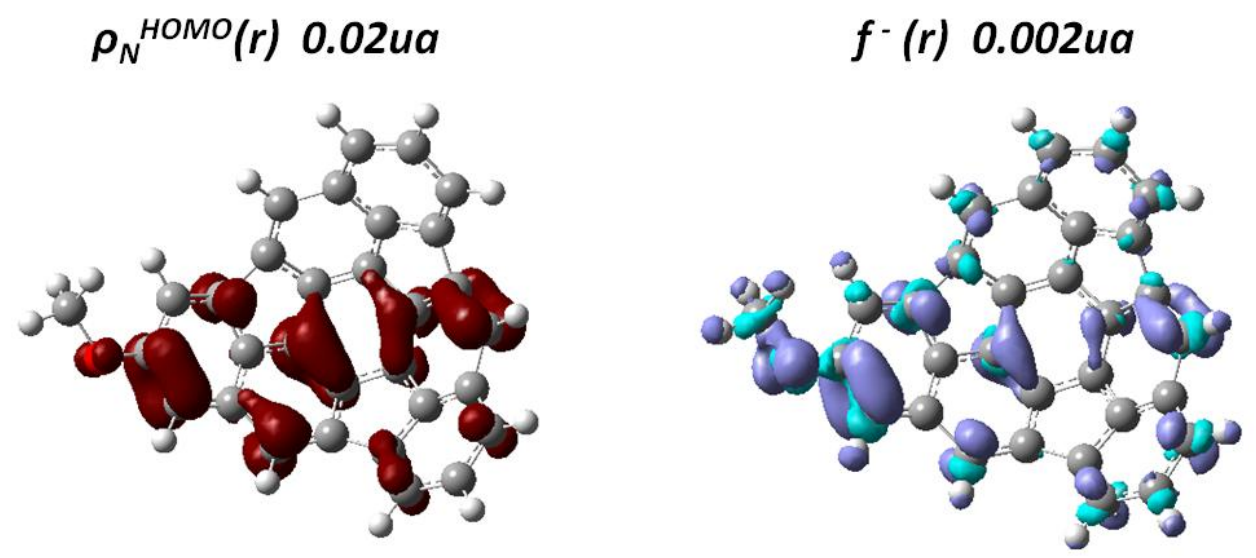

$\rho_{N}{ }^{L U M O}(r) 0.02 u a$
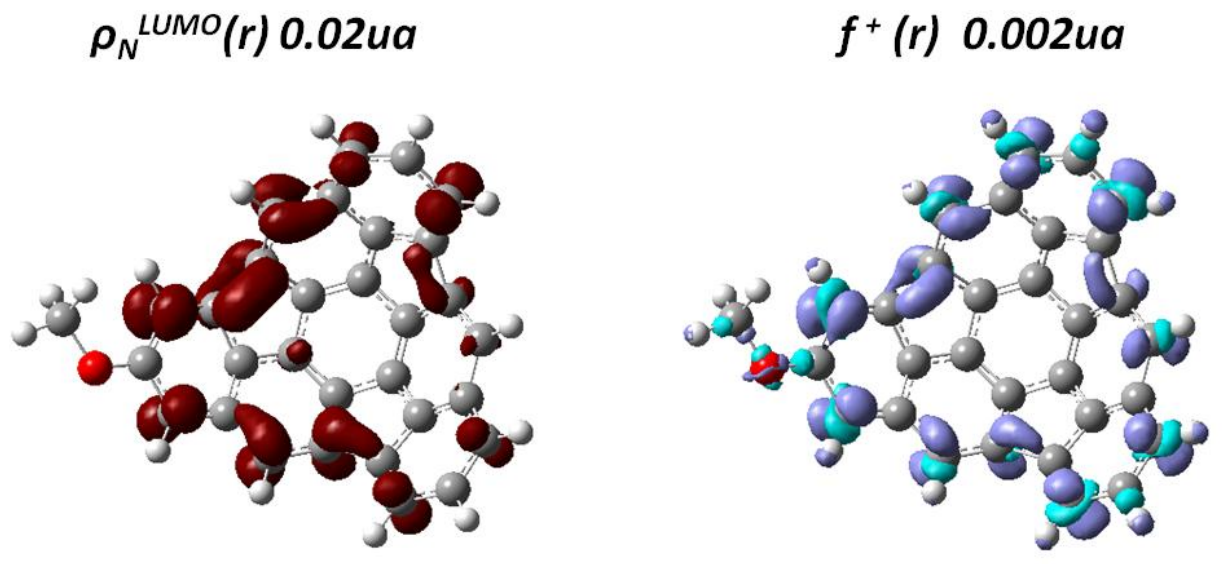

$\Delta f(r) 0.003 u a$

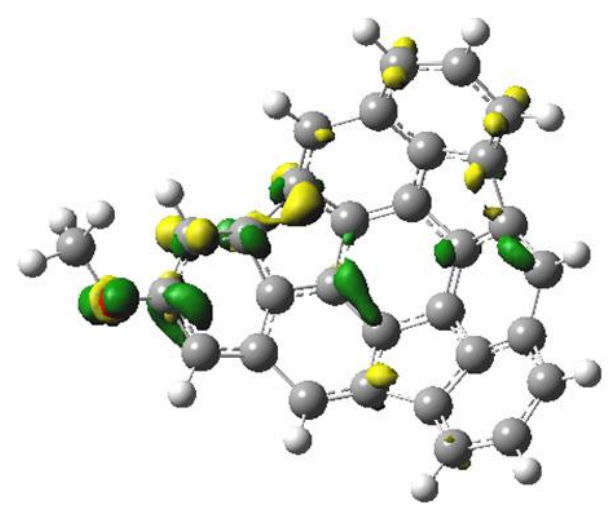


Fragmento 2 mono-sustituido con $\mathrm{CF}_{3}$
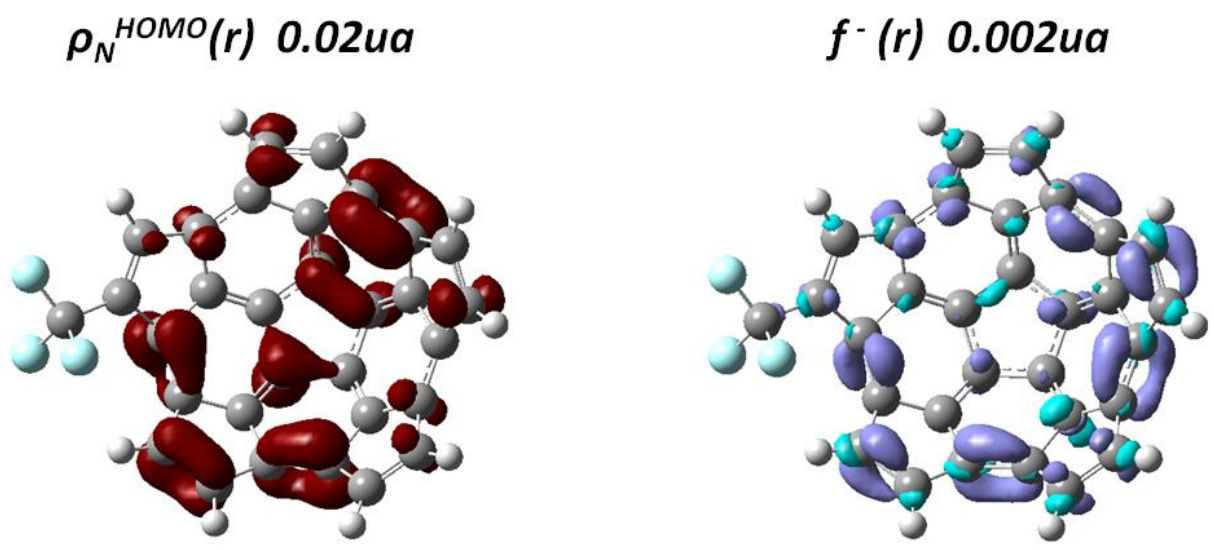

$\rho_{N}{ }^{\mathrm{LUMO}}(r) 0.02 \mathrm{ua}$
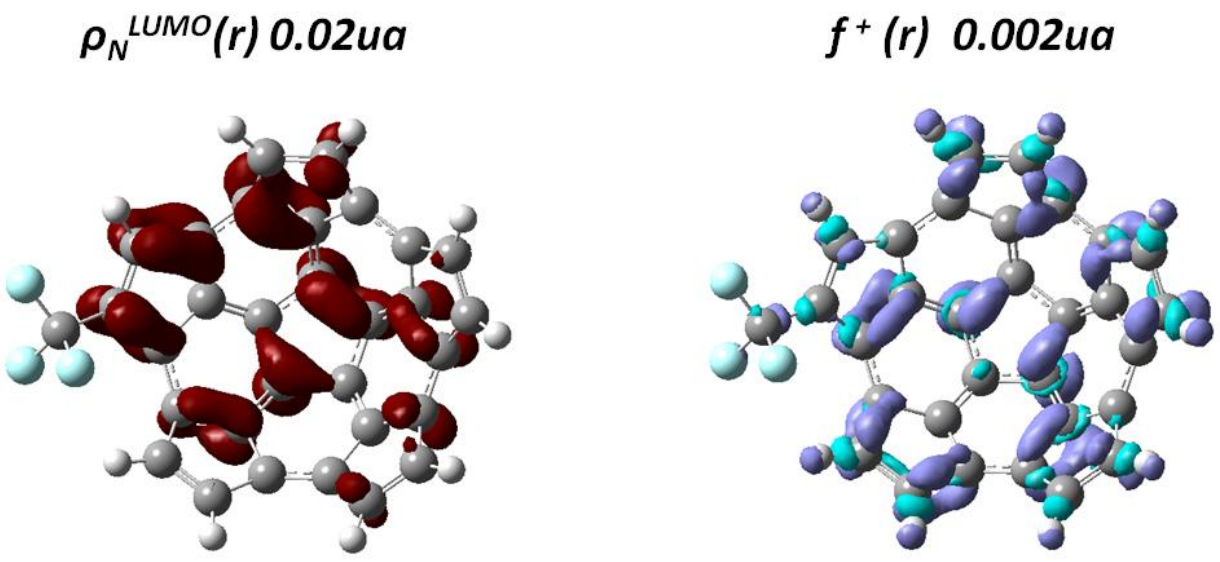

$\Delta f(r) 0.005 u a$

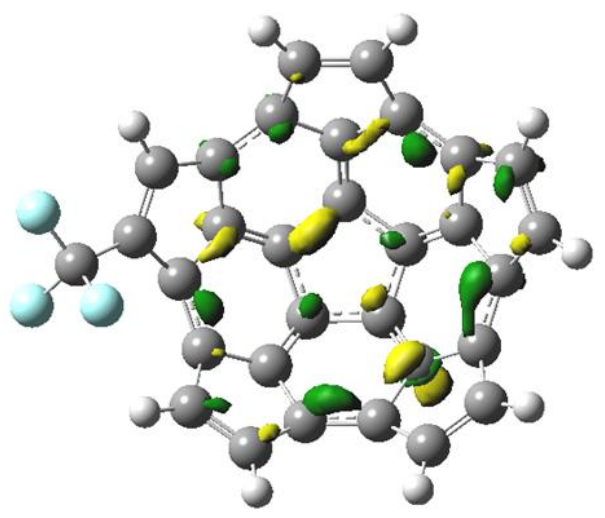


Fragmento 2 mono-sustituido con $\mathrm{CHO}$
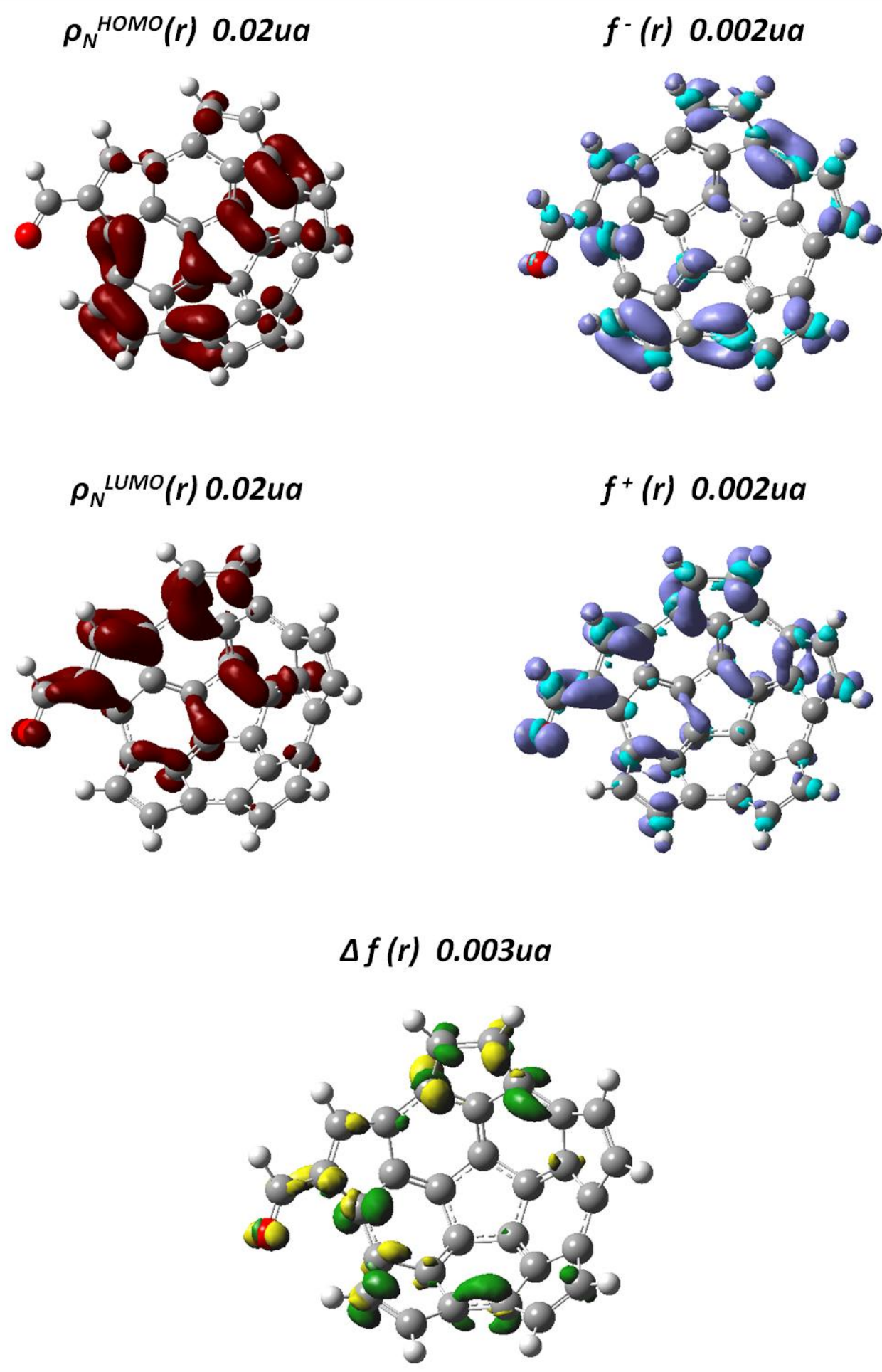
Fragmento 2 mono-sustituido con $\mathrm{CN}$
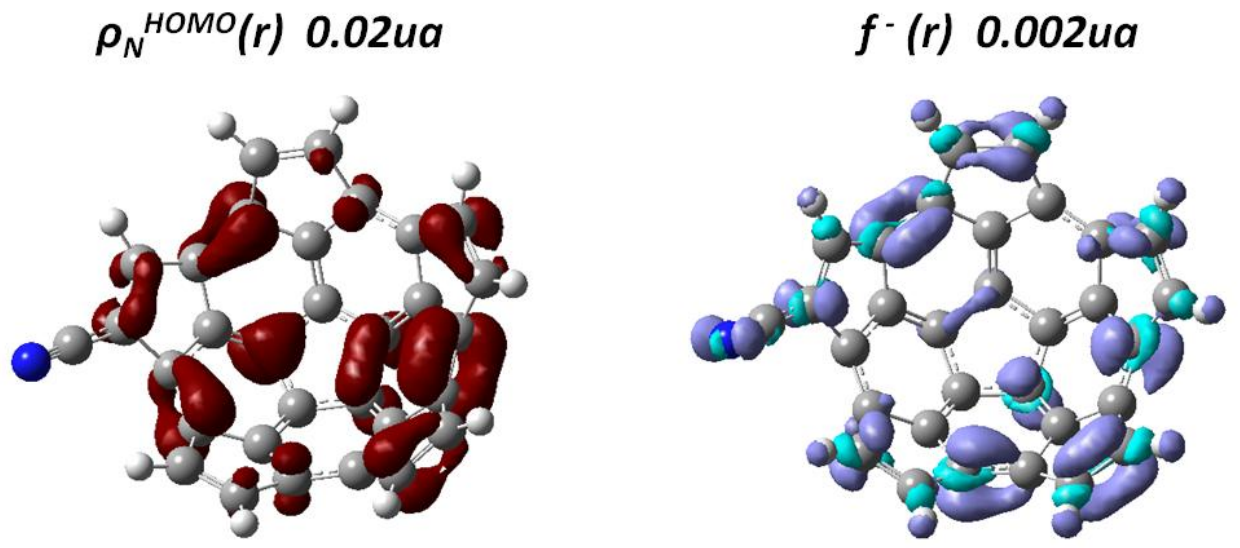

$\rho_{N}{ }^{L U M O}(r) 0.02 u a$
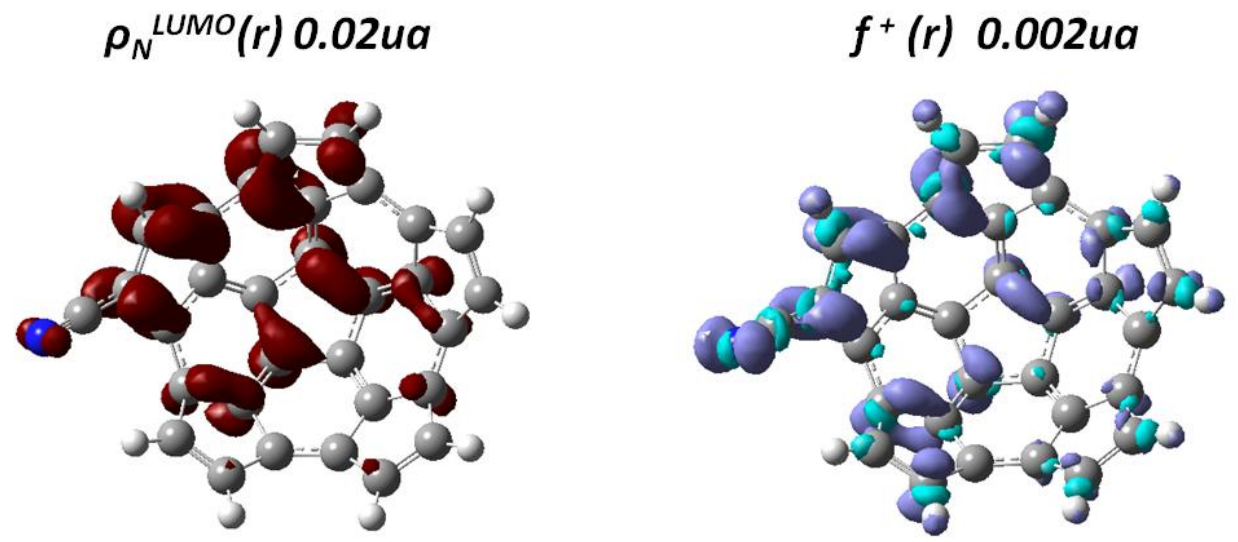

$\Delta f(r) 0.003 u a$

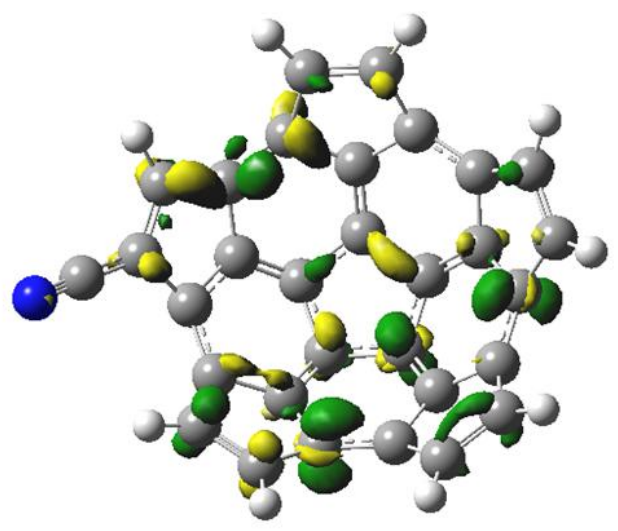




\section{Fragmento 2 mono-sustituido con $\mathrm{COOH}$}
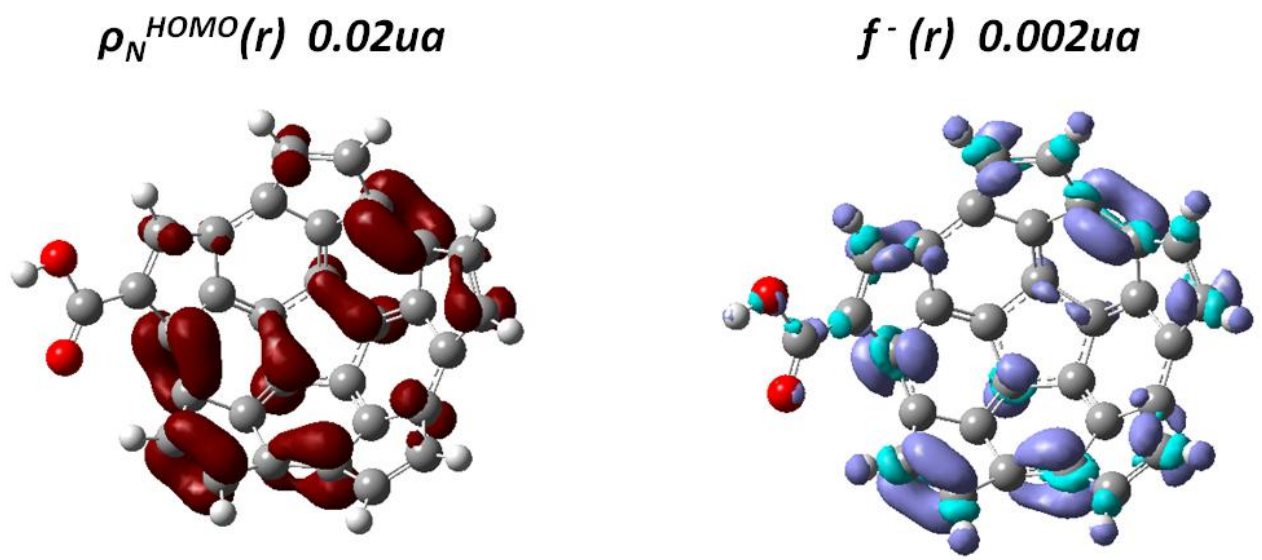

$\rho_{N}{ }^{\mathrm{LUMO}}(r) 0.02 \mathrm{ua}$

$$
f^{+}(r) 0.002 u a
$$
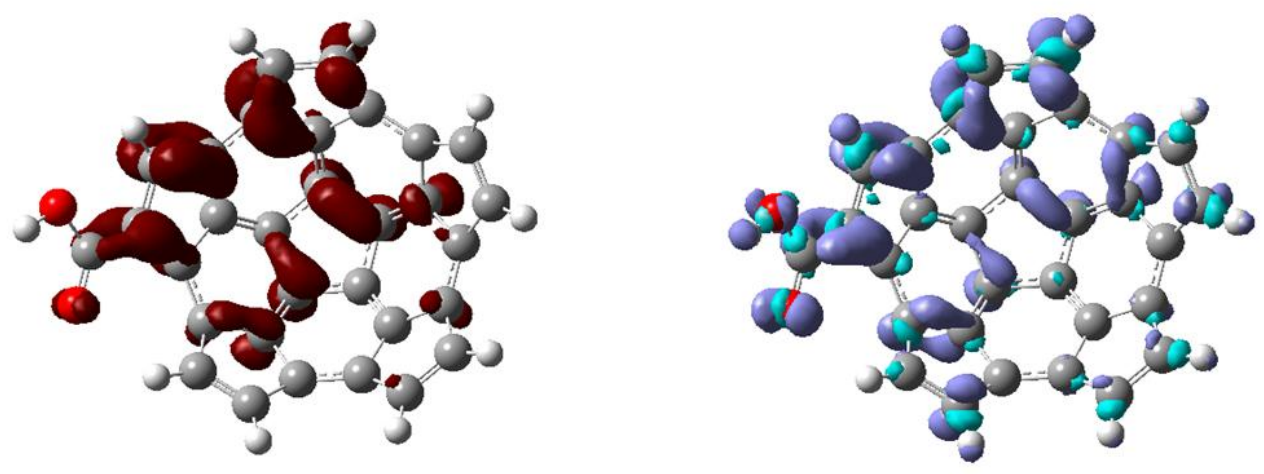

$\Delta f(r) 0.003 u a$

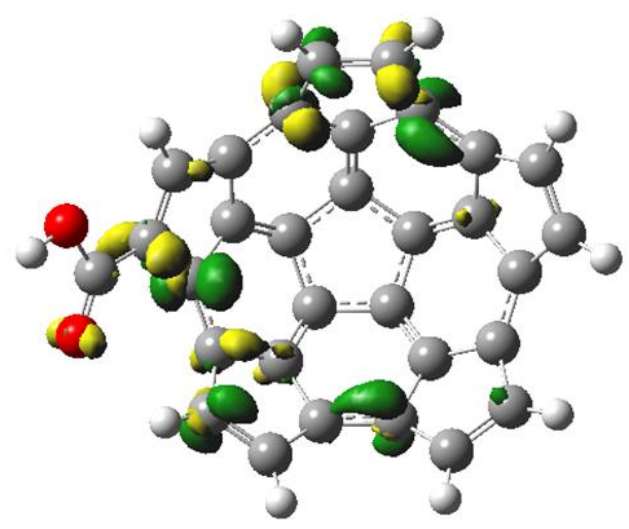


Fragmento 2 mono-sustituido con $\mathrm{F}$
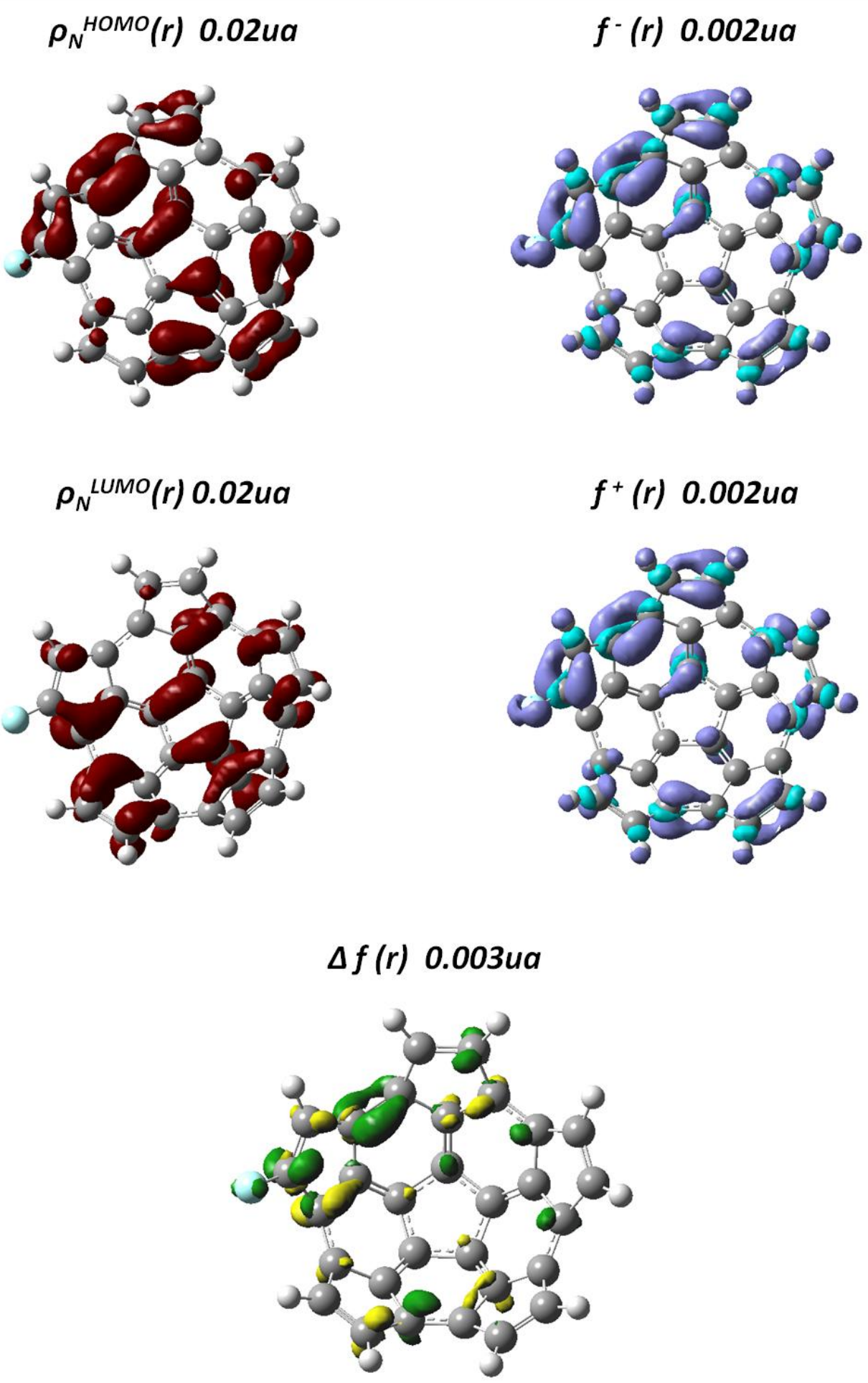
Fragmento 2 mono-sustituido con $i-P r$
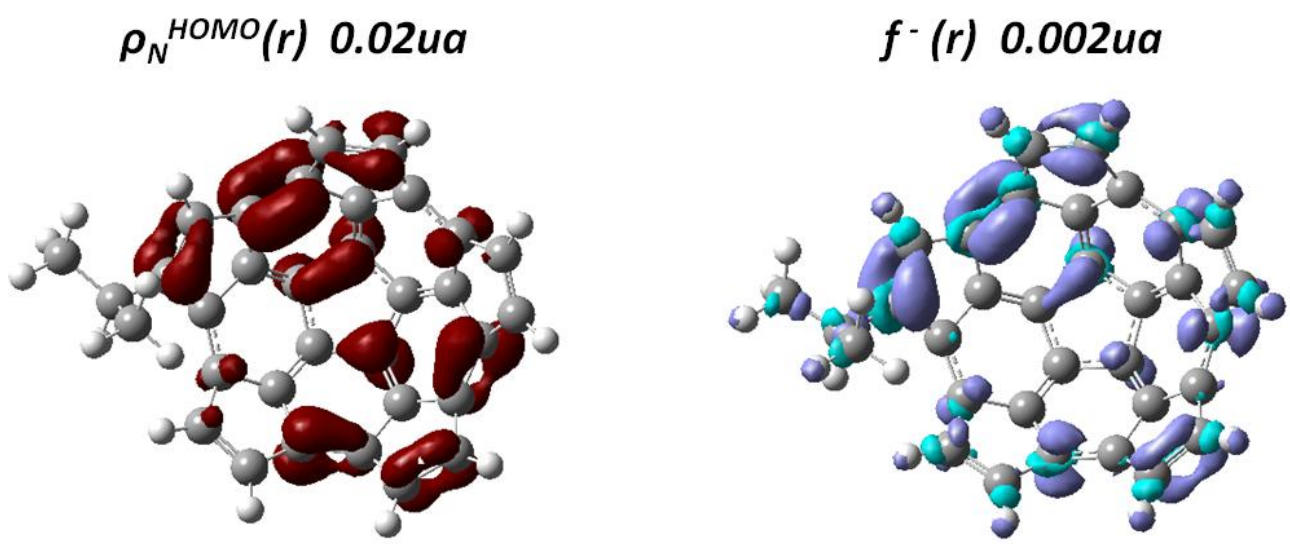

$\rho_{N}{ }^{\mathrm{LUMO}}(r) 0.02 \mathrm{ua}$

$f^{+}(r) 0.002 u a$
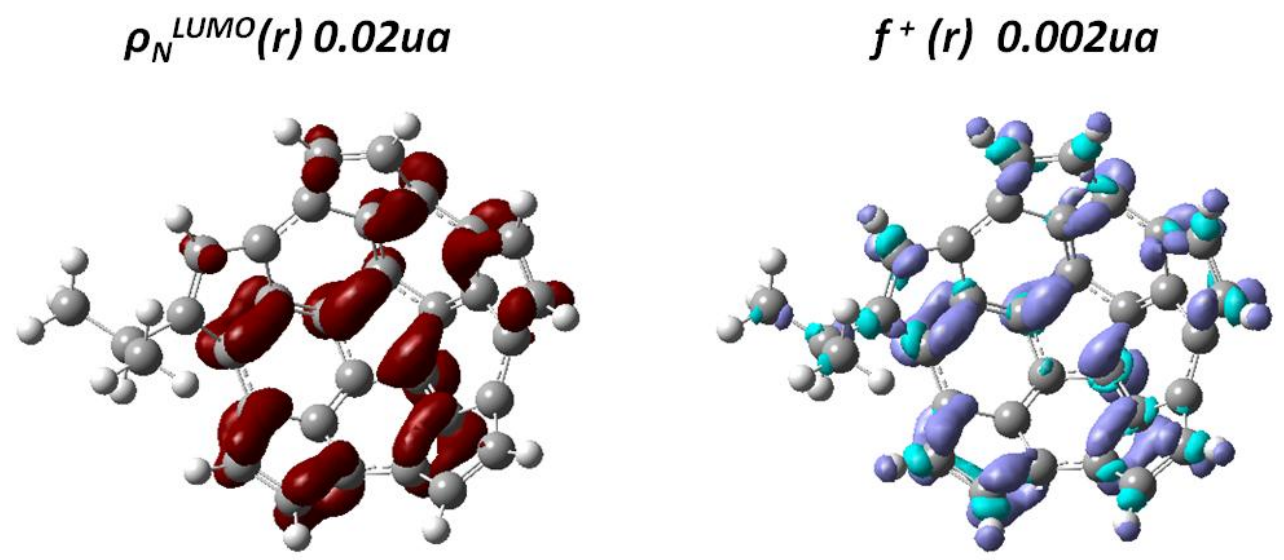

\section{$\Delta f(r) 0.003 u a$}

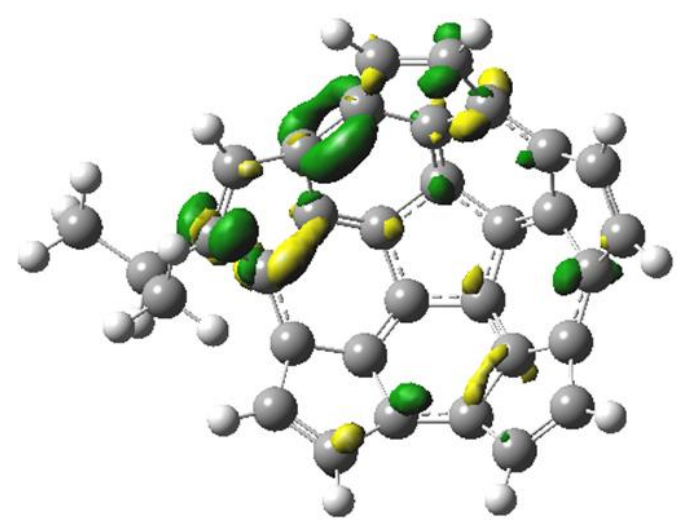


Fragmento 2 mono-sustituido con Me
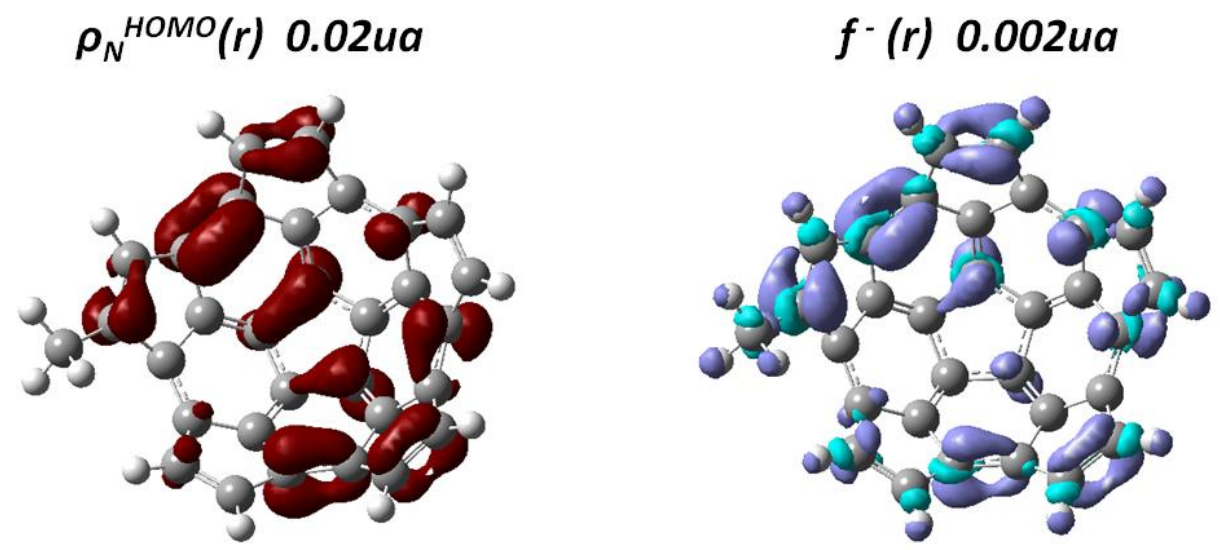

$\rho_{N}{ }^{\mathrm{LUMO}}(r) 0.02 \mathrm{ua}$
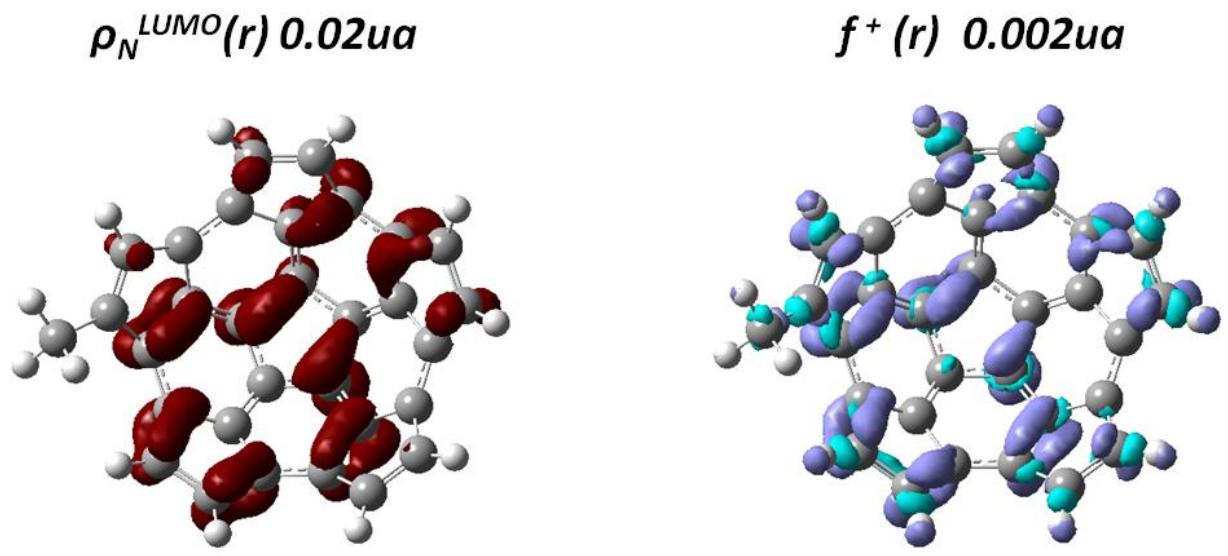

$\Delta f(r) 0.003 u a$

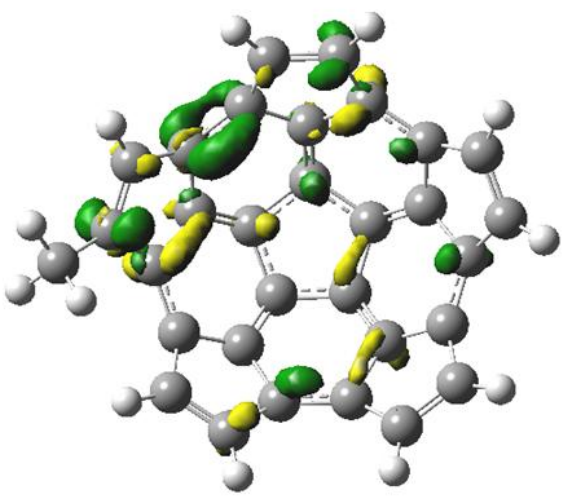


Fragmento 2 mono-sustituido con $\mathrm{NH}_{2}$
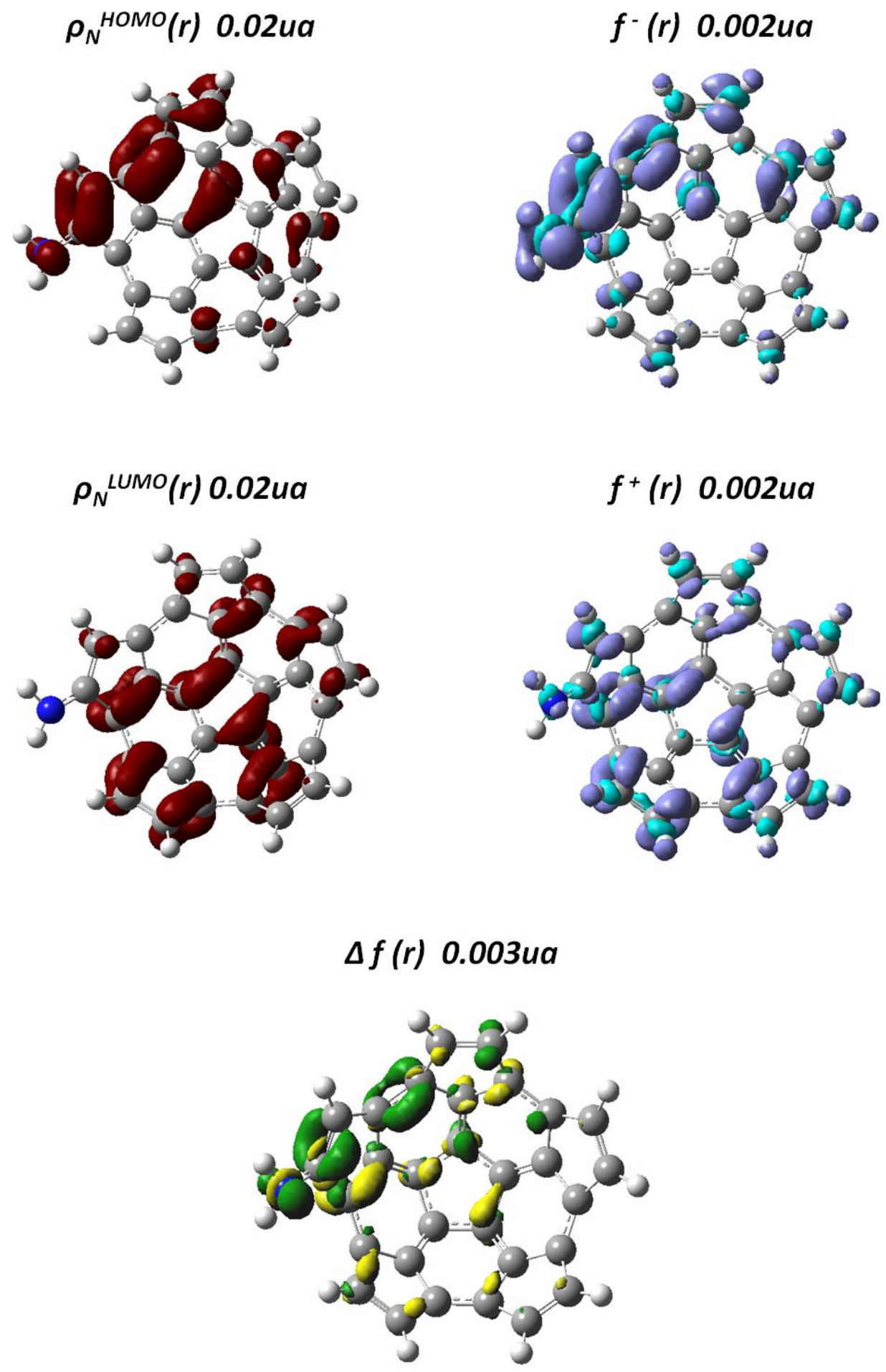
Fragmento 2 mono-sustituido con $\mathrm{NO}_{2}$
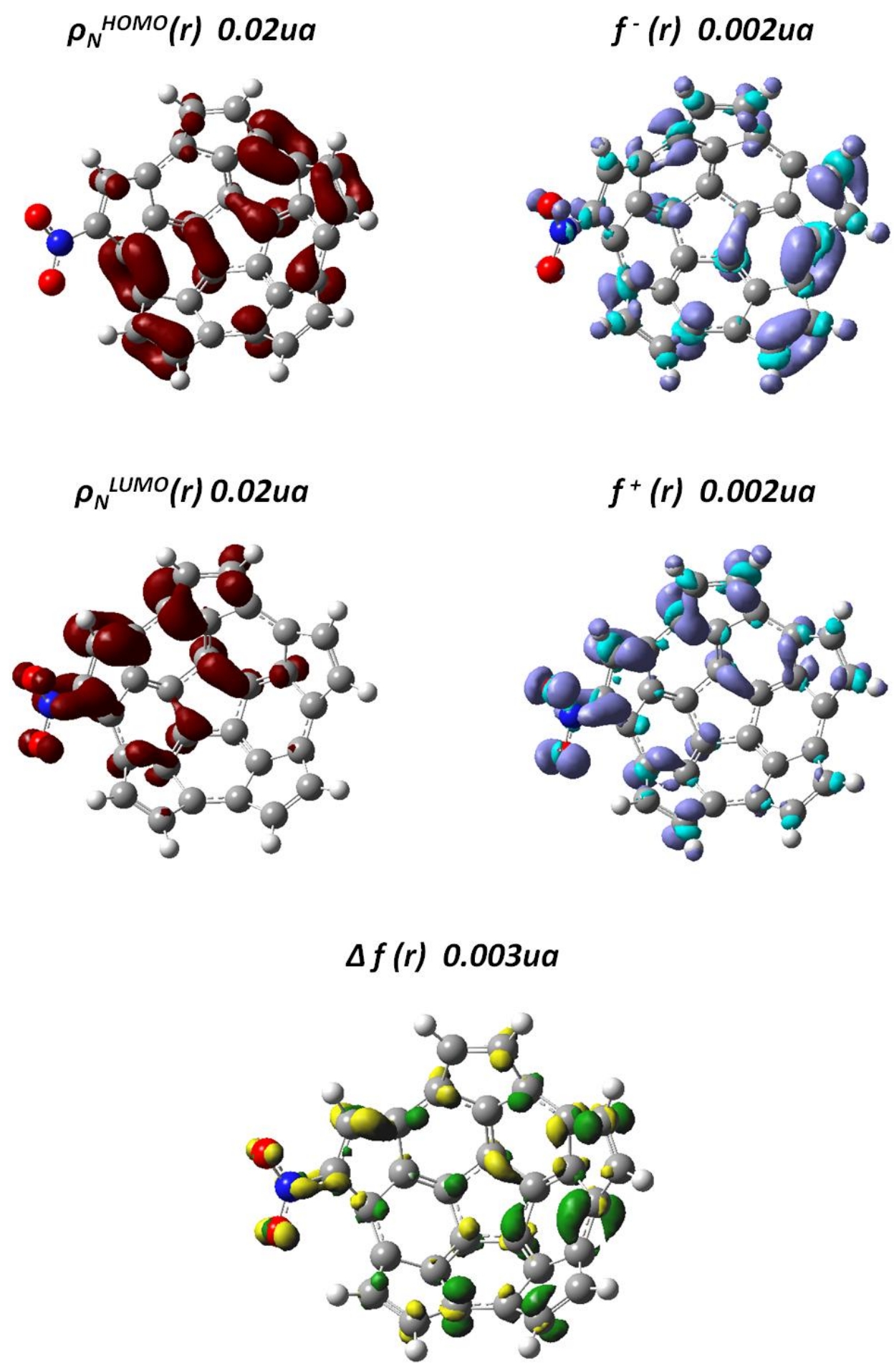


\section{Fragmento 2 mono-sustituido con $\mathrm{OH}$}
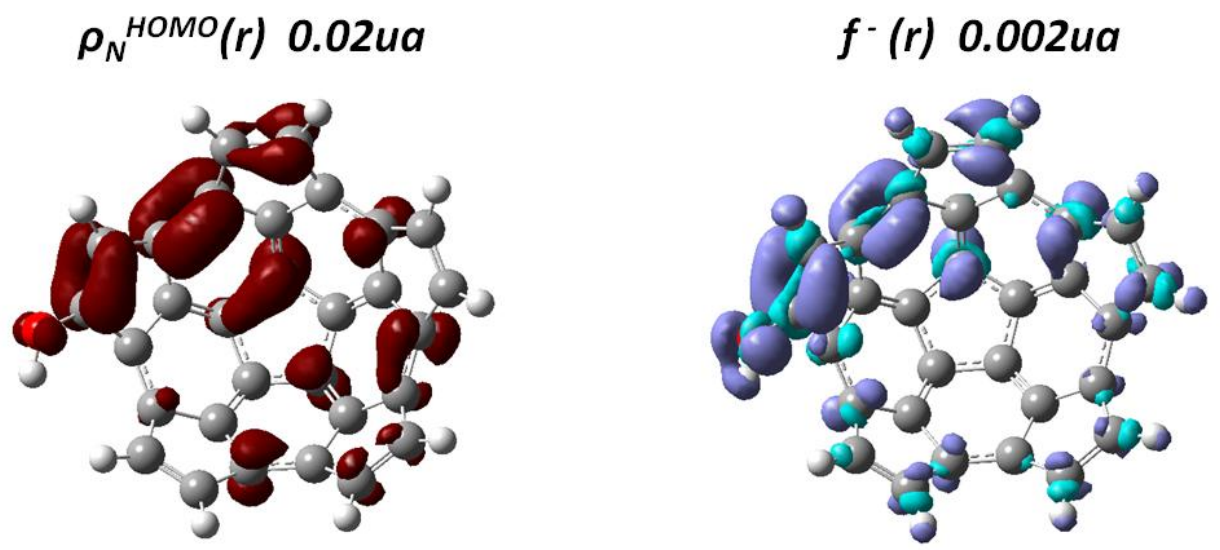

$$
\rho_{N}^{\text {LUMO }}(r) 0.02 u a
$$
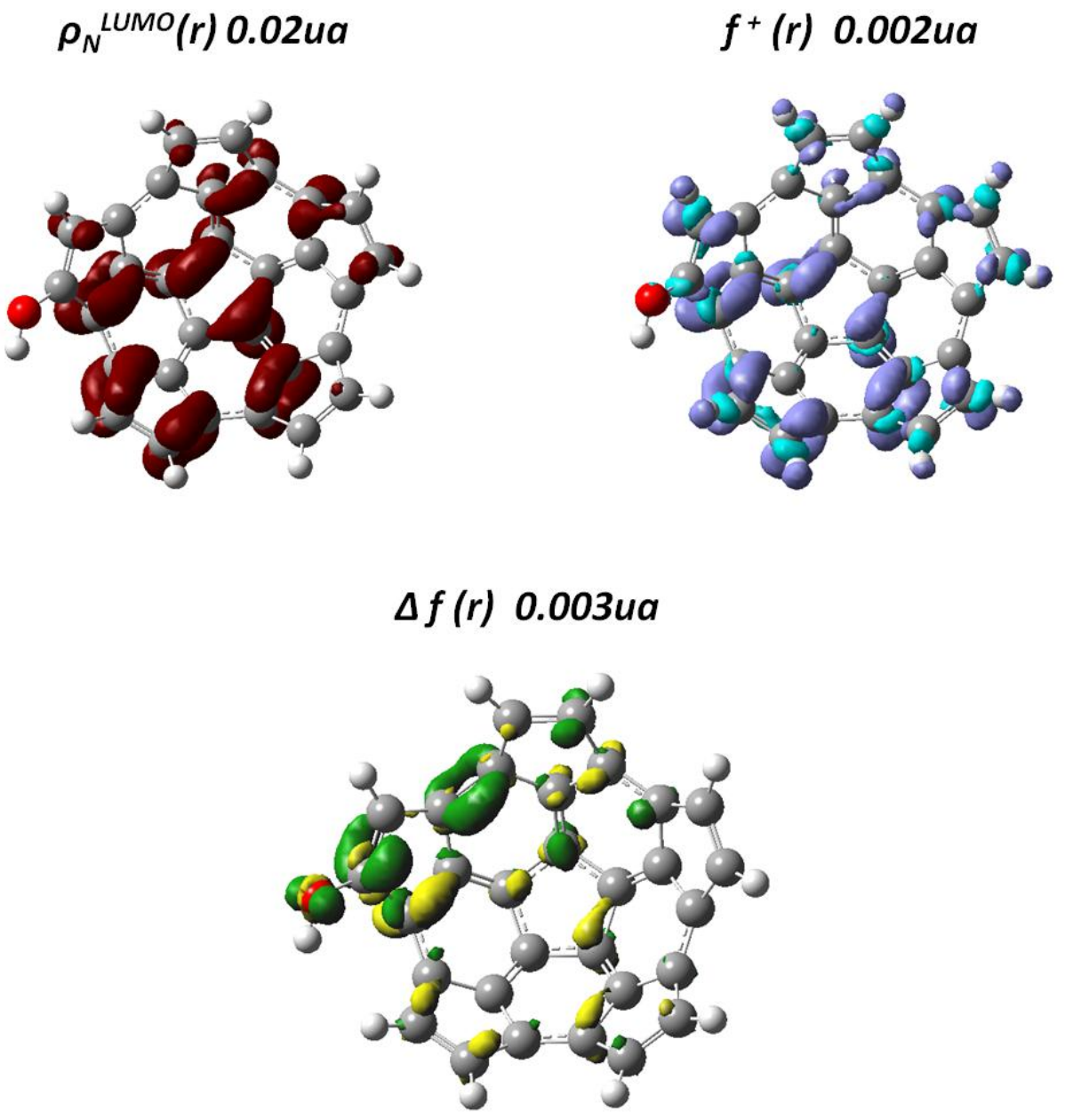
Fragmento 2 mono-sustituido con OMe
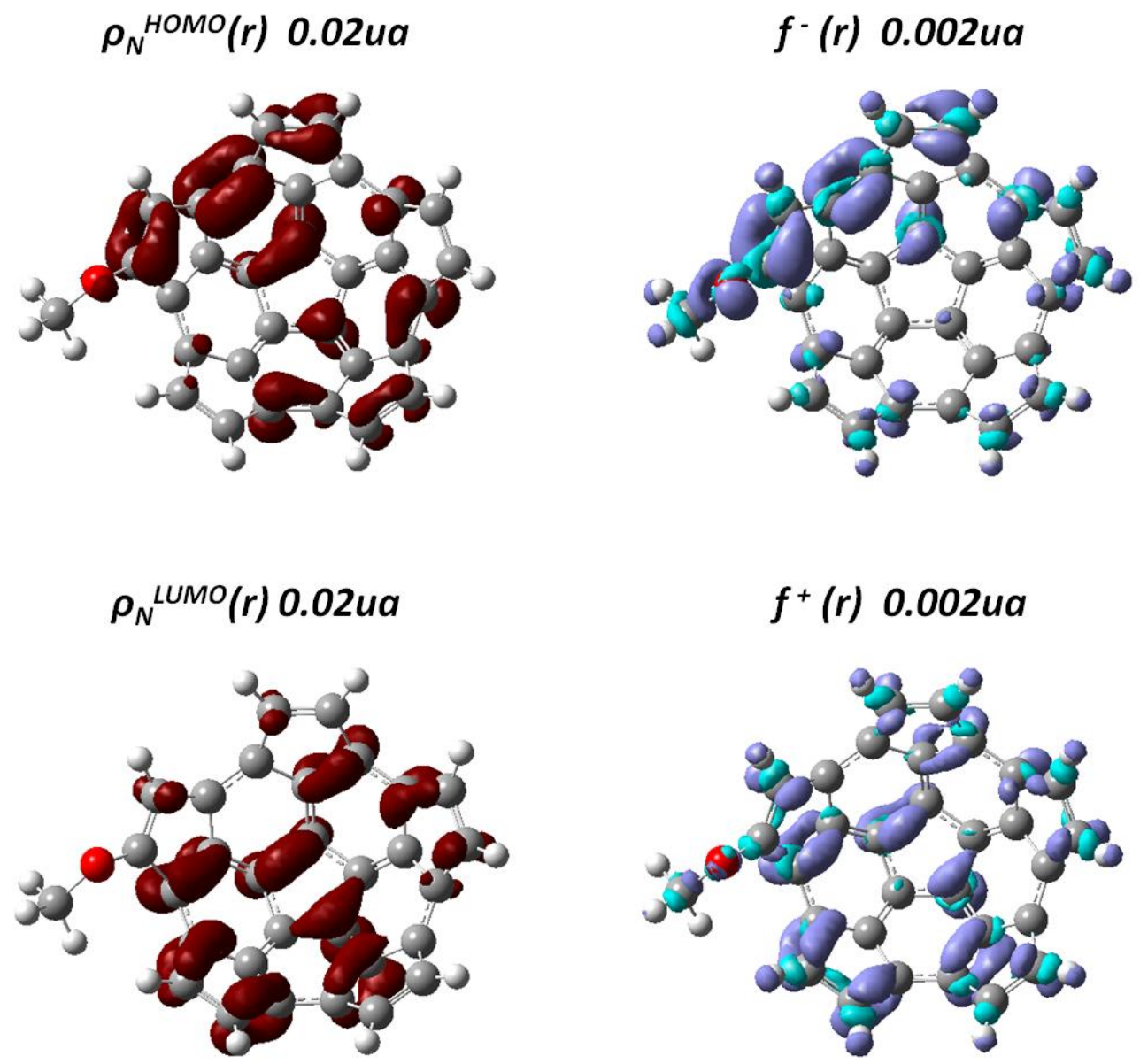

$\Delta f(r) 0.003 u a$

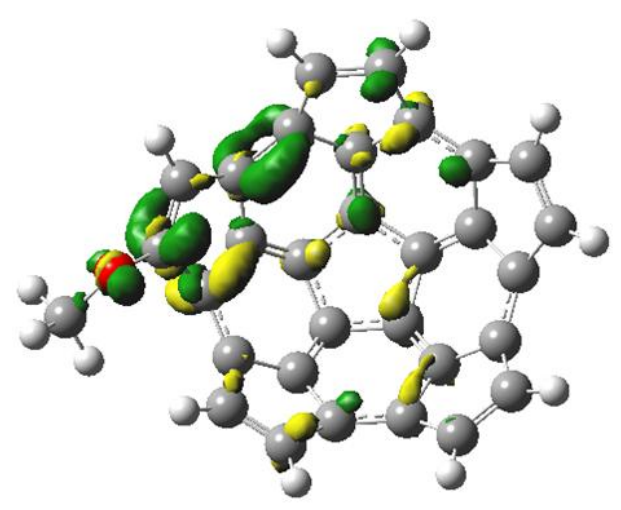


Fragmento 1 di-sustituido con $\mathrm{CF}_{3}$
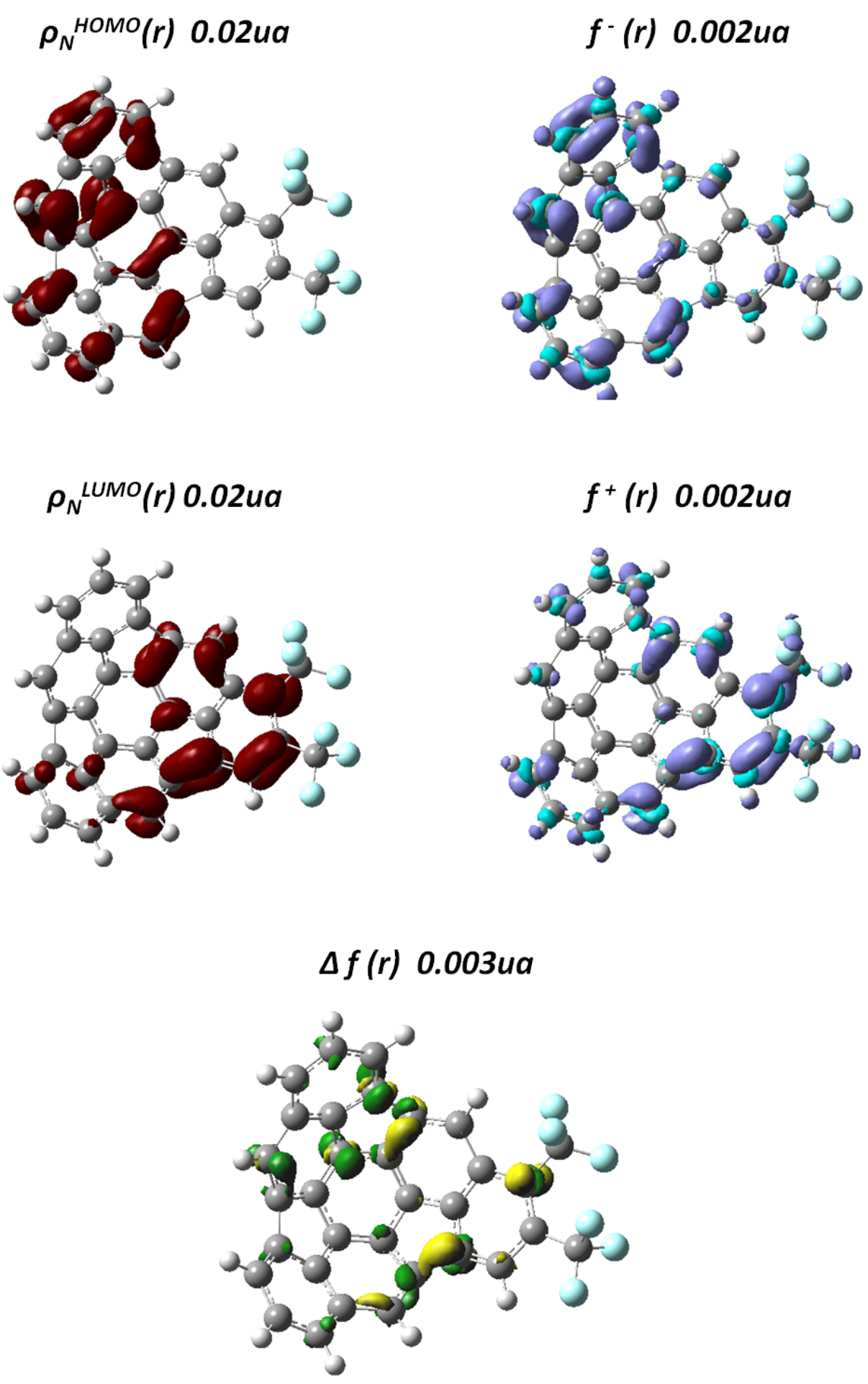
Fragmento 1 di-sustituido con $\mathrm{CHO}$
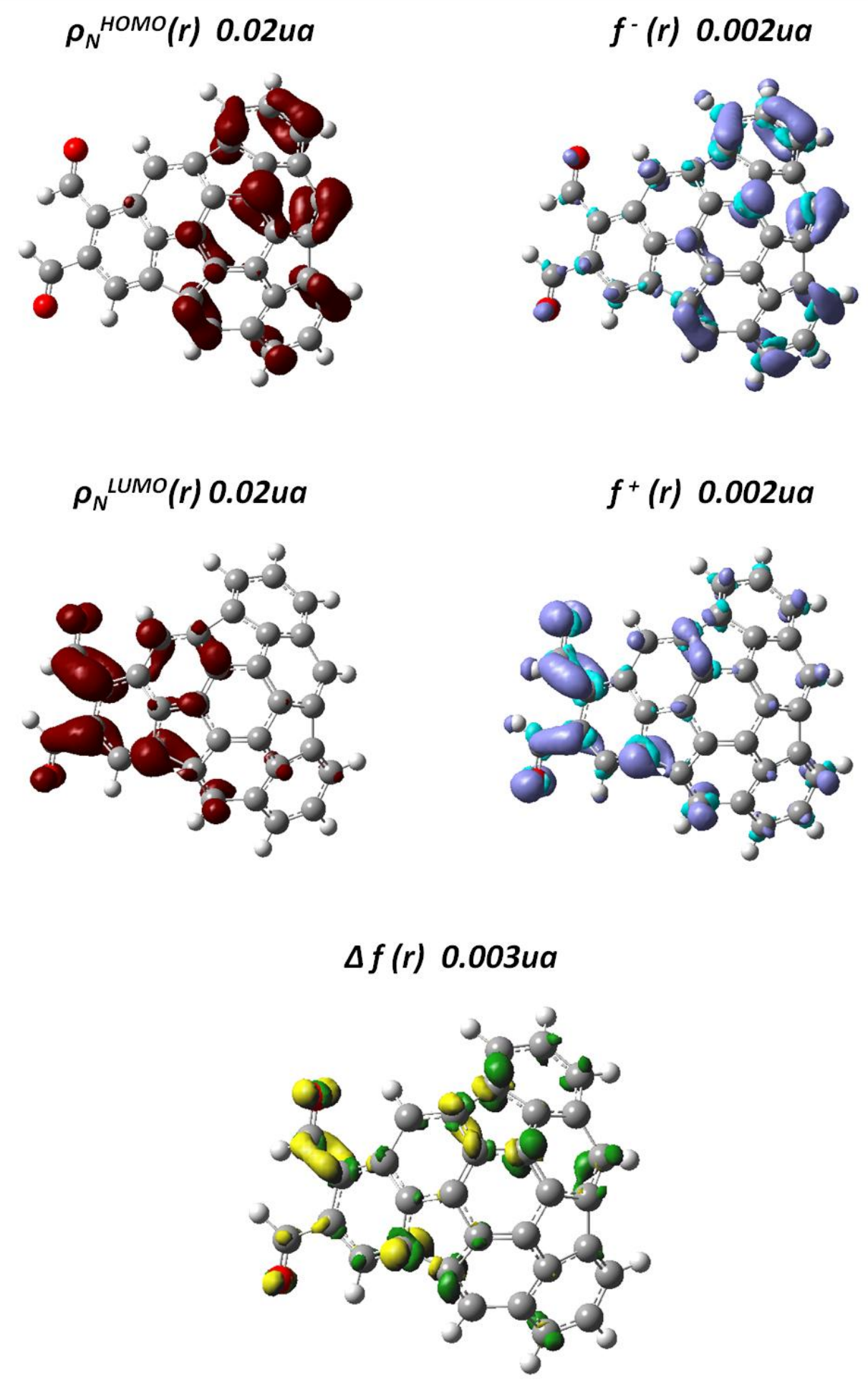


\section{Fragmento 1 di-sustituido con CN}
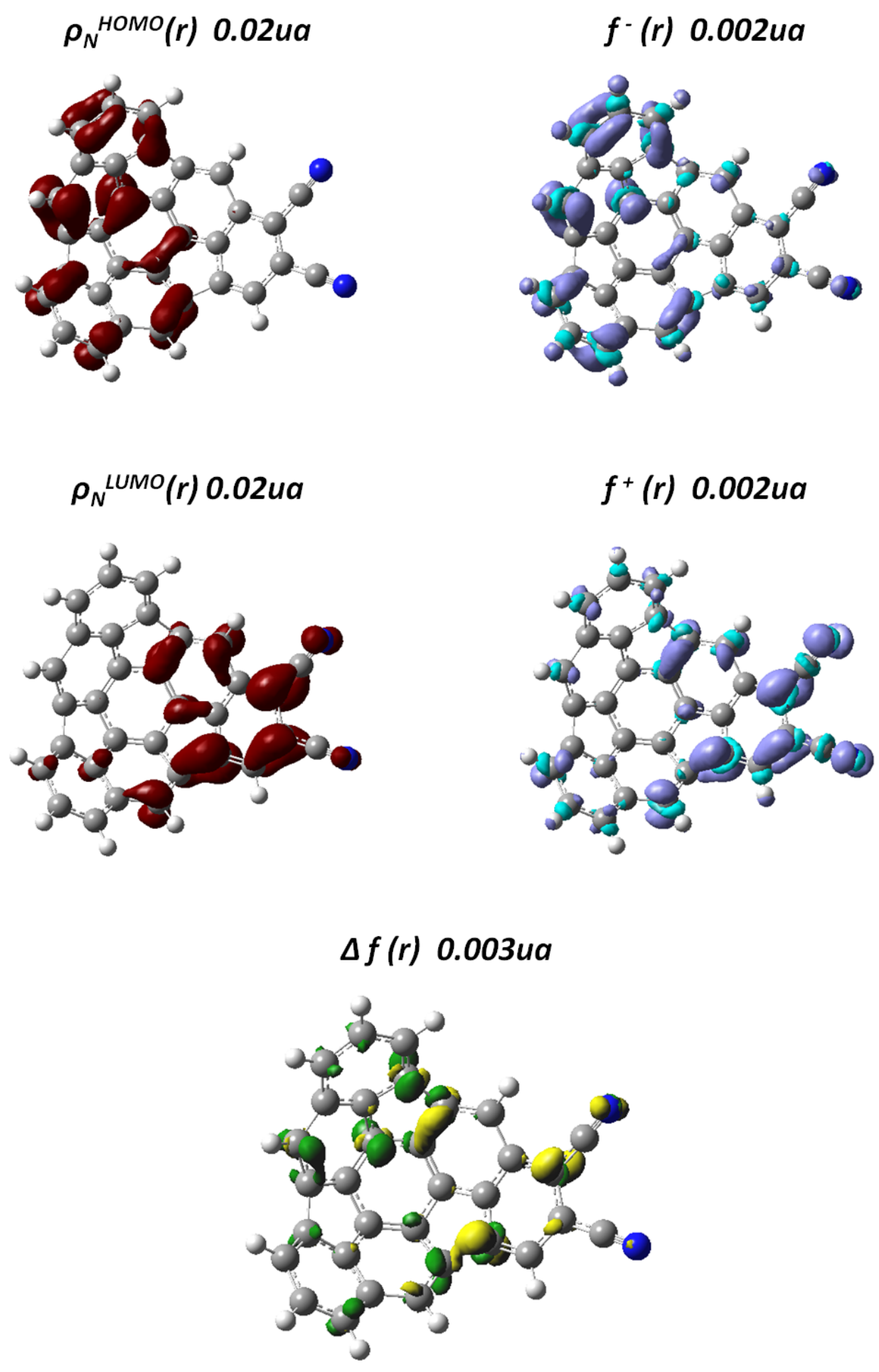
Fragmento 1 di-sustituido con $\mathrm{COOH}$
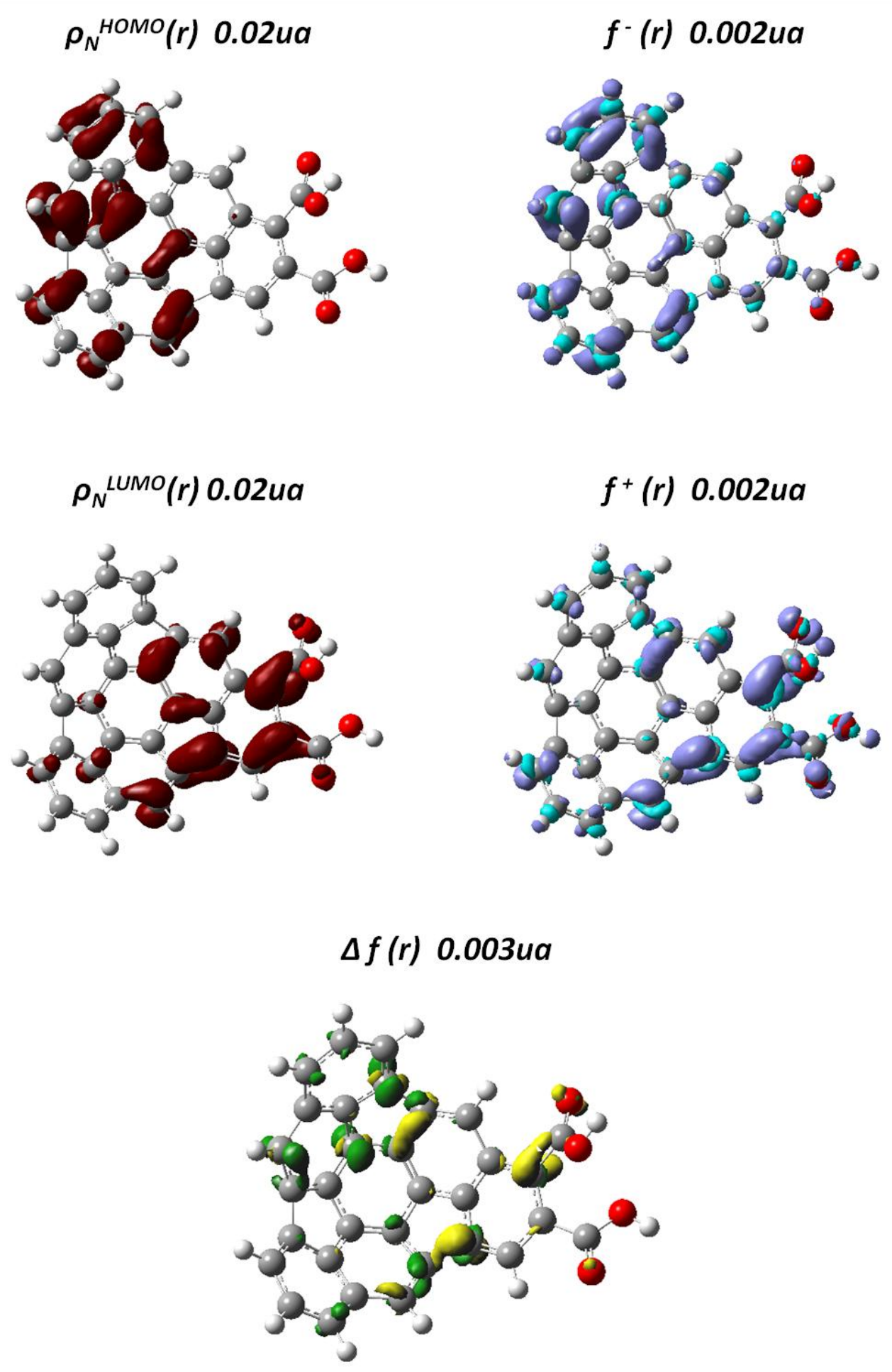
Fragmento 1 di-sustituido con $F$
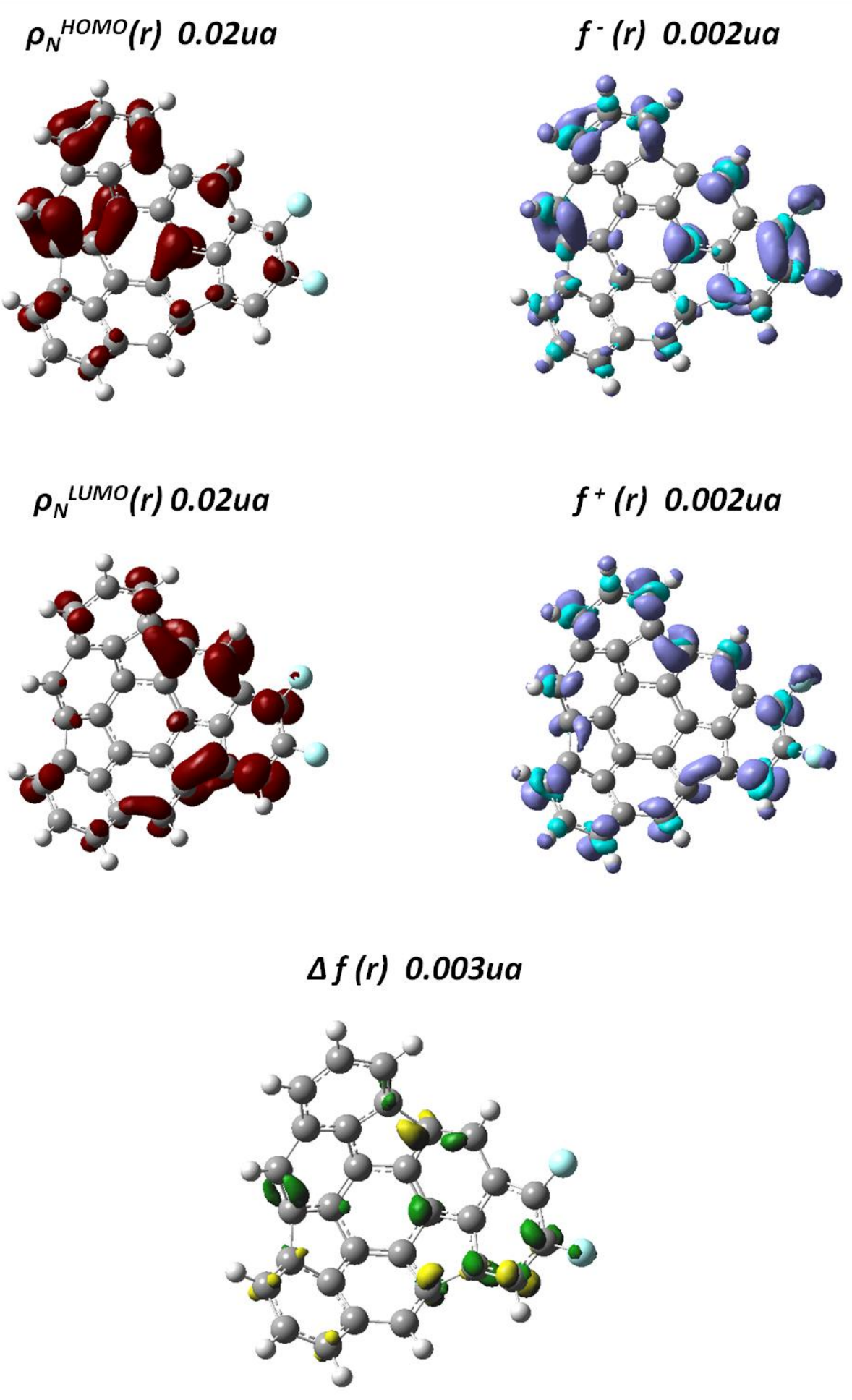
Fragmento 1 di-sustituido con $i-P r$
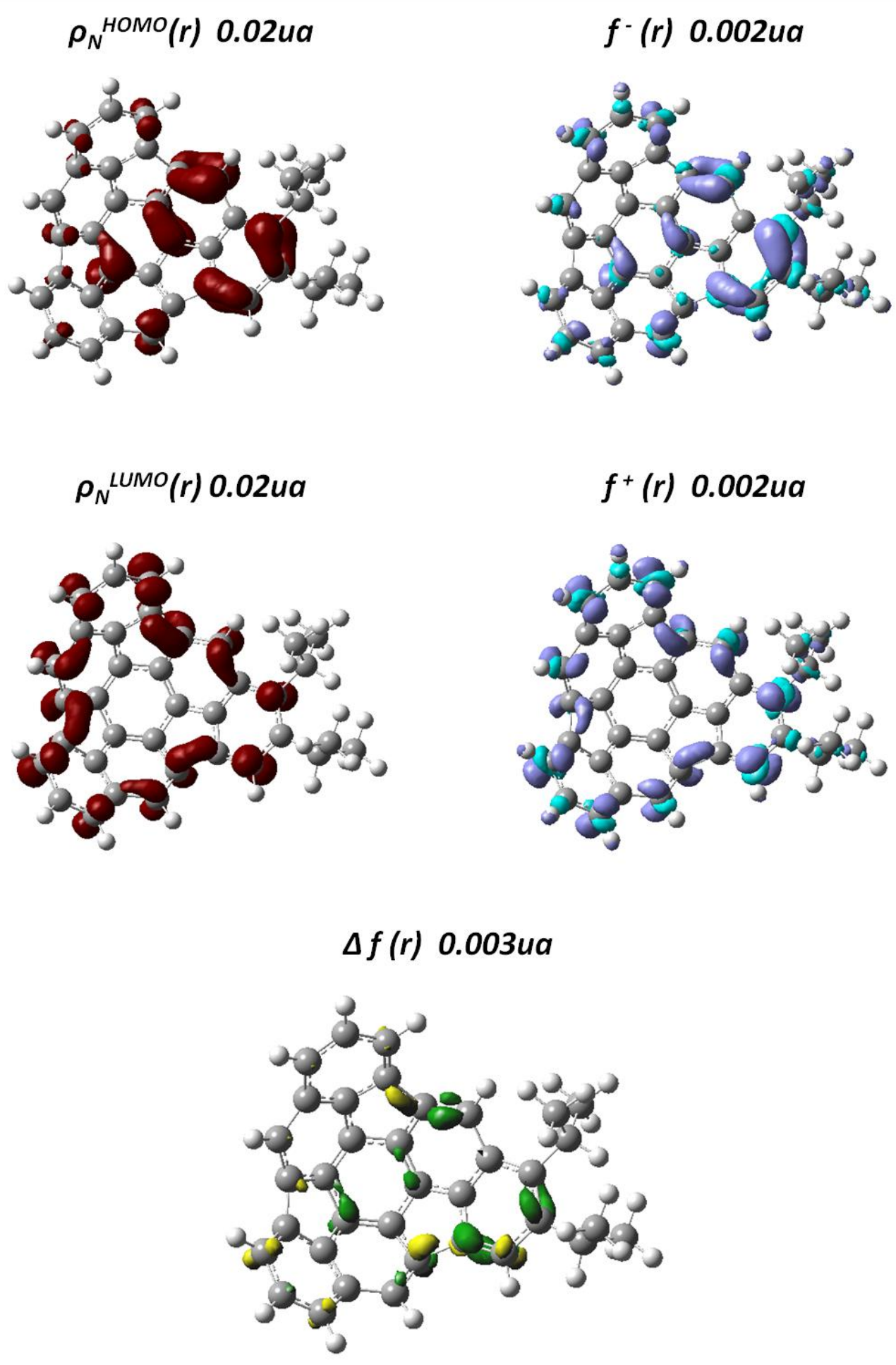
Fragmento 1 di-sustituido con Me
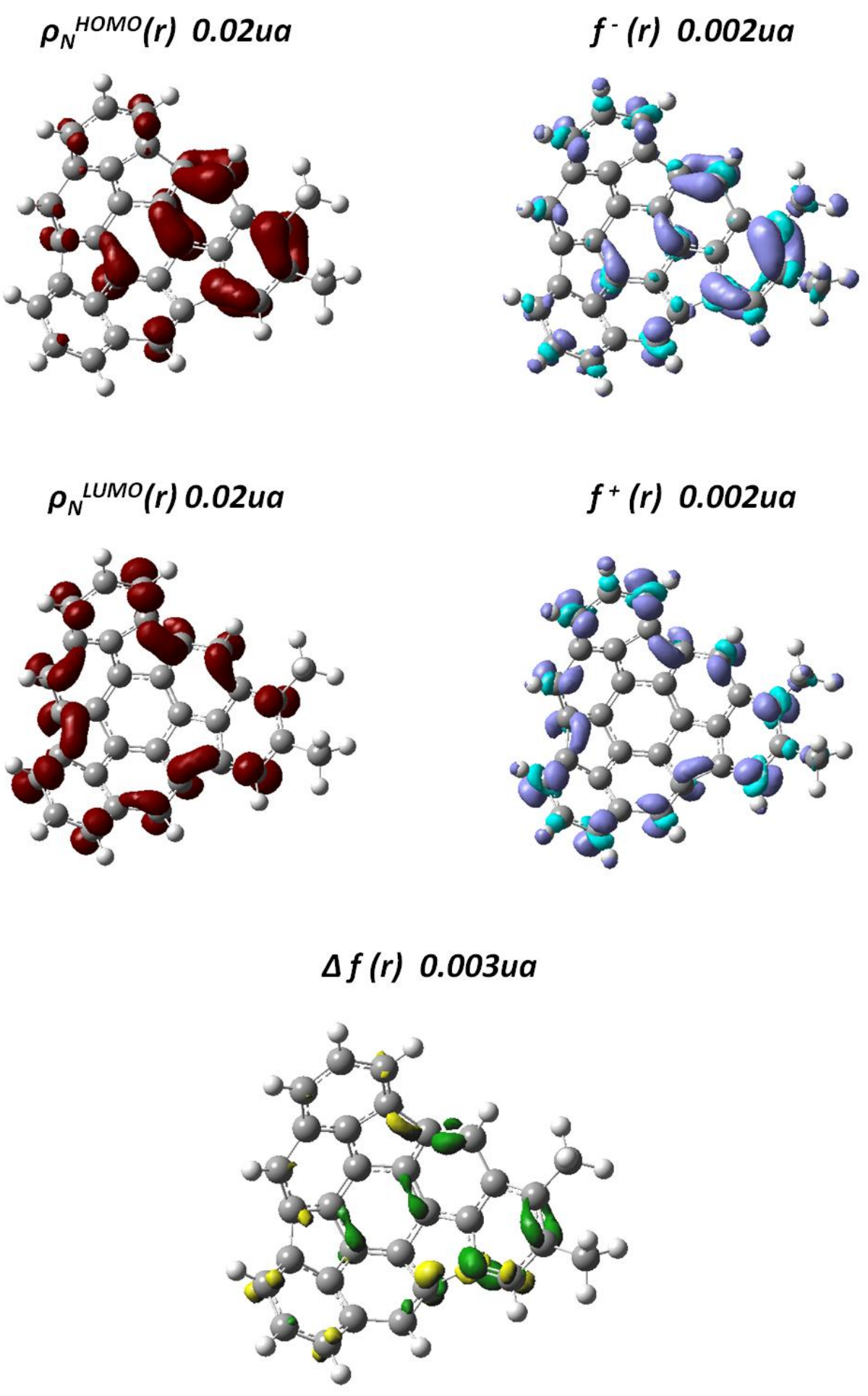
Fragmento 1 di-sustituido con $\mathrm{NH}_{2}$
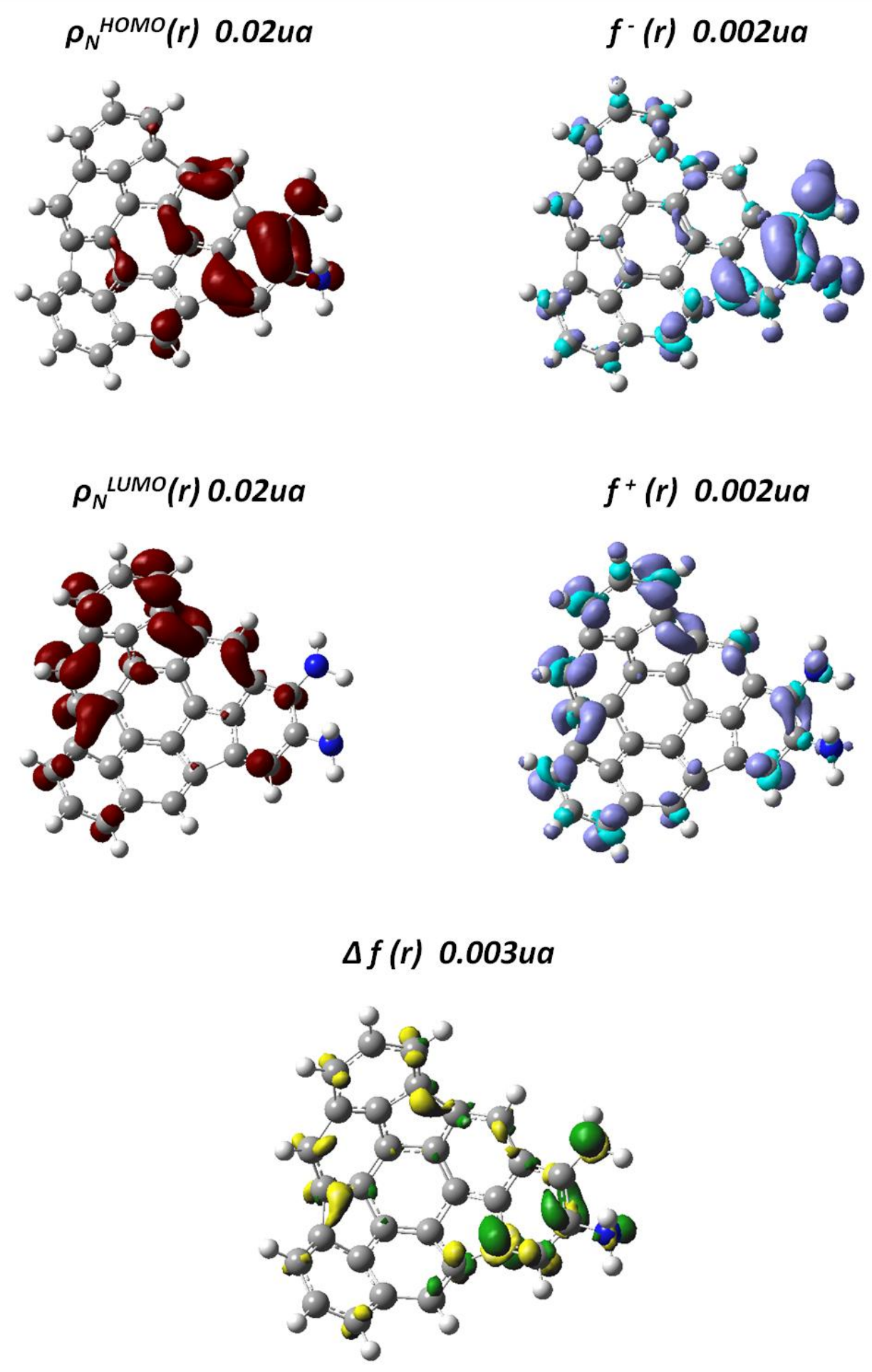
Fragmento 1 di-sustituido con $\mathrm{NO}_{2}$
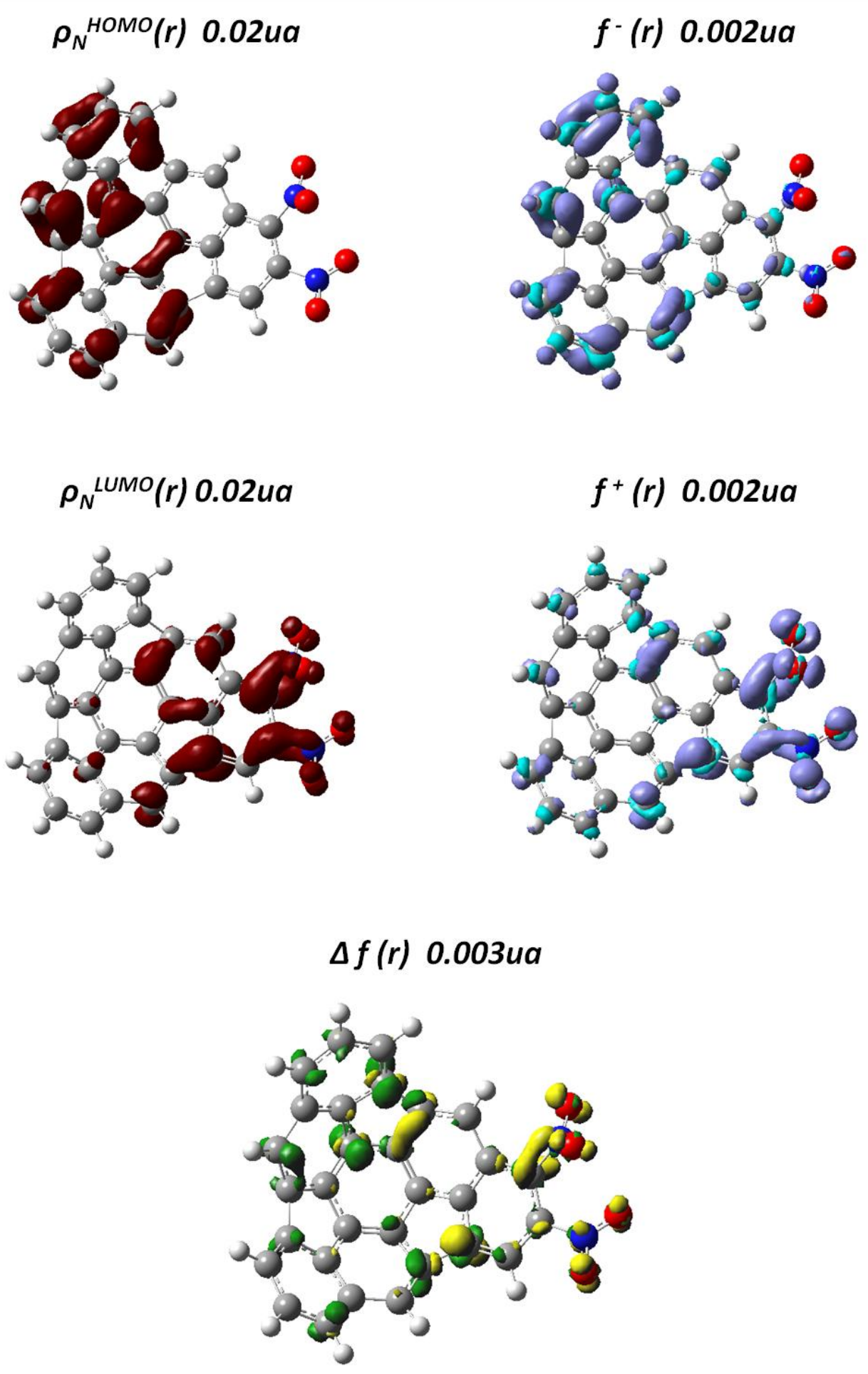
Fragmento 1 di-sustituido con $\mathrm{OH}$
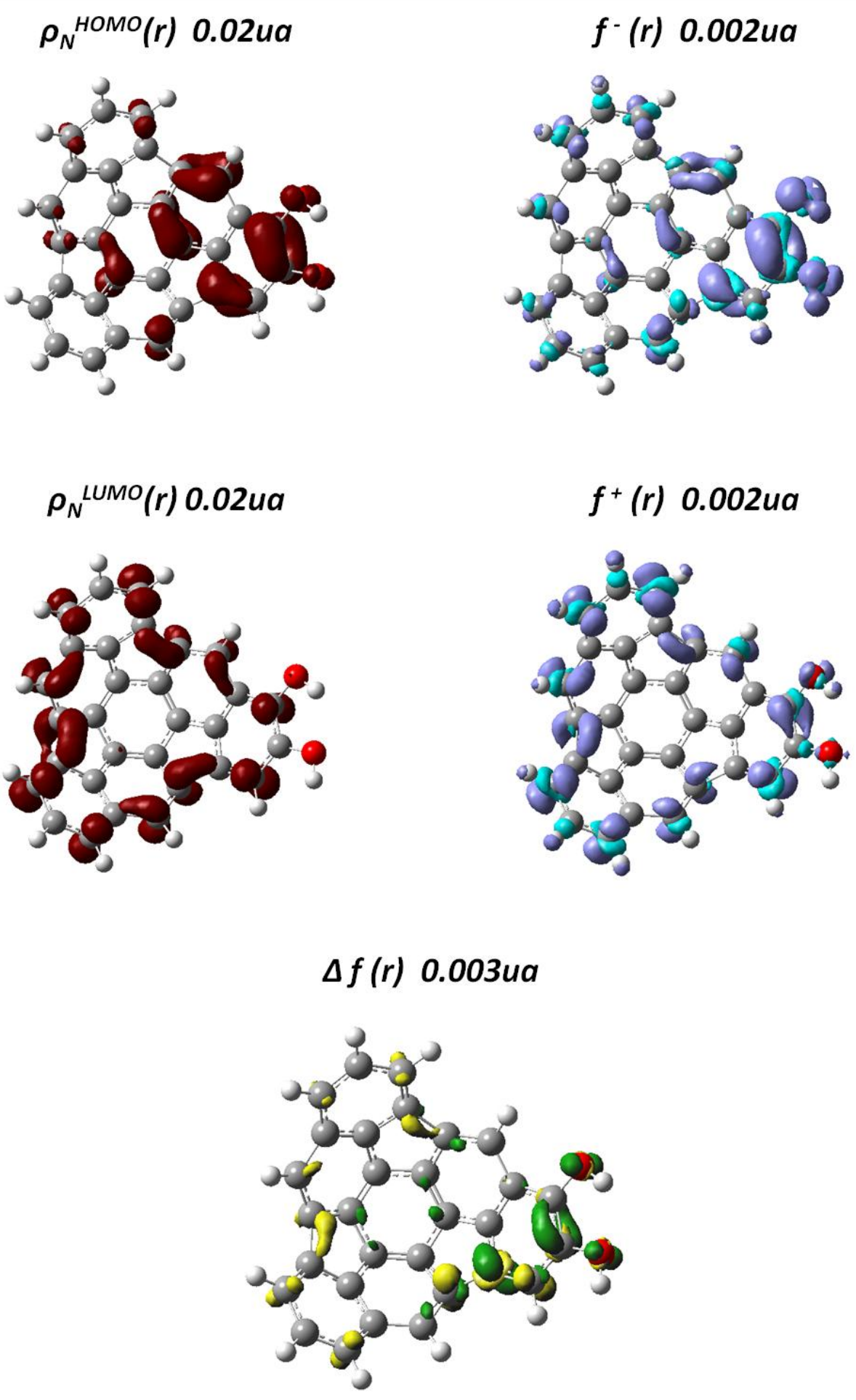
Fragmento 1 di-sustituido con OMe
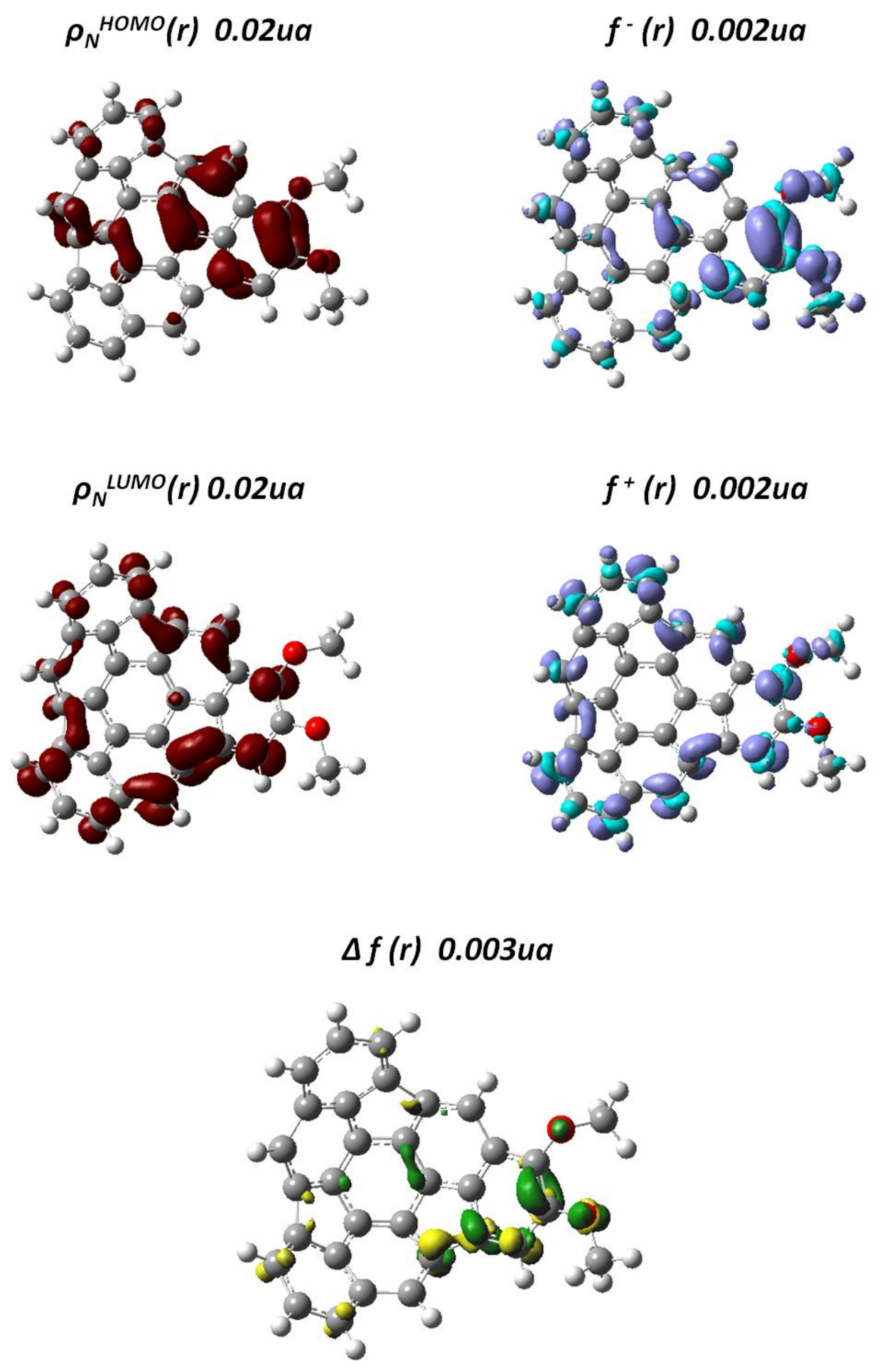
Fragmento 2 di -sustituido con $\mathrm{CF}_{3}$
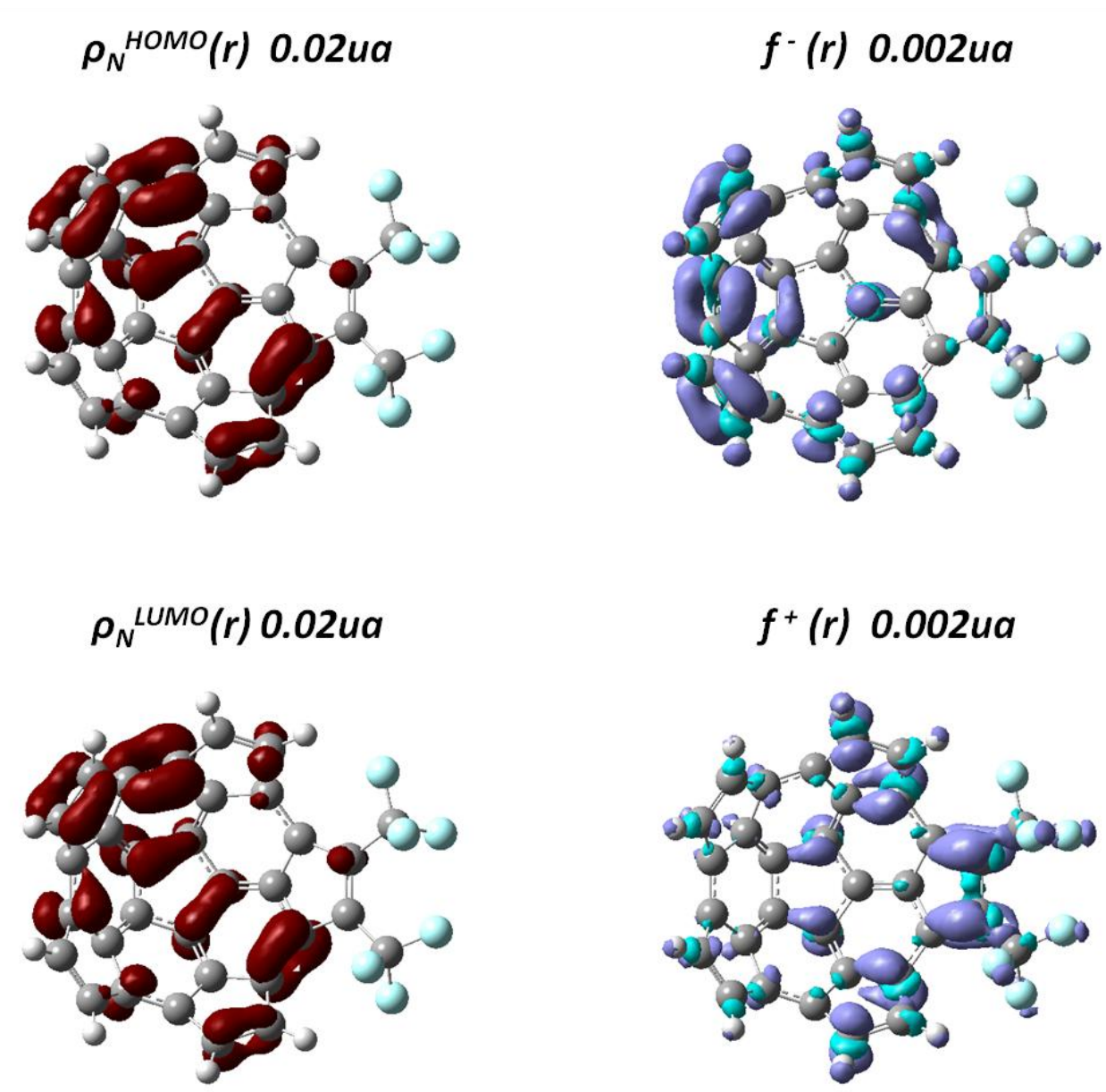

\section{$\Delta f(r) 0.003 u a$}

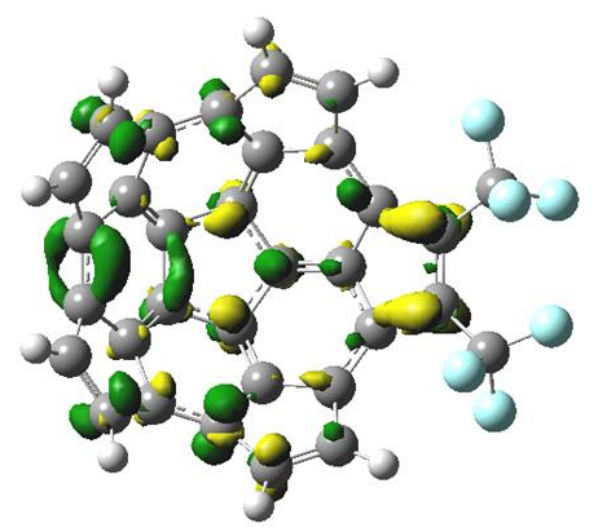


Fragmento 2 di-sustituido con $\mathrm{CHO}$
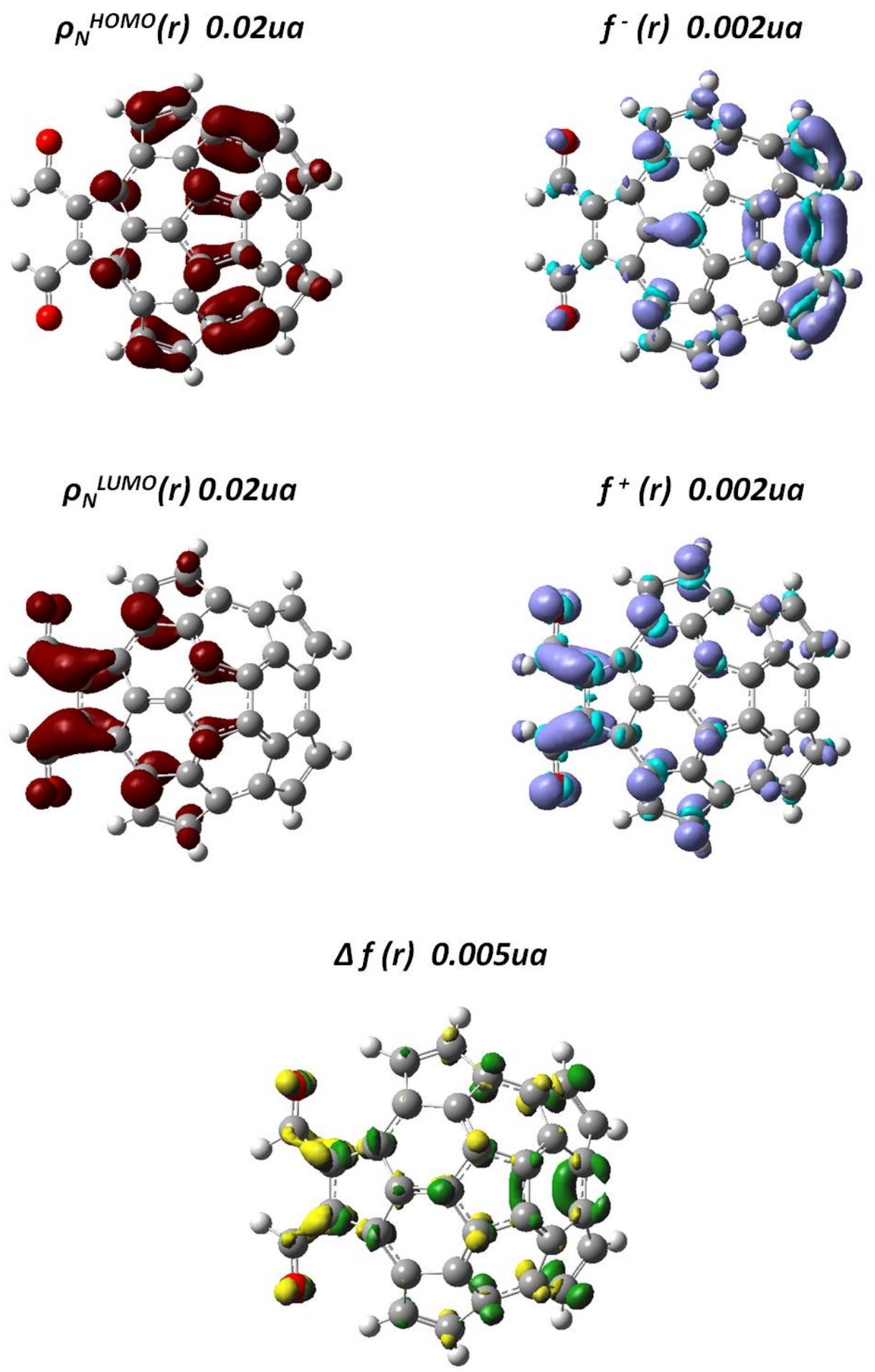
Fragmento 2 di-sustituido con $\mathrm{CN}$
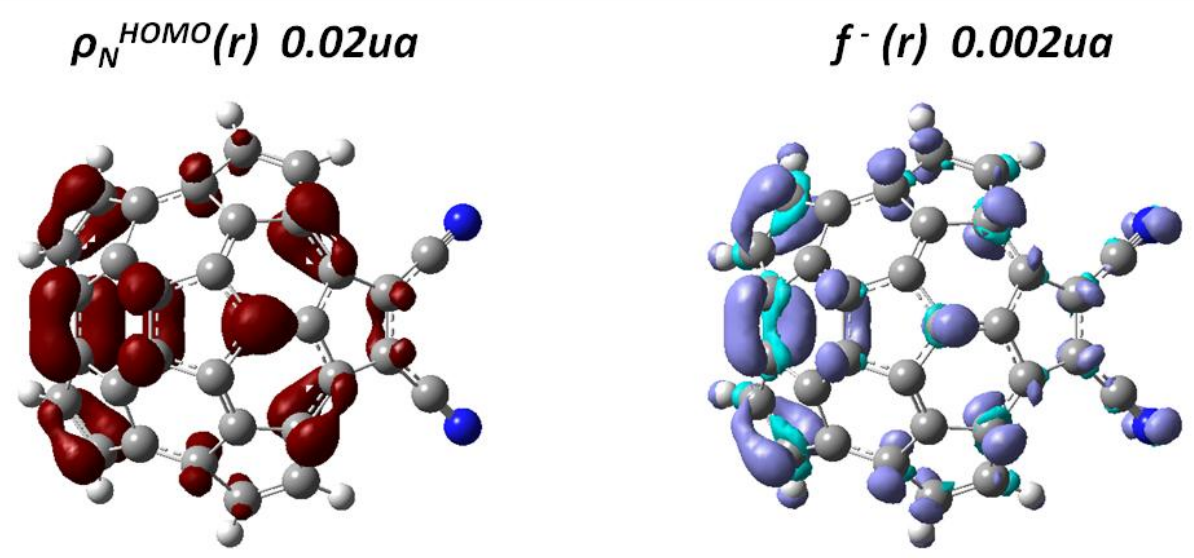

$$
\rho_{N}{ }^{L U M O}(r) 0.02 u a
$$
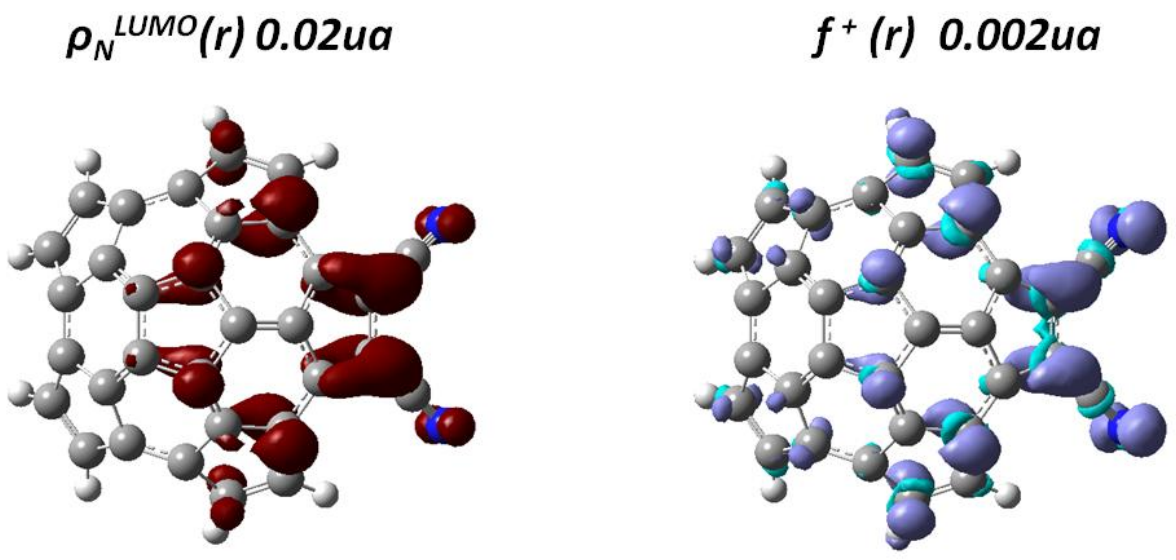

$\Delta f(r) 0.003 u a$

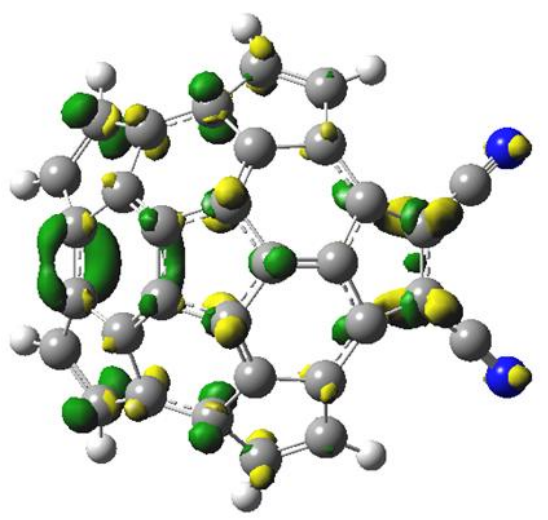


Fragmento 2 di-sustituido con $\mathrm{COOH}$
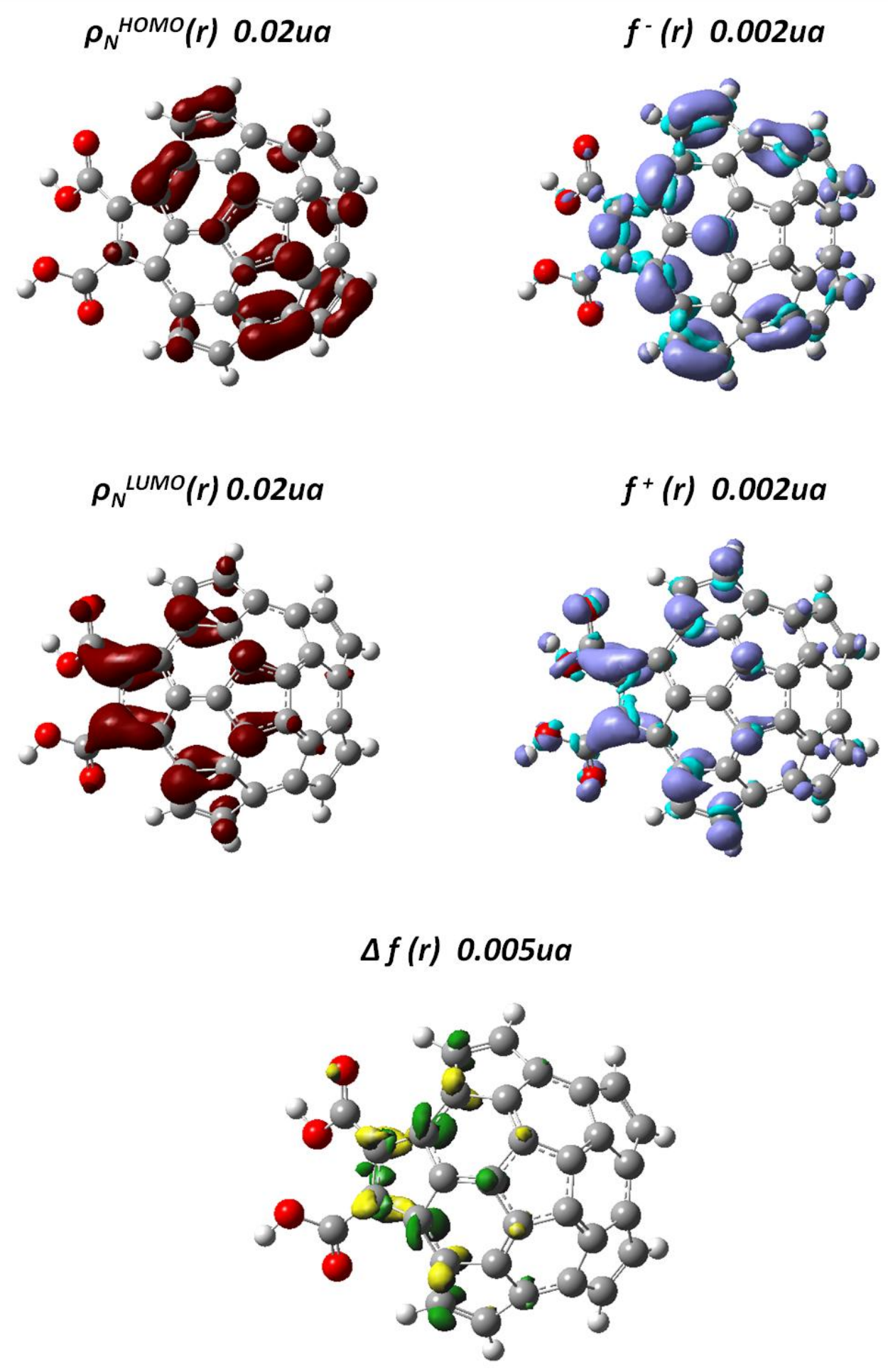
Fragmento 2 di-sustituido con $F$
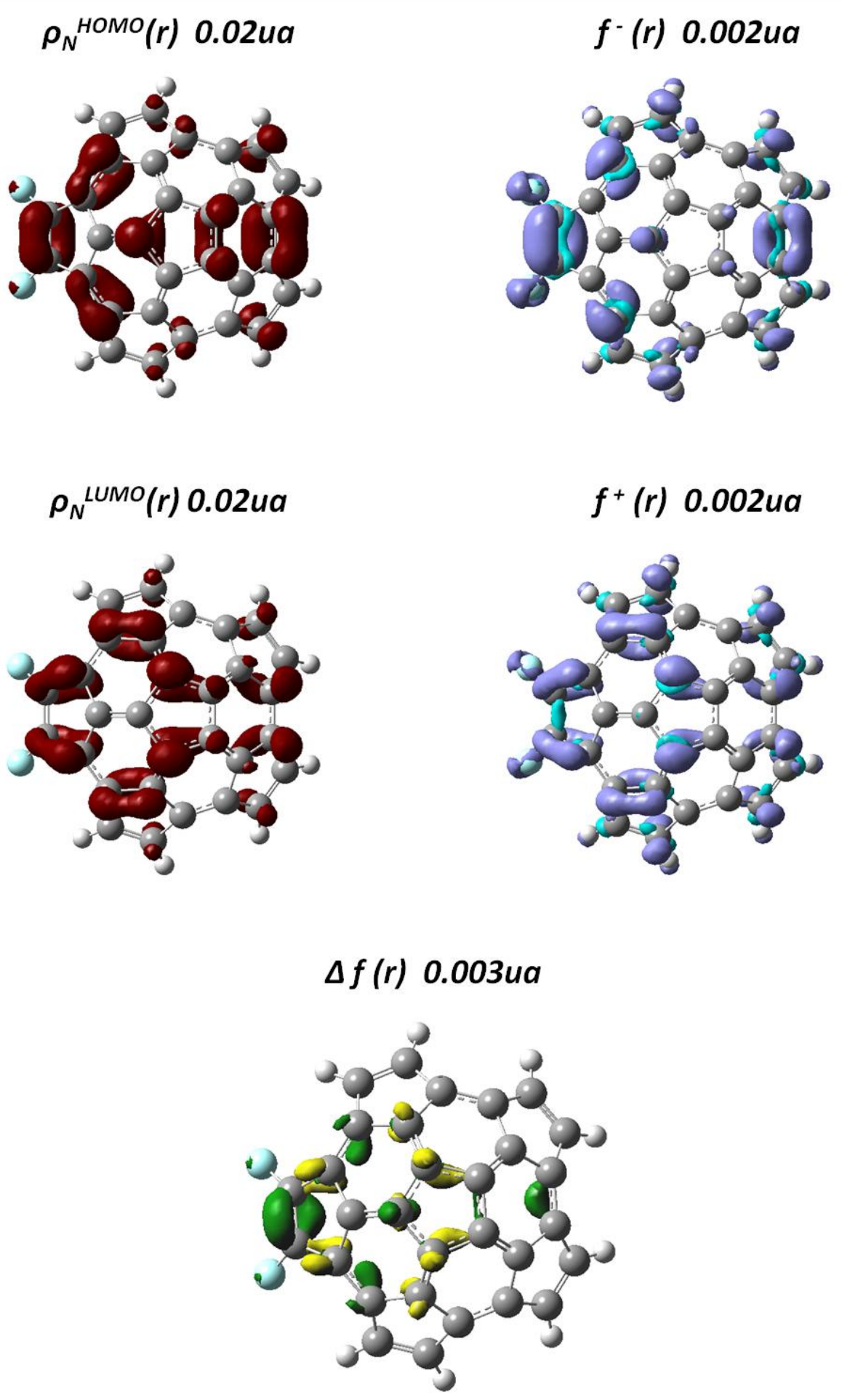
Fragmento 2 di-sustituido con $i-P r$
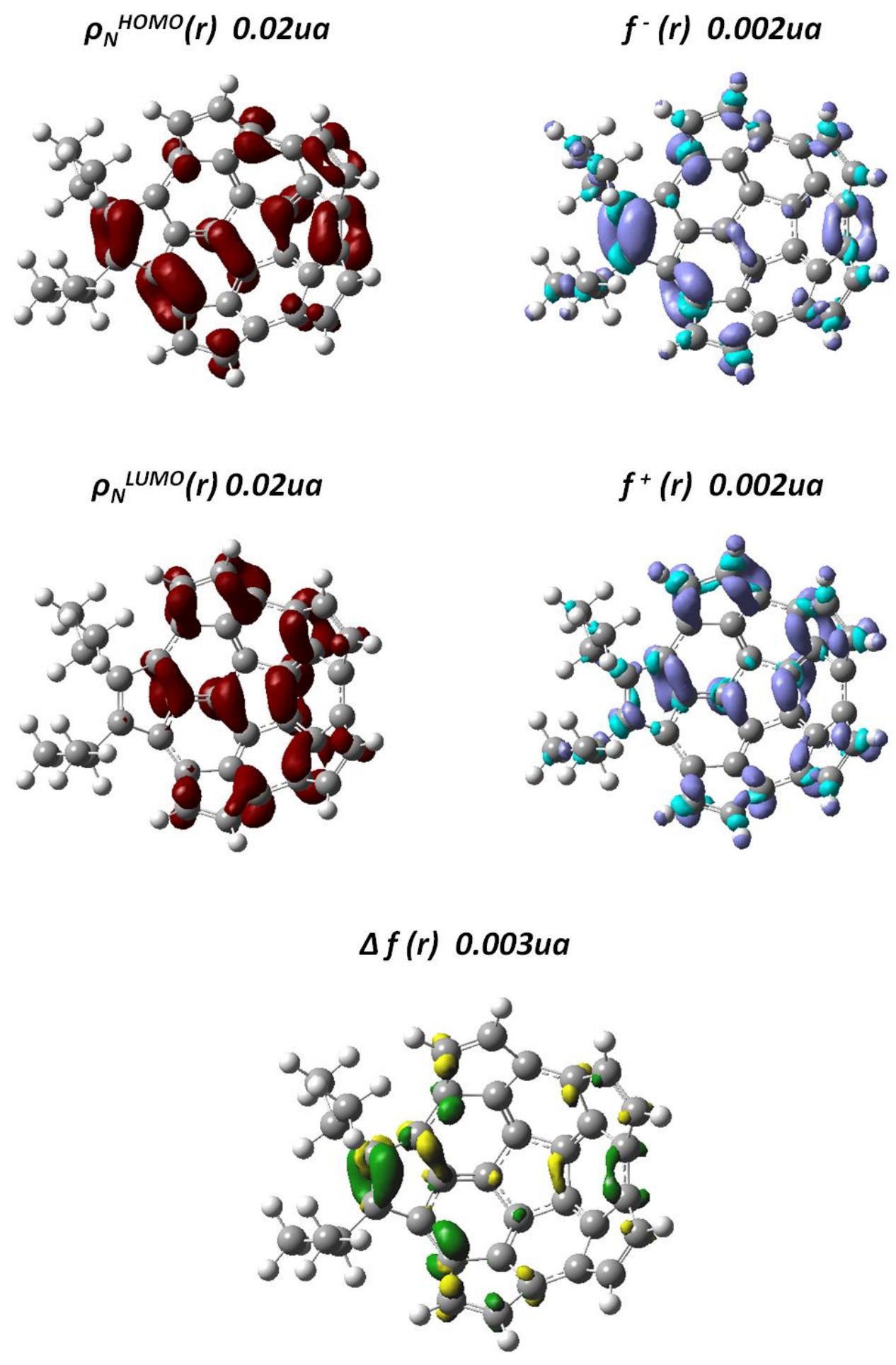
Fragmento 2 di-sustituido con Me
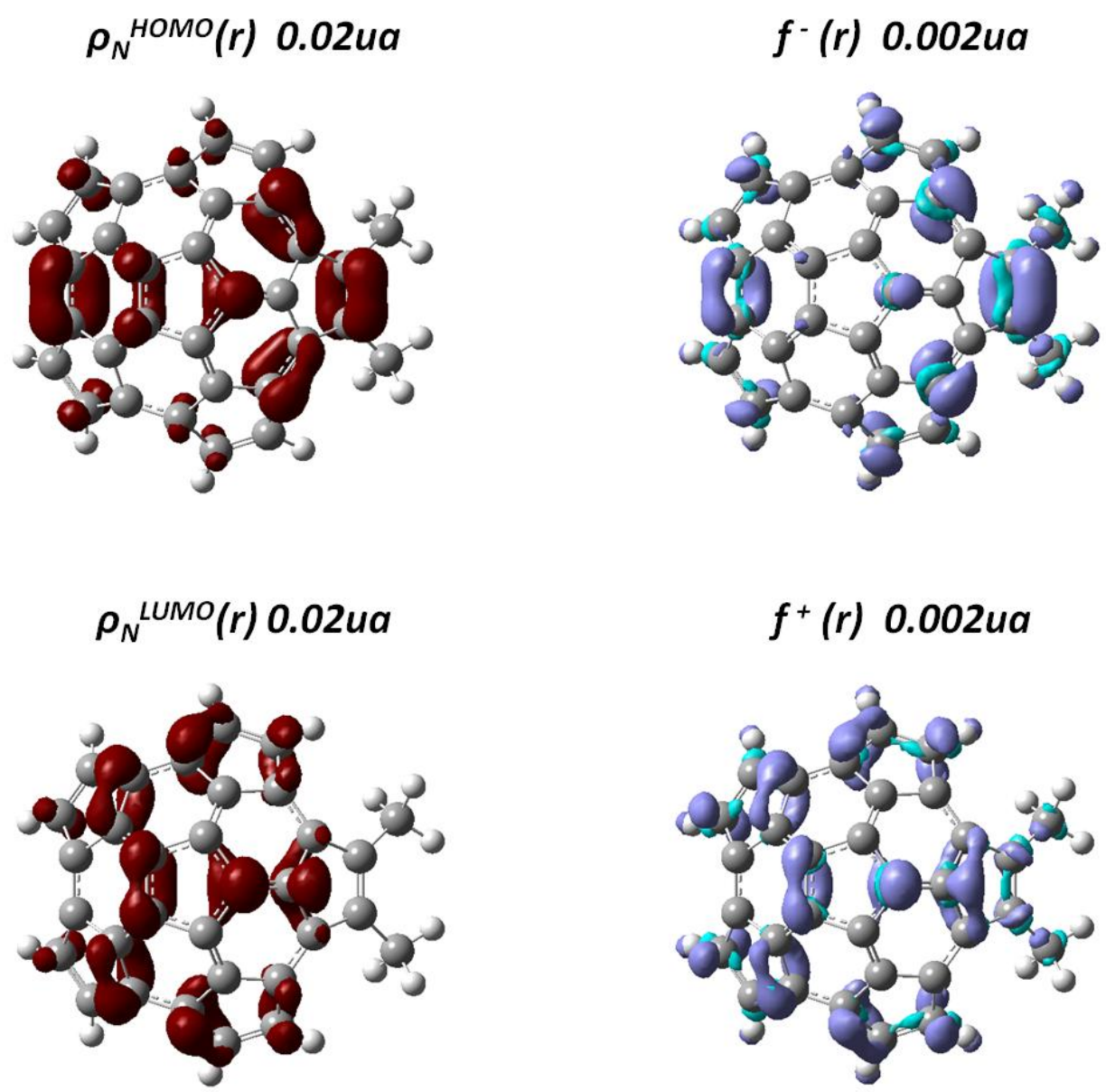

$\Delta f(r) 0.003 u a$

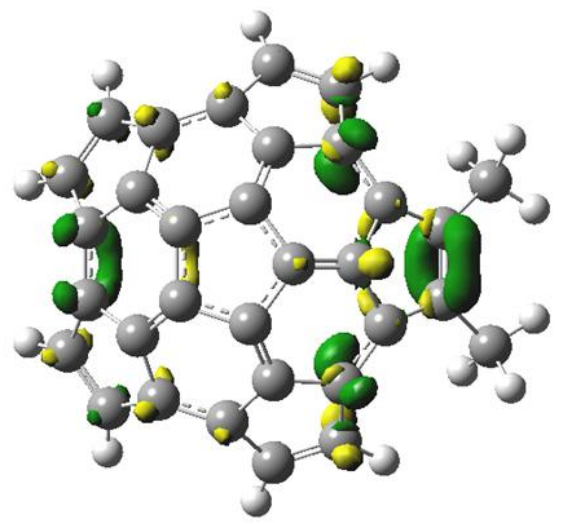


Fragmento 2 di-sustituido con $\mathrm{NH}_{2}$
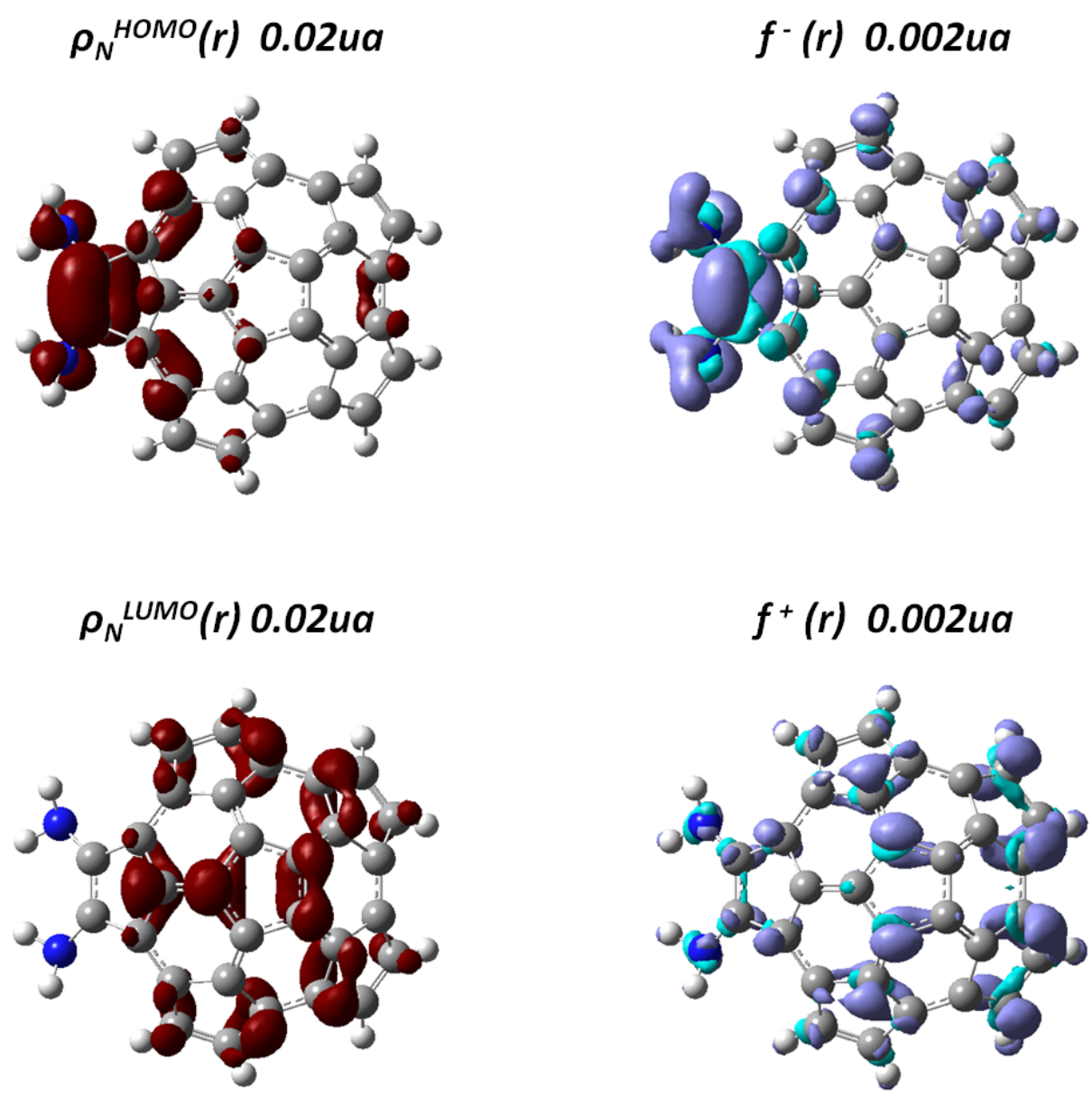

$\Delta f(r) 0.005 u a$

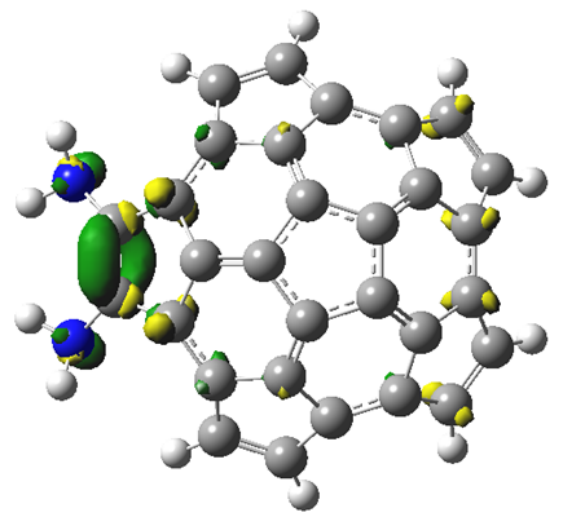


Fragmento 2 di-sustituido con $\mathrm{NO}_{2}$
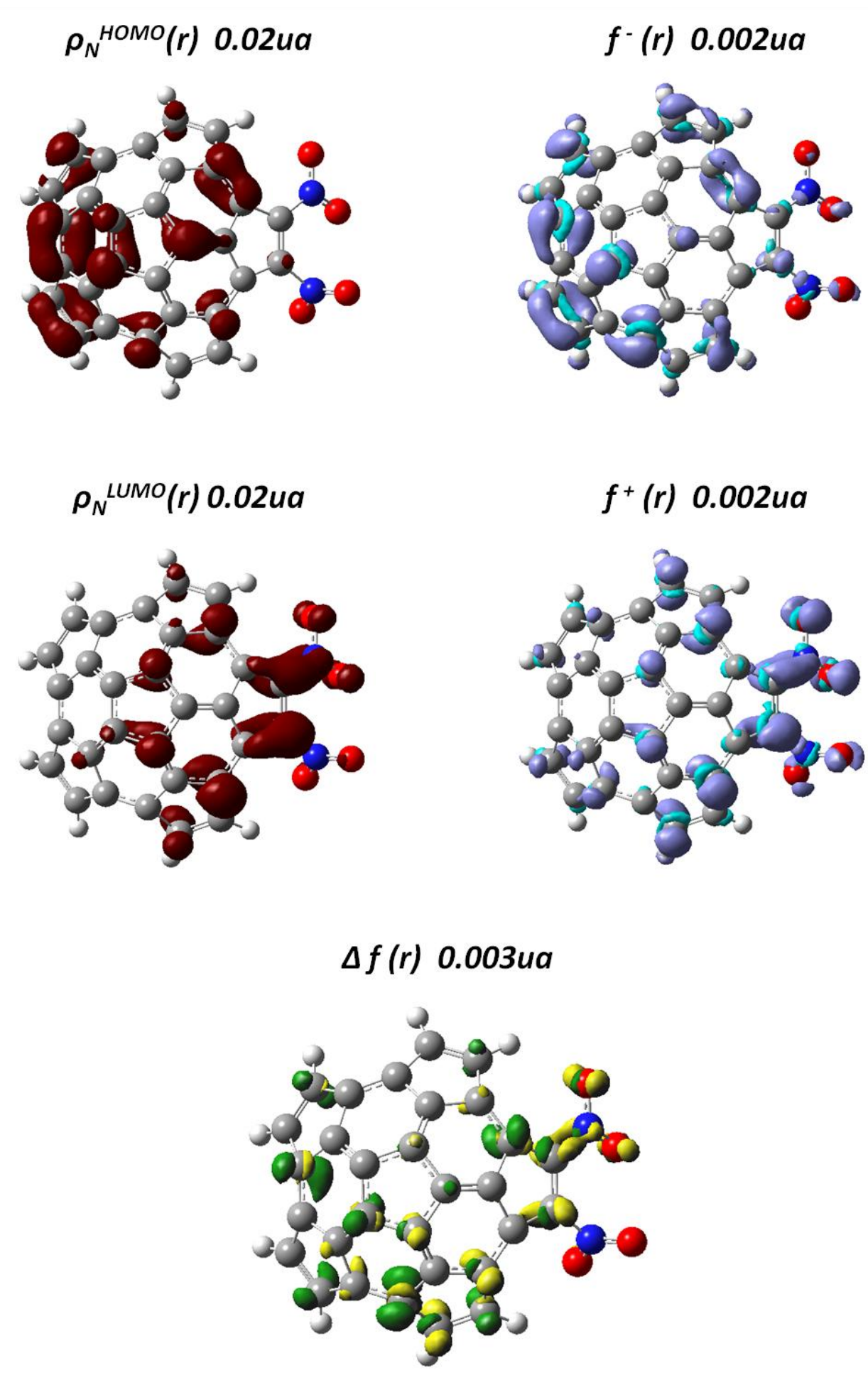
Fragmento 2 di-sustituido con $\mathrm{OH}$
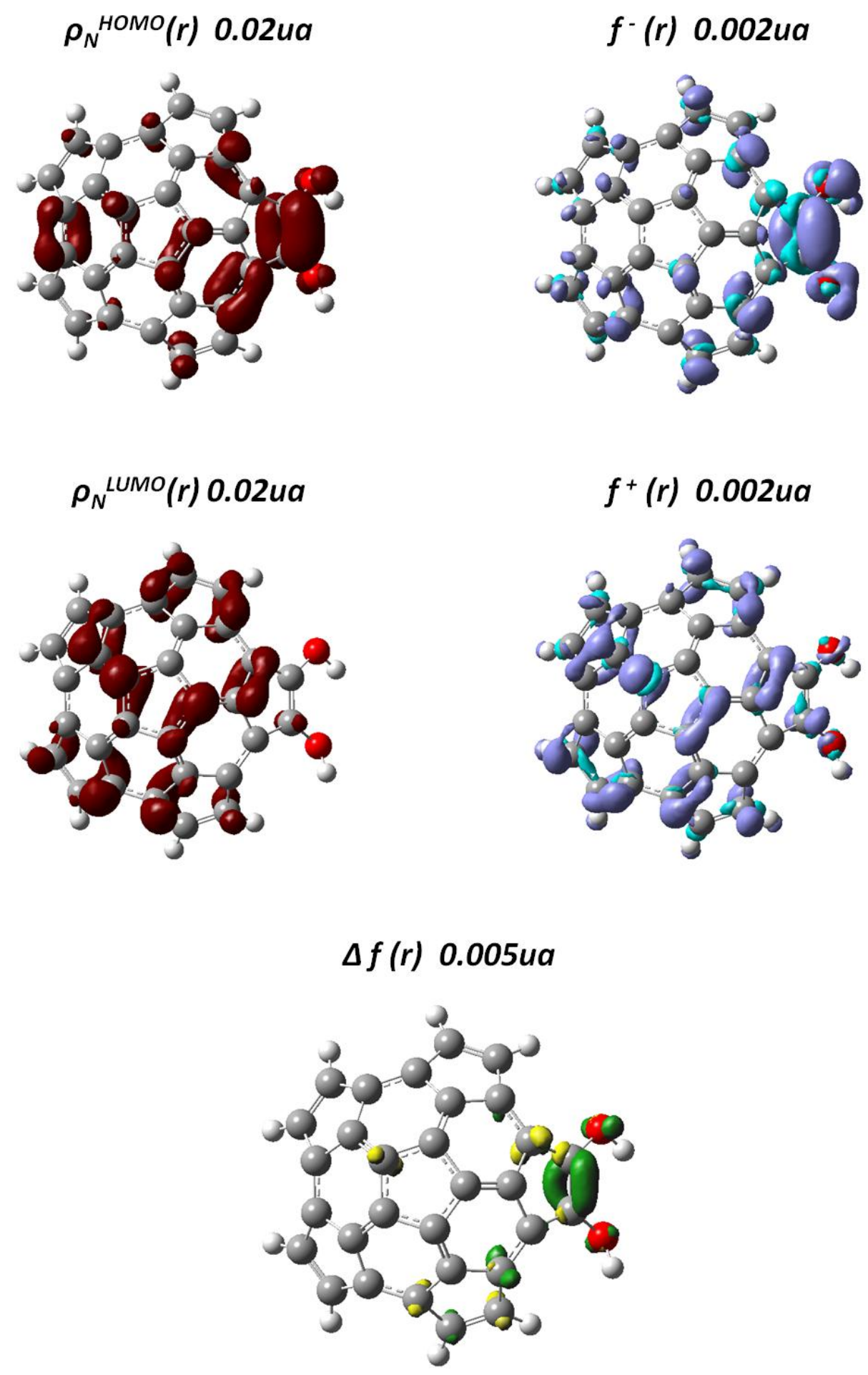
Fragmento 2 di-sustituido con OMe
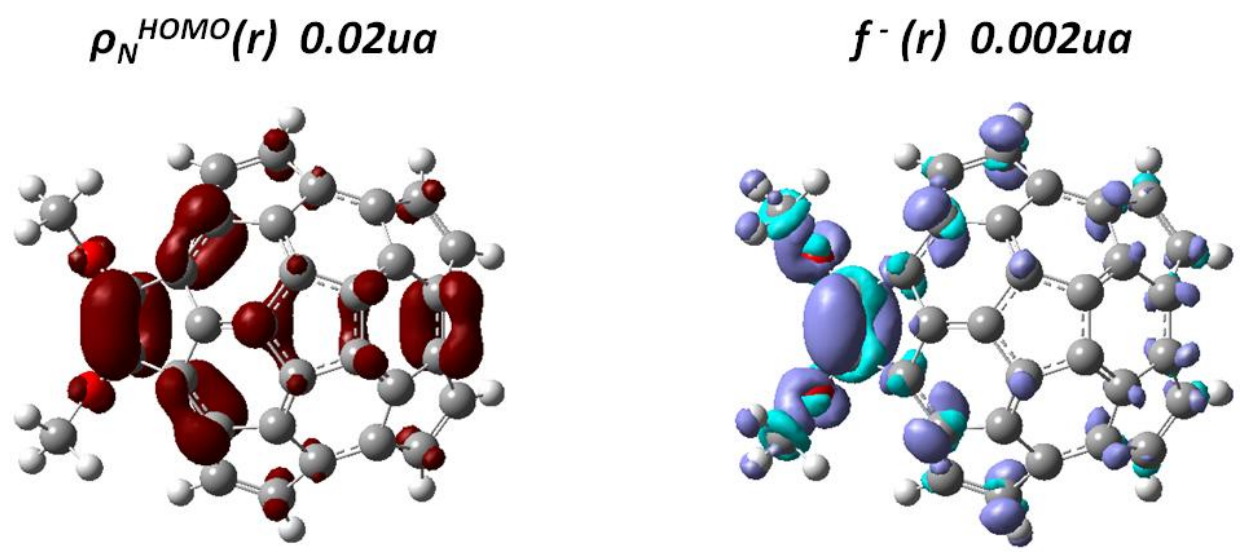

$$
\rho_{N}^{\text {LUMO }}(r) 0.02 u a
$$
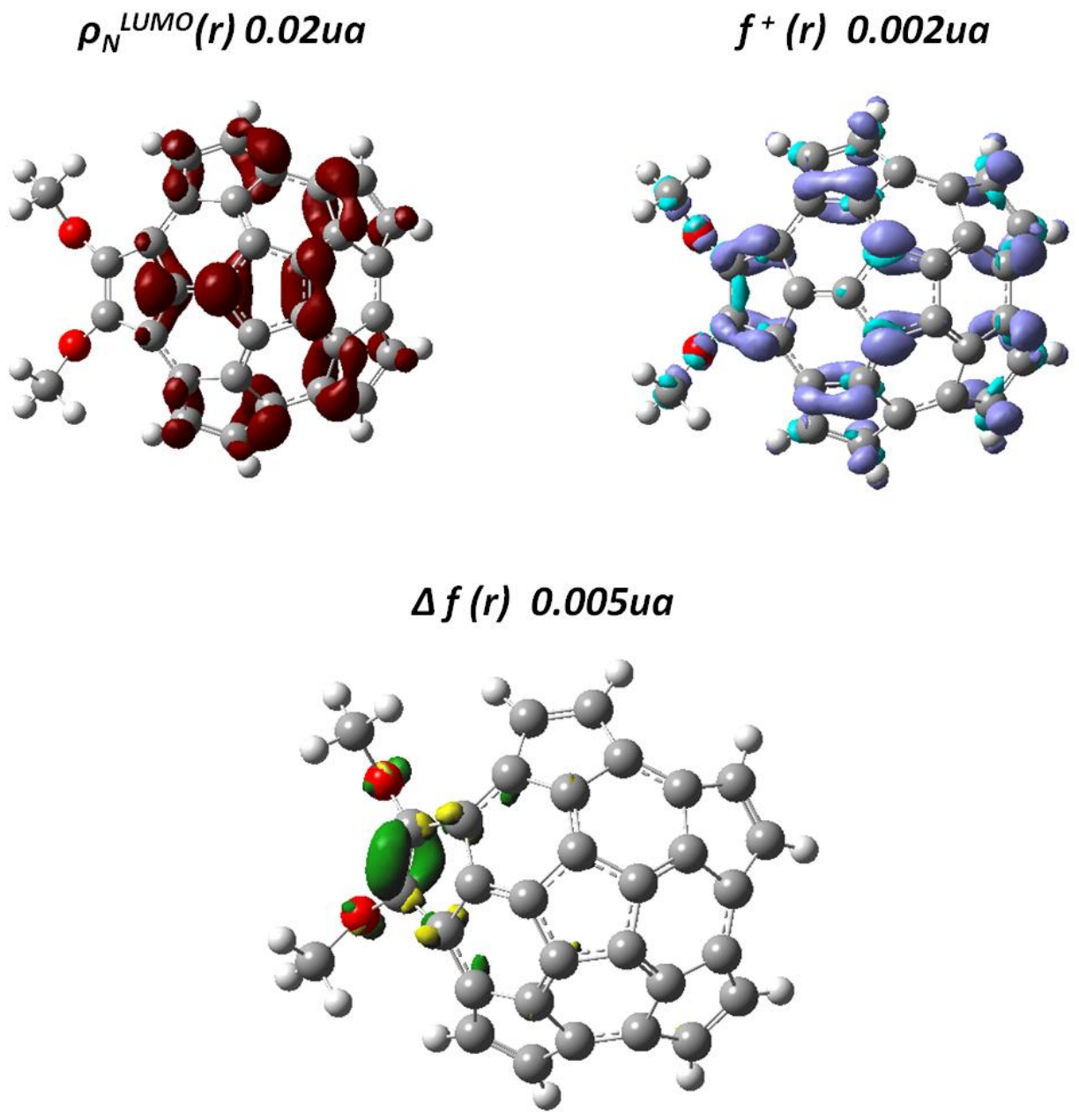


\section{Anexo 3. \\ Parámetros de reactividad}

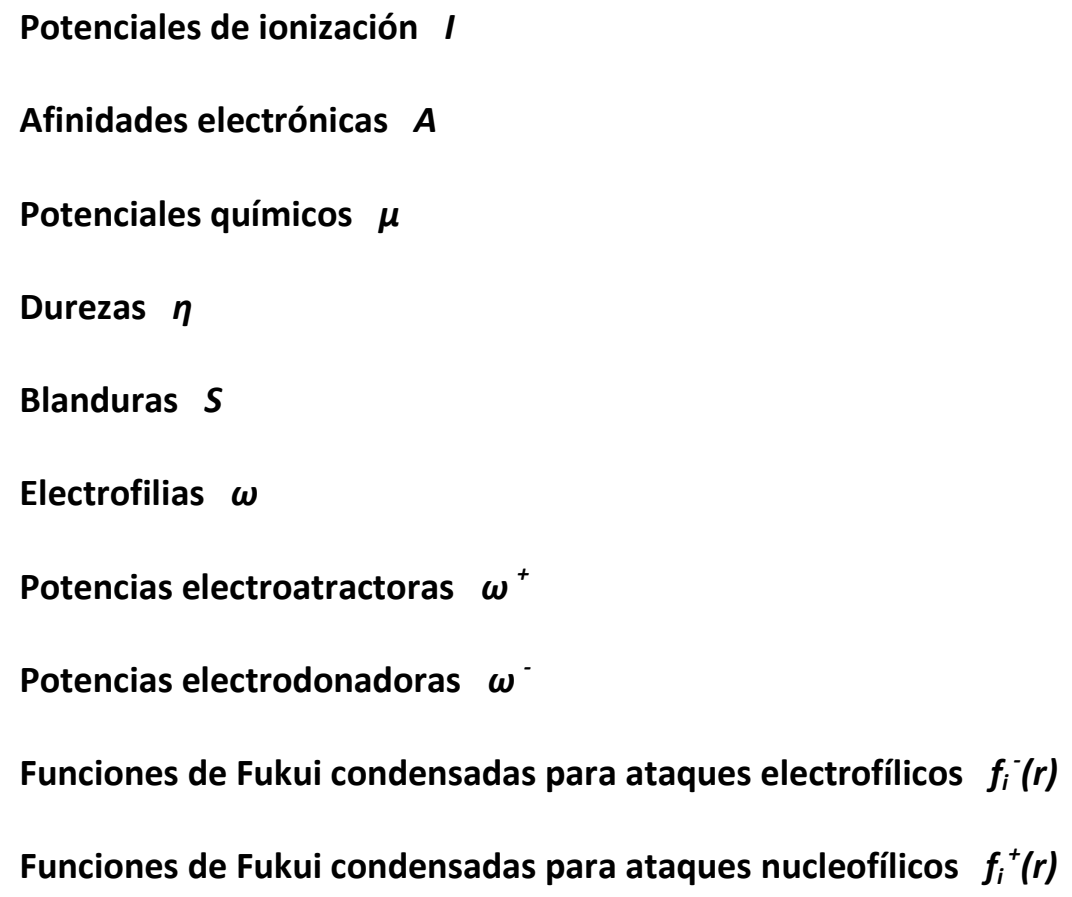




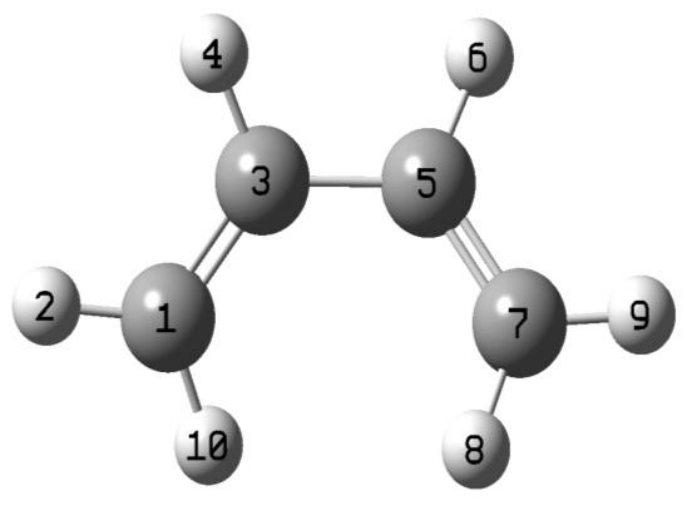

\section{Butadieno}

\begin{tabular}{cc}
\hline $\mathrm{I} \mathrm{eV}$ & $\mathbf{8 . 7 5}$ \\
$\mathrm{A} \mathrm{eV}$ & -1.68 \\
$\mu \mathrm{eV}$ & -3.54 \\
$\eta \mathrm{eV}$ & 10.43 \\
$\mathrm{~S} \mathrm{eV}$ & 0.10 \\
$\omega \mathrm{eV}$ & 0.60 \\
$\omega^{+} \mathrm{eV}$ & 0.80 \\
$\omega^{-} \mathrm{eV}$ & 3.62
\end{tabular}
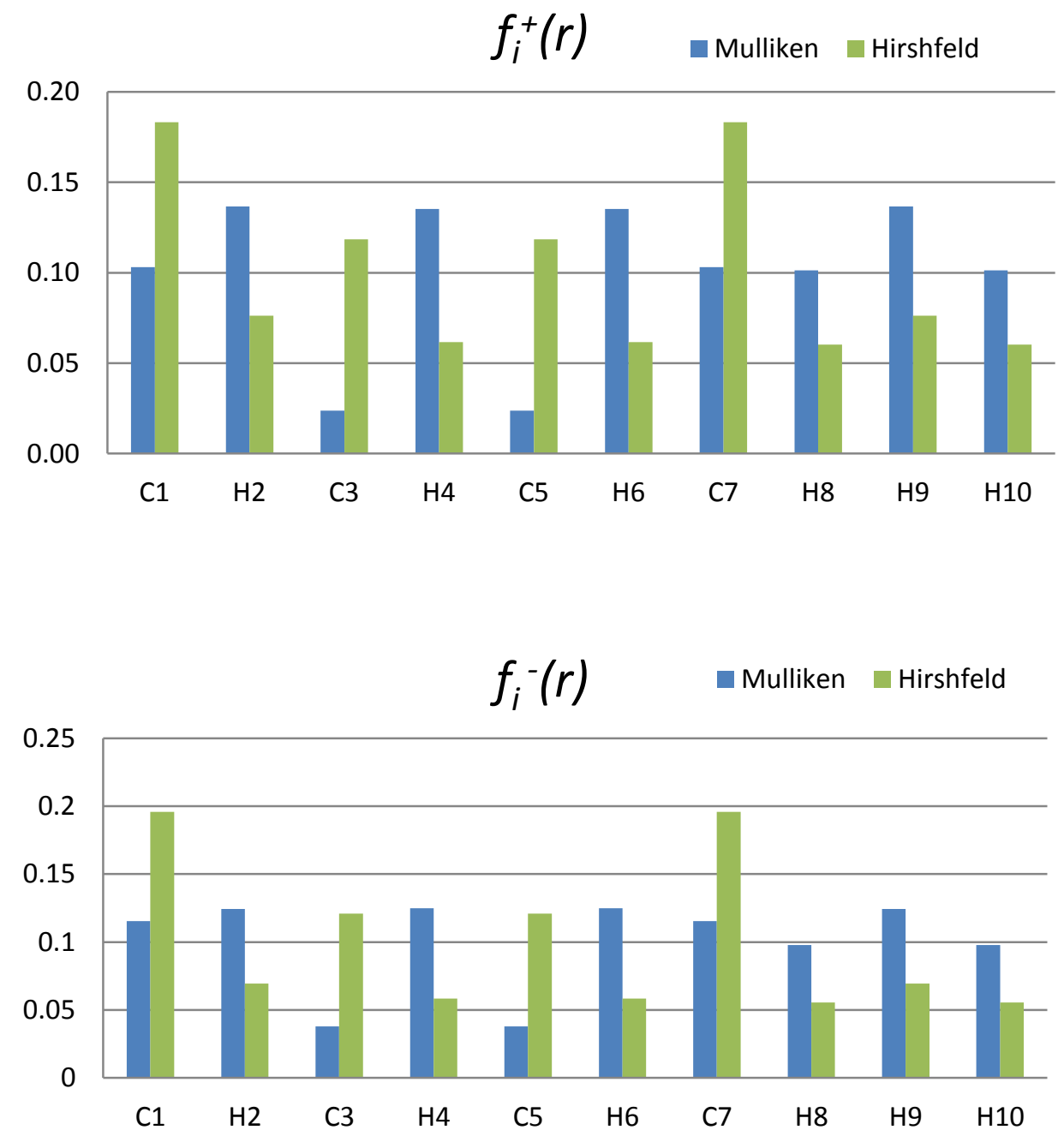


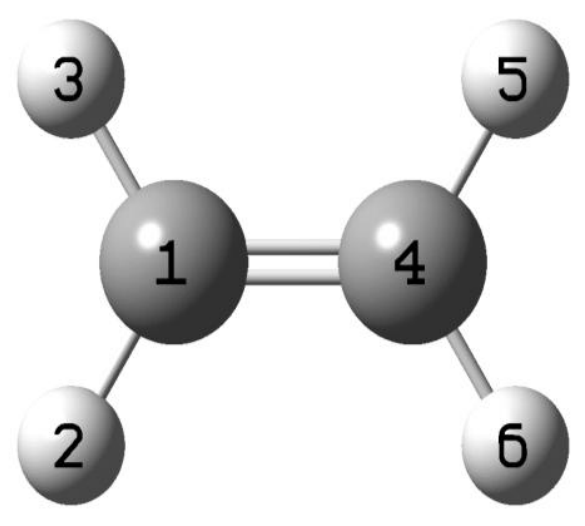

\section{Etileno}

\begin{tabular}{cc}
\hline $\mathrm{I} \mathrm{eV}$ & $\mathbf{1 0 . 3 8}$ \\
$\mathrm{A} \mathrm{eV}$ & -3.31 \\
$\mu \mathrm{eV}$ & -3.54 \\
$\eta \mathrm{eV}$ & 13.68 \\
$\mathrm{~S} \mathrm{eV}$ & 0.07 \\
$\omega \mathrm{eV}$ & 0.49 \\
$\omega^{+} \mathrm{eV}$ & 0.001 \\
$\omega^{-} \mathrm{eV}$ & 3.54
\end{tabular}

$f_{i}{ }^{+}(r) \quad$ Mulliken $\square$ Hirshfeld

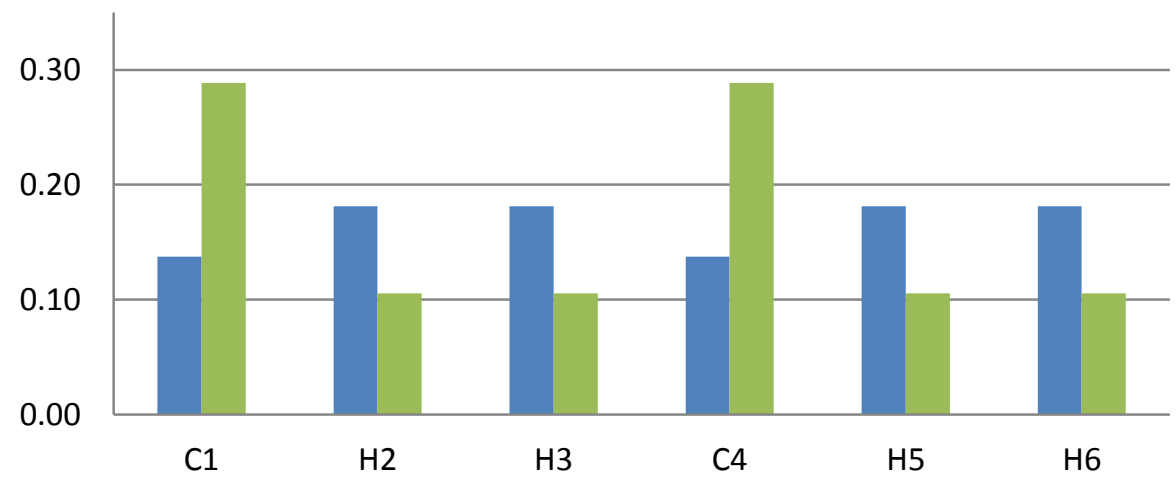

$f_{i}{ }^{-}(r) \quad$ Mulliken Hirshfeld

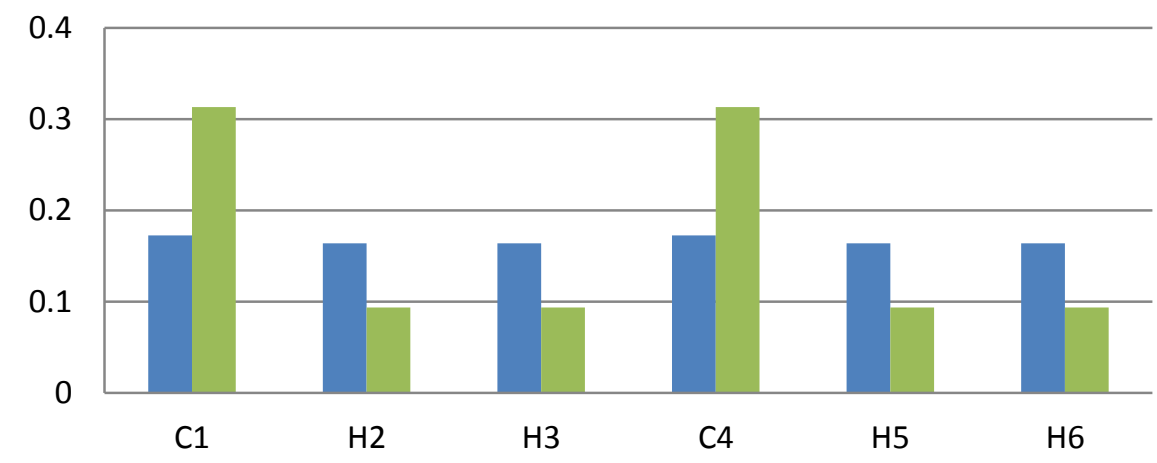




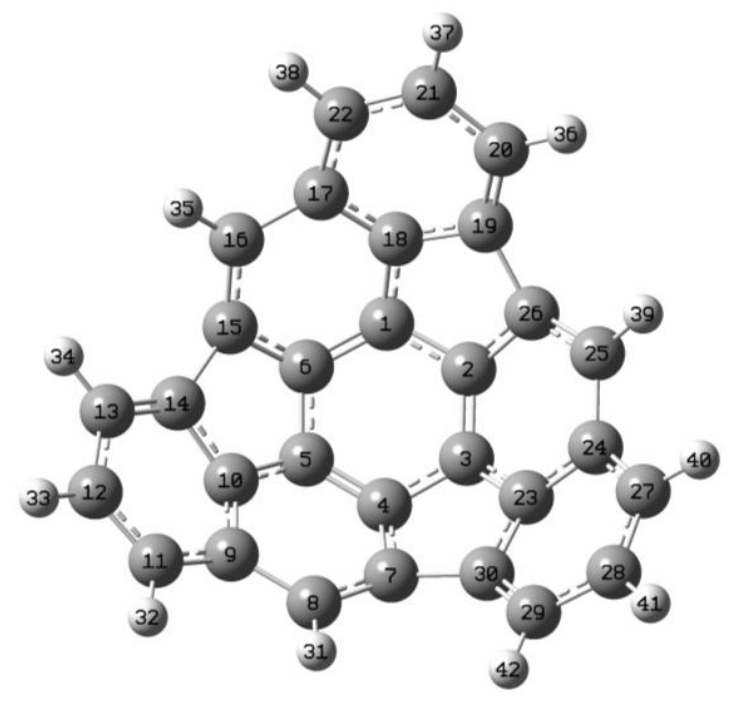

Fragmento 1

\begin{tabular}{cc}
\hline $\mathrm{I} \mathrm{eV}$ & $\mathbf{7 . 0 0}$ \\
$\mathrm{A} \mathrm{eV}$ & 0.60 \\
$\mu \mathrm{eV}$ & -3.80 \\
$\eta \mathrm{eV}$ & 6.39 \\
$\mathrm{~S} \mathrm{eV}$ & 0.16 \\
$\omega \mathrm{eV}$ & 1.13 \\
$\omega^{+} \mathrm{eV}$ & 0.76 \\
$\omega^{-} \mathrm{eV}$ & 4.56
\end{tabular}

0.06
0.05
0.04
0.03
0.02
0.01
0

$f_{i}^{+}(r)$

mulliken $\quad$ Hirshfeld

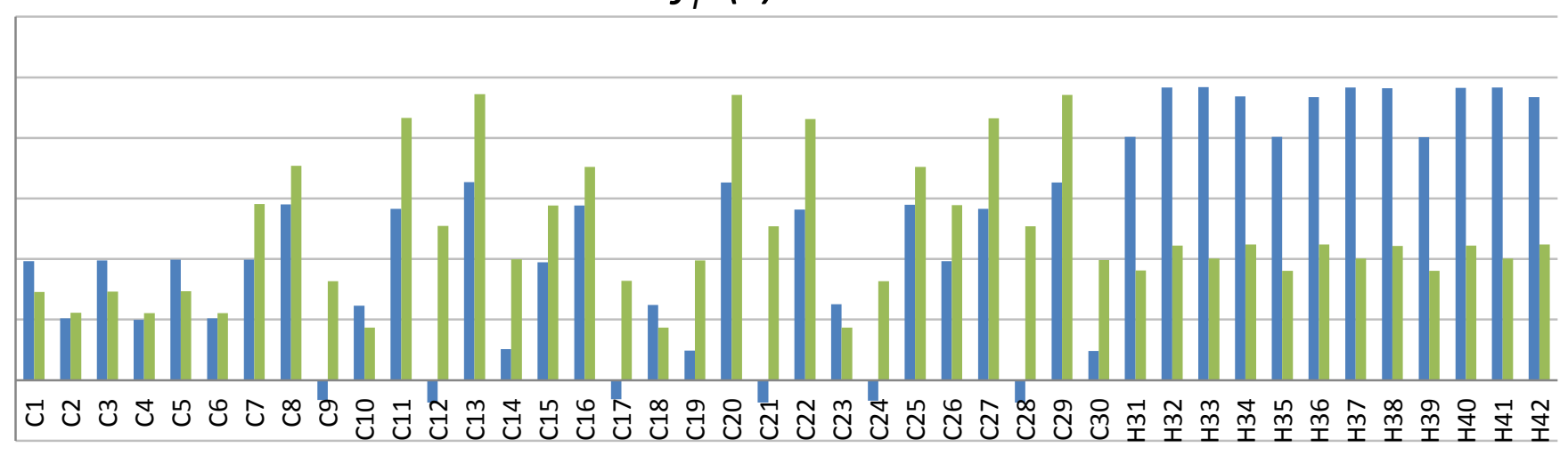

0.07
0.06
0.05
0.04
0.03
0.02
0.01
0
-0.01

$f_{i}^{-}(r)$

- Mulliken Hirshfeld

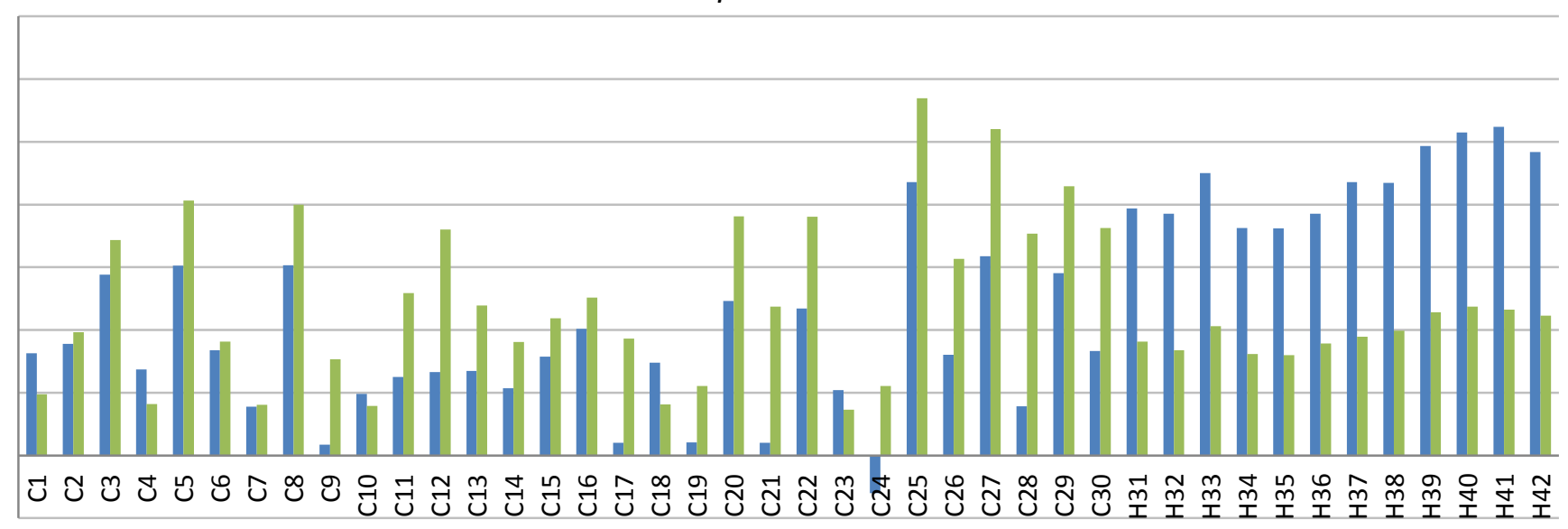




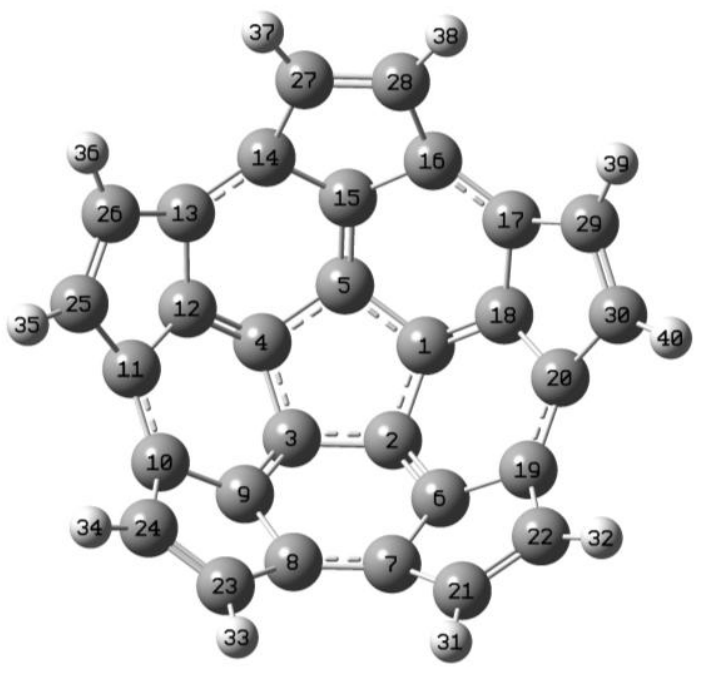

\section{Fragmento 2}

$\begin{array}{cc}\mathrm{I} \mathrm{eV} & 6.95 \\ \mathrm{~A} \mathrm{eV} & 1.09 \\ \mu \mathrm{eV} & -4.02 \\ \eta \mathrm{eV} & 5.86 \\ \mathrm{~S} \mathrm{eV} & 0.17 \\ \omega \mathrm{eV} & 1.38 \\ \omega^{+} \mathrm{eV} & 1.11 \\ \omega^{-} \mathrm{eV} & 5.13\end{array}$

0.06
0.05
0.04
0.03
0.02
0.01
0

$f_{i}^{+}(r)$

Mulliken Hirshfeld

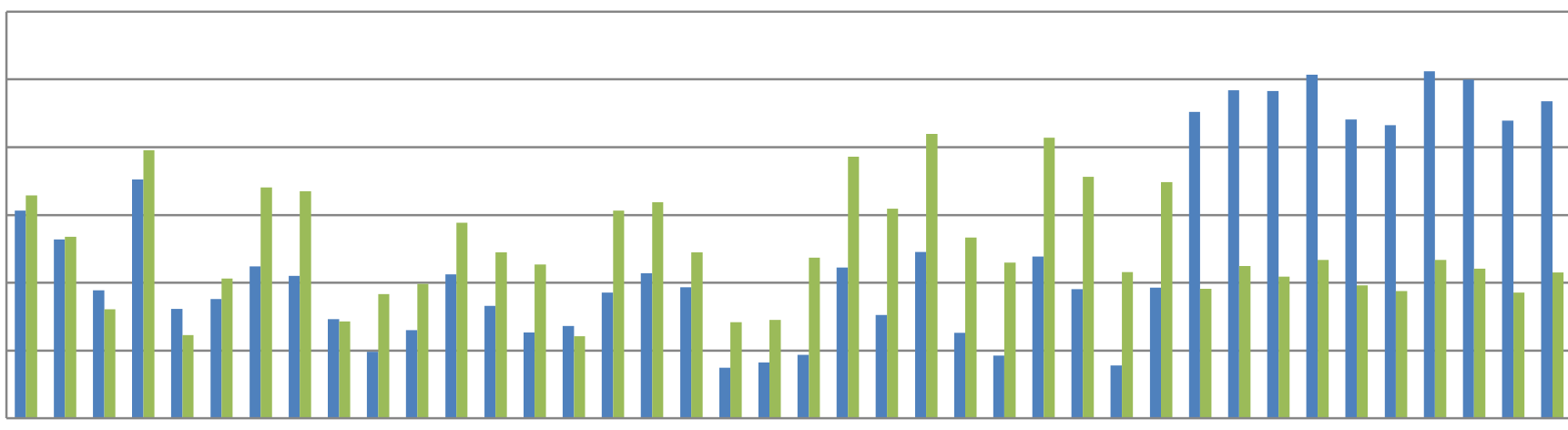

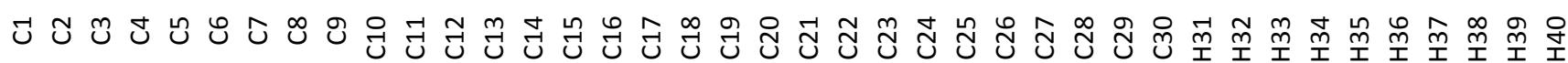

$f_{i}^{-}(r)$

Mulliken $\square$ Hirshfeld

0.06
0.05
0.04
0.03
0.02
0.01
0

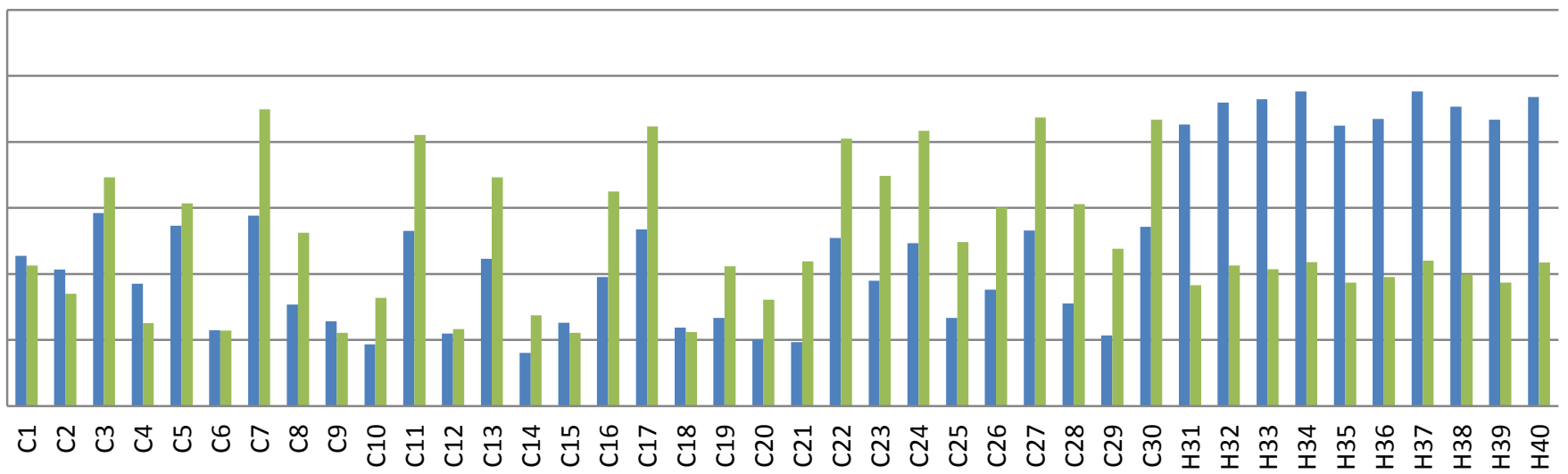




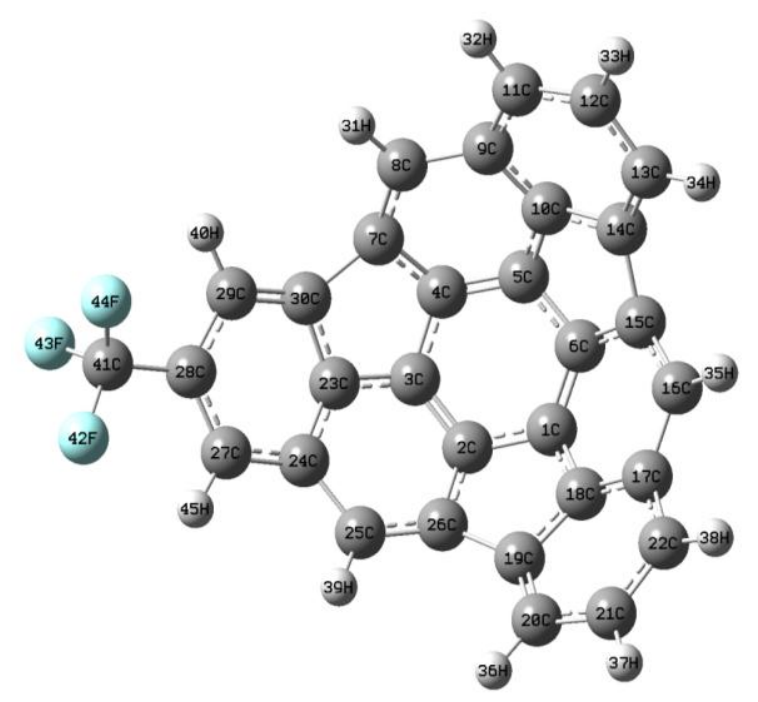

Fragmento 1 mono-sustituido con $\mathrm{CF}_{3}$

\begin{tabular}{cc}
\hline I eV & $\mathbf{7 . 1 8}$ \\
$\mathrm{A} \mathrm{eV}$ & 0.86 \\
$\mu \mathrm{eV}$ & -4.02 \\
$\eta \mathrm{eV}$ & 6.32 \\
$\mathrm{~S} \mathrm{eV}$ & 0.16 \\
$\omega \mathrm{eV}$ & 1.28 \\
$\omega^{+} \mathrm{eV}$ & 0.94 \\
$\omega^{-} \mathrm{eV}$ & 4.96
\end{tabular}

$\square f_{i}{ }^{+}(r) \quad \square f_{i}^{-}(r)$

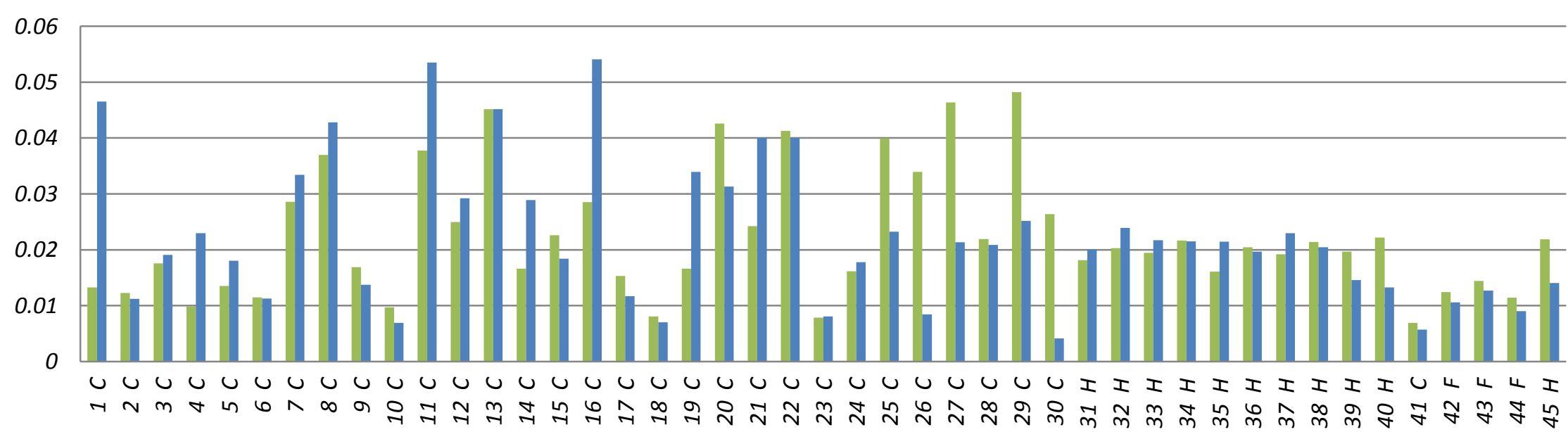




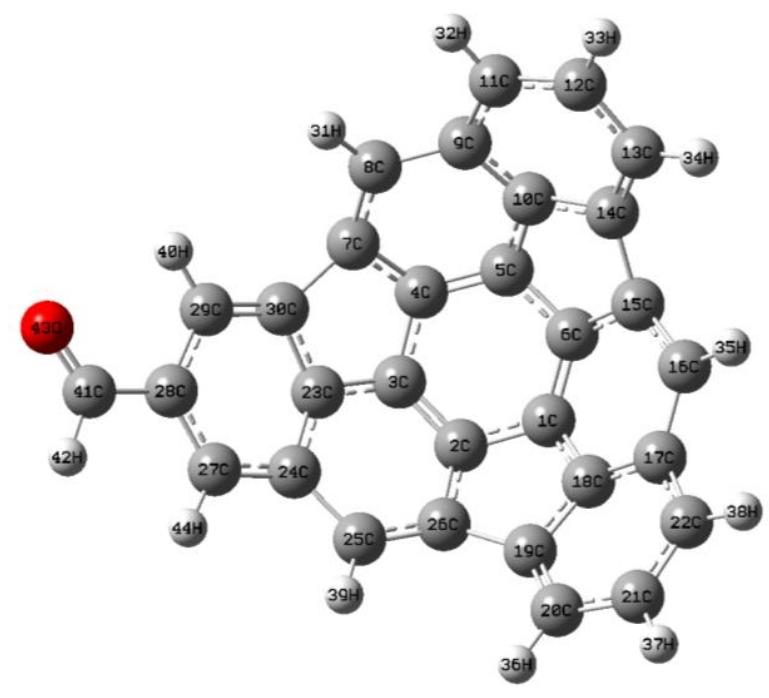

\section{Fragmento 1 mono-sustituido con $\mathrm{CHO}$}

\begin{tabular}{cc}
\hline $\mathrm{I} \mathrm{eV}$ & $\mathbf{7 . 1 8}$ \\
$\mathrm{A} \mathrm{eV}$ & 0.94 \\
$\mu \mathrm{eV}$ & -4.06 \\
$\eta \mathrm{eV}$ & 6.24 \\
$\mathrm{~S} \mathrm{eV}$ & 0.16 \\
$\omega \mathrm{eV}$ & 1.32 \\
$\omega^{+} \mathrm{eV}$ & 1.00 \\
$\omega^{-} \mathrm{eV}$ & 5.06
\end{tabular}

$\square f_{i}{ }^{+}(r) \quad \square f_{i}^{-}(r)$

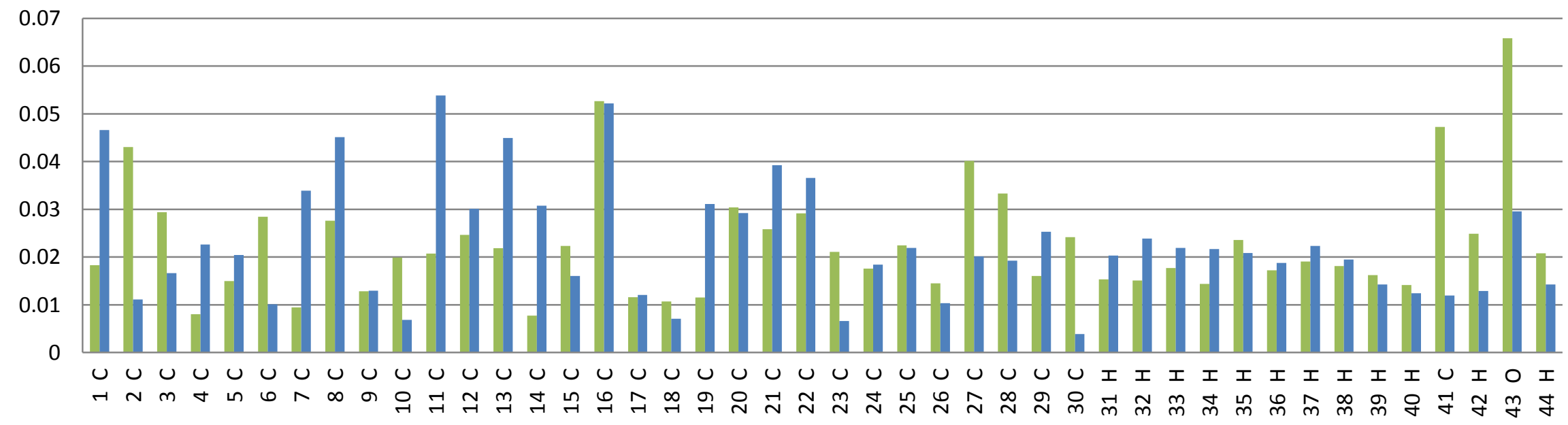




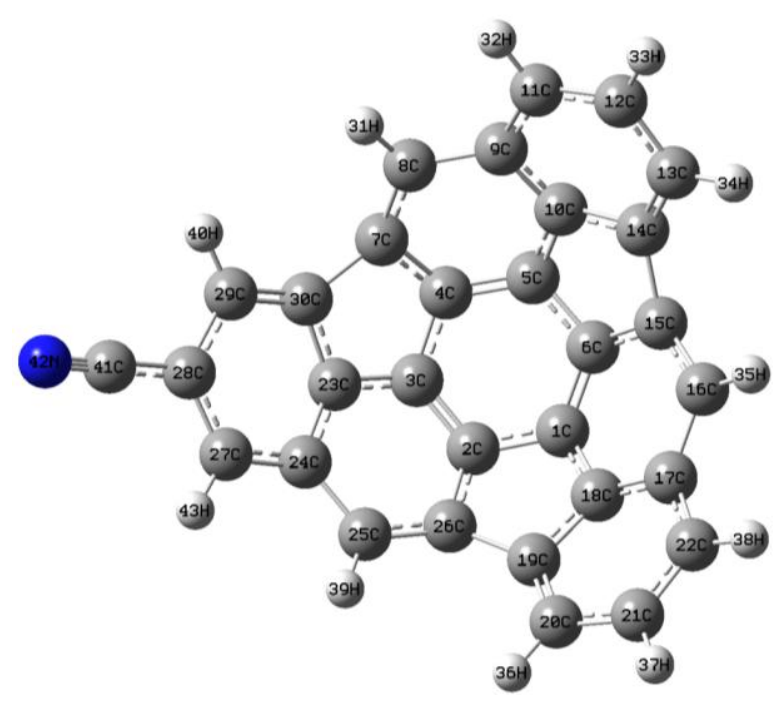

\section{Fragmento 1 mono-sustituido con $\mathrm{CN}$}

$\begin{array}{cc}\mathrm{I} \mathrm{eV} & \mathbf{7 . 2 8} \\ \mathrm{A} \mathrm{eV} & 1.03 \\ \mu \mathrm{eV} & -4.15 \\ \eta \mathrm{eV} & 6.25 \\ \mathrm{~S} \mathrm{eV} & 0.16 \\ \omega \mathrm{eV} & 1.38 \\ \omega^{+} \mathrm{eV} & 1.07 \\ \omega^{-} \mathrm{eV} & 5.23\end{array}$

\section{$\square f_{i}^{+}(r) \quad \square f_{i}^{-}(r)$}

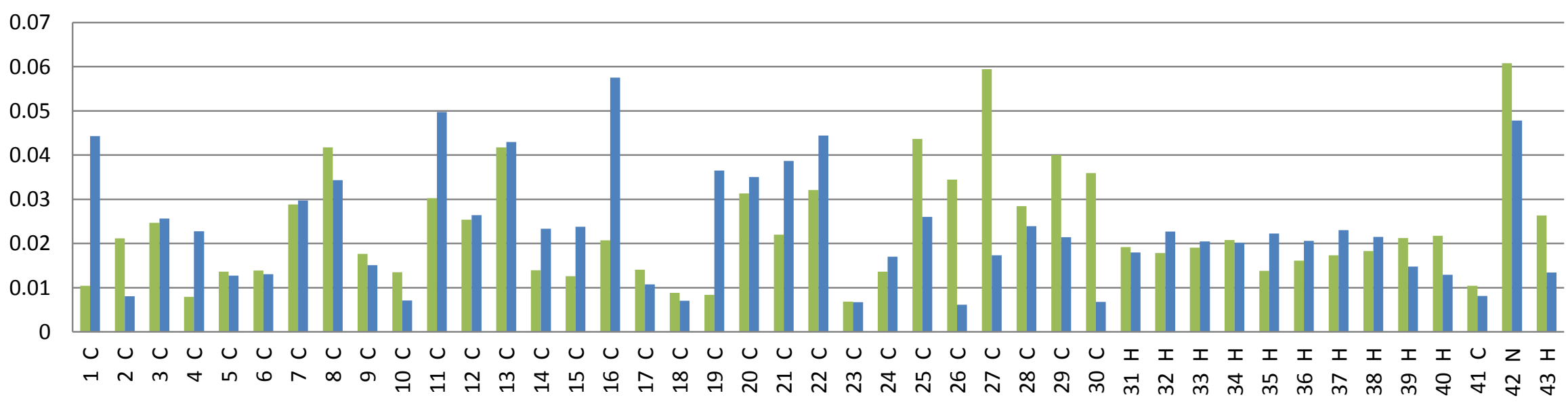




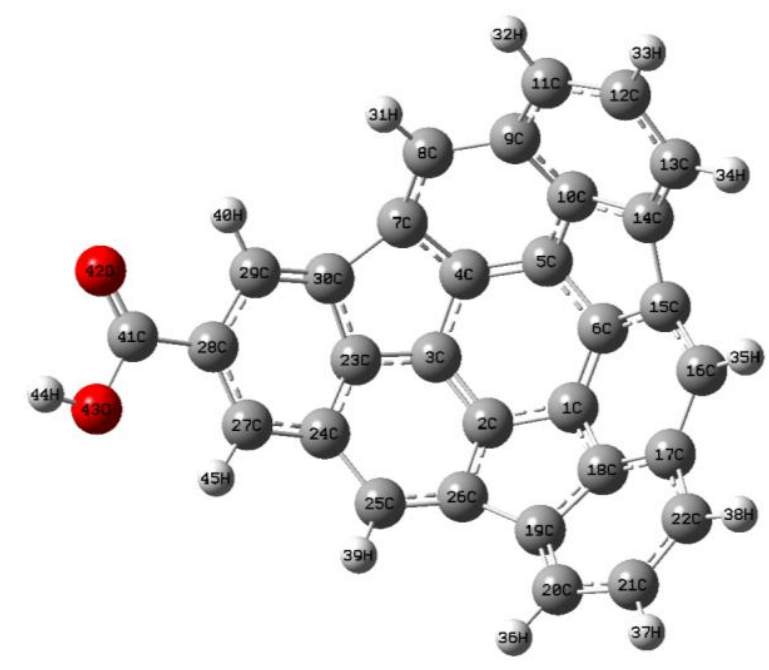

Fragmento 1 mono-sustituido con $\mathrm{COOH}$

\begin{tabular}{cc}
\hline $\mathrm{I} \mathrm{eV}$ & $\mathbf{7 . 1 2}$ \\
$\mathrm{A} \mathrm{eV}$ & 0.82 \\
$\mu \mathrm{eV}$ & -3.97 \\
$\eta \mathrm{eV}$ & 6.29 \\
$\mathrm{~S} \mathrm{eV}$ & 0.16 \\
$\omega \mathrm{eV}$ & 1.25 \\
$\omega^{+} \mathrm{eV}$ & 0.91 \\
$\omega^{-} \mathrm{eV}$ & 4.88
\end{tabular}

$\square f_{i}^{+}(r) \quad \square f_{i}^{-}(r)$

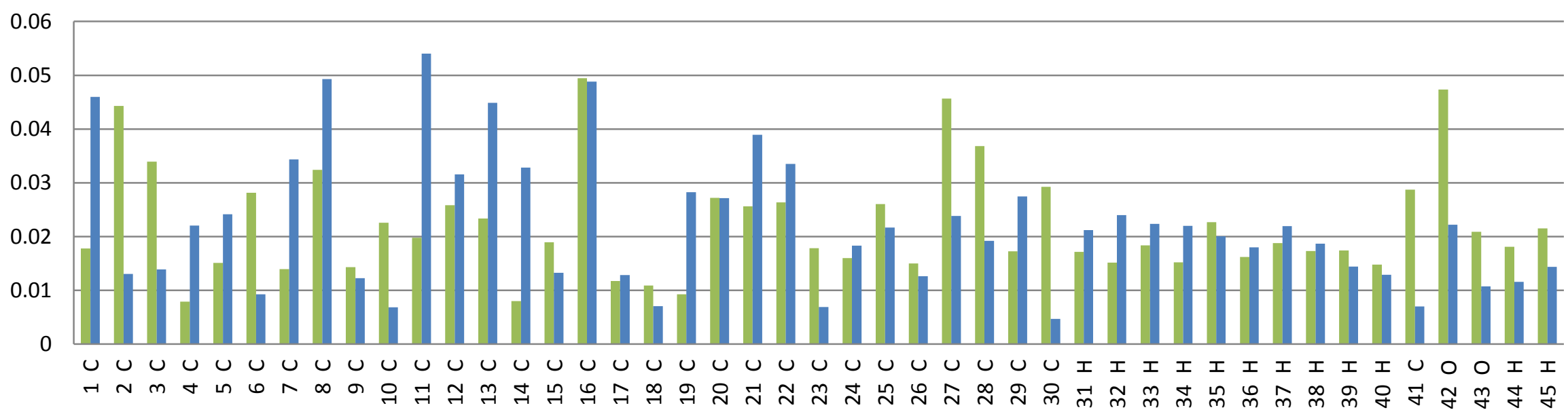




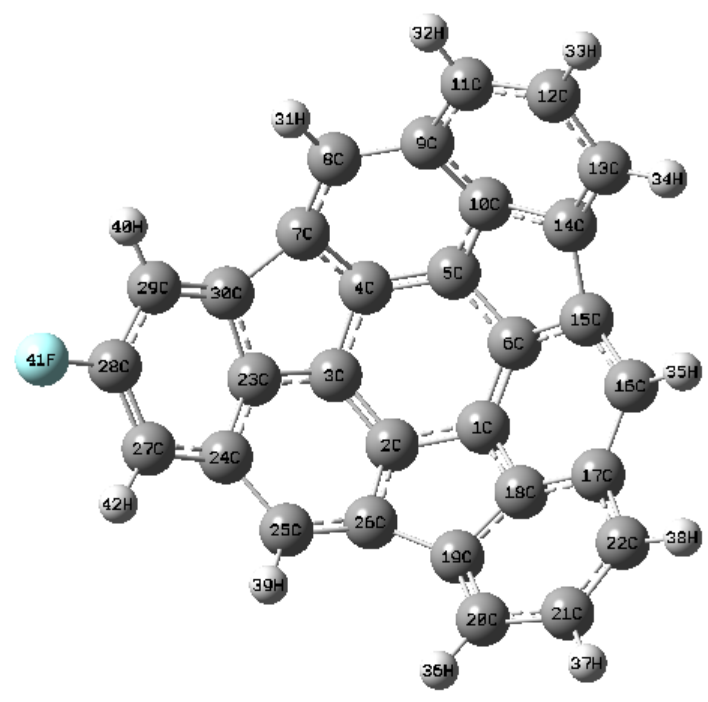

Fragmento 1 mono-sustituido con $\mathrm{F}$

\begin{tabular}{cc}
\hline $\mathrm{I} \mathrm{eV}$ & $\mathbf{7 . 0 6}$ \\
$\mathrm{A} \mathrm{eV}$ & 0.76 \\
$\mu \mathrm{eV}$ & -3.91 \\
$\eta \mathrm{eV}$ & 6.30 \\
$\mathrm{~S} \mathrm{eV}$ & 0.16 \\
$\omega \mathrm{eV}$ & 1.21 \\
$\omega^{+} \mathrm{eV}$ & 0.86 \\
$\omega^{-} \mathrm{eV}$ & 4.77
\end{tabular}

$\square f_{i}{ }^{+}(r) \quad \square f_{i}^{-}(r)$

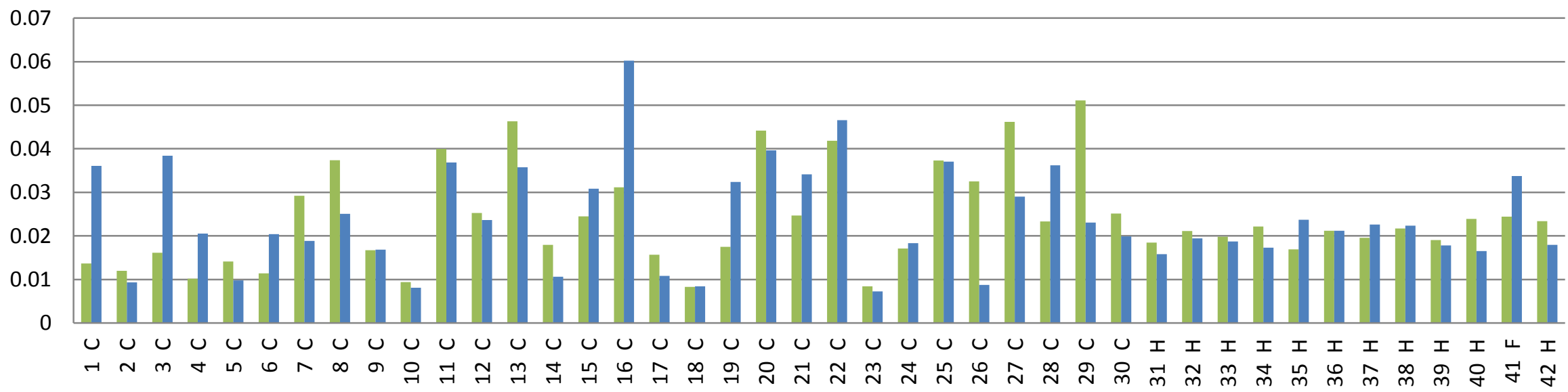




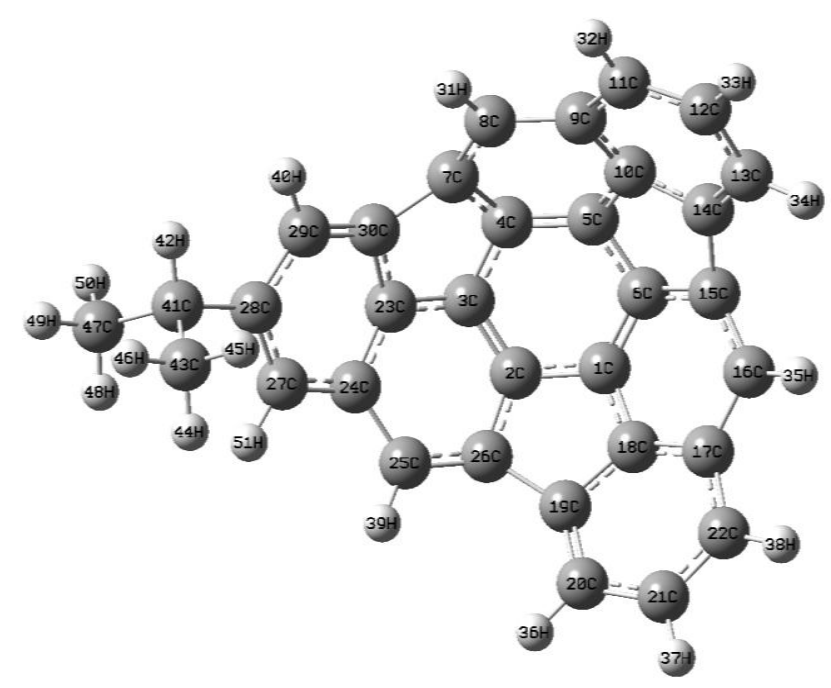

\section{Fragmento 1 mono-sustituido con $i-\mathrm{Pr}$}

\begin{tabular}{cc}
\hline $\mathrm{l} \mathrm{eV}$ & $\mathbf{6 . 8 8}$ \\
$\mathrm{A} \mathrm{eV}$ & 0.62 \\
$\mu \mathrm{eV}$ & -3.75 \\
$\eta \mathrm{eV}$ & 6.26 \\
$\mathrm{~S} \mathrm{eV}$ & 0.16 \\
$\omega \mathrm{eV}$ & 1.12 \\
$\omega^{+} \mathrm{eV}$ & 0.76 \\
$\omega^{-} \mathrm{eV}$ & 4.51
\end{tabular}

\section{$\quad f_{i}{ }^{+}(r) \quad \square f_{i}^{-}(r)$}

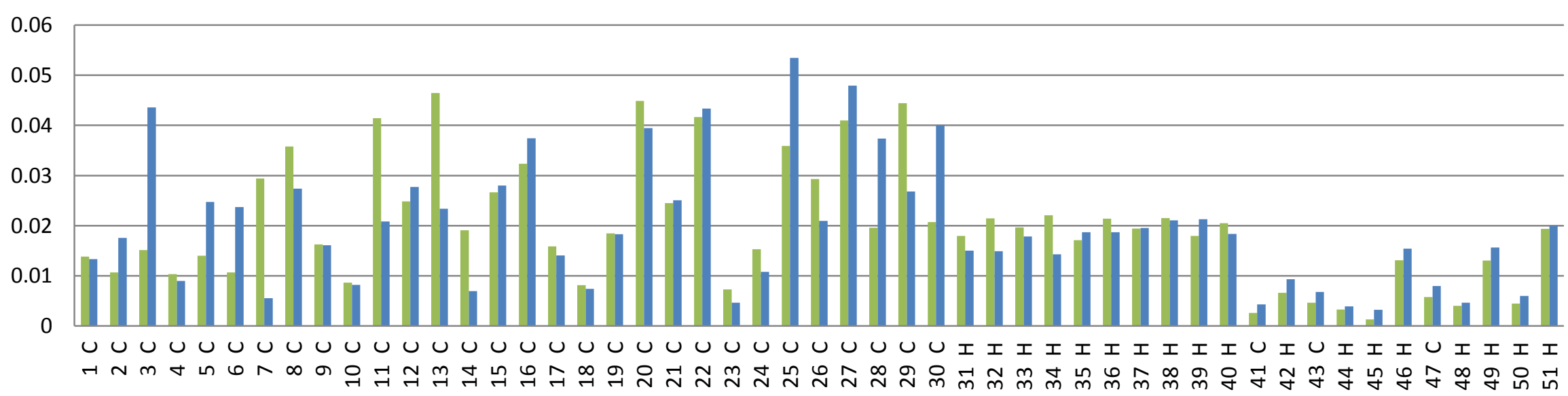




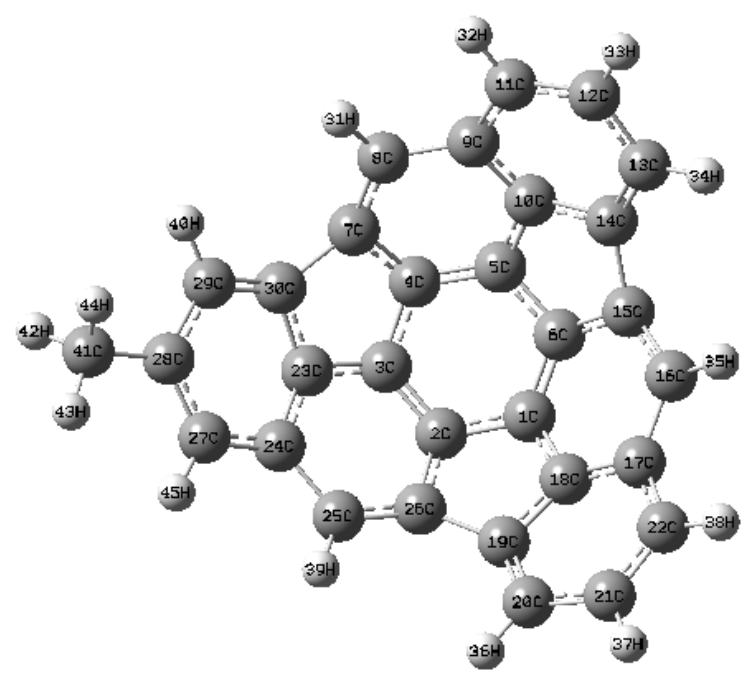

\section{Fragmento 1 mono-sustituido con Me}

\begin{tabular}{cc}
\hline $\mathrm{I} \mathrm{eV}$ & $\mathbf{6 . 9 2}$ \\
$\mathrm{A} \mathrm{eV}$ & 0.60 \\
$\mu \mathrm{eV}$ & -3.76 \\
$\eta \mathrm{eV}$ & 6.32 \\
$\mathrm{~S} \mathrm{eV}$ & 0.16 \\
$\omega \mathrm{eV}$ & 1.12 \\
$\omega^{+} \mathrm{eV}$ & 0.75 \\
$\omega^{-} \mathrm{eV}$ & 4.51
\end{tabular}

\section{$\square f_{i}{ }^{+}(r) \quad \square f_{i}^{-}(r)$}

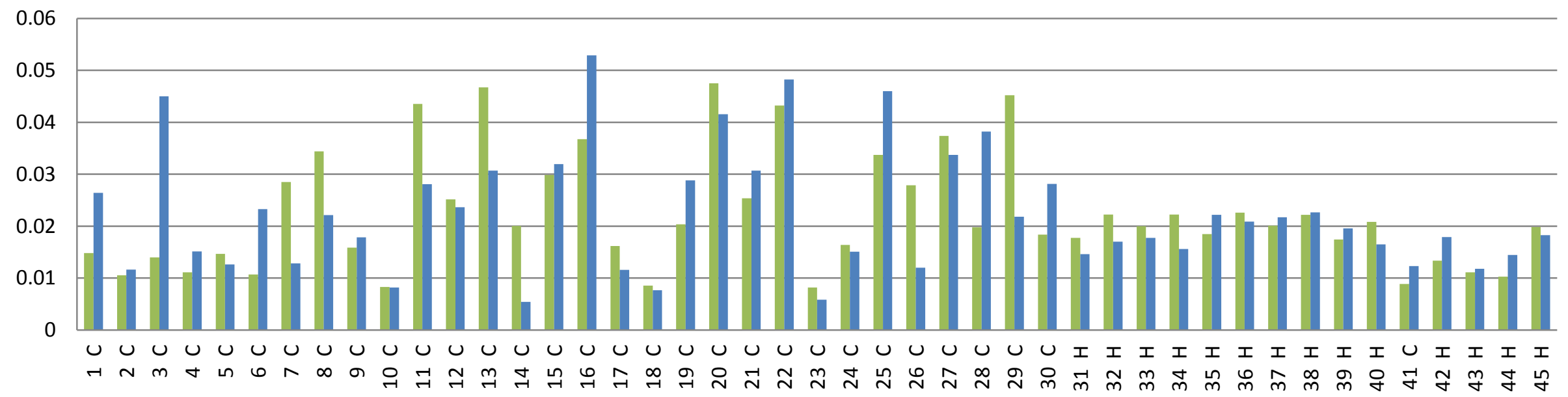




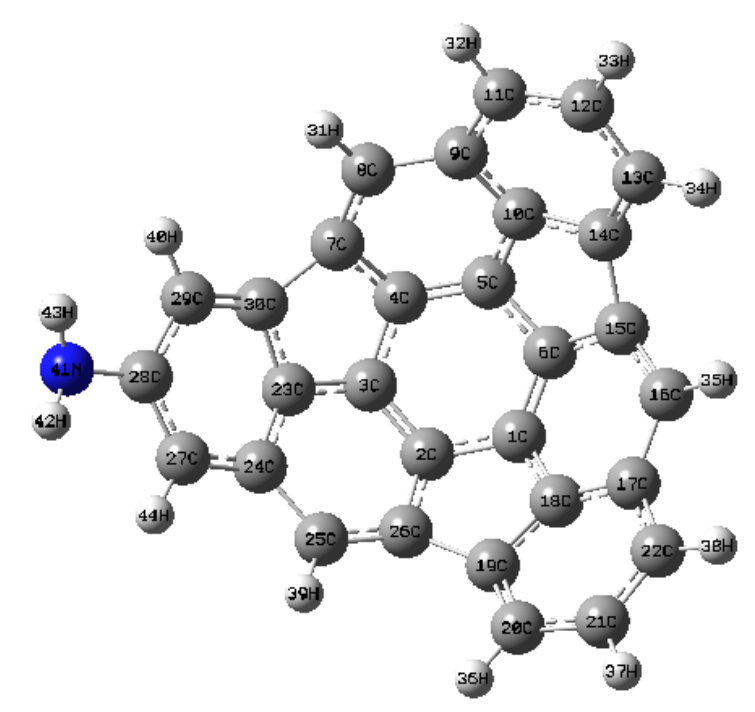

Fragmento 1 mono-sustituido con $\mathrm{NH}_{2}$

\begin{tabular}{cc}
\hline $\mathrm{I} \mathrm{eV}$ & $\mathbf{6 . 5 6}$ \\
$\mathrm{A} \mathrm{eV}$ & 0.55 \\
$\mu \mathrm{eV}$ & -3.55 \\
$\eta \mathrm{eV}$ & 6.01 \\
$\mathrm{~S} \mathrm{eV}$ & 0.17 \\
$\omega \mathrm{eV}$ & 1.05 \\
$\omega^{+} \mathrm{eV}$ & 0.70 \\
$\omega^{-} \mathrm{eV}$ & 4.25
\end{tabular}

$\square f_{i}{ }^{+}(r) \quad \square f_{i}{ }^{-}(r)$

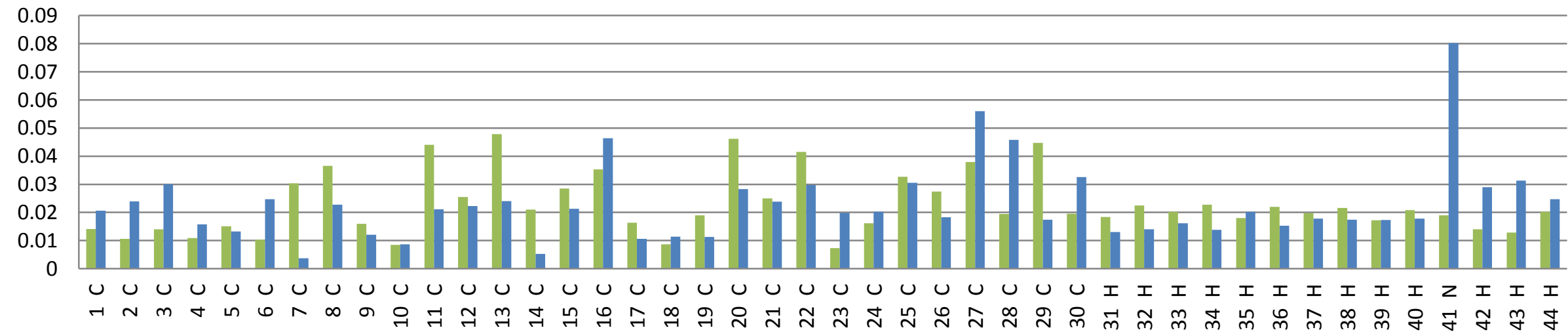




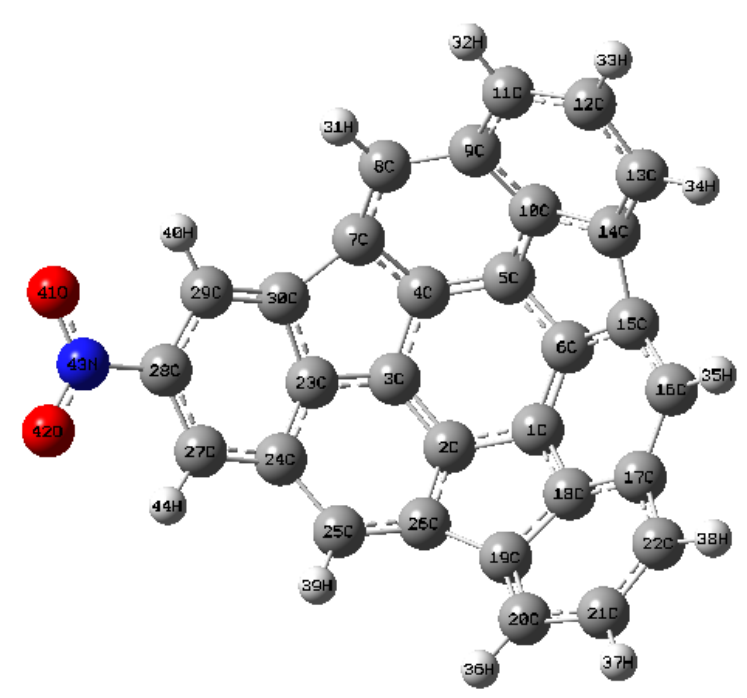

Fragmento 1 mono-sustituido con $\mathrm{NO}_{2}$

\begin{tabular}{cc}
\hline $\mathrm{I} \mathrm{eV}$ & $\mathbf{7 . 3 2}$ \\
$\mathrm{A} \mathrm{eV}$ & 1.23 \\
$\mu \mathrm{eV}$ & -4.28 \\
$\eta \mathrm{eV}$ & 6.09 \\
$\mathrm{~S} \mathrm{eV}$ & 0.16 \\
$\omega \mathrm{eV}$ & 1.50 \\
$\omega^{+} \mathrm{eV}$ & 1.24 \\
$\omega^{-} \mathrm{eV}$ & 5.52
\end{tabular}

$\square f_{i}^{+}(r) \quad \square f_{i}^{-}(r)$

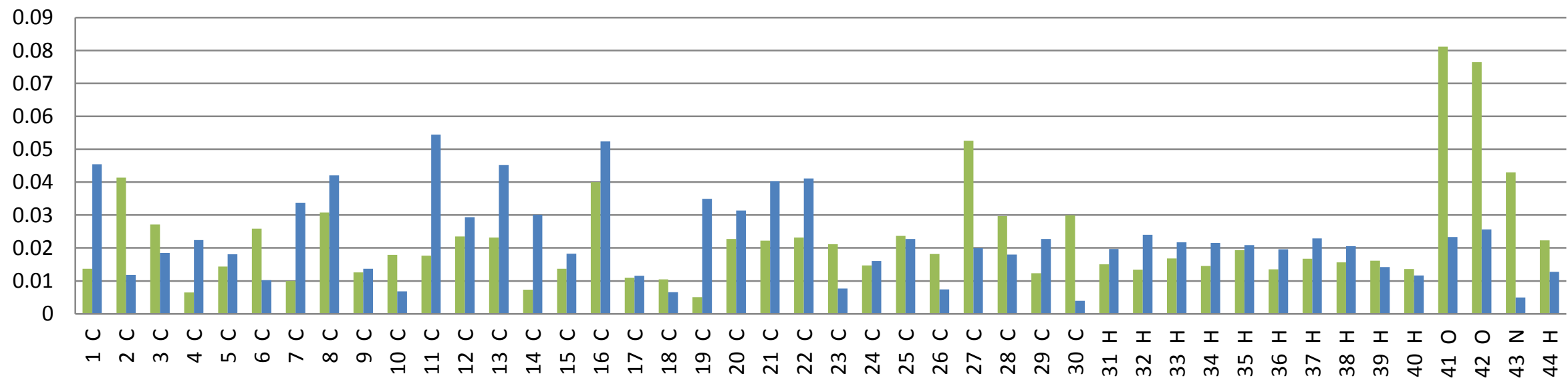




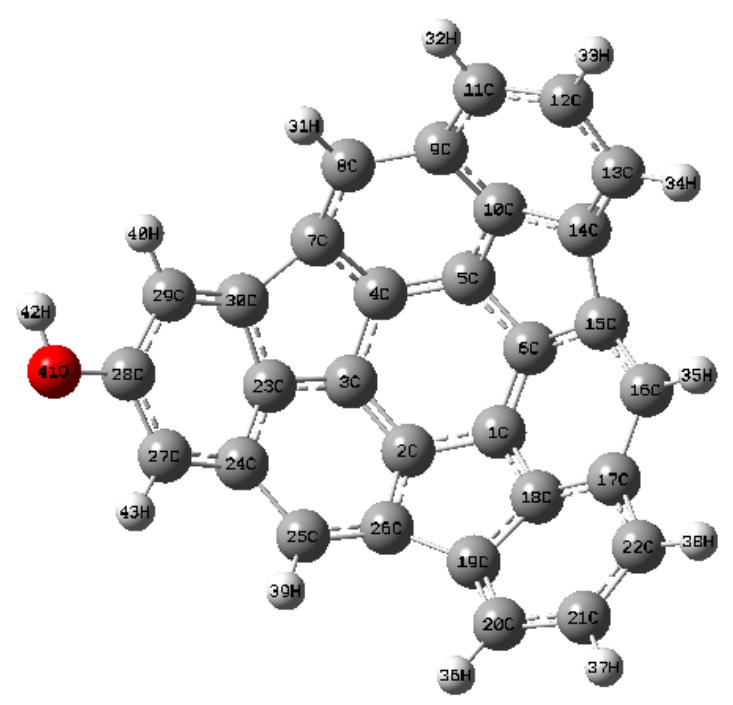

Fragmento 1 mono-sustituido con $\mathrm{OH}$

\begin{tabular}{cc}
\hline $\mathrm{I} \mathrm{eV}$ & $\mathbf{6 . 8 1}$ \\
$\mathrm{A} \mathrm{eV}$ & 0.65 \\
$\mu \mathrm{eV}$ & -3.73 \\
$\eta \mathrm{eV}$ & 6.16 \\
$\mathrm{~S} \mathrm{eV}$ & 0.16 \\
$\omega \mathrm{eV}$ & 1.13 \\
$\omega^{+} \mathrm{eV}$ & 0.78 \\
$\omega^{-} \mathrm{eV}$ & 4.51
\end{tabular}

\section{$\square f_{i}^{+}(r) \quad \square f_{i}^{-}(r)$}

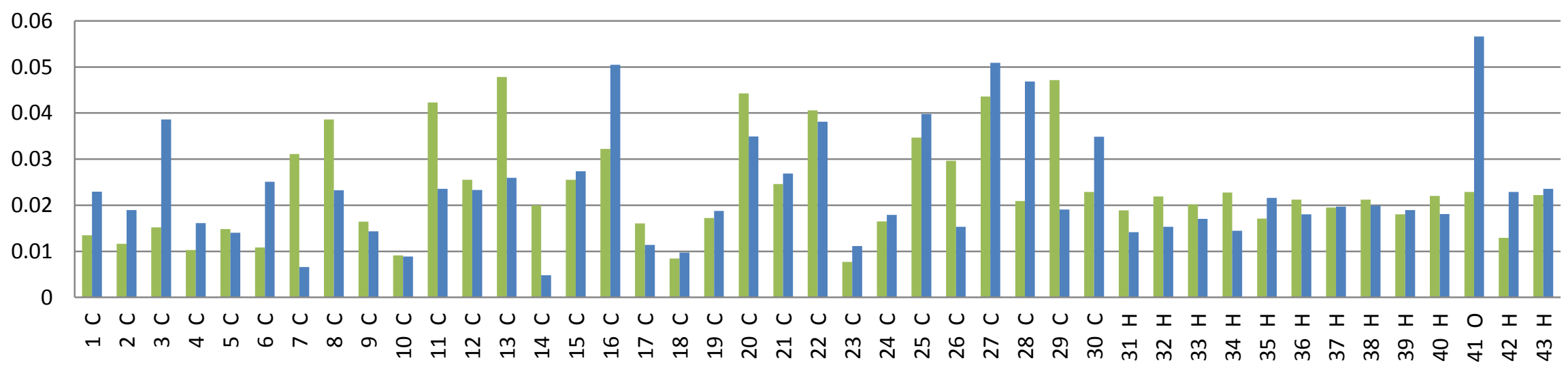




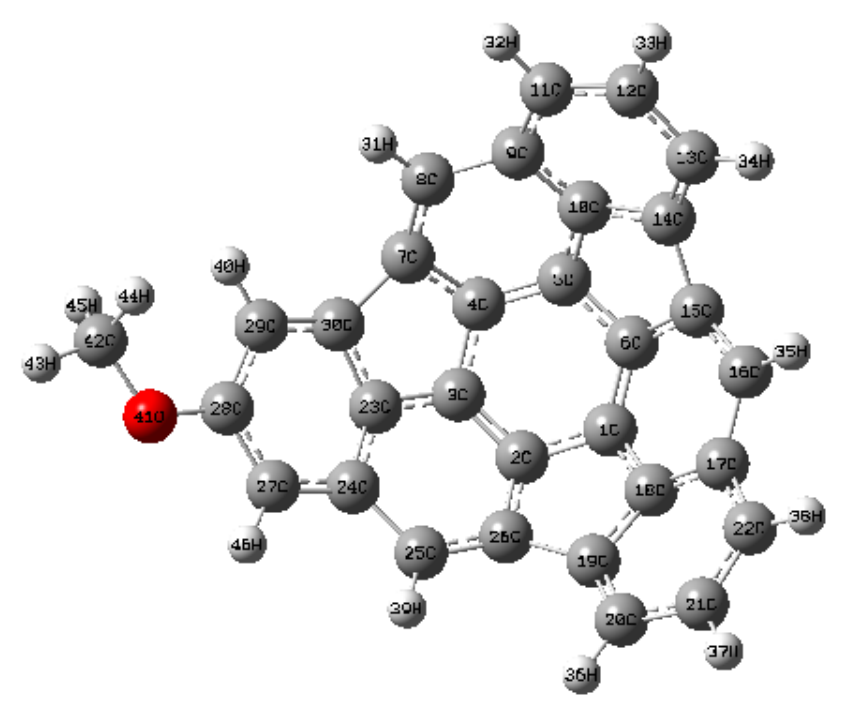

Fragmento 1 mono-sustituido con OMe

\begin{tabular}{cc}
\hline $\mathrm{l} \mathrm{eV}$ & $\mathbf{6 . 7 2}$ \\
$\mathrm{A} \mathrm{eV}$ & 0.62 \\
$\mu \mathrm{eV}$ & -3.67 \\
$\eta \mathrm{eV}$ & 6.09 \\
$\mathrm{~S} \mathrm{eV}$ & 0.16 \\
$\omega \mathrm{eV}$ & 1.10 \\
$\omega^{+} \mathrm{eV}$ & 0.75 \\
$\omega^{-} \mathrm{eV}$ & 4.42
\end{tabular}

\section{$\square f_{i}^{+}(r) \quad f_{i}{ }^{-}(r)$}

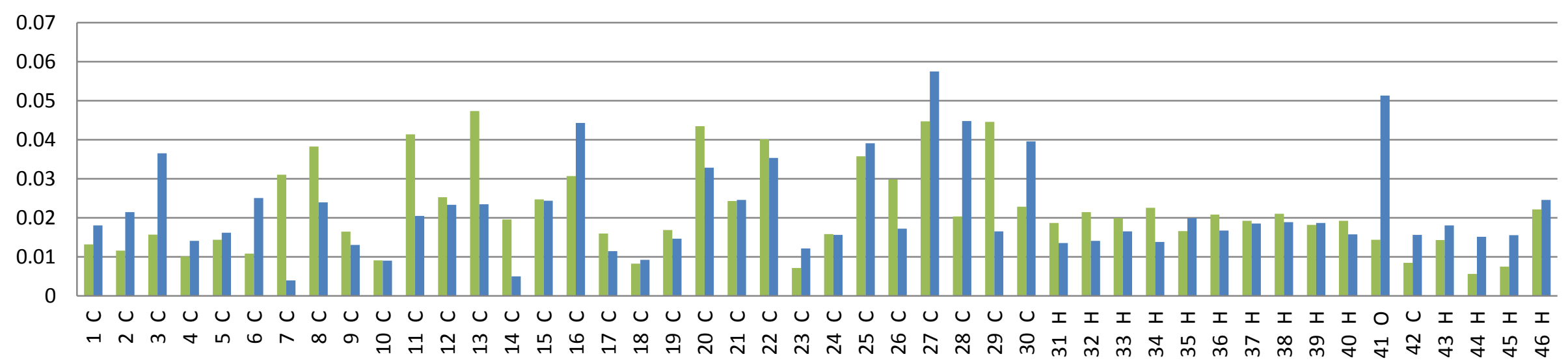




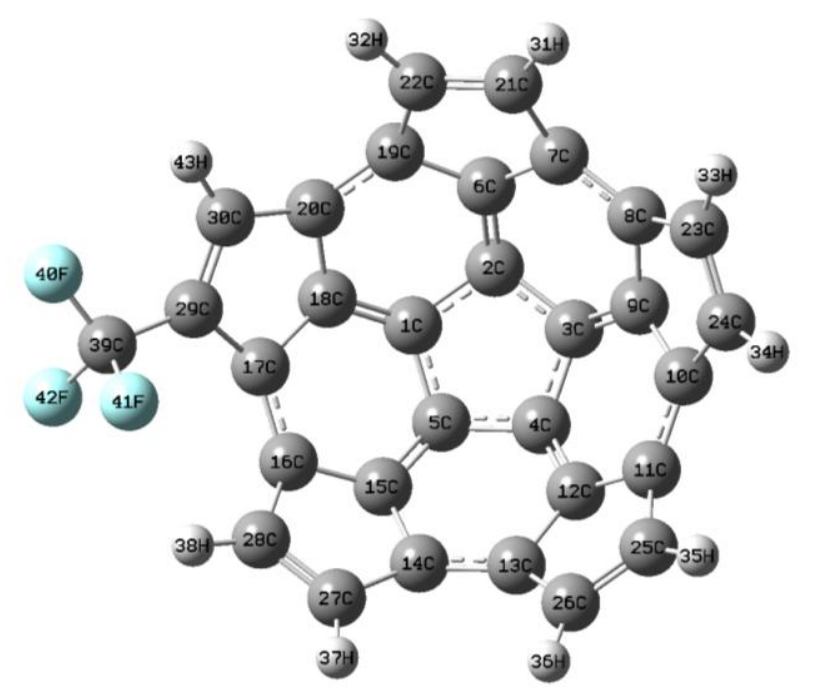

Fragmento 2 mono-sustituido con $\mathrm{CF}_{3}$

$\begin{array}{cc}\mathrm{I} \mathrm{eV} & \mathbf{9 . 1 7} \\ \mathrm{A} \mathrm{eV} & 1.32 \\ \mu \mathrm{eV} & -5.24 \\ \eta \mathrm{eV} & 7.85 \\ \mathrm{~S} \mathrm{eV} & 0.13 \\ \omega \mathrm{eV} & 1.75 \\ \omega^{+} \mathrm{eV} & 1.37 \\ \omega^{-} \mathrm{eV} & 6.61\end{array}$

\section{$\square f_{i}{ }^{+}(r) \quad \square f_{i}{ }^{-}(r)$}

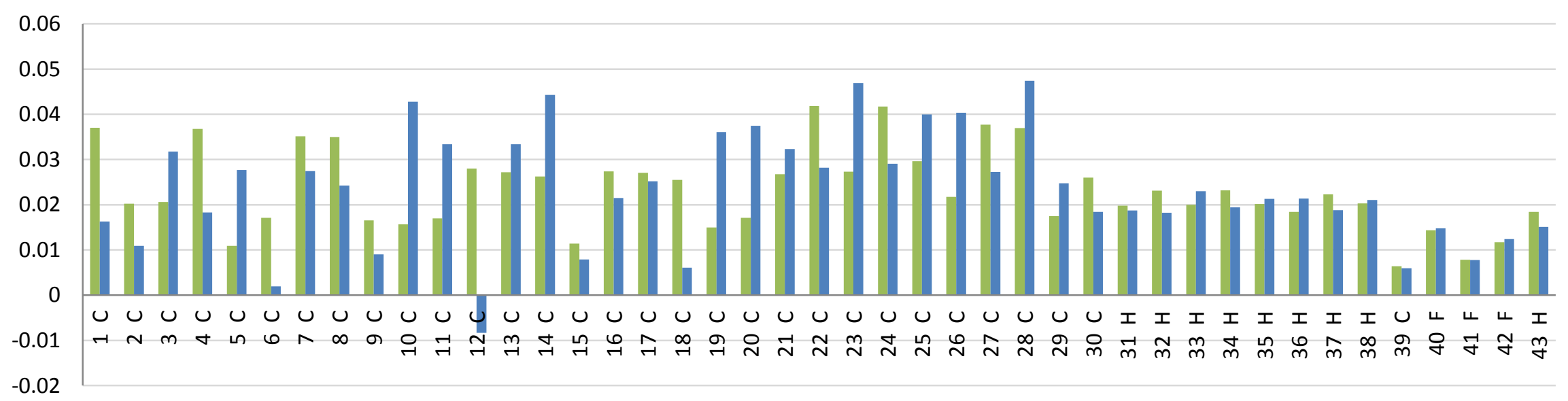




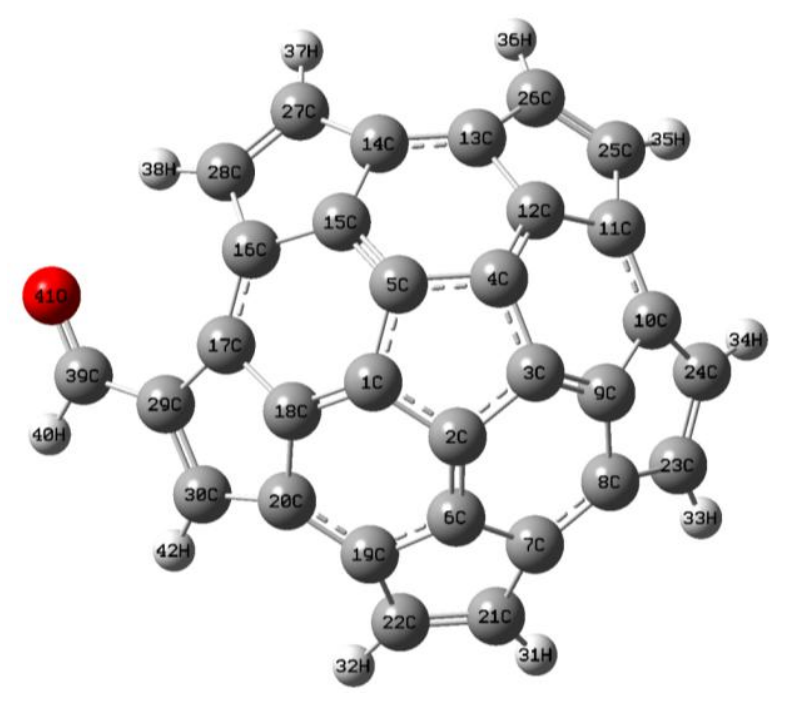

Fragmento 2 mono-sustituido con $\mathrm{CHO}$

\begin{tabular}{cc}
\hline $\mathrm{I} \mathrm{eV}$ & $\mathbf{7 . 1 2}$ \\
$\mathrm{A} \mathrm{eV}$ & 1.59 \\
$\mu \mathrm{eV}$ & -4.36 \\
$\eta \mathrm{eV}$ & 5.53 \\
$\mathrm{~S} \mathrm{eV}$ & 0.18 \\
$\omega \mathrm{eV}$ & 1.71 \\
$\omega^{+} \mathrm{eV}$ & 1.60 \\
$\omega^{-} \mathrm{eV}$ & 5.95
\end{tabular}

$\quad f_{i}{ }^{+}(r) \quad \square f_{i}{ }^{-}(r)$

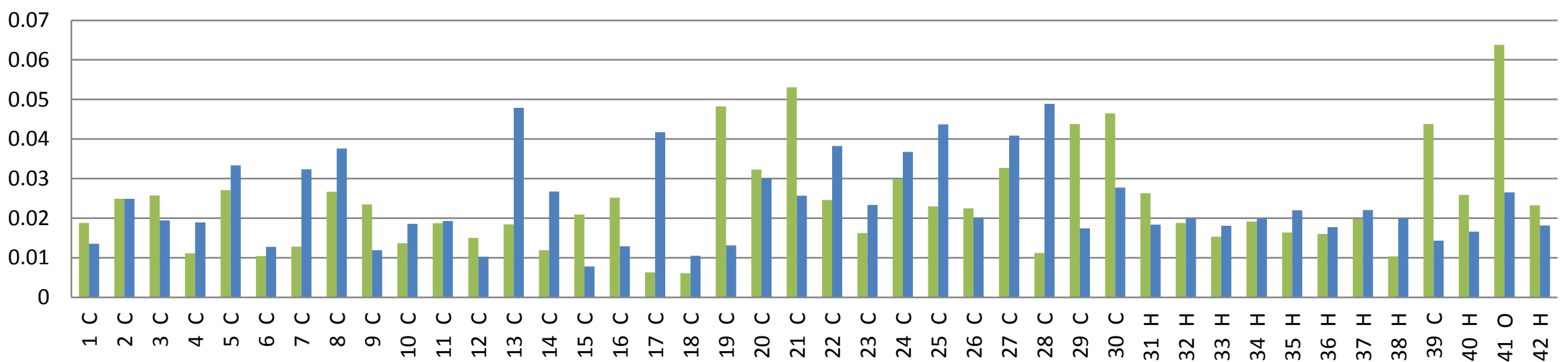




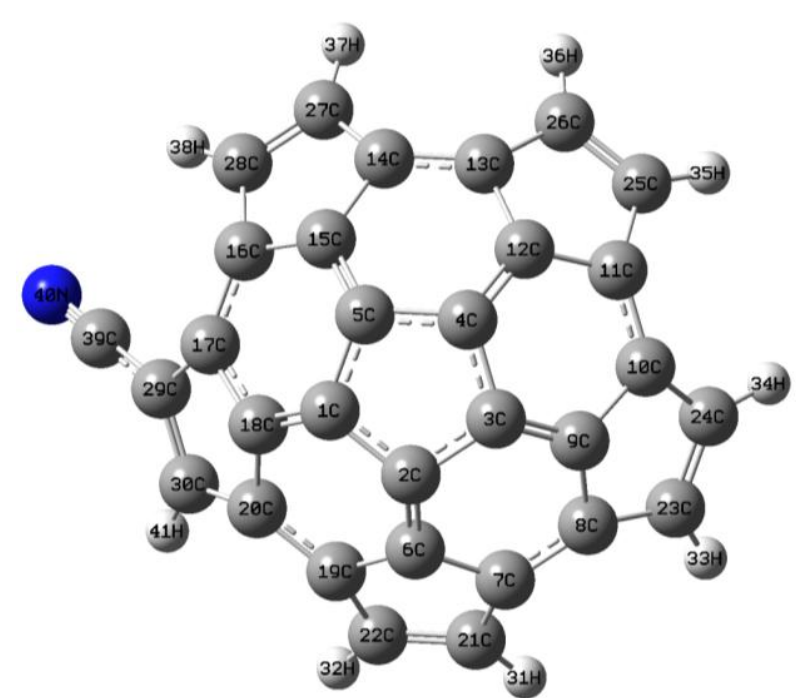

Fragmento 2 mono-sustituido con $\mathrm{CN}$

\begin{tabular}{cr}
\hline $\mathrm{I} \mathrm{eV}$ & $\mathbf{7 . 2 5}$ \\
$\mathrm{A} \mathrm{eV}$ & 1.64 \\
$\mu \mathrm{eV}$ & -4.44 \\
$\eta \mathrm{eV}$ & 5.61 \\
$\mathrm{~S} \mathrm{eV}$ & 0.18 \\
$\omega \mathrm{eV}$ & 1.76 \\
$\omega^{+} \mathrm{eV}$ & 1.65 \\
$\omega^{-} \mathrm{eV}$ & 6.09
\end{tabular}

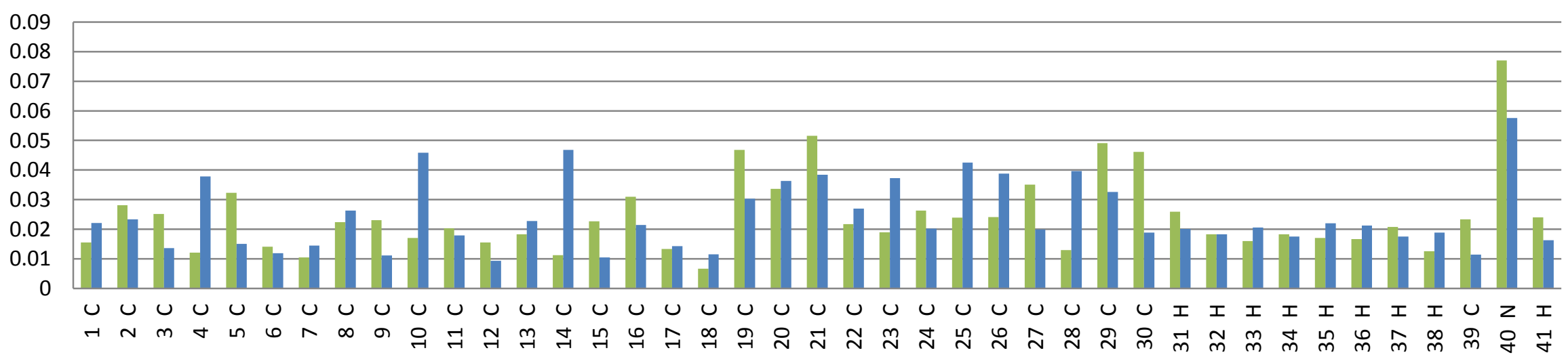




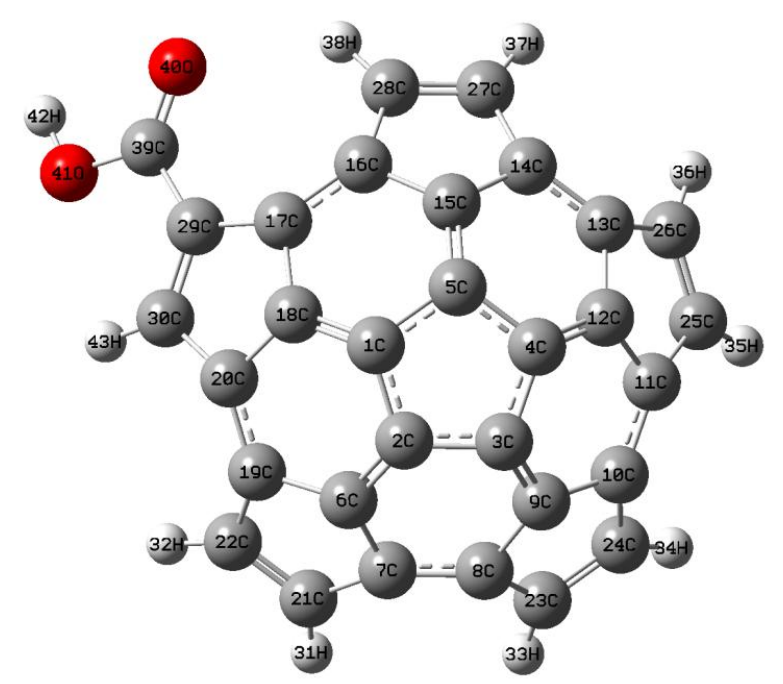

Fragmento 2 mono-sustituido con $\mathrm{COOH}$

\begin{tabular}{cc}
\hline $\mathrm{I} \mathrm{eV}$ & $\mathbf{7 . 0 5}$ \\
$\mathrm{A} \mathrm{eV}$ & 1.48 \\
$\mu \mathrm{eV}$ & -4.27 \\
$\eta \mathrm{eV}$ & 5.58 \\
$\mathrm{~S} \mathrm{eV}$ & 0.18 \\
$\omega \mathrm{eV}$ & 1.63 \\
$\omega^{+} \mathrm{eV}$ & 1.48 \\
$\omega^{-} \mathrm{eV}$ & 5.74
\end{tabular}

\section{$\quad f_{i}{ }^{+}(r) \quad \square f_{i}{ }^{-}(r)$}

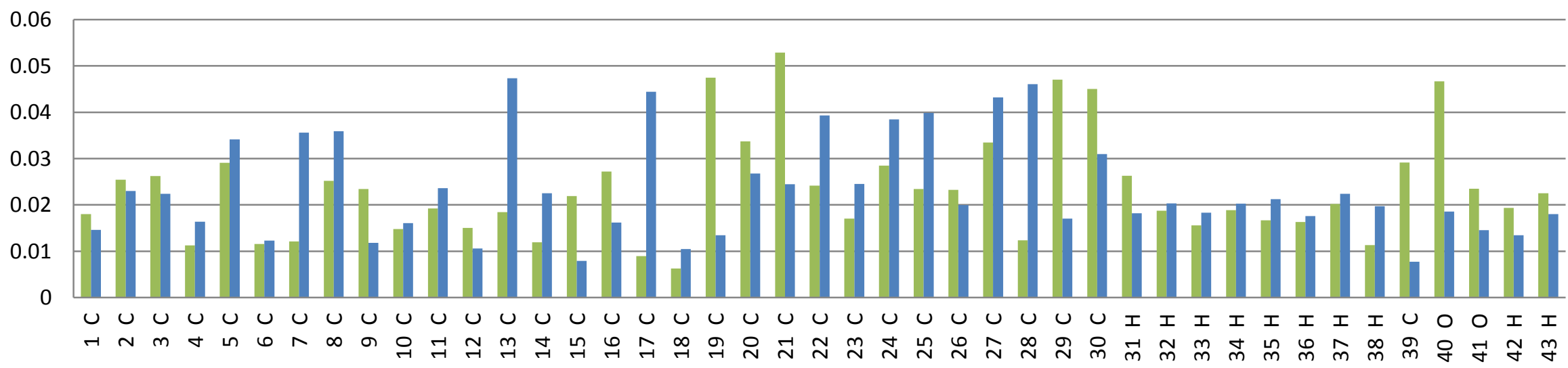




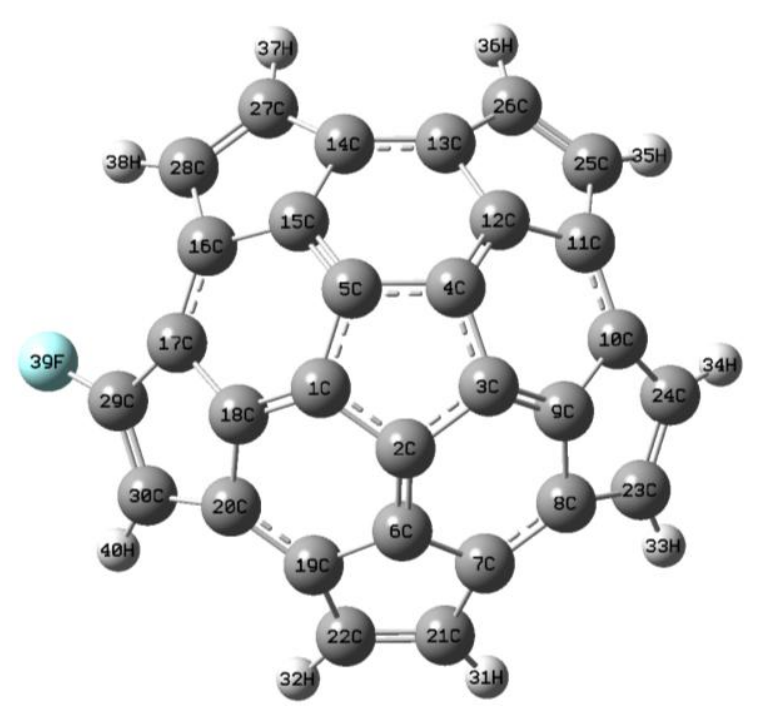

Fragmento 2 mono-sustituido con $F$

\begin{tabular}{cc}
\hline $\mathrm{I} \mathrm{eV}$ & 6.99 \\
$\mathrm{~A} \mathrm{eV}$ & 1.23 \\
$\mu \mathrm{eV}$ & -4.11 \\
$\eta \mathrm{eV}$ & 5.76 \\
$\mathrm{~S} \mathrm{eV}$ & 0.17 \\
$\omega \mathrm{eV}$ & 1.47 \\
$\omega^{+} \mathrm{eV}$ & 1.24 \\
$\omega^{-} \mathrm{eV}$ & 5.35
\end{tabular}

\section{$\square f_{i}^{+}(r) \quad \square f_{i}^{-}(r)$}

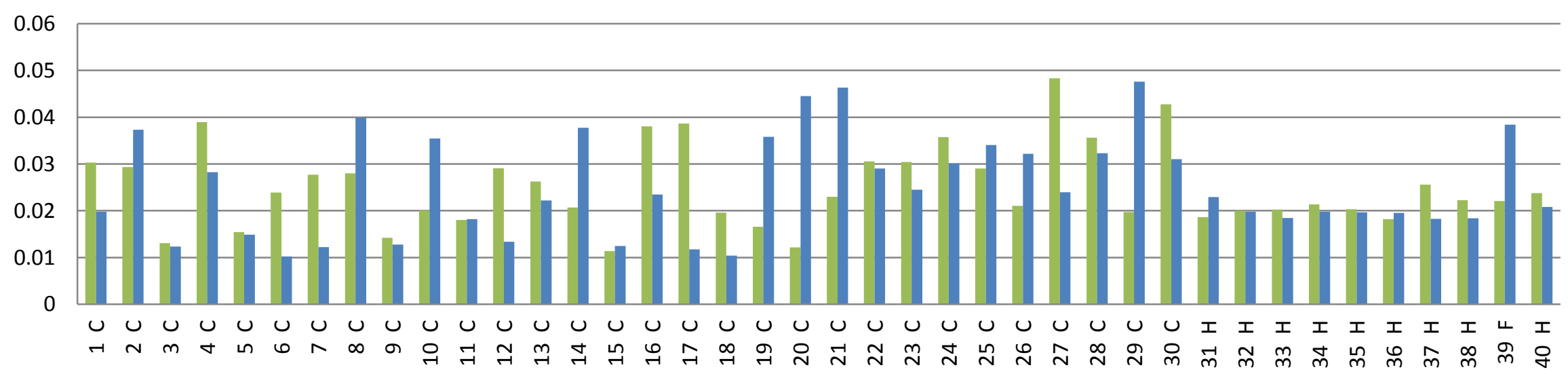




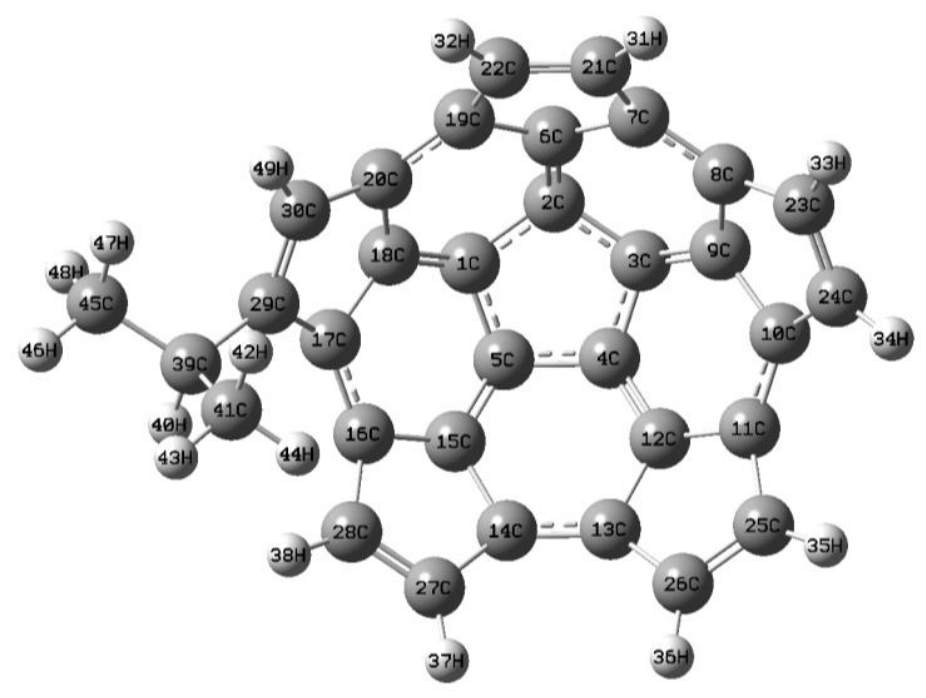

Fragmento 2 mono-sustituido con $i$-Pr

\begin{tabular}{cc}
\hline $\mathrm{I} \mathrm{eV}$ & $\mathbf{6 . 8 1}$ \\
$\mathrm{A} \mathrm{eV}$ & 1.10 \\
$\mu \mathrm{eV}$ & -3.96 \\
$\eta \mathrm{eV}$ & 5.72 \\
$\mathrm{~S} \mathrm{eV}$ & 0.17 \\
$\omega \mathrm{eV}$ & 1.37 \\
$\omega^{+} \mathrm{eV}$ & 1.12 \\
$\omega^{-} \mathrm{eV}$ & 5.07
\end{tabular}

$\square f_{i}{ }^{+}(r) \quad \square f_{i}{ }^{-}(r)$

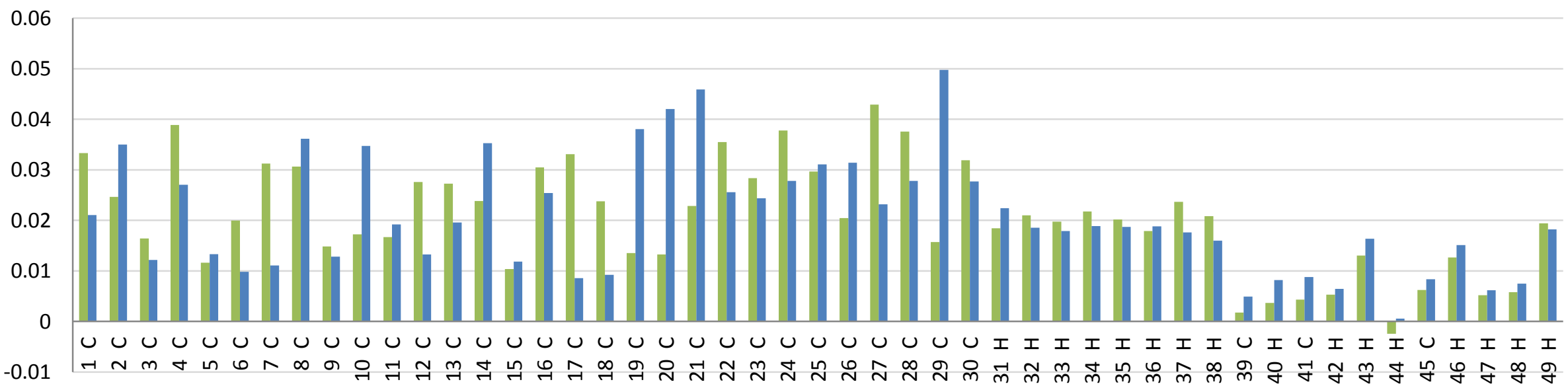




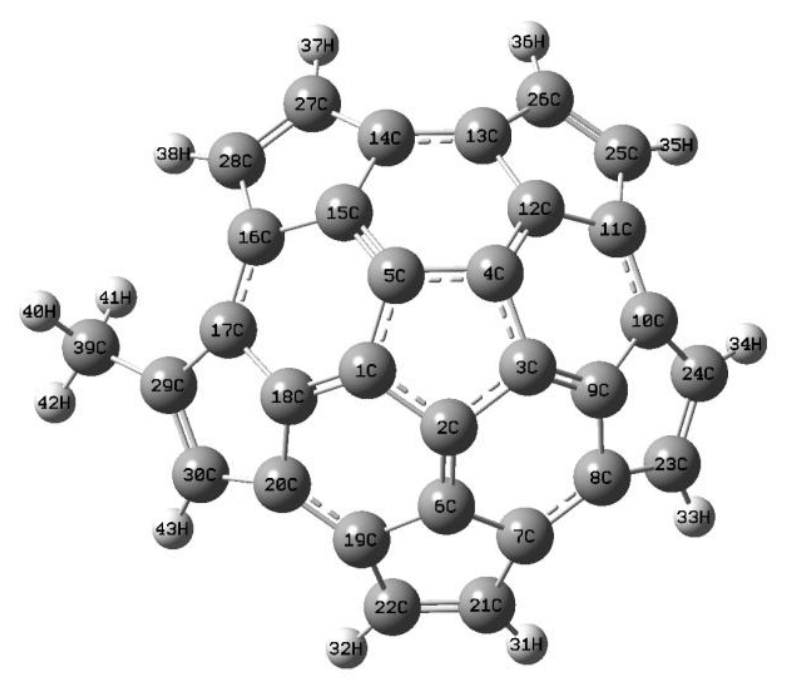

Fragmento 2 mono-sustituido con Me

\begin{tabular}{cc}
\hline $\mathrm{I} \mathrm{eV}$ & $\mathbf{6 . 8 5}$ \\
$\mathrm{A} \mathrm{eV}$ & 1.08 \\
$\mu \mathrm{eV}$ & -3.97 \\
$\eta \mathrm{eV}$ & 5.77 \\
$\mathrm{~S} \mathrm{eV}$ & 0.17 \\
$\omega \mathrm{eV}$ & 1.36 \\
$\omega^{+} \mathrm{eV}$ & 1.10 \\
$\omega^{-} \mathrm{eV}$ & 5.07
\end{tabular}

\section{$\square f_{i}^{+}(r) \quad \square f_{i}^{-}(r)$}

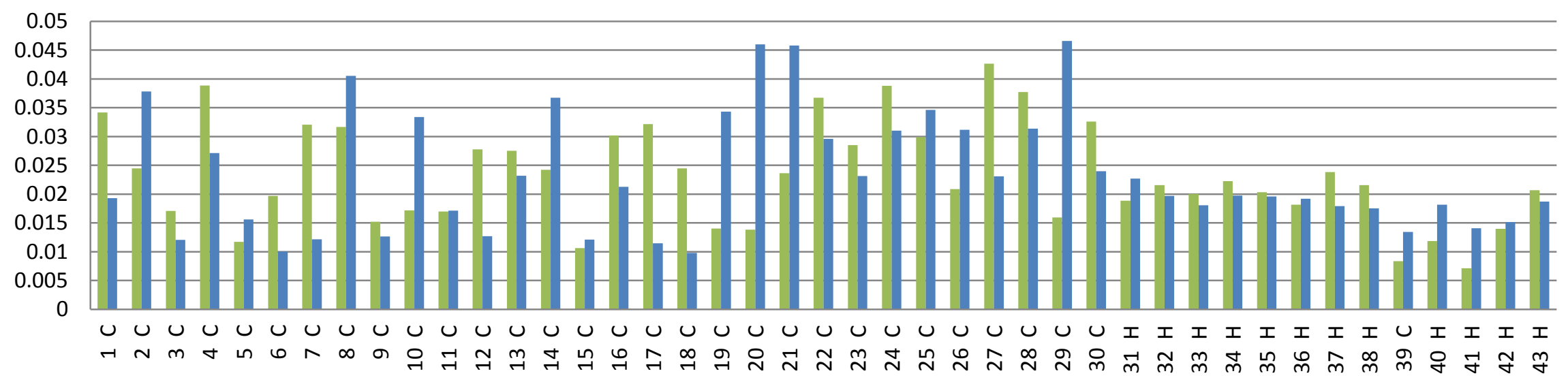




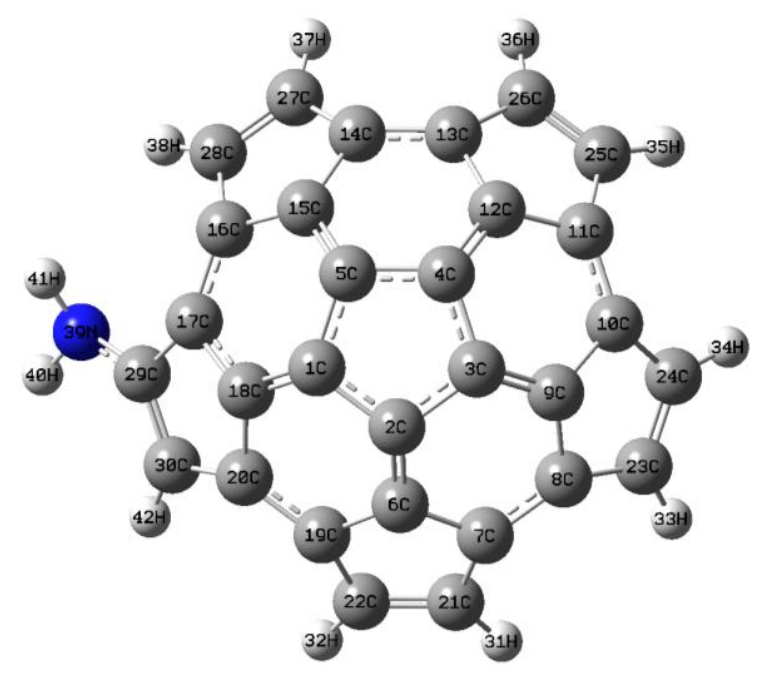

Fragmento 2 mono-sustituido con $\mathbf{N H}_{2}$

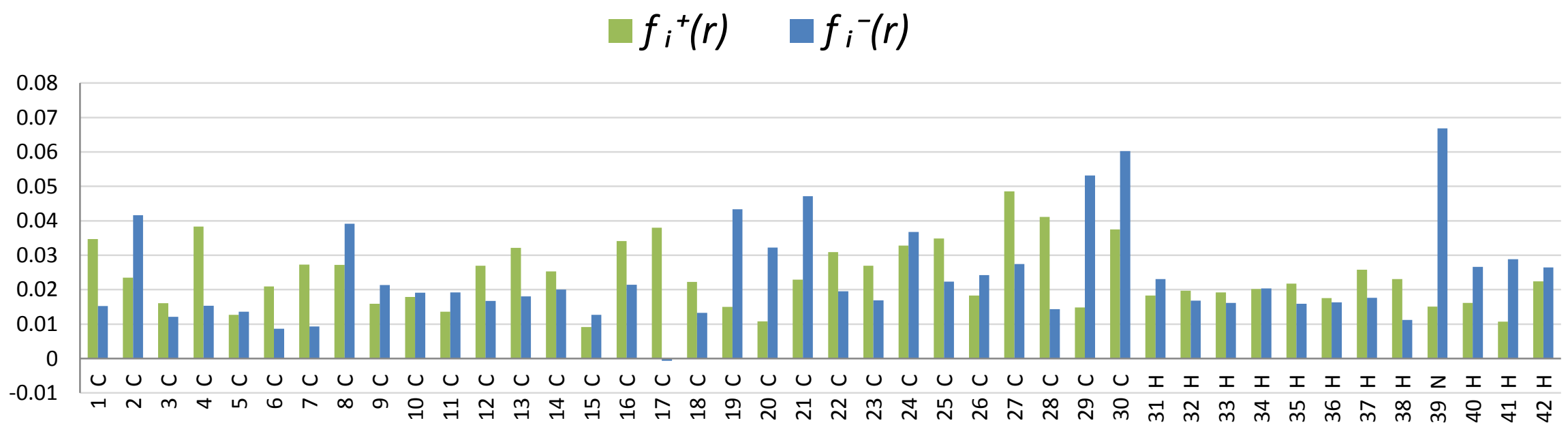




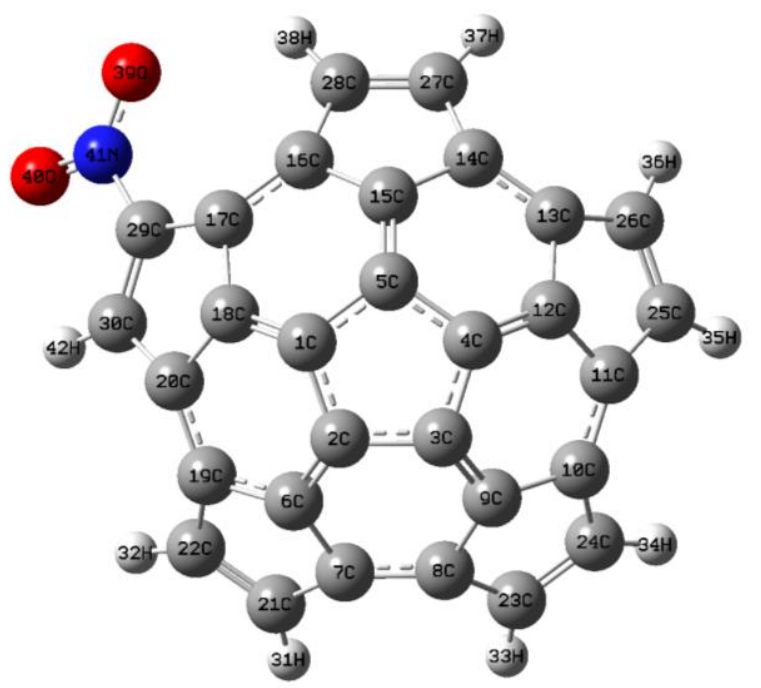

Fragmento 2 mono-sustituido con $\mathrm{NO}_{2}$

\begin{tabular}{cc}
\hline $\mathrm{I} \mathrm{eV}$ & $\mathbf{7 . 3 3}$ \\
$\mathrm{A} \mathrm{eV}$ & 1.87 \\
$\mu \mathrm{eV}$ & -4.60 \\
$\eta \mathrm{eV}$ & 5.45 \\
$\mathrm{~S} \mathrm{eV}$ & 0.18 \\
$\omega \mathrm{eV}$ & 1.94 \\
$\omega^{+} \mathrm{eV}$ & 1.92 \\
$\omega^{-} \mathrm{eV}$ & 6.52
\end{tabular}

$\square f_{i}^{+}(r) \quad \square f_{i}^{-}(r)$

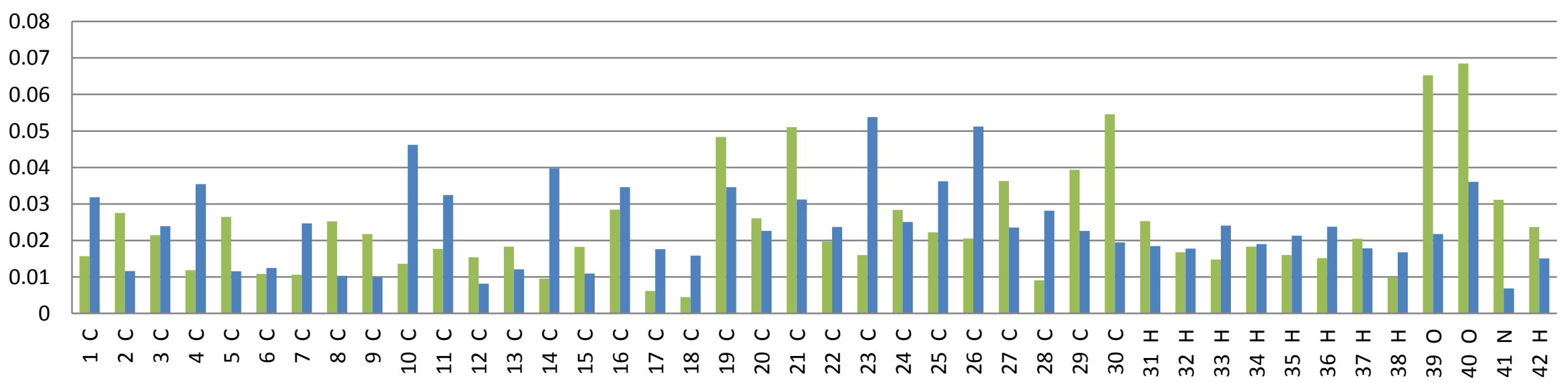




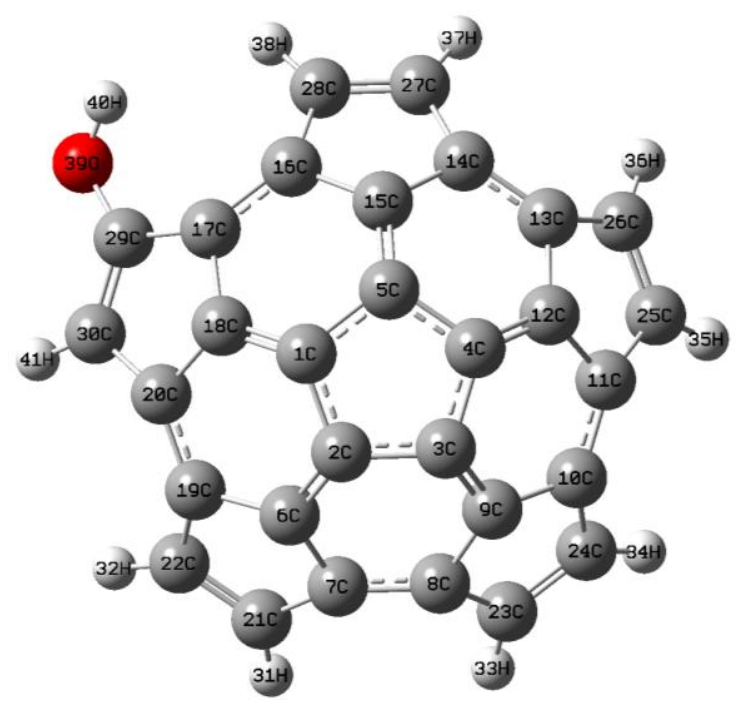

\section{Fragmento 2 mono-sustituido con $\mathrm{OH}$}

\begin{tabular}{cr}
\hline $\mathrm{I} \mathrm{eV}$ & $\mathbf{6 . 7 4}$ \\
$\mathrm{A} \mathrm{eV}$ & 1.17 \\
$\mu \mathrm{eV}$ & -3.95 \\
$\eta \mathrm{eV}$ & 5.57 \\
$\mathrm{~S} \mathrm{eV}$ & 0.18 \\
$\omega \mathrm{eV}$ & 1.40 \\
$\omega^{+} \mathrm{eV}$ & 1.18 \\
$\omega^{-} \mathrm{eV}$ & 5.13
\end{tabular}

\section{$\square f_{i}^{+}(r) \quad \square f_{i}^{-}(r)$}

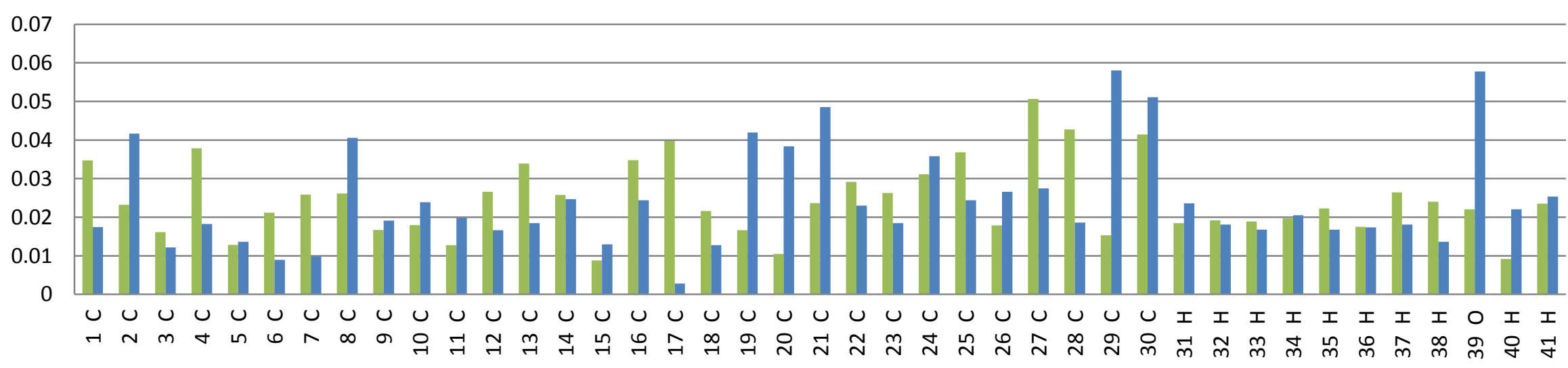




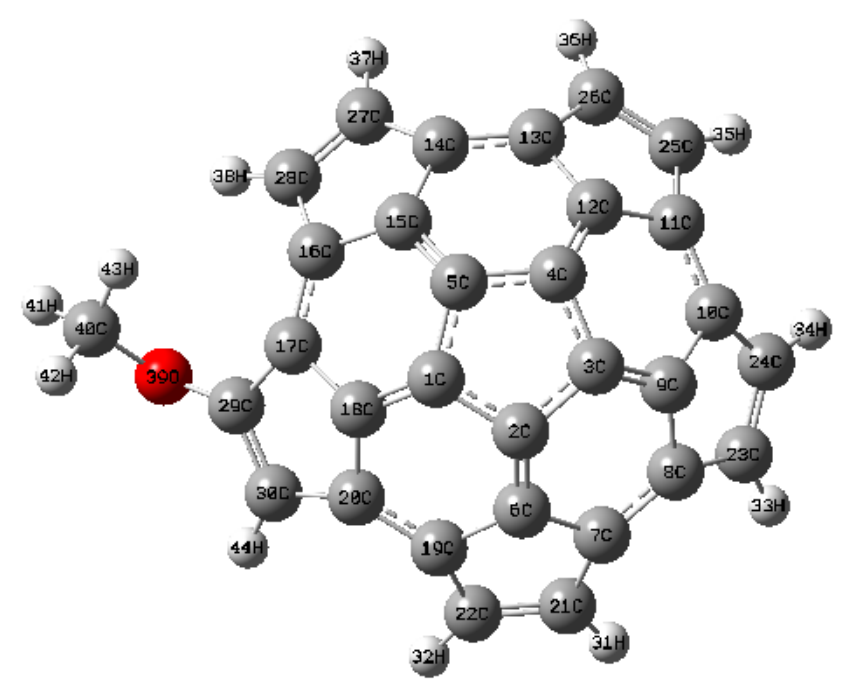

Fragmento 2 mono-sustituido con OMe

\begin{tabular}{cc}
\hline I eV & 6.76 \\
A eV & 1.12 \\
$\mu$ eV & -3.94 \\
$\eta$ eV & 5.63 \\
S eV & 0.18 \\
$\omega$ eV & 1.38 \\
$\omega^{+}$eV & 1.14 \\
$\omega^{-}$eV & 5.08
\end{tabular}

$\square f_{i}^{+}(r) \quad \square f_{i}^{-}(r)$

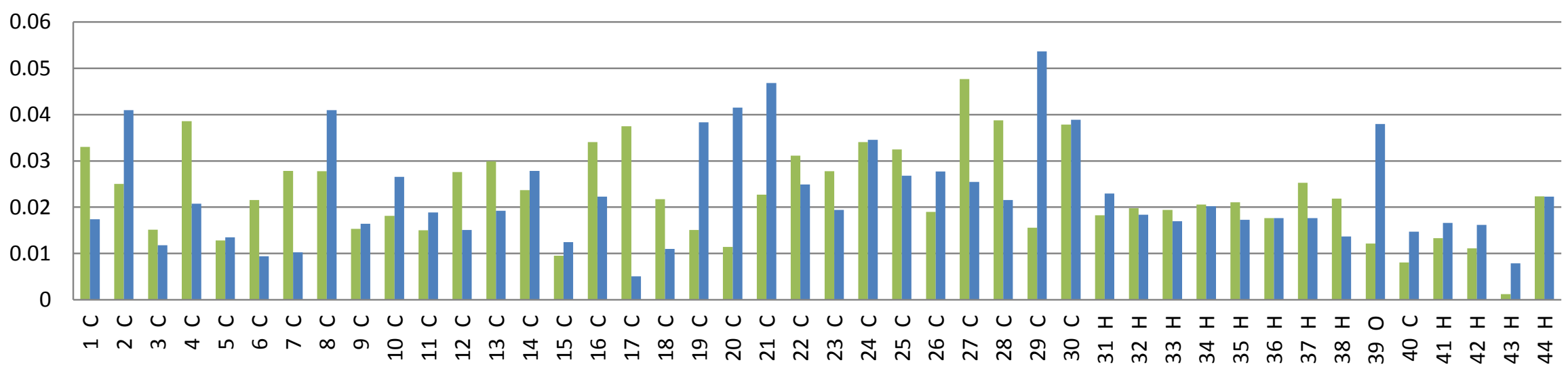




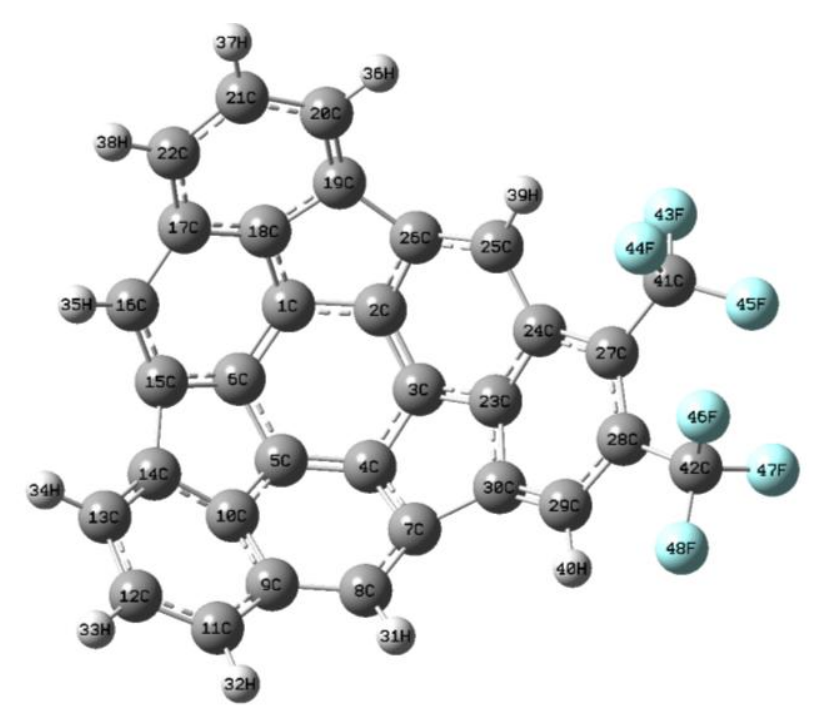

Fragmento 1 di-sustituido con $\mathrm{CF}_{3}$

$\begin{array}{cc}\mathrm{I} \mathrm{eV} & \mathbf{7 . 3 1} \\ \mathrm{A} \mathrm{eV} & 1.18 \\ \mu \mathrm{eV} & -4.24 \\ \eta \mathrm{eV} & 6.13 \\ \mathrm{~S} \mathrm{eV} & 0.16 \\ \omega \mathrm{eV} & 1.47 \\ \omega^{+} \mathrm{eV} & 1.20 \\ \omega^{-} \mathrm{eV} & 5.44\end{array}$

$\square f_{i}{ }^{+}(r) \quad \square f_{i}{ }^{-}(r)$

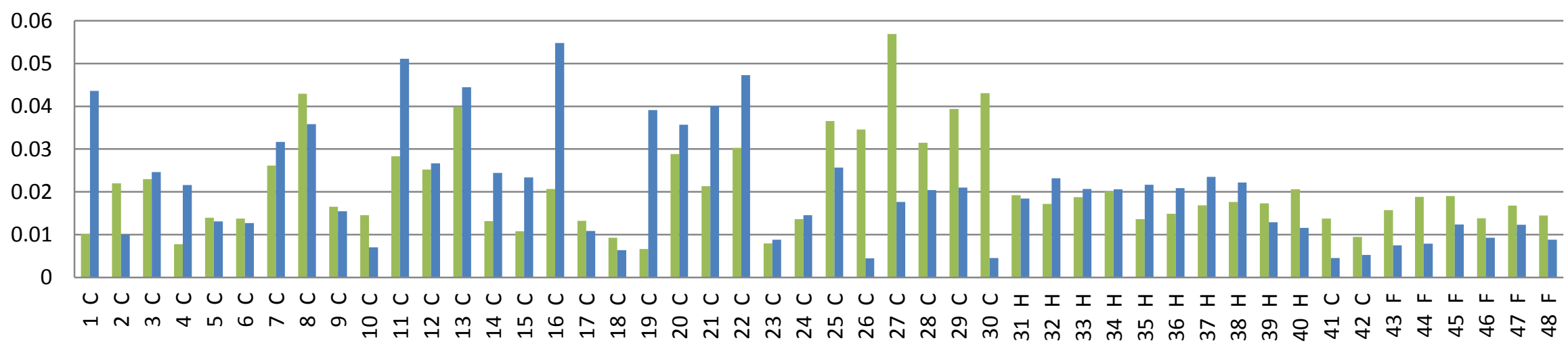




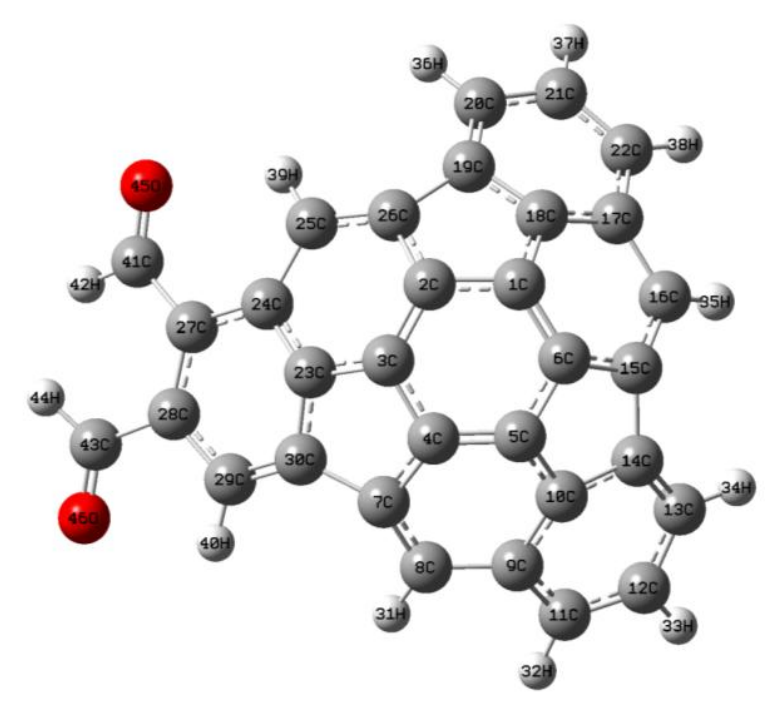

\section{Fragmento 1 di-sustituido con $\mathrm{CHO}$}

\begin{tabular}{cc}
\hline $\mathrm{I} \mathrm{eV}$ & $\mathbf{7 . 3 9}$ \\
$\mathrm{A} \mathrm{eV}$ & 1.44 \\
$\mu \mathrm{eV}$ & -4.41 \\
$\eta \mathrm{eV}$ & 5.95 \\
$\mathrm{~S} \mathrm{eV}$ & 0.17 \\
$\omega \mathrm{eV}$ & 1.64 \\
$\omega^{+} \mathrm{eV}$ & 1.44 \\
$\omega^{-} \mathrm{eV}$ & 5.86
\end{tabular}

$\square f_{i}{ }^{+}(r) \quad \square f_{i}{ }^{-}(r)$

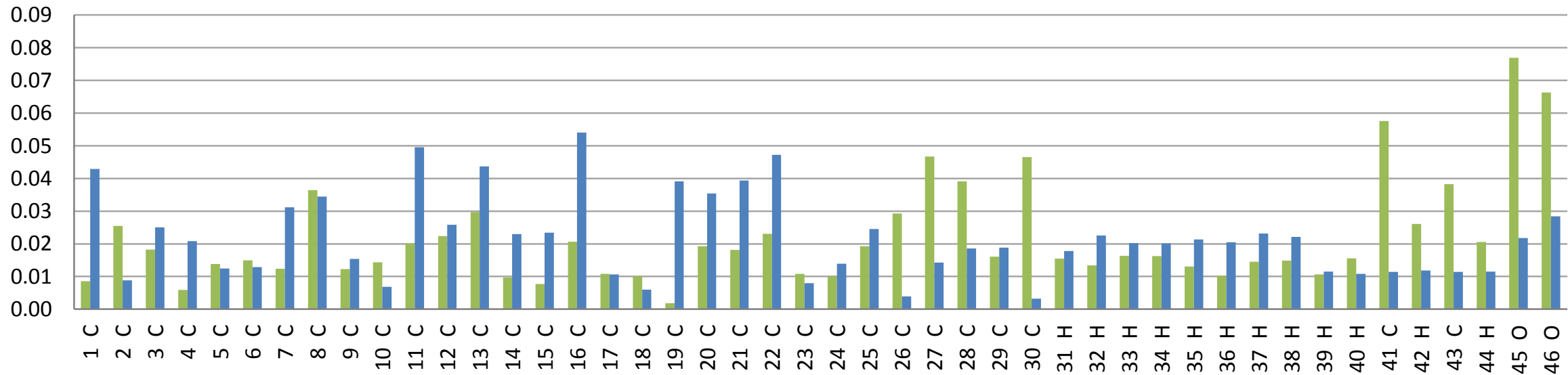


Fragmento 1 di-sustituido con $\mathrm{CN}$

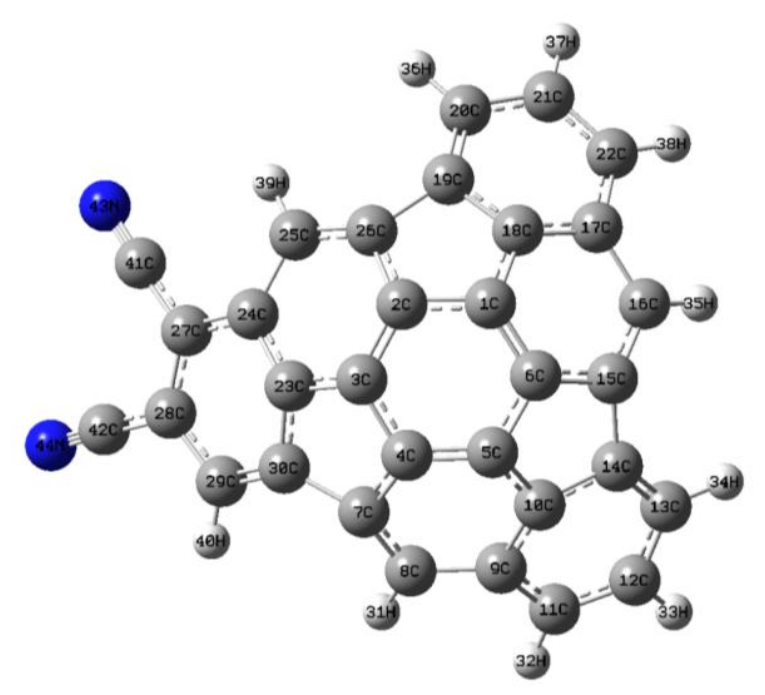

\begin{tabular}{cc}
\hline $\mathrm{I} \mathrm{eV}$ & $\mathbf{7 . 5 0}$ \\
$\mathrm{A} \mathrm{eV}$ & 1.63 \\
$\mu \mathrm{eV}$ & -4.56 \\
$\eta \mathrm{eV}$ & 5.87 \\
$\mathrm{~S} \mathrm{eV}$ & 0.17 \\
$\omega \mathrm{eV}$ & 1.77 \\
$\omega^{+} \mathrm{eV}$ & 1.63 \\
$\omega^{-} \mathrm{eV}$ & 6.19
\end{tabular}

\section{$\square f_{i}^{+}(r) \quad \square f_{i}^{-}(r)$}

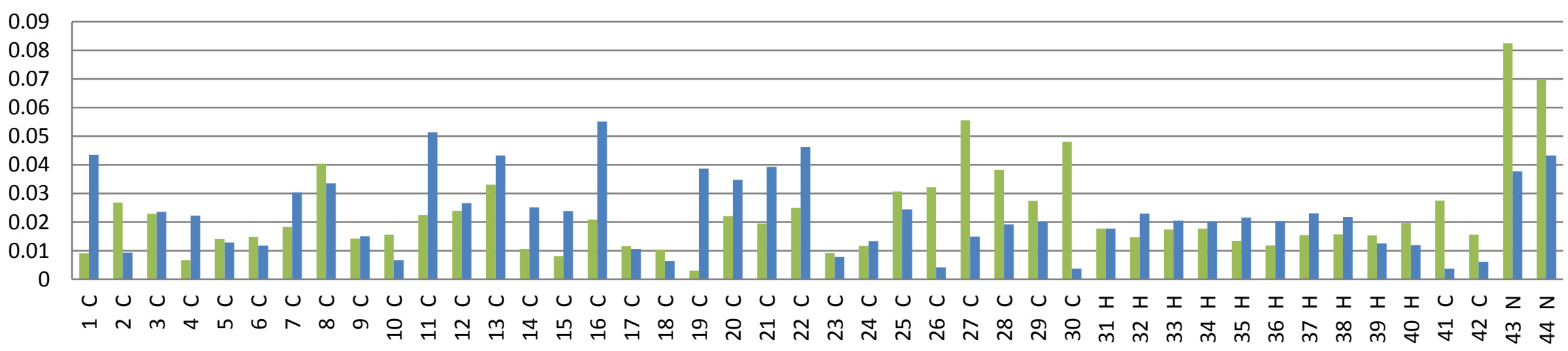




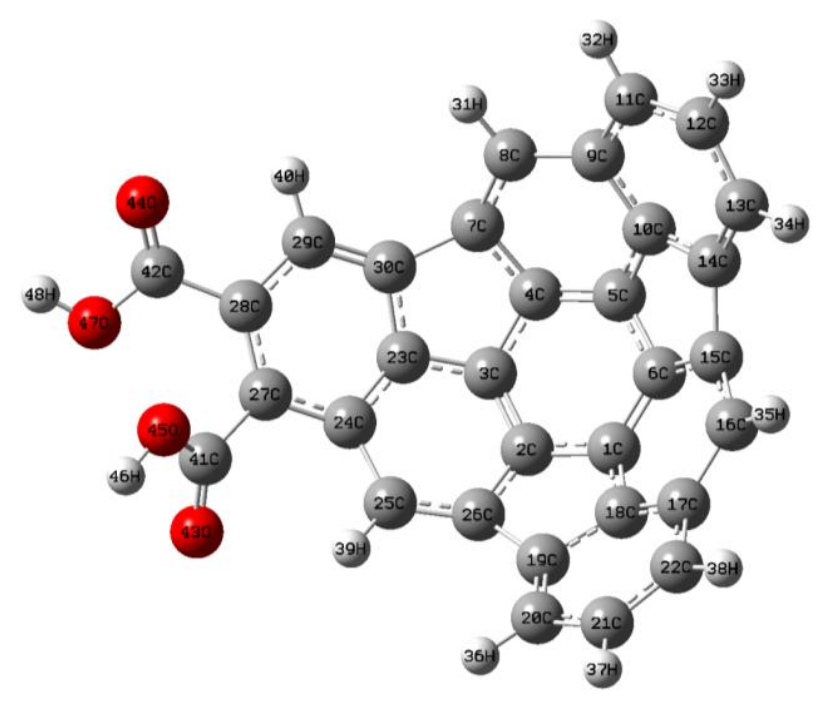

Fragmento 1 di-sustituido con $\mathrm{COOH}$

\begin{tabular}{cc}
\hline $\mathrm{I} \mathrm{eV}$ & $\mathbf{7 . 1 8}$ \\
$\mathrm{A} \mathrm{eV}$ & 1.15 \\
$\mu \mathrm{eV}$ & -4.16 \\
$\eta \mathrm{eV}$ & 6.03 \\
$\mathrm{~S} \mathrm{eV}$ & 0.17 \\
$\omega \mathrm{eV}$ & 1.44 \\
$\omega^{+} \mathrm{eV}$ & 1.17 \\
$\omega^{-} \mathrm{eV}$ & 5.34
\end{tabular}

$\square f_{i}{ }^{+}(r) \quad \square f_{i}^{-}(r)$

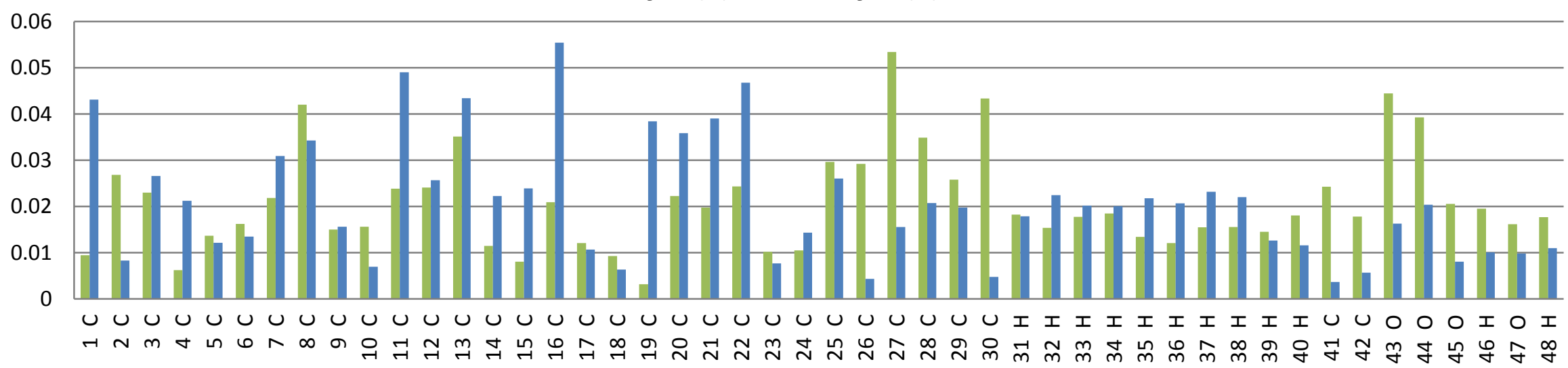




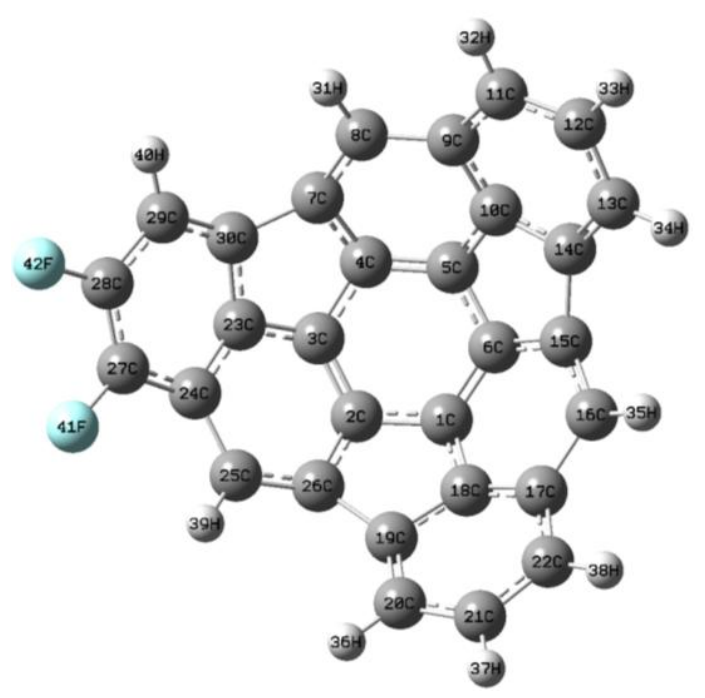

Fragmento 1 di-sustituido con $F$

\begin{tabular}{cc}
\hline $\mathrm{l} \mathrm{eV}$ & $\mathbf{7 . 1 2}$ \\
$\mathrm{A} \mathrm{eV}$ & 0.80 \\
$\mu \mathrm{eV}$ & -3.96 \\
$\eta \mathrm{eV}$ & 6.31 \\
$\mathrm{~S} \mathrm{eV}$ & 0.16 \\
$\omega \mathrm{eV}$ & 1.24 \\
$\omega^{+} \mathrm{eV}$ & 0.90 \\
$\omega^{-} \mathrm{eV}$ & 4.86 \\
\end{tabular}

\section{$\square f_{i}{ }^{+}(r) \quad \square f_{i}{ }^{-}(r)$}

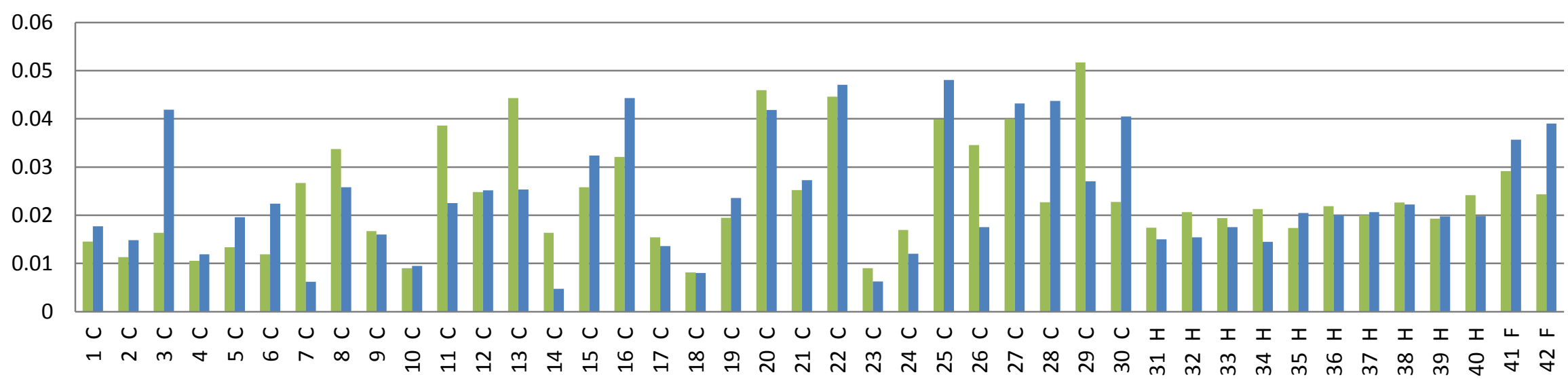




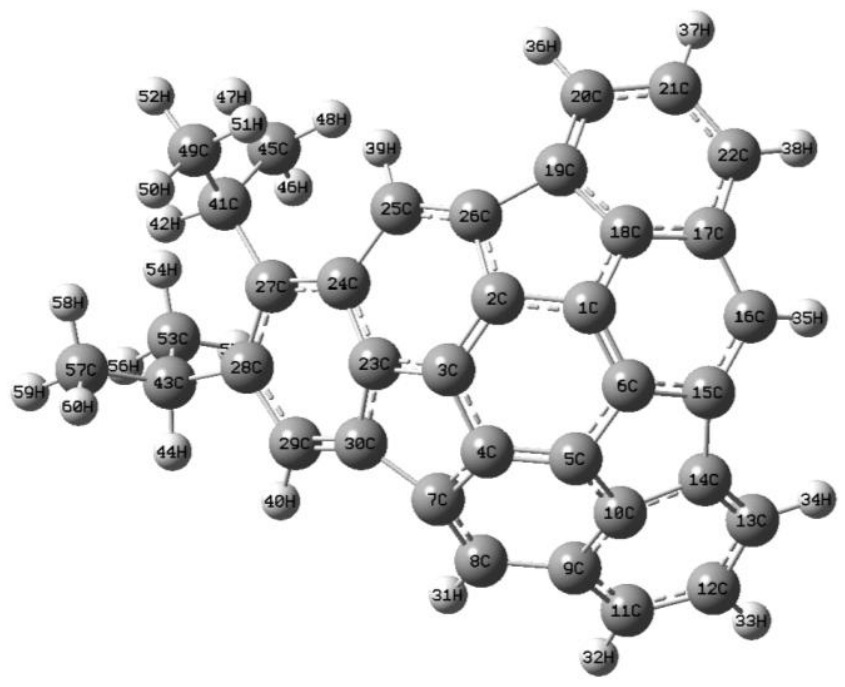

Fragmento 1 di-sustituido con $i-P r$

$\begin{array}{cc}\mathrm{I} \text { eV } & 6.77 \\ \mathrm{~A} \mathrm{eV} & 0.62 \\ \mu \mathrm{eV} & -3.69 \\ \eta \mathrm{eV} & 6.15 \\ \mathrm{~S} \mathrm{eV} & 0.16 \\ \omega \mathrm{eV} & 1.11 \\ \omega^{+} \mathrm{eV} & 0.76 \\ \omega^{-} \mathrm{eV} & 4.45\end{array}$

$\square f_{i}{ }^{+}(r) \quad \square f_{i}{ }^{-}(r)$

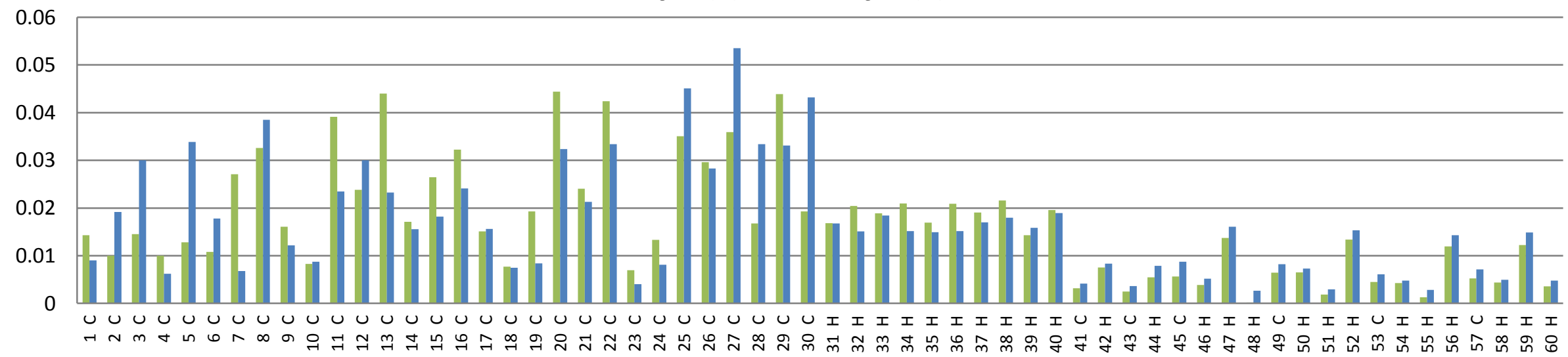




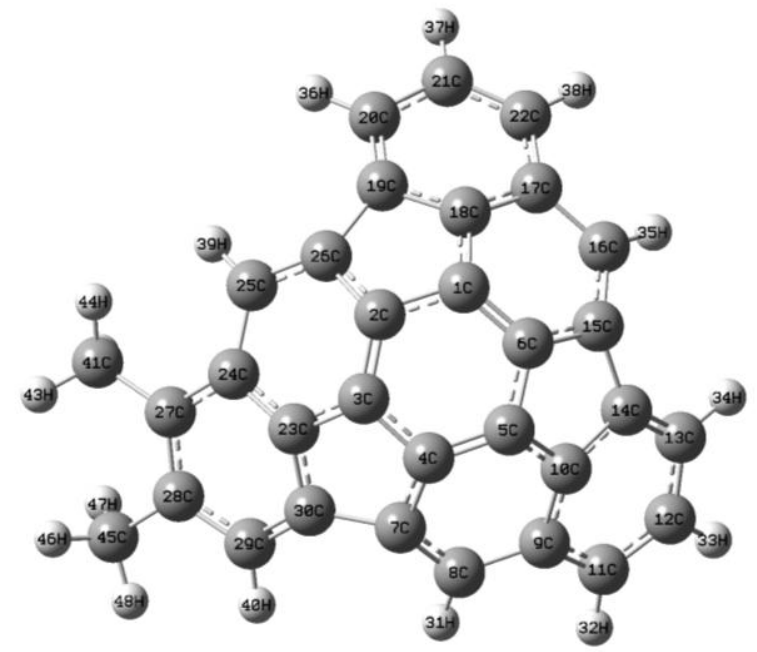

Fragmento 1 di-sustituido con Me

\begin{tabular}{cc}
\hline $\mathrm{l} \mathrm{eV}$ & 6.79 \\
$\mathrm{~A} \mathrm{eV}$ & 0.57 \\
$\mu \mathrm{eV}$ & -3.68 \\
$\eta \mathrm{eV}$ & 6.22 \\
$\mathrm{~S} \mathrm{eV}$ & 0.16 \\
$\omega \mathrm{eV}$ & 1.09 \\
$\omega^{+} \mathrm{eV}$ & 0.73 \\
$\omega^{-} \mathrm{eV}$ & 4.41
\end{tabular}

\section{$\square f_{i}{ }^{+}(r) \quad \square f_{i}{ }^{-}(r)$}

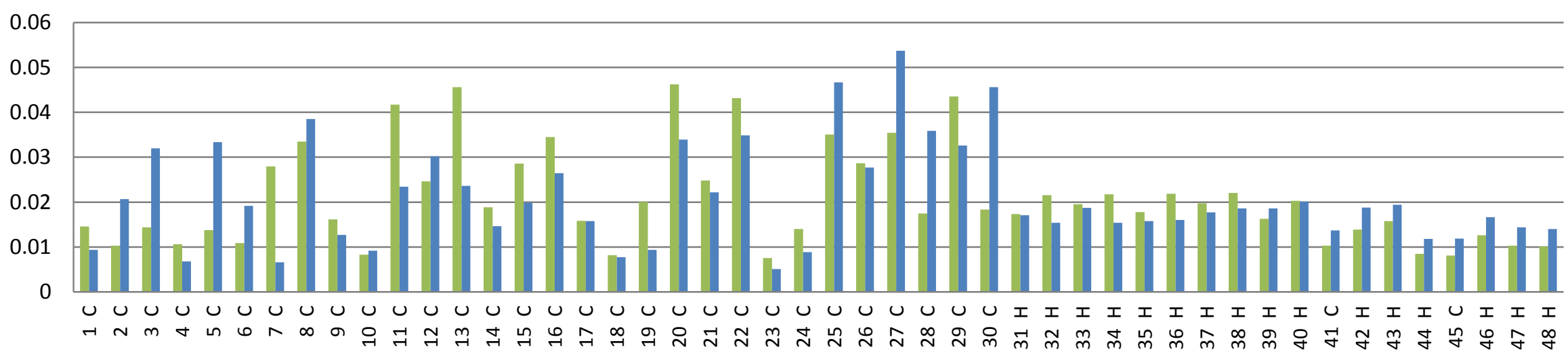


Fragmento 1 di-sustituido con $\mathrm{NH}_{2}$

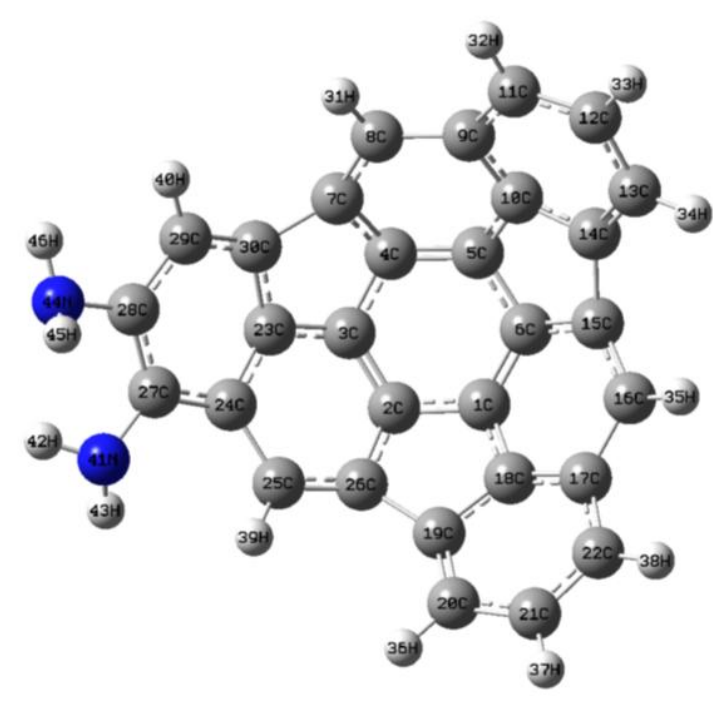

\begin{tabular}{cc} 
& \\
\hline $\mathrm{I} \mathrm{eV}$ & $\mathbf{6 . 2 5}$ \\
$\mathrm{A} \mathrm{eV}$ & 0.44 \\
$\mu \mathrm{eV}$ & -3.35 \\
$\eta \mathrm{eV}$ & 5.81 \\
$\mathrm{~S} \mathrm{eV}$ & 0.17 \\
$\omega \mathrm{eV}$ & 0.97 \\
$\omega^{+} \mathrm{eV}$ & 0.62 \\
$\omega^{-} \mathrm{eV}$ & 3.97
\end{tabular}

\section{$\square f_{i}{ }^{+}(r) \quad \square f_{i}{ }^{-}(r)$}

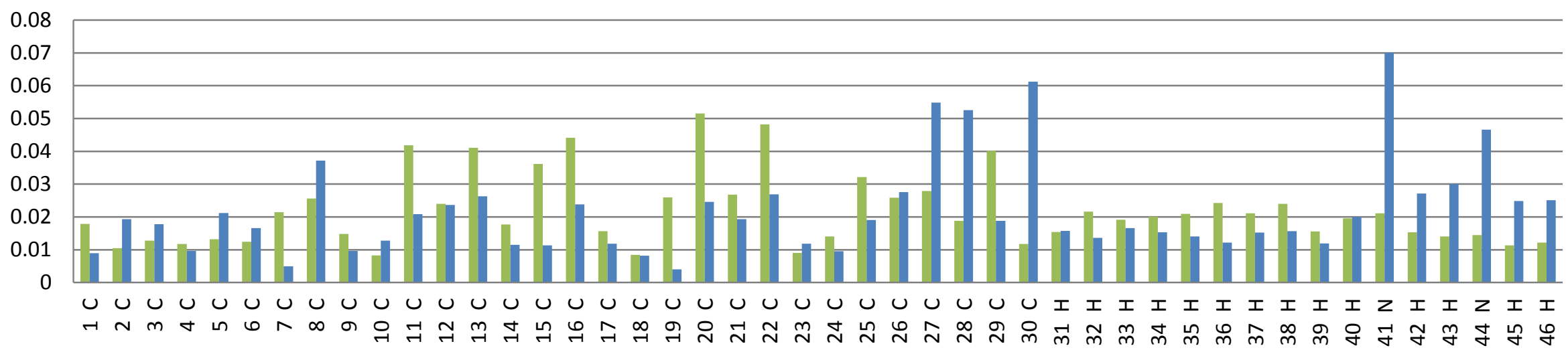




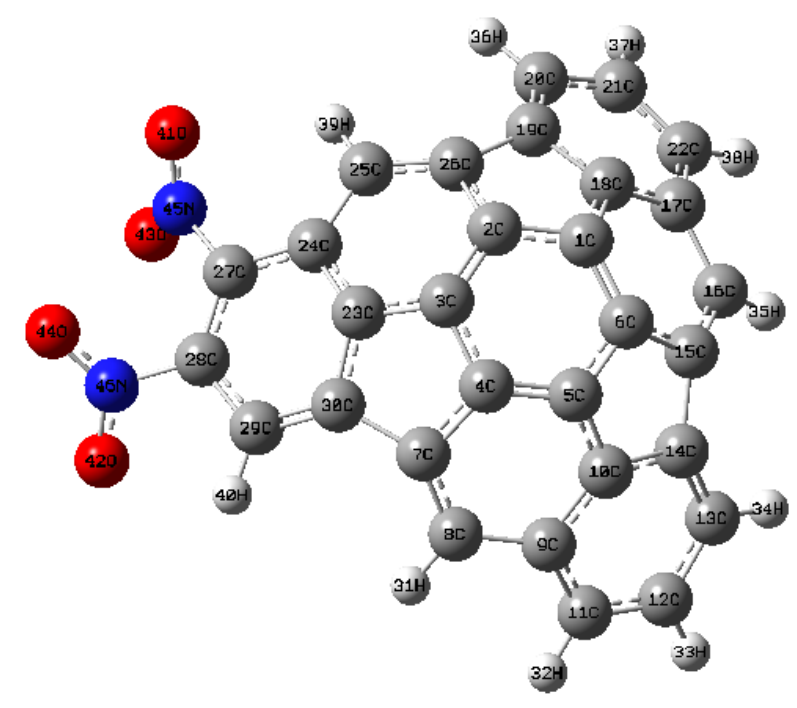

Fragmento 1 di-sustituido con $\mathrm{NO}_{2}$

\begin{tabular}{cc}
\hline $\mathrm{I} \mathrm{eV}$ & $\mathbf{7 . 5 5}$ \\
$\mathrm{A} \mathrm{eV}$ & 1.80 \\
$\mu \mathrm{eV}$ & -4.67 \\
$\eta \mathrm{eV}$ & 5.74 \\
$\mathrm{~S} \mathrm{eV}$ & 0.17 \\
$\omega \mathrm{eV}$ & 1.90 \\
$\omega^{+} \mathrm{eV}$ & 1.83 \\
$\omega^{-} \mathrm{eV}$ & 6.50
\end{tabular}

$\square f_{i}^{+}(r) \quad \square f_{i}^{-}(r)$

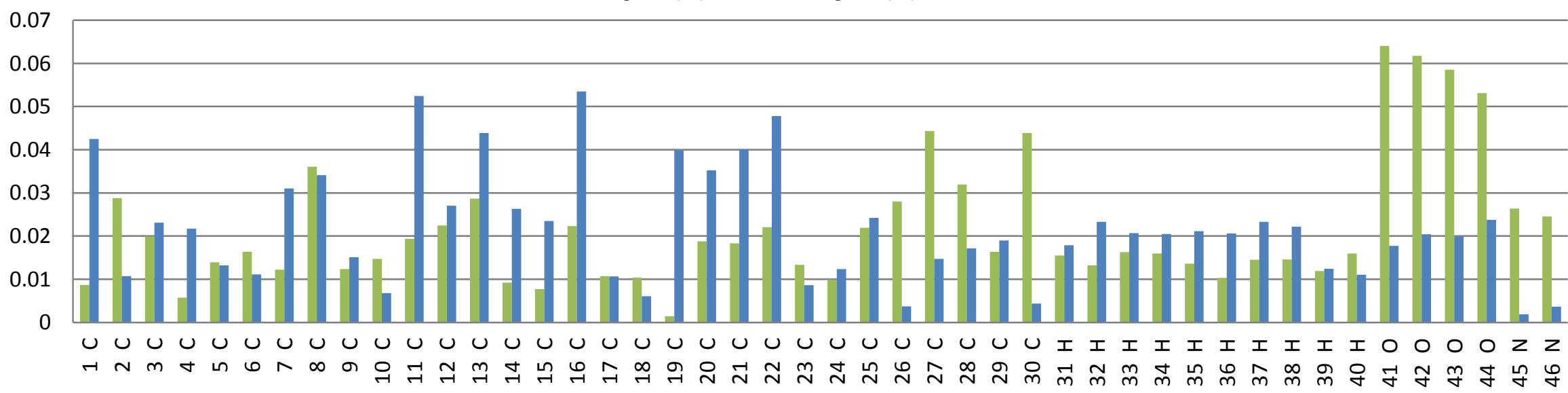




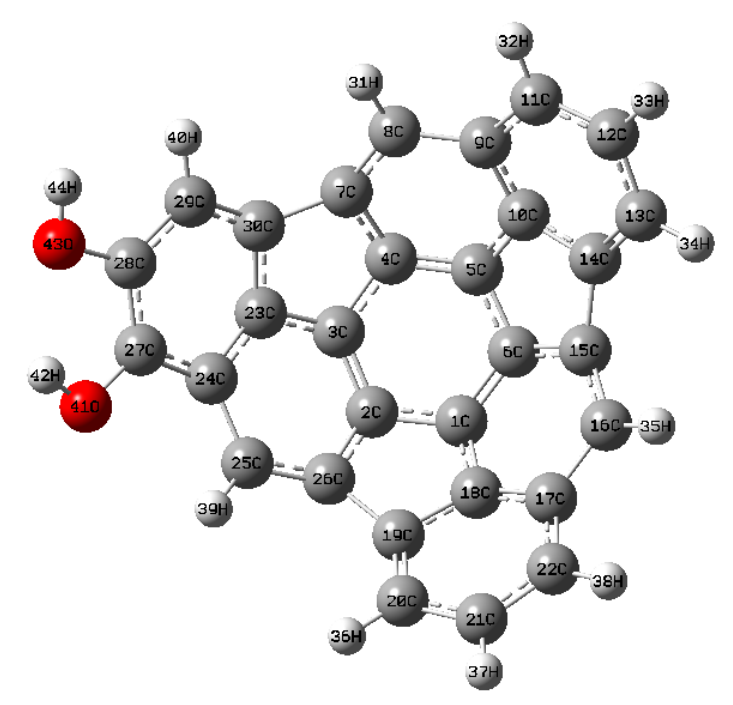

Fragmento 1 di-sustituido con $\mathrm{OH}$

\begin{tabular}{cc}
\hline $\mathrm{I} \mathrm{eV}$ & $\mathbf{6 . 5 8}$ \\
$\mathrm{A} \mathrm{eV}$ & 0.54 \\
$\mu \mathrm{eV}$ & -3.56 \\
$\eta \mathrm{eV}$ & 6.05 \\
$\mathrm{~S} \mathrm{eV}$ & 0.17 \\
$\omega \mathrm{eV}$ & 1.05 \\
$\omega^{+} \mathrm{eV}$ & 0.69 \\
$\omega^{-} \mathrm{eV}$ & 4.25
\end{tabular}

\section{$\square f_{i}{ }^{+}(r) \quad \square f_{i}{ }^{-}(r)$}

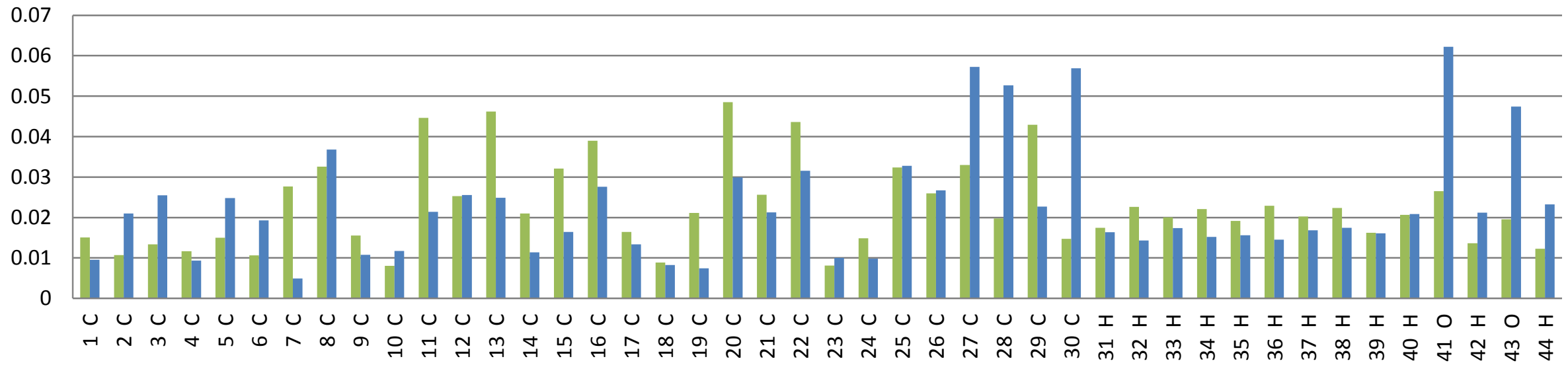




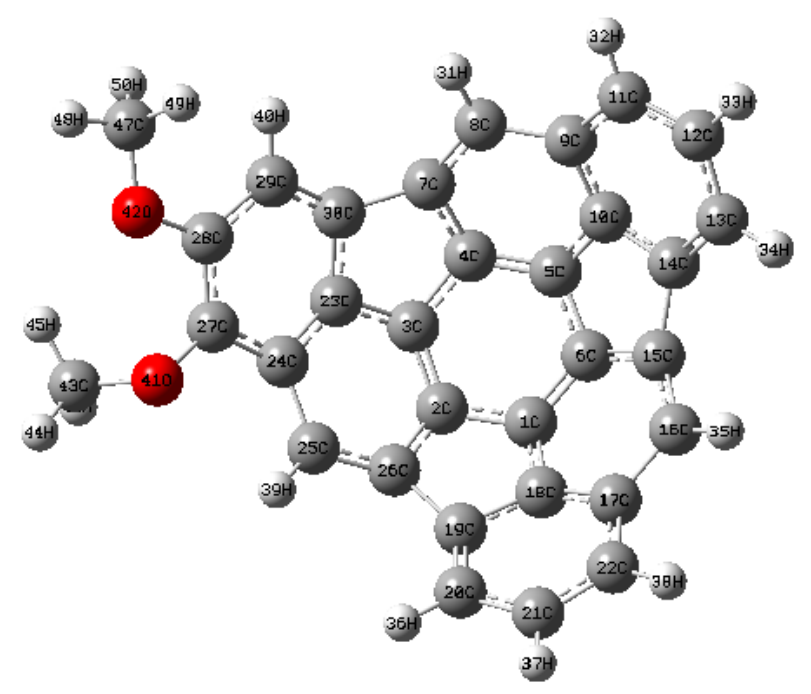

Fragmento 1 di-sustituido con OMe

$\begin{array}{cc}\mathrm{I} \mathrm{eV} & \mathbf{6 . 5 7} \\ \mathrm{A} \mathrm{eV} & 0.57 \\ \mu \mathrm{eV} & -3.57 \\ \eta \mathrm{eV} & 6.00 \\ \mathrm{~S} \mathrm{eV} & 0.17 \\ \omega \mathrm{eV} & 1.06 \\ \omega^{+} \mathrm{eV} & 0.72 \\ \omega^{-} \mathrm{eV} & 4.29\end{array}$

$f_{i}^{+}(r) \quad \square f_{i}^{-}(r)$

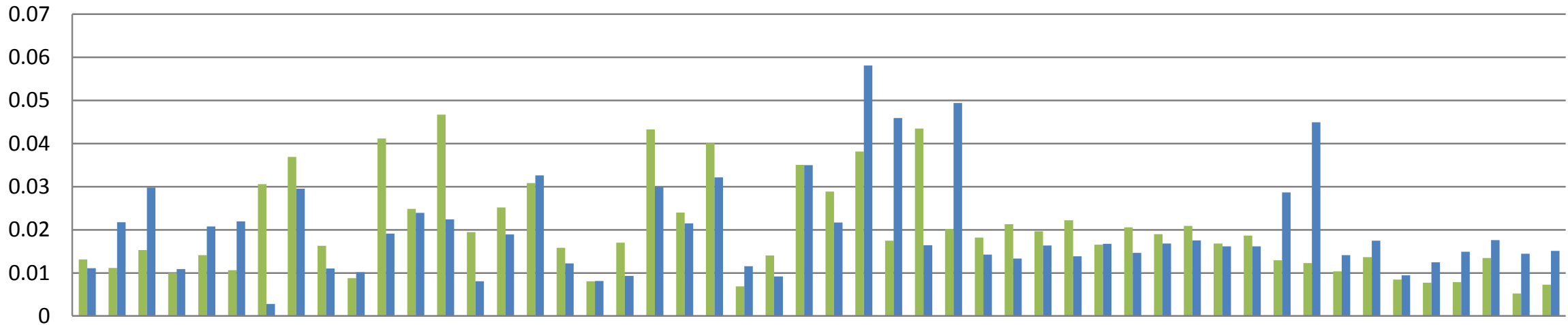

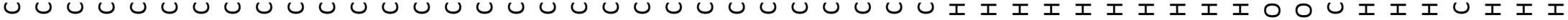

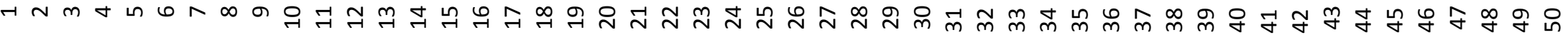




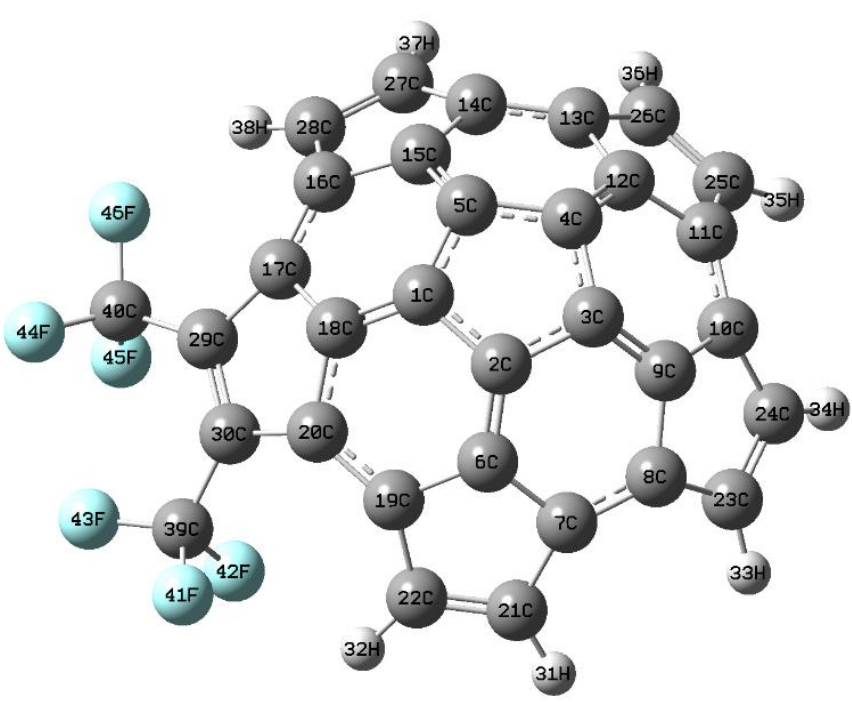

Fragmento 2 di-sustituido con $\mathrm{CF}_{3}$

$\begin{array}{cc}\mathrm{I} \text { eV } & \mathbf{7 . 3 1} \\ \mathrm{A} \mathrm{eV} & 1.72 \\ \mu \mathrm{eV} & -4.52 \\ \eta \mathrm{eV} & 5.59 \\ \mathrm{~S} \mathrm{eV} & 0.18 \\ \omega \mathrm{eV} & 1.82 \\ \omega^{+} \mathrm{eV} & 1.74 \\ \omega^{-} \mathrm{eV} & 6.25\end{array}$

$\square f_{i}^{+}(r) \quad \square f_{i}^{-}(r)$

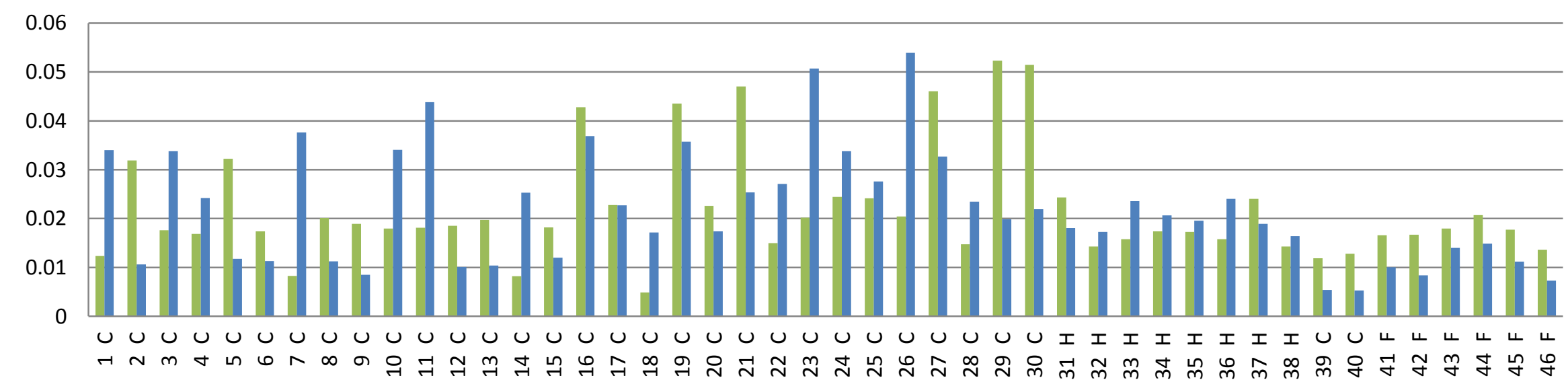




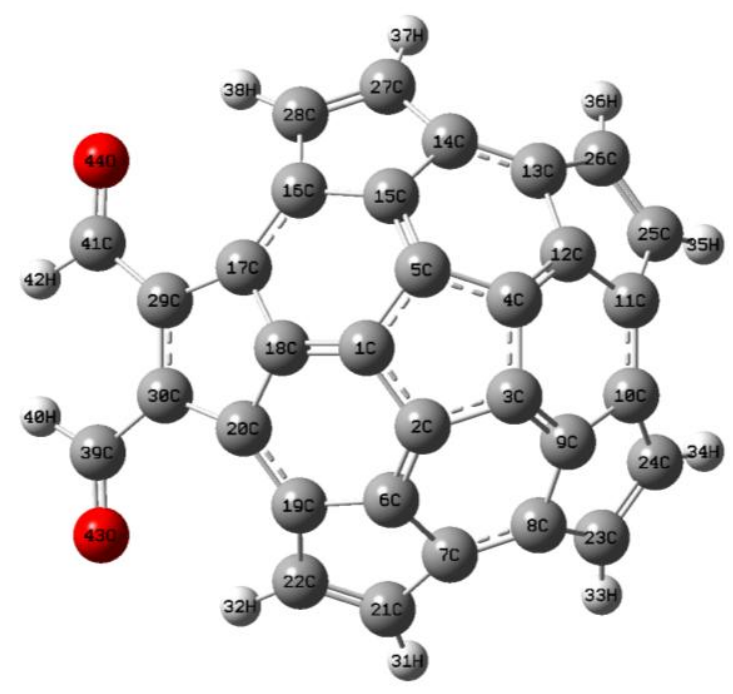

Fragmento 2 di-sustituido con $\mathrm{CHO}$

\begin{tabular}{cc}
\hline $\mathrm{I} \mathrm{eV}$ & $\mathbf{7 . 7 2}$ \\
$\mathrm{A} \mathrm{eV}$ & 2.09 \\
$\mu \mathrm{eV}$ & -4.68 \\
$\eta \mathrm{eV}$ & 5.18 \\
$\mathrm{~S} \mathrm{eV}$ & 0.19 \\
$\omega \mathrm{eV}$ & 2.11 \\
$\omega^{+} \mathrm{eV}$ & 2.21 \\
$\omega^{-} \mathrm{eV}$ & 6.89
\end{tabular}

\section{$\square f_{i}^{+}(r) \quad \square f_{i}^{-}(r)$}

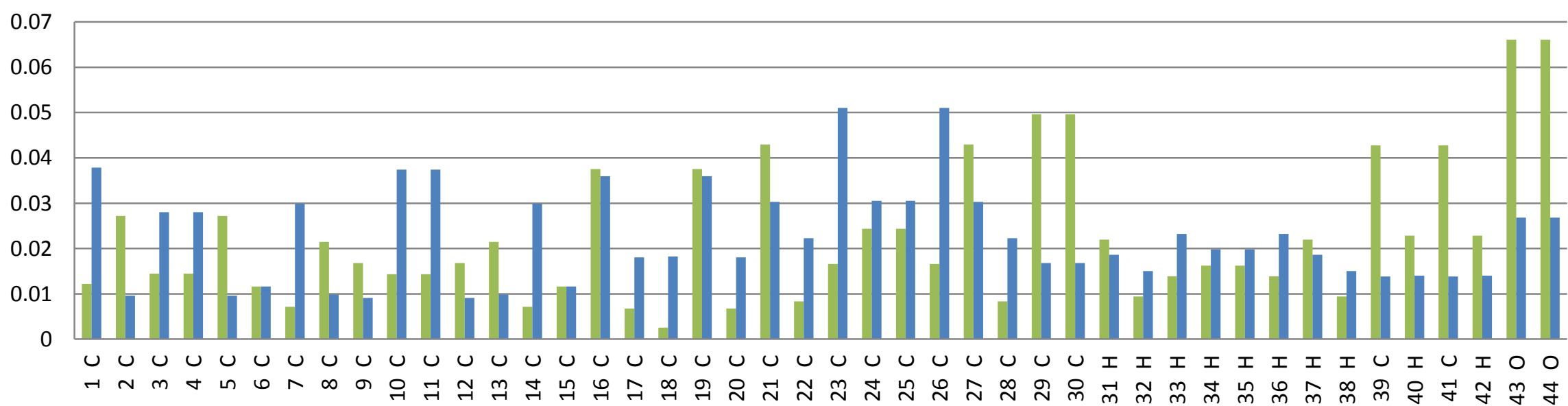




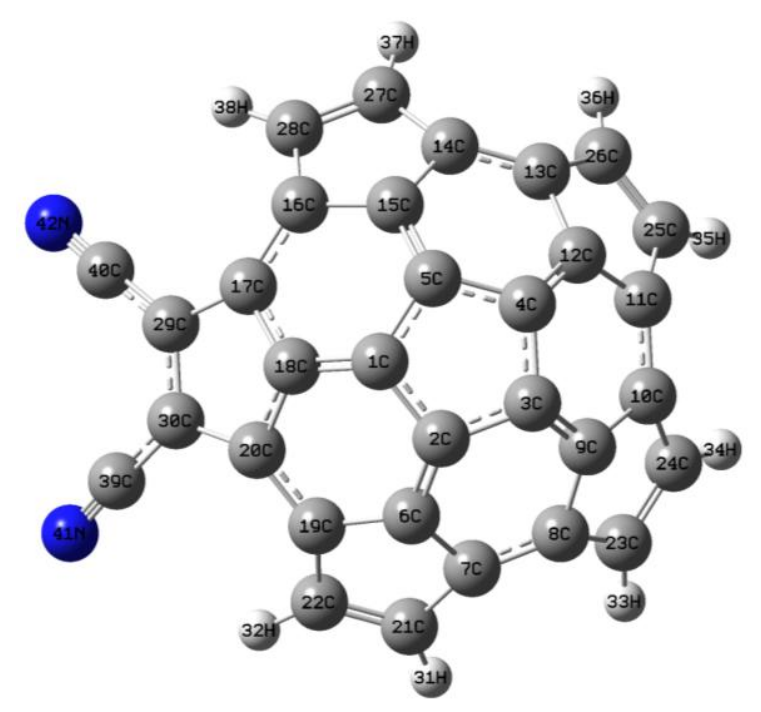

Fragmento 2 di-sustituido con CN

\begin{tabular}{cc}
\hline $\mathrm{I} \mathrm{eV}$ & $\mathbf{7 . 5 1}$ \\
$\mathrm{A} \mathrm{eV}$ & 2.17 \\
$\mu \mathrm{eV}$ & -4.84 \\
$\eta \mathrm{eV}$ & 5.34 \\
$\mathrm{~S} \mathrm{eV}$ & 0.19 \\
$\omega \mathrm{eV}$ & 2.19 \\
$\omega^{+} \mathrm{eV}$ & 2.30 \\
$\omega^{-} \mathrm{eV}$ & 7.14
\end{tabular}

$\square f_{i}{ }^{+}(r) \quad \square f_{i}^{-}(r)$

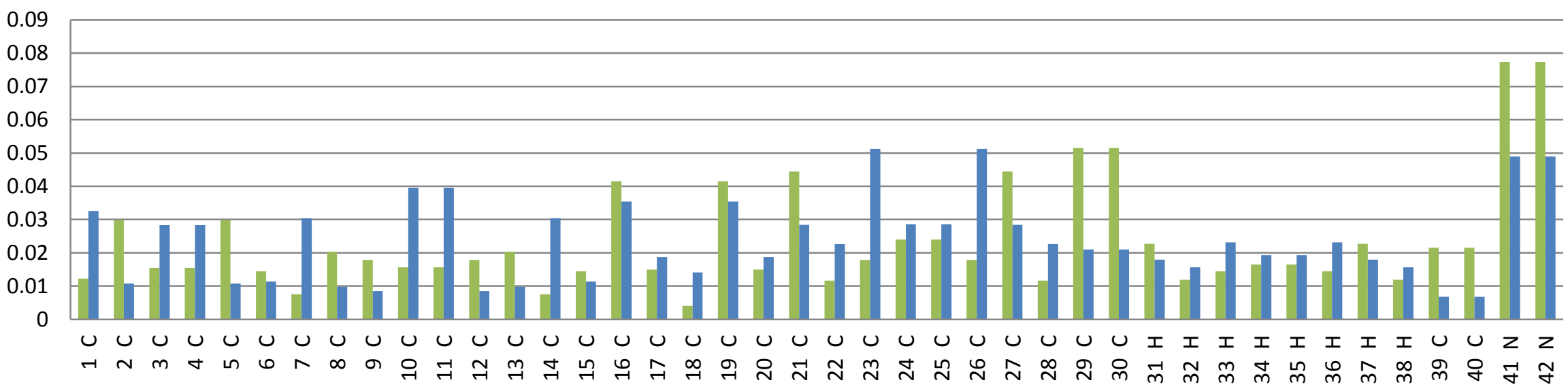




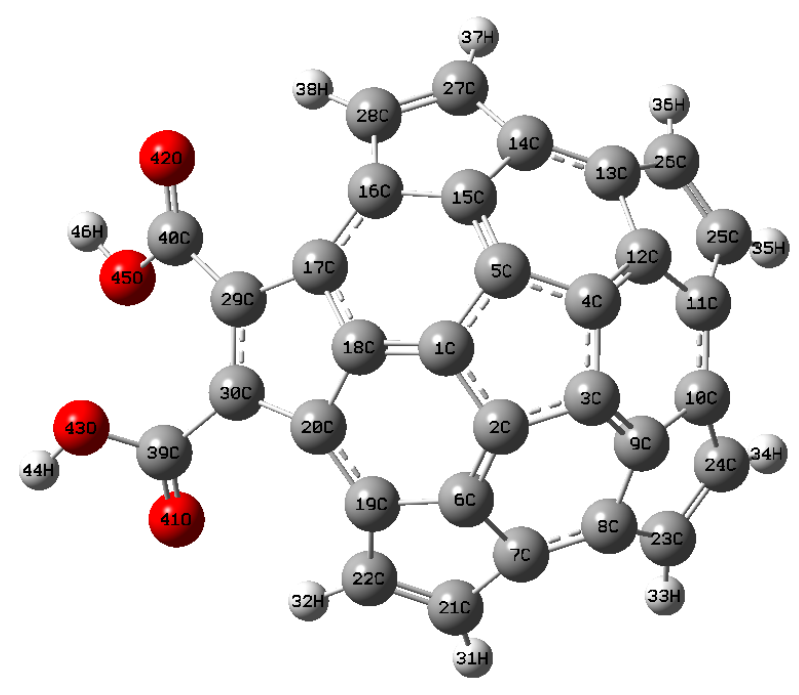

Fragmento 2 di-sustituido con $\mathrm{COOH}$

$\begin{array}{cc}\mathrm{I} \mathrm{eV} & \mathbf{8 . 8 6} \\ \mathrm{A} \mathrm{eV} & 1.70 \\ \mu \mathrm{eV} & -5.28 \\ \eta \mathrm{eV} & 7.16 \\ \mathrm{~S} \mathrm{eV} & 0.14 \\ \omega \mathrm{eV} & 1.94 \\ \omega^{+} \mathrm{eV} & 1.70 \\ \omega^{-} \mathrm{eV} & 6.98\end{array}$

$\square f_{i}{ }^{+}(r) \quad \square f_{i}{ }^{-}(r)$

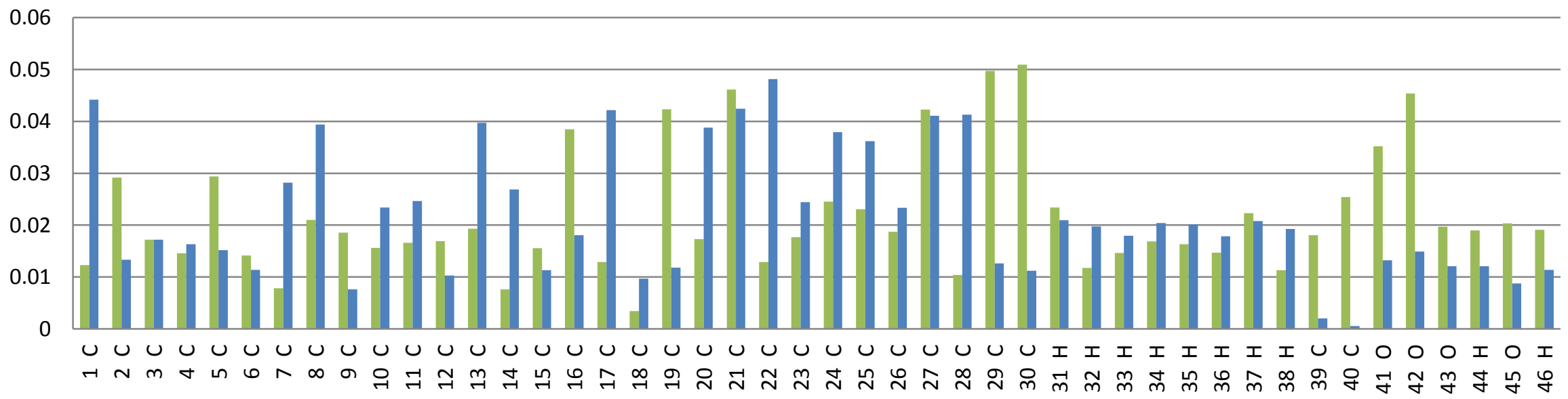




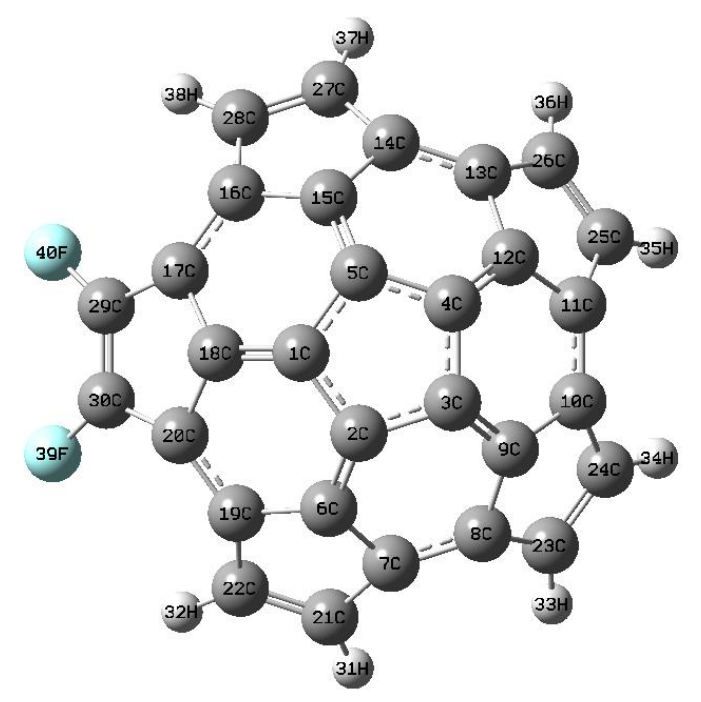

\section{Fragmento 2 di-sustituido con $F$}

\begin{tabular}{cc}
\hline $\mathrm{I} \mathrm{eV}$ & $\mathbf{7 . 0 6}$ \\
$\mathrm{A} \mathrm{eV}$ & 1.28 \\
$\mu \mathrm{eV}$ & -4.17 \\
$\eta \mathrm{eV}$ & 5.78 \\
$\mathrm{~S} \mathrm{eV}$ & 0.17 \\
$\omega \mathrm{eV}$ & 1.51 \\
$\omega^{+} \mathrm{eV}$ & 1.29 \\
$\omega^{-} \mathrm{eV}$ & 5.46
\end{tabular}

\section{$\square f_{i}^{+}(r) \quad \square f_{i}^{-}(r)$}

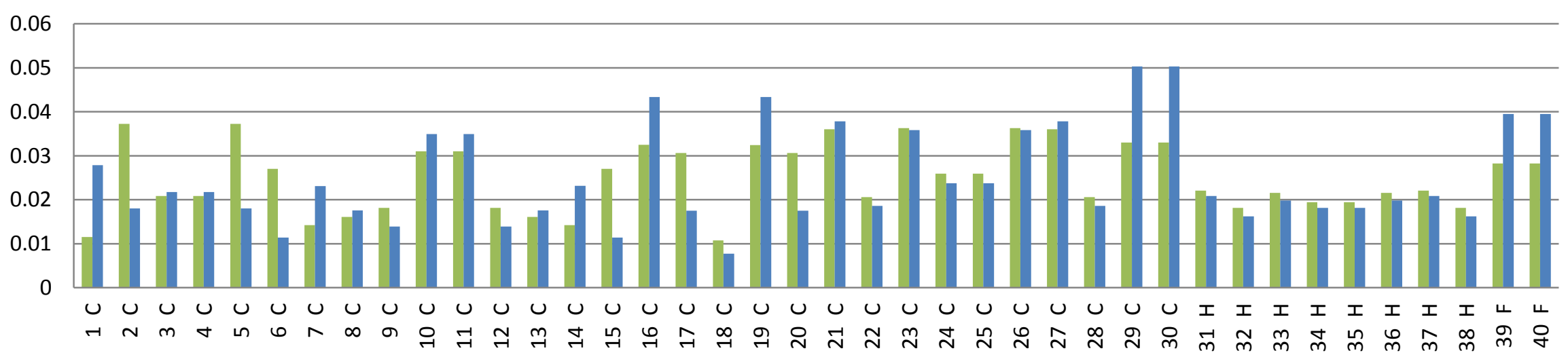




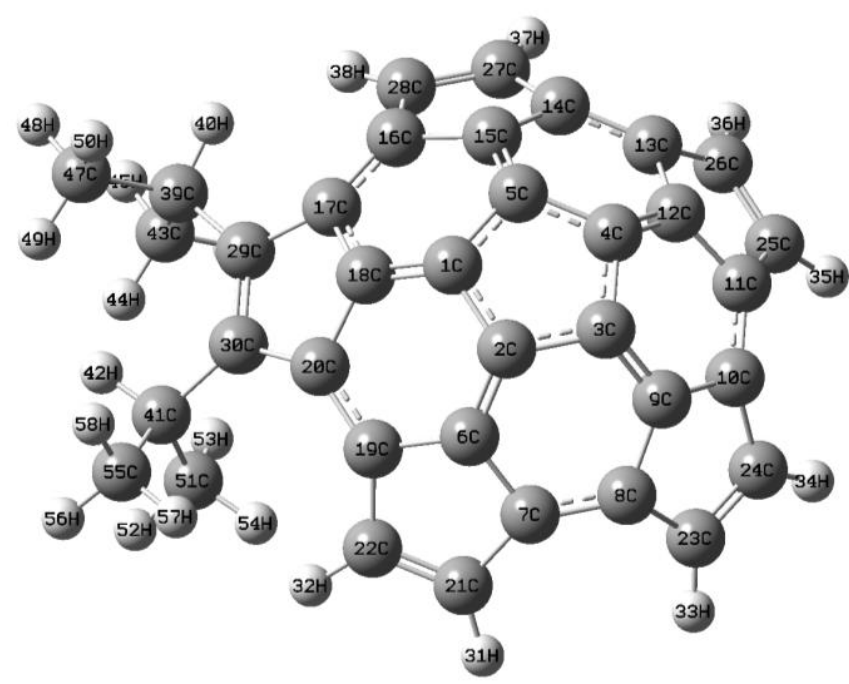

Fragmento 2 di-sustituido con $i$-Pr

\begin{tabular}{cc}
\hline $\mathrm{I} \mathrm{eV}$ & $\mathbf{6 . 7 2}$ \\
$\mathrm{A} \mathrm{eV}$ & 1.10 \\
$\mu \mathrm{eV}$ & -3.91 \\
$\eta \mathrm{eV}$ & 5.62 \\
$\mathrm{~S} \mathrm{eV}$ & 0.18 \\
$\omega \mathrm{eV}$ & 1.36 \\
$\omega^{+} \mathrm{eV}$ & 1.12 \\
$\omega^{-} \mathrm{eV}$ & 5.03
\end{tabular}

$\square f_{i}^{+}(r) \quad \square f_{i}^{-}(r)$

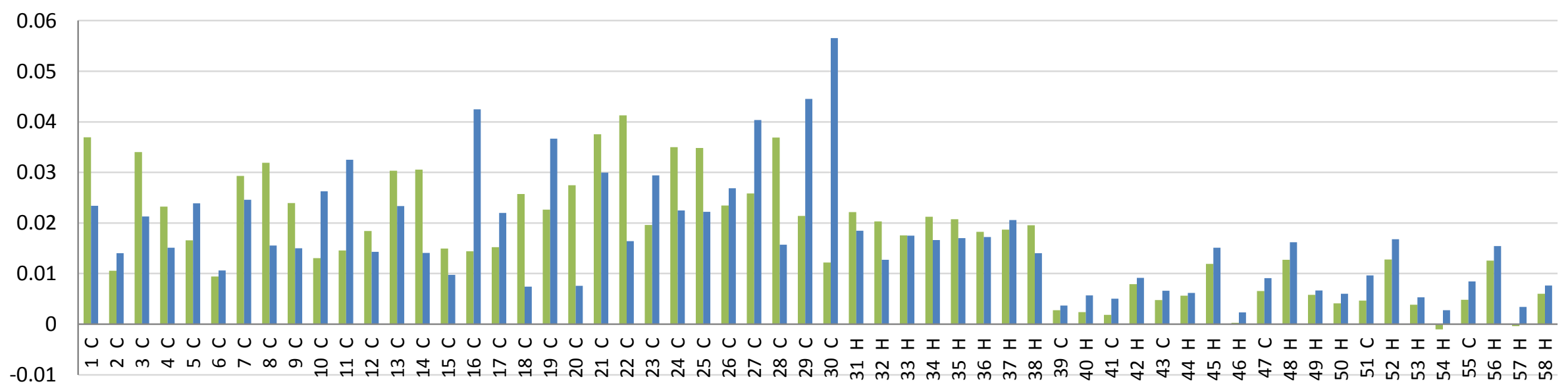




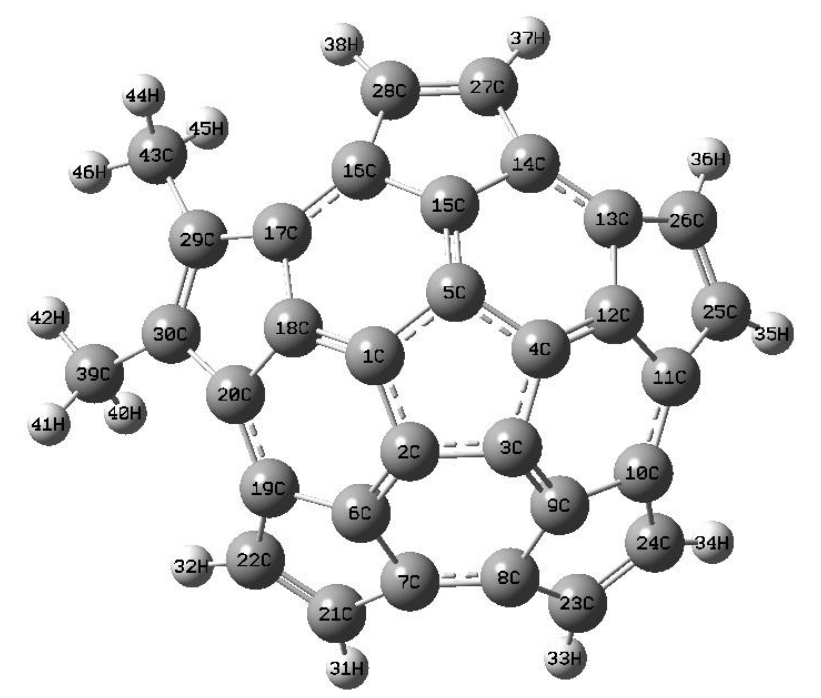

\section{Fragmento 2 di-sustituido con Me}

\begin{tabular}{cc}
\hline $\mathrm{I} \mathrm{eV}$ & 6.79 \\
$\mathrm{~A} \mathrm{eV}$ & 1.04 \\
$\mu \mathrm{eV}$ & -3.92 \\
$\eta \mathrm{eV}$ & 5.75 \\
$\mathrm{~S} \mathrm{eV}$ & 0.17 \\
$\omega \mathrm{eV}$ & 1.34 \\
$\omega^{+} \mathrm{eV}$ & 1.07 \\
$\omega^{-} \mathrm{eV}$ & 4.99
\end{tabular}

\section{$\square f_{i}^{+}(r) \quad \square f_{i}^{-}(r)$}

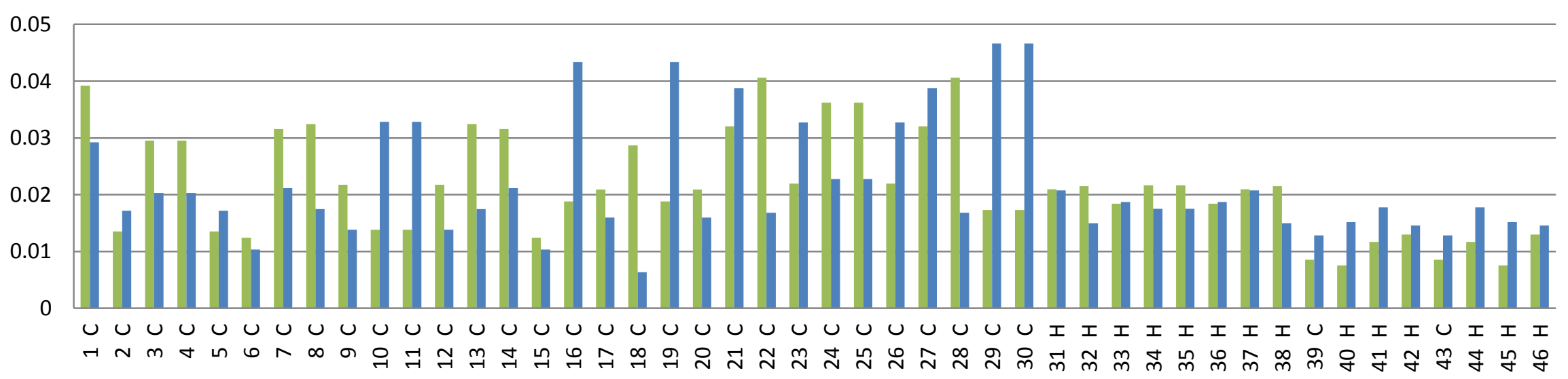




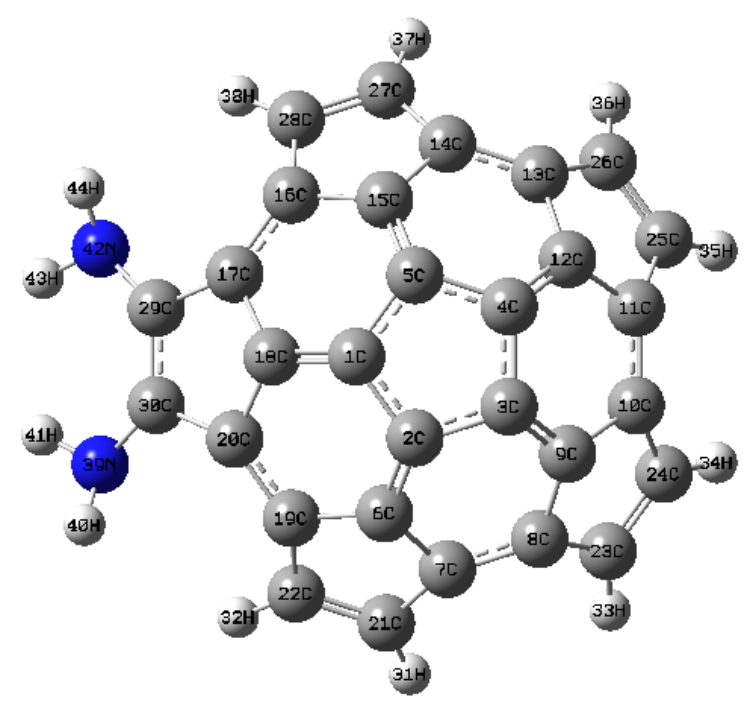

Fragmento 2 di-sustituido con $\mathrm{NH}_{2}$

$\begin{array}{cr}\mathrm{I} \mathrm{eV} & \mathbf{6 . 0 5} \\ \mathrm{A} \mathrm{eV} & 0.81 \\ \mu \mathrm{eV} & -3.43 \\ \eta \mathrm{eV} & 5.24 \\ \mathrm{~S} \mathrm{eV} & 0.19 \\ \omega \mathrm{eV} & 1.12 \\ \omega^{+} \mathrm{eV} & 0.86 \\ \omega^{-} \mathrm{eV} & 4.29\end{array}$

$\square f_{i}^{+}(r) \quad \square f_{i}{ }^{-}(r)$

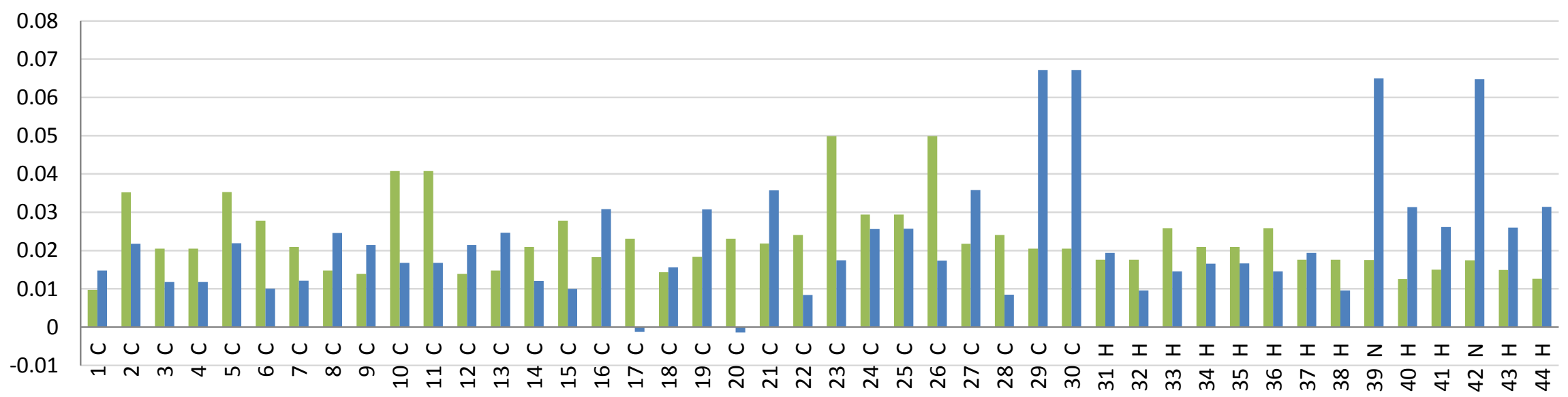




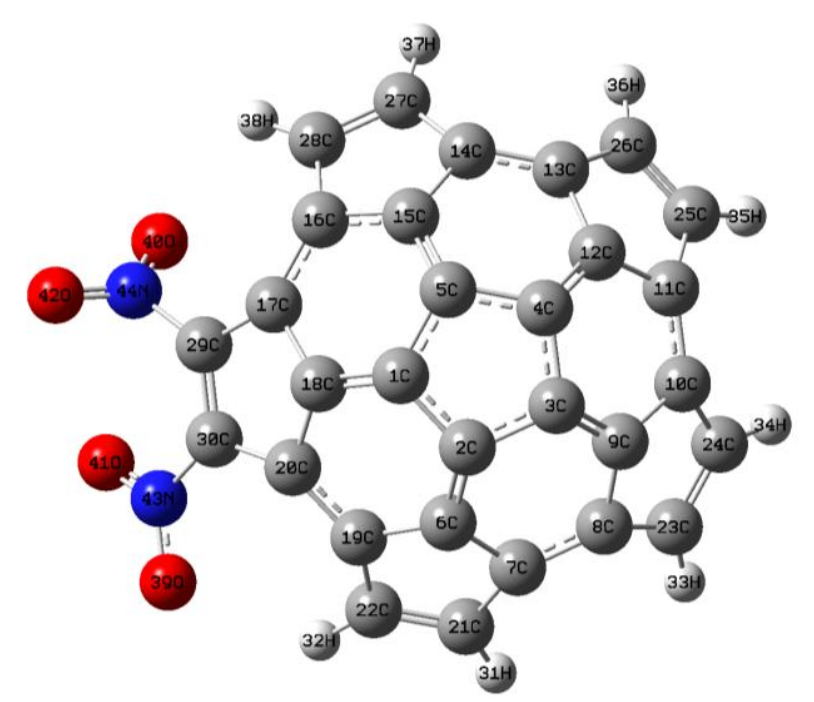

Fragmento 2 di-sustituido con $\mathbf{N O}_{2}$

\begin{tabular}{cc}
\hline $\mathrm{I} \mathrm{eV}$ & $\mathbf{7 . 5 6}$ \\
$\mathrm{A} \mathrm{eV}$ & 2.30 \\
$\mu \mathrm{eV}$ & -4.93 \\
$\eta \mathrm{eV}$ & 5.25 \\
$\mathrm{~S} \mathrm{eV}$ & 0.19 \\
$\omega \mathrm{eV}$ & 2.31 \\
$\omega^{+} \mathrm{eV}$ & 2.49 \\
$\omega^{-} \mathrm{eV}$ & 7.42
\end{tabular}

$\square f_{i}^{+}(r) \quad \square f_{i}^{-}(r)$

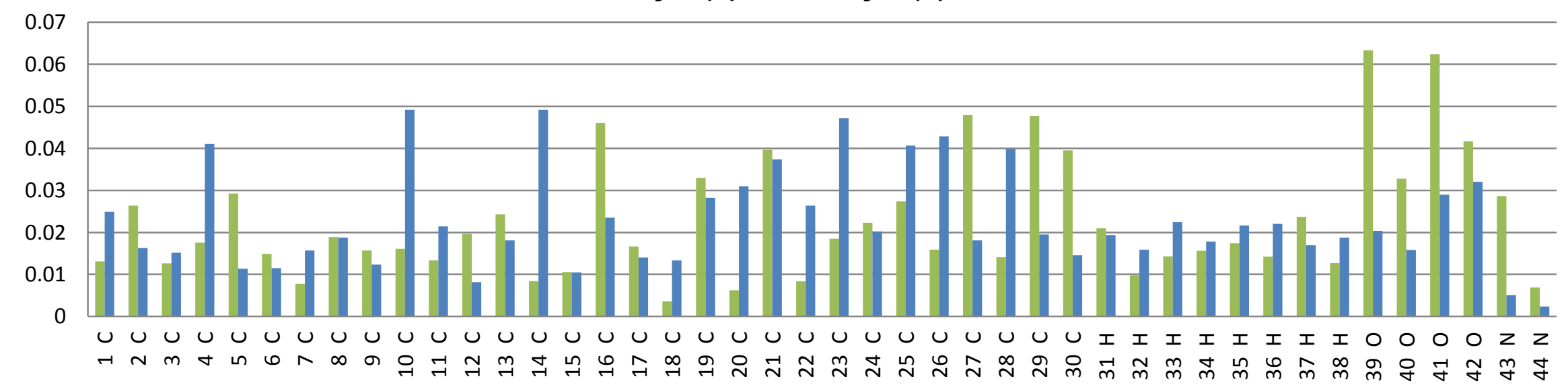




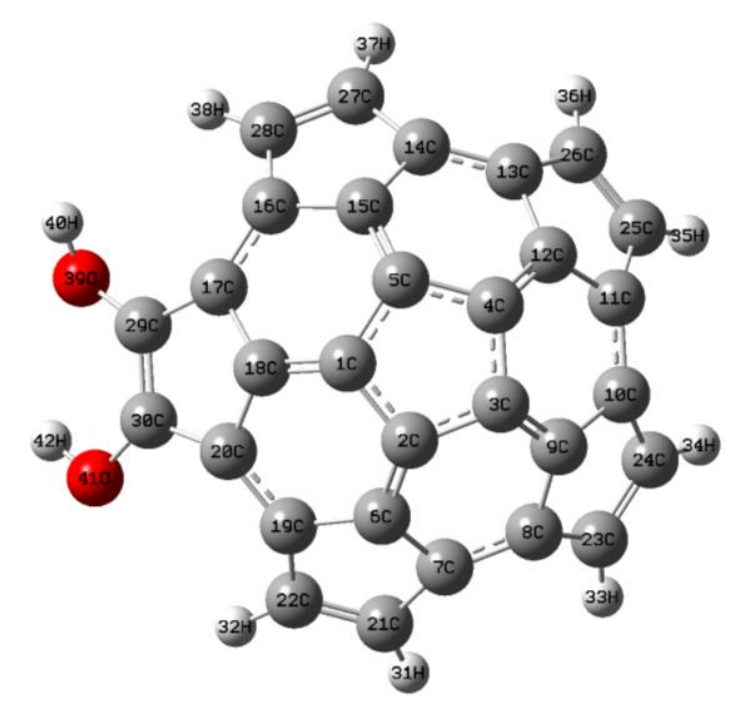

Fragmento 2 di-sustituido con $\mathrm{OH}$

$\begin{array}{cr}\mathrm{I} \mathrm{eV} & \mathbf{6 . 5 5} \\ \mathrm{A} \mathrm{eV} & 1.03 \\ \mu \mathrm{eV} & -3.79 \\ \eta \mathrm{eV} & 5.51 \\ \mathrm{~S} \mathrm{eV} & 0.18 \\ \omega \mathrm{eV} & 1.30 \\ \omega^{+} \mathrm{eV} & 1.05 \\ \omega^{-} \mathrm{eV} & 4.84\end{array}$

\section{$\square f_{i}{ }^{+}(r) \quad \square f_{i}{ }^{-}(r)$}

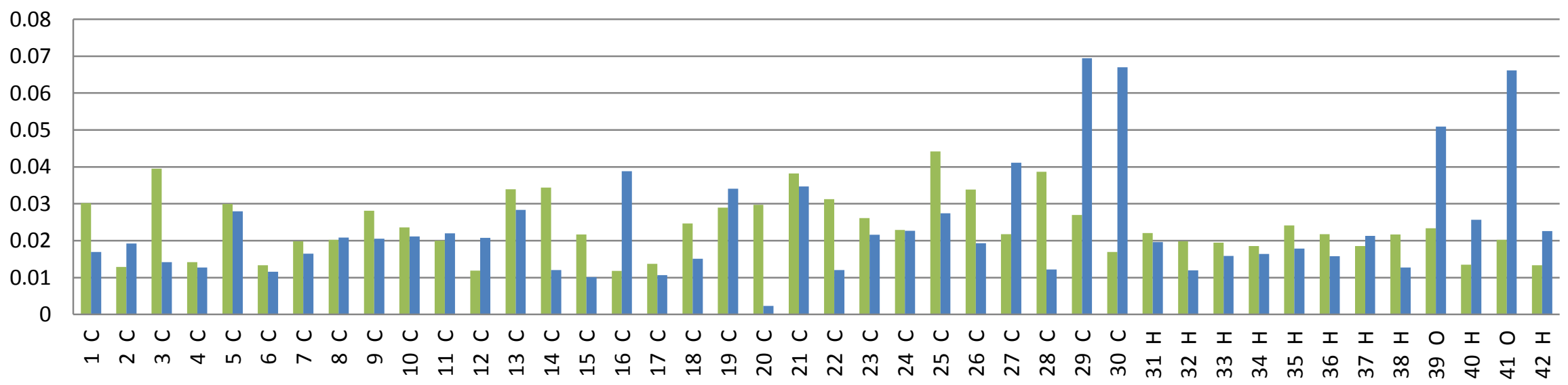




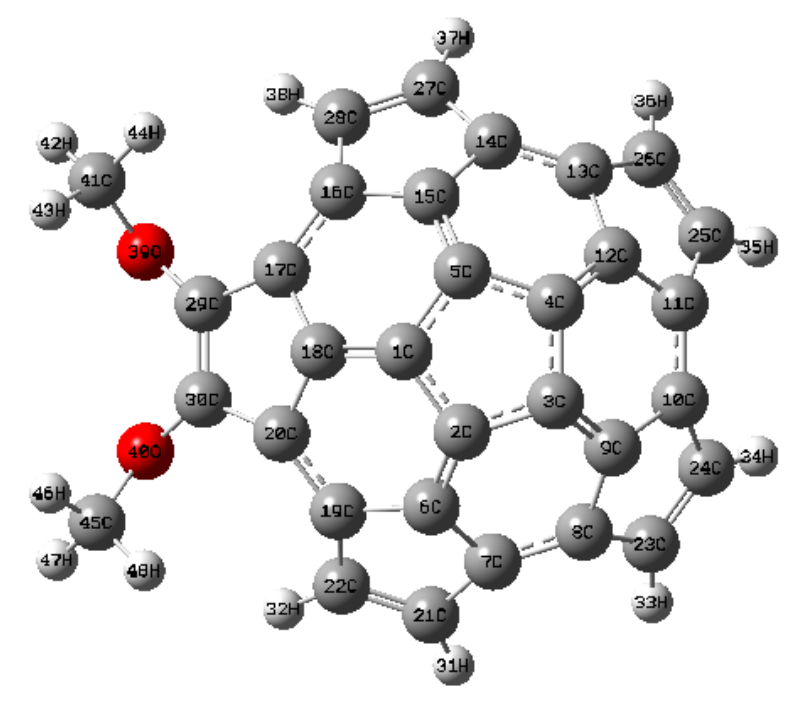

Fragmento 2 di-sustituido con $\mathrm{OMe}$

\begin{tabular}{cc}
\hline $\mathrm{I} \mathrm{eV}$ & 6.60 \\
$\mathrm{~A} \mathrm{eV}$ & 1.06 \\
$\mu \mathrm{eV}$ & -3.83 \\
$\eta \mathrm{eV}$ & 5.55 \\
$\mathrm{~S} \mathrm{eV}$ & 0.18 \\
$\omega \mathrm{eV}$ & 1.32 \\
$\omega^{+} \mathrm{eV}$ & 1.08 \\
$\omega^{-} \mathrm{eV}$ & 4.90
\end{tabular}

\section{$\square f_{i}^{+}(r) \quad \square f_{i}^{-}(r)$}

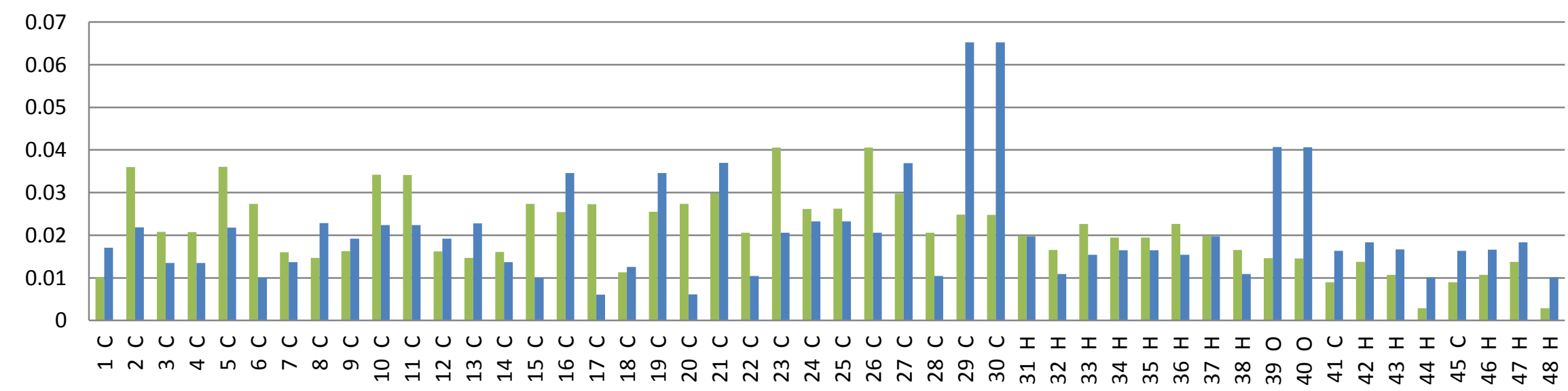




\title{
molecules
}

ISSN 1420-3049

www.mdpi.com/journal/molecules

Article

\section{Growth of Fullerene Fragments Using the Diels-Alder Cycloaddition Reaction: First Step towards a $\mathrm{C}_{60}$ Synthesis by Dimerization}

\author{
Martha Mojica ${ }^{1}$, Francisco Méndez ${ }^{1, *}$ and Julio A. Alonso ${ }^{2}$ \\ 1 Departamento de Química, División de Ciencias Básicas e Ingeniería, Universidad Autónoma \\ Metropolitana, Unidad Iztapalapa, A.P. 55-534, México, D.F., 09340, Mexico; \\ E-Mail: cbi209382312@xanum.uam.mx \\ 2 Departamento de Física Teórica, Atómica y Óptica, Facultad de Ciencias, Universidad de Valladolid, \\ E-47011Valladolid, Spain; E-Mail: jaalonso@fta.uva.es \\ * Author to whom correspondence should be addressed; E-Mail: fm@xanum.uam.mx; \\ Tel.: +52-55-5804 6417; Fax: +52-55-5804 6415.
}

Received: 25 December 2012; in revised form: 20 January 2013 / Accepted: 5 February 2013 / Published: 13 February 2013

\begin{abstract}
Density Functional Theory has been used to model the Diels-Alder reactions of the fullerene fragments triindenetriphenilene and pentacyclopentacorannulene with ethylene and 1,3-butadiene. The purpose is to prove the feasibility of using Diels-Alder cycloaddition reactions to grow fullerene fragments step by step, and to dimerize fullerene fragments, as a way to obtain $\mathrm{C}_{60}$. The dienophile character of the fullerene fragments is dominant, and the reaction of butadiene with pentacyclopentacorannulene is favored.
\end{abstract}

Keywords: Diels-Alder cycloaddition; fullerene fragments; transition states; triindenetriphenilene; pentacyclopentacorannulene

\section{Introduction}

After the discovery of $\mathrm{C}_{60}$ by Kroto et al. in 1985 [1], its chemical synthesis has been an intensely pursued target. Krätschmer et al. [2] developed in 1990 a technique to produce $\mathrm{C}_{60}$ based on the vaporization of graphite on a helium atmosphere, and later Scott and coworkers [3] synthesized $\mathrm{C}_{60}$ by the pyrolysis of the polycyclic aromatic hydrocarbon precursor $\mathrm{C}_{60} \mathrm{H}_{27} \mathrm{Cl}_{3}$. However, the chemical synthesis of $\mathrm{C}_{60}$ cannot be considered a solved problem [4] due to the inherent disadvantages of both 
techniques. In the first case, the low yield and the problems of separating and purifying the products make the fullerene synthesis inefficient and expensive [5]. In the second, the low yield problem can be solved by using a Pt catalyst [6], however, the pyrolytic treatment employed makes necessary the creation of new methodologies to synthesize fullerenes.

One particularly seductive and promising strategy is the polymerization of $\mathrm{C}_{60}$ fragments, especially the dimerization of two identical hemispherical hydrocarbons, $\mathrm{C}_{30} \mathrm{H}_{\mathrm{x}}$ [7-11]. In this case the curvature is already present in the fragments, and the main challenge is to find a way to "stitch" the fragments together to give the closed fullerene. Obviously, the choice of those fragments is a key condition for the success of this methodology. Using "La Coupe du Roi" method [12], Geneste and coworkers [9] found nine isometric $\mathrm{C}_{30}$ fragments derived from $\mathrm{C}_{60}$, and they determined that the triindenetriphenilene and the pentacyclopentacorannulene fragments (labeled $\mathbf{1}$ and $\mathbf{2}$ in Figure 1, respectively) were the most stable fragments.

Figure 1. Triindenetriphenilene (1) and pentacyclopentacorannulene (2).

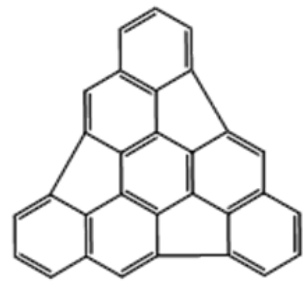

1

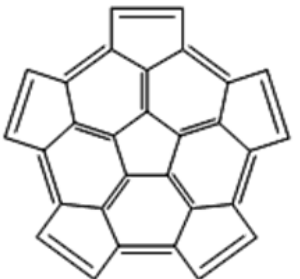

2

Recently, Scott and coworkers [13] have used the Diels-Alder (DA) cycloaddition reaction to synthesize carbon nanotubes in solution, with single chiralities and uniform diameters. Addition of an acetylene dienophile molecule to the bay regions on the rim of a buckybowl (a fragment of a buckyball acting as the end-cap of a nanotube), or an aromatic belt, leads to the nanotube growth by the formation of new six-membered rings [14]. They also calculated the activation energies for the addition of acetylene to different aromatic hydrocarbons and observed that the activation energy barrier decreases as the size of the hydrocarbon molecule increases. Moreover, they proposed the DA reaction of 7,14-dimesitylbisanthene and acetylene to experimentally probe the methodology, and concluded that the reaction was relatively easy under mild conditions. Latter they used a benzyne molecule as masked acetylene to achieve the addition of two rings at once [15]. This evidence suggested us the idea of using the DA cycloaddition reaction in the synthesis of $\mathrm{C}_{60}$. In this paper we describe the calculations that we have performed to study the DA cycloaddition reactions between fragments 1 and 2 with ethylene (e) and butadiene (b) molecules, because of the opportunity of forming new six-membered carbon rings in the rims of the two fragments. The study supports the feasibility of a systematic method to obtain fullerenes by DA cycloadditions to a starting fragment, and also suggests the possibility of assembling fullerenes by dimerization of fragments. 


\section{Results and Discussion}

The structures of fragments $\mathbf{1}$ and $\mathbf{2}$ were analyzed and the regions that behave as a diene or a dienophile have been identified (see Figure 2). In fragment $\mathbf{1}$, these alternating regions are separated by one or two bonds, while in fragment 2 the regions are adjacent and share carbon atoms; this is clearly appreciated in the resonance structures $\mathbf{2} \mathbf{a}$ and $\mathbf{2 b}$.

Figure 2. Regions in which the fragments $\mathbf{1}$ and $\mathbf{2}$ behave as a diene (blue color), or as a dienophile (red color).

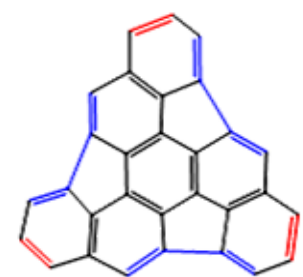

1

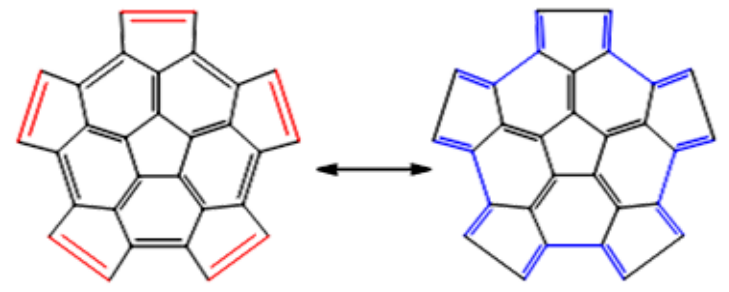

2b

Based on the structure of fragments 1 and 2, we have studied two main reactions. In the first case the DA reaction is carried out with 1,3-butadiene as the diene and the fragments $\mathbf{1}$ or $\mathbf{2}$ as the dienophile (Figure 3, panels $\mathbf{1}$ and $\mathbf{2 a}$ ). In the second case, the DA reaction is between ethylene as the dienophile and fragments $\mathbf{1}$ or $\mathbf{2}$ as the diene (Figure 3, panels $\mathbf{1}$ and $\mathbf{2 b}$ ). We have also analyzed the preference for the addition on the concave (endo) or convex (exo) surfaces of the fragments.

Figure 3. DA reaction of the fragments 1 and 2 with a diene (1,3-butadiene) and a dienophile (ethylene).

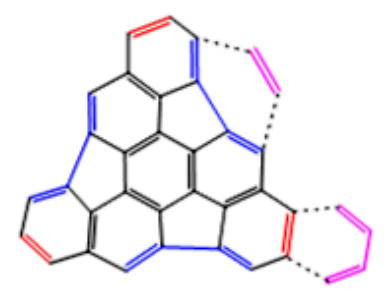

1

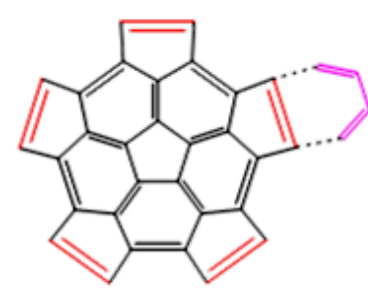

2a

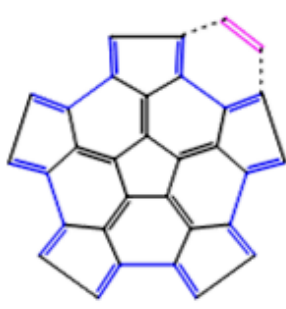

$2 \mathbf{b}$

\subsection{Frontier Molecular Orbital Analysis}

On the basis of Frontier Molecular Orbitals (FMO) theory [16,17], the Diels-Alder reaction proceeds through the interaction of the Highest Occupied Molecular Orbital (HOMO) of one of the molecules and the Lowest Unoccupied Molecular Orbital (LUMO) of the other molecule. When the interaction is between the HOMO of the diene and the LUMO of the dienophile, the reaction is called Normal Electron Demand Diels-Alder (NEDDA) reaction, and when the interaction is between the HOMO of the dienophile and the LUMO of the diene the reaction is called Inverse Electron Demand Diels-Alder (IEDDA) reaction [18]. As the energy difference between the two involved orbitals (HOMO-LUMO gap) decreases, the reaction should be easier. 
Table 1 reports the energies of the HOMO and LUMO of the e and $\mathbf{b}$ molecules and the fragments $\mathbf{1}$ and 2, obtained from the canonical orbitals calculated at the HF/6-31G(d,p)// B3LYP/6-31G(d,p) level of theory. That is, the geometries were optimized at the B3LYP/6-31G(d,p) level and then a calculation at the HF/6-31G(d,p) level was performed to obtain the orbital energies of the canonical orbitals. These orbital energies are in good agreement with the HOMO and LUMO energies calculated by Park et al. [19] with the MP2 method [20]. Table 2 shows the HOMO-LUMO gaps for the NEDDA and IEDDA reactions, that is, $\Delta \mathrm{E}_{\mathrm{NEDDA}}=\mathrm{E}_{\text {LUMOdienophile }}-\mathrm{E}_{\text {HOMOdiene, }}$ and $\Delta \mathrm{E}_{\text {IEDDA }}=\mathrm{E}_{\text {LUMOdiene }}-$ $\mathrm{E}_{\mathrm{HOMOdienopile}}$, and also the difference $\delta \Delta \mathrm{E}=\Delta \mathrm{E}_{\text {IEDDA }}-\Delta \mathrm{E}_{\mathrm{NEDDA}}$. Values of $\delta \Delta \mathrm{E}<0$ indicate that an IEDDA reaction is preferred, and values of $\delta \Delta \mathrm{E}>0$ indicate the preference for a NEDDA reaction. The largest IEDDA and NEDDA HOMO-LUMO gaps occur for the reference reaction between e and b. The gaps for the reactions between fragments $\mathbf{1}$ and $\mathbf{2}$ with $\mathbf{e}$ and $\mathbf{b}$ are smaller, and consequently these reactions should be easier. In addition, the reactions of $\mathbf{e}$ or $\mathbf{b}$ with fragment $\mathbf{2}$ are preferred over the corresponding reactions with fragment $\mathbf{1}$. Moreover, the reactions of fragments $\mathbf{1}$ and $\mathbf{2}$ with $\mathbf{b}$ are favored over the corresponding reactions of fragments $\mathbf{1}$ and $\mathbf{2}$ with $\mathbf{e}$.

Table 1. HOMO and LUMO energies (in eV) of ethylene, 1,3-butadiene and the fragments $\mathbf{1}$ and 2.

\begin{tabular}{ccc}
\hline Molecule & $\mathbf{E}_{\text {HOMO }}$ & $\mathbf{E}_{\text {LUMO }}$ \\
\hline Ethylene & $-10.11(-10.07)^{\mathrm{a}}$ & $4.88(4.87)^{\mathrm{a}}$ \\
Butadiene & $-8.77(-8.82)^{\mathrm{a}}$ & $3.45(3.59)^{\mathrm{a}}$ \\
1 & -7.28 & 1.38 \\
2 & -7.18 & 0.67 \\
\hline \multicolumn{3}{c}{}
\end{tabular}

Table 2. HOMO-LUMO gaps, in $\mathrm{eV}$, for the NEDDA and IEDDA reactions of fragments 1 and 2 with ethylene and 1,3-butadiene. $\triangle \mathrm{E}_{\mathrm{NEDDA}}=\mathrm{E}_{\mathrm{LUMOdienophile}}-\mathrm{E}_{\text {HOMOdiene }}$ and $\Delta \mathrm{E}_{\mathrm{IEDDA}}=\mathrm{E}_{\mathrm{LUMOdiene}}-\mathrm{E}_{\mathrm{HOMOdienopile}}$. Also, $\delta \Delta \mathrm{E}=\Delta \mathrm{E}_{\mathrm{IEDDA}}-\Delta \mathrm{E}_{\mathrm{NEDDA}}$. Results for reaction $\mathbf{e}+\mathbf{b}$ are also included.

\begin{tabular}{cccc}
\hline Reaction & $\boldsymbol{\Delta} \mathbf{E}_{\mathbf{N E D D A}}$ & $\boldsymbol{\Delta} \mathbf{E}_{\text {IEDDA }}$ & $\boldsymbol{\delta} \boldsymbol{\Delta} \mathbf{E}$ \\
\hline $\mathrm{e}+\mathrm{b}$ & $13.66(13.69)^{\mathrm{a}}$ & $13.56(13.66)^{\mathrm{a}}$ & -0.09 \\
$1+\mathrm{e}$ & 12.17 & 11.49 & -0.67 \\
$2+\mathrm{e}$ & 12.06 & 10.78 & -1.27 \\
$1+\mathrm{b}$ & 10.15 & 10.73 & 0.58 \\
$2+\mathrm{b}$ & 9.45 & 10.63 & 1.18 \\
\hline \multicolumn{4}{c}{${ }^{\mathrm{a}}$ from ref. [19]. }
\end{tabular}

We have also calculated the HOMO-LUMO gaps for the dimerization reaction between two fragments of type 1, and between two fragments of type 2 (see Figure 4). The values obtained, $8.66 \mathrm{eV}$ and $7.85 \mathrm{eV}$, respectively, are lower than the values for the reactions shown in Table 2. This suggests that the dimerization reactions $\mathbf{1}+\mathbf{1}$ and $\mathbf{2}+\mathbf{2}$ should be possible, the dimerization of fragment $\mathbf{2}$ being preferred over the dimerization of fragment 1 . Moreover, these values indicate that both dimerization reactions should be easier than the reaction between $\mathbf{e}$ and $\mathbf{b}$. However, chemical reactions do not only depend on electronic effects [21], and an analysis of kinetic and thermodynamic factors is required for the complete understanding of the proposed reactions. 
Figure 4. Dimerization reaction between two fragments of type $1(1+1)$, and between two fragments of type $2(2+2)$.

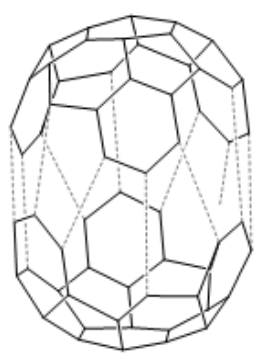

$1+1$

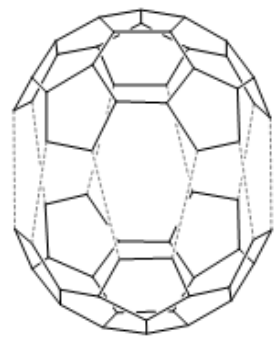

$2+2$

\subsection{Kinetic and Thermodynamic Analysis}

The transition state structures of the proposed reactions are shown in Figure 5, and the transition distances $r_{1}$ and $r_{2}$ are given in Table 3. Small values of $\Delta r=r_{1}-r_{2}$ suggest synchronous reactions while large values of $\Delta \mathrm{r}$ suggest asynchronous reactions. The reactions of $\mathbf{e}$ onto the convex surface of fragments $\mathbf{1}$ and $\mathbf{2}$ will be the most asynchronous ones, followed by the reactions of $\mathbf{b}$ onto the concave and convex surfaces of fragment $\mathbf{1}$. The reaction of $\mathbf{b}$ onto the convex surface of fragment $\mathbf{2}$ will be fully synchronous $(\Delta r=0)$, as well as the reaction of $\mathbf{e}$ and $\mathbf{b}$; moreover, the transition distances in both reactions are equal $(2.27 \AA)$. This value is in good agreement with the values reported by Houk et al. [22] for the reaction between $\mathbf{e}$ and $\mathbf{b}$.

Figure 5. Transition state structures for the reactions of (a) e onto the concave surface of $\mathbf{1}$; (b) e onto the concave surface of 2; (c) e onto the convex surface of 1; (d) e onto the convex surface of $\mathbf{2}$; (e) b onto the concave surface of $\mathbf{1}$; (f) b onto the concave surface of $\mathbf{2}$; (g) $\mathbf{b}$ onto the convex surface of $\mathbf{1}$; and (h) $\mathbf{b}$ onto the convex surface of $\mathbf{2}$.

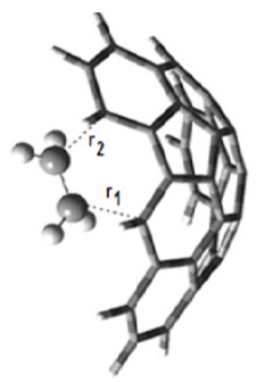

a

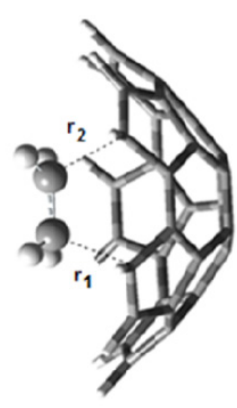

$\mathbf{b}$

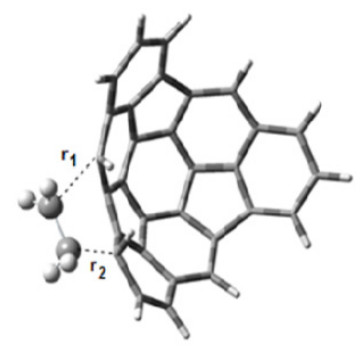

c

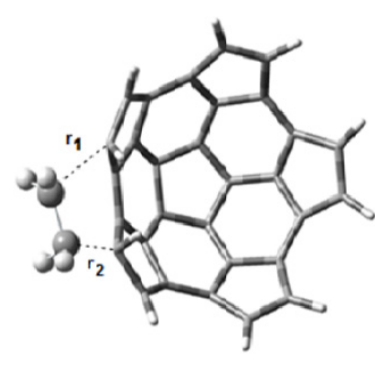

d

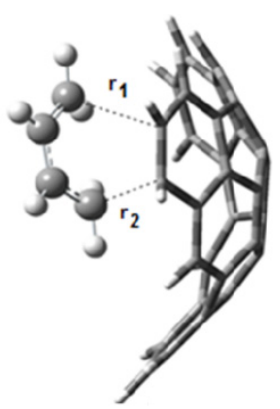

e

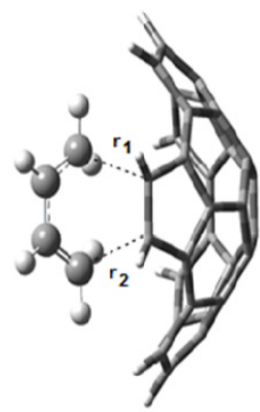

f

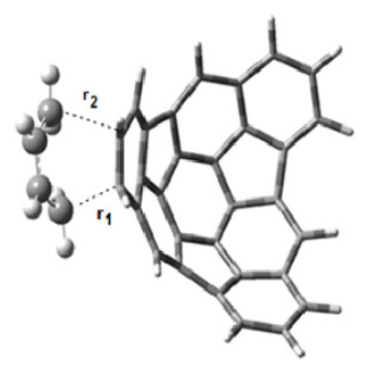

g

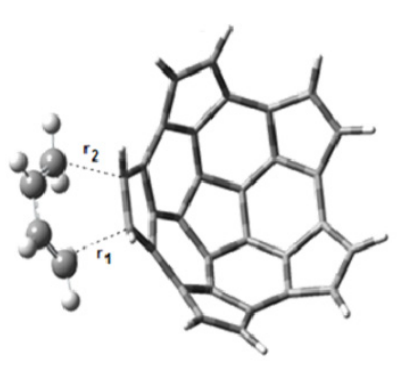

h 
Table 3. Distances $r_{1}$ and $r_{2}$, in $\AA$, for the transition states shown in Figure 5. Also $\Delta r=r_{1}-r_{2}$.

\begin{tabular}{cccc}
\hline $\mathbf{T S}$ & $\mathbf{r}_{\mathbf{1}}$ & $\mathbf{r}_{\mathbf{2}}$ & $\boldsymbol{\Delta} \mathbf{r}$ \\
\hline $\mathrm{e}+\mathrm{b}$ & $2.27(2.21)^{\mathrm{a}}$ & $2.27(2.21)^{\mathrm{a}}$ & 0 \\
1 + e concave & 2.09 & 2.07 & 0.02 \\
2 + e concave & 2.07 & 2.06 & 0.01 \\
1 + e convex & 2.41 & 1.68 & 0.73 \\
2 + e convex & 2.31 & 1.63 & 0.67 \\
$1+$ b concave & 2.49 & 1.92 & 0.58 \\
$2+$ b concave & 2.28 & 2.25 & 0.03 \\
$1+$ b convex & 2.36 & 2.02 & 0.34 \\
$2+$ b convex & 2.27 & 2.27 & 0 \\
\hline
\end{tabular}

${ }^{a}$ from reference [22].

Table 4 summarizes the calculated activation energies $E_{a}$, the standard activation Gibbs energies $\Delta G_{a}$, standard activation enthalpies $\Delta H_{a}$, and standard activation entropies $\Delta S_{a}$ for the eight transition states of Figure 5, and for the reaction between $\mathbf{e}$ and $\mathbf{b}$. The interactions with ethylene are favored on the concave surface of the fragments, while the reactions with butadiene are preferred on the convex surface. Butadiene, being a larger molecule, gives rise to steric effects that make the reaction over the concave surface difficult, leading thus to a lower activation barrier for the approach onto the convex surface. The calculated activation energy of reaction $\mathbf{e}+\mathbf{b}$ is $24.82 \mathrm{kcal} \mathrm{mol}^{-1}$. Therefore, reactions with equal or lower activation energies should proceed easily. The reaction of $\mathbf{b}$ onto the convex surface of fragment 2 shows the lowest activation energy, $22.61 \mathrm{kcal} \mathrm{mol}^{-1}$, which means that this reaction would be possible under mild conditions. The activation energy for the same reaction on the concave surface is $8 \mathrm{kcal}$ per mol higher, because of the steric repulsion between $\mathbf{b}$ and the electron cloud at the concave surface of the fragment. This makes the interaction difficult. All the other reactions have higher activation energies. Therefore, those reactions would require a larger amount of energy to proceed, and this could be translated into higher temperatures and pressures, which make the experimental conditions similar to those employed in the traditional synthesis methods. Considering the reactions of $\mathbf{e}$ and $\mathbf{b}$ with fragments $\mathbf{1}$ and $\mathbf{2}$, in general the reactivity trend predicted by FMO is similar to that derived from $E_{a}$, except for the relative ordering of the reactions of $\mathbf{e}$ with fragments $\mathbf{1}$ and 2 . The reactions in which the fragment $\mathbf{2}$ acts as diene have higher $E_{a}$ values compared to reactions in which the fragment $\mathbf{1}$ acts as diene. Following Scott's ideas, we have also calculated the transition state for the reaction of fragment $\mathbf{2}$ with acetylene as the dienophile. The activation energy of this reaction, $E_{a}=40.07 \mathrm{kcal} \mathrm{mol}^{-1}$, is close to that of the reaction of fragment 2 with ethylene, and this suggests that the use of acetylene as a dienophile is not adequate for the growth of the fragment. 
Table 4. Activation energy $E_{a}$, standard activation Gibbs energy $\Delta G_{a}$, standard activation enthalpy $\Delta H_{a}$, and standard activation entropy $T \Delta S_{a}$ for Diels-Alder reactions of $\mathbf{e}$ and $\mathbf{b}$ with fragments $\mathbf{1}$ and 2. Data for the Diels-Alder reaction between $\mathbf{e}$ and $\mathbf{b}$ is given as reference.

\begin{tabular}{|c|c|c|c|c|}
\hline Reaction & $\begin{array}{c}E_{a} \\
\left(\mathrm{kcal} \mathrm{mol}^{-1}\right)\end{array}$ & $\begin{array}{c}\Delta G_{a} \\
\left(\mathrm{kcal} \mathrm{mol}^{-1}\right)\end{array}$ & $\begin{array}{c}\Delta H_{a} \\
\left(\mathrm{kcal} \mathrm{mol}^{-1}\right)\end{array}$ & $\begin{array}{c}T \Delta S_{a} \\
\left(\mathrm{kcal} \mathrm{mol}^{-1}\right)\end{array}$ \\
\hline$e+b$ & $\begin{array}{c}24.8(27.5)^{\mathrm{a}} \\
(24.2-26.7)^{\mathrm{b}} \\
(25.9)^{\mathrm{c}}\end{array}$ & 36.35 & $\begin{array}{c}23.45(24.2)^{\mathrm{d}} \\
(24.9)^{\mathrm{e}}\end{array}$ & $\begin{array}{c}-12.91(-13.32)^{\mathrm{f}} \\
(-12.76)^{\mathrm{g}}\end{array}$ \\
\hline $1+$ e concave & 40.71 & 52.88 & 39.62 & -13.25 \\
\hline $2+$ e concave & 45.63 & 57.21 & 44.70 & -12.51 \\
\hline $1+\mathrm{e}$ convex & 67.89 & 80.22 & 66.75 & -13.47 \\
\hline $2+\mathrm{e}$ convex & 73.54 & 85.84 & 72.35 & -13.49 \\
\hline $1+b$ concave & 39.01 & 51.86 & 38.36 & -13.49 \\
\hline $2+b$ concave & 31.05 & 43.53 & 30.43 & -13.10 \\
\hline $1+b$ convex & 33.11 & 46.03 & 32.47 & -13.56 \\
\hline $2+\mathrm{b}$ convex & 22.61 & 35.52 & 22.02 & -13.51 \\
\hline
\end{tabular}

${ }^{\mathrm{a}}$ Experimental $E_{a}$ from ref. [23]; ${ }^{\mathrm{b}}$ Experimental values from $E_{a}$ of cyclohexene cycloreversion and the experimental heat of reaction [23]; ${ }^{\mathrm{c}}$ Calculated $E_{a}$ from ref. [23]; ${ }^{\mathrm{d}}$ Experimental $\Delta H_{a}$ from ref. [24];

${ }^{\mathrm{e}}$ Calculated $\Delta H_{a}$ from ref. [24]; ${ }^{\mathrm{f}}$ Experimental value of $T \Delta S_{a}$ from ref. [24], ${ }^{\mathrm{g}}$ Calculated value of $T \Delta S_{a}$ from ref. [24].

The Intrinsic Reaction Coordinate (IRC) determines the energy as well as the geometry of the reacting system [25,26]. The energy change along the IRC gives the potential barrier shape [27]. Figure 6 summarizes the IRCs of the proposed reactions. The analysis of the reaction mechanisms shows that the transition state structures $($ IRC $=0$ ) leads to the DA cycloadducts in the forward direction and the reagents in the reverse direction without any evidence of intermediates or complex structures along the reaction paths (Figure 6). The IRC summarizes the trend predicted by FMO (except for the relative ordering of the reactions of $\mathbf{e}$ with fragments $\mathbf{1}$ and $\mathbf{2}$ ) and those derived from $E_{a}$.

Figure 6. Plots of energy $\left(\mathrm{kcal} \mathrm{mol}^{-1}\right)$ versus the IRC (in bohr/amu ${ }^{1 / 2}$ ) for the proposed reactions.

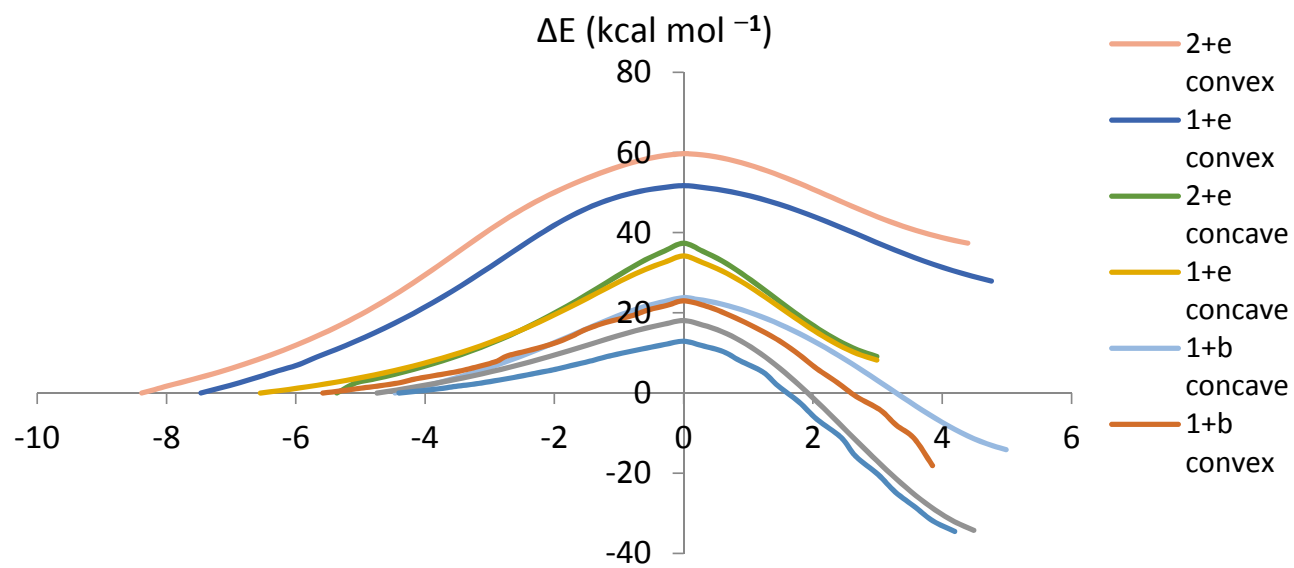

The thermodynamic parameters for the reactions studied are collected in Table 5. All those reactions are entropically disfavored. The reactions of fragments $\mathbf{1}$ and $\mathbf{2}$ with $\mathbf{b}$ are exothermic (negative $\Delta H_{r}$ ), while the reactions with e are endothermic (positive $\Delta H_{r}$ ). Only the reference reaction 
$\mathbf{e}+\mathbf{b}$ and the reactions between fragment $\mathbf{2}$ and $\mathbf{b}$ are spontaneous, having negative values of $\Delta G_{r}$. These reactions indicate the dienophilic character of fragment 2 . This result reinforces the possibility of synthesizing larger fullerene fragments starting with fragment $\mathbf{2}$, and it also suggests the dimerization of fragment $\mathbf{2}$, that is, the reaction $\mathbf{2}+\mathbf{2}$, as a promising method to produce $\mathrm{C}_{60}$.

Table 5. Standard Gibbs energies $\Delta G_{r}$, standard enthalpies $\Delta H_{r}$, and standard entropies $T \Delta S_{r}$ of reaction.

\begin{tabular}{|c|c|c|c|}
\hline Reaction & $\Delta G_{r}\left(\mathrm{kcal} \mathrm{mol}^{-1}\right)$ & $\Delta H_{r}\left(\mathrm{kcal} \mathrm{mol}^{-1}\right)$ & $T \Delta S_{r}\left(\mathrm{kcal} \mathrm{mol}^{-1}\right)$ \\
\hline $\mathrm{e}+\mathrm{b}$ & -23.76 & $-37.82(-37.9)^{\mathrm{a}}(-36.6)^{\mathrm{b}}$ & $-14.06(-14.1)^{\mathrm{c}}$ \\
\hline $1+$ e concave & 16.03 & 1.73 & -14.29 \\
\hline $2+$ e concave & 21.90 & 7.86 & -14.04 \\
\hline $1+\mathrm{e}$ convex & 49.53 & 35.36 & -14.17 \\
\hline $2+\mathrm{e}$ convex & 60.84 & 46.82 & -14.02 \\
\hline $1+\mathrm{b}$ concave & 6.62 & -8.49 & -15.11 \\
\hline $2+\mathrm{b}$ concave & -10.41 & -25.50 & -15.09 \\
\hline $1+\mathrm{b}$ convex & 10.34 & -4.28 & -14.62 \\
\hline $2+\mathrm{b}$ convex & -13.05 & -27.88 & -14.82 \\
\hline
\end{tabular}

${ }^{\mathrm{a}}$ Experimental $\Delta H_{r}$ from reference [24]; ${ }^{\mathrm{b}}$ Calculated $\Delta H_{r}$ from reference [24]; ${ }^{\mathrm{c}}$ Calculated value of $T \Delta S_{r}$ from reference [24].

Following the scheme proposed by Scott, we have studied the dehydrogenation reactions of the adducts formed by the Diels-Alder reactions. Dehydrogenation leads to the aromatization of the adducts. The dehydrogenation reactions are shown in Figure 7, and the standard Gibbs energies $\Delta G_{r}$, enthalpies $\Delta H_{r}$, and entropies $T \Delta S_{r}$ of reaction are given in Table 6. We observe that the reactions with fragment 2 are endothermic (positive $\Delta H_{r}$ ), except for the reaction with e onto the convex surface. On the other hand the reactions with fragment $\mathbf{1}$ are exothermic, with the exception of the reaction with $\mathbf{e}$ onto the concave surface. However, all the reactions are spontaneous (negative $\Delta G_{r}$ ) because of the strongly favorable entropy contribution $\left(T \Delta S_{r} \approx 17 \mathrm{kcal} \mathrm{mol}^{-1}\right)$. The values of $\Delta G_{r}$ for the aromatization of the adducts formed in the reactions between $\mathbf{e}$ and fragments $\mathbf{1}$ and $\mathbf{2}$ over the convex surface are more negative because of the lower stability of those adducts.

Table 6. Standard Gibbs energy $\Delta G_{r}$, standard enthalpy $\Delta H_{r}$, and standard entropy $T \Delta S_{r}$ of the dehydrogenation reaction.

\begin{tabular}{|c|c|c|c|}
\hline System & $\Delta \mathrm{G}_{r}\left(\mathrm{kcal} \mathrm{mol}^{-1}\right)$ & $\Delta \mathbf{H}_{r}\left(\mathrm{kcal} \mathrm{mol}^{-1}\right)$ & $\mathrm{T} \Delta \mathrm{S}_{\mathrm{r}}\left(\mathrm{kcal} \mathrm{mol}^{-1}\right)$ \\
\hline $\mathrm{e}+\mathrm{b}$ & 0.09 & $17.20(21.13)^{\mathrm{a}}$ & 17.12 \\
\hline $1+$ e concave & -11.34 & 5.93 & 17.27 \\
\hline $2+$ e concave & -12.23 & 4.96 & 17.19 \\
\hline $1+\mathrm{e}$ convex & -44.85 & -27.70 & 17.15 \\
\hline $2+\mathrm{e}$ convex & -51.16 & -33.99 & 17.17 \\
\hline $1+\mathrm{b}$ concave & -22.84 & -5.58 & 17.26 \\
\hline $2+b$ concave & -13.12 & 4.14 & 17.25 \\
\hline $1+b$ convex & -26.56 & -9.80 & 16.77 \\
\hline $2+b$ convex & -10.48 & 6.51 & 16.99 \\
\hline
\end{tabular}

${ }^{\mathrm{a}}$ Experimental value of $\Delta H_{r}$ value obtained from the hydrogenation reaction [28]. 
Figure 7. Dehydrogenation reactions to aromatize the adducts obtained from the Diels-Alder reactions of a) $\mathbf{1}+\mathbf{e}$, b) $\mathbf{1}+\mathbf{b}$, c) $\mathbf{2}+\mathbf{e}$ and d) $\mathbf{2}+\mathbf{b}$.

a
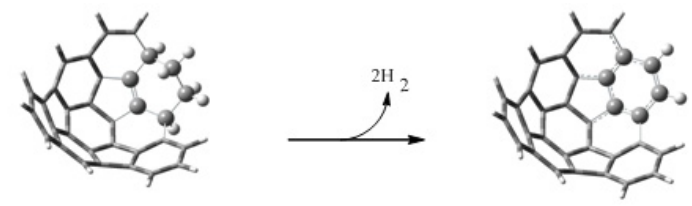

b

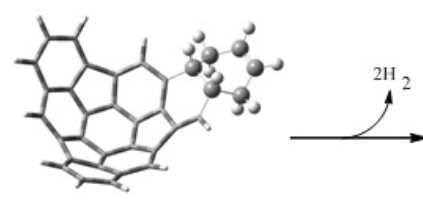

$\mathbf{c}$
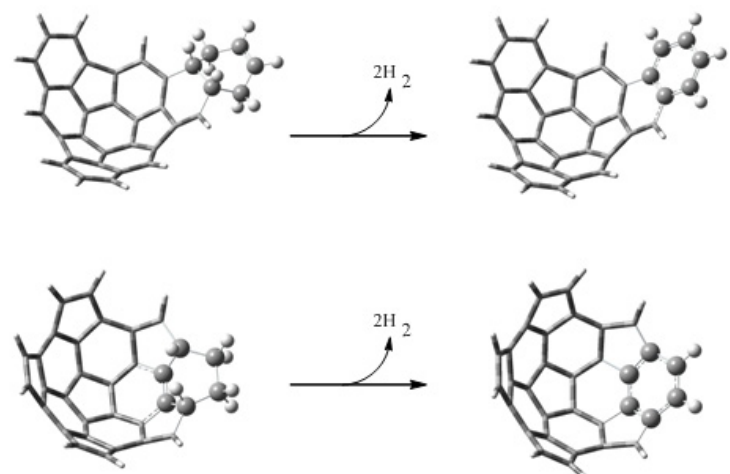

d

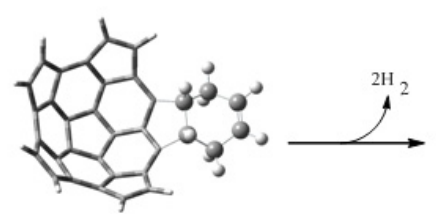

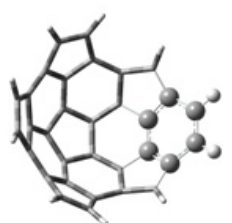

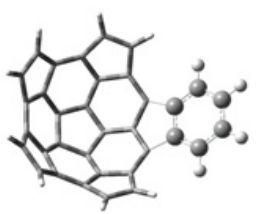

\section{Experimental}

The calculations described in this work have been carried out with the Gaussian 03 (G03) program package [29]. The geometries were fully optimized at the B3LYP/6-31G(d,p) level of theory. This method and basis set have been widely used in the study of fullerene reactivity [30,31], leading to good agreement with geometrical parameters of fullerene fragments obtained by X-ray diffraction [32]. The methodology has proven to describe satisfactorily the DA cycloaddition reactions over fullerenes and fullerene fragments $[33,34]$. Therefore, the kinetic and thermodynamic parameters were calculated at the B3LYP/6-31G(d,p) level of theory as well. The transition states (TS) were located with the QST2 G03 optimization option of the code; for all of them, vibrational frequency analyses were carried out. A single imaginary frequency was located for each transition state. All frequencies are real for the minima. The electronic energies of minima and transition states were corrected by the inclusion of zero-point energies. The Intrinsic Reaction Coordinates (IRCs) were determined from the corresponding TS using the IRC G03 keyword. In each case the FORWARD and REVERSE sections were calculated independently.

\section{Conclusions}

The calculations performed show the feasibility of the Diels-Alder reaction as a method to increase the size of fullerene fragments step by step. We have studied the Diels-Alder reactions of ethylene and butadiene with two fragments, triindenetriphenilene (Fragment 1) and pentacyclopentacorannulene (Fragment 2). The Frontier Molecular Orbitals analysis indicates that the reactions in which the 
fullerene fragments behave as a dienophile are preferred, and this was confirmed by the kinetic and thermodynamic analysis. The transition states indicate that the interactions with ethylene are favored on the concave surface of the fragments, while the reactions with butadiene, a larger molecule, are preferred on the convex surface.

Even if fragment $\mathbf{1}$ is more stable than fragment $\mathbf{2}$ owing to its lower pyramidalization of the bonds, the most favorable addition reactions are those of butadiene with fragment $\mathbf{2}$. In this particular case, the activation barriers are lower than for other reactions, and in addition, the free energies of reaction are negative, in contrast with the positive values for other reactions. The negative values of the free energy for the dehydrogenation reactions of the adducts favor the growth of the fragments. Motivated by these results and others $[35,36]$, experimental studies of the reactivity of the current systems are underway.

\section{Acknowledgments}

Work supported by MEC of Spain and the European Regional Development Fund (project MAT2011-22781), Junta de Castilla y León (project VA158A11-2) and CONACyT-México (project grants 180523 and 163234). M.M. acknowledges CONACyT-México for scholarship 228923. We are especially grateful to Hugo Jimenez for many discussions. We acknowledge LSCVP-UAMIMéxico for supercomputer time.

\section{References}

1. Kroto, H.W.; Heath, J.R.; O’Brien, S.C.; Curl, R.F.; Smalley, R.E. C60: Bukminsterfullerene. Nature 1985, 318, 162-163.

2. Krätschmer, W.; Fostiropoulos, K.; Huffman, D.R. The infrared and ultraviolet absorption spectra of laboratory-produced carbon dust: Evidence of the presence of the $\mathrm{C}_{60}$ molecule. Chem. Phys. Lett. 1990, 170, 167-170.

3. Scott, L.T.; Boorum, M.M.; McMahon, B.J.; Hagen, S.; Mack, J.; Blank, J.; Wegner, H.; de Meijere, A. A rational synthesis of $\mathrm{C}_{60}$. Science 2002, 295, 1500-1503.

4. Scott, L.T.; Petrukhina, M.A. Preface. In Fragments of Fullerenes and Carbon Nanotubes: Designed Synthesis, Unusual Reactions and Coordination Chemistry; Scott, L.T., Petrukhina, M.A., Eds.; John Wiley \& Sons: Hoboken, NJ, USA, 2012; pp. vii-X.

5. Krätschmer, W.; Lamb, L.D.; Fostiropoulos, K.; Huffman, D.R. Solid $\mathrm{C}_{60}$ : A new form of carbon. Nature 1990, 347, 354-358.

6. Otero, G.; Biddau, G.; Sánchez-Sánchez, C.; Caillard, R.; López, M.F.; Rogero, C.; Palomares, F.J.; Cabello, N.; Basanta, M.A.; Ortega, J.; et al. Fullerenes from aromatic precursors by surface-catalysed cyclodehydrogenation. Nature 2008, 454, 865-868.

7. Scott, L.T. Methods for the chemical synthesis of fullerenes. Angew. Chem. Int. Ed. 2004, 43, 4994-5007.

8. Tsefrikas, V.M.; Scott, L.T. Geodesic polyarenes by flash vacuum pyrolysis. Chem. Rev. 2006, 106, 4868-4884.

9. Geneste, F.; Moradpour, A.; Dive, G.; Peeters, D.; Malthête, J.; Sadoc, J.-F. Retrosynthetic analysis of fullerene $\mathrm{C}_{60}$ : Structure, stereochemistry, and calculated stability of $\mathrm{C}_{30}$ fragments. J. Org. Chem. 2002, 67, 605-607. 
10. Abdourazak, A.H.; Marcinow, Z.; Sygula, A.; Sygula, R.; Rabideau, P.W. Buckybowls 2. Toward the total synthesis of Bukminsterfullerene (C60): Benz[5,6]-as-indaceno-[3,2,1,8,7mnopqr]indeno[4,3,2,1-cdef]chrysene. J. Am. Chem. Soc. 1995, 117, 6410-6411.

11. Hagen, S.; Bratcher, M.S.; Erickson, M.S.; Zimmermann, G.; Scott, L.T. Novel syntheses of three $\mathrm{C}_{30} \mathrm{H}_{12}$ bowl-shaped polycyclic aromatic hydrocarbons. Angew. Chem. Int. Ed. 1997, 36, 406-408.

12. Anet, F.A.L.; Muira, S.S.; Siegel, J.; Mislow, K. La Coupe du Roi and its relevance to stereochemistry. Combination of two homochiral molecules to give an achiral product. J. Am. Chem. Soc. 1983, 105, 1419-1926.

13. Scott, L.T. Polycyclic aromatic hydrocarbon bowls, baskets, balls and tubes: Challenging targets for chemical synthesis. Polycyclic Aromat. Compd. 2010, 30, 247-259.

14. Fort, E.H.; Donovan, P.M.; Scott, L.T. Diels-Alder reactivity of polycyclic aromatic hydrocarbon bay regions: Implications for metal-free growth of single-chirality carbon nanotubes. J. Am. Chem. Soc. 2009, 131, 16006-16007.

15. Fort, E.H.; Scott, L.T. Gas-phase Diels-Alder cycloaddition of benzyne to an aromatic hydrocarbon bay region: Groundwork for the selective solvent-free growth of armchair carbon nanotubes. Tetrahedron Lett. 2011, 52, 2051-2053.

16. Fukui, K.; Yonezawa, T.; Shingu, H. A molecular-orbital theory of reactivity in aromatic hydrocarbons. J. Chem. Phys. 1952, 20, 722-725.

17. Fukui, K.; Yonezawa, T.; Nagata, C.; Shingu, H. Molecular-orbital theory of orientation in aromatic, heteroaromatic, and other conjugated molecules. J. Chem. Phys. 1954, 22, 1433-1442.

18. Flemig, I. Molecular Orbitals and Organic Chemical Reactions; John Wiley \& Sons Ltd: London, UK, 2009; pp. 224-242.

19. Park, Y.S.; Lee, B.-S.; Lee, I. Density functional theory studies of hetero-Diels-Alder reactions. New J. Chem. 1999, 27, 707-715.

20. Hehre, W.J.; Radom, L.; Schleyer, P.v.R.; Pople, J.A. Ab Initio Molecular Orbital Theory; Wiley: New York, NY, USA, 1986; Chapter 4.

21. Mendoza, J.A.; García-Pérez, E.; Jiménez-Vázquez, H.A.; Tamariz, J. Effect of aryl substituents on the reactivity of the captodative olefins 1-acetylvinyl arenecarboxylates. J. Mex. Chem. Soc. 2006, 50, 47-56.

22. Houk, K.N.; Lin, Y.-T.; Brown, F.K. Evidence for the concerted mechanism of the Diels-Alder reaction of butadiene with ethylene. J. Am. Chem. Soc. 1986, 108, 554-556.

23. Houk, K.N.; Loncharich, R.J.; Blake, J.F.; Jorgensen, W.L. Substituent effects and transition structures for Diels-Alder reactions of butadiene and cyclopentadiene with cyanoalkenes. J. Am. Chem. Soc. 1989, 111, 9172-9176.

24. Guner, V.; Khuong, K.S.; Leach, A.G.; Lee, P.S.; Bartberger, M.D.; Houk, K.N. A standard set of pericyclic reactions of hydrocarbons for the benchmarking of computational methods: The performance of ab initio, Density functional, CASSCF, CASPT2, and CBS-QB3 methods for the prediction of activation barriers, Reaction energetics, and transition state geometries. J. Phys. Chem. A 2003, 107, 11445-11459.

25. Ishida, K.; Morokuma, K.; Komornic, A. The intrinsic reaction coordinate. An ab inito calculation for $\mathrm{HNC} \rightarrow \mathrm{HCN}$ and $\mathrm{H}-+\mathrm{CH} 4 \rightarrow \mathrm{CH} 4+\mathrm{H}-$. J. Chem. Phys. 1977, 66, 2153-2156.

26. Fukui, K. The paths of chemical reaction-The IRC approach. Acc. Chem. Res. 1981, 14, 363-368. 
27. Gonzalez, C.; Schlegel, H.B. An improved algorithm for reaction path following. J. Chem. Phys. 1989, 90, 2154-2161.

28. Kistiakowsky, G.B.; Ruhoff, J.R.; Smith, H.A.; Vaughan, W.E. Heats of organic reactions. IV. Hydrogenation of some dienes and of benzene. J. Am. Chem. Soc. 1936, 58, 146-153.

29. Frisch, M.J.; Trucks, G.W.; Schlegel, H.B.; Scuseria, G.E.; Robb, M.A.; Cheeseman, J.R.; Zakrzewski, V.G.; Montgomery, J.A., Jr.; Stratmann, R.E.; Burant, J.C.; et al. GAUSSIAN 03 (Revision A.9); Gaussian, Inc.: Pittsburgh, PA, USA, 2003.

30. Lee, K.H.; Lee, C.; Kang, J.; Park, S.S.; Lee, J.; Lee, S.K.; Bohme, D.K. Preferential site of attack on fullerene cations: Frontier orbitals and rate coefficients. J. Phys. Chem. A 2006, 110, 11730-11733.

31. Martinez, J.I.; Moncada, J.L.; Larenas, J.M. The dual descriptor to measure local reactivity on Buckminster fullerenes: An analysis within the framework of conceptual DFT. J. Mol. Model. 2010, 16, 1825-1832.

32. Petrukhina, M.A.; Andreini, K.W.; Mack, J.; Scott, L.T. X-ray quality geometries of geodesic polyarenes from theoretical calculations: What levels of theory are reliable? J. Org. Chem. 2005, $70,5713-5716$.

33. Osuna, S.; Morera, J.; Cases, M.; Morokuma, K.; Solà, M. Diels-Alder reaction between ciclopentadiene and C60: An analysis of the performance of the ONIOM method for the study of chemical reactivity in fullerenes and nanotubes. J. Phys. Chem. A 2009, 113, 9721-9726.

34. Ikuma, N.; Susami, Y.; Oshima, T. Kinetics and regioselectivity in the Diels-Alder reaction of fulleroids vs. methanofullerene and $\mathrm{C}_{60}$. Org. Biomol. Chem. 2010, 8, 1394-1398.

35. Suárez-Moreno, G.V.; González-Zamora, E.; Méndez, F. Oxazole as an electron-deficient diene in the Diels-Alder reaction. Org. Lett. 2011, 13, 6358-6361.

36. Damoun, S.; Van de Woude, G.; Méndez, F.; Geerlings, P. Local softness as a regioselectivity indicator in [4+2] cycloaddition reactions. J. Phys. Chem. A 1997, 101, 886-893.

Sample Availability: Not available.

(C) 2013 by the authors; licensee MDPI, Basel, Switzerland. This article is an open access article distributed under the terms and conditions of the Creative Commons Attribution license (http://creativecommons.org/licenses/by/3.0/). 
July 2013 • Volume $26 \bullet$ Issue No. 7

Pages 525-610 • ISSN 0894-3230

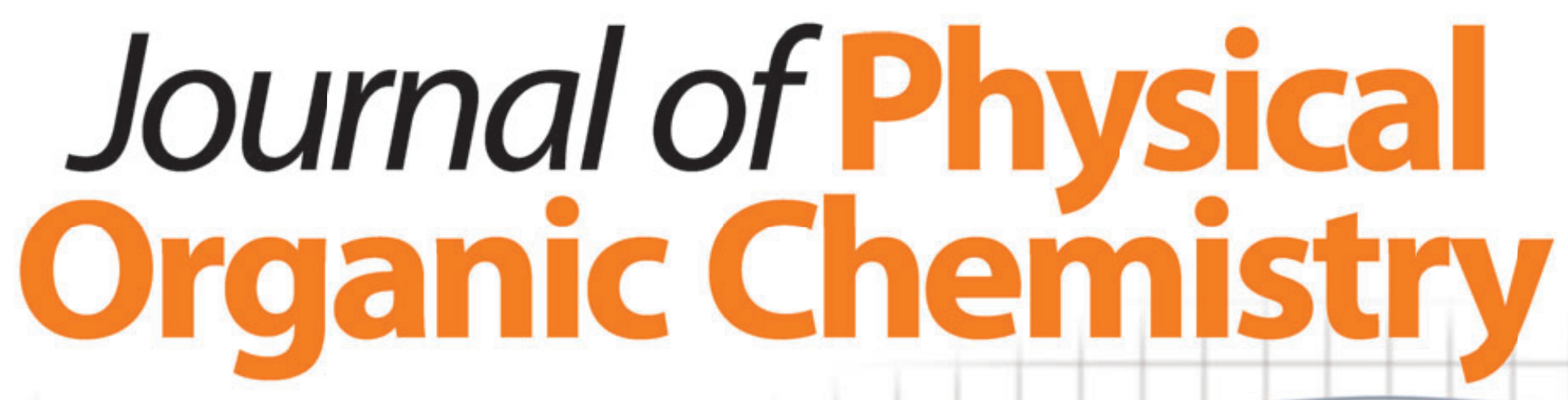
wileyonlinelibrary.com/journal/poc

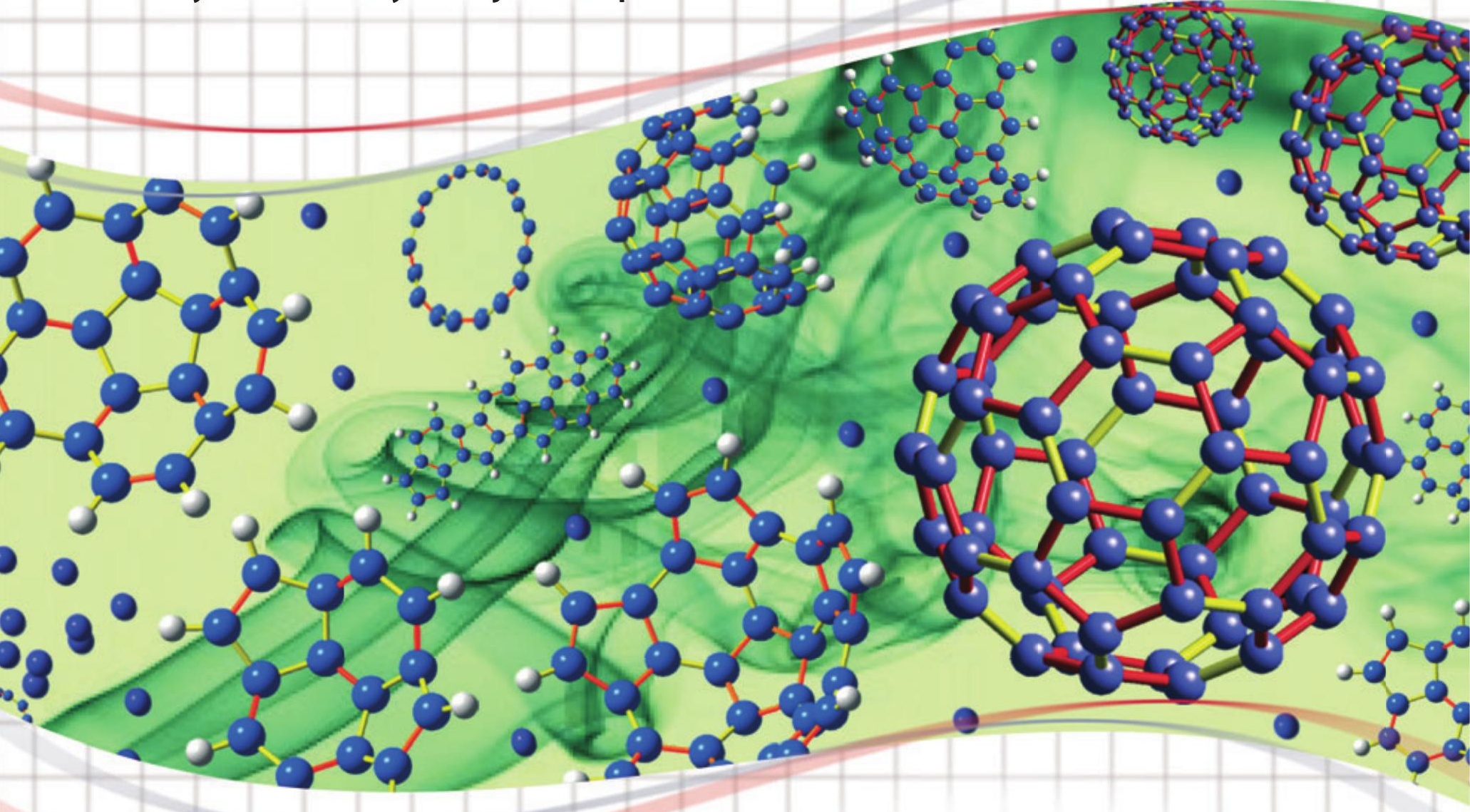

In this Issue:

Synthesis of fullerenes

by M. Mojica et al.

Early Excellence Profile

Prof. Hermann A. Wegner 


\title{
Synthesis of fullerenes
}

\section{Martha Mojica ${ }^{a}$, Julio A. Alonso ${ }^{b}$ and Francisco Méndez ${ }^{a *}$}

\begin{abstract}
We present a review of the methods most frequently used for the synthesis of fullerenes and the changes that these methods have experienced since 1985 when Kroto and co-workers discovered $C_{60}$. We also analyze the most important models that explain the mechanism of the formation of fullerenes in carbon soot, as well as the new methodologies that lead to the rational chemical synthesis of fullerenes and of fullerene fragments as precursors. Copyright ๑ 2013 John Wiley \& Sons, Ltd.
\end{abstract}

Keywords: Carbon clusters; chemical synthesis; corannulene; fullerenes; hemifullerenes

\section{INTRODUCTION}

Since the discovery of $C_{60}$ in $1985^{[1]}$ fullerenes have been the subject of many studies. Fullerenes have been employed as drug delivery systems, ${ }^{[2,3]}$ nanosensors, ${ }^{[4]}$ antioxidants, ${ }^{[5,6]}$ solar cell construction materials, ${ }^{[7,8]}$ and in many other applications, because of their electronic properties ${ }^{[9,10]}$ and chemical reactivity. ${ }^{[11]}$ Fullerenes are constituted by an even number of $\mathrm{sp}^{2}$ hybridized carbon atoms that form 12 pentagonal rings and $m$ hexagonal rings, where $m=(n-20) / 2$ and $n$ is the number of carbon atoms in the molecule. $C_{60}$ is the smallest fullerene that fulfills the isolated pentagon rule, which states that the pentagons should be separated from each other by hexagons to avoid the inherent instability associated to fused pentagons. ${ }^{[12-14]}$ The carbon atoms of $C_{60}$ form a truncated icosahedron where each atom is placed at a vertex. This geometric arrangement, formed by 12 pentagons and 20 hexagons, makes all carbon atoms equivalent.

The structural determination by $\mathrm{X}$-ray diffraction of $\mathrm{C}_{60}$ and some of its derivatives reveals the existence of two different types of bonds, which are indicated in Fig. 1 . The 66 bonds connect atoms common to two adjacent hexagons. These 66 bonds have a length of $1.38 \AA$. The 56 bonds connect atoms common to a pentagon-hexagon pair. The bond length is $1.45 \AA$. The pentagonal rings allow for the introduction of the curvature, which is necessary for the closing of the carbon cage. The curvature of the fullerene structure has the effect that the three bonds of any carbon atom with its three neighbor atoms are not in a plane. ${ }^{[15]}$ The pyramidalization modifies the hybridization from the pure $\mathrm{sp}^{2}$ hybridization characteristic of planar graphene to an intermediate between $\mathrm{sp}^{2}$ and $\mathrm{sp}^{3}$. That is, it results in a gain in $p$ character, in which the $p$ lobes extend further outside the surface than they do into the interior of the sphere; in addition, $\pi$ orbitals gain some $s$ character. ${ }^{[16]}$ These changes exert an important effect on a number of properties, contributing, for instance, to the high electron affinity of the molecule. The pyramidalization is also responsible for the increase of the chemical reactivity of the fullerene toward addition reactions compared with that of a planar graphene layer. ${ }^{[17]}$

To study the properties and the reactivity of fullerenes, sizable quantities of fullerenes are required. Moreover, the design and development of new materials and technologies based on fullerenes demand isomerically pure fullerenes in high quantities. Fullerenes are traditionally produced by the arc-discharge vaporization of graphite, by chemical vapor deposition methods, and by combustion processes. However, these methods are not very efficient, and continued refinements and improvements in this area are needed. For this reason, the study of new synthetic methods is a key factor in the advancement of fullerene science. We can say that the field of fullerenes has provided some intriguing challenges and opportunities, where one of the most important is to develop a generalized synthetic route with high yields and purity. In this work, we review the experimental methods used to produce fullerenes. The Synthesis of Fullerenes by Vaporization of a Carbon Source section presents the synthesis of fullerenes by vaporization of a carbon source; this is the technique that led to the discovery of $C_{60}$. The Models to Explain the Formation of Fullerenes section discusses the models that have been proposed to explain the formation of fullerenes in the carbon vaporization experiments. A completely different way to produce fullerenes is chemical synthesis; this is a very active field at present, which is introduced in Chemical Synthesis section. Finally, in Other Proposed Methods section, we present other methods, which do not fit into the aforementioned categories, although they bear some relation with them.

\section{SYNTHESIS OF FULLERENES BY VAPORIZATION OF A CARBON SOURCE}

In the course of experiments aimed at understanding the mechanisms by which long-chain carbon molecules form in the interstellar space, Kroto et al. ${ }^{[1]}$ vaporized graphite by laser irradiation, producing a remarkably stable cluster consisting of 60 carbon atoms. This experiment led to an outburst of interest in the

\footnotetext{
* Correspondence to: Francisco Méndez, Departamento de Química, División de Ciencias Básicas e Ingeniería, Universidad Autónoma Metropolitana-Iztapalapa. E-mail: fm@xanum.uam.mx
}

a M. Mojica, F. Méndez

Departamento de Química, División de Ciencias Básicas e Ingeniería, Universidad Autónoma Metropolitana-Iztapalapa, 09340 México D. F., México

\section{b J. A. Alonso}

Departamento de Física Teórica, Atómica y Óptica, Universidad de Valladolid, 47011 Valladolid, Spain 


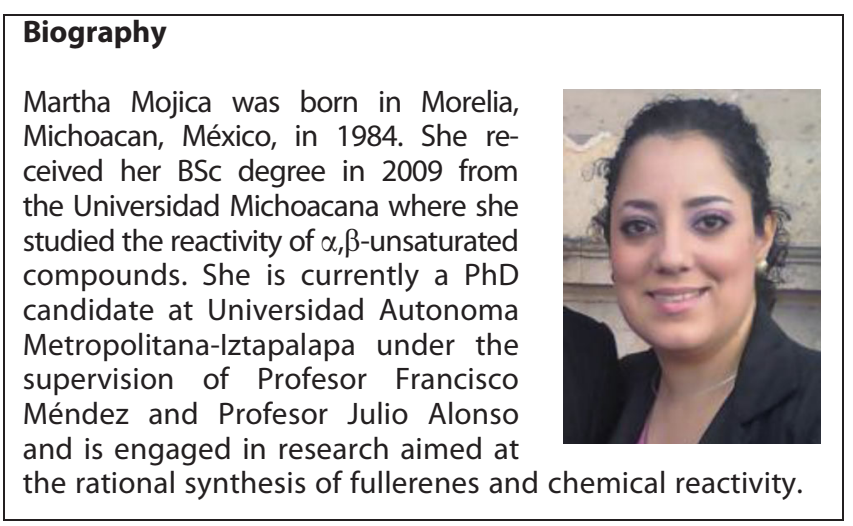

\section{Biography}

Julio A. Alonso has been Professor of Physics since 1988 at the UVA in Spain. He received his BSc degree in 1971 and $\mathrm{PhD}$ degree in 1975 from the UVA. He was a research associate from 1975 to 1980 in the University of Pennsylvania, with Professor L. A. Girifalco. From 1980 to 1988 , he was an associate professor at the UVA. He has been a visiting scientist at Imperial College, Max-Planck Institut, University of

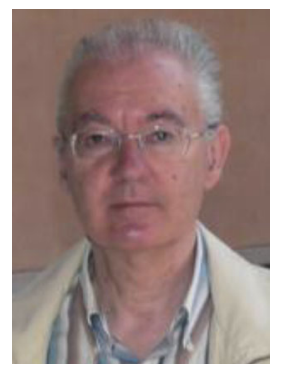
Osnabruck, University of Oxford, ICTP, UEA, and Queen's University. His research includes density functional theory, amorphous materials, atomic clusters, and hydrogen storage.

\section{Biography}

Francisco Méndez is a Professor of the Departamento de Química at the UAM-I, México. He obtained a BSc in 1982 at the Universidad Michoacana, an MS degree in 1987 at the CIEA-IPN, and a PhD in 1993 at the UAM-I. He spent a sabbatical at the VUB University, and he has been a visiting professor at the UCLA, Queen's University, UNAM, DIPC, and UVA. His research interests span chemical reactivity and density functional theory.

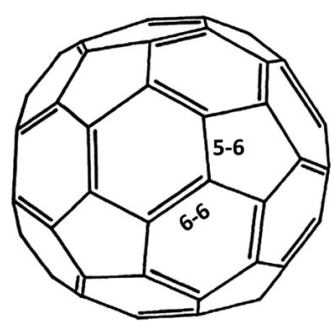

Figure 1. The two types of bond, 66 and 56 , in $C_{60}$

chemistry of $C_{60}$. Five years after the discovery of $C_{60}$, Krätschmer and co-workers ${ }^{[18]}$ developed the first method to obtain $C_{60}$ in usable quantities. This method consists of the vaporization of graphite rods by resistive heating in an $\mathrm{He}$ atmosphere. Krätschmer and co-workers made a real breakthrough in the fullerene science; the access to sizable quantities of $C_{60}$ changed the way in which fullerenes were studied. Moreover, their synthesis allowed the establishment of fullerene chemistry. However, this method is not very efficient, with yields lower than $1 \%$, and it requires harsh reaction conditions (temperatures around $1300^{\circ} \mathrm{C}$ and pressure of $\left.1 \mathrm{kbar}\right) .{ }^{[19]}$ This method produces $C_{60}$ and $C_{70}$, but it gives very low quantities of larger fullerenes such as $C_{84}$, and requires great effort to purify the samples (more than 20 high-performance liquid chromatography cycles for $\left.\mathrm{C}_{84}\right)^{\left[{ }^{[20]}\right.}$ Because of the uncontrollable character of the reaction, it is not possible to obtain a single fullerene or a specific isomer.

Since then, the scientific community has been interested in finding new methods that could be controlled to facilitate the synthesis of larger fullerenes and specific isomers with high yields and low costs. However, nowadays, the methods used for obtaining fullerenes in commercial scale are based on the vaporization of graphite by pyrolysis, radio-frequency-plasma, or arc discharge-plasma techniques. ${ }^{[21]}$ All these methods have the same disadvantages as the one proposed by Krätschmer. The process of production of functionalized fullerenes for use, for instance, in organic solar cells, takes several steps illustrated in Fig. 2: synthesis of the fullerenes, separation, purification, and functionalization. Anctil and co-workers ${ }^{[22]}$ have analyzed the economic and environmental impacts of the process, quantifying the total raw material and the embodied energy necessary for fullerene production. The energy used in the synthesis and the separation of $C_{60}$ can reach up to $106.9 \mathrm{GJ} / \mathrm{kg}$ of $C_{60}$ (for comparison, the average annual electricity consumption for a US home in 2010 was about $41 \mathrm{GJ}^{[23]}$ ), and if we add the energy needed for purification and functionalization, the total energy can suffer a threefold increase, making fullerenes expensive. This is why a variety of new techniques have been tested to synthesize $C_{60}$, and different theoretical methods have been proposed to explain the process of synthesis.

\section{MODELS TO EXPLAIN THE FORMATION OF FULLERENES}

Following the discovery of fullerenes, scientists have tried to explain the process of fullerene formation. General theory indicates that the carbon source is first vaporized into the smallest units, that is, carbon atoms, and probably carbon dimers, which then go through a series of reactions and, in a limited range of pressure and temperature, recombine to produce fullerenes. ${ }^{[1]} \mathrm{How}$ ever, this simple idea is not a satisfactory explanation of the process of formation of fullerenes, and different models have been proposed to explain it. An earlier report of models for fullerene formation has been published by Goroff. ${ }^{[24]}$

The icospiral particle nucleation scheme was the first theory proposed to explain the formation of fullerenes. ${ }^{[2,26]}$ In this model, shown in Fig. 3, the icospiral nucleation proceeds starting with a corannulene-like $C_{20}$ molecule, whose structure is a pentagon surrounded by five hexagons. This highly reactive structure grows and tends to form nautilus-like open spiral shells by accretion of small carbon fragments that are mopped up by adsorption on the surface of such shells. ${ }^{[27,28]}$ The occasional statistical closure of a network with the correct disposition of pentagons explains the formation of fullerenes. Other embryos do not close and grow further, forming quasi-spiral soot particles, such as the ones found by lijima ${ }^{[29]}$ by high-resolution electron microscopy (Fig. 4). 


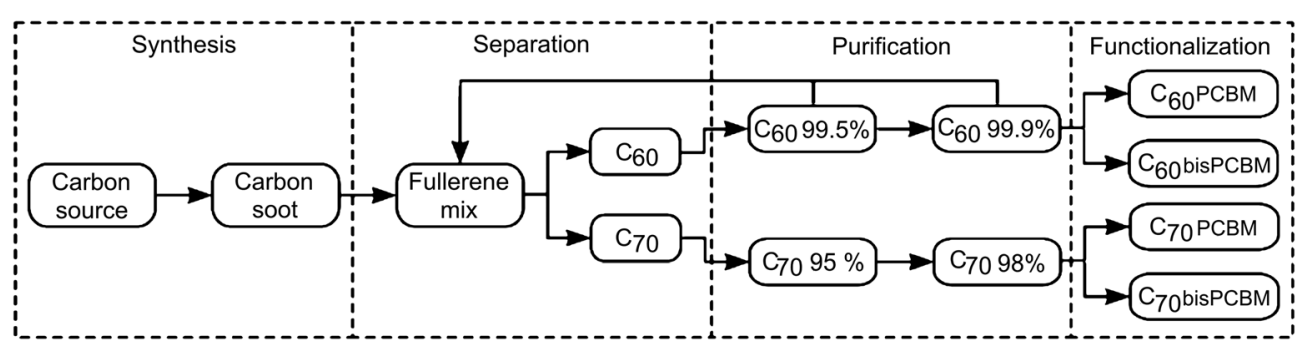

Figure 2. A flowchart of the process for the production of modified fullerene compounds for use as functional materials in organic solar cells. The process takes several steps: synthesis of the fullerenes, separation, purification, and functionalization. In this example, the functionalization of the $\mathrm{C}_{60}$ fullerenes consists in adding either one or two methyl ester functional groups to form [6,6]-phenyl-C61-butyric acid methyl ester (PCBM), or bis [6,6]-phenyl-C61-butyric acid methyl ester (bis-PCBM), and the same for $C_{70}$. Reproduced from A. Anctil, C. W. Babbitt, R. P. Raffaelle, and B. Landi, J. Environ. Sci. Technol., 2011, 45, 2353, with permission from the American Chemical Society

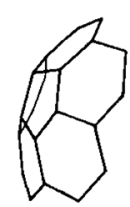

(a)

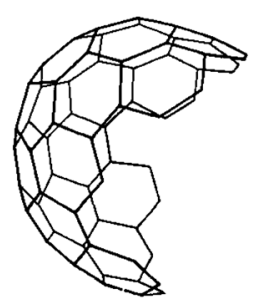

(b)

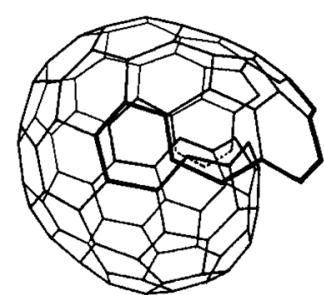

(c)

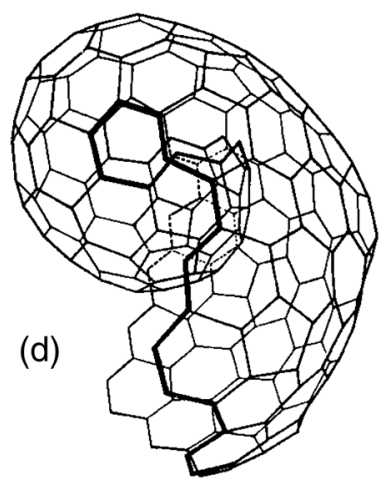

Figure 3. Icospiral nucleation process for the formation of fullerenes. The process starts with a saucer-shaped corannulene $C_{20}$ (a). This reactive cluster grows by the accretion of small carbon fragments. A half shell is formed in (b). Further growth leads to (c), where an edge bypass has occurred and then to (d), where the second shell is forming under epitaxial control. Statistical closure at stage (c) is proposed as the explanation for fullerene formation. Reproduced from H. W. Kroto and K. McKay, Nature, 1988, 331, 328, with permission from Nature Publishing Group

However, there is evidence in conflict with this hypothesis. ${ }^{[30]}$ In addition, according to the icospiral model, fullerene formation, which occurs in an expanding He jet, takes $10^{-4} \mathrm{~s}$ to complete, whereas experimental work $^{[31]}$ concludes that fullerenes are created on a much shorter time scale of $10^{-12}-10^{-9} \mathrm{~s}$.

In 1991, Heath proposed a mechanism in which the formation of fullerenes takes place in four steps. ${ }^{[32]}$ The mechanism is shown in Fig. 5. In the first step, the carbon atoms formed by the vaporization of graphite start forming carbon chains of lengths up to $C_{10}$. The second step consists of the growth from chains to monocyclic rings $\left(\mathrm{C}_{10} \mathrm{C}_{20}\right)$. In a third step, the growth and production of three-dimensional carbon networks take place, and finally, the fourth step consists of the growth of small fullerene cages via a shell-closing mechanism that exclusively produces $C_{60}, C_{70}$, and larger fullerenes as the stable products. In 1992, Wakabayashi and co-workers ${ }^{[33,34]}$ proposed a growth mechanism for $C_{60}$ and $C_{70}$. They suggested that fullerenes are formed following a "ring-stacking model", in which a closed cage is formed by a sequential stacking of the appropriate number of even-numbered carbon rings, without any loss of carbon atoms, as shown in Fig. 6 . They considered a $C_{10}$ ring as an initial precursor that deforms into a molecule formed by two hexagons with eight dangling bonds. This molecule is subsequently stacked with $C_{18}$ to eliminate all the initial dangling bonds and to create new ones, whose number decreases in subsequent steps by the stacking of $C_{18}, C_{12}$, and $C_{2}$ molecules, finally resulting in a $C_{60}$ cage. A similar scheme was proposed for the formation of $C_{70}$ and larger fullerenes.
Another scheme for the formation of fullerenes is the annealing of carbon clusters ${ }^{[35,36]}$ (Fig. 7). Those carbon clusters may be chains and rings that stick together and then anneal, ${ }^{[37]}$ bi-cycles or tri-cycles consisting of 34 to 60 atoms, ${ }^{[38,39]}$ larger clusters, or even other fullerenes. ${ }^{[40]}$ In this mechanism, the annealing of carbon clusters into fullerenes leads to the emission of atoms or small clusters. Two ways were proposed for the annealing of carbon clusters into fullerenes. In the first mechanism, the formation of fullerenes occurs through a process of sequential isomeric transformations. The second involves the crystallization of a liquid-like cluster. Lozovik and Popov ${ }^{[36]}$ proposed a mechanism involving the formation of liquid carbon clusters, which then crystallize into fullerenes with the emission of atoms and very small clusters. In their experiments, Hunter et al. ${ }^{[35]}$ observed that laser vaporization of graphite generates some $\mathrm{C}_{60}^{+}$cluster ions. A fraction of these are fullerenes, and the rest are a mixture of roughly planar polycyclic polyyne ring isomers. By annealing, most of the nonfullerene $\mathrm{C}_{60}^{+}$ions transform into intact fullerenes; however, a part suffers fragmentation.

Later, Askhabov proposed that fullerenes emerge from an intermediate phase (or hidden phase) existing at the nanoscale level. ${ }^{[1,42]}$ Askhabov proposed that clusters of this intermediate phase, which he called quatarons, arise under non-equilibrium conditions. Quatarons are viewed as dynamic structures with specific sizes, constantly changing their forms. Physically, these are pre-crystallization clusters of the intermediate phase. In this scheme, liquid quatarons initially arise in a supersaturated medium, and then, owing to the minimization of the energy 

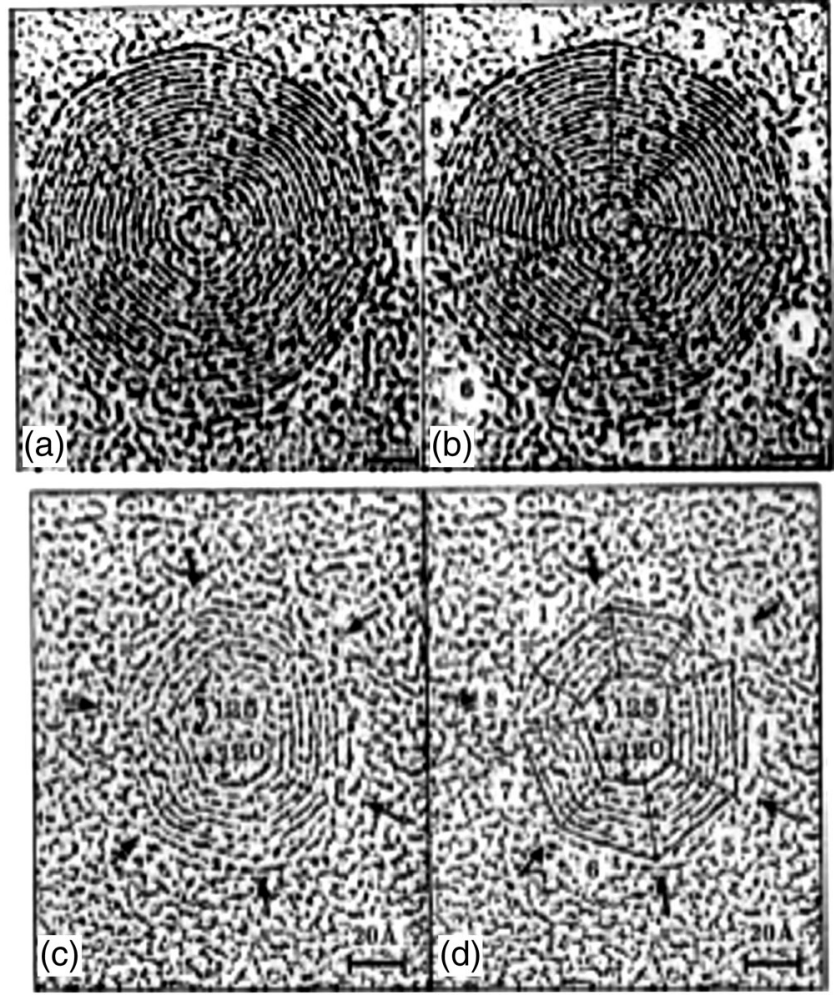

Figure 4. Particles imaged by lijima using high resolution electron microscopy. The images show evidence of a polyhedral concentric shell structure. Below, the contours are delineated. Reproduced from H. Kroto, Science, 1988, 242, 1139, with permission from the American Association for the Advancement of Science by the formation of bonds between the atoms forming the quataron, they transform into rigid clusters with fixed interatomic distances such as fullerenes. However, the quataron scheme is controversial and has not been accepted because of the lack of evidence in support of this scheme.

On the basis of the results of quantum chemical molecular dynamics simulations of the dynamics of the self-assembling in a hot carbon vapor far from thermodynamic equilibrium, Irle and co-workers ${ }^{[43]}$ proposed a mechanism called the shrinking hot giant (SHG) road to fullerene formation, in which fullerenes are formed by the shrinking of giant fullerenes $\left(C_{n}\right.$ with $\left.n \geq 90\right)$. They proposed that the SHG mechanism consists of two main steps: the size-up step, where the giant fullerene grows from the carbon vapor following a scheme similar to that proposed by Heath, ${ }^{[32]}$ and the size-down step, where the giant fullerene shrinks down to the sizes of $C_{60}$ and $C_{70}$ by irreversible elimination of $C_{2}$ molecules, favored by the vibrationally excited, highly irregular, and defective nature of the giant fullerene cages. Irle and co-workers proposed that this combined mechanism explains the formation of $C_{60}$ in a chaotic hot-reaction system.

\section{CHEMICAL SYNTHESIS}

Many different methods have been proposed with the aim of synthesizing $C_{60}$. Unfortunately, only a few of them have been successful. For example, one particularly attractive strategy is the assembling of two identical hemispherical hydrocarbons. ${ }^{[4]}$ However, the methods for fusing such molecules do not currently exist. The major challenge in the synthesis of fullerenes is the introduction of curvatures or pyramidalizations in the carbon network.

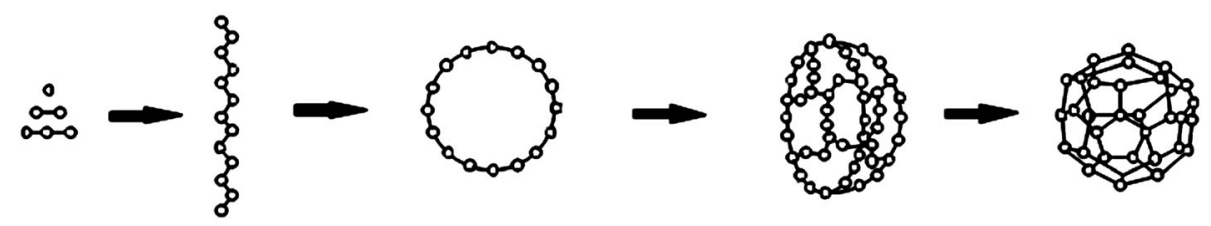

Figure 5. Four-step mechanism proposed by Heath for the formation of fullerenes. Reproduced from J. R. Heath, Fullerenes: Synthesis, Properties and Chemistry of Large Carbon Clusters. Editors G. S. I. Hammond and V. J. Kuck. American Chemical Society. Washington, DC, 1991, p. 1, with permission from the American Chemical Society

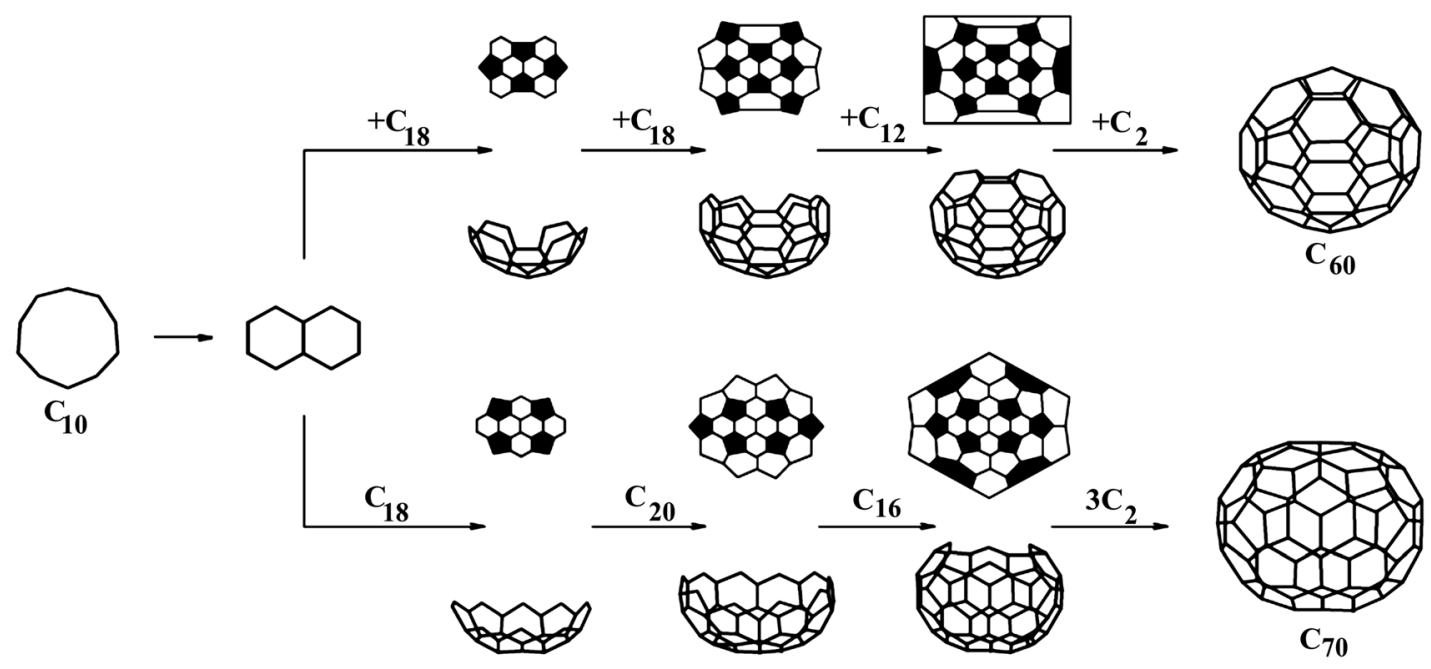

Figure 6. Schematic picture of the ring-stacking model for the assembly of $C_{60}$ and $C_{70}$. Reproduced from T. Wakabayashi and Y. Achiba, Chem. Phys. Lett., 1992, 190, 465, with permission from Elsevier 


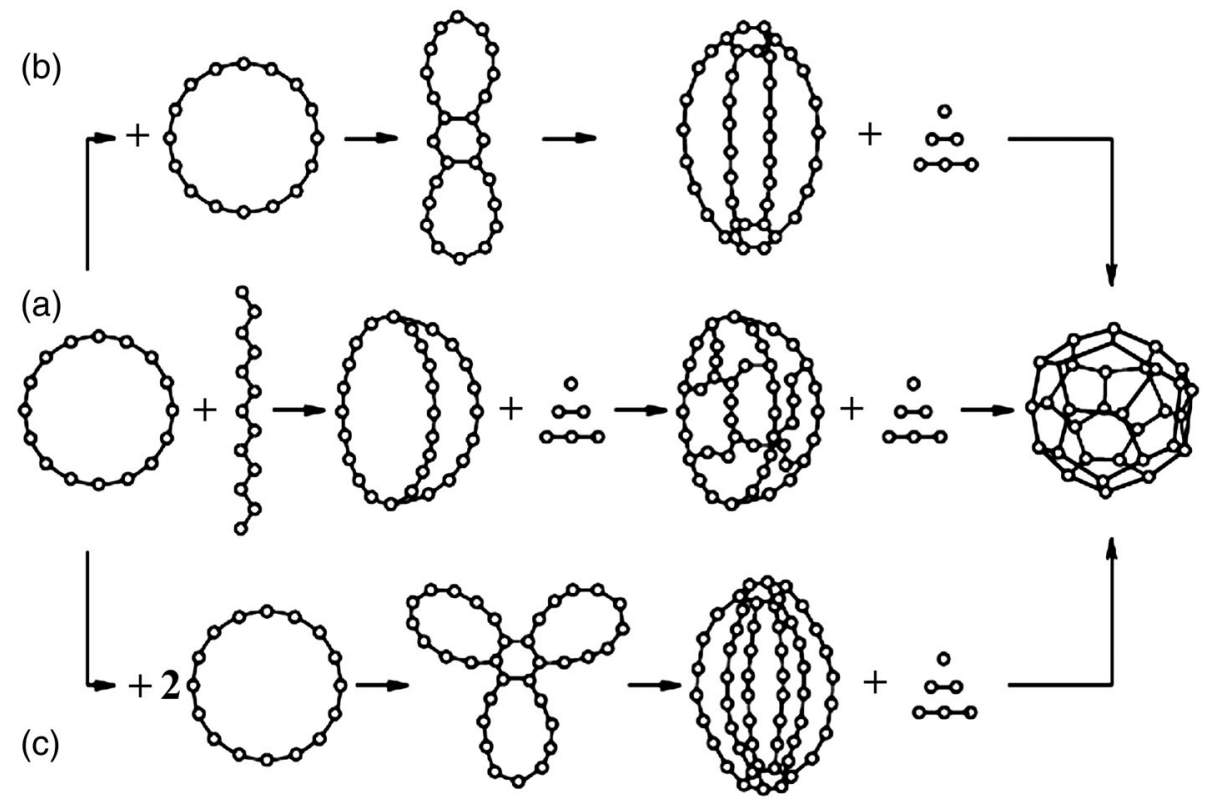

Figure 7. Scheme of growth of a carbon cluster accounting for the following stages: chain-ring-three-dimensional polycyclic cluster-transformation to the fullerene. Different ways for the formation of a three-dimensional polycyclic cluster are shown: (a) a chain and a monocyclic ring form a threedimensional tricyclic cluster, transforming into the three-dimensional polycyclic cluster; (b) two monocyclic rings form a plane bicyclic cluster, transforming into the three-dimensional polycyclic cluster; (c) three monocyclic rings form a plane tricyclic cluster, transforming into the three dimensional polycyclic cluster. Reproduced from Y. E. Lozovik and A. M. Popov, Physics-Uspekhi, 1997, 40, 717, with permission from the Institute of Physics

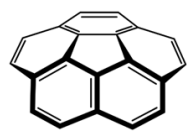

Figure 8. Structure of corannulene

Barth and Lawton ${ }^{[45]}$ were the first to introduce curvatures in an aromatic molecule when they synthesized the corannulene molecule. The structure of corannulene is shown in Fig. 8. However, the synthesis process was complicated, and the yields were too low (0.04\%), so it was forgotten until 1991 when Scott and co-workers ${ }^{[46]}$ proposed a new synthesis of corannulene that employs flash vacuum pyrolysis (FVP) for the ring-closure step. This method was further improved ${ }^{[47]}$ into a three-step synthesis, shown in Fig. 9. ${ }^{[48]}$ The first step is a Knoevenagel-Diels-Alder reaction between acenaphthoquinone, heptane-2,4,6-trione, and norbornadiene to give 7,10-diacetylfluoranthene, with $70-75 \%$ yield. The second step consists of the conversion of the acetyl groups to 1 -clorovinyl side chains, with $80-85 \%$ yield. In the last step, FVP generates terminal alkynes in situ by thermal elimination of $\mathrm{HCl}$ from the $\alpha$-chlorovinyl chains, leading to the formation of corannulene, with $35-40 \%$ yield. Scott and co-workers also studied other fragments of fullerenes ${ }^{[49]}$ and proposed that fragments

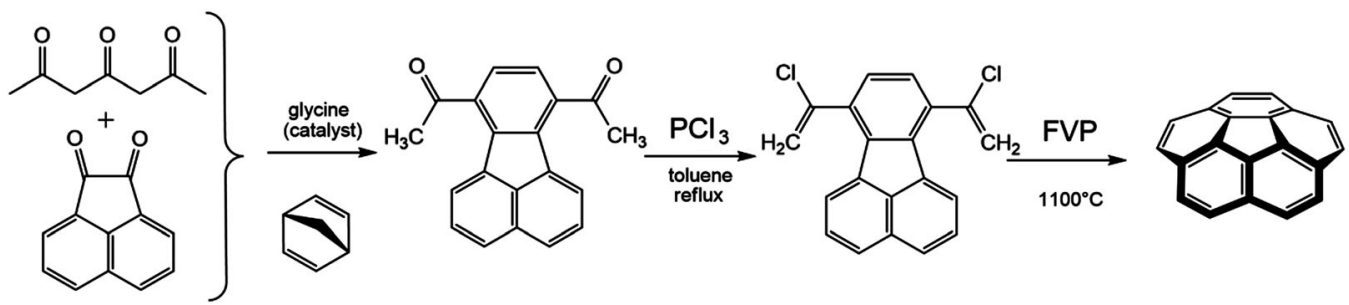

Figure 9. Synthesis of corannulene in three steps. Reproduced from L. T. Scott, Polycyclic Aromatic Compounds, 2010, 30, 247, with permission from Taylor and Francis. FVP, flash vacuum pyrolysis
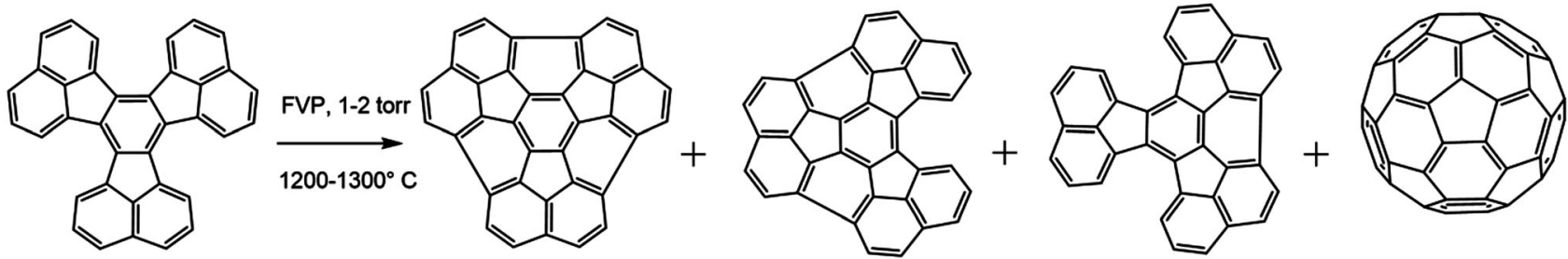

Figure 10. Products obtained in the pyrolysis of decacyclene. The triple closed $\mathrm{C}_{36} \mathrm{H}_{12}$ hydrocarbon (triacenaphthotriphenylene), the double closed $\mathrm{C}_{36} \mathrm{H}_{14}$ hydrocarbon, the single closed $\mathrm{C}_{36} \mathrm{H}_{16}$ hydrocarbon, and $\mathrm{C}_{60}$. FVP, flash vacuum pyrolysis 


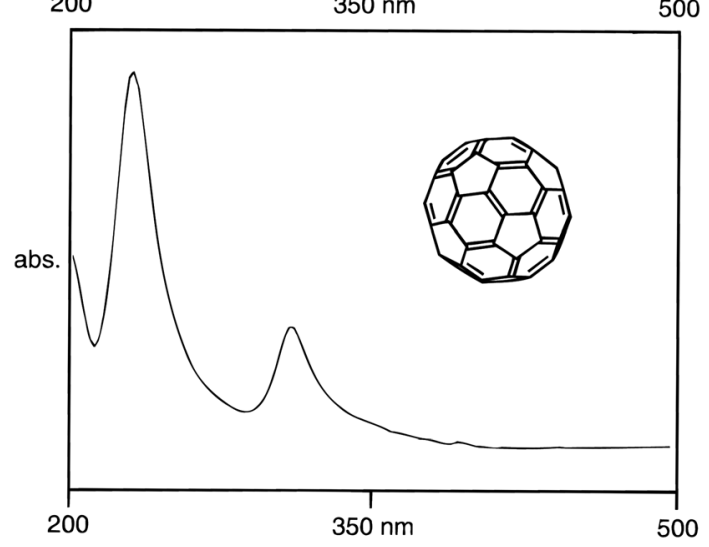

Figure 11. UV-Vis absorption spectra for triacenaphthotriphenylene and $\mathrm{C}_{60}$. Reproduced from L. T. Scott, M. S. Bratcher, and S. Hagen, J. Am. Chem. Soc., 1996, 118, 8743, with permission from the American Chemical Society

whose electronic character was more similar to that of $C_{60}$ should favor the formation of $\mathrm{C}_{60}$. They studied the pyrolysis of decacyclene at $1200-1300^{\circ} \mathrm{C}$ with the aim of obtaining triacenaphthotriphenylene $\left(\mathrm{C}_{36} \mathrm{H}_{12}\right)$ by a triple intermolecular ring closure mediated by the decacyclene dehydrogenation (Fig. 10). They also obtained the doubly closed $\mathrm{C}_{36} \mathrm{H}_{14}$ hydrocarbon, as well as the singly closed $\mathrm{C}_{36} \mathrm{H}_{16}$ hydrocarbon and traces of $\mathrm{C}_{60}$ within this temperature range, and determined that pyrolysis at lower temperatures $\left(1100^{\circ} \mathrm{C}\right.$ or less) is not able to provide the energy necessary to achieve the threefold dehydrogenation of decacyclene. They also observed that the ultraviolet absorption spectrum of the triacenaphthotriphenylene resembles that of $C_{60}$ (Fig. 11). The similarity of the spectrum could be a way to quantify how much a fragment resembles $C_{60}$.

A retrosynthetic analysis of $C_{60}$ splits open the cage and leads to relatively strain-free threefold symmetric polycyclic aromatic hydrocarbons. Following this analysis, Plater ${ }^{[50,51]}$ observed a series of fragments that could be isolated and expected that those fragments would act as construction blocks for $\mathrm{C}_{60}$. He proposed three main fragments: decacyclene $\left(\mathrm{C}_{36} \mathrm{H}_{18}\right)$, tribenzodecacyclene $\left(\mathrm{C}_{48} \mathrm{H}_{24}\right)$, and trinaphtodecacyclene $\left(\mathrm{C}_{60} \mathrm{H}_{30}\right)$ shown in Fig. 12, which should react by pyrolysis to form $\mathrm{C}_{60}$, and suggested that halogenated fragments could favor the reaction. These robust polycyclic precursors undergo endothermic ring coupling reactions (lines with arrows in Fig. 12) that would lead to the desired fullerenes and fullerene fragments. Latter, Ansems and Scott ${ }^{[52]}$ proposed a path to synthesize triacenaphthotriphenylene from the pyrolysis of trichlorodecacyclene with a yield close to $30 \%$. This method was employed as a prototype for the synthesis of larger geodesic molecules from halogenated polycyclic aromatic hydrocarbons. They suggested that once the curvature has been introduced in an aromatic system, the subsequent closure by dehydrogenation occurs generally with no need of additional promoters. Since then, the pyrolysis of different precursors has been used to synthesize a number of curved polycyclic aromatic hydrocarbons identified as fullerene fragments ${ }^{[53]}$ (Fig. 13). However, the intrinsic disadvantages of FVP have led to the necessity of proposing non-pyrolytic methods to synthesize these structures, methods that have been called "wet" synthesis or synthesis in a solution.

Seiders and co-workers ${ }^{[54]}$ proposed the synthesis of corannulene based on the reductive coupling mediated by low-valence titanium (Fig. 14). In this method, the corannulene core is formed by the internal cyclization of the benzylic bromides of the precursor by reductive coupling with $\mathrm{TiCl}_{3} / \mathrm{LiAlH}_{4}$ and the subsequent dehydrogenation of the obtained

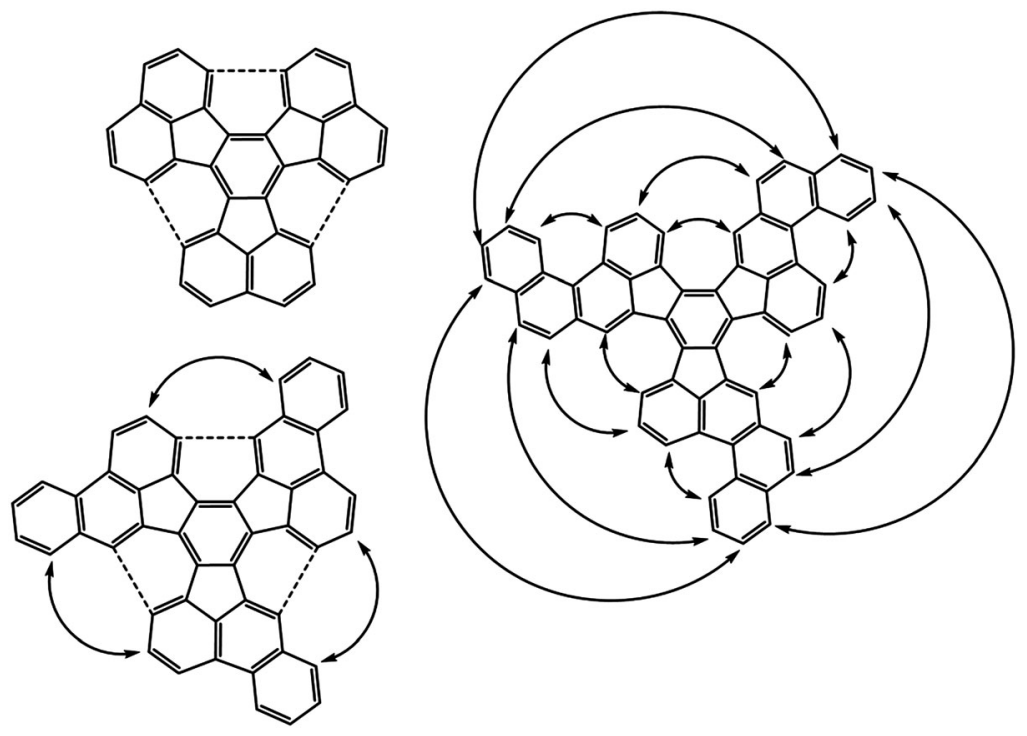

Figure 12. Fragments proposed by Plater toward the retro-synthetic analysis of $C_{60}$. Reproduced from J. M. Plater, J. Chem. Soc. Perkin Trans. 1, 1997, 19, 2897, with permission from the Royal Chemical Society 
<smiles>c1cc2ccc3ccc4ccc5ccc6ccc1c1c2c3c4c5c61</smiles>

1<smiles></smiles>

2<smiles></smiles>

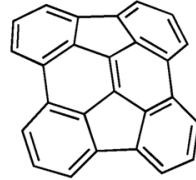

4

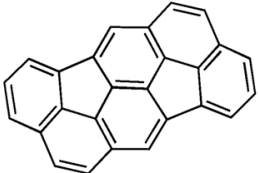

5

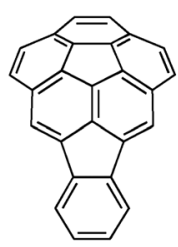

6

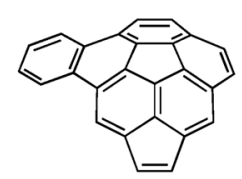

7<smiles>c1ccc2c(c1)c1ccc3ccc4ccc5c6ccccc6c5c4c3c21</smiles>

12

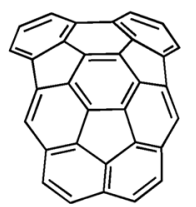

17

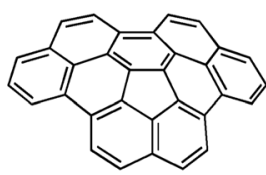

22

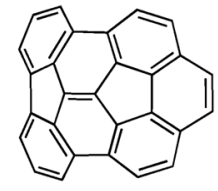

8

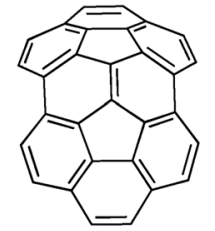

13

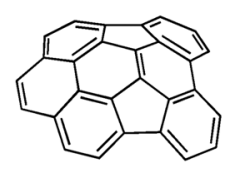

9

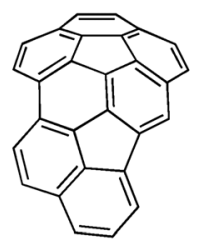

10

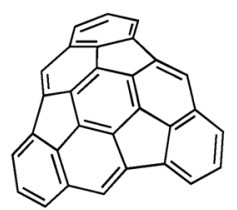

14

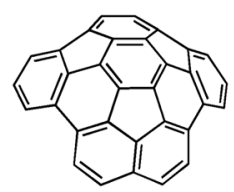

18

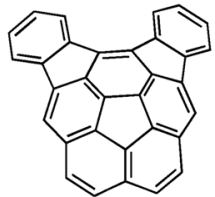

19

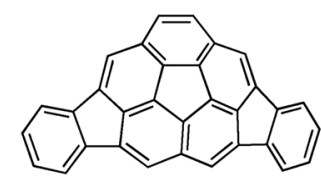

20<smiles>c1ccc2c(c1)c1ccc3c1c1ccc4ccc2c4c3c2ccccc12</smiles>

11

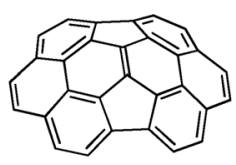

15

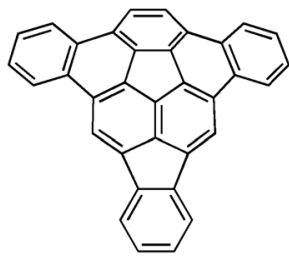

25

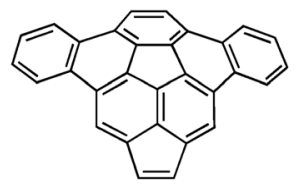

16<smiles></smiles>

23

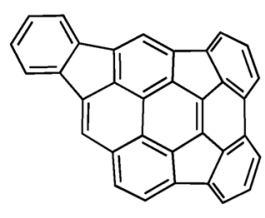

24

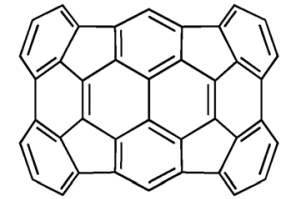

28

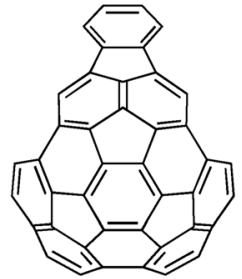

29<smiles>c1cc2sc3ccc4sc5ccc6sc7ccc1sc2c3c4c5c76</smiles>

30

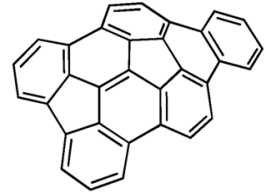

21

27

Figure 13. Curved polycyclic aromatic hydrocarbons that have been synthesized by flash vacuum pyrolysis (FVP). Reproduced from V. M. Tsefrikas and L. T. Scott, Chem. Rev., 2006, 106, 4868, with permission from the American Chemical Society

mixture of cis-dimethyltetrahydrocorannulenes and transdimethyltetrahydrocorannulenes, to give dimethylcorannulene in $18 \%$ yield. This method was employed by Sygula and Rabideau ${ }^{[55]}$ to synthesize the hemifullerene $\mathrm{C}_{30} \mathrm{H}_{12}$ (labeled 13 in Fig. 13). In this case, the reductive coupling of the dodecabromide precursor by using low-valence vanadium instead of titanium compounds (Fig. 15) led to the hemifullerene $\mathrm{C}_{30} \mathrm{H}_{12}$ in a modest $20 \%$ yield. The disadvantages of this method are that it requires high dilution techniques and reaction times of 2 to 4 days. Moreover, a clean atmosphere, free of moisture and oxygen, is needed, which makes 

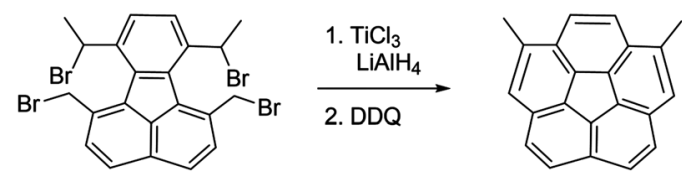

$(18 \%)$

Figure 14. Synthesis of corannulene proposed by Seiders and co-workers, based on the reductive coupling of 1,6-bis(bromomethyl)-7,10-bis (1-bromoethyl)fluoranthene and the subsequent reduction. Reproduced from A. Sygula and P. W. Rabideau, J. Am. Chem. Soc., 1998, 120, 12666, with permission from the American Chemical Society. DDQ, 2,3-dichloro-5, 6-dicyano-I,4-benzoquinone

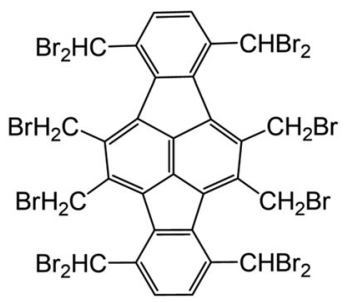
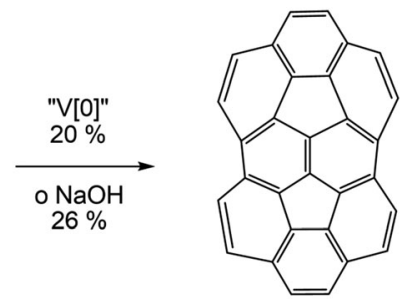

Figure 15. Scheme of the synthesis of the hemifullerene $\mathrm{C}_{30} \mathrm{H}_{12}$ (labeled 13 in Fig. 13), by Sygula and Rabidau, using the method developed by Seiders et al. Reproduced from A. Sygula and P. W. Rabideau, J. Am. Chem. Soc., 1998, 120, 12666, with permission from the American Chemical Society

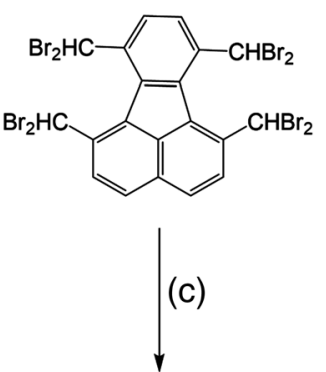<smiles>Brc1c(Br)c2ccc3c(Br)c(Br)c4ccc5ccc1c1c5c4c3c21</smiles>

(a)

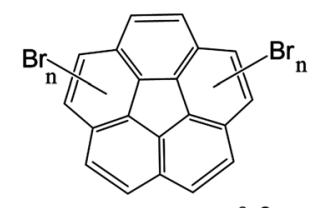

(b)

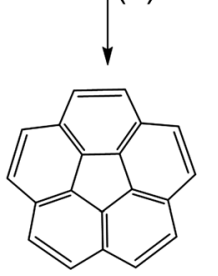

Figure 16. Synthesis of corannulene proposed by Sygula and Rabideau. Conditions: (a) acetone/water (3:1), NaOH, reflux $1 \mathrm{~h}$. (b) $n$-BuLi, THF, $-78^{\circ} \mathrm{C}$, $30 \mathrm{~min}$, diluted $\mathrm{HCl}$. The combined yield of the two steps is $50-55 \%$. (c) Dioxane/water (2.5:1), $\mathrm{NaOH}$, reflux 15 min, yield 83\%. (d) LiAlH4, DDQ, yield $30 \%$. Reproduced from A. Sygula and P. Rabideau, J. Am. Chem. Soc., 2000, 122,6323 , with permission from the American Chemical Society. THF, tetrahydrofuran; DDQ, 2,3-dichloro-5,6-dicyano-l,4-benzoquinone

even more difficult the application of the method. In 2000, Sygula y Rabideau $^{[56]}$ discovered a method to synthesize corannulene. This method, shown in Fig. 16, is based on the treatment of 1,6,7,10tetrakis(dibromomethyl)fluorantene with sodium hydroxide and the subsequent reduction to obtain corannulene with a combined yield of $55 \%$. In the first step, $\mathrm{NaOH}$ favors the deprotonation of the dibromomethyl group, leading to a bromocarbanion. Then, a viable option is the formation of a bromocarbene from the carbanion that could be inserted into the $\mathrm{CBr}$ bond of the adjacent dibromomethyl group, followed by $\mathrm{HBr}$ elimination. The bromine<smiles>CC(=O)c1c(CBr)c(C(C)=O)c2c(c1CBr)-c1c(CBr)ccc3ccc(CBr)c-2c13</smiles>
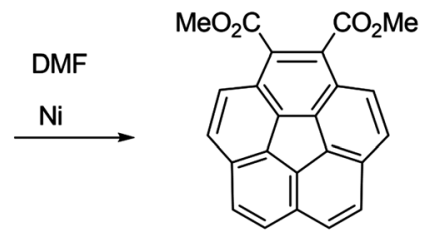

$(60 \%)$

Figure 17. Synthesis of 1,2-corannulene dicarboxylate proposed by Sygula and co-workers. Reproduced from A. Sygula, S. D. Karlen, R. Sygula, and P. W. Rabideau, Organic Letters, 2002, 4, 3135, with permission from the American Chemical Society. DMF, dimethylformamide

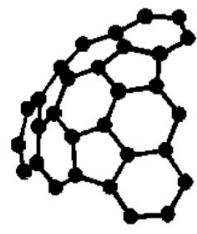

14

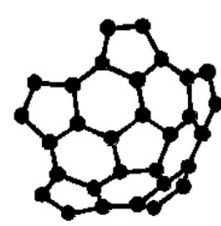

34

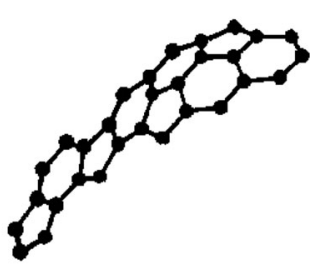

37

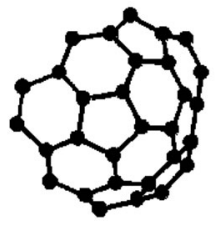

32

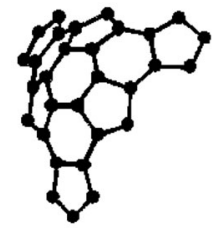

35

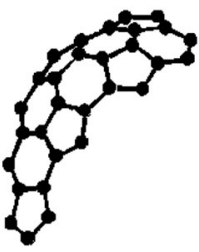

38

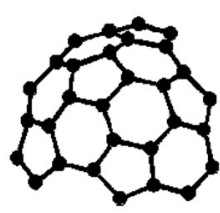

33

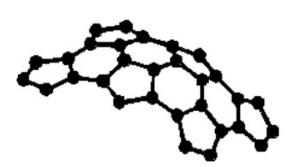

36

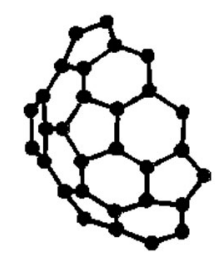

39
Figure 18. Structures of the isometric hemifullerenes found by Geneste and co-workers. Reproduced from F. Geneste, A. Moradpour, G. Dive, D. Peeters, J. Malthête, and J. F. Sadoc, J. Org. Chem., 2002, 67, 605, with permission from the American Chemical Society

atoms in the tetrabromocorannulene formed could be replaced by methyl groups or trimethylsilylacetylene that will allow the formation of new structures. Later, Sygula and co-workers proposed another method to synthesize 1,2-corannulene dicarboxylate (Fig. 17) that employed $\mathrm{Ni}$ in an intermolecular reductive coupling. ${ }^{[57]}$ The major advantage of this method is that it provides an easy way to obtain substituted corannulenes by the substitution of the carbomethoxy groups.

On the basis of the idea of the dimerization of two isometric $C_{30}$ fragments to obtain $C_{60}$, Geneste et al. ${ }^{[58]}$ investigated all the isometric fragments derived from $C_{60}$ by using the method known as "la coupe du roi" (a segmentation method to divide an apple in two chiral halves), used by Anet and co-workers ${ }^{[59]}$ in the study of the fragmentation and recombination of different molecules. Geneste and co-workers found the nine isometric 
structures (Fig. 18), which include a heterochiral fragment (labeled 14), four homochiral (32-35), and four achiral (36-39) segments. They calculated the stabilization energy and the curvature of the fragments and determined that Structure $\mathbf{1 4}$ is the most stable hemifullerene, followed by Structure 34, which is also the most curved hemifullerene. However, they do not propose a way to fuse those fragments to form the $\mathrm{C}_{60}$ molecule. We have not found further work reporting a method to achieve the dimerization.

In 2002, Scott and co-workers ${ }^{[60]}$ reported the first rational synthesis of $C_{60}$. They synthesized $C_{60}$ from the pyrolysis of $\mathrm{C}_{60} \mathrm{H}_{27} \mathrm{Cl}_{3}$ (Fig. 19). The pyrolysis of the chlorinated precursors at $1100^{\circ} \mathrm{C}$ induces the carbon-carbon bond formation shown by the arrows in Fig. 19 mediated by the formation and rearrangement of aryl radicals (* in Fig. 19) promoted by the presence of chlorine atoms in the molecule. The stitching of the arms of the molecule leads specifically to the formation of $\mathrm{C}_{60}$; no other fullerenes can be formed in this process. However, the yield was lower than $1 \%$ because the reactive molecule was decomposed by the harsh reaction conditions, which led Scott and co-workers to suggest that different radical promoters might improve the yields. Kabdulov et al. proposed the use of fluorine as a radical promoter ${ }^{[61]}$ on the same $\mathrm{C}_{60} \mathrm{H}_{30}$ precursor, besides chlorine or bromine. The proposed hexa-fluorinated and nona-fluorinated precursors are shown in Fig. 20. In the
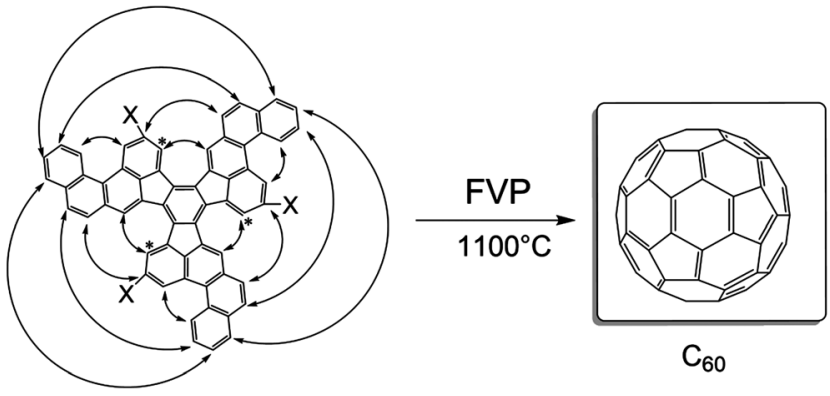

$\mathrm{X}=\mathrm{H}, \mathrm{Cl}$

Figure 19. Final step in the synthesis of $C_{60}$. Curved lines indicate where the new bonds are formed. Reproduced from L. T. Scott, M. M. Boorum, B. J. McMahon, S. Hagen, J. Mack, J. Blank, H. Wegner, and A. de Meijere, Science, 2002, 295, 1500, with permission from the American Association for the Advancement of Science. FVP, flash vacuum pyrolysis

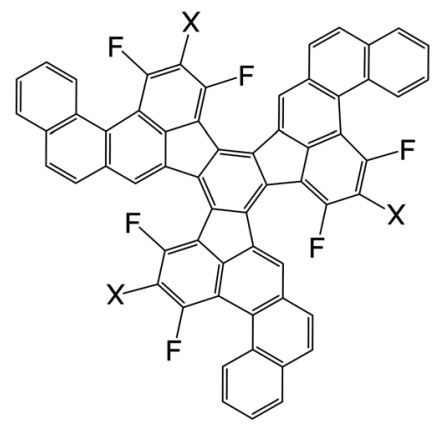

$\mathrm{X}=\mathrm{H}, \mathrm{F}$

Figure 20. Hexa-fluorinated (XH) and nona-fluorinated (XF) precursors proposed by Kabdulov et al. The radical promoters are fluorine atoms. Reproduced from M. A. Kabdulov, K. Y. Amsharov, and M. Jansen, Tetrahedron, 2010, 66, 8587, with permission from Elsevier hexa-fluorinated precursor $(\mathrm{XH})$, the fluorine atoms located on the bay regions of the molecule are eliminated to introduce a curvature that favors the subsequent thermal dehydrogenation that leads to $C_{60}$, and in the nona-fluorinated molecule (XF), the loss of the six first fluorine atoms leads to new bay regions where an HF elimination is possible. Fluorine should improve the reaction yields, because the addition of this atom to the precursor molecule does not increase substantially the molecular weight: a fact that enables the sublimation of the molecule. Moreover, the CF bond is relatively stable and thus reducing the decomposition of the precursor. Also, because of its size, the $\mathrm{F}$ atom can be introduced in the most sterically impeded regions.

In 2008, Otero and co-workers ${ }^{[62]}$ proposed the use of Pt surfaces to catalyze the dehydrogenation of the planar $\mathrm{C}_{60} \mathrm{H}_{30}$ precursor and thus improve the yield of the reactions proposed by

(a)
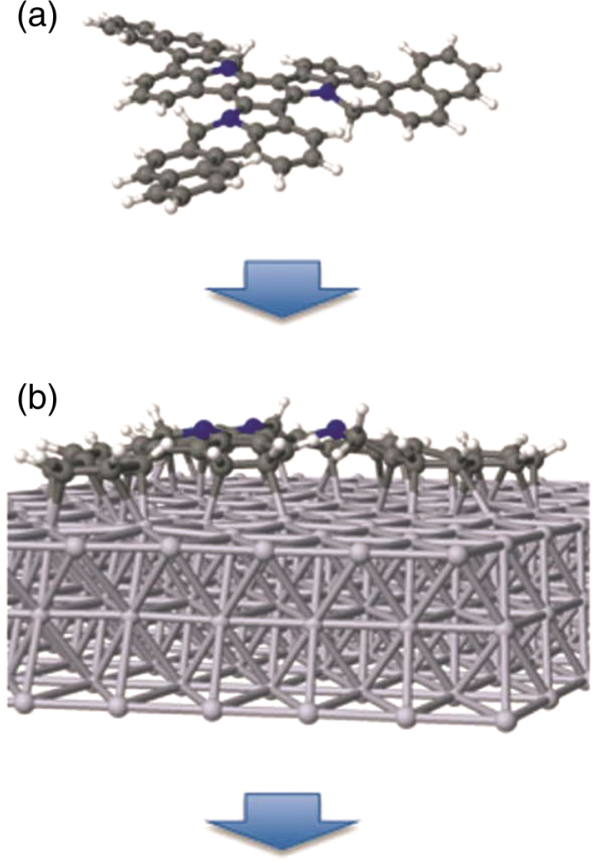

(c)

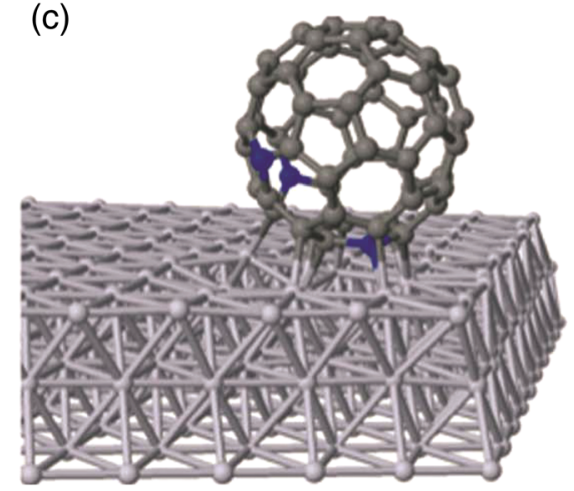

Figure 21. Formation of the closed $\mathrm{C}_{57} \mathrm{~N}_{3}$ triazofullerene, starting with the planar $\mathrm{C}_{57} \mathrm{~N}_{3} \mathrm{H}_{33}$ precursor molecule, which is first adsorbed at $300 \mathrm{~K}$ on the platinum (111) surface and then heated at $750 \mathrm{~K}$. Blue balls represent the nitrogen atoms in the structure. Reproduced from G. Otero, G. Biddau, C. Sánchez-Sánchez, R. Caillard, M. F. López, C. Rogero, F. J. Palomares, N. Cabello, M. A. Basanta, J. Ortega, J. Méndez, A. M. Echavarren, R. Pérez, B. Gómez-Lor, and J. A. Martín-Gago, Nature, 2008, 454, 865, with permission from Nature Publishing Group 
Scott and co-workers. Pt favors the dehydrogenation of the precursor molecule, allowing the lowering of the reaction temperature $(750 \mathrm{~K})$. Moreover, the interaction of the precursor molecule with the Pt surface fixes the precursor molecule onto the surface, eliminating the geometric and collision factors of the reaction. All those features make it possible to obtain yields close to $100 \%$. Fig. 21 shows the process for $\mathrm{C}_{57} \mathrm{H}_{33} \mathrm{~N}_{3}$, which produces the closed $\mathrm{C}_{57} \mathrm{~N}_{3}$ triazafullerene by heating at $750 \mathrm{~K}$. In addition, from calculations using the "nudge elastic band" method, ${ }^{[63]}$ Otero et al. proposed a general pathway for the cyclization of $\mathrm{C}_{60} \mathrm{H}_{30}$ (Fig. 22). The process starts with the cyclization of the center bonds, which produces curvature that approaches the molecule arms, favoring the subsequent formation of the other bonds. Soon after this work, Amsharov et al. ${ }^{[64]}$ studied the dehydrogenation reaction of a series of substituted benzophenantrenes to form 2,13-benzofluoranthene. This reaction was considered a prototype reaction in the formation of fullerenes. Amsharov et al. proposed a series of pathways based on free radicals and probed different radical promoters, as shown in Fig. 23. They used $\mathrm{Cl}(\mathbf{4 0 a})$ and $\mathrm{F}(\mathbf{4 0 d})$ atoms as radical promoters, as well as the homolitic cleavage of a $\mathrm{CH}$ bond from the methyl (40b) and phenyl (40c) groups. The rearrangement of the radicals formed, leading to the desired benzofluoranthene (41), is shown in that figure. They also proposed two routes not mediated by radicals. In the first route, the elimination of HF from $40 \mathrm{~d}$ leads to benzynes (49 and 50) that evolve to benzofluoranthene (41) and benzopyrene (43). In the second, a 1,5-elimination of HF from 40e leads directly to benzofluoranthene (41). Experimental data for the reactions showed in Fig. 23 indicate that the reaction of 40 to 41 is the thermodynamically and kinetically favored, instead of the reactions based on radical rearrangement. The synthesis of 2,13-benzofluoranthene gives a guideline for the analysis of the last step on the fullerene synthesis, allowing to propose and understand new methods of synthesis.

\section{OTHER PROPOSED METHODS}

Using the symmetry properties of $\mathrm{C}_{60}$, Yasuda ${ }^{[65]}$ proposed the synthesis of tetrahedral precursors formed by benzene rings and acetylene rods, which are converted to $C_{60}$ by intramolecular multimember ring formation reactions. The synthesis of such highly reactive precursors is shown in Fig. 24, where two different tetrahedral precursors are obtained from successive coupling reactions of halogenated benzenes and acetylene derivatives. These tetrahedral analogs have the same symmetry of $\mathrm{C}_{60}$ and all the carbon atoms needed to form it; they also could be substituted with heteroatoms, leading to heterofullerenes such as $\mathrm{C}_{48} \mathrm{~N}_{12}$. The high reactivity of the precursors arising from the halogen atoms in the benzene molecules and the acetylene derivatives is the base of the success of this method.

Rubin et al. ${ }^{[6]]}$ and Tobe et al. ${ }^{[67]}$ independently proposed that the use of carbon-rich acetylenic spherical macrocycles (Fig. 25) should lead to $C_{60}$ by a polyyne cyclization mechanism. The advantage of this method is that it only involves bond-forming reactions, avoiding costly bond-breaking and bond-rearrangement processes that are thought to take place during the formation of $\mathrm{C}_{60}$ from evaporated carbon. ${ }^{[68]}$ However, even if some fullerene ions have been identified in the laser desorption mass spectrum of those precursors, the attempts to obtain $\mathrm{C}_{60}$ have been unsuccessful. This indicates that reorganization of the precursors is needed. ${ }^{[69]}$

In a theoretical study, Viñes and Görling ${ }^{[70]}$ have analyzed the possibility of synthesizing fullerenes from simple precursors such as methane and ethylene by using supported metallic nanoparticles as templates. In this case, the formation of fullerenes is induced by the specific structure and the electronic properties of the template nanoparticles, which act, in addition, as catalysts during the process. The conditions that have to be met for a successful fullerene synthesis are (i) the precursor molecules have to be fully dehydrogenated by the metal nanoparticles; (ii) the carbon atoms and the intermediate carbon compounds have to remain at the metal nanoparticle; and (iii) the formation of fullerenes must be energetically favorable compared with that of other carbon structures, in particular graphene fragments. The particular case studied by Viñes and Görling was the synthesis of $C_{60}$ on Pt nanoparticles. Ethylene on Pt (111) single crystals dehydrogenates at temperatures above $400 \mathrm{~K} .^{[71]}$ Nanometric Pt nanoparticles dehydrogenate methane at temperatures between 460 and $500 \mathrm{~K}^{\left[{ }^{[72]} \text { However, }\right.}$ molecular beam experiments combined with density functional calculations $^{[73]}$ indicate that dehydrogenation of methane at the edges and corners of small Pt nanoparticles can occur at lower temperatures because of the small energy barriers. The second condition is fulfilled when the metallic nanoparticles are supported on basic $(\mathrm{MgO}$ and $\mathrm{BaO})$ or inert $\left(\mathrm{Al}_{2} \mathrm{O}_{3}\right)$ oxides.

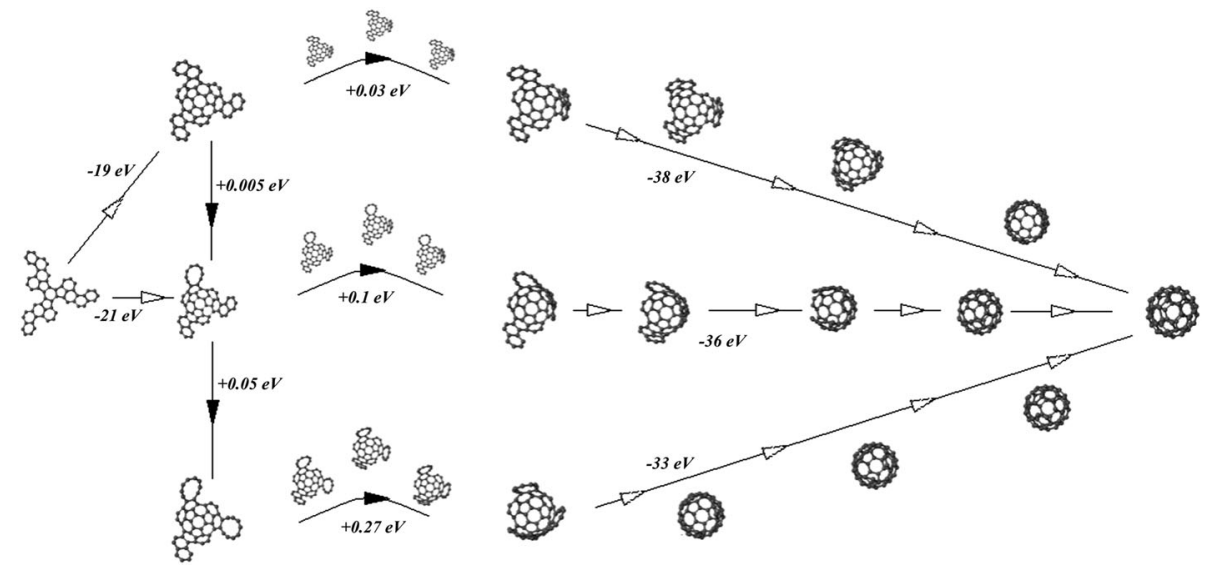

Figure 22. Key steps to the formation of a fullerene cage, following complete dehydrogenation of $\mathrm{C}_{60} \mathrm{H}_{30}$. Reproduced from G. Otero, G. Biddau, C. Sánchez-Sánchez, R. Caillard, M. F. López, C. Rogero, F. J. Palomares, N. Cabello, M. A. Basanta, J. Ortega, J. Méndez, A. M. Echavarren, R. Pérez, B. Gómez-Lor, and J. A. Martín-Gago, Nature, 2008, 454, 865, supplementary information, with permission from Nature Publishing Group 


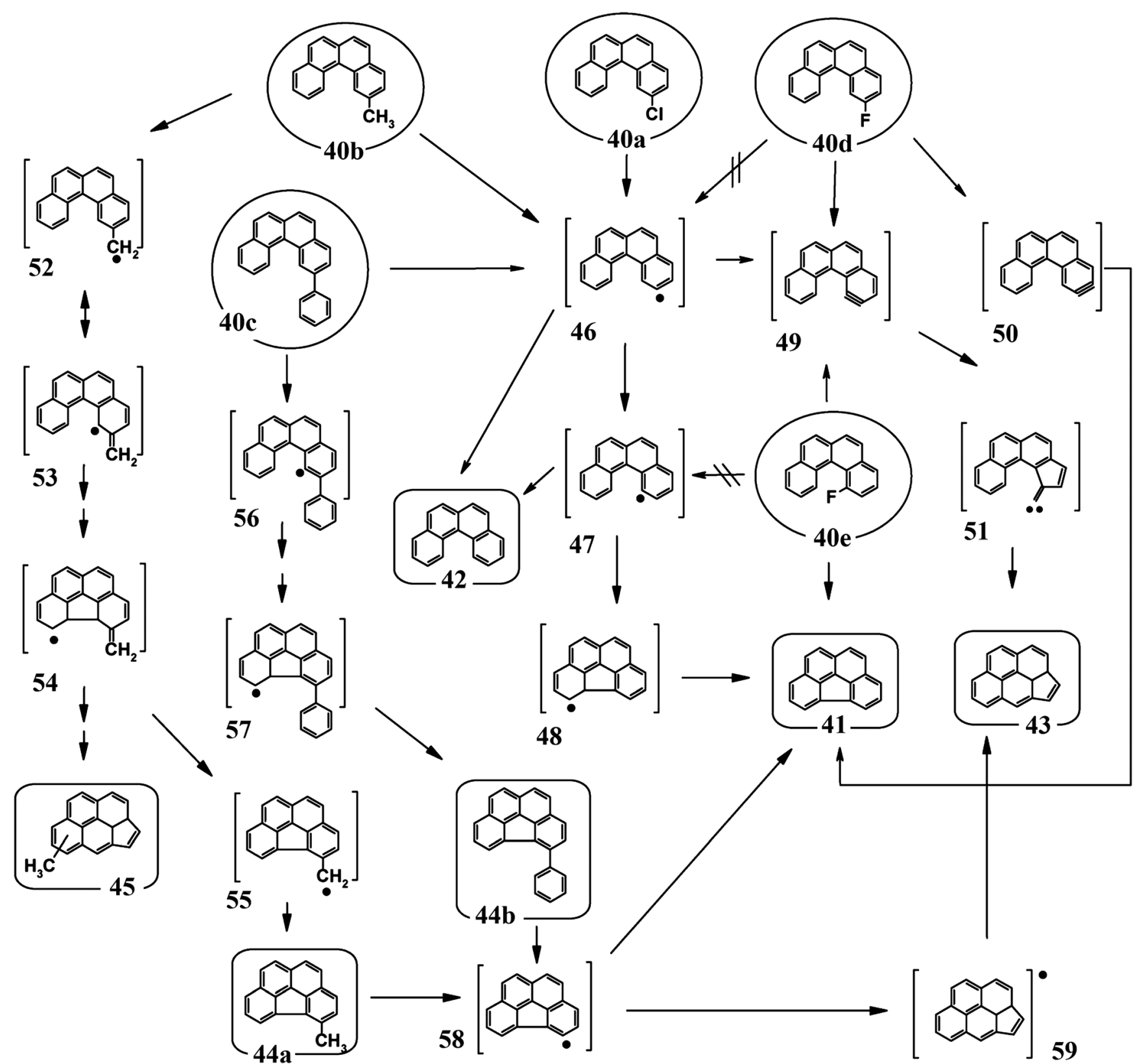

Figure 23. Proposed mechanisms for the condensation of substituted benzophenantrenes into benzofluoranthene under flash-vacuum pyrolysis. Reproduced from K. Y. Amsharov, M. A. Kabdulov, and M. Jansen, Eur. J. Org. Chem., 2009, 6328, with permission from Wiley-VCH

With respect to the third condition, the calculations of Viñes and Görling have shown that the cohesive energy per atom of $C_{60}$ is higher than those of isolated graphene flakes with less than 200 atoms; that is, $\mathrm{C}_{60}$ is thermodynamically more stable than those flakes. To study the formation of fullerenes on Pt nanoparticles, they optimized various carbon structures on $\mathrm{Pt}_{79}$, including disperse carbon atoms, graphene-like and fullerene-like structures, and the results are shown in Fig. 26. For low coverage, $n<13$, the carbon atoms are dispersed over the particle facets. For $13<n$ 24, graphene-like structures are preferred, although fullerene-like structures are close in energy, and for $n>30$, the carbon atoms prefer to adopt fullerene-like structures. It is expected that the formation of fullerenes can occur at reaction temperatures lower than those in other methods because the carbon atoms can diffuse on the surface of the nanoparticle at the temperatures of the dehydrogenation reactions.
On the basis of the fact that $\mathrm{C}_{60}$, like most organic compounds, readily converts to $\mathrm{CO}_{2}$ under oxidative conditions, Chen and Lou ${ }^{[74]}$ synthesized $C_{60}$ by the inverse reaction, the reduction of $\mathrm{CO}_{2}$ with metallic lithium at $700{ }^{\circ} \mathrm{C}$ and $100 \mathrm{MPa}$, obtaining a yield of $0.2 \%$ (Fig. 27). These authors demonstrated that the inverse conversion of $\mathrm{CO}_{2}$ to $\mathrm{C}_{60}$ depends on the temperature and pressure. This method is, however, not a cheap source of $\mathrm{C}_{60}$. Other temperature and pressure ranges lead to different products such as diamond, carbon nanotubes, carbon nanospheres, or amorphous carbon. Chen and Lou also suggest that single-carbon radicals such as the $\mathrm{CO}_{2}{ }^{\circ}$ radical anion, carbenes, or methyl radicals are involved as intermediates in the formation of $\mathrm{C}_{60}$ by this method. On the other hand, Chuvilin et $a{ }^{\left[{ }^{[75]}\right.}$ proposed the formation of $\mathrm{C}_{60}$ from graphene under transmission electron microscopy (TEM) conditions. They studied graphene sheets by TEM and observed that while exposed to an 


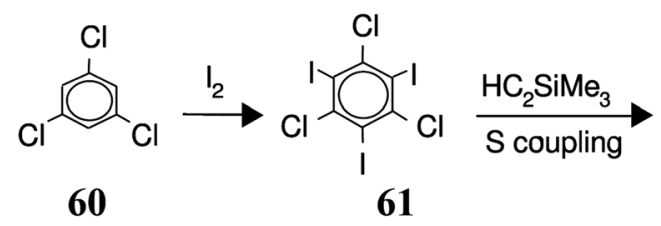

60 61

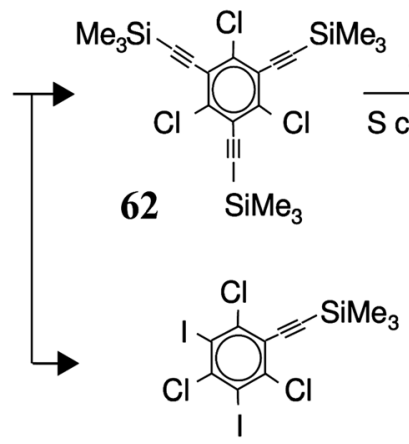

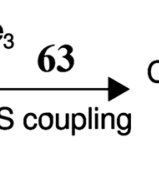

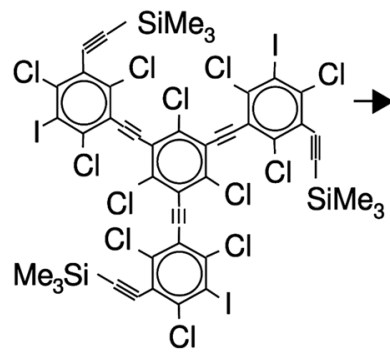

64

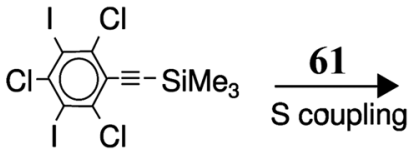

63

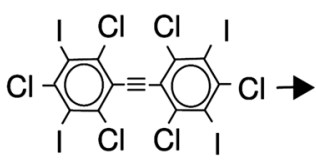

66

63

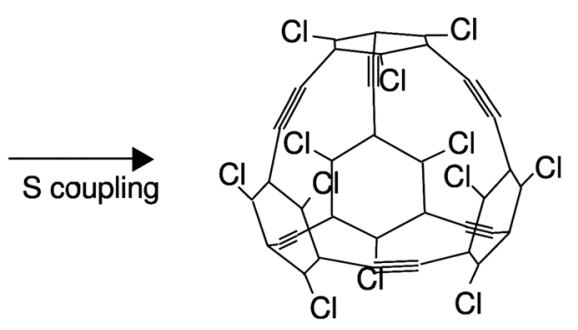

(a)

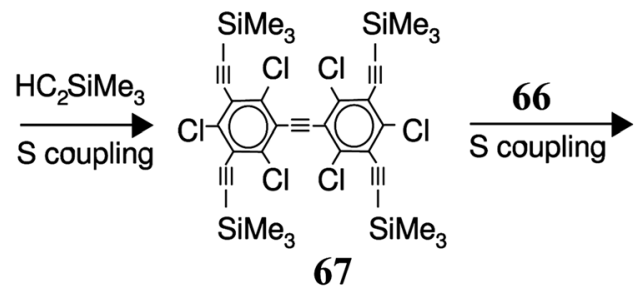

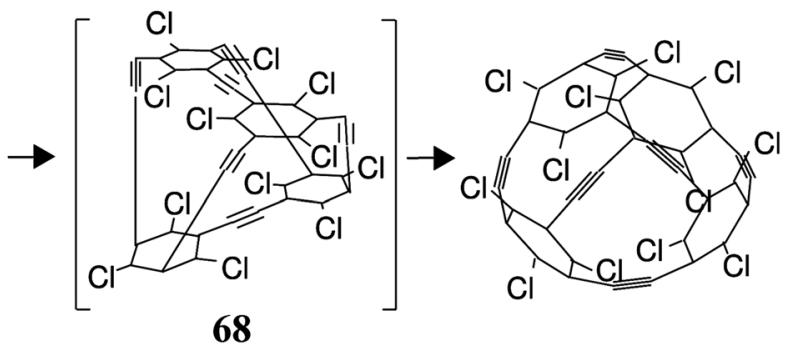

69

Figure 24. Synthesis of two tetrahedron analogs by successive Sonogashira coupling (intramolecular and intermolecular couplings) of halogenated benzene and ethyne derivatives. (a) Synthesis of tetrahedral precursor $\mathbf{6 5}$ by intramolecular coupling of 1,3,5-tri(phenylethynyl)benzene (64). (b) Synthesis of tetrahedral precursor 69 by intermolecular coupling between 1,2-diphenylethyne derivatives (67). Reproduced from A. Yasuda, Carbon, 2005, 43, 889, with permission from Elsevier
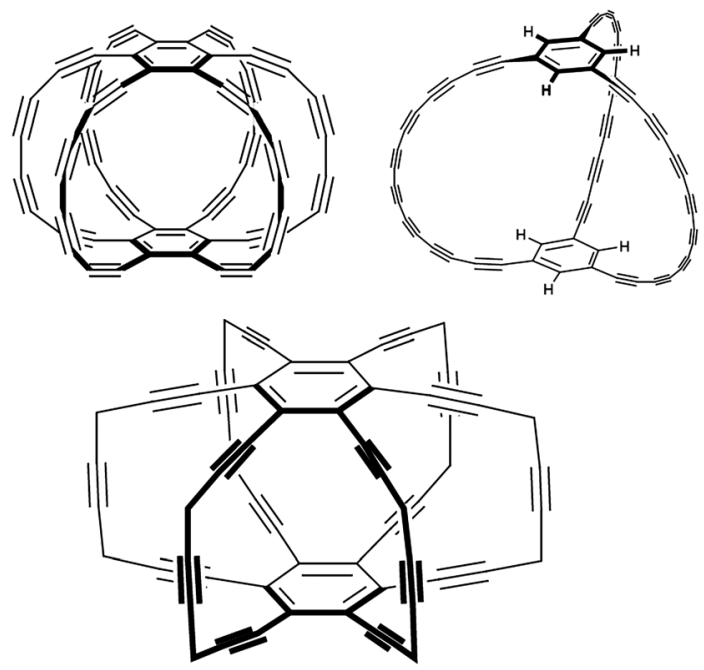

Figure 25. Carbon-rich acetylenic spherical macrocycles proposed by Rubin et al. and Tobe et al. as intermediates in the fullerene synthesis by polyyne cyclization

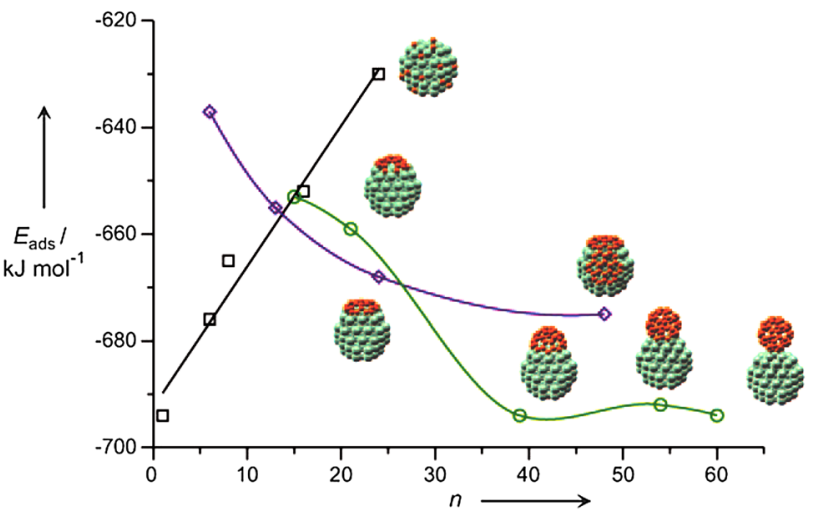

Figure 26. Carbon structures on a $\mathrm{Pt}_{79}$ nanoparticle. Shown are the adsorption energies $E_{\text {ads }}$ per carbon atom as a function of the number $n$ of carbon atoms for three different carbon patterns: completely dispersed carbon atoms (squares), hexagonal graphene-like structures on the facets of the Pt particle (rhombuses), and fullerene-like structures on a given facet (circles). Orange and green spheres represent $\mathrm{C}$ and $\mathrm{Pt}$ atoms, respectively. Reproduced from F. Viñes and A. Görling, Angew. Chem. Int. Ed., 2011, 50, 4611, with permission from Wiley-VCH 


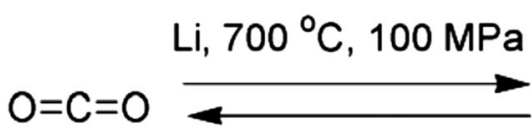

(O)

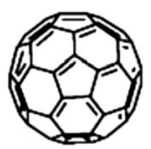

Figure 27. Formation of $\mathrm{C}_{60}$ by the reduction of $\mathrm{CO}_{2}$ with metallic lithium. Reproduced from C. Chen and Z. Lou, J. of Supercritical Fluids, 2009, 50, 42, with permission from Elsevier

electron beam of $80 \mathrm{keV}$, the edges of the graphene sheets change continuously, leading to the formation of $\mathrm{C}_{60}$. The high energy of the electron beam is transferred to the carbon atoms, producing the fragmentation of the graphene sheets in flake-like structures that finally produce fullerene molecules.

\section{CONCLUSIONS}

After the pioneering work of Scott and co-workers, ${ }^{[44,46,47]}$ it has been possible to synthesize $C_{60}$ following a chemical rationalization process. However, a general and practical procedure for the chemical synthesis of fullerenes is still to be discovered. On the basis of the variety of methods to obtain $C_{60}$ presently existing, different mechanisms that explain the formation of fullerenes in carbon soot can be proposed. In our opinion, the complete understanding of the cyclization process is a key step to rationalize the synthesis of fullerenes. Another urgent need is the study of fullerene fragments as possible precursor molecules in the ambitious synthesis by dimerization. ${ }^{[76]}$ In the words of Scott and Petrukhina ${ }^{[77]}$, "There is clearly still a long way to go before the routine synthesis of isomerically pure fullerenes - from the ground up - can be considered a solved problem."

\section{Acknowledgements}

The work of one of us, J. A. A., was supported by MEC of Spain and the European Regional Development Fund (Grant MAT2011-22781), and by Junta de Castilla y León (Grant VA158A11-2). F. M. acknowledges support from CONACyT (Grants 180523 and 163234) and a visitor fellowship from the University of Valladolid. M. M. thanks CONACyT for Scholarship 228923. We thank Prof. Hugo Jiménez (ENBC-IPN, México) for helpful discussions.

\section{REFERENCES}

[1] H. W. Kroto, J. R. Heath, S. C. O'Brien, R. R. Curl, R. E. Smalley, Nature 1985, 318, 162.

[2] M. Gallo, A. Favila, D. Glossman-Mitnik, Chem. Phys. Lett. 2007, $447,105$.

[3] K. A. Gonzalez, L. J. Wilson, W. Wu, G. H. Nancollas, Bioorg. Med. Chem. 2002, 10, 1991.

[4] A. Modi, N. Koratkar, E. Lass, B. Wei, P. M. Ajayan, Nature 2003, 424,171

[5] C. Fumelli, A. Marconi, S. Salvioli, E. Straface, W. Malorni, A. M. Offidani, R. Pellicciari, G. Schettini, A. Giannetti, D. Monti, C. Franceschi, C. Pincelli, J. Invest. Dermatol. 2000, 115(5), 835.

[6] M. Lens, Recent Pat. Biotechnol. 2011, 5, 67.

[7] S. R. Chae, M. Therezien, J. F. Budarz, L. Wessel, S. Lin, Y. Xiao, M. R. Wiesner, J. Nanopart. Res. 2011, 13, 5121.

[8] D. Ray, C. Belin, F. Hui, B. Fabre, P. Hapiot, D. M. Bassani, Chem. Commun. 2011, 47, 2547.

[9] I. Yanov, J. Leszczynski, Theoretical and Computational Chemistry, Vol. 15: Computational Materials Science (Ed: J. Leszczynski), Elsevier, Amsterdam, 2004, p. 85.

[10] J. A. Alonso, Structure and Properties of Atomic Nanoclusters, Imperial College Press, London, 2005, pp. 333-354.
[11] D. S. Sabirov, R. G. Bulakov, S. L. Khufsan, Arkivoc 2011, VIII, 200.

[12] T. G. Schmalz, W. A. Seitz, D. J. Klein, G. E. Hite, Chem. Phys. Lett. 1986, 130, 203.

[13] D. Vukicevic, M. Randic, Chem. Phys. Lett. 2005, 401, 446.

[14] M. Randic, H. W. Kroto, D. J. Vukicevic, J. Chem. Inf. Model. 2007, $487,897$.

[15] R. C. Haddon, J. Am. Chem. Soc. 1997, 119, 1797.

[16] R. C. Haddon, Science 1993, 261, 1545.

[17] T. C. Dinadayalane, J. Leszczynski, Struct. Chem. 2010, 21, 1155.

[18] W. Krätschmer, L. D. Lamb, K. Fostiropoulos, D. R. Huffman, Nature 1990, 347, 354.

[19] W. Krätschmer, K. Fostiropoulos, D. R. Huffman, Chem. Phys. Lett. 1990, 170, 167.

[20] T. J. S. Dennis, T. Kai, T. Tomiyama, T. H. Shinohara, T. Yoshida, Y. Kobayashi, H. Ishiwatari, Y. Miyake, K. Kikuchi, Y. Achiba, J. Phys. Chem. A 1999, 103, 8747.

[21] N. I. Alekseev, B. M. Filippov, I. V. Basargin, A. I. Sedov, J. Eng. Phys. Thermophys 2011, 84, 1087.

[22] A. Anctil, C. W. Babbitt, R. P. Raffaelle, B. Landi, J. Environ. Sci. Technol. 2011, 45, 2353.

[23] U. S. Energy Information Administration. http://www.eia.gov/tools/ faqs/faq.cfm?id=97\&t=3

[24] N. S. Goroff, Acc. Chem. Res. 1996, 29, 77.

[25] Q. L. Zhang, S. C. O'Brien, J. R. Heath, Y. Liu, R. F. Curl, H. W. Kroto, R. E. Smalley, J. Phys. Chem. 1986, 90, 525.

[26] R. F. Curl, R. E. Smalley, Science 1988, 242, 1017.

[27] H. W. Kroto, Science 1988, 242, 1139.

[28] H. W. Kroto, K. McKay, Nature 1988, 331, 328.

[29] S. lijima, J. Cryst. Growth 1980, 50, 675.

[30] L. B. Ebert, Science 1989, 247, 1468.

[31] G. Brinkmalm, D. Barofsky, P. Demirev, D. Feny, P. Hakansson, R. E. Johnson, C. T. Reimann, B. U. R. Sundqvist, Chem. Phys. Lett. 1992, 191, 345.

[32] J. R. Heath, Fullerenes: Synthesis, Properties and Chemistry of Large Carbon Clusters (Eds: G. S. I. Hammond, V. J. Kuck), American Chemical Society, Washington, DC, 1991, p. 1.

[33] T. Wakabayashi, Y. Achiba, Chem. Phys. Lett. 1992, 190, 465.

[34] T. Wakabayashi, H. Shiromaru, K. Kikuchi, Y. Achiba, Chem. Phys. Lett. 1993, 201, 470.

[35] J. Hunter, J. Fye, M. F. Jarrold, Science 1993, 260, 784.

[36] Y. E. Lozovik, A. M. Popov, Phys. Usp. 1997, 40, 717.

[37] S. W. McElvany, M. M. Ross, N. S. Goroff, F. Diederich, Science 1993, 259, 1594.

[38] G. von Helden, N. G. Gotts, M. T. Bowers, Nature 1993, 363, 60.

[39] J. Hunter, J. Fye, M. F. Jarrold, J. Phys. Chem. 1993, 97, 3460.

[40] C. Yeretzian, K. Hansen, F. Diederich, R. L. Whetten, Nature 1992, 359, 44.

[41] A. M. Askhabov, Phys. Solid State 2005, 47, 1186.

[42] A. M. Askhabov, Zap. Vseross. Mineral. O-va. 2004, 133, 108.

[43] S. Irle, G. Zheng, Z. Wang, K. Morokuma, J. Phys. Chem. B 2006, $110,14531$.

[44] L. T. Scott, Angew. Chem. Int. Ed. 2004, 43, 4994.

[45] W. E. Barth, R. G. Lawton, J. Am. Chem. Soc. 1966, 88, 380.

[46] L. T. Scott, M. M. Hasheim, D. T. Meyer, H. B. Warren, J. Am. Chem. Soc. 1991, 113, 7082.

[47] L. T. Scott, P. Ch. Cheng, M. M. Hasheim, M. S. Bratcher, D. T. Meyer, H. B. Warren, J. Am. Chem. Soc. 1997, 119, 10963.

[48] L. T. Scott, Polycyclic Aromat. Compd. 2010, 30, 247.

[49] L. T. Scott, M. S. Bratcher, S. Hagen, J. Am. Chem. Soc. 1996, 118, 8743.

[50] M. J. Plater, J. Chem. Soc. Perkin Trans. 1 1997, 19, 2897.

[51] M. J. Plater, J. Chem. Soc. Perkin Trans. 1 1997, 19, 2903.

[52] R. B. M. Ansems, L. T. Scott, J. Am. Chem. Soc. 2000, 122, 2719.

[53] V. M. Tsefrikas, L. T. Scott, Chem. Rev. 2006, 106, 4868.

[54] T. J. Seiders, K. K. Baldridge, J. S. Siegel, J. Am. Chem. Soc. 1996, $118,2754$.

[55] A. Sygula, P. W. Rabideau, J. Am. Chem. Soc. 1998, 120, 12666.

[56] A. Sygula, P. Rabideau, J. Am. Chem. Soc. 2000, 122, 6323.

[57] A. Sygula, S. D. Karlen, R. Sygula, P. W. Rabideau, Org. Lett. 2002, 4, 3135.

[58] F. Geneste, A. Moradpour, G. Dive, D. Peeters, J. Malthête, J. F. Sadoc, J. Org. Chem. 2002, 67, 605.

[59] F. A. L. Anet, S. S. Miura, J. Siegel, K. Mislow, J. Am. Chem. Soc. 1983, 105,1419

[60] L. T. Scott, M. M. Boorum, B. J. McMahon, S. Hagen, J. Mack, J. Blank, H. Wegner, A. de Meijere, Science 2002, 295, 1500. 
[61] M. A. Kabdulov, K. Y. Amsharov, M. Jansen, Tetrahedron 2010, $66,8587$.

[62] G. Otero, G. Biddau, C. Sánchez-Sánchez, R. Caillard, M. F. López, C. Rogero, F. J. Palomares, N. Cabello, M. A. Basanta, J. Ortega, J. Méndez, A. M. Echavarren, R. Pérez, B. Gómez-Lor, J. A. Martín-Gago, Nature 2008, 454, 865.

[63] G. Henkelman, B. P. Ubesuaga, H. J. Jonsson, J. Chem. Phys. 2000, $113,9901$.

[64] K. Y. Amsharov, M. A. Kabdulov, M. Jansen, Eur. J. Org. Chem. 2009; 6328.

[65] A. Yasuda, Carbon 2005, 43, 889.

[66] Y. Rubin, T. C. Parker, S. J. Pastor, S. Jalisatgi, C. Boulle, C. C. Wilkins, Angew. Chem. Int. Ed. 1998, 37, 1226.

[67] Y. Tobe, N. Nakagawa, K. Naemura, T. Wakabayashi, T. Shida, Y. Achiba, J. Am. Chem. Soc. 1998, 120, 4544.

[68] Y. Rubin, Chem. Eur. J. 1997, 3, 1009.
[69] U. H. F. Bunz, Y. Rubin, Y. Tobe, Chem. Soc. Rev. 1999, 28, 107.

[70] F. Viñes, A. Görling, Angew. Chem. Int. Ed. 2011, 50, 4611.

[71] J. R. Creighton, J. M. White, Surf. Sci. 1983, 129, 327.

[72] C. Papp, B. Tränkenschuh, R. Streber, T. Fuhrmann, R. Denecke, H. P. Steinrück, J. Phys. Chem. C 2007, 111, 2177.

[73] F. Viñes, Y. Lykhach, T. Staudt, M. P. A. Lorenz, C. Papp, H. P. Steinrück, J. Libuda, K. M. Neyman, A. G örling, Chem. Eur. J. 2010, 16, 6530 .

[74] C. Chen, Z. Lou, J. Supercrit. Fluids 2009, 50, 42.

[75] A. Chuvilin, U. Kaiser, E. Bichoutskaia, N. A. Besley, A. N. Khlobystov, Nat. Chem. 2010, 2, 450.

[76] M. Mojica, F. Méndez, J. A. Alonso, Molecules 2013, 18, 2243.

[77] L. T. Scott, M. A. Petrukhina, Preface in Fragments of Fullerenes and Carbon Nanotubes: Designed Synthesis, Unusual Reactions and Coordination Chemistry, (Eds: L. T. Scott, M. A. Petrukhina), John Wiley \& Sons, Inc, Hoboken, New Jersey, 2012, pp. vii-x. 


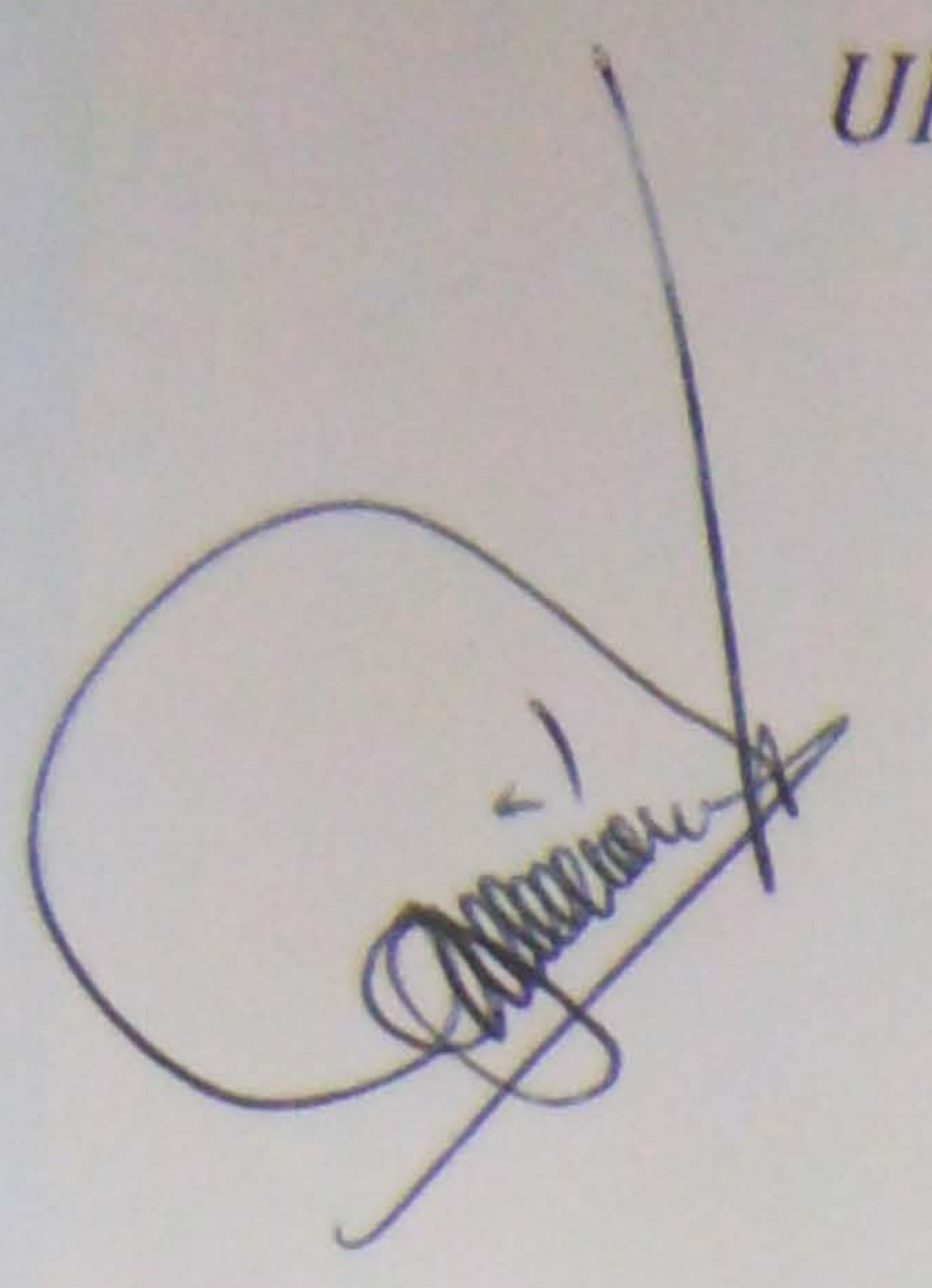

\author{
POSGRADO EN QUIMICA
}

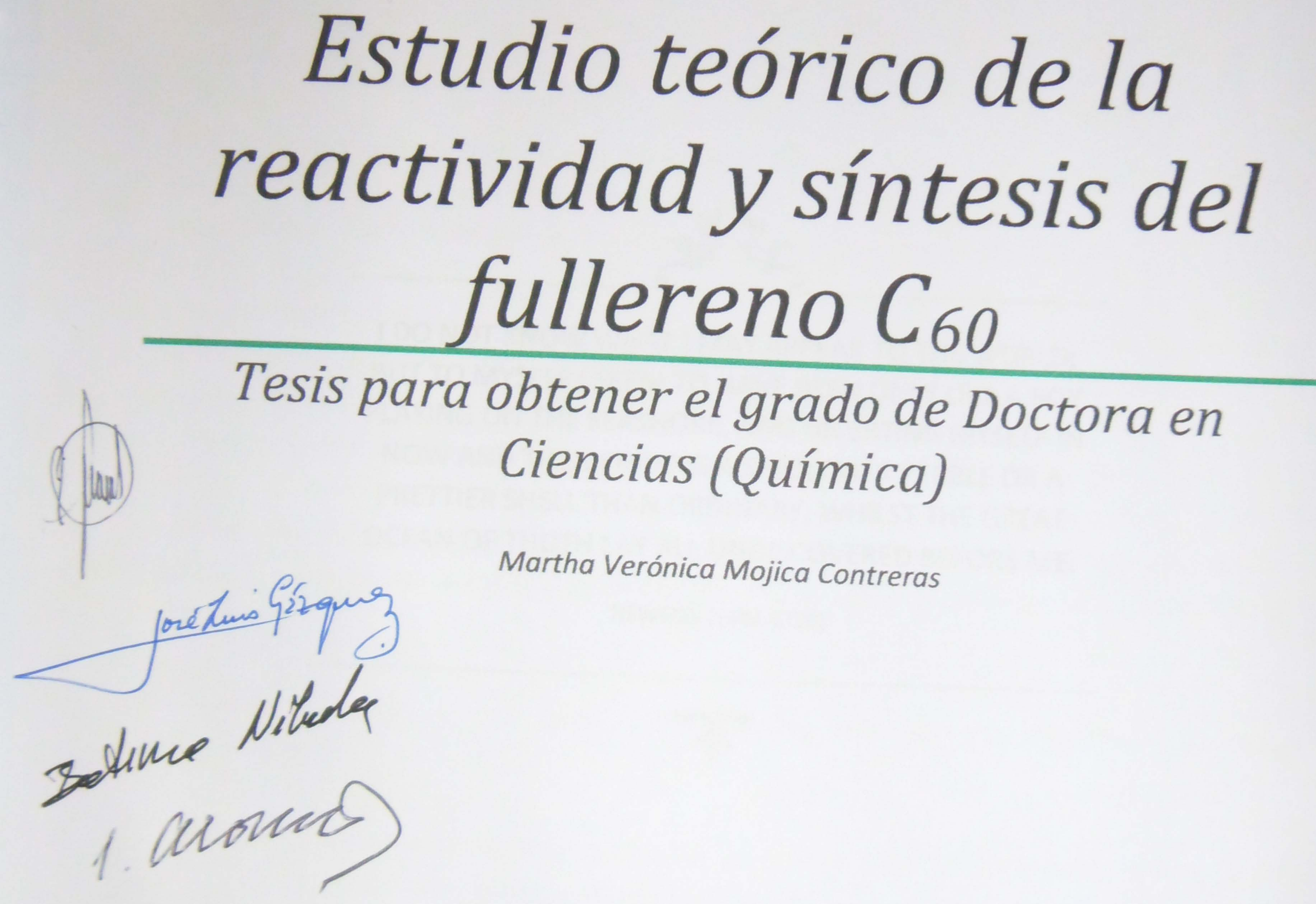

Francisco Méndez Ruiz

Profesor Titular C

Universidad Autónoma Metropolitana Iztapalapa

Julio Alfonso Alonso Martín

Catedrático de Universidad

Universidad de Valladolid 